\title{
Community-Facility Survey Questionnaire for the Indonesia Family Life Survey, Wave 5
}

JOHN STRAUSS, FIRMAN WITOELAR AND BONDAN SIKOKI

RAND Labor \& Population

WR-1143/4-NIA/NICHD

March 2016

RAND working papers are intended to share researchers' latest findings and to solicit informal peer review. They have been approved for circulation by RAND Labor and Population but have not been formally edited or peer reviewed. Unless otherwise indicated, working papers can be quoted and cited without permission of the author, provided the source is clearly referred to as a working paper. RAND's publications do not necessarily reflect the opinions of its research clients and sponsors. RAND® is a registered trademark. 
For more information on this publication, visit www.rand.org/pubs/working_papers/WR1143z4.html

Published by the RAND Corporation, Santa Monica, Calif.

(C) Copyright 2016 RAND Corporation

RAND $^{\star}$ is a registered trademark

\section{Limited Print and Electronic Distribution Rights}

This document and trademark(s) contained herein are protected by law. This representation of RAND intellectual property is provided for noncommercial use only. Unauthorized posting of this publication online is prohibited. Permission is given to duplicate this document for personal use only, as long as it is unaltered and complete. Permission is required from RAND to reproduce, or reuse in another form, any of its research documents for commercial use. For information on reprint and linking permissions, please visit www.rand.org/pubs/permissions.html.

The RAND Corporation is a research organization that develops solutions to public policy challenges to help make communities throughout the world safer and more secure, healthier and more prosperous. RAND is nonprofit, nonpartisan, and committed to the public interest.

RAND's publications do not necessarily reflect the opinions of its research clients and sponsors.

Support RAND

Make a tax-deductible charitable contribution at

www.rand.org/giving/contribute

www.rand.org 


\section{INDONESIAN FAMILY LIFE SURVEY 2014 \\ COMMUNITY AND FACILITY \\ PEOPLE CHARACTERISTICS BOOK IA}

SECTIONS : LK, IR, A, B, C, D, E, F, CP 
SECTION LK : CONTROL SHEET

\begin{tabular}{||c|c||}
\hline \multicolumn{1}{|c|}{ SAMPLING INFORMATION } & CODE \\
\hline LK01. Province__ & \\
\hline LK02. Kabupaten/Kota & \\
\hline LK03. Kecamatan & \\
\hline LK04. Village/Urban Township /Nagari & \\
\hline LK05. Region: 1. Urban 2. Rural & \\
\hline
\end{tabular}

\begin{tabular}{||c|c||}
\hline \multicolumn{1}{|c|}{ SUPERVISION: } & CODE \\
\hline LK15. Name of Interviewer : & \\
\hline LK17. Name of Local Supervisor: & \\
\hline LK19. Name of Field Coordinator: & \\
\hline
\end{tabular}

\section{VILLAGE/TOWNSHIP ADDRESS}

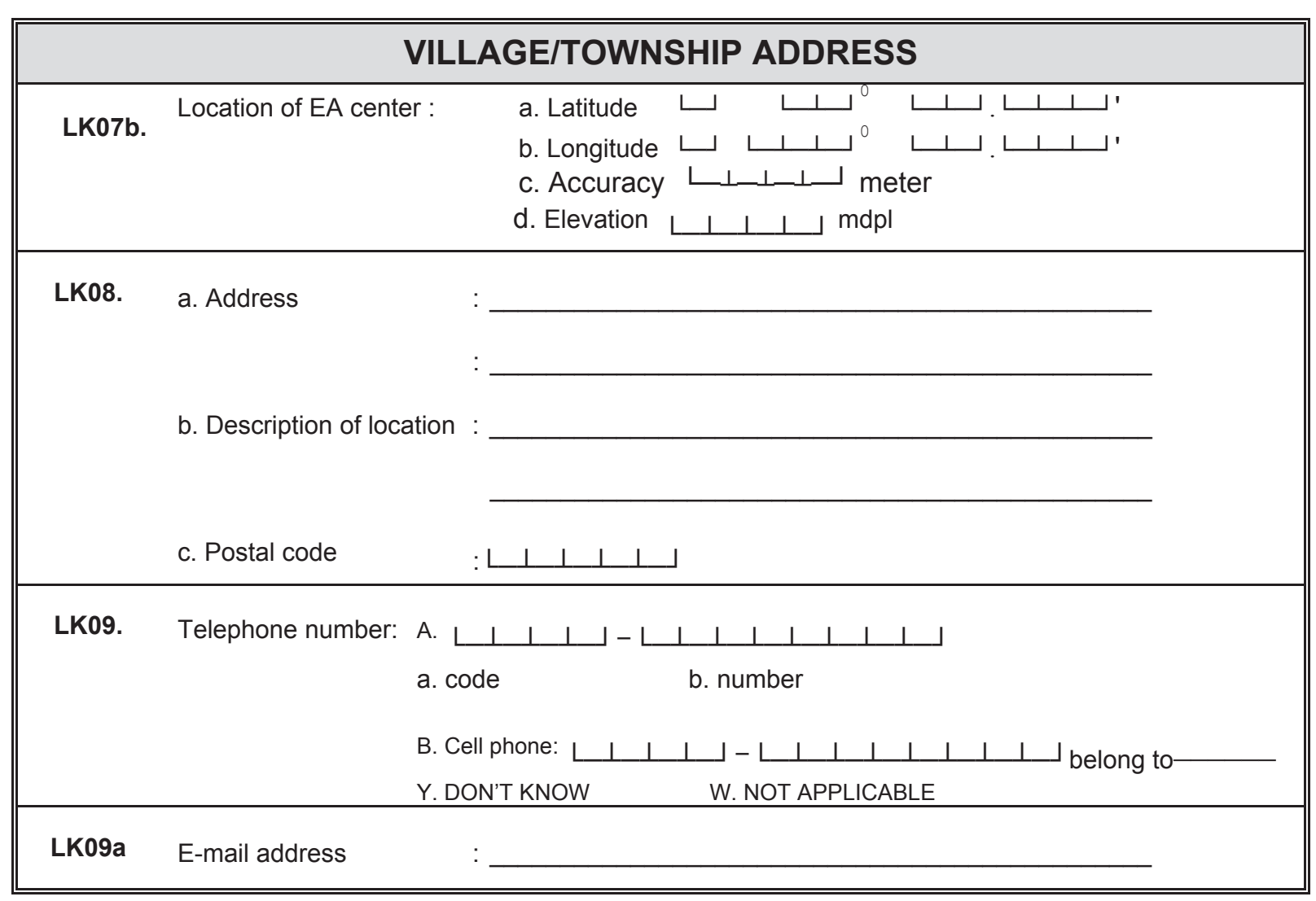

\begin{tabular}{|l|l||}
\hline LK19x. CAPI CHECK: IS THIS A TWIN EA? & $\begin{array}{l}\text { 1. Yes } \rightarrow \text { CK1 } \\
\text { 3. No }\end{array}$ \\
\hline
\end{tabular}




\section{SECTION IR : IDENTITY OF RESPONDENT}

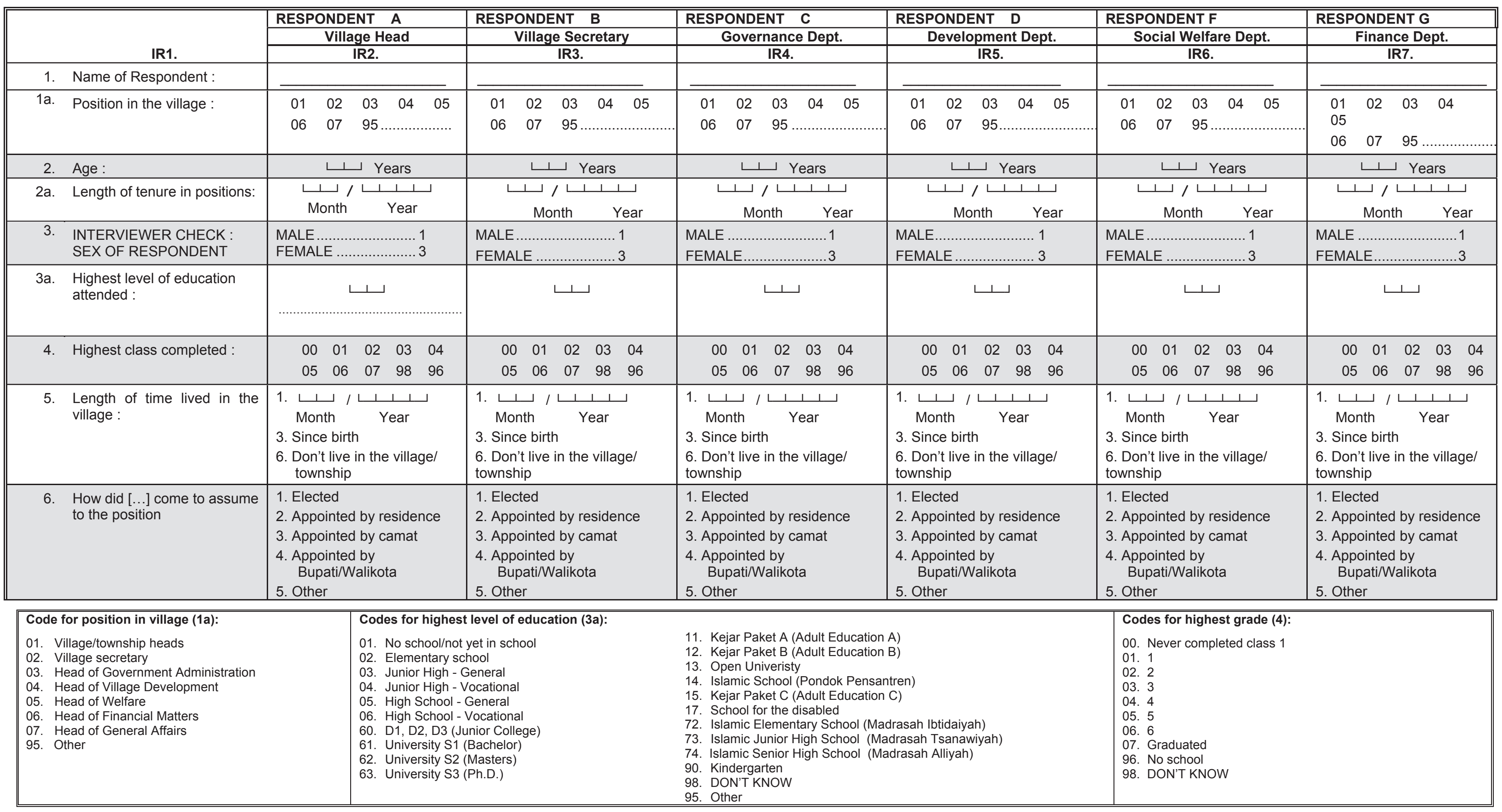




\section{SECTION IR : IDENTITY OF RESPONDENT}

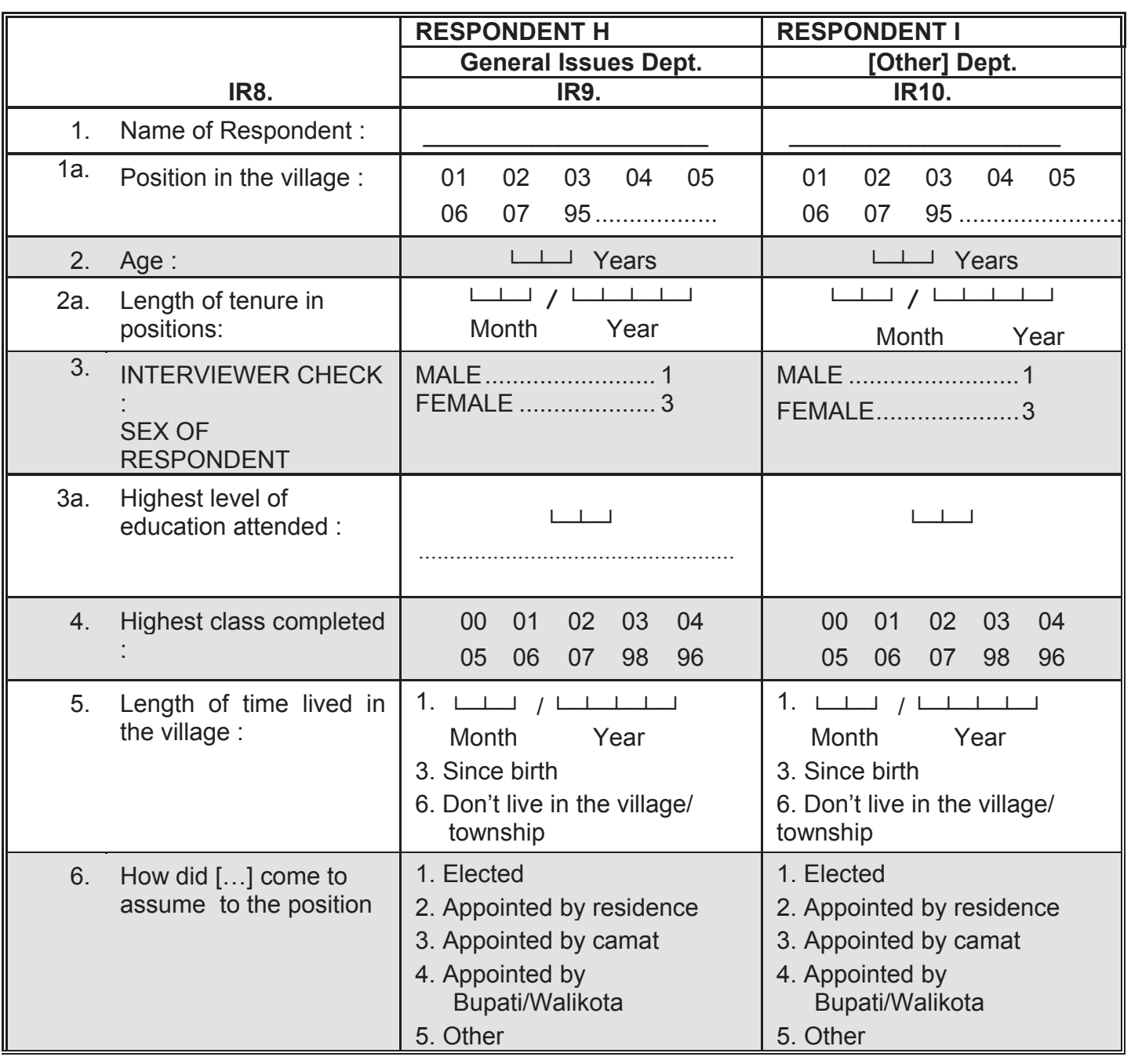

\begin{tabular}{|l|l|l|}
\hline Code for position in village (1a): & Codes for highest level of education (3a): & 11. Kejar Paket A (Adult Education A) \\
08. Village/township heads & 01. No school/not yet in school & 12. Kejar Paket B (Adult Education B) \\
09. Village secretary & 02. Elementary school & 13. Open Univeristy \\
010. Head of Government Administration & 03. Junior High - General & 14. Islamic School (Pondok Pensantren) \\
011. Head of Village Development & 04. Junior High - Vocational & 15. Kejar Paket C (Adult Education C) \\
012. Head of Welfare & 05. High School - General & 17. School for the disabled \\
013. Head of Financial Matters & 06. High School - Vocational & 72. Islamic Elementary School (Madrasah Ibtidaiyah) \\
014. Head of General Affairs & 60. D1, D2, D3 (Junior College) & 73. Islamic Junior High School (Madrasah Tsanawiyah) \\
95. Other & 61. University S1 (Bachelor) & 74. Islamic Senior High School (Madrasah Alliyah) \\
& 62. University S2 (Masters) & 90. Kindergarten \\
& 63. University S3 (Ph.D.) & 98. DON'T KNOW \\
& & 95. Other
\end{tabular}

Codes for highest grade (4)

00. Never completed class 1

01. 1

3. 3

Islamic Schol (Pondok Pensantren)

5. Kejar Paket C (Adult Education C)

72. Islamic Elementary School (Madrasah Ibtidaiyah)

mic Junior High School (Madrasah Tsanawiyah)

Kindergarten

05. 5

77. Graduated

8. DON'T KNOW 


\section{SECTION A : TRANSPORTATION AND INFRASTRUCTURE}

Now, we would like to ask about the transportation facilities in this village.

\begin{tabular}{|c|c|}
\hline IR1 & IR2 \\
\hline $\begin{array}{l}\text { 1_A. Name of Respondent: } \\
\text { CAPI WILL SKIP TO B1 IF THE SAME NAME HAS } \\
\text { ALREADY BEEN ENTERED EARLIER } \\
\end{array}$ & \\
\hline Position in the village : & $\begin{array}{ccccc}01 & 02 & 03 & 04 & 05 \\
06 & 07 & 95 & \ldots \ldots \ldots \ldots \ldots . .\end{array}$ \\
\hline 2_A. & $\downarrow$ Years \\
\hline Length of tenure in positions: & 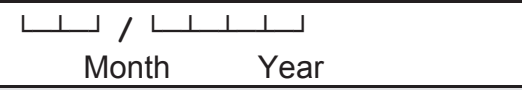 \\
\hline $\begin{array}{ll}\text { 3_A. INTERVIEWER CHECK: } & \text { INEX OF RESPONDENT } \\
& \text { SEX REOER }\end{array}$ & 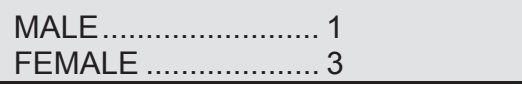 \\
\hline Highest level of education attended: & $\sqcup$ \\
\hline 4_A. Highest class completed: & $\begin{array}{lllll}00 & 01 & 02 & 03 & 04 \\
05 & 06 & 07 & 98 & 96 \\
\end{array}$ \\
\hline Length of time lived in the village : & 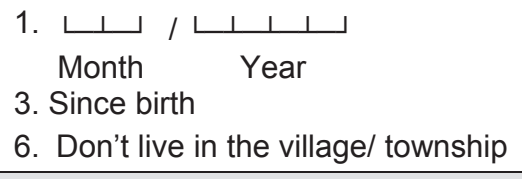 \\
\hline 6_A. How did [...] come to assume to the position & $\begin{array}{l}\text { 1. Elected } \\
\text { 2. Appointed by residence } \\
\text { 3. Appointed by camat } \\
\text { 4. Appointed by Bupati/Walikota } \\
\text { 5. Other }\end{array}$ \\
\hline
\end{tabular}

\section{Code for position in village (1a_A):}

01. Village/township heads

02. Village secretary

ernment Administration

. Head of Village Development

06. Head of Financial Matters

07. Head of General Affairs

95. Other

\section{Codes for highest level of education (3a_A)}

01. No school/not yet in school

2. Elementary school

04. Junior High - Vecat

05. High School - General

06. High School - Vocational

60. D1, D2, D3 (Junior College)

61. University S1 (Bachelor)

62. University S2 (Masters)
63. University S3 (Ph.D.)
11. Kejar Paket $A$ (Adult Education $A$ )

2. Kejar Paket $B$ (Adult Education $B$ )

14. Islamic School (Pondok Pensantren)

15. Kejar Paket C (Adult Education C)

72.

73. Islamic Junior High School (Madrasah Tsanawiya

98. DON'T KNOW

98. DON'T
95. Other
Codes for highest grade (4_A):

00. Never completed class 1

01. 1

03. 3

04. 4

06. 6

07. Graduated

98. DON'T KNOW 
SECTION A : TRANSPORTATION AND INFRASTRUCTURE

INTERVIEWER NOTE: THE OFFICE OF THE HEAD OF VILLAGE/TOWNSHIP IS THE REFERENCE POINT CONCERNING DISTANCE.

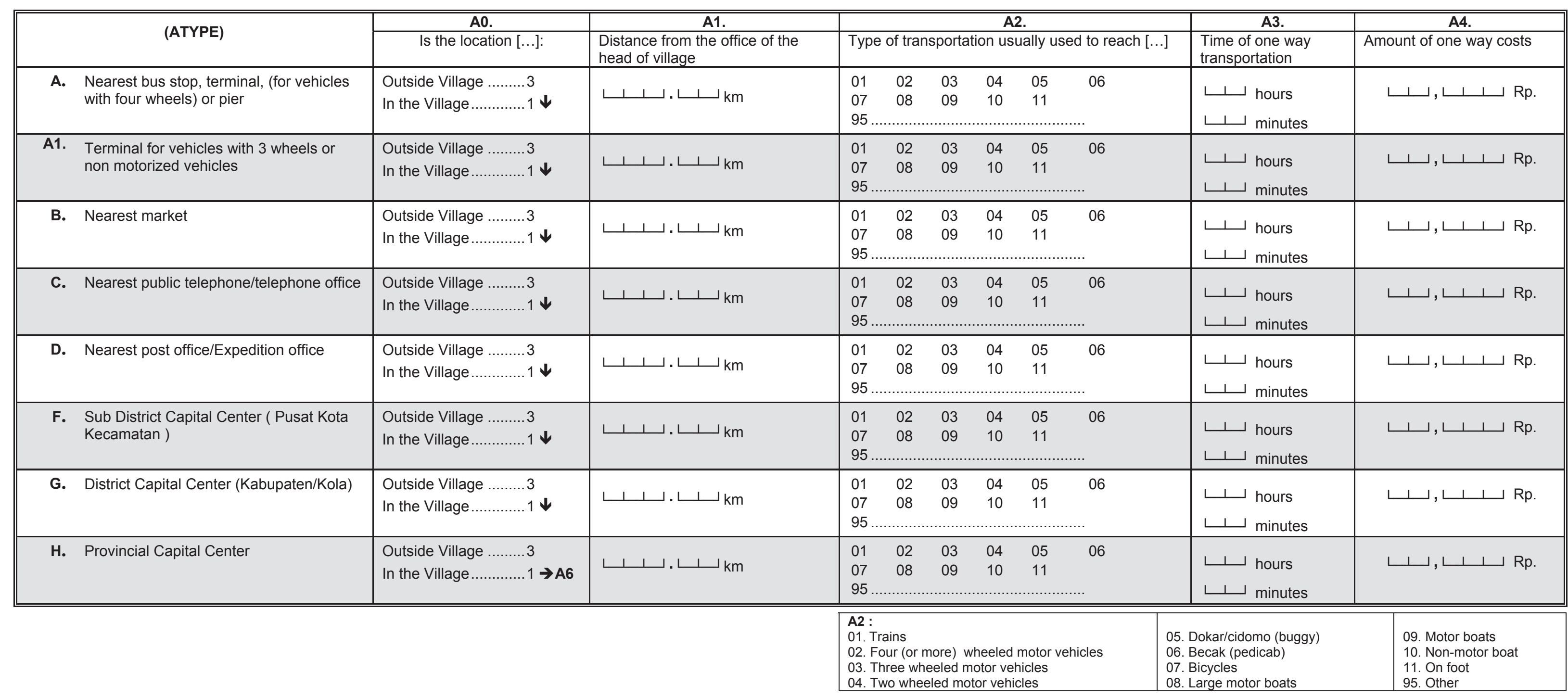




\section{SECTION A : TRANSPORTATION AND INFRASTRUCTURE}

\begin{tabular}{|c|c|c|}
\hline A6. & $\begin{array}{l}\text { Is there any public three wheeled } \\
\text { or four wheeled or motor boat } \\
\text { service in this village? }\end{array}$ & 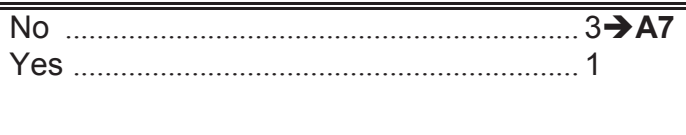 \\
\hline A6a. & $\begin{array}{l}\text { In what year did the first public } \\
\text { motor vehicle service start } \\
\text { operating? }\end{array}$ & 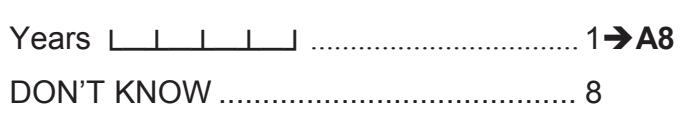 \\
\hline A6b. & $\begin{array}{l}\text { For how many years has this } \\
\text { service been operating? }\end{array}$ & DON'T KNOW \\
\hline A7. & $\begin{array}{l}\text { In what year did the nearest public } \\
\text { transportation service to this village } \\
\text { start to operate? }\end{array}$ & 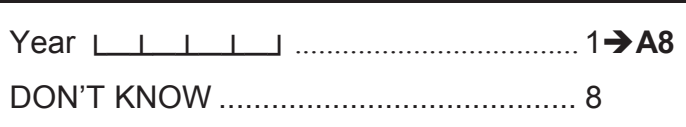 \\
\hline A7a. & $\begin{array}{l}\text { For how many years has this } \\
\text { service been operating? }\end{array}$ & 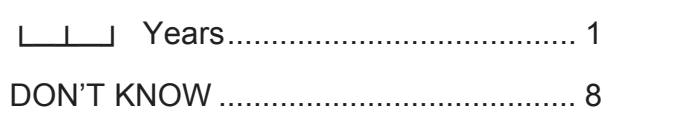 \\
\hline A8. & $\begin{array}{l}\text { What is the predominant type of } \\
\text { road in this village? }\end{array}$ & 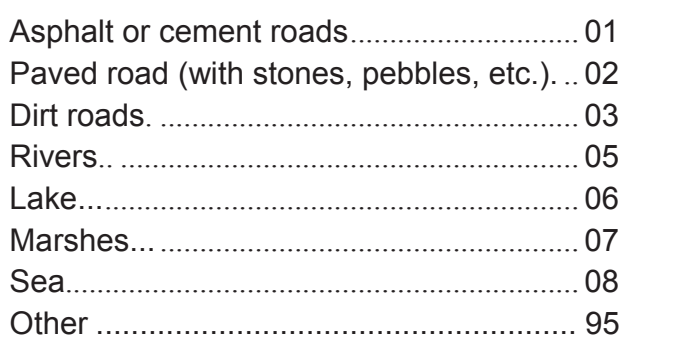 \\
\hline
\end{tabular}

\begin{tabular}{|c|}
\hline 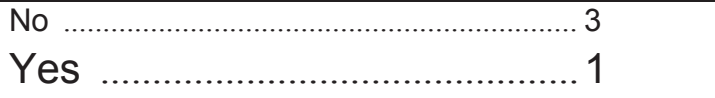 \\
\hline 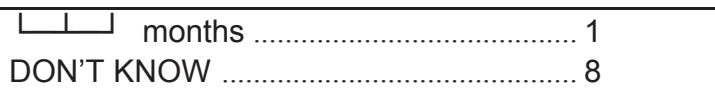 \\
\hline 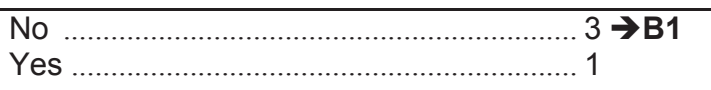 \\
\hline 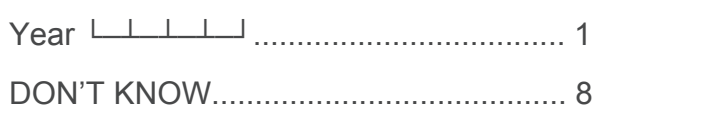 \\
\hline 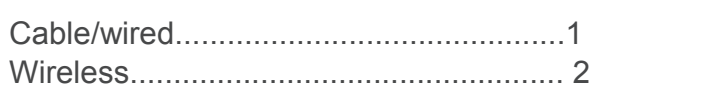 \\
\hline $\begin{array}{l}\text { A. Sending and receiving emails } \\
\text { B. Sending and receiving documents/attachments } \\
\text { C. Browsing/searching (e.g. news portal, Google) } \\
\text { D. E-government } \\
\text { E. Video/streaming (e.g. Youtube) } \\
\text { V. Other }\end{array}$ \\
\hline
\end{tabular}




\section{SECTION B : AVAILABILITY OF ELECTRICITY}

Section B Respondent:

\begin{tabular}{|c|c|c|}
\hline & IR1 & IR2 \\
\hline & $\begin{array}{l}\text { Name of Respondent: } \\
\text { CAPI WILL SKIP TO B1 IF THE SAME NAME HAS } \\
\text { ALREADY BEEN ENTERED EARLIER }\end{array}$ & \\
\hline $1 a_{-} B$ & Position in the village : & $\begin{array}{ccccc}01 & 02 & 03 & 04 & 05 \\
06 & 07 & 95 & \ldots \ldots \ldots \ldots \ldots . .\end{array}$ \\
\hline 2_B. & Age : & $\sqcup$ Years \\
\hline 2a_B & Length of tenure in positions: & 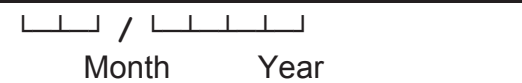 \\
\hline 3_B. & $\begin{array}{l}\text { INTERVIEWER CHECK : } \\
\text { SEX OF RESPONDENT }\end{array}$ & $\begin{array}{l}\text { MALE } \ldots \ldots \ldots \ldots \ldots \ldots \ldots \ldots . . . .1 \\
\text { FEMALE …………..... } 3\end{array}$ \\
\hline 3a_B & Highest level of education attended: & 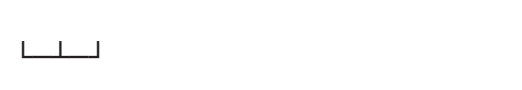 \\
\hline 4_B. & Highest class completed : & $\begin{array}{lllll}00 & 01 & 02 & 03 & 04 \\
05 & 06 & 07 & 98 & 96 \\
\end{array}$ \\
\hline 5_B. & Length of time lived in the village : & 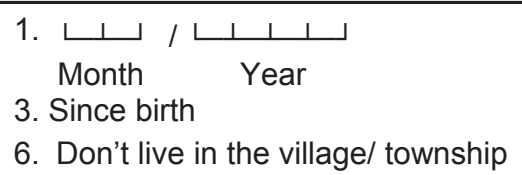 \\
\hline 6_B. & How did $[\ldots]$ come to assume to the position & $\begin{array}{l}\text { 1. Elected } \\
\text { 2. Appointed by residence } \\
\text { 3. Appointed by camat } \\
\text { 4. Appointed by Bupati/Walikota } \\
\text { 5. Other }\end{array}$ \\
\hline
\end{tabular}

Code for position in village (1a_B)

08. Village/township heads

09. Village secretary

ment Administration

011. Head of Village Development

012. Head of Welfare

013. Head of Financial Matters

95. Other
11. Kejar Paket $\mathrm{A}$ (Adult Education $\mathrm{A}$ )

2. Kejar Paket B (Adult Education B)

14. Islamic School (Pondok Pensantren)

15. Kejar Paket C (Adult Education C)

72. Islamic Elementary School (Madrasah Ibtidaiyah)

74. Islamic Junior High School (Madrasah Tsanawiyah)

98. DON'T KNOW

95. Other
Codes for highest grade (4_B):

00. Never completed class 1

02.2

03.3

04.4

05. 5

06. 6

07. Graduated

98. DON'T KNOW 


\section{SECTION B : AVAILABILITY OF ELECTRICITY}

Now, we would like to ask you about the availability of electricity in this village.

\begin{tabular}{|c|c|}
\hline B1. Is there any electricity available in this village? & 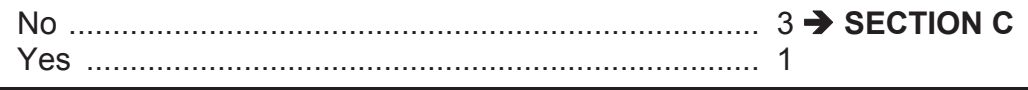 \\
\hline B1a. What percent of the households in this village use electricity? & 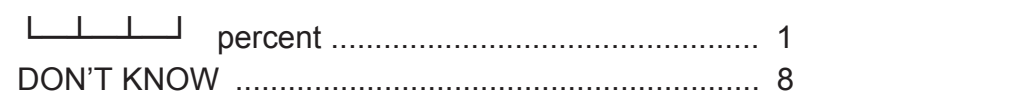 \\
\hline
\end{tabular}

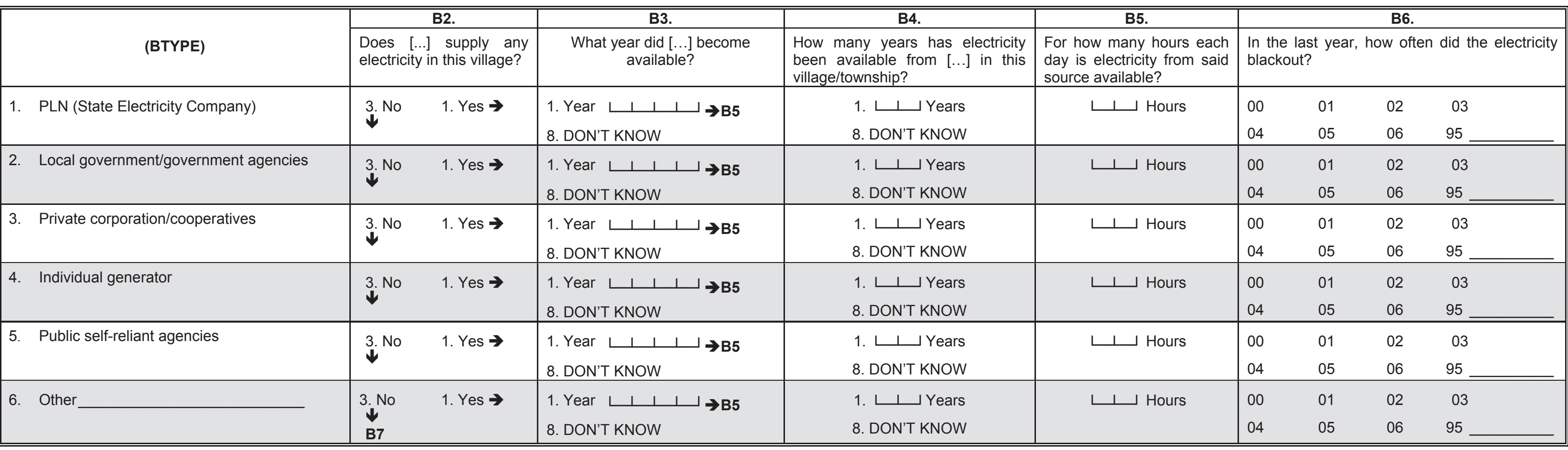

B6. :

00. Never

01. Everyday, at least once a day

02. Every week at least once a week

03. Every two weeks, at least once every two weeks

04. Every month, at least once a month

05. In the rainy season

06. Less than once a month

95. Other 


\section{SECTION B : AVAILABILITY OF ELECTRICITY}

B7. Of the above electricity sources, please mention the one main source:

Individual Generators.

$04 \rightarrow B 8$

PLN (State Electricity Company)

01

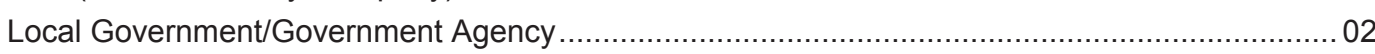

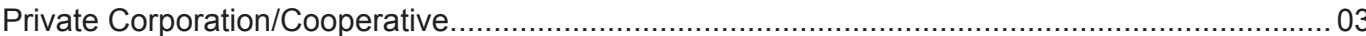

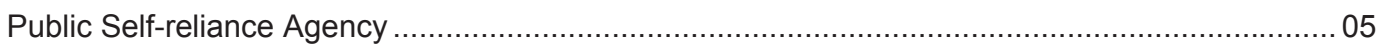

B7a. Generally, what quantity of electricity is available for household in this village?

Other

. .95

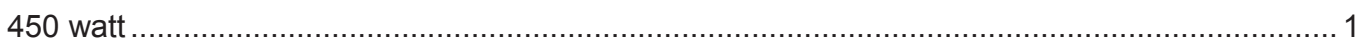

900 watt $\ldots \ldots \ldots \ldots \ldots \ldots \ldots \ldots \ldots \ldots . . .2$

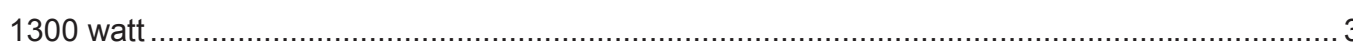

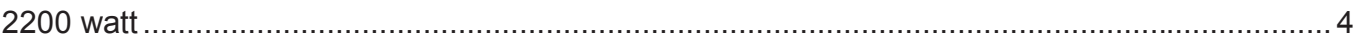

Other

B8. In comparison to 2007 has electricity in this village been [...]?

Much more available

Somewhat more available

About the same

Somewhat less available

Much less available

B8a. In comparison to 2007 how often does outage occur?

More often .......

Same.

Less often .......

NOT APPLICABLE

(a)


SECTION C : WATER SOURCE AND SANITATION

Section C Respondent:

\begin{tabular}{|c|c|c|}
\hline 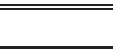 & 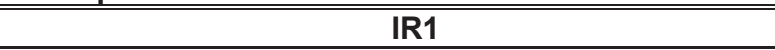 & IR2 \\
\hline $1+C$. & $\begin{array}{l}\text { Name of Respondent: } \\
\text { CAPI WILL SKIP TO C1 IF THE SAME NAME HAS } \\
\text { ALREADY BEEN ENTERED EARLIER }\end{array}$ & \\
\hline 1a_c. & Position in the village : & $\begin{array}{ccccc}01 & 02 & 03 & 04 & 05 \\
06 & 07 & 95 & & \ldots \ldots \ldots \ldots . \\
\end{array}$ \\
\hline 2_C. & Age : & $\perp$ Years \\
\hline 2a_C. & Length of tenure in positions: & 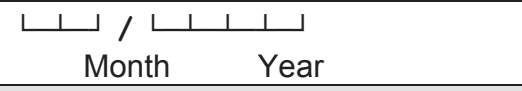 \\
\hline 3_C. & $\begin{array}{l}\text { INTERVIEWER CHECK : } \\
\text { SEX OF RESPONDENT }\end{array}$ & $\begin{array}{l}\text { MALE } \ldots \ldots \ldots \ldots \ldots \ldots . . . .1 \\
\text { FEMALE .................... } 3\end{array}$ \\
\hline 3a_c. & Highest level of education attended: & $\sqcup$ \\
\hline 4_C. & Highest class completed & $\begin{array}{lllll}00 & 01 & 02 & 03 & 04 \\
05 & 06 & 07 & 98 & 96 \\
\end{array}$ \\
\hline 5_C. & Length of time lived in the village: & 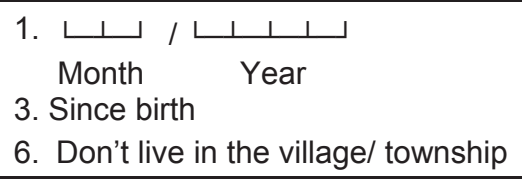 \\
\hline 6_C. & How did $[\ldots]$ come to assume to the position & $\begin{array}{l}\text { 1. Elected } \\
\text { 2. Appointed by residence } \\
\text { 3. Appointed by camat } \\
\text { 4. Appointed by Bupati/Walikota } \\
\text { 5. Other }\end{array}$ \\
\hline
\end{tabular}

\section{Code for position in village (1a_C)}

01. Village/township heads

02. Village secretary

03. Head of Government Administ
04. Head of Village Development

05. Head of Welfare

07. Head of Financial Matters

95. Other

\section{Codes for highest level of education (3a_C)}
01. No school/not yet in school
02. Elementary school
03. Junior High - General
04 . Junior High - Vocational
04. Junior High - Vocationa
05. High School - General
60. D1 D2 D3 (Junior College)
61. University $S 1$ (Bachelor)
62. University S2 (Masters)
63. University S3 (Ph.D.)

11. Kejar Paket A (Adult Education $A$ )

12. Kjar Paket B (Adult Education B)

14. Islamic School (Pondok Pensantren)

15. Kejar Paket C (Adult Education C)

17. School for the disabled

72. Islamic Elementary School (Madrasah Ibtidaiyah)

73. Islamic Junior High School (Madrasah Tsanawiyah)

74. Islamic Senior High School (Madrasah Alliyah)

90. Kindergarten

98. DON'T KNOW

\section{Codes for highest grade (4_C)}

00 . Never completed class 1

01. 1

03. 3

04. 4

06. 6

07. Graduated

6. No school

9. DON'T KNOW 


\section{SECTION C : WATER SOURCE AND SANITATION}

We want to ask about water resources and sanitation in this village/township.

\begin{tabular}{|c|c|c|c|c|}
\hline $\mathrm{Co.}$ & \multicolumn{2}{|c|}{ C1. } & C2. & C4. \\
\hline WATER SOURCE & \multicolumn{2}{|c|}{$\begin{array}{l}\text { Where does the village population get } \\
\text { water from }[\ldots] \text { for drinking and } \\
\text { cooking? }\end{array}$} & $\begin{array}{l}\text { Among the aforementioned water sources for drinking } \\
\text { and cooking sources, which is the predominant source? }\end{array}$ & $\begin{array}{l}\text { Among the aforementioned water sources for bathing and } \\
\text { washing water source, which is the predominant source? }\end{array}$ \\
\hline 1a. PAM piped water directly to the house & 1. Yes & 3. No & $1 a$ & $1 \mathrm{a}$ \\
\hline 1b. PAM piped water from a public hydrant/stand pipe & 1. Yes & 3. No & $1 \mathrm{~b}$ & $1 b$ \\
\hline 1c. PAM piped water purchased from a vendor & 1. Yes & 3. No & $1 \mathrm{c}$ & 1c \\
\hline 2. Pump water (electrical/manual) & 1. Yes & 3. No & 2 & 2 \\
\hline 3. Well water & 1. Yes & 3. No & 3 & 3 \\
\hline 4. Spring water & 1. Yes & 3. No & 4 & 4 \\
\hline 5. Rain water & 1. Yes & 3. No & 5 & 5 \\
\hline 6. River water & 1. Yes & 3. No & 6 & 6 \\
\hline 7. Lake water & 1. Yes & 3. No & 7 & 7 \\
\hline 10. Water purchased from a vendor (non-PAM) & 1. Yes & 3. No & 10 & \\
\hline 8a. Other, drinking and cooking water & 1. Yes & 3. No & $8 a$ & \\
\hline 8b. Other, bathing and washing water & 1. Yes & 3. No & & $8 \mathrm{~b}$ \\
\hline
\end{tabular}




\section{SECTION C : WATER SOURCE AND SANITATION}

\begin{tabular}{|c|c|c|}
\hline C6. & $\begin{array}{l}\text { In what year was the piped water } \\
\text { system/PAM introduced into this } \\
\text { village? }\end{array}$ & 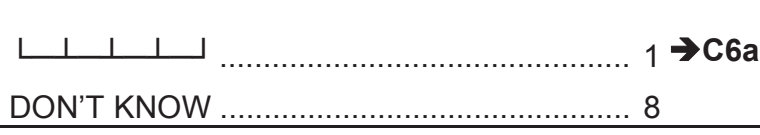 \\
\hline C61. & $\begin{array}{l}\text { For how many years has there been } \\
\text { piped water system in this village? }\end{array}$ & 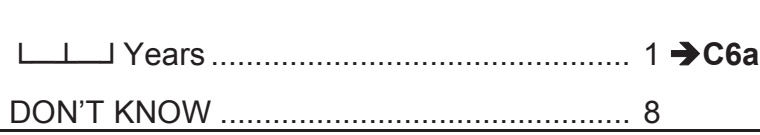 \\
\hline C6a. & $\begin{array}{l}\text { What is the main water source for the } \\
\text { piped water system/PAM that flows in } \\
\text { the village? }\end{array}$ & 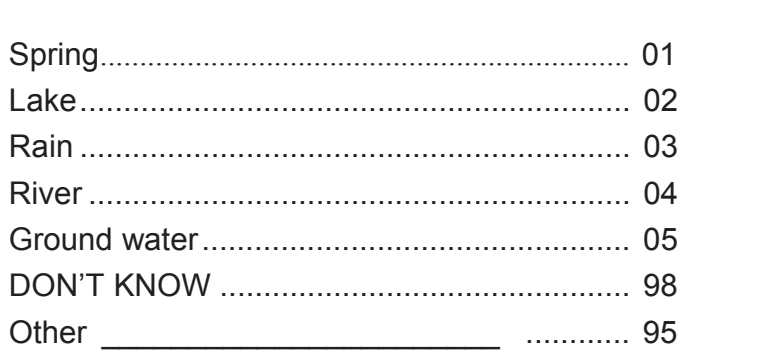 \\
\hline C6b. & $\begin{array}{l}\text { Which of the following are involved in } \\
\text { providing water to this community? }\end{array}$ & 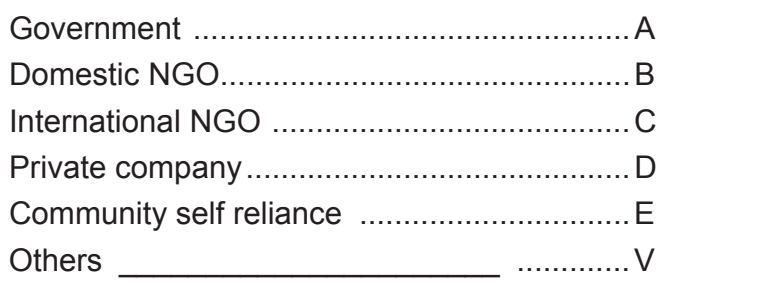 \\
\hline C7. & $\begin{array}{l}\text { In the last year, have there been } \\
\text { disruptions in the water service? }\end{array}$ & 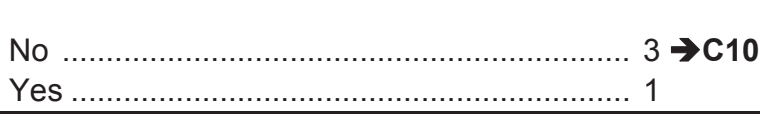 \\
\hline C8. & How often? & 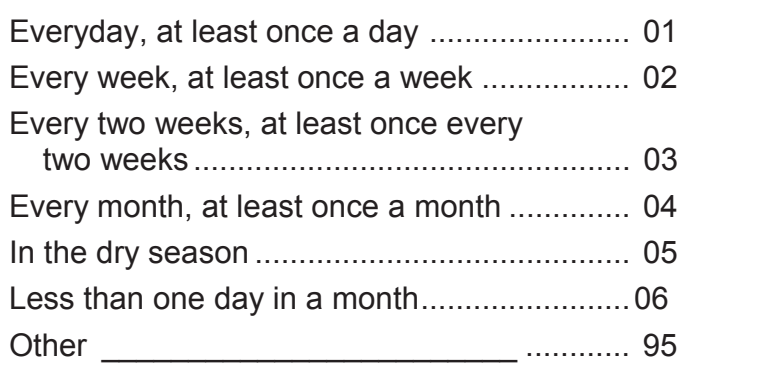 \\
\hline C8a. & $\begin{array}{l}\text { Have disruptions to or problems with } \\
\text { the water supply been more or less } \\
\text { frequent now compare to } 12 \text { months } \\
\text { ago? }\end{array}$ & 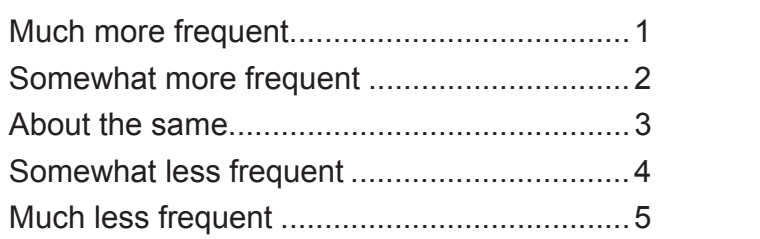 \\
\hline
\end{tabular}

\begin{tabular}{|c|c|c|}
\hline C10. & $\begin{array}{l}\text { Are water needs for drinking and } \\
\text { cooking met every dry season in this } \\
\text { village? }\end{array}$ & 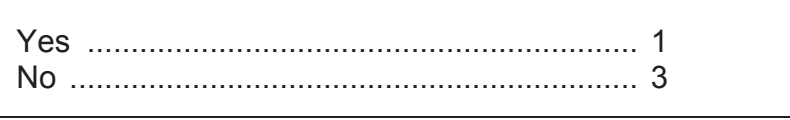 \\
\hline C10a. & $\begin{array}{l}\text { In comparison to } 12 \text { months ago, has } \\
\text { the time it takes to obtain safe drinking } \\
\text { water changed? }\end{array}$ & 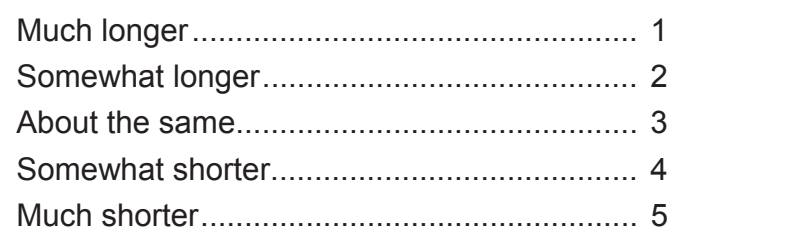 \\
\hline C13. & $\begin{array}{l}\text { Is there any system of sewage } \\
\text { channels/gutters in this village? }\end{array}$ & 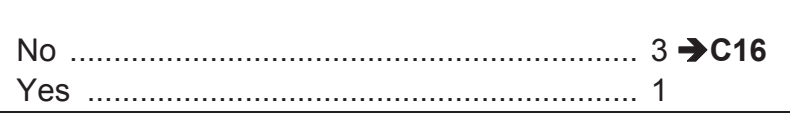 \\
\hline C14. & $\begin{array}{l}\text { In what year did the sewage system } \\
\text { become available? }\end{array}$ & 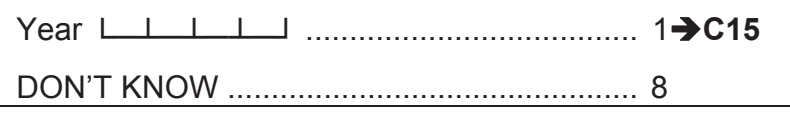 \\
\hline C14a. & $\begin{array}{l}\text { How many years has the sewage } \\
\text { system been available in this village? }\end{array}$ & 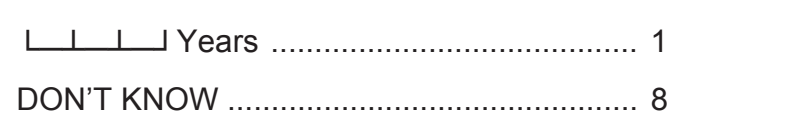 \\
\hline C15. & $\begin{array}{l}\text { Do all the households in the village } \\
\text { use the sewage system? }\end{array}$ & 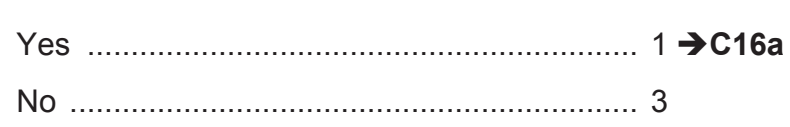 \\
\hline C16. & $\begin{array}{l}\text { In what places do the households in } \\
\text { this village dispose of their } \\
\text { households' sewage? } \\
\text { (CHOOSE ALL THAT APPLY) }\end{array}$ & 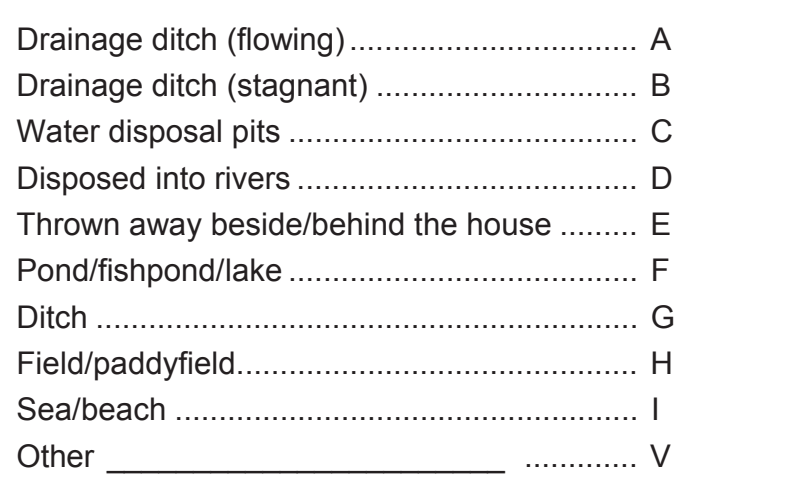 \\
\hline
\end{tabular}


SECTION C : WATER SOURCE AND SANITATION

\begin{tabular}{|c|c|c|}
\hline C16a. & C17. & C18. \\
\hline TOILET FACILITIES & Where does the village population defecate? & Among the aforementioned places which is the predominant one? \\
\hline 01. Private toilet with septic tank & 1. Yes & 1 \\
\hline 02. Private toilet without septic tank & 1. Yes & 2 \\
\hline 03. Common toilet shared with neighbors & 1. Yes & 3 \\
\hline 04. Public lavatory (MCK) & 1. Yes & 4 \\
\hline 05. In the river/gutter & 1. Yes & 5 \\
\hline 06. In the garden/paddyfield & 1. Yes & 6 \\
\hline 07. Sewer & 1. Yes & 7 \\
\hline 08. Pond/fishpond & 1. Yes & 8 \\
\hline 09. Animal stable & 1. Yes & 9 \\
\hline 10. Sea/lake & 1. Yes & 10 \\
\hline 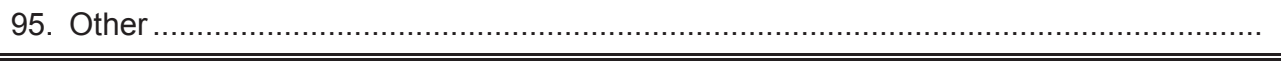 & 1. Yes & 11 \\
\hline C18a. & C19. & C19a. \\
\hline VARIOUS TYPES OF GARBAGE DISPOSALS & Where does the village population dispose of garbage? & Among the aforementioned places which is the predominant one? \\
\hline 01. Disposed in garbage cans/barrels, carried away by sanitation worker & 1. Yes & $1 \rightarrow \mathrm{C} 20 \mathrm{a}$ \\
\hline 02. Burned & 1. Yes & $2 \rightarrow \mathrm{DO}$ \\
\hline 03. Thrown into the creek/river & 1. Yes & $3 \rightarrow \mathrm{DO}$ \\
\hline 04. Dumped onto the yards/in the garden, let decompose & 1. Yes & $4 \rightarrow \mathrm{D} 0$ \\
\hline 05. Thrown into pits & 1. Yes & $5 \rightarrow \mathrm{DO}$ \\
\hline 06. Forest/Mountain & 1. Yes & $6 \rightarrow \mathrm{D} 0$ \\
\hline 07. Sea/lake/beach & 1. Yes & $7 \rightarrow \mathrm{D} 0$ \\
\hline 08. Field/paddyfield & 1. Yes & $8 \rightarrow \mathrm{D} 0$ \\
\hline 95. Other & 1. Yes & $9 \rightarrow$ DO \\
\hline
\end{tabular}




\section{SECTION C : WATER SOURCE AND SANITATION}

C20a. In what year did the garbage start to be collected by sanitation workers?

1.

$1 \rightarrow \mathrm{C} 20 \mathrm{c}$

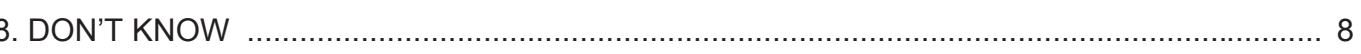

C20b. How many years has the garbage been collected by sanitation workers in this village?

$\perp+1$ Years

DON'T KNOW

KNOW .m.n.

C20c. Who manages the garbage collection?

(CIRCLE ALL THAT APPLY)

Private group

Public self-reliant

Other 


\section{SECTION D : AGRICULTURE AND INDUSTRY}

Section D Respondent:

\begin{tabular}{|c|c|}
\hline 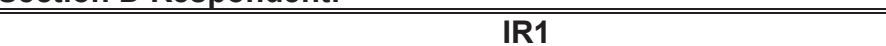 & IR2 \\
\hline $\begin{array}{l}\text { 1_D. Name of Respondent: } \\
\text { CAPI WILL SKIP TO D1 IF THE SAME NAME HAS } \\
\text { ALREADY BEEN ENTERED EARLIER }\end{array}$ & \\
\hline 1a_D. Position in the village : & $\begin{array}{rrrrr}01 & 02 & 03 & 04 & 05 \\
06 & 07 & 95 & \ldots \ldots \ldots \ldots \ldots . .\end{array}$ \\
\hline 2_D. Age : & $\sqcup \perp$ Years \\
\hline 2a_D. Length of tenure in positions: & 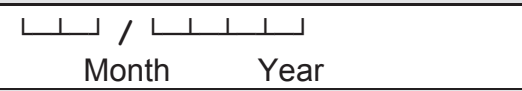 \\
\hline $\begin{array}{ll}\text { 3_D. } & \text { INTERVIEWER CHECK : } \\
& \text { SEX OF RESPONDENT }\end{array}$ & $\begin{array}{l}\text { MALE } \ldots \ldots \ldots \ldots \ldots \ldots \ldots \ldots . . .1 \\
\text { FEMALE ……........... } 3\end{array}$ \\
\hline 3a_D. Highest level of education attended: & $\sqcup$ \\
\hline 4_D. Highest class completed: & $\begin{array}{lllll}00 & 01 & 02 & 03 & 04 \\
05 & 06 & 07 & 98 & 96\end{array}$ \\
\hline 5_D. Length of time lived in the village : & 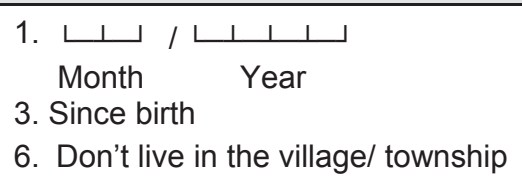 \\
\hline 6_D. How did [...] come to assume to the position & $\begin{array}{l}\text { 1. Elected } \\
\text { 2. Appointed by residence } \\
\text { 3. Appointed by camat } \\
\text { 4. Appointed by Bupati/Walikota } \\
\text { 5. Other }\end{array}$ \\
\hline
\end{tabular}

\section{Code for position in village (1a_D)}

01. Village/township heads

02. Village secretary
03. Head of Government Administration

03. Head of Government Administ
04 . Head of Village Development

05. Head of Welfare

07. Head of Financial Matters

95. Other

\section{Codes for highest level of education (3a_D)}
01. No school/not yet in school
02. Elementary school
03. Junior High - General
04. Junior High - Vocational
05. High School - General
66. High School - Vocalional
61. University S1 (Bachelor)
62. University $S 2$ (Masters)
62. University S2 (Masters)
63. University S3 (Ph.D.)

11. Kejar Paket A (Adult Education A)

14. Kejar Paket B (Adult Education B)

14. Islamic School (Pondok Pensantren)

15. Kejar Paket C (Adult Education C)

17. School for the disabled

72. Islamic Elementary School (Madrasah Ibtidaiyah)

73. Islamic Junior High School (Madrasah Tsanaw

74. Islamic Senior High School (Madrasah Alliyah)

90. Kindergarten

98. DON'T KNOW
Codes for highest grade (4_D):

00. Never completed class 1

01. 1

03. 3

04. 4

06. 5

07. Graduated

6. No school

98. DON'T KNOW 


\section{SECTION D : AGRICULTURE AND INDUSTRY}

Now, we would like to ask about the agriculture and industry in this village.

\begin{tabular}{|c|c|}
\hline Do. Are there any crops cultivated by members of this community? & 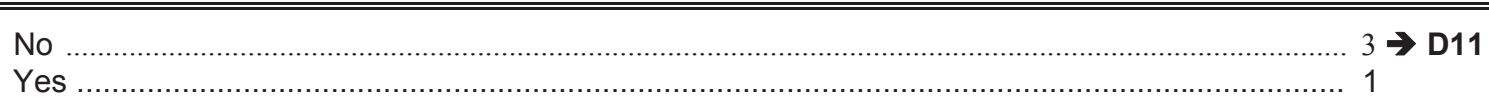 \\
\hline
\end{tabular}

\begin{tabular}{|c|c|c|c|c|c|}
\hline (D3TYPE) & D3. & D3a. & D4. & D5. & D6. \\
\hline $\begin{array}{l}\text { D2. Mention three main crops } \\
\text { cultivated in this village in the last } 12 \\
\text { months? }\end{array}$ & $\begin{array}{l}\text { Was there any } \\
\text { harvest of }[\ldots] \text { in the } \\
\text { last } 12 \text { months? }\end{array}$ & $\begin{array}{l}\text { INTERVIEWER NOTE: IS } \\
\text { [...] AN ANNUAL CROP? }\end{array}$ & $\begin{array}{l}\text { How many times was } \\
{[\ldots . .] \text { harvested in this }} \\
\text { village in the last } 12 \\
\text { months? }\end{array}$ & $\begin{array}{l}\text { In general, is this crop sold } \\
\text { (including bartering) or for } \\
\text { personal consumption? }\end{array}$ & $\begin{array}{l}\text { IF RICE IS LISTED IN D.2: } \\
\text { What was the variety (type) of rice planted in this village in the last } 12 \\
\text { months? }\end{array}$ \\
\hline 1. لــ & $\begin{array}{l}\text { Yes .................... } 1 \\
\text { No ................. } 3\end{array}$ & 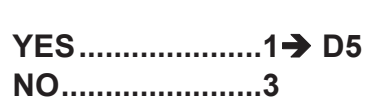 & 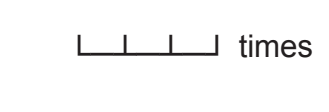 & $\begin{array}{l}\text { 1. Sold } \\
\text { 3. Personal consumption }\end{array}$ & $\begin{array}{l}\text { 1. Rice variety : } \begin{array}{llllllllll}01 & 02 & 03 & 04 & 05 & 06 & 07 & 08 & 09 & 95 \\
\text { 3. Not rice } & & & & & & & & & \end{array}\end{array}$ \\
\hline 2. ᄂ & $\begin{array}{l}\text { Yes } \ldots \ldots \ldots \ldots \ldots \ldots \ldots \ldots . . .1 \\
\text { No } \ldots \ldots \ldots \ldots \ldots \ldots \ldots \ldots \ldots \ldots \ldots \ldots \ldots \ldots\end{array}$ & 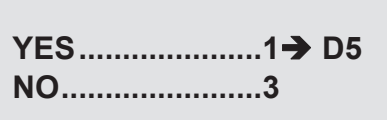 & 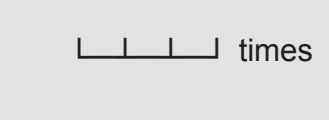 & $\begin{array}{l}\text { 1. Sold } \\
\text { 3. Personal consumption }\end{array}$ & $\begin{array}{l}\text { 1. Rice variety : } \begin{array}{llllllllll}01 & 02 & 03 & 04 & 05 & 06 & 07 & 08 & 09 & 95 \\
\text { 3. Not rice } & & & & & & & & & \end{array}\end{array}$ \\
\hline 3. $\llcorner\perp$ & $\begin{array}{l}\text { Yes } \ldots \ldots \ldots \ldots \ldots \ldots \ldots \ldots \ldots \ldots \ldots \ldots \ldots \ldots \\
\text { No } \ldots \ldots \ldots \ldots \ldots \ldots \ldots \ldots \ldots . .3\end{array}$ & 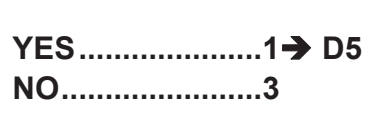 & 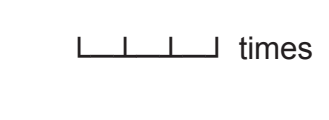 & $\begin{array}{l}\text { 1. Sold } \\
\text { 3. Personal consumption }\end{array}$ & $\begin{array}{l}\text { 1. Rice variety : } \begin{array}{llllllllll}01 & 02 & 03 & 04 & 05 & 06 & 07 & 08 & 09 & 95 \\
\text { 3. Not rice } & & & & & & & & & \end{array}\end{array}$ \\
\hline
\end{tabular}

\begin{tabular}{|c|c|c|c|}
\hline \multicolumn{4}{|c|}{ CODE D3TYPE (D2) } \\
\hline 01. & Cassava & 12. & Coffee \\
\hline 02. & Sweet potatoes & 13. & Sugarcane \\
\hline 03. & Peanuts & 14. & Tobacco \\
\hline 04. & Cashews and other nuts & 15. & Rubber \\
\hline 05. & Soybean & 16. & Wood \\
\hline 06. & Corn & 22. & Other vegetables \\
\hline 07. & Chili & 23. & Other fruits \\
\hline 08. & Red onion & 90. & Rice \\
\hline 09. & Coconut & 96. & NO OTHER CROP \\
\hline 10. & Bananas & 95. & Other \\
\hline 11. & Spice & & \\
\hline
\end{tabular}

CODE D6
01. Cianjur kepala
02. Cianjur slip
03. Setra
04. Saigon Bandung
05. Muncil
06. IR64
07. IR42
08. Ketan putih (white sticky rice)
09. Ketan hitam (black sticky rice)

95 Others 


\section{SECTION D : AGRICULTURE AND INDUSTRY}

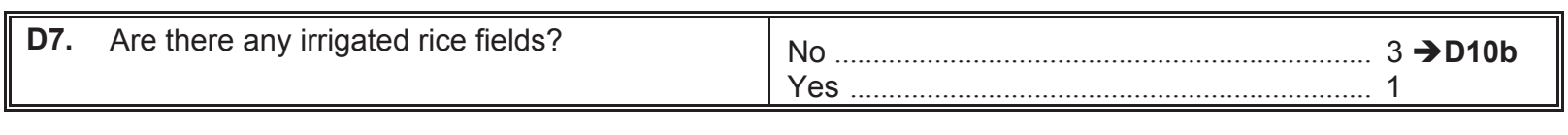

\begin{tabular}{|c|c|c|}
\hline \multirow{2}{*}{$\begin{array}{l}\text { TYPE OF IRRIGATION } \\
\text { (D1TYPE) }\end{array}$} & D8. & D9. \\
\hline & $\begin{array}{l}\text { Is there }[\ldots] \text { irrigation in } \\
\text { this village? }\end{array}$ & $\begin{array}{l}\text { How many hectares of rice fields in the village are } \\
\text { watered by }[\ldots] \text { irrigation system? }\end{array}$ \\
\hline 1. Technical & $\begin{array}{l}\text { 3. No 1. Yes } \\
\Downarrow\end{array}$ & 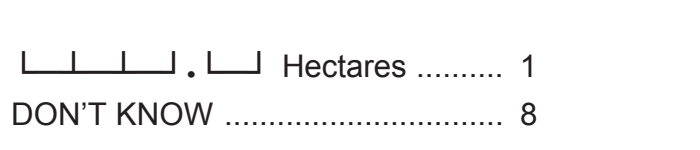 \\
\hline 2. Semi technical & $\begin{array}{l}\text { 3. No 1. Yes } \\
\Downarrow\end{array}$ & 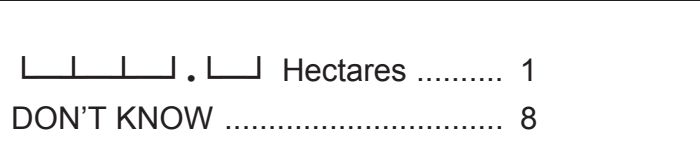 \\
\hline 3. Simple & $\begin{array}{l}\text { 3. No 1. Yes } \\
\Downarrow\end{array}$ & 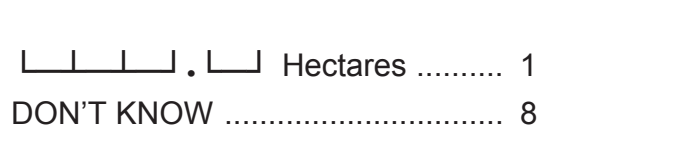 \\
\hline 3a. Tube well/pump & $\begin{array}{l}\text { 3. No 1. Yes } \\
\downarrow \\
\text { D8a }\end{array}$ & 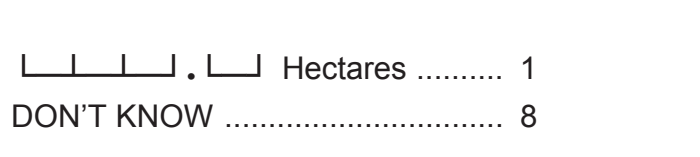 \\
\hline
\end{tabular}

\begin{tabular}{|c|c|c|}
\hline \multirow{2}{*}{$\begin{array}{l}\text { TYPE OF RICEFIELD } \\
\text { (D1TYPE) }\end{array}$} & D8a. & D9a. \\
\hline & $\begin{array}{l}\text { Is there }[\ldots] \text { rice field in } \\
\text { this village? }\end{array}$ & $\begin{array}{l}\text { How many hectares of rice fields in the village are } \\
\text { watered by }[\ldots . .] \text { ? }\end{array}$ \\
\hline 4. Rain water & 3. No 1. Yes & 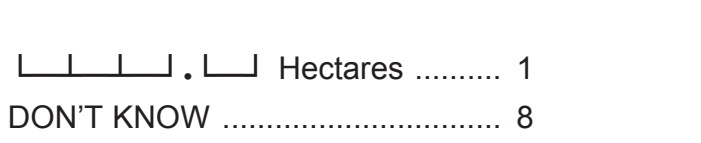 \\
\hline 5. Tidal & $\begin{array}{l}\text { 3. No 1. Yes } \\
\downarrow \\
\text { D10a }\end{array}$ & 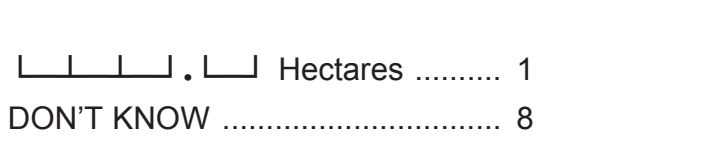 \\
\hline
\end{tabular}

D10a. How many percentage of

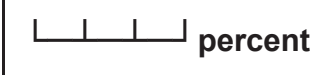

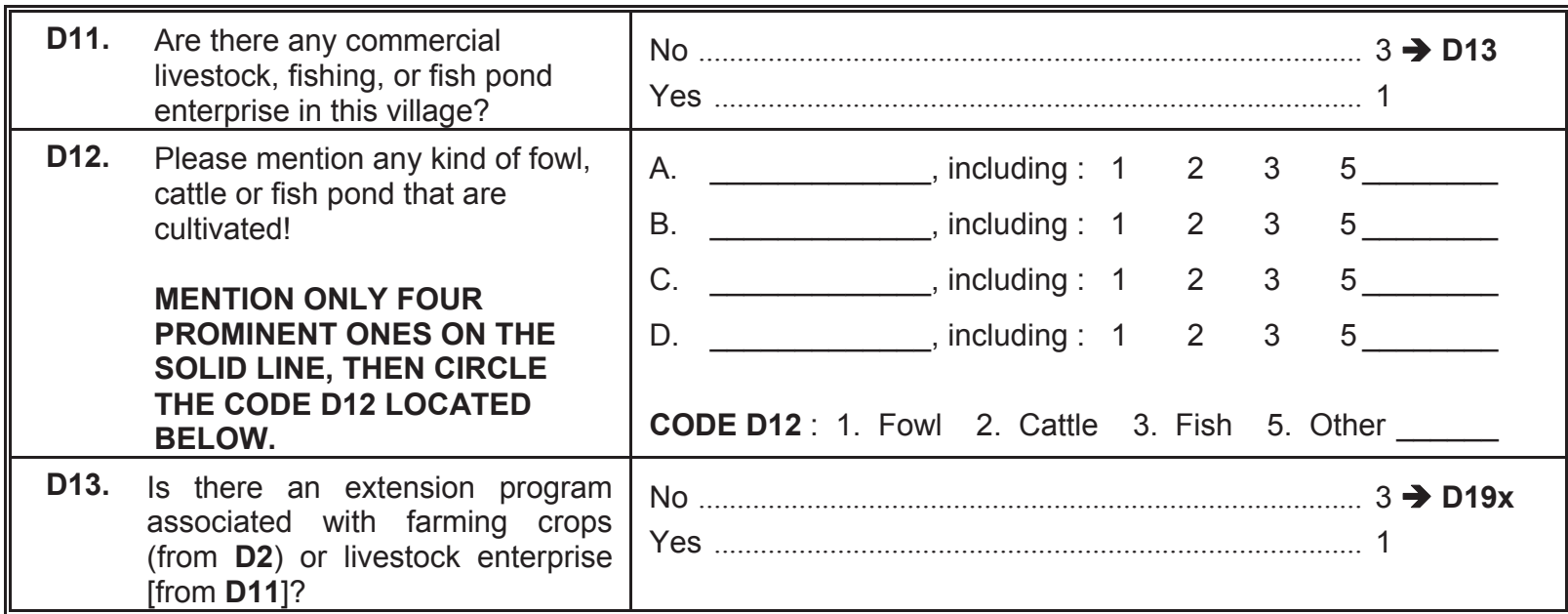

\begin{tabular}{|c|c|c|}
\hline D10b. & $\begin{array}{l}\text { How often does the irrigation } \\
\text { canals get cleaned (from dirt, } \\
\text { sand, garbage)? }\end{array}$ & $\begin{array}{l}\text { At least once a month } \\
\text { At least once every } 6 \text { month } \\
\text { At least once every year } \\
\text { Never }\end{array}$ \\
\hline
\end{tabular}




\section{SECTION D : AGRICULTURE AND INDUSTRY}

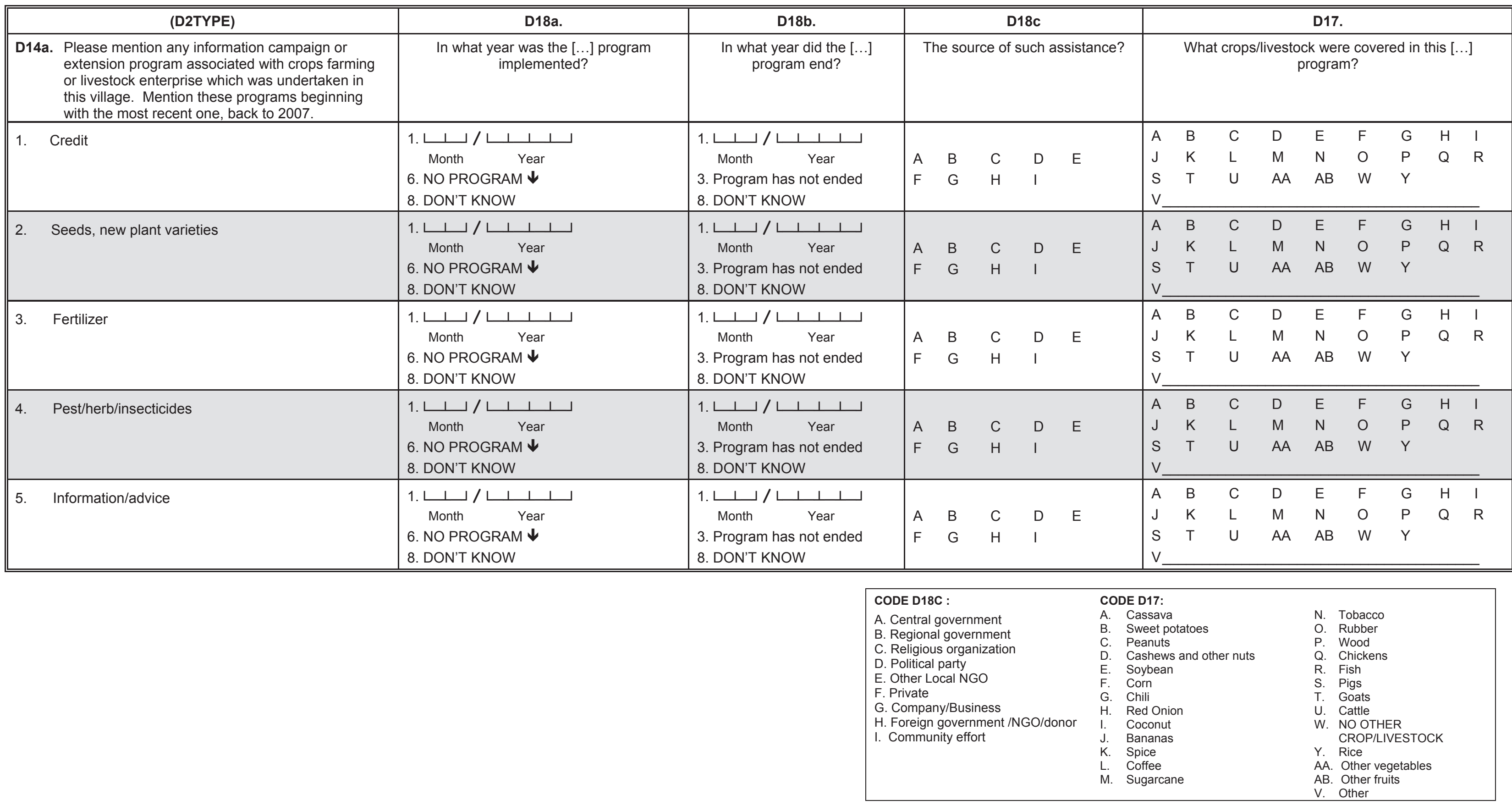




\section{SECTION D : AGRICULTURE AND INDUSTRY}

Now we will ask about wages of farm labor and sharecropping in this village.

\begin{tabular}{|c|c|c|c|c|c|c|}
\hline & \multicolumn{2}{|r|}{ D19x. CROP D2 LINE 1} & \multicolumn{2}{|r|}{ D22x. CROP D2 LINE 2} & \multicolumn{2}{|r|}{ D25x. CROP D2 LINE 3} \\
\hline & CROP1 & $\begin{array}{l}\text { INTERVIEWER CHECK: } \\
\text { IF D2 LINE1 = "NONE" } \rightarrow \text { D28a }\end{array}$ & CROP2 & $\begin{array}{l}\text { INTERVIEWER CHECK: } \\
\text { IF D2 LINE2 = "NONE" } \rightarrow \text { D28a }\end{array}$ & CROP3 & $\begin{array}{l}\text { INTERVIEWER CHECK: } \\
\text { IF D2 LINE3 = "NONE" } \rightarrow \text { D28a }\end{array}$ \\
\hline $\begin{array}{l}\text { How much are the current wages for the farm laborers, according to to } \\
\text { the type of work and the type of laborer ? }\end{array}$ & D19. & & D22. & & D25. & \\
\hline $\begin{array}{l}\text { A.11 } \\
\text { a. Harvesting by Adult males }\end{array}$ & D19A. & $\begin{array}{l}\text { 1. } \downarrow \perp, \downarrow \perp \_\_ \text {Rupiah } \\
\text { 6. NONE } \\
\text { 8. DON'T KNOW }\end{array}$ & D22A. & $\begin{array}{l}\text { 1. } \downarrow \perp, \downarrow \perp \_\_ \text {Rupiah } \\
\text { 6. NONE } \\
\text { 8. DON'T KNOW }\end{array}$ & D25A. & $\begin{array}{l}\text { 1. } \downarrow \perp, \downarrow \perp \_\_ \text {Rupiah } \\
\text { 6. NONE } \\
\text { 8. DON'T KNOW }\end{array}$ \\
\hline b. Harvesting by Adult females & D19B. & $\begin{array}{l}\text { 1. } \sqcup \perp, \downarrow \perp \perp \_ \text {Rupiah } \\
\text { 6. NONE } \\
\text { 8. DON'T KNOW }\end{array}$ & D22B. & $\begin{array}{l}\text { 1. } \downarrow \perp, \downarrow \perp \perp \_ \text {Rupiah } \\
\text { 6. NONE } \\
\text { 8. DON'T KNOW }\end{array}$ & D25B. & $\begin{array}{l}\text { 1. } \square \perp, \perp \perp \perp \_ \text {Rupiah } \\
\text { 6. NONE } \\
\text { 8. DON'T KNOW }\end{array}$ \\
\hline c. Harvesting by Children & D19C. & 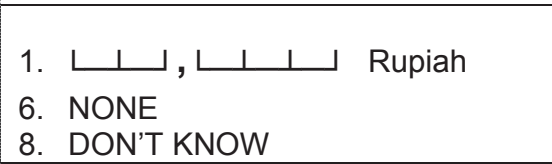 & D22C. & $\begin{array}{l}\text { 1. } \downarrow \perp, \downarrow \perp \_\_ \text {Rupiah } \\
\text { 6. NONE } \\
\text { 8. DON'T KNOW }\end{array}$ & D25C. & $\begin{array}{l}\text { 1. } \downarrow \perp, \downarrow \perp \perp \perp \_ \text {Rupiah } \\
\text { 6. NONE } \\
\text { 8. DON'T KNOW }\end{array}$ \\
\hline Is the type of work usually given by piece to the workers in this village? & D20. & 1. Yes & D23. & $\begin{array}{l}\text { 3. No } \\
\downarrow \\
\text { D24a }\end{array}$ & D26. & $\begin{array}{l}\text { 3. No } \\
\downarrow \\
\text { D27a }\end{array}$ \\
\hline How many rupiah on average are paid for each hectare of $[\ldots]$ work? & D21. & 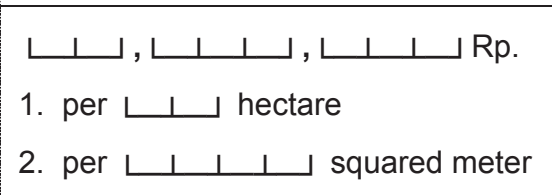 & D24. & 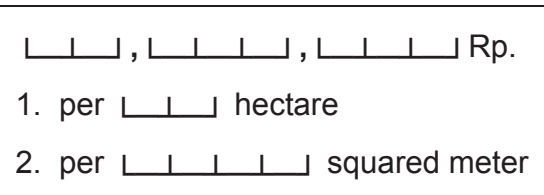 & D27. & 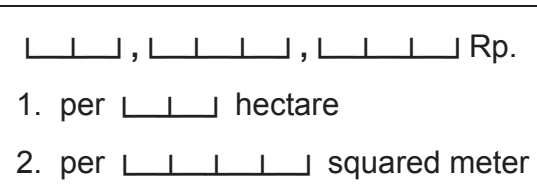 \\
\hline Is there a tradition of share-cropping (this crop) in this village? & D21a. & $\begin{array}{l}\text { 3. No } \rightarrow \text { D22x (CROP2) } \\
\text { 1. Yes }\end{array}$ & D24a. & $\begin{array}{l}\text { 3. No } \rightarrow \text { D25x (CROP3) } \\
\text { 1. Yes }\end{array}$ & D27a. & $\begin{array}{l}\text { 3. No } \rightarrow \mathbf{D 2 8 a} \\
\text { 1. Yes }\end{array}$ \\
\hline $\begin{array}{l}\text { How is the harvest usually shared in this village? } \\
\text { (FILL OUT SHARE-RATIO BETWEEN SHARECROPPER AND } \\
\text { LANDOWNER, EXAMPLE } 1 / 4 \text { AND } 3 / 4 \text { ) }\end{array}$ & D21b. & a. $\underset{\text { sharecropper }}{\longleftarrow} \quad \stackrel{\sqcup .}{\longleftarrow}$ & D24b. & a. $\underset{\text { sharecropper }}{\longleftarrow} \quad \stackrel{\sqcup . L}{L}$ & D27b. & a. $\underset{L}{L} \quad$ b. $\underset{\text { sharecropper }}{\longleftarrow}$ \\
\hline
\end{tabular}


SECTION D : AGRICULTURE AND INDUSTRY

D28a. Is there a factory (plant) in this village including cottage/home industry? (INCLUDE HOME INDUSTRY)

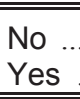

$\rightarrow \mathrm{D} 35$

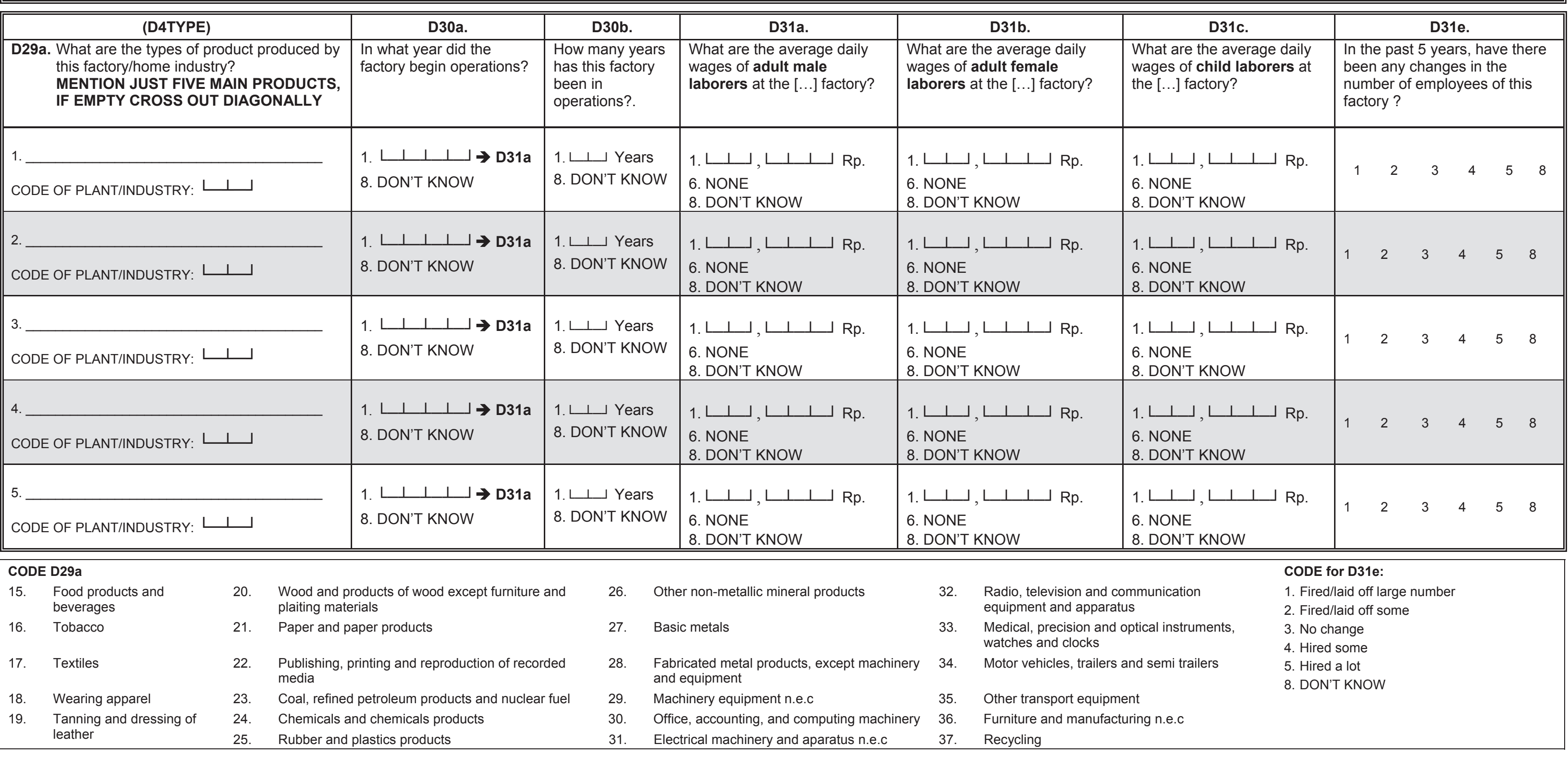




\section{SECTION D : AGRICULTURE AND INDUSTRY}

\begin{tabular}{|c|c|c|}
\hline D35. & $\begin{array}{l}\text { In the past } 12 \text { months, have there been } \\
\text { any public works projects in this } \\
\text { village/township? }\end{array}$ & $\begin{array}{l}\text { No } \\
\text { Yes }\end{array}$ \\
\hline D36. & $\begin{array}{l}\text { What are the wages of adult male } \\
\text { laborers per day in the public works } \\
\text { project? } \\
\text { (NOTE: IF MORE THAN ONE, } \\
\text { ESTIMATE FOR THE SCHEME THAT } \\
\text { HAS EMPLOYED THE MOST } \\
\text { PEOPLE) }\end{array}$ & 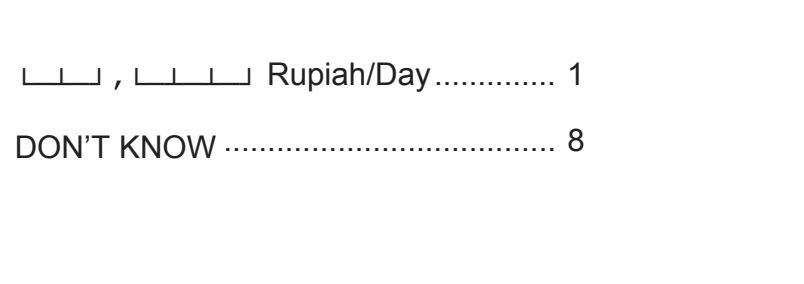 \\
\hline D37. & $\begin{array}{l}\text { What are the wages of adult female } \\
\text { laborers per day in the infrastructure } \\
\text { and facility project? }\end{array}$ & 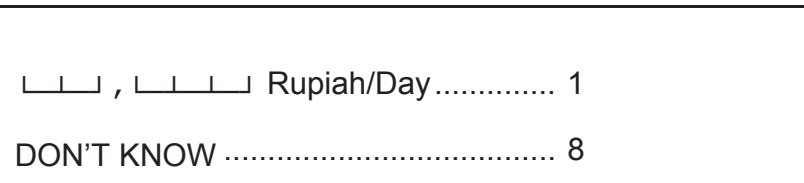 \\
\hline D38. & $\begin{array}{l}\text { Please estimate the number of people } \\
\text { employed by these projects. }\end{array}$ & 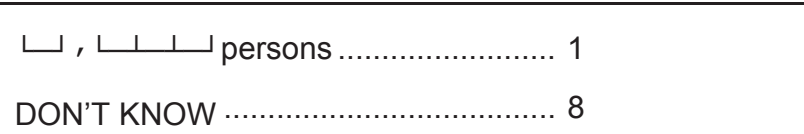 \\
\hline D39. & $\begin{array}{l}\text { Please estimate the average number of } \\
\text { days that someone employed on these } \\
\text { projects worked. }\end{array}$ & 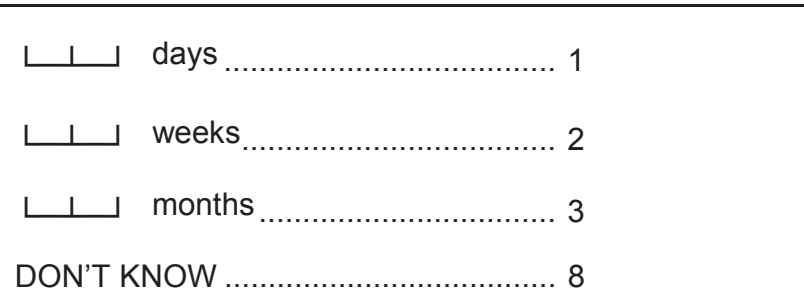 \\
\hline
\end{tabular}




\section{SECTION E : HISTORY AND CLIMATE}

Section E Respondent:

\begin{tabular}{|c|c|c|}
\hline & IR1 & IR2 \\
\hline $1 \_$. & $\begin{array}{l}\text { Name of Respondent: } \\
\text { CAPI WILL SKIP TO E1 IF THE SAME NAME HAS } \\
\text { ALREADY BEEN ENTERED EARLIER } \\
\end{array}$ & \\
\hline 1a_E. & Position in the village : & $\begin{array}{rrrrr}01 & 02 & 03 & 04 & 05 \\
06 & 07 & 95 & \ldots \ldots \ldots \ldots . . \\
\end{array}$ \\
\hline 2_E. & Age : & $\downarrow$ Years \\
\hline 2a_E. & Length of tenure in positions: & 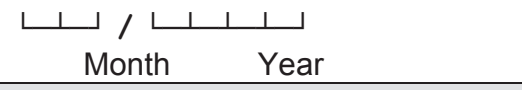 \\
\hline 3_E. & $\begin{array}{l}\text { INTERVIEWER CHECK : } \\
\text { SEX OF RESPONDENT }\end{array}$ & 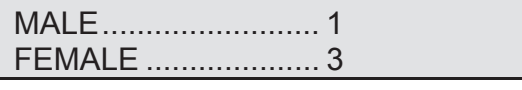 \\
\hline 3a_E. & Highest level of education attended: & $\downarrow$ \\
\hline 4_E. & Highest class completed & $\begin{array}{lllll}00 & 01 & 02 & 03 & 04 \\
05 & 06 & 07 & 98 & 96 \\
\end{array}$ \\
\hline 5_E. & Length of time lived in the village : & 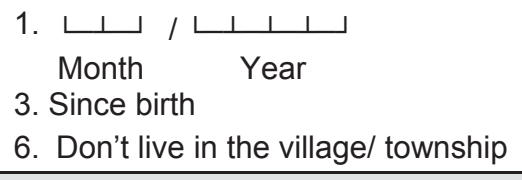 \\
\hline 6_E. & How did $[\ldots]$ come to assume to the position & $\begin{array}{l}\text { 1. Elected } \\
\text { 2. Appointed by residence } \\
\text { 3. Appointed by camat } \\
\text { 4. Appointed by Bupati/Walikota } \\
\text { 5. Other }\end{array}$ \\
\hline
\end{tabular}

\section{Code for position in village (1a_E):}

01. Village/township heads

02. Village secretary

04. Head of Government Administration

05. Head of Welfare

06. Head of Financial Matters

07. Head of General Affairs

95. Other

\begin{abstract}
01. No school/not yet in schoo
01. No school/not yet in
02. Elementary school

02. Elementary school
03. Junior High - General

04. Junior High - Vocational

05. High School - General

60. D1, D2, D3 (Junior College)

61. University S1 (Bachelor)

63. University S3 (Ph.D.)
\end{abstract}

11. Kejar Paket A (Adult Education A)

12. Kejar Paket $B$ (Adult Education $B$ )

13. Open Univeristy

14. Islamic School (Pondok Pensantren)

15. Kejar Paket C (Adult Education C)

17. School for the disabled

72. Islamic Elemiontary School (Madrasah Ibtidaiyah)

74. Islamic Senior High School (Madrasah Tsanawiy

90. Kindergarten

98. DON'T KNOI
95. Other
Codes for highest grade (4 E):

00. Never completed class 1

01.

2.

04. 4

05. 5

06. 6

07. Graduated

96. No school
98. DON'T KNOW 


\section{SECTION E : HISTORY AND CLIMATE}

We would like to ask about history and important events in this village.

E1a. Has this village, kecamatan, kabupaten, or province ever changed its name since 2007?

No

$3 \rightarrow \mathrm{E}$

E2a. How many times has this village/township, sub district, district/city or province changed its name since 2007 ?

$\downarrow \downarrow$ times (USE LINE E2 TO E5 TO RECORD EACH NAME CHANGES)

\section{(E1TYPE)}

E2. What was the month/year

of this change of name?

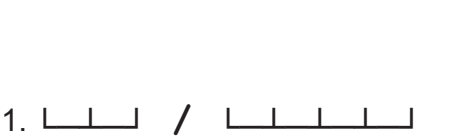

Month / Year

2. ᄂ $\longleftarrow / L \perp \perp$

Month / Year

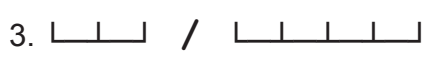

Month / Year
What were the old names of the old village, kecamatan, kabupaten and province, and the new names since 2007 ?

a.1. Village 3.Township

b.1. Sub District

c.1. City 3.District

d.1. Province

a.1. Village 3.Township

b.1. Sub District

c.1. City 3.District

d.1. Province

a.1. Village 3.Township

b.1. Sub District

c.1. City 3.District

d.1. Province

\begin{tabular}{|c|c|}
\hline A. Old Name & \\
\hline & $\begin{array}{l}\text { a.1. Village 3.Township } \\
\text { b.1. Sub District } \\
\text { c.1. City 3.District } \\
\text { d.1. Province }\end{array}$ \\
\hline & $\begin{array}{l}\text { a.1. Village 3.Township } \\
\text { b.1. Sub District } \\
\text { c.1. City 3.District } \\
\text { d.1. Province }\end{array}$ \\
\hline & $\begin{array}{l}\text { a.1. Village 3.Township } \\
\text { b.1. Sub District } \\
\text { c.1. City 3.District } \\
\text { d.1. Province }\end{array}$ \\
\hline
\end{tabular}

c.1. City 3.Distric

d.1. Province
E4.

What was the main reason for the change of names of

this village?

\begin{tabular}{|c|c|}
\hline B. New Name & this \\
\hline $\bar{\square}$ & \\
\hline $\bar{\square}$ & \\
\hline & \\
\hline &
\end{tabular}

Did the geographical size of the

village expand, shrink, or

remain the same when the name changed?

\section{Expand}

2. Shrink

3. No change

\begin{tabular}{ll|l}
5 & 2. Shrink \\
3. No change
\end{tabular}

\section{CODE FOR E4:}

1. Establishment of a new village

2. Expansion/splitting off of old village

3. Merging of villages
4. Expansion/splitting off of kecamatan, kabupaten, province 5. Other 


\section{SECTION E : HISTORY AND CLIMATE}

E6. Has this village ever changed in geographical size, without having to
change its name since 2007?

E7. When was there shrinking or expansion in the geographical size of the village/township?

\begin{tabular}{|c|c|c|c|c|}
\hline & 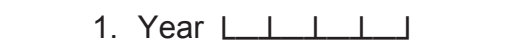 & & 1. Expansion & 3. Shrinking \\
\hline & 2. Year $\llcorner\perp \perp \perp \perp$ & & 1. Expansion & 3. Shrinking \\
\hline & 3. Year $\llcorner\perp \perp \perp \perp$ & & 1. Expansion & 3. Shrinking \\
\hline E8. & $\begin{array}{l}\text { In what months of } 2014 \text { did the rainy } \\
\text { season occur? }\end{array}$ & $\begin{array}{l}\text { A. January } \\
\text { B. February } \\
\text { C. March } \\
\text { D. April } \\
\text { E. May } \\
\text { F. June }\end{array}$ & & $\begin{array}{l}\text { G. July } \\
\text { H. August } \\
\text { I. September } \\
\text { J. October } \\
\text { K. November } \\
\text { L. December }\end{array}$ \\
\hline E9. & $\begin{array}{l}\text { Normally, in what months does the } \\
\text { rainy season take place? }\end{array}$ & $\begin{array}{l}\text { A. January } \\
\text { B. February } \\
\text { C. March } \\
\text { D. April } \\
\text { E. May } \\
\text { F. June }\end{array}$ & & $\begin{array}{l}\text { G. July } \\
\text { H. August } \\
\text { I. September } \\
\text { J. October } \\
\text { K. November } \\
\text { L. December }\end{array}$ \\
\hline
\end{tabular}


E10. Mention the year and the type of important events that have occurred in this village since 2007, such as introduction of electricity, building of infrastructure, and the like.

\section{[WRITE DOWN THE EVENT AND CIRCLE THE EVENT TYPE. IF EMPTY, CROSS OUT DIAGONALLY].}

\section{W. NO IMPORTANT EVENT/PROGRAM $\rightarrow$ SECTION F}

A.

1. New road opening

4. Construction of new school

7. First time mobile phone can be used in the village

10. Market construction

2. Construction of new road

5. Introduction of electricity

8. Internet (e.g. opening of the first internet shop)

11. Others infrastructure construction

3. Construction of new health facilities 6. Introduction of telephone (land line) 9. Irrigation construction 95. Other
1. New road opening
4. Construction of new schoo
7. First time mobile phone can be used in the village
10. Market construction
2. Construction of new road
5. Introduction of electricity
8. Internet (e.g. opening of the first internet shop)
11. Others infrastructure construction 95. Other

3. Construction of new health facilities 6. Introduction of telephone (land line) 9. Irrigation construction
1. New road opening
4. Construction of new school
2. Construction of new road
5. Introduction of electricity
8. Internet (e.g. opening of the firstinternet shop)
10. Market construction
3. Construction of new health facilities 6. Introduction of telephone (land line) 9. Irrigation construction
1. Others infrastructure construction 95. Other
1. New road openin
4. Construction of new school
7. First time mobile phone can be used in the village
$\begin{array}{lll}\text { 2. Construction of new road } & \text { 5. Introduction of electricity } & \text { 8. Internet (e.g. opening of the first internet shop) }\end{array}$
10. Market construction
3. Construction of new health facilities 6. Introduction of telephone (land line) 9. Irrigation construction
11. Others infrastructure construction 95. Other
1. New road opening
4. Construction of new school
7. First time mobile phone can be used in the village
3. Construction of new health facilities 6 . Introduction of telephone (land line) $\quad$ 9. Irrigation construction
10. Market construction
11. Others infrastructure construction 95. Other

\begin{tabular}{|c|c|}
\hline $\begin{array}{l}\text { In what year did the } \\
\text { event occur? }\end{array}$ & $\begin{array}{l}\text { What percent of the population } \\
\text { was affected by this event? }\end{array}$ \\
\hline ᄂ & $\begin{array}{l}\text { 1. }<25 \% \\
\text { 2. } 25-75 \% \\
\text { 3. }>75 \%\end{array}$ \\
\hline لـ & $\begin{array}{l}\text { 1. }<25 \% \\
\text { 2. } 25-75 \% \\
\text { 3. }>75 \%\end{array}$ \\
\hline$\left\llcorner\begin{array}{llll}\perp & \perp & \perp\end{array}\right.$ & $\begin{array}{l}\text { 1. }<25 \% \\
\text { 2. } 25-75 \% \\
\text { 3. }>75 \%\end{array}$ \\
\hline لـ & $\begin{array}{l}\text { 1. }<25 \% \\
\text { 2. } 25-75 \% \\
\text { 3. }>75 \%\end{array}$ \\
\hline 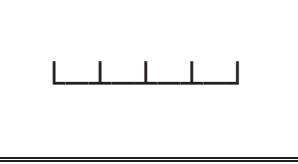 & $\begin{array}{l}\text { 1. }<25 \% \\
\text { 2. } 25-75 \% \\
\text { 3. }>75 \% \\
\end{array}$ \\
\hline
\end{tabular}

3. $>75 \%$ 


\section{SECTION F : NATURAL DISASTER}

Section F Respondent:

\begin{tabular}{|c|c|}
\hline IR1 & IR2 \\
\hline $\begin{array}{l}\text { 1_F. Name of Respondent: } \\
\text { CAPI WILL SKIP TO F01 IF THE SAME NAME HAS } \\
\text { ALREADY BEEN ENTERED EARLIER }\end{array}$ & \\
\hline 1a_F. $\quad$ Position in the village : & $\begin{array}{ccccc}01 & 02 & 03 & 04 & 05 \\
06 & 07 & 95 & \ldots \ldots \ldots \ldots \ldots . .\end{array}$ \\
\hline 2_F. Age: & $\sqcup$ Years \\
\hline 2a_F. Length of tenure in positions: & 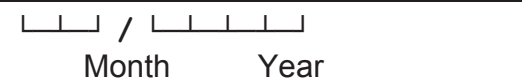 \\
\hline $\begin{array}{ll}\text { 3_F. INTERVIEWER CHECK : } & \text { SEX OF RESPONDENT } \\
& \text { SEX RE }\end{array}$ & 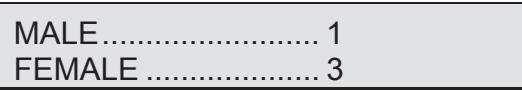 \\
\hline 3a_F. Highest level of education attended: & $\sqcup-$ \\
\hline 4_F. Highest class completed: & $\begin{array}{lllll}00 & 01 & 02 & 03 & 04 \\
05 & 06 & 07 & 98 & 96 \\
\end{array}$ \\
\hline 5_F. Length of time lived in the village : & 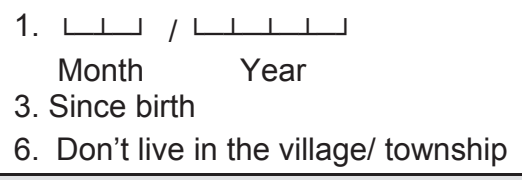 \\
\hline 6_F. How did $[\ldots]$ come to assume to the position & $\begin{array}{l}\text { 1. Elected } \\
\text { 2. Appointed by residence } \\
\text { 3. Appointed by camat } \\
\text { 4. Appointed by Bupati/Walikota } \\
\text { 5. Other }\end{array}$ \\
\hline
\end{tabular}

\section{Code for position in village (1a $F$ )}

01. Village/township heads

02. Village secretary

04. Head of Government Administration

05. Head of Welfare

06. Head of Financial Matters

07. Head of General Affairs

95. Other

\begin{abstract}
01. No school/not yet in school
02. Elementary school
03. Junior High - General
04. Junior High - Vocationa

04. Junior High - Vocationa
05 . High School - General

06. High School - Vocational

60. D1, D2, D3 (Junior College)

61. University $\mathrm{S1}$ (Bachelor)

2. University S2 (Masters)

63. University S3 (Ph.D.)
\end{abstract}

11. Kejar Paket A (Adult Education A)

2. Kejar Paket B (Adult Education B)

14. Islamic Siveristy

15. Kejar Paket C (Adult Education C)

7. School for the disabled

72. Islamic Elemontary School (Madrasah Ibtidaiyah)

74. Islamic Senior High School (Madrasah Tsanawiyah)

98. DON'T KNOW

95. Other
Codes for highest grade (4 F):

00. Never completed class 1

01.

02. 2

04. 4

06.

07. Graduated

98. DON'T KNOW 


\section{SECTION F : NATURAL DISASTER}

Now I would like to ask you about natural disasters that occurred in this village in the last five years

\begin{tabular}{|c|c|c|c|c|c|c|}
\hline & \multirow[b]{2}{*}{ (FTYPE) } & & & \multirow{2}{*}{$\begin{array}{c}\text { F02. } \\
\text { How many times has }[\ldots] \\
\text { disaster occurred in this village? }\end{array}$} & \multirow{2}{*}{$\begin{array}{c}\text { F03. } \\
\text { When was the most severe } \\
{[\ldots . .] \text { disaster occurred? }}\end{array}$} & \multirow{2}{*}{$\begin{array}{c}\text { F03a } \\
\text { How many casualties were } \\
\text { resulted from the most severe } \\
\text { disaster? }\end{array}$} \\
\hline & & $\begin{array}{r}\text { Were } \\
\text { disasters[ }\end{array}$ & $\begin{array}{l}\text { any natural } \\
\text { his village in the } \\
\text { years? }\end{array}$ & & & \\
\hline A. & Flood & $\begin{array}{l}\text { 3. No } \\
\Downarrow\end{array}$ & 1. Yes $\rightarrow$ & $\sqcup \downarrow$ times & لـ & $\downarrow \downarrow \downarrow \perp \_$لـ lives \\
\hline B. & Earthquake & $\begin{array}{l}\text { 3. No } \\
\Downarrow\end{array}$ & 1. Yes $\rightarrow$ & $\llcorner\perp ل$ times & 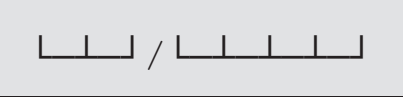 & $\left\llcorner\perp \perp \_\right.$لـ lives \\
\hline C. & Landslide & $\begin{array}{l}\text { 3. No } \\
\Downarrow\end{array}$ & 1. Yes $\rightarrow$ & $\sqcup \downarrow$ times & 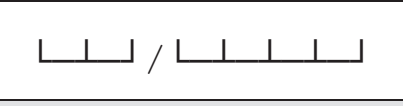 & ــــ lives \\
\hline D. & Volcano Eruption & 3. No & 1. Yes $\rightarrow$ & $\llcorner\perp$ times & لـ & $\downarrow \downarrow \downarrow \perp \_$lives \\
\hline E. & Tsunami & 3. No & 1. Yes $\rightarrow$ & $\llcorner\perp \downarrow$ times & $\sqcup \downarrow$ & 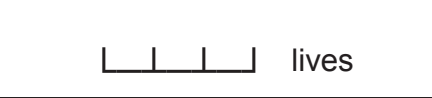 \\
\hline $\mathrm{F}$. & Drought & 3. No & 1. Yes $\rightarrow$ & $\sqcup \downarrow$ times & $\sqcup \downarrow$ & $\downarrow \perp \perp \_$lives \\
\hline G. & Forest fire & 3. No & 1. Yes $\rightarrow$ & $\llcorner\perp$ times & 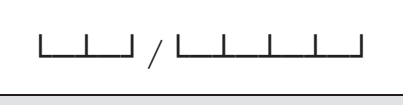 & 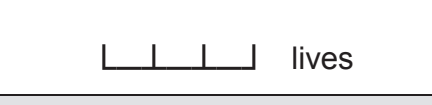 \\
\hline $\mathrm{H}$. & Fire & $\begin{array}{l}\text { 3. No } \\
\downarrow \\
\text { F04x }\end{array}$ & 1. Yes $\rightarrow$ & $\sqcup \downarrow$ times & 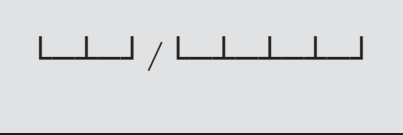 & 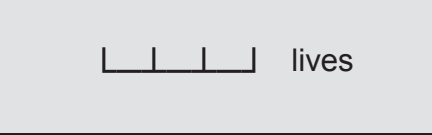 \\
\hline
\end{tabular}




\section{SECTION F : NATURAL DISASTER}

Now we would like to ask you about the damage caused by natural disasters to infrastructures and facilities in this village.

\begin{tabular}{|c|c|c|c|c|c|c|c|c|}
\hline \multicolumn{4}{|c|}{ F04X. INTERVIEWER CHECK F01 ROWS A-H: ONE OF F01 ROWS A-H = 1? } & 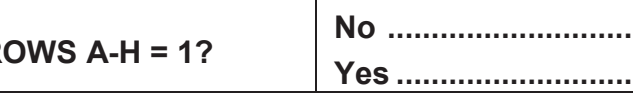 & \multicolumn{3}{|c|}{ No Yes } & \multirow[b]{2}{*}{ F08. } \\
\hline \multirow[b]{2}{*}{$\begin{array}{c}\text { (FTYPE) } \\
\text { TRANSPORTATION } \\
\text { AND } \\
\text { INFRASTRUCTURE } \\
\text { TYPE }\end{array}$} & \multicolumn{2}{|c|}{ F04. } & F04a & F04b & F05. & F06. & \begin{tabular}{|c|} 
F07. \\
\end{tabular} & \\
\hline & \multicolumn{2}{|c|}{$\begin{array}{l}\text { is there any damage } \\
\text { of }[\ldots] \text { in this village } \\
\text { caused by the } \\
\text { disasters? }\end{array}$} & $\begin{array}{l}\text { How bad was } \\
\text { the damage? }\end{array}$ & $\begin{array}{l}\text { How much was the damage loss caused by } \\
\text { the disaster? }\end{array}$ & $\begin{array}{l}\text { Have } \\
\text { repairs } \\
\text { been } \\
\text { made to } \\
\text { the } \\
\text { damage? }\end{array}$ & By Whom? & Relative to before the disaster, in what condition are the $[\ldots]$ now? & $\begin{array}{l}\text { In light of } \\
\text { the repairs } \\
\text { done so } \\
\text { far how } \\
\text { satisfied } \\
\text { are you? }\end{array}$ \\
\hline a. Roads & $\begin{array}{l}\text { 3. No } \\
\text { Yes } \rightarrow \\
\downarrow\end{array}$ & $\begin{array}{l}\text { 6. NA } 1 . \\
\Downarrow\end{array}$ & $\begin{array}{l}\text { 1. very } \\
\text { severe } \\
\text { 2. severe } \\
\text { 3. medium } \\
\text { 4. light } \\
\text { 5. very light }\end{array}$ & 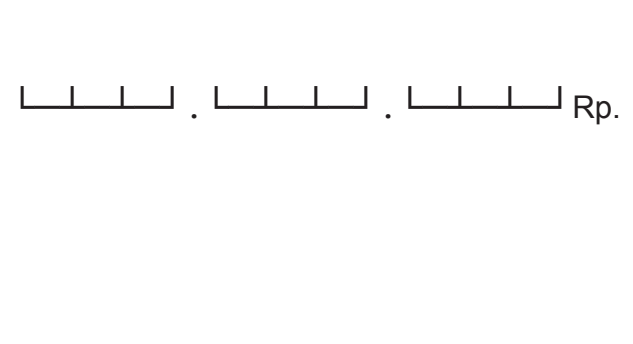 & $\begin{array}{l}\text { 3. No } \\
\text { 1. Yes } \\
\rightarrow \\
\downarrow\end{array}$ & $\begin{array}{lll}A & B & C \\
& D^{-} & \\
& H & \end{array}$ & $\begin{array}{l}\text { 1. Better than they were before } \\
\text { 2. About the same as before } \\
\text { 3. Worst than they were before }\end{array}$ & $\begin{array}{l}\text { 1. Satisfied } \\
2 . \\
\text { Somewhat } \\
\text { satisfied } \\
3 . \\
\text { Somewhat } \\
\text { unsatisfied } \\
4 . \\
\text { Unsatisfied }\end{array}$ \\
\hline b. Bridges & $\begin{array}{l}\text { 3. No } \\
\text { Yes } \rightarrow \\
\downarrow\end{array}$ & $\begin{array}{l}\text { 6. NA } 1 . \\
\downarrow\end{array}$ & $\begin{array}{l}\text { 1. very } \\
\text { severe } \\
\text { 2. severe } \\
\text { 3. medium } \\
\text { 4. light } \\
\text { 5. very light }\end{array}$ & 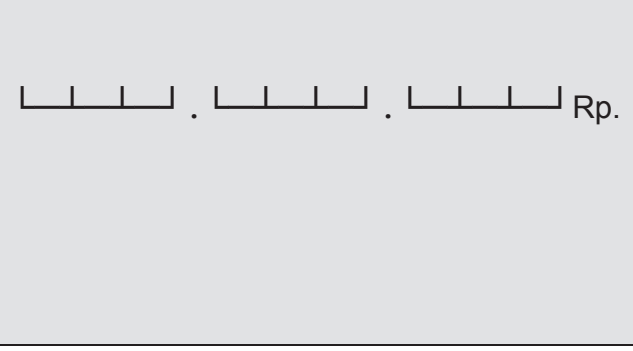 & $\begin{array}{l}\text { 3. No } \\
\text { 1. Yes } \\
\rightarrow \\
\downarrow\end{array}$ & 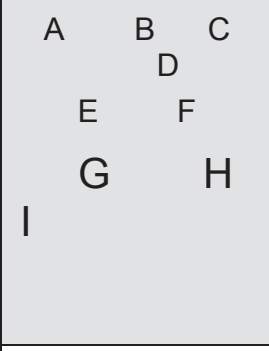 & $\begin{array}{l}\text { 1. Better than they were before } \\
\text { 2. About the same as before } \\
\text { 3. Worst than they were before }\end{array}$ & $\begin{array}{l}\text { 1. Satisfied } \\
2 . \\
\text { Somewhat } \\
\text { satisfied } \\
\text { 3. } \\
\text { Somewhat } \\
\text { unsatisfied } \\
4 . \\
\text { Unsatisfied }\end{array}$ \\
\hline c. Ports & $\begin{array}{l}\text { 3. No } \\
\text { Yes } \rightarrow \\
\downarrow\end{array}$ & $\begin{array}{l}\text { 6. NA } 1 . \\
\downarrow\end{array}$ & $\begin{array}{l}\text { 1. very } \\
\text { severe } \\
\text { 2. severe } \\
\text { 3. medium } \\
\text { 4. light } \\
\text { 5. very light }\end{array}$ & 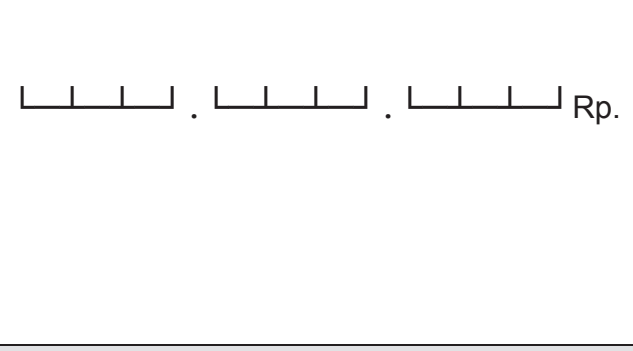 & $\begin{array}{l}\text { 3. No } \\
\text { 1. Yes } \\
\Rightarrow \\
\downarrow\end{array}$ & 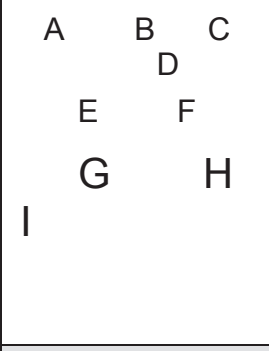 & $\begin{array}{l}\text { 1. Better than they were before } \\
\text { 2. About the same as before } \\
\text { 3. Worst than they were before }\end{array}$ & $\begin{array}{l}\text { 1. Satisfied } \\
2 . \\
\text { Somewhat } \\
\text { satisfied } \\
\text { 3. } \\
\text { Somewhat } \\
\text { unsatisfied } \\
4 . \\
\text { Unsatisfied }\end{array}$ \\
\hline d. Airports & $\begin{array}{l}\text { 3. No } \\
\text { Yes } \rightarrow \\
\downarrow\end{array}$ & $\begin{array}{l}\text { 6. NA } 1 \text {. } \\
\downarrow\end{array}$ & $\begin{array}{l}\text { 1. very } \\
\text { severe } \\
\text { 2. severe } \\
\text { 3. medium } \\
\text { 4. light } \\
\text { 5. very light }\end{array}$ & 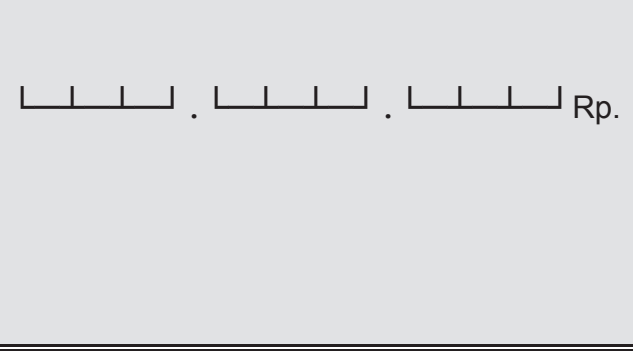 & $\begin{array}{l}\text { 3. No } \\
\text { 1. Yes } \\
\rightarrow \\
\downarrow\end{array}$ & 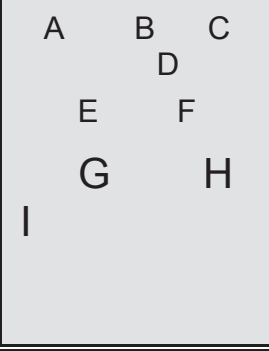 & $\begin{array}{l}\text { 1. Better than they were before } \\
\text { 2. About the same as before } \\
\text { 3. Worst than they were before }\end{array}$ & $\begin{array}{l}\text { 1. Satisfied } \\
2 . \\
\text { Somewhat } \\
\text { satisfied } \\
3 . \\
\text { Somewhat } \\
\text { unsatisfied } \\
4 . \\
\text { Unsatisfied }\end{array}$ \\
\hline
\end{tabular}


SECTION F : NATURAL DISASTER

\begin{tabular}{|c|c|c|c|c|c|c|c|c|}
\hline $\begin{array}{l}\text { e. Railways/Rail } \\
\text { Station }\end{array}$ & $\begin{array}{l}\text { 3. No } \\
\text { Yes } \rightarrow \\
\downarrow\end{array}$ & $\begin{array}{l}\text { 6. NA } 1 . \\
\downarrow\end{array}$ & $\begin{array}{l}\text { 1. very } \\
\text { severe } \\
\text { 2. severe } \\
\text { 3. medium } \\
\text { 4. light } \\
\text { 5. very light }\end{array}$ & 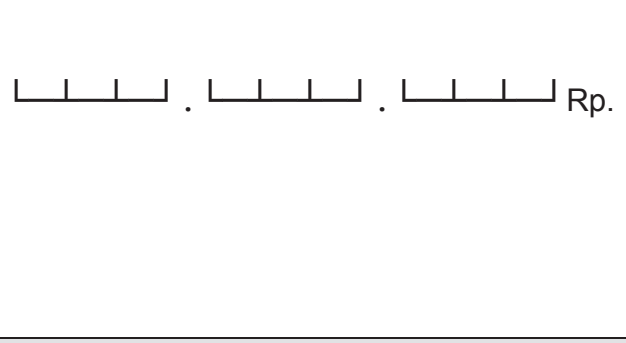 & $\begin{array}{l}\text { 3. No } \\
\text { 1. Yes } \\
\rightarrow \\
\downarrow\end{array}$ & $\begin{array}{ccc}A & B & C \\
& & D \\
& E & F \\
& G & H\end{array}$ & $\begin{array}{l}\text { 1. Better than they were before } \\
\text { 2. About the same as before } \\
\text { 3. Worst than they were before }\end{array}$ & $\begin{array}{l}\text { 1. Satisfied } \\
\text { 2. } \\
\text { Somewhat } \\
\text { satisfied } \\
\text { 3. } \\
\text { Somewhat } \\
\text { unsatisfied } \\
\text { 4. } \\
\text { Unsatisfied }\end{array}$ \\
\hline f. Schools & $\begin{array}{l}\text { 3. No } \\
\text { Yes } \rightarrow \\
\downarrow\end{array}$ & $\begin{array}{l}\text { 6. NA } 1 . \\
\downarrow\end{array}$ & $\begin{array}{l}\text { 1. very } \\
\text { severe } \\
\text { 2. severe } \\
\text { 3. medium } \\
\text { 4. light } \\
\text { 5. very light }\end{array}$ & $\downarrow \downarrow$ & $\begin{array}{l}\text { 3. No } \\
\text { 1. Yes } \\
\rightarrow \\
\downarrow\end{array}$ & $\begin{array}{ccc}A & B & C \\
& & D \\
& G & H \\
& & \end{array}$ & $\begin{array}{l}\text { 1. Better than they were before } \\
\text { 2. About the same as before } \\
\text { 3. Worst than they were before }\end{array}$ & $\begin{array}{l}\text { 1. Satisfied } \\
2 . \\
\text { Somewhat } \\
\text { satisfied } \\
3 . \\
\text { Somewhat } \\
\text { unsatisfied } \\
4 . \\
\text { Unsatisfied }\end{array}$ \\
\hline & & & & & \multicolumn{3}{|c|}{$\begin{array}{l}\text { Code for F06: } \\
\text { A. Central government } \\
\text { B. Regional government } \\
\text { C. Religious organization }\end{array}$} & \\
\hline
\end{tabular}




\section{SECTION F : NATURAL DISASTER}

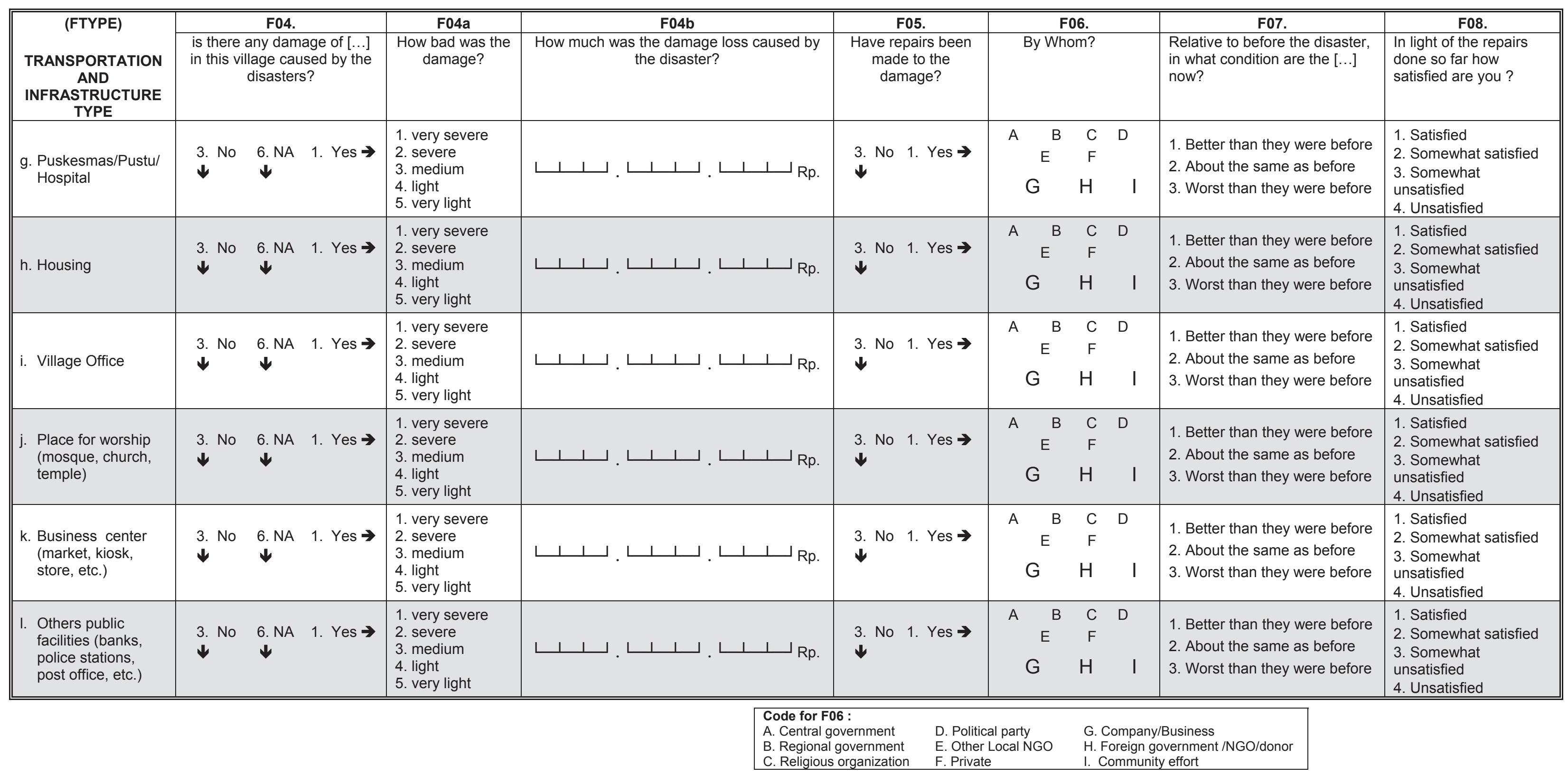




\section{SECTION F : NATURAL DISASTER}

F09. Has there been a briefing about disaster preparedness to the people of the village?

F10. Who gave the briefing about disaster preparedness?

\section{(CIRCLE ALL THAT APPLY)}

F11. Has there been a training about disaster preparedness in this village?

F12. What has been prepared by the village to anticipate potential natura disaster?

\section{(CIRCLE ALL THAT APPLY)}

No ………......... $3 \rightarrow$ F11

DON'T KNOW

Yes

District (Kabupaten/Kota) official........... A

Village official ...................................... B

Community leaders ………………....... C

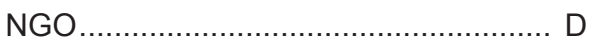

Students/University ................................. E

Community organization ......................... F

Political Party .......................................... G

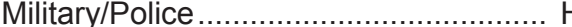

No ……..................................... $3 \rightarrow$ E15a

Yes..................................................... 1

Food storage ...................................... A

Volunteer …………………………….... B

Shelter .................................................. C

Early warning system .............................. D

Evacuation route ................................ E

Information center/Command post ....... F

Command post officer

Medical post....................................... $\mathrm{H}$

Fund .............................................. I

Bunker................................................ J

NO PREPARATION

W

F13. What is the amount of village budge allocated to increase disaster preparedness?

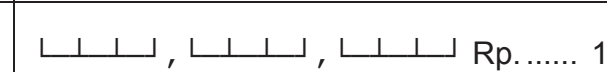

None ................................................... 3

DON'T KNOW
E15a. ever experienced any smoke clouds (from forest fire)?

15b In the past 5 years, how many times did you see smoke clouds ( from forest fire)?

E16a. When did this village experienced smoke clouds for the first time?

17a. When did this village experienced smoke clouds for the last time?

A

A

C

F

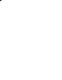

W

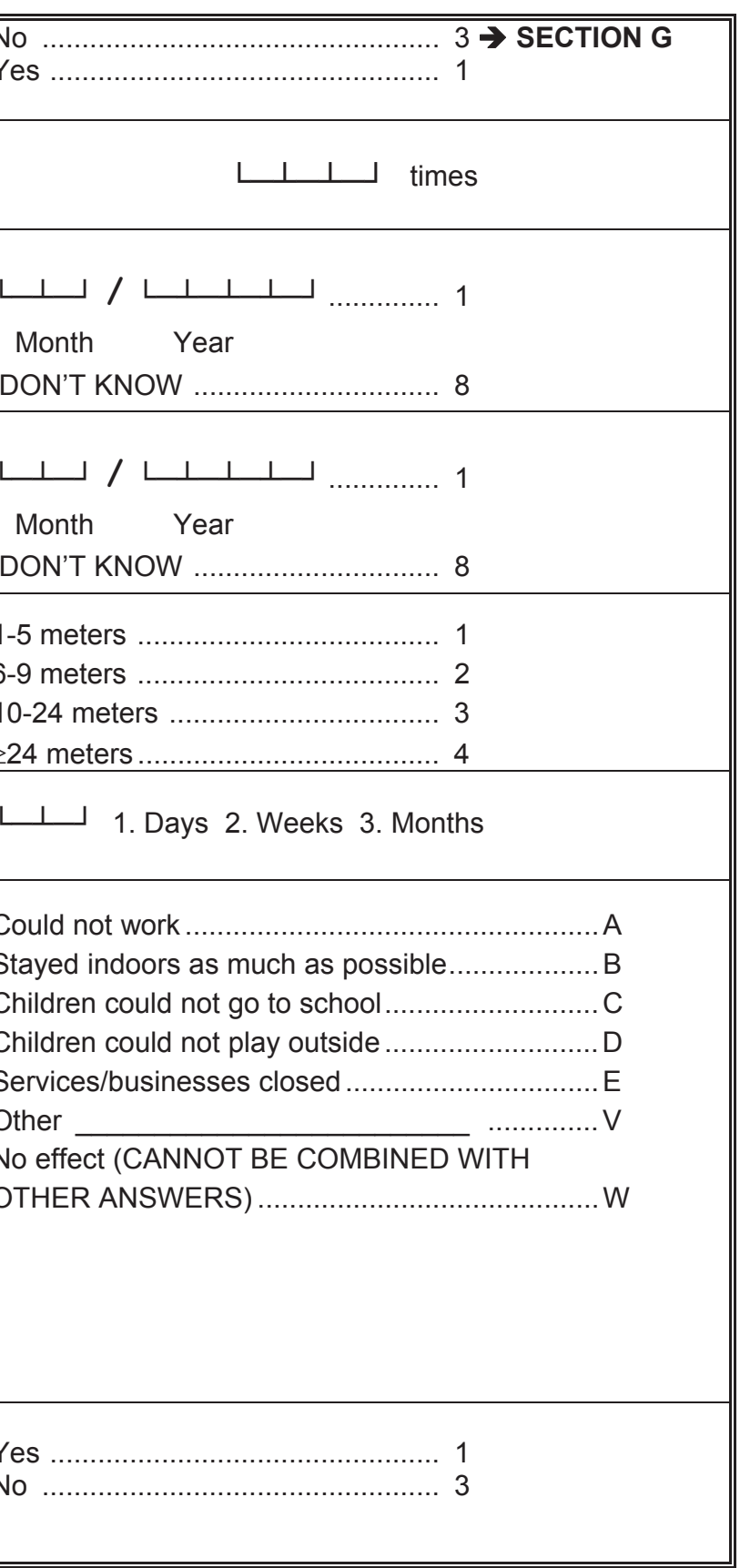

E28. At the time when the smoke cloud were at their worst (in the last 6 months), did residents in your community have to wear masks?

\begin{tabular}{|c|c|c|}
\hline E26a. & $\begin{array}{l}\text { Approximately how long does the } \\
\text { smoke last before it stopped } \\
\text { completely? }\end{array}$ & لــ 1. Days 2. Weeks 3. Months \\
\hline E27. & $\begin{array}{l}\text { At the time when the smoke clouds } \\
\text { were at their worst in the last } 6 \\
\text { months, how were the normal daily } \\
\text { activities of residents of your } \\
\text { community affected? } \\
\text { (CIRCLE ALL THAT APPLY) }\end{array}$ & 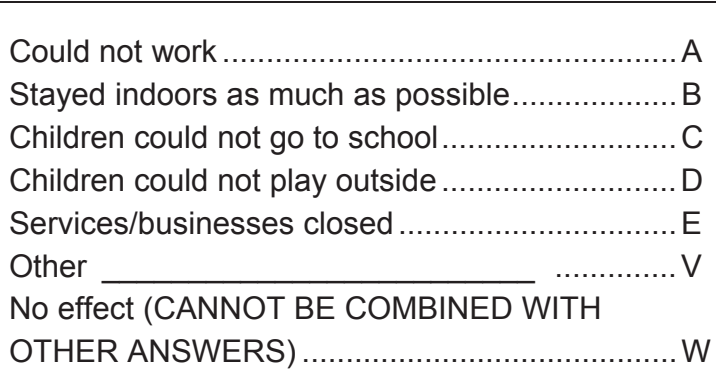 \\
\hline E28. & $\begin{array}{l}\text { At the time when the smoke clouds } \\
\text { were at their worst (in the last } 6 \\
\text { months), did residents in your } \\
\text { community have to wear masks? }\end{array}$ & Yes ……… \\
\hline
\end{tabular}


SECTION F : NATURAL DISASTER 


\section{SECTION CP: INTERVIEWER NOTES}

\begin{tabular}{|c|c|c|c|c|c|c|}
\hline LANGMAIN (CK1). & \multicolumn{2}{|l|}{ Interview was entirely/mostly conducted in what language? } & \multicolumn{4}{|l|}{ 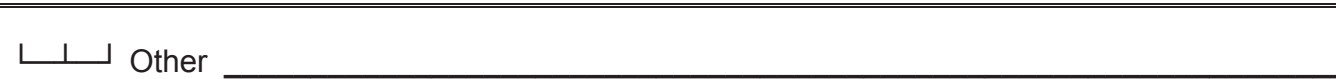 } \\
\hline LANGOTHR (CK2.) & Other languaged used (if any): & & Iـ Other & & & \\
\hline \multicolumn{7}{|l|}{ LANGUAGE CODE: } \\
\hline 00. Bahasa Indonesia & 04. Batak & Sasak & 12. Makassar & 16. Toraja & & Lampung \\
\hline 01. Java & 05. Bugis & Minang & 13. Nias & 17. Lahat & 95. & Other, \\
\hline 02. Sunda & 06. Cina & 10. Banjar & 14. Palembang & 18. Other South Sumatera & 96. & NA \\
\hline 03. Bali & 07. Madura & 11. Bima & 15. Sumbawa & 19. Betawi & & \\
\hline
\end{tabular}

\begin{tabular}{|c|c|c|c|c|}
\hline RESULT (FP3). RESULTS OF INTERVIEW & REASON (FP4). REASON FOR ANSWERING “2” / “3” IN RESULT. & \multicolumn{3}{|c|}{ FP6. MONITORING BY SUPERVISOR } \\
\hline $\begin{array}{l}\text { 1. Completed } \rightarrow \text { FP6 } \\
\text { 2. Partly completed } \\
\text { 3. Not completed } \\
\text { 4. Twin EA with EA } \downarrow \perp \rightarrow F P 6\end{array}$ & $\begin{array}{l}\text { 1. Respondent was travelling/not in location } \\
\text { 2. Respondent was too busy } \\
\text { 3. Respondent refused }\end{array}$ & & $\begin{array}{l}\text { Observed (sup_obs)..........1 } \\
\text { Checked (sup_edit) ...........1 } \\
\text { Verified (sup_veri) ............1 }\end{array}$ & $\begin{array}{l}\text { No } \\
3 \\
3 \\
3\end{array}$ \\
\hline
\end{tabular}

\section{INTERVIEWER NOTE:}




\section{INDONESIAN FAMILY LIFE SURVEY 2014 \\ COMMUNITY AND FACILITY \\ PEOPLE CHARACTERISTICS BOOK IB}

SECTIONS : G, I, J, PMKD, SW, CP

NAME OF DESA/KELURAHAN :

EA / BOOK TYPE

$\perp \perp / \mathrm{B}_{\mid} \mathrm{K}_{1} 1$

COV1X. CAPI CHECK: IS THIS A TWIN EA?

1. Yes $\rightarrow$ CK1 


\section{SECTION G : SAVING AND BORROWING}

\begin{tabular}{|c|c|}
\hline IR1 & IR2 \\
\hline $\begin{array}{l}\text { 1_G. Name of Respondent: } \\
\text { CAPI WILL SKIP TO G3a IF THE SAME NAME HAS } \\
\text { ALREADY BEEN ENTERED EARLIER }\end{array}$ & \\
\hline Position in the village : & $\begin{array}{ccccc}01 & 02 & 03 & 04 & 05 \\
06 & 07 & 95 & \ldots \ldots \ldots \ldots \ldots . .\end{array}$ \\
\hline 2_G. Age: & $\downarrow$ Years \\
\hline 2a_G. Length of tenure in positions: & 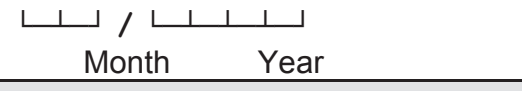 \\
\hline $\begin{array}{ll}\text { 3_G. INTERVIEWER CHECK : } & \text { SEX OF RESPONDENT } \\
& \text { SESPE }\end{array}$ & 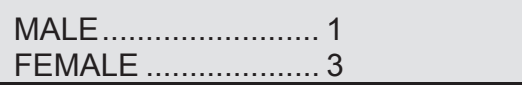 \\
\hline 3a_G. Highest level of education attended: & $\sqcup$ \\
\hline 4_G. Highest class completed: & $\begin{array}{lllll}00 & 01 & 02 & 03 & 04 \\
05 & 06 & 07 & 98 & 96 \\
\end{array}$ \\
\hline 5_G. Length of time lived in the village : & $\begin{array}{l}\text { 1. } L_{\text {Month }} / L_{\text {Year }} \\
\text { 3. Since birth } \\
\text { 6. Don't live in the village/ township }\end{array}$ \\
\hline 6_G. How did $[\ldots]$ come to assume to the position & $\begin{array}{l}\text { 1. Elected } \\
\text { 2. Appointed by residence } \\
\text { 3. Appointed by camat } \\
\text { 4. Appointed by Bupati/Walikota } \\
\text { 5. Other }\end{array}$ \\
\hline
\end{tabular}

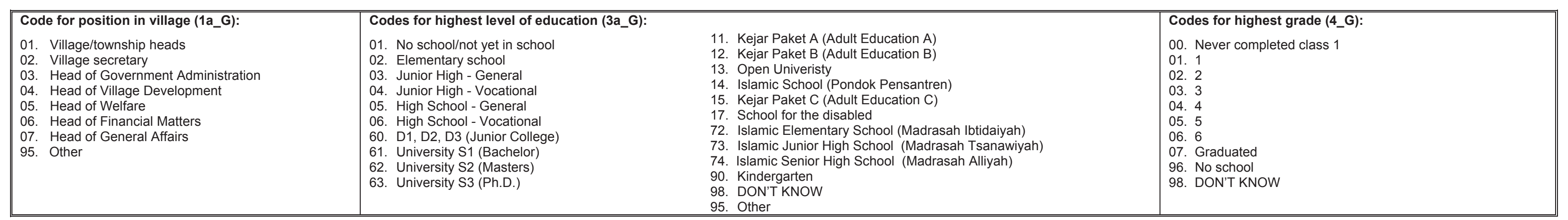




\section{SECTION G : SAVING AND BORROWING}

Now, we want to ask about saving and borrowing habit in this village.

\begin{tabular}{|c|c|c|c|c|c|}
\hline \multirow{2}{*}{ (GTYPE) } & \multicolumn{2}{|l|}{ G3a. } & \multicolumn{3}{|c|}{ G3c. } \\
\hline & $\begin{array}{l}\text { Is there }[\ldots] \text { in or outside this village } \\
\text { where people go to, to borrow and save } \\
\text { money? }\end{array}$ & $\begin{array}{l}\text { How many kilometers is it from the } \\
\text { community center to this institution? }\end{array}$ & \multicolumn{3}{|c|}{ What types of services are available at this institution $[\ldots]$ ? } \\
\hline 1. Bank BRI (Bank Rakyat Indonesia) & $\begin{array}{l}\text { 1. In the village } \rightarrow \mathbf{G} 3 \mathbf{c} \\
\text { 3. Outside the village } \\
\text { 6. N/A } \downarrow \\
\text { 8. DON'T KNOW } \downarrow\end{array}$ & 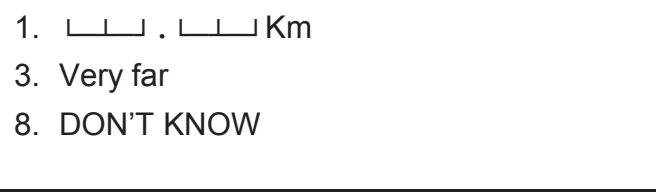 & $\begin{array}{ll}\text { A. } & \text { Savings account } \\
\text { B. Checking account } \\
\text { C. Deposit certificate } \\
\text { D. Letter of credit } \\
\text { E. } & \text { Business loan }\end{array}$ & $\begin{array}{ll}\text { F. } & \text { Vehicle loan } \\
\text { G. House loan } \\
\text { H. Personal loan (education, } \\
\text { I. Health) } \\
\text { J. Mealth/life insurance } \\
\text { J. Micro credit } \\
\end{array}$ & Y. DON'T KNOW \\
\hline 2. People Credit Bank (BPR) & $\begin{array}{l}\text { 1. In the village } \rightarrow \mathbf{G} 3 \mathbf{c} \\
\text { 3. Outside the village } \\
\text { 6. N/A } \downarrow \\
\text { 8. DON'T KNOW } \downarrow\end{array}$ & $\begin{array}{l}\text { 1. } \sqcup \perp . \sqcup \sqcup \mathrm{Km} \\
\text { 3. Very far } \\
\text { 8. DON'T KNOW }\end{array}$ & $\begin{array}{ll}\text { A. } & \text { Savings account } \\
\text { B. Checking account } \\
\text { C. Deposit certificate } \\
\text { D. Letter of credit } \\
\text { E. Business loan }\end{array}$ & $\begin{array}{ll}\text { F. Vehicle loan } \\
\text { G. House loan } \\
\text { H. Personal loan (education, } \\
\text { I. Health) } \\
\text { J. Mealth/life insurance } \\
\text { j. Micro credit }\end{array}$ & Y. DON'T KNOW \\
\hline 3. Village Credit Institution $(L K D)$ & $\begin{array}{l}\text { 1. In the village } \rightarrow \mathbf{G} 3 \mathbf{c} \\
\text { 3. Outside the village } \\
\text { 6. N/A } \downarrow \\
\text { 8. DON'T KNOW } \downarrow\end{array}$ & $\begin{array}{l}\text { 1. } \sqcup \sqcup . \sqcup \sqcup \mathrm{Km} \\
\text { 3. Very far } \\
\text { 8. DON'T KNOW }\end{array}$ & $\begin{array}{ll}\text { A. } & \text { Savings account } \\
\text { B. Checking account } \\
\text { C. Deposit certificate } \\
\text { D. Letter of credit } \\
\text { E. Business loan } \\
\end{array}$ & $\begin{array}{ll}\text { F. } & \text { Vehicle loan } \\
\text { G. House loan } \\
\text { H. Personal loan (education, } \\
\text { I. Health) } \\
\text { J. Mealth/life insurance } \\
\text { J. Micro credit } \\
\end{array}$ & Y. DON'T KNOW \\
\hline 4. Village Unit Cooperative (KUD) & $\begin{array}{l}\text { 1. In the village } \rightarrow \mathbf{G} 3 \mathbf{c} \\
\text { 3. Outside the village } \\
\text { 6. N/A } \downarrow \\
\text { 8. DON'T KNOW } \downarrow\end{array}$ & $\begin{array}{l}\text { 1. } \sqcup \perp . \sqcup \sqcup \mathrm{Km} \\
\text { 3. Very far } \\
\text { 8. DON'T KNOW }\end{array}$ & $\begin{array}{ll}\text { A. } & \text { Savings account } \\
\text { B. Checking account } \\
\text { C. Deposit certificate } \\
\text { D. Letter of credit } \\
\text { E. Business loan }\end{array}$ & $\begin{array}{ll}\text { F. } & \text { Vehicle loan } \\
\text { G. House loan } \\
\text { H. Personal loan (education, } \\
\text { I. health) } \\
\text { j. Mellife insurance } \\
\text { J. Micro credit } \\
\end{array}$ & $\begin{array}{l}\text { Y. DON'T KNOW } \\
\text { W. NA }\end{array}$ \\
\hline $\begin{array}{l}\text { 5. Other Formal Cooperative (THE } \\
\text { MAIN ONE) }\end{array}$ & $\begin{array}{l}\text { 1. In the village } \rightarrow \mathbf{G} 3 \mathbf{c} \\
\text { 3. Outside the village } \\
\text { 6. N/A } \downarrow \\
\text { 8. DON'T KNOW } \downarrow\end{array}$ & $\begin{array}{l}\text { 1. } \sqcup \sqcup . \sqcup \sqcup \mathrm{Km} \\
\text { 3. Very far } \\
\text { 8. DON'T KNOW }\end{array}$ & $\begin{array}{ll}\text { A. } & \text { Savings account } \\
\text { B. } & \text { Checking account } \\
\text { C. Deposit certificate } \\
\text { D. Letter of credit } \\
\text { E. } & \text { Business loan }\end{array}$ & $\begin{array}{ll}\text { F. } & \text { Vehicle loan } \\
\text { G. } & \text { House loan } \\
\text { H. Personal loan (education, } \\
\text { health) } \\
\text { I. Health/life insurance } \\
\text { J. Micro credit } \\
\end{array}$ & Y. DON'T KNOW \\
\hline $\begin{array}{l}\text { 6. State/private bank (THE MAIN } \\
\text { ONE) }\end{array}$ & $\begin{array}{l}\text { 1. In the village } \rightarrow \mathbf{G} 3 \mathbf{c} \\
\text { 3. Outside the village } \\
\text { 6. N/A } \downarrow \\
\text { 8. DON'T KNOW } \downarrow\end{array}$ & $\begin{array}{l}\text { 1. } \downarrow \text { لـــ Km } \\
\text { 3. Very far } \\
\text { 8. DON'T KNOW }\end{array}$ & $\begin{array}{ll}\text { A. } & \text { Savings account } \\
\text { B. } & \text { Checking account } \\
\text { C. Deposit certificate } \\
\text { D. Letter of credit } \\
\text { E. } & \text { Business loan }\end{array}$ & $\begin{array}{ll}\text { F. } & \text { Vehicle loan } \\
\text { G. House loan } \\
\text { H. Personal loan (education, } \\
\text { I. Health) } \\
\text { J. Mealth/life insurance } \\
\text { J. Micro credit } \\
\end{array}$ & Y. DON'T KNOW \\
\hline 7. Baitul Maal Wat Tamwil (BMT) & $\begin{array}{l}\text { 1. In the village } \rightarrow \mathbf{G} 3 \mathbf{c} \\
\text { 3. Outside the village } \\
\text { 6. N/A } \Downarrow \\
\text { 8. DON'T KNOW } \downarrow\end{array}$ & $\begin{array}{l}\text { 1. } \downarrow\lrcorner \sqcup \sqcup \sqcup \mathrm{Km} \\
\text { 3. Very far } \\
\text { 8. DON'T KNOW }\end{array}$ & $\begin{array}{ll}\text { A. } & \text { Savings account } \\
\text { B. } & \text { Checking account } \\
\text { C. } & \text { Deposit certificate } \\
\text { D. } & \text { Letter of credit } \\
\text { E. } & \text { Business loan }\end{array}$ & $\begin{array}{ll}\text { F. } & \text { Vehicle loan } \\
\text { G. House loan } \\
\text { H. Personal loan (education, } \\
\text { I. Health) } \\
\text { J. Mealth/life insurance } \\
\text { J. Micro credit } \\
\end{array}$ & Y. DON'T KNOW \\
\hline 8. Syariah/Islamic Bank & $\begin{array}{l}\text { 1. In the village } \rightarrow \mathbf{G} 3 \mathbf{c} \\
\text { 3. Outside the village } \\
\text { 6. N/A } \downarrow \\
\text { 8. DON'T KNOW } \downarrow\end{array}$ & 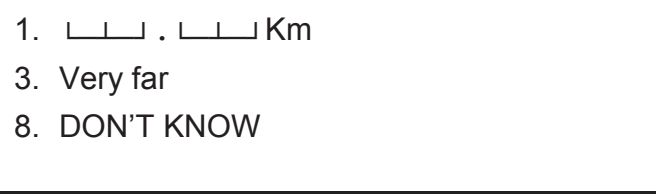 & $\begin{array}{ll}\text { A. } & \text { Savings account } \\
\text { B. } & \text { Checking account } \\
\text { C. } & \text { Deposit certificate } \\
\text { D. } & \text { Letter of credit } \\
\text { E. } & \text { Business loan }\end{array}$ & $\begin{array}{ll}\text { F. } & \text { Vehicle loan } \\
\text { G. House loan } \\
\text { H. Personal loan (education, } \\
\text { health) } \\
\text { I. Health/life insurance } \\
\text { j. Micro credit }\end{array}$ & Y. DON'T KNOW \\
\hline
\end{tabular}




\section{SECTION G : SAVING AND BORROWING}

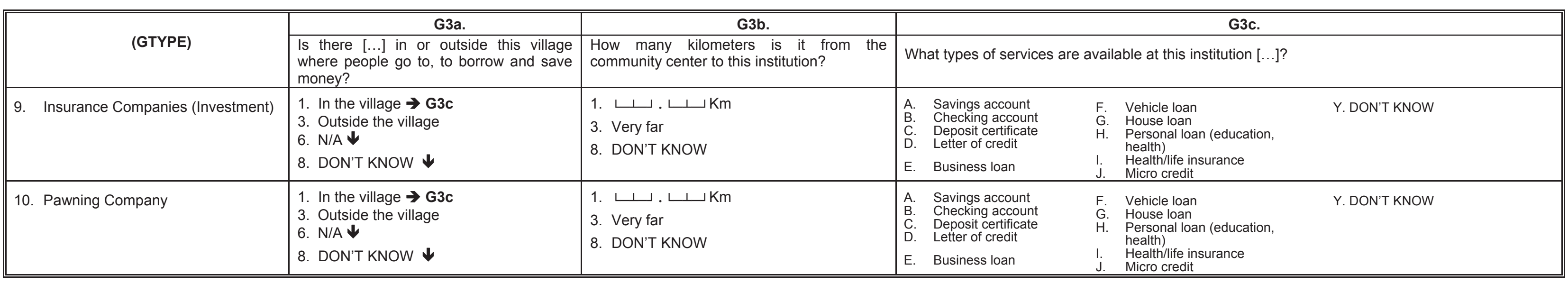

G6. In this village is there credit available from sources other than financial institutions, such as 


\section{SECTION I : THE PRESENCE OF SCHOOL}

Section I Respondent:

\begin{tabular}{|c|c|}
\hline 2 & IR2 \\
\hline $\begin{array}{l}\text { 1_I. Name of Respondent : } \\
\text { CAPI WILL SKIP TO I13 IF THE SAME NAME HAS } \\
\text { ALREADY BEEN ENTERED EARLIER } \\
\end{array}$ & \\
\hline 1a_l. Position in the village : & $\begin{array}{lllll}01 & 02 & 03 & 04 & 05 \\
06 & 07 & 95 & \ldots \ldots \ldots \ldots \ldots . .\end{array}$ \\
\hline 2_l. Age: & $\downarrow$ Years \\
\hline 2a_l. Length of tenure in positions: & 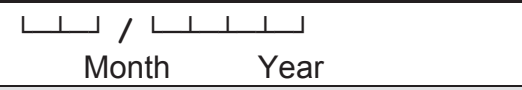 \\
\hline $\begin{array}{ll}\text { 3_ I. } & \text { INTERVIEWER CHECK : } \\
& \text { SEX OF RESPONDENT }\end{array}$ & $\begin{array}{l}\text { MALE } \ldots \ldots \ldots \ldots \ldots \ldots \ldots \ldots \ldots \ldots . . .1 \\
\text { FEMALE } \ldots \ldots \ldots \ldots \ldots \ldots \ldots \ldots \ldots \ldots \ldots \ldots \\
\end{array}$ \\
\hline 3a_I. Highest level of education attended & 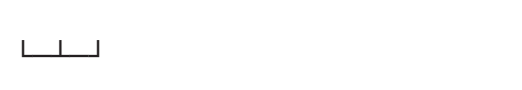 \\
\hline 4_I. Highest class completed: & $\begin{array}{lllll}00 & 01 & 02 & 03 & 04 \\
05 & 06 & 07 & 98 & 96 \\
\end{array}$ \\
\hline 5_I. Length of time lived in the village: & 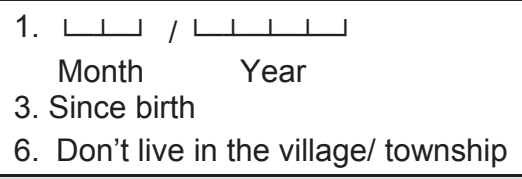 \\
\hline $6 \_$I. How did $[\ldots]$ come to assume to the position & $\begin{array}{l}\text { 1. Elected } \\
\text { 2. Appointed by residence } \\
\text { 3. Appointed by camat } \\
\text { 4. Appointed by Bupati/Walikota } \\
\text { 5. Other }\end{array}$ \\
\hline
\end{tabular}

\section{Code for position in village (1a I):}

01. Village/township heads

02. Village secretary

Government Administration

04. Head of Village Development

05. Head of Welfare

06. Head of Financial Matters
07. Head of General Affairs

95. Other
01. No school/not yet in school
02 . Elementary school
02. Elementary school
04. Junior High - Vocational
05. High School - General
06. High School - Vocational
60. D1, D2, D3 (Junior College)
61. University S1 (Bachelor)
62. University S2 (Masters)
63. University S3 (Ph.D.)

11. Kejar Paket A (Adult Education $A$ )

12. Kejar Paket $B$ (Adult Education $B$ )

13. Open Univeristy

14. Islamic School (Pondok Pensantren)

15. Kejar Paket C (Adult Education C)

72. Islamic Elementary School (Madrasah Ibtidaiyah)

73. Islamic Junior High School (Madrasah Tsanawiya

98. DON'T KNOW

95. Other
Codes for highest grade (4 I):

00. Never completed class 1

01. 1

03.3

04.4

05. 5

06. 6

07. Graduated

98. DON'T KNOW 


\section{SECTION I : THE PRESENCE OF SCHOOL}

We would like to ask about the presence of Elementary School, Junior High School and Senior High School.

\begin{tabular}{|c|c|c|c|c|c|}
\hline & A.11 & A1. PRE ELEMENTARY SCHOOL & A. ELEMENTARY SCHOOL & B. JUNIOR HIGH SCHOOL & C. SENIOR HIGH SCHOOL \\
\hline I13. & $\begin{array}{l}\text { How many [...] schools are used by } \\
\text { the local population? }\end{array}$ & $\begin{array}{l}\text { 6. NONE } \rightarrow \text { I13 COLUMN A } \\
\text { 1. لـ schools } \\
\text { 8. DON'T KNOW }\end{array}$ & $\begin{array}{l}\text { 6. NONE } \rightarrow \text { I13 COLUMN B } \\
\text { 1. ــ J schools } \\
\text { 8. DON'T KNOW }\end{array}$ & $\begin{array}{l}\text { 6. NONE } \rightarrow \text { I13 COLUMN C } \\
\text { 1. } \downarrow \text { I schools } \\
\text { 8. DON'T KNOW }\end{array}$ & $\begin{array}{l}\text { 6. NONE } \rightarrow \mathrm{J} 10 \mathrm{a} \\
\text { 1. Lــ J schools } \\
\text { 8. DON'T KNOW }\end{array}$ \\
\hline \multirow[t]{20}{*}{$\begin{array}{l}\text { I14. } \\
\text { I14a. }\end{array}$} & $\begin{array}{l}\text { Please list the names of the } \\
\text { schools. }\end{array}$ & $\begin{array}{l}\text { I14. I14a. I14b } \\
\text { 1. } \longleftarrow \quad \sqcup\end{array}$ & $\begin{array}{l}\text { I14. I14a. } \\
1 . \quad \sqcup\end{array}$ & $\begin{array}{l}\text { I14. I14a. } \\
1 . \quad \sqcup\end{array}$ & $\begin{array}{r}\text { I14a. } \\
\square\end{array}$ \\
\hline & \multirow{3}{*}{$\begin{array}{l}\text { INTERVIEWER'S NOTE: } \\
\text { INDICATE WHETHER THE } \\
\text { SCHOOL IS LOCATED WITHIN } \\
\text { THIS VILLAGE OR OUTSIDE } \\
\text { THIS VILLAGE. }\end{array}$} & Kode fasilitas $\llcorner\perp \perp \sqcup \sqcup \downarrow \perp$ & Kode fasilitas $\llcorner\perp \perp \sqcup \sqcup \sqcup \perp \perp$ & Kode fasilitas $\llcorner\perp \perp \sqcup \sqcup \sqcup \downarrow \downarrow$ & Kode fasilitas $\llcorner\perp \perp \sqcup \sqcup \sqcup \perp \perp$ \\
\hline & & 2. & 2. & 2. $\longleftarrow$ & 2. \\
\hline & & Kode fasilitas $\llcorner\perp \perp \sqcup \sqcup \sqcup 1 \perp$ & Kode fasilitas $\llcorner\perp \perp \sqcup \sqcup \sqcup \perp \perp$ & Kode fasilitas $\llcorner\perp \perp \sqcup \sqcup \sqcup \perp \perp$ & Kode fasilitas $\llcorner\perp \perp \sqcup \sqcup \sqcup \perp \perp$ \\
\hline & \multirow{3}{*}{$\begin{array}{l}\text { CODE 14a: } \\
\text { 1. IF SCHOOL IS IN THIS } \\
\text { VILLAGE } \\
\text { 3. IF SCHOOL IS NOT IN THIS } \\
\text { VILLAGE }\end{array}$} & 3. & 3. $\longrightarrow$ & 3. $\longleftarrow$ & \multirow{2}{*}{$\begin{array}{l}\text { 3. } \\
\text { Kode fasilitas }\llcorner\perp \sqcup \sqcup \sqcup \perp \perp \sqcup\end{array}$} \\
\hline & & Kode fasilitas $\llcorner\perp \perp \sqcup \sqcup \sqcup \perp \perp \neg$ & Kode fasilitas $\llcorner\perp \perp \sqcup \sqcup \sqcup \perp \perp \sqcup$ & Kode fasilitas $\llcorner\perp \perp \sqcup \sqcup \sqcup \perp \perp \sqcup$ & \\
\hline & & $\begin{array}{l}\text { 4. } \\
\text { Kode fasilitas } \sqcup \perp \sqcup \sqcup \sqcup \sqcup \sqcup\end{array}$ & $\begin{array}{l}\text { 4. } \\
\text { Kode fasilitas } \sqcup \perp \sqcup \sqcup \sqcup \perp \perp \sqcup\end{array}$ & $\begin{array}{l}\text { 4. } \\
\text { Kode fasilitas } \sqcup \perp \perp \sqcup \sqcup \perp \perp \sqcup\end{array}$ & $\begin{array}{l}\text { 4. } \\
\text { Kode fasilitas }\llcorner\perp \perp \sqcup \sqcup \perp \perp \sqcup\end{array}$ \\
\hline & \multirow{5}{*}{$\begin{array}{l}\text { CODE 14b: } \\
\text { 1. PLAYGROUP } \\
\text { 2. KINDERGARTEN } \\
\text { AFTER THE RESPONDENT } \\
\text { MENTIONS ALL NAMES, CHECK } \\
\text { SAR. }\end{array}$} & 5. & 5. & 5. & 5. \\
\hline & & Kode fasilitas $\llcorner\perp \perp \sqcup \sqcup \sqcup \perp \perp$ & Kode fasilitas $\llcorner\perp \perp \sqcup \sqcup \sqcup \perp \perp$ & Kode fasilitas $\llcorner\perp \perp\llcorner\sqcup \sqcup \perp \perp$ & Kode fasilitas $\llcorner\perp \perp \sqcup \sqcup \sqcup \perp \perp$ \\
\hline & & 6. & 6. & 6. & 6. \\
\hline & & Kode fasilitas $\llcorner\perp \perp \sqcup \sqcup \sqcup \perp \perp$ & Kode fasilitas $\llcorner\perp \perp \sqcup \sqcup \sqcup \perp \perp$ & Kode fasilitas $\llcorner\perp \perp \sqcup \sqcup \sqcup \perp \perp$ & Kode fasilitas $\llcorner\perp \perp \sqcup \sqcup \sqcup \perp \perp$ \\
\hline & & 7. & 7. $\longleftarrow$ & 7. $\longleftarrow$ & 7. \\
\hline & \multirow{8}{*}{$\begin{array}{l}\text { IF A NAME MENTIONED IS NOT } \\
\text { AT SAR, RECORD AT SAR AND } \\
\text { ASK ALL QUESTIONS OF SAR. }\end{array}$} & Kode fasilitas $\llcorner\perp \perp \sqcup \sqcup \sqcup \perp$ & Kode fasilitas $\llcorner\perp \perp \sqcup \sqcup \perp \perp$ & Kode fasilitas $\llcorner\perp \perp \sqcup \sqcup \sqcup \perp \perp$ & Kode fasilitas $\llcorner\perp \perp \sqcup \sqcup \sqcup \perp \perp$ \\
\hline & & 8. $\longleftarrow \sqcup \sqcup$ & 8. & 8. & 8. \\
\hline & & Kode fasilitas $\llcorner\perp \perp \sqcup \sqcup \sqcup \perp \perp$ & Kode fasilitas $\llcorner\perp \perp \sqcup \sqcup \sqcup \mid$ & Kode fasilitas $\llcorner\perp \perp \sqcup \sqcup \sqcup \perp \perp$ & Kode fasilitas $\llcorner\perp \perp \sqcup \sqcup \sqcup \perp \perp$ \\
\hline & & 9. & 9. & 9. & \multirow{2}{*}{ Kode fasilitas $\llcorner\perp \perp \sqcup \sqcup \sqcup \perp \sqcup$} \\
\hline & & Kode fasilitas $\llcorner\perp \perp \sqcup \sqcup \sqcup \perp \perp$ & Kode fasilitas $\llcorner\perp \perp \sqcup \sqcup \sqcup \sqcup$ & Kode fasilitas $\llcorner\perp \perp \sqcup \sqcup \sqcup \sqcup$ & \\
\hline & & 10. & 10. & 10. & \multirow{3}{*}{ 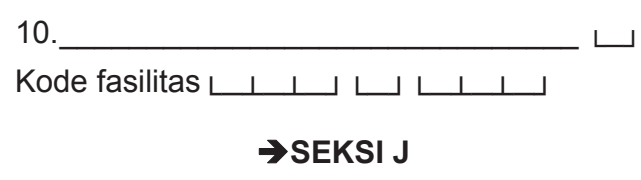 } \\
\hline & & Kode fasilitas $\llcorner\perp \perp \sqcup \sqcup \sqcup \perp \perp$ & Kode fasilitas $\llcorner\perp \perp \sqcup \sqcup\llcorner\perp$ & Kode fasilitas $\llcorner\perp\lrcorner \sqcup \sqcup \downarrow$ & \\
\hline & & $\Rightarrow I 13$ KOLOM SD & - I 13 KOLOM SMP & $\Rightarrow 13$ KOLOM SMA & \\
\hline
\end{tabular}


SECTION J : HISTORY OF THE PRESENCE OF HEALTH FACILITIES

Section I Respondent:

\begin{tabular}{|c|c|}
\hline 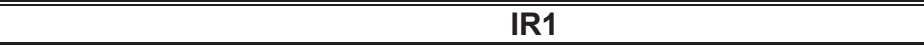 & IR2 \\
\hline $\begin{array}{l}\text { 1_J. Name of Respondent : } \\
\text { CAPI WILL SKIP TO J10a IF THE SAME NAME HAS } \\
\text { ALREADY BEEN ENTERED EARLIER }\end{array}$ & \\
\hline Position in the village : & $\begin{array}{ccccc}01 & 02 & 03 & 04 & 05 \\
06 & 07 & 95 & \ldots \ldots \ldots \ldots \ldots . .\end{array}$ \\
\hline 2_J. Age: & $\llcorner$ Years \\
\hline 2a_J. Length of tenure in positions: & 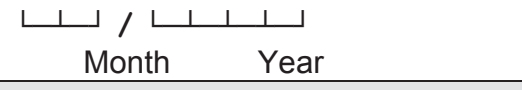 \\
\hline $\begin{array}{ll}3 \_J . & \text { INTERVIEWER CHECK : } \\
& \text { SEX OF RESPONDENT } \\
\end{array}$ & 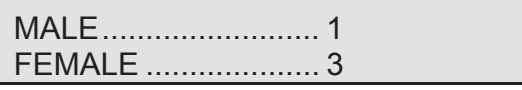 \\
\hline 3a_J. Highest level of education attended: & $\sqcup$ \\
\hline 4_J. Highest class completed: & $\begin{array}{lllll}00 & 01 & 02 & 03 & 04 \\
05 & 06 & 07 & 98 & 96 \\
\end{array}$ \\
\hline 5_ J. Length of time lived in the village: & $\begin{array}{l}\text { 1. }{ }_{\text {Month }} / \sqcup \text { Year } \\
\text { 3. Since birth } \\
\text { 6. Don't live in the village/ township }\end{array}$ \\
\hline $6 \_$J. How did $[\ldots]$ come to assume to the position & $\begin{array}{l}\text { 1. Elected } \\
\text { 2. Appointed by residence } \\
\text { 3. Appointed by camat } \\
\text { 4. Appointed by Bupati/Walikota } \\
\text { 5. Other }\end{array}$ \\
\hline
\end{tabular}

\section{Code for position in village (1a_J):}

\section{Village/township head}

03. Head of Government Administration

04. Head of Village Development

05. Head of Welfare

06. Head of Financial Matter

97. Other

\section{Codes for highest level of education (3a_J):}
01. No school/not yet in school
02. Elementary school
03. Junior High - General
04. Junior High - Vocational
05. High School - General
06. High School - Vocational
61. University $\mathrm{S} 1$ (Bacheler)
62. University $S 2$ (Masters)
63. University S3 (Ph.D.)

11. Kejar Paket A (Adult Education A)

2. Kejar Paket B (Adult Education B)

13. Open Univeristy

(Pondok Pensantren)

15. Kejar Paket C (Adult Education C)

72. Islamic Elementary School (Madrasah Ibtidaiyah)

73. Islamic Junior High

74. Islamic Senior High School (Madrasah Alliyah)

90. Kindergarten

95. Other
Codes for highest grade (4_J):

00 . Never completed class 1

01. 1

02.

03. 3

05. 5

07. Graduated

9. $\mathrm{No}$ school

96. No school
98. DON'T KNOW 
SECTION J : HISTORY OF THE PRESENCE OF HEALTH FACILITIES

Next, we want to ask about the presence of health facilities.

\begin{tabular}{|c|c|c|}
\hline J10a. & $\begin{array}{l}\text { Is there a Village Midwife (Bidan Desa) } \\
\text { located in this village? }\end{array}$ & 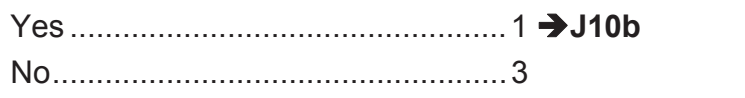 \\
\hline J10a1. & $\begin{array}{l}\text { Has there ever been a Village Midwife in } \\
\text { this village? }\end{array}$ & $\begin{array}{l}\text { No } \\
\text { Yes }\end{array}$ \\
\hline J10a2. & $\begin{array}{l}\text { When did the Village Midwife leave this } \\
\text { village? }\end{array}$ & 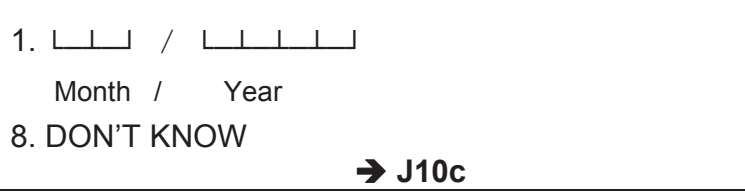 \\
\hline J10b. & $\begin{array}{l}\text { How many Village Midwives are located in } \\
\text { this village? }\end{array}$ & $\begin{array}{l}\text { 1. } ـ \perp \text { people } \\
\text { 8. DON'T KNOW }\end{array}$ \\
\hline J10c. & $\begin{array}{l}\text { In what year did the first Village Midwife } \\
\text { begin to practice in this village? }\end{array}$ & $\begin{array}{l}\text { 1. Year }\llcorner\perp \perp \perp \perp \rightarrow \mathbf{J 1 0 e} \\
\text { 8. DON'T KNOW }\end{array}$ \\
\hline J10d. & $\begin{array}{l}\text { How many years has the Village Midwife } \\
\text { begun to practice in this village? }\end{array}$ & $\begin{array}{l}\text { 1. } \longleftarrow \text { \ } \sqcup \text { years } \\
\text { 8. DON'T KNOW }\end{array}$ \\
\hline J10e. & $\begin{array}{l}\text { What type of support does/did the (first) } \\
\text { Village Midwife receive from the local } \\
\text { people or the village office? }\end{array}$ & $\begin{array}{ll}\text { A. } & \text { NONE } \\
\text { B. Housing } \\
\text { C. Food } \\
\text { D. A place to practice } \\
\text { E. Cash } \\
\text { V. } \\
\end{array}$ \\
\hline J10h. & $\begin{array}{l}\text { Where does the (first) Village Midwife } \\
\text { originate from? }\end{array}$ & $\begin{array}{ll}\text { Vill.: } & \text { 1. } \\
& \text { 3. Same location } \\
& \text { 8. DON'T KNOW } \\
\text { Kec: } & \text { 1. } \\
& \text { 3. Same location } \\
& \text { 8. DON'T KNOW } \\
\text { Kab: } & \text { 1. } \\
& \text { 3. Same location } \\
& \text { 8. DON'T KNOW } \\
\text { Prov : } & \text { 1. } \\
& \text { 3. Same location } \\
& \text { 8. DON'T KNOW } \\
\end{array}$ \\
\hline J19. & $\begin{array}{l}\text { In this village is there a Delivery Post (Pos } \\
\text { Bersalin Desa/Polindes)? }\end{array}$ & $\begin{array}{l}\text { No } \\
\text { Yes }\end{array}$ \\
\hline
\end{tabular}

\begin{tabular}{|c|c|c|}
\hline J19a. & $\begin{array}{l}\text { In what year was the first Polindes } \\
\text { established in this village? }\end{array}$ & 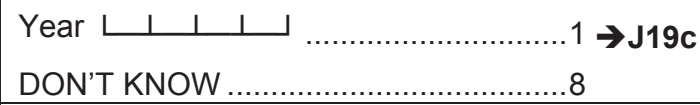 \\
\hline J19b. & $\begin{array}{l}\text { How many years has the Polindes } \\
\text { operated in this village? }\end{array}$ & 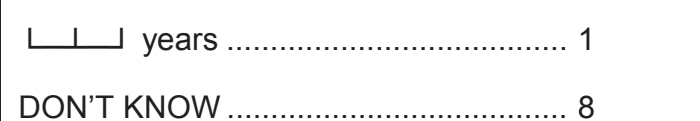 \\
\hline J19c. & How many beds does the Polindes have? & 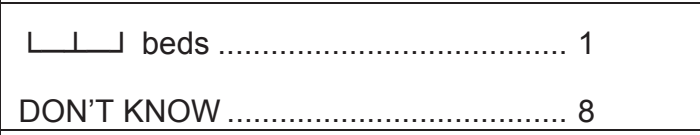 \\
\hline J11. & $\begin{array}{l}\text { How many Health Posts (Posyandu) exist } \\
\text { in this village? }\end{array}$ & 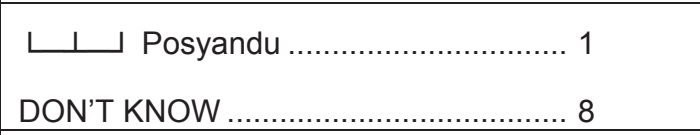 \\
\hline J13. & $\begin{array}{l}\text { How many times did staff from } \\
\text { Puskesmas visit the Posyandu last year? }\end{array}$ & 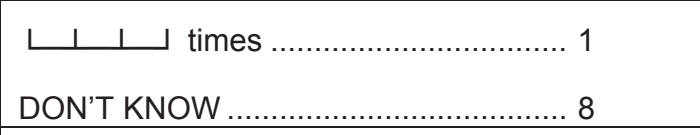 \\
\hline J13a. & $\begin{array}{l}\text { In comparison to the previous years, have } \\
\text { visits in the past } 12 \text { months from the } \\
\text { Puskesmas staff changed? }\end{array}$ & 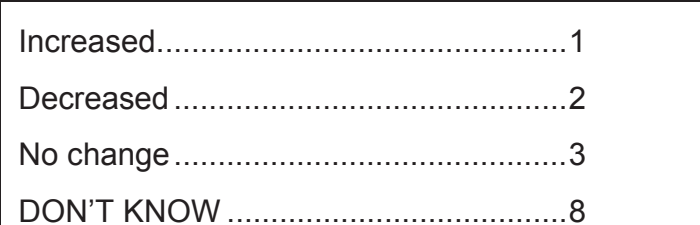 \\
\hline
\end{tabular}




\section{SECTION J : HISTORY OF THE PRESENCE OF HEALTH FACILITIES}

\begin{tabular}{|c|c|c|}
\hline J21a. & $\begin{array}{l}\text { Is there any Health Posts for elderly ( } \\
\text { Posyandu Lansia ) in this village? }\end{array}$ & 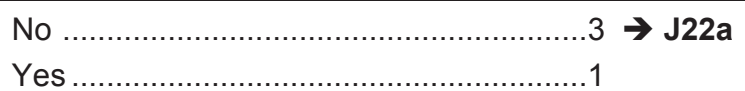 \\
\hline J21b. & $\begin{array}{l}\text { How many Health Posts for elderly ( } \\
\text { Posyandu Lansia ) are located in this } \\
\text { village? }\end{array}$ & 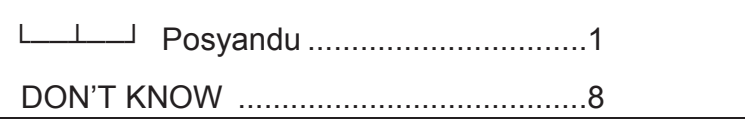 \\
\hline J21c. & $\begin{array}{l}\text { In what year did the first Health Posts for } \\
\text { elderly ( Posyandu Lansia) begin to } \\
\text { practice in this village? }\end{array}$ & 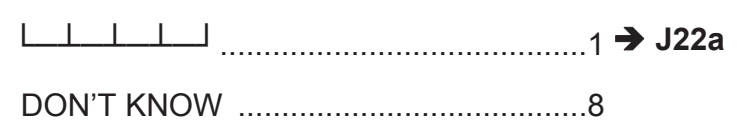 \\
\hline J21d. & $\begin{array}{l}\text { How many years has Health Posts for } \\
\text { elderly ( Posyandu Lansia) begun to } \\
\text { practice in this village? }\end{array}$ & 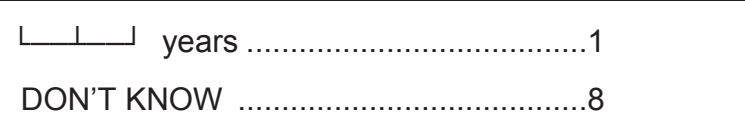 \\
\hline J22a. & Is there any Bidan Delima in this village? & $\begin{array}{l}\text { No } \\
\text { Yes }\end{array}$ \\
\hline J22b. & $\begin{array}{l}\text { How many Bidan Delima are located in } \\
\text { this village? }\end{array}$ & 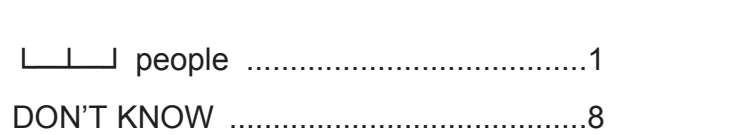 \\
\hline
\end{tabular}

\begin{tabular}{|c|c|c|}
\hline J22c. & $\begin{array}{l}\text { In what year did the first many Bidan } \\
\text { Delima begin to practice in this village? }\end{array}$ & 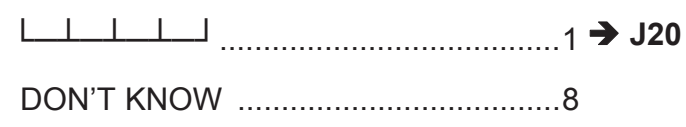 \\
\hline J22d. & $\begin{array}{l}\text { How many years has the Bidan Delima } \\
\text { begin to practice in this village? }\end{array}$ & 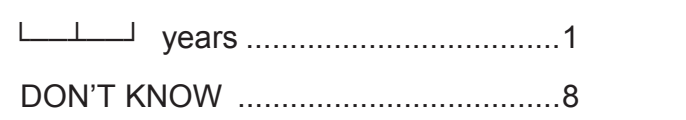 \\
\hline J20. & $\begin{array}{l}\text { Is there a Medicine Post (Pos Obat Desa) } \\
\text { in this village? }\end{array}$ & Yes No \\
\hline J24. & $\begin{array}{l}\text { Since } 2007 \text {, has there ever been any } \\
\text { mass immunization/vaccination for the } \\
\text { people? }\end{array}$ & Yes No \\
\hline
\end{tabular}


SECTION J : HISTORY OF THE PRESENCE OF HEALTH FACILITIES

\begin{tabular}{|c|c|c|c|c|c|c|c|c|}
\hline & (JTYPE) & $\begin{array}{l}\text { Aa. Integrated } \\
\text { Community Health } \\
\text { Post (Posyandu) }\end{array}$ & $\begin{array}{l}\text { Ab. Integrated } \\
\text { Community Health } \\
\text { Post for the } \\
\text { elderly (Posyandu } \\
\text { Lansia) }\end{array}$ & $\begin{array}{l}\text { A. Health Centers } \\
\text { (Puskesmas) and } \\
\text { Health Sub-centers } \\
\text { (Puskesmas } \\
\text { Pembantu/Pustu) }\end{array}$ & $\begin{array}{l}\text { B. Private health } \\
\text { practitioner such as } \\
\text { doctors, clinics, } \\
\text { medical posts, } \\
\text { maternity clinics, } \\
\text { nurses, midwives, } \\
\text { medical assistants, } \\
\text { and other } \\
\text { paramedical } \\
\text { workers }\end{array}$ & $\begin{array}{l}\text { C. Traditional health } \\
\text { workers, such as: } \\
\text { midwives, Chinese } \\
\text { healer (sinshe), } \\
\text { shaman, etc. }\end{array}$ & $\begin{array}{l}\text { D. Public or private } \\
\text { hospital }\end{array}$ & Ba. Pharmacy \\
\hline J26. & $\begin{array}{l}\text { With regards to the }[\ldots] \\
\text { available to the community in } \\
\text { this village/township, how many } \\
{[\ldots] \text { can be used by the people }} \\
\text { from this village/township? }\end{array}$ & $\begin{array}{l}\text { 1. } \downarrow \perp \text { facilities } \\
\text { 8. DON'T KNOW } \\
\text { 6. NONE } \rightarrow \text { J26Ab }\end{array}$ & 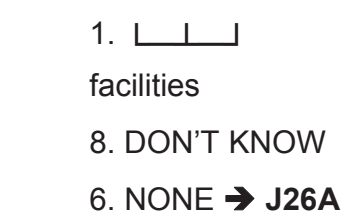 & $\begin{array}{l}\text { 1. } ـ \perp \text { facilities } \\
\text { 8. DON'T KNOW } \\
\text { 6. NONE } \rightarrow \mathbf{J} 26 \mathrm{~B}\end{array}$ & 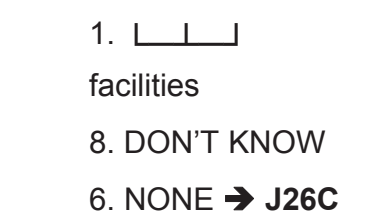 & 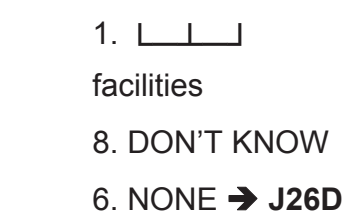 & 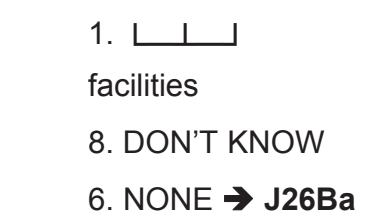 & $\begin{array}{l}\text { 1. } ـ \perp \text { facilities } \\
\text { 8. DON'T KNOW } \\
\text { 6.NONE } \rightarrow \text { SECTION } \\
\text { PMKD }\end{array}$ \\
\hline J27a & $\begin{array}{l}\text { What are the names of the } \\
\text { facilities? } \\
\text { INTERVIEWER'S NOTE: } \\
\text { INDICATE WHETHER } \\
\text { FACILITY IS LOCATED } \\
\text { WITHIN THIS VILLAGE OR } \\
\text { OUTSIDE THIS VILLAGE, } \\
\text { CODE FOR J27a: } \\
\text { 1. IF FACILITY IS IN THIS } \\
\text { VILLAGE } \\
\text { 3. IF FACILITY IS NOT IN } \\
\text { THIS VILLAGE }\end{array}$ & 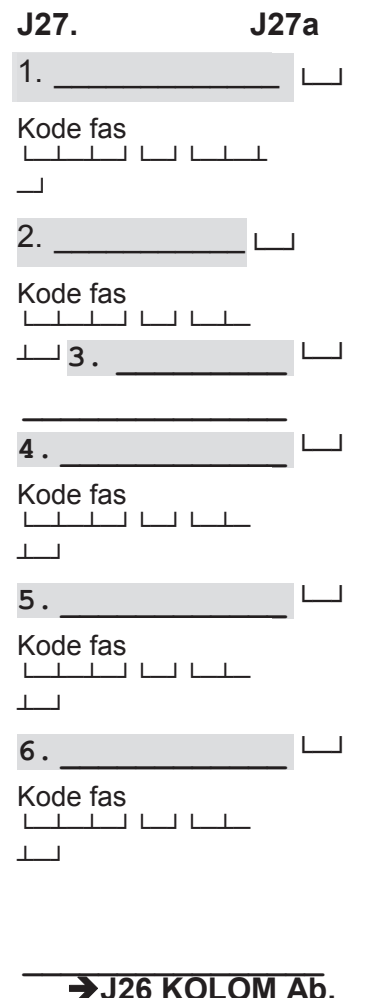 & 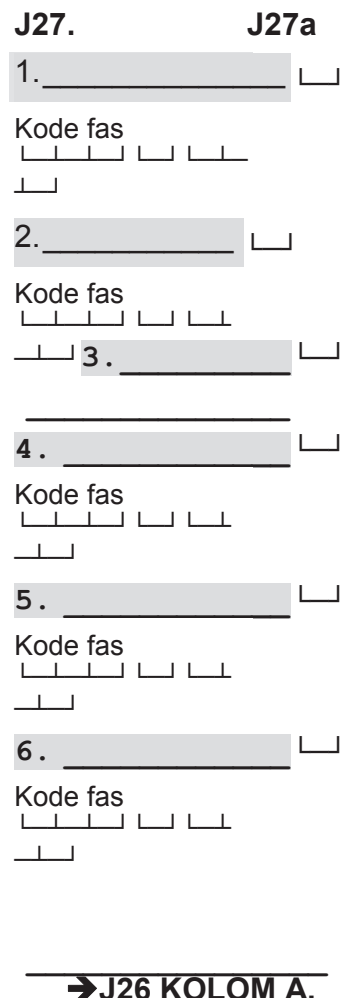 & 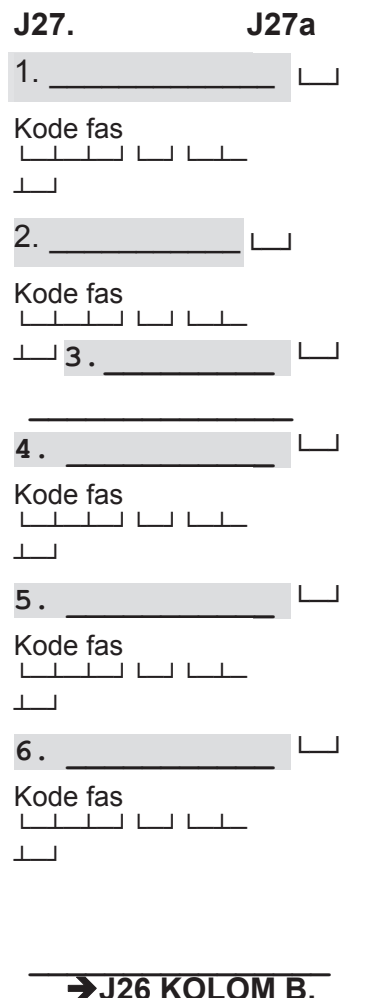 & 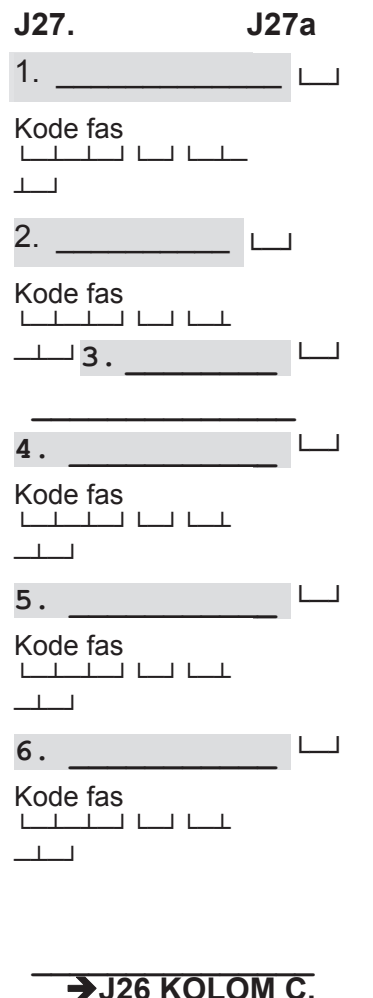 & 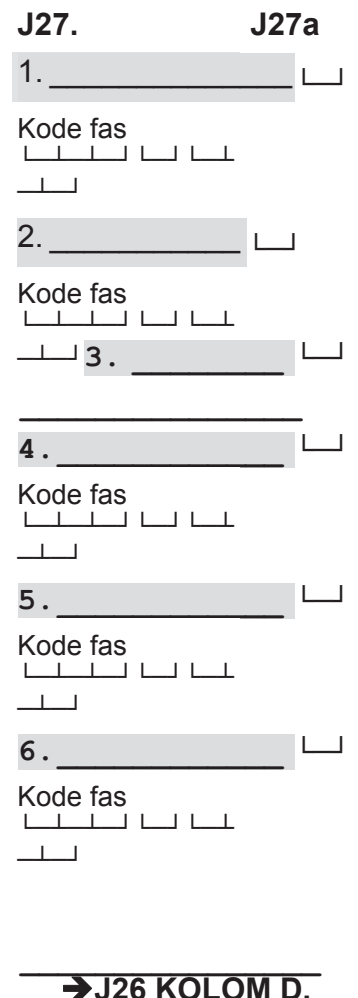 & 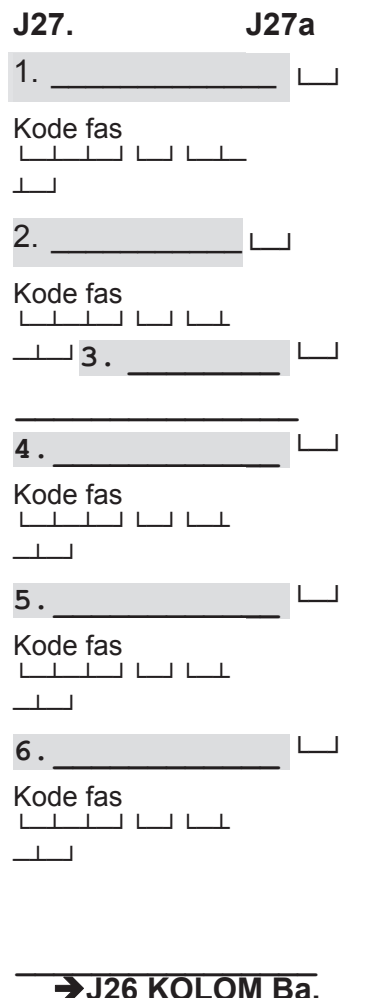 & 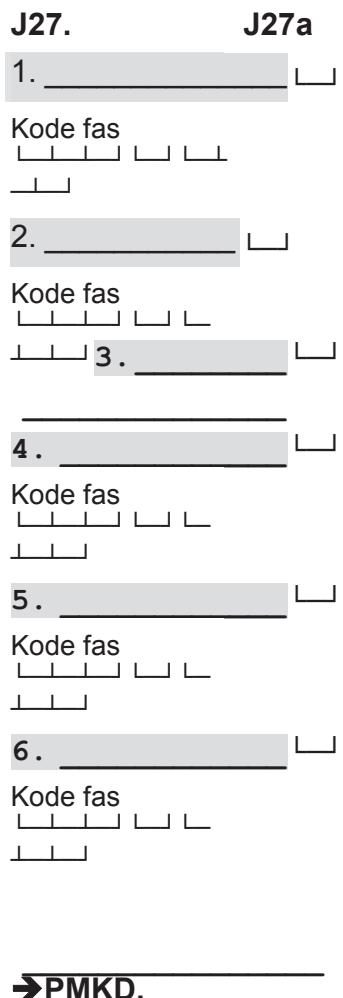 \\
\hline
\end{tabular}




\section{SECTION PMKD : CITIZEN PARTICIPATION}

Section PMKD Respondent:

\begin{tabular}{|c|c|c|}
\hline 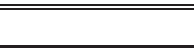 & - & IR2 \\
\hline 1_PMKD. & $\begin{array}{l}\text { Name of Respondent: } \\
\text { CAPI WILL SKIP TO PMKD2 IF THE SAME NAME HAS } \\
\text { ALREADY BEEN ENTERED EARLIER }\end{array}$ & \\
\hline 1a_PMKD. & Position in the village : & $\begin{array}{ccccc}01 & 02 & 03 & 04 & 05 \\
06 & 07 & 95 & \ldots \ldots \ldots \ldots \ldots . .\end{array}$ \\
\hline 2_PMKD. & Age & $\sqcup$ Years \\
\hline 2a_PMKD. & Length of tenure in positions: & 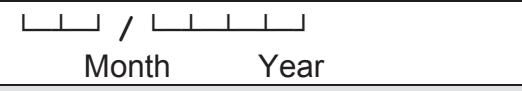 \\
\hline 3_PMKD. & $\begin{array}{l}\text { INTERVIEWER CHECK : } \\
\text { SEX OF RESPONDENT }\end{array}$ & $\begin{array}{l}\text { MALE } \ldots \ldots \ldots \ldots \ldots \ldots \ldots \ldots . . .1 \\
\text { FEMALE ………….... } 3\end{array}$ \\
\hline 3a_PMKD. & Highest level of education attended: & 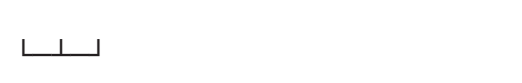 \\
\hline 4_PMKD. & Highest class completed & $\begin{array}{lllll}00 & 01 & 02 & 03 & 04 \\
05 & 06 & 07 & 98 & 96\end{array}$ \\
\hline 5_PMKD. & Length of time lived in the village & 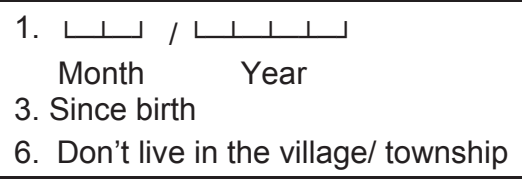 \\
\hline 6_PMKD. & How did $[\ldots]$ come to assume to the position & $\begin{array}{l}\text { 1. Elected } \\
\text { 2. Appointed by residence } \\
\text { 3. Appointed by camat } \\
\text { 4. Appointed by Bupati/Walikota } \\
\text { 5. Other }\end{array}$ \\
\hline
\end{tabular}

\section{Code for position in village (1a_PMKD)}

\section{Village/township head}

03. Head of Government Administration

04. Head of Village Development

05. Head of Welfare

06. Head of Financial Matter

95. Other

\begin{abstract}
Codes for highest level of educat
01. No school/not yet in schoo

02. Elementary school

03. Junior High - General

04. Junior High - Vocational

05. High School - General

06. High School - Vocational
60. D1, D2, D3 (Junior College)

61. University 11 (Bachelor)

62. University S2 (Masters)

63. University $\mathrm{S} 3(\mathrm{Ph} . \mathrm{D}$.)
\end{abstract}

11. Kejar Paket A (Adult Education $A$ )

2. Kejar Paket $B$ (Adult Education B)

14. Open Univeristy

15. Kejar Paket C (Adult

17. School for the disabled

72. Islamic Elementary School (Madrasah Ibtidaiyah)

73. Islamic Junior High School (Madrasah Tsanawiyah)

74. Islamic Senior High School (Madrasah Alliyah)

90. Kindergarten

95. Other

\section{Codes for highest grade (4_PMKD):}

00. Never completed class 1

01. 2

03. 3

04. 4

05. 5

07. Graduated

96. No school

8. DON'T KNOW 


\section{SECTION PMKD : CITIZEN PARTICIPATION}

Next, we want to ask about people's activities in this village.

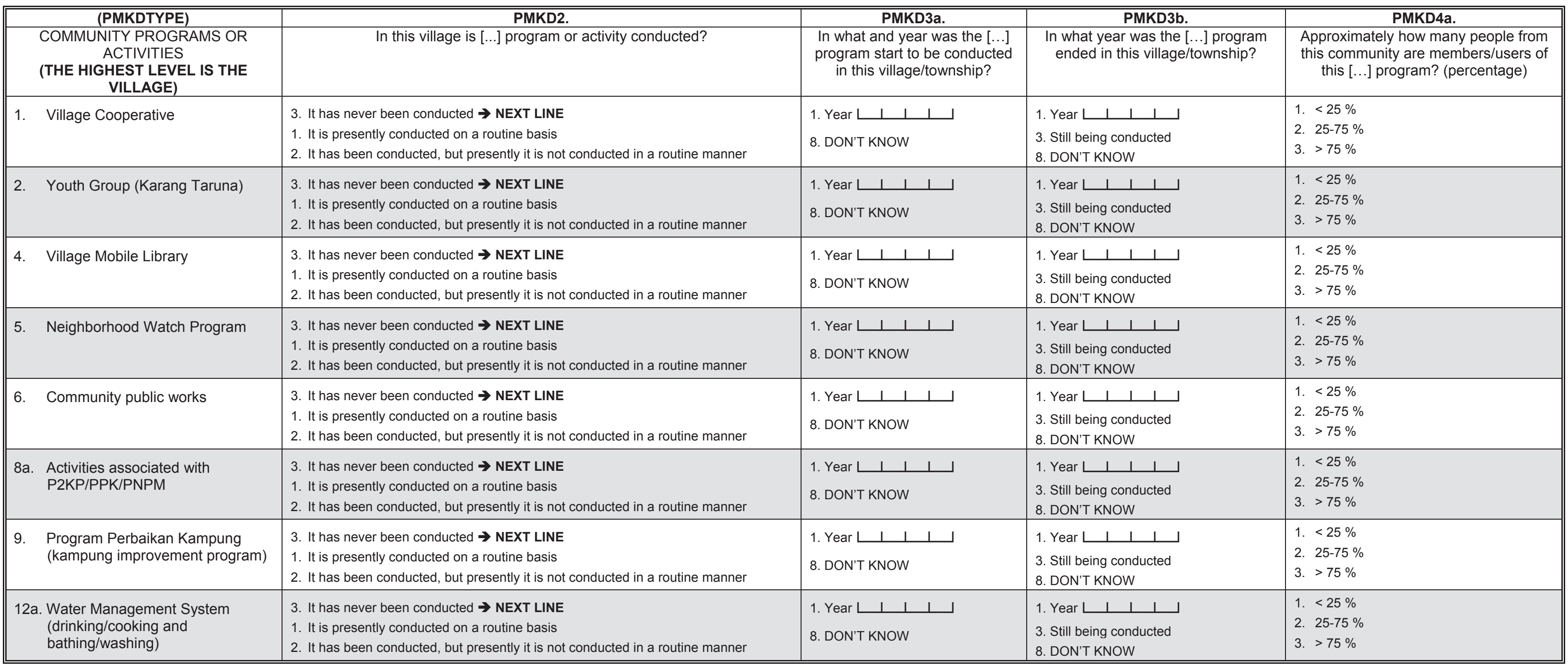




\section{SECTION PMKD : CITIZEN PARTICIPATION}

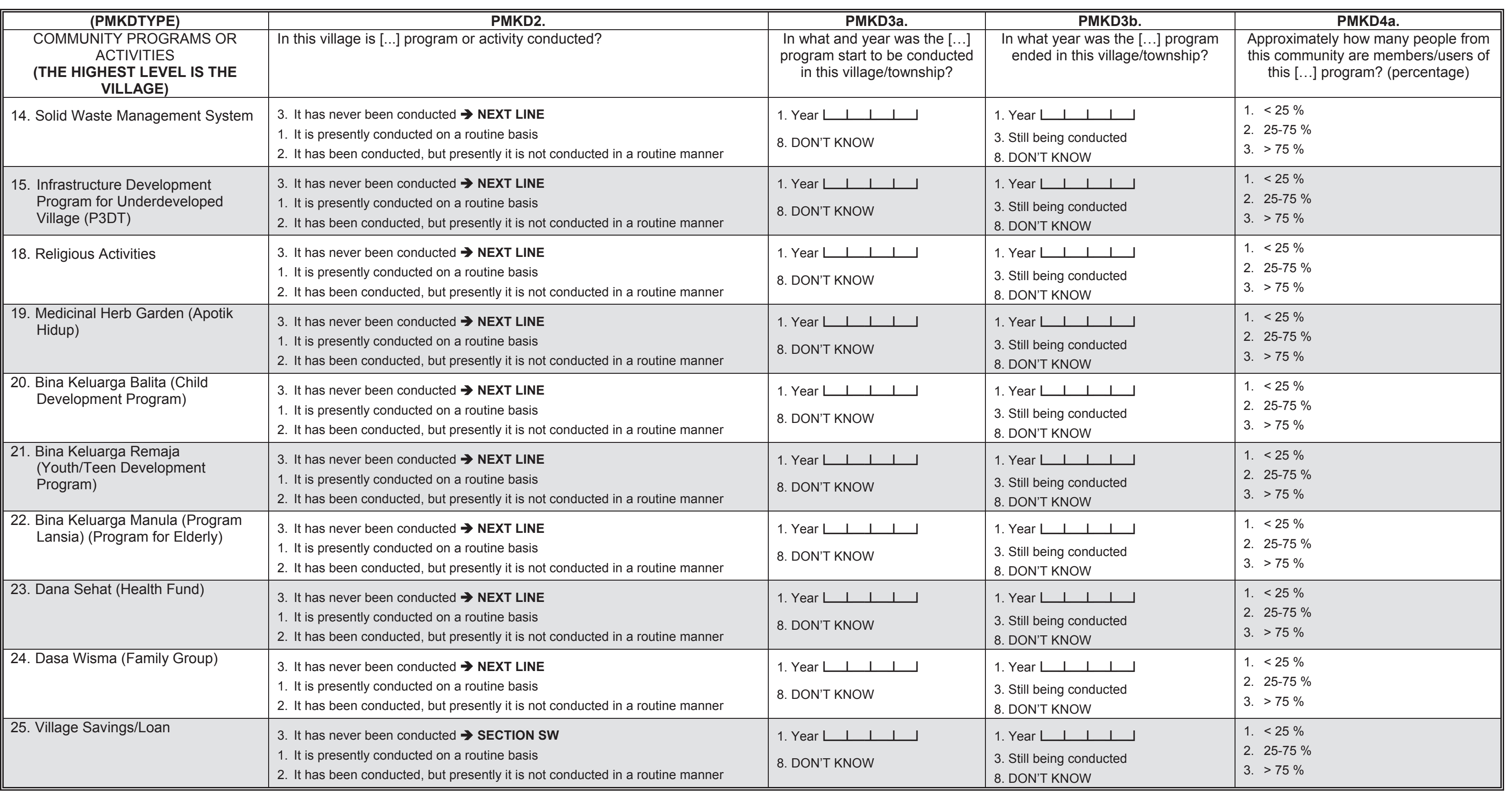


SECTION SW: SUBJECTIVE WELL-BEING

Section SW Respondent:

\begin{tabular}{|c|c|}
\hline 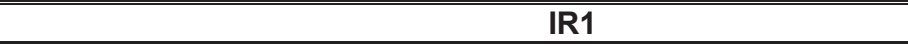 & IR2 \\
\hline $\begin{array}{l}\text { 1_SW. Name of Respondent: } \\
\text { CAPI WILL SKIP TO SW01 IF THE SAME NAME } \\
\text { HAS ALREADY BEEN ENTERED EARLIER } \\
\end{array}$ & \\
\hline 1a_SW. Position in the village : & $\begin{array}{ccccc}01 & 02 & 03 & 04 & 05 \\
06 & 07 & 95 & \ldots \ldots \ldots \ldots . . \\
\end{array}$ \\
\hline 2_sW. & $\downarrow$ \ـears \\
\hline 2a_SW. Length of tenure in positions: & 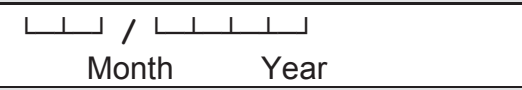 \\
\hline $\begin{array}{ll}3 \text { SW. } & \text { INTERVIEWER CHECK : } \\
& \text { SEX OF RESPONDENT } \\
\end{array}$ & 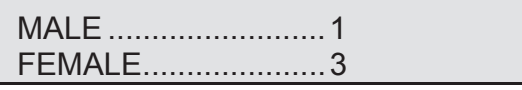 \\
\hline 3a_SW. Highest level of education attended: & $\sqcup$ \\
\hline 4_SW. Highest class completed : & $\begin{array}{lllll}00 & 01 & 02 & 03 & 04 \\
05 & 06 & 07 & 98 & 96 \\
\end{array}$ \\
\hline 5_SW. Length of time lived in the village & $\begin{array}{l}\text { 1. } \underbrace{}_{\text {Month }} / \sqcup \text { Year } \\
\text { 3. Since birth } \\
\text { 6. Don't live in the village/ township }\end{array}$ \\
\hline 6_SW. How did $[\ldots]$ come to assume to the position & $\begin{array}{l}\text { 1. Elected } \\
\text { 2. Appointed by residence } \\
\text { 3. Appointed by camat } \\
\text { 4. Appointed by Bupati/Walikota } \\
\text { 5. Other }\end{array}$ \\
\hline
\end{tabular}

\begin{tabular}{|c|c|c|c|}
\hline Code for position in village (1a_SW): & \multicolumn{2}{|c|}{ Codes for highest level of education (3a SW): } & \\
\hline $\begin{array}{l}\text { 01. Village/township heads } \\
\text { 02. Village secretary } \\
\text { 03. Head of Government Administration } \\
\text { 04. Head of Village Development } \\
\text { 05. Head of Welfare } \\
\text { 06. Head of Financial Matters } \\
07 \text {. Head of General Affairs } \\
\text { 95. Other }\end{array}$ & $\begin{array}{l}\text { 01. No school/not yet in school } \\
\text { 02. Elementary school } \\
\text { 03. Junior High - General } \\
\text { 04. Junior High - Vocational } \\
\text { 05. High School - General } \\
\text { 06. High School - Vocational } \\
\text { 60. D1, D2, D3 (Junior College) } \\
\text { 61. University S1 (Bachelor) } \\
\text { 62. University S2 (Masters) } \\
\text { 63. University S3 (Ph.D.) }\end{array}$ & $\begin{array}{l}\text { 11. Kejar Paket A (Adult Education A) } \\
\text { 12. Kejar Paket B (Adult Education B) } \\
\text { 13. Open Univeristy } \\
\text { 14. Islamic School (Pondok Pensantren) } \\
\text { 15. Kejar Paket C (Adult Education C) } \\
\text { 17. School for the disabled } \\
\text { 72. Islamic Elementary School (Madrasah Ibtidaiyah) } \\
\text { 73. Islamic Junior High School (Madrasah Tsanawiyah) } \\
\text { 74. Islamic Senior High School (Madrasah Alliyah) } \\
\text { 90. Kindergarten } \\
\text { 98. DON'T KNOW } \\
\text { 95. Other }\end{array}$ & $\begin{array}{l}\text { 00. Never completed class } 1 \\
01.1 \\
\text { 02. } 2 \\
\text { 03. } 3 \\
\text { 04. } 4 \\
\text { 05. } 5 \\
\text { 06. } 6 \\
\text { 07. Graduated } \\
\text { 96. No school } \\
\text { 98. DON'T KNOW }\end{array}$ \\
\hline
\end{tabular}




\section{SECTION SW: SUBJECTIVE WELL-BEING}

Now, we would like to know about the subjective well-being of the people in this village.

sw01. If you consider a scale of 1 to 6 , where 1 represents the village where the population is poorest, and 6 represents the the village where the population is richest, what is the number that best represents your village, considering the curren conditions of people in your village?

Richest

SW02. What is the number that best represents your village at 5 years ago?

1

3

45

6

8. DON'T KNOW

8. DON'T KNOW

SW03. Considering the current conditions of people in your village, what number do you think will best describe the conditions of the people in your village 5 years from now?

Poorest

Richest

$\begin{array}{llllll}1 & 2 & 3 & 4 & 5 & 6\end{array}$

8. DON'T KNOW 


\section{SECTION CP: INTERVIEWER NOTES}

\begin{tabular}{|c|c|c|c|c|c|c|c|}
\hline LANGMAIN (CK1). & \multicolumn{2}{|l|}{ Interview was entirely/mostly conducted in what language? } & \multicolumn{5}{|l|}{ ـــ other } \\
\hline LANGOTHR (CK2). & Other languaged used (if any): & & \multicolumn{5}{|l|}{ 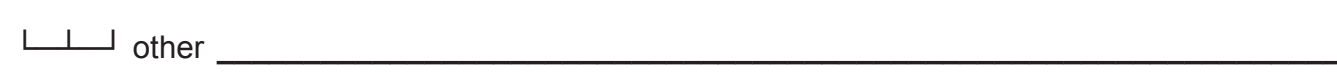 } \\
\hline \multicolumn{8}{|l|}{ LANGUAGE CODE: } \\
\hline 00. Bahasa Indonesia & 04. Batak & Sasak & 12. Makassar & 16. & Toraja & & Lampung \\
\hline 01. Java & 05. Bugis & 09. Minang & 13. Nias & 17. & Lahat & 95. & Other, \\
\hline 02. Sunda & 06. Cina & Banjar & 14. Palembang & 18. & Other South Sumatera & 96. & NA \\
\hline 03. Bali & 07. Madura & 11. Bima & 15. Sumbawa & 19. & Betawi & & \\
\hline
\end{tabular}

\begin{tabular}{|c|c|c|c|}
\hline RESULT (FP3). RESULTS OF INTERVIEW & REASON (FP4). REASON FOR ANSWERING “2” I "3" IN RESULT. & FP6. MONITORING BY SUPERVISOR & \\
\hline $\begin{array}{l}\text { 1. Completed } \rightarrow \text { FP6 } \\
\text { 2. Partly completed } \\
\text { 3. Not completed } \\
\text { 4. Twin EA with EA } \downarrow \perp \text { LPP6 }\end{array}$ & $\begin{array}{l}\text { 1. Respondent was travelling/not in location } \\
\text { 2. Respondent was too busy } \\
\text { 3. Respondent refused }\end{array}$ & $\begin{array}{ll}\text { a. } & \text { Observed (sup_obs) .......... } 1 \\
\text { b. } & \text { Checked (sup_edit)........... } 1 \\
\text { c. } & \text { Verified (sup_veri).............. } 1\end{array}$ & $\begin{array}{l}\text { No } \\
3 \\
3 \\
3\end{array}$ \\
\hline
\end{tabular}

\section{INTERVIEWER NOTE:}




\section{INDONESIAN FAMILY LIFE SURVEY 2014 \\ COMMUNITY AND FACILITY \\ PEOPLE CHARACTERISTICS BOOK IC}

\section{SECTIONS : PAP, PPS, GD, TR, FP, CP}

NAME OF DESA/KELURAHAN :

EA / BOOK TYPE

$\perp \perp / \mathrm{B}_{\mid} \mathrm{K}_{1} 1$

CoV1X. CAPI CHECK: IS THIS A TWIN EA?

1. Yes $\rightarrow$ CK1

3. No 


\section{SECTION PAP : POVERTY ALLEVIATION PROGRAM}

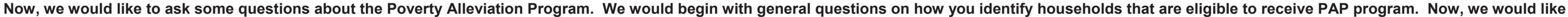
to ask some questions about the Poverty Alleviation Program.

\begin{tabular}{|c|c|c|}
\hline & IR1 & IR2 \\
\hline 1_PAP. & $\begin{array}{l}\text { Name of Respondent: } \\
\text { CAPI WILL SKIP TO B1 IF THE SAME NAME HAS } \\
\text { ALREADY BEEN ENTERED EARLIER }\end{array}$ & \\
\hline 1a_PAP & Position in the village : & $\begin{array}{ccccc}01 & 02 & 03 & 04 & 05 \\
06 & 07 & 95 & \ldots \ldots \ldots \ldots \ldots\end{array}$ \\
\hline 2_PAP & Age & $\perp$ Y Years \\
\hline 2a_PAP & Length of tenure in positions: & 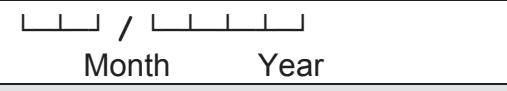 \\
\hline 3_PAP & $\begin{array}{l}\text { INTERVIEWER CHECK : } \\
\text { SEX OF RESPONDENT }\end{array}$ & $\begin{array}{l}\text { MALE } \ldots \ldots \ldots \ldots \ldots \ldots \ldots . . .1 \\
\text { FEMALE ..................... } 3\end{array}$ \\
\hline 3a_PAP & Highest level of education attended: & \llcorner \\
\hline 4_PAP & Highest class completed: & $\begin{array}{lllll}00 & 01 & 02 & 03 & 04 \\
05 & 06 & 07 & 98 & 96 \\
\end{array}$ \\
\hline 5_PAP & Length of time lived in the village & 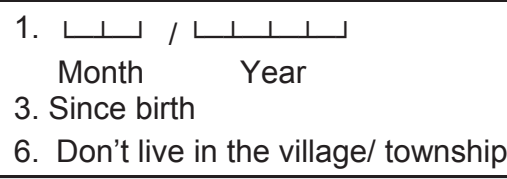 \\
\hline 6_PAP & How did $[\ldots]$ come to assume to the position & $\begin{array}{l}\text { 1. Elected } \\
\text { 2. Appointed by residence } \\
\text { 3. Appointed by camat } \\
\text { 4. Appointed by Bupati/Walikota } \\
\text { 5. Other }\end{array}$ \\
\hline
\end{tabular}

\begin{tabular}{||l|ll|}
\hline Code for position in village (1a_PAP): & Codes for highest level of education (3a_PAP): & \\
01. Village/township heads & 01. No school/not yet in school & 11. Kejar Paket A (Adult Education A) \\
02. Village secretary & 02. Elementary school & 12. Kejar Paket B (Adult Education B) \\
03. Head of Government Administration & 03. Junior High - General & 13. Open Univeristy \\
04. Head of Village Development & 04. Junior High - Vocational & 14. Islamic School (Pondok Pensantren) \\
05. Head of Welfare & 05. High School - General & 15. Kejar Paket C (Adult Education C) \\
06. Head of Financial Matters & 06. High School - Vocational & 17. School for the disabled \\
07. Head of General Affairs & 60. D1, D2, D3 (Junior College) & 72. Islamic Elementary School (Madrasah Ibtidaiyah) \\
95. Other & 61. University S1 (Bachelor) & 73. Islamic Junior High School (Madrasah Tsanawiyah) \\
& 62. University S2 (Masters) & 74. Islamic Senior High School (Madrasah Alliyah) \\
& 63. University S3 (Ph.D.) & 90. Kindergarten \\
& & 98. DON'T KNOW \\
\hline
\end{tabular}




\section{SECTION PAP : POVERTY ALLEVIATION PROGRAM}

\begin{tabular}{|c|c|c|}
\hline PAP01a. & $\begin{array}{l}\text { Does your village has its own mechanism } \\
\text { to identify the households as poor } \\
\text { households that are eligible for a poverty } \\
\text { alleviation program }\end{array}$ & 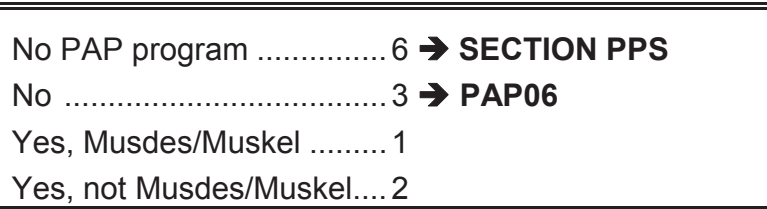 \\
\hline PAP02aa & $\begin{array}{l}\text { In this system, what criteria are used to } \\
\text { identify families who deserve to receive } \\
\text { poverty alleviation programs }\end{array}$ & $\begin{array}{l}\text { CIRCLE ALL THAT APPLY } \\
\text { A. Floor area per household members / family <8 m2 } \\
\text { B. Ground floor type / board / low quality } \\
\text { C. Bamboo wall types, low quality boards } \\
\text { D. There is no facility defecation (latrines) } \\
\text { E. Source of drinking water instead of fresh water } \\
\text { F. Used instead of electric lighting } \\
\text { G. Fuel used is wood / charcoal } \\
\text { H. Frequency of meals in a day is less than } 2 \text { times a } \\
\text { day } \\
\text { I. Not afford to buy meat / chicken / milk in a week } \\
\text { J. Can not afford to buy new clothes for each member } \\
\text { of the household } \\
\text { K. Not afford to go to the health center / clinic } \\
\text { L. Employment of household heads are smallholder } \\
\text { farmers, fishermen, planters } \\
\text { M. Education of household head is not school / } \\
\text { Unfinished Primary Education } \\
\text { N. Ownership of assets / valuables maximum } \\
\text { Rp.500.000, - } \\
\text { O. elderly } \\
\text { P. Underprivileged families } \\
\text { Q. Family Welfare } 1 \text { (KS-1) } \\
\text { R. bad health } \\
\text { S. widower } \\
\text { T. widow } \\
\text { U. Do not have a steady job } \\
\text { AA. No wealth } \\
\text { V. Others } \\
\text { Y. DON'T KNOW }\end{array}$ \\
\hline
\end{tabular}

\begin{tabular}{||l|l||}
\hline \hline PAP03. Among these residents, who determines & CIRCLE ALL THAT APPLY \\
which households are eligible to receive & A. Village head \\
& PAP? \\
& B. Village official/staff \\
& C. PLKB \\
& D. Village midwife \\
& E. Puskesmas staff \\
& F. Community figure \\
& G. Activist \\
& H. PKK \\
& I. NGO \\
& J. LKMD \\
& K. Head of RT/RW \\
& V. Other \\
\hline
\end{tabular}

\begin{tabular}{|c|c|c|}
\hline PAP04. & $\begin{array}{l}\text { Among these residents, who do you think } \\
\text { is more appropriate to be involved in the } \\
\text { listing and selection of those eligible to } \\
\text { receive the assistance? }\end{array}$ & $\begin{array}{l}\text { CIRCLE ALL THAT APPLY } \\
\text { A. Village head } \\
\text { B. Village official/staff } \\
\text { C. PLKB } \\
\text { D. Village midwife } \\
\text { E. Puskesmas staff } \\
\text { F. Community figure } \\
\text { G. Activist } \\
\text { H. PKK } \\
\text { I. NGO } \\
\text { J. LKMD } \\
\text { K. Head of RT/RW } \\
\text { V. Other } \\
\text { Y. DON'T KNOW }\end{array}$ \\
\hline PAP06. & $\begin{array}{l}\text { If it was felt that a household was eligible } \\
\text { to receive PAP program, but household } \\
\text { was not on the recipient list, is there any } \\
\text { mechanism in which the household can } \\
\text { apply to be a recipient of the program? }\end{array}$ & $\begin{array}{l}\text { 1. Report to RT/RW head } \\
\text { 2. Report to village } \\
\text { 3. Report to kecamatan } \\
\text { 4 Depends on type of PAP program } \\
\text { 6. No mechanism } \\
\text { 8. DON'T KNOW }\end{array}$ \\
\hline
\end{tabular}




\section{SECTION PAP : POVERTY ALLEVIATION PROGRAM}

\begin{tabular}{|c|c|c|c|c|c|c|c|c|}
\hline \multirow{2}{*}{\multicolumn{2}{|c|}{$\begin{array}{l}\text { TYPE OF POVERTY PROGRAM } \\
\text { (PAP1TYPE) }\end{array}$}} & \multirow[b]{2}{*}{$\begin{array}{c}\text { PAP7. } \\
\text { Was there } \\
\quad[\ldots] \\
\text { program in } \\
\text { this village } \\
\text { since } \\
2007 ?\end{array}$} & \multirow{2}{*}{$\begin{array}{l}\text { PAP7a. } \\
\text { When was the first time the } \\
\text { program begin? }\end{array}$} & \multirow{2}{*}{$\begin{array}{c}\text { PAP7c. } \\
\text { Is the program still running? }\end{array}$} & \multirow{2}{*}{$\begin{array}{c}\text { PAP7d. } \\
\text { When did the program stop? }\end{array}$} & \multirow{2}{*}{$\begin{array}{l}\text { PAP8 } \\
\text { What percent of households } \\
\text { were recipients/participants } \\
\text { of the program? }\end{array}$} & \multirow[b]{2}{*}{\begin{tabular}{l}
\multicolumn{1}{c}{ PAP14. } \\
Before the [...] program \\
was implemented, has \\
there been information \\
campaign of the program \\
to the community in this \\
village?
\end{tabular}} & PAP17a. \\
\hline & & & & & & & & $\begin{array}{l}\text { Has there been a } \\
\text { complaint/protest from the } \\
\text { community with respect to } \\
\text { the selection of those } \\
\text { eligible to receive }[\ldots . . .] \text { ? }\end{array}$ \\
\hline 1. & $\begin{array}{l}\text { Jamkesda ( Regional health } \\
\text { insurance ) }\end{array}$ & $\begin{array}{l}\text { 1. Yes } \\
\text { 3. No } \downarrow\end{array}$ & 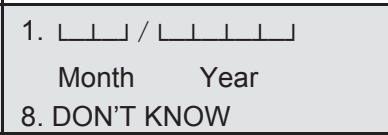 & $\begin{array}{l}\text { 1. Yes } \rightarrow \text { PAP8 } \\
\text { 3. No } \\
\text { 8. DK }\end{array}$ & 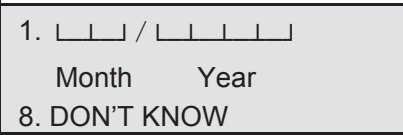 & $\begin{array}{l}\text { 1. } \sqcup \perp \perp \sqcup \% \\
\text { 8. DON'T KNOW }\end{array}$ & $\begin{array}{l}\text { 1. Yes } \\
\text { 3. No }\end{array}$ & $\begin{array}{l}\text { 1. Yes } \\
\text { 3. No }\end{array}$ \\
\hline 2. & $\begin{array}{l}\text { Jamkesmas ( Public health } \\
\text { insurance) }\end{array}$ & $\begin{array}{l}\text { 1. Yes } \\
\text { 3. No } \downarrow\end{array}$ & 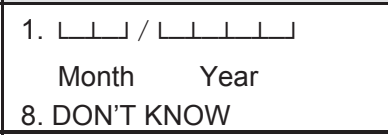 & $\begin{array}{l}\text { 1. Yes } \rightarrow \text { PAP8 } \\
\text { 3. No } \\
\text { 8. DK }\end{array}$ & 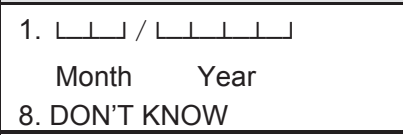 & $\begin{array}{l}\text { 1. } \sqcup \perp \perp \sqcup \% \\
\text { 8. DON'T KNOW }\end{array}$ & $\begin{array}{l}\text { 1. Yes } \\
\text { 3. No }\end{array}$ & $\begin{array}{l}\text { 1. Yes } \\
\text { 3. No }\end{array}$ \\
\hline $2 a$ & Jampersal ( Delivery insurance) & $\begin{array}{ll}\text { 1. } & \text { Yes } \\
\text { 3. No } \downarrow\end{array}$ & 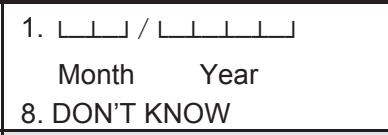 & $\begin{array}{l}\text { 1. Yes } \rightarrow \text { PAP8 } \\
\text { 3. No } \\
\text { 8. DK }\end{array}$ & 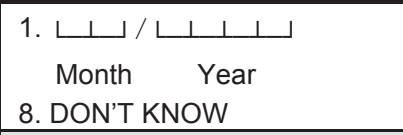 & $\begin{array}{l}\text { 1. } \sqcup \perp \perp \sqcup \% \\
\text { 8. DON'T KNOW }\end{array}$ & $\begin{array}{l}\text { 1. Yes } \\
\text { 3. No }\end{array}$ & $\begin{array}{l}\text { 1. Yes } \\
\text { 3. No }\end{array}$ \\
\hline $2 b$ & JKN (National Health Insurance) & $\begin{array}{l}\text { 1. Yes } \\
\text { 3. No } \downarrow\end{array}$ & 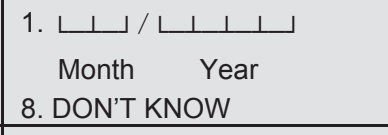 & $\begin{array}{l}\text { 1. Yes } \rightarrow \text { PAP8 } \\
\text { 3. No } \\
\text { 8. DK }\end{array}$ & 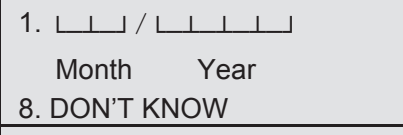 & $\begin{array}{l}\text { 1. } \sqcup \perp \perp\lrcorner \% \\
\text { 8. DON'T KNOW }\end{array}$ & $\begin{array}{l}\text { 1. Yes } \\
\text { 3. No }\end{array}$ & $\begin{array}{l}\text { 1. Yes } \\
\text { 3. No }\end{array}$ \\
\hline $2 c$ & & & & & & & & \\
\hline 3. & Raskin (Rice for the poor) & $\begin{array}{l}\text { 1. Yes } \\
\text { 3. No } \downarrow\end{array}$ & 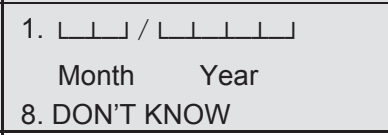 & $\begin{array}{l}\text { 1. Yes } \rightarrow \text { PAP8 } \\
\text { 3. No } \\
\text { 8. DK }\end{array}$ & 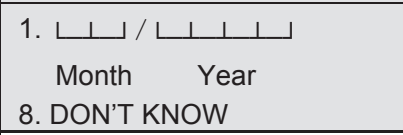 & $\begin{array}{l}\text { 1. } \sqcup \perp \sqcup \% \\
\text { 8. DON'T KNOW }\end{array}$ & $\begin{array}{l}\text { 1. Yes } \\
\text { 3. No }\end{array}$ & $\begin{array}{l}\text { 1. Yes } \\
\text { 3. No }\end{array}$ \\
\hline 4. & Market Operation for Rice & $\begin{array}{l}\text { 1. Yes } \\
\text { 3. No } \downarrow\end{array}$ & 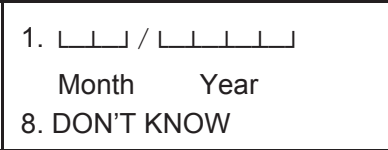 & $\begin{array}{l}\text { 1. Yes } \rightarrow \text { PAP8 } \\
\text { 3. No } \\
\text { 8. DK }\end{array}$ & 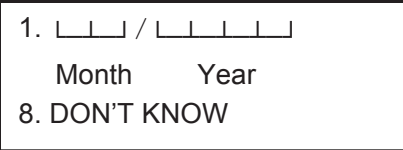 & & & \\
\hline 5. & $\begin{array}{l}\text { Market Operation for Other } \\
\text { goods }\end{array}$ & $\begin{array}{ll}\text { 1. } \text { Yes } \\
\text { 3. No } \downarrow\end{array}$ & 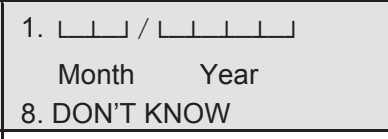 & $\begin{array}{l}\text { 1. Yes } \rightarrow \text { PAP8 } \\
\text { 3. No } \\
\text { 8. DK } \\
\end{array}$ & 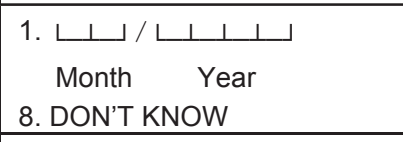 & & & \\
\hline 6. & $\begin{array}{l}\text { PKPS BBM - SLT } \\
\text { (Unconditional Cash Transfer) }\end{array}$ & $\begin{array}{l}\text { 1. Yes } \\
\text { 3. No } \downarrow\end{array}$ & 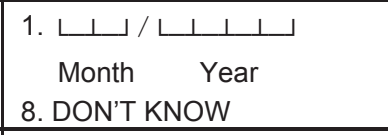 & $\begin{array}{l}\text { 1. Yes } \rightarrow \text { PAP8 } \\
\text { 3. No } \\
\text { 8. DK }\end{array}$ & 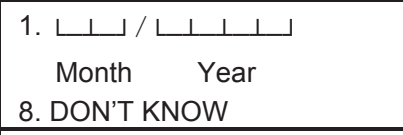 & $\begin{array}{l}\text { 1. } \sqcup \perp \perp \text { \% } \\
\text { 8. DON'T KNOW }\end{array}$ & $\begin{array}{l}\text { 1. Yes } \\
\text { 3. No }\end{array}$ & $\begin{array}{l}\text { 1. Yes } \\
\text { 3. No }\end{array}$ \\
\hline 7. & $\begin{array}{l}\text { Keluarga Harapan (Conditional } \\
\text { Cash Transfer) }\end{array}$ & $\begin{array}{l}\text { 1. Yes } \\
\text { 3. No } \downarrow\end{array}$ & 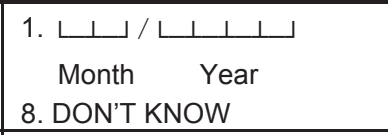 & $\begin{array}{l}\text { 1. Yes } \rightarrow \text { PAP8 } \\
\text { 3. No } \\
\text { 8. DK } \\
\end{array}$ & 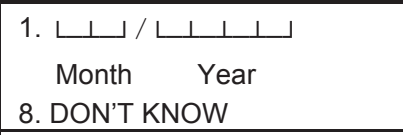 & $\begin{array}{l}\text { 1. } \sqcup \perp \sqcup \% \\
\text { 8. DON'T KNOW }\end{array}$ & $\begin{array}{l}\text { 1. Yes } \\
\text { 3. No }\end{array}$ & $\begin{array}{l}\text { 1. Yes } \\
\text { 3. No }\end{array}$ \\
\hline & PNPM Mandiri & $\begin{array}{l}\text { 1. Yes } \\
\text { 3. No } \downarrow\end{array}$ & 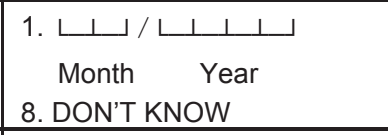 & $\begin{array}{l}\text { 1. Yes } \rightarrow \text { PAP8 } \\
\text { 3. No } \\
\text { 8. DK }\end{array}$ & 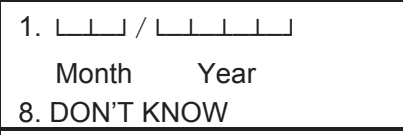 & $\begin{array}{l}\text { 1. } \sqcup \perp \perp\lrcorner \% \\
\text { 8. DON'T KNOW }\end{array}$ & $\begin{array}{l}\text { 1. Yes } \\
\text { 3. No }\end{array}$ & $\begin{array}{l}\text { 1. Yes } \\
\text { 3. No }\end{array}$ \\
\hline & $\begin{array}{l}\text { BLSM ( Direct Cash Assistance } \\
\text { for the People ) }\end{array}$ & $\begin{array}{l}\text { 1. Yes } \\
\text { 3. No } \downarrow\end{array}$ & 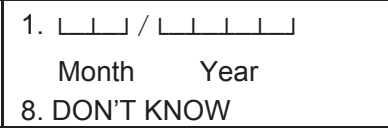 & $\begin{array}{l}\text { 1. Yes } \rightarrow \text { PAP8 } \\
\text { 3. No } \\
\text { 8. DK }\end{array}$ & 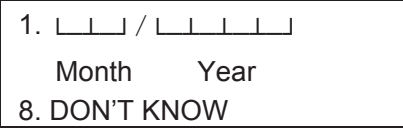 & $\begin{array}{l}\text { 1. } \sqcup \perp \perp \sqcup \% \\
\text { 8. DON'T KNOW }\end{array}$ & $\begin{array}{l}\text { 1. Yes } \\
\text { 3. No }\end{array}$ & $\begin{array}{l}\text { 1. Yes } \\
\text { 3. No }\end{array}$ \\
\hline
\end{tabular}


SECTION PAP : POVERTY ALLEVIATION PROGRAM

\begin{tabular}{|c|c|c|c|c|c|c|c|c|}
\hline 10. & $\begin{array}{l}\text { BSM ( Cash transfer for poor } \\
\text { student ) }\end{array}$ & $\begin{array}{l}\text { 1. Yes } \\
\text { 3. No } \downarrow\end{array}$ & 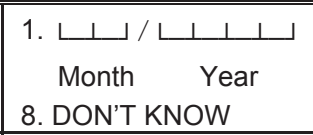 & $\begin{array}{l}\text { 1. Yes } \rightarrow \text { PAP8 } \\
\text { 3. No } \\
\text { 8. DK }\end{array}$ & 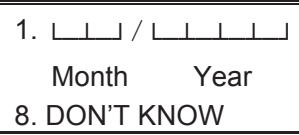 & $\begin{array}{l}\text { 1. } \perp \perp \_\% \\
\text { 8. DON'T KNOW }\end{array}$ & $\begin{array}{l}\text { 1. Yes } \\
\text { 3. No }\end{array}$ & $\begin{array}{l}\text { 1. Yes } \\
\text { 3. No }\end{array}$ \\
\hline 11. & $\begin{array}{l}\text { Askesos ( Social Welfare } \\
\text { Insurance ) }\end{array}$ & $\begin{array}{l}\text { 1. Yes } \\
\text { 3. No } \downarrow\end{array}$ & 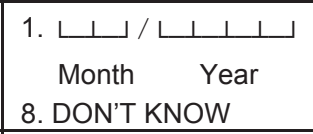 & $\begin{array}{l}\text { 1. Yes } \rightarrow \text { PAP8 } \\
\text { 3. No } \\
\text { 8. DK }\end{array}$ & 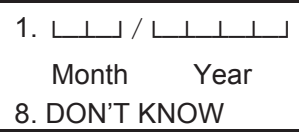 & & & \\
\hline 12. & $\begin{array}{l}\text { PPA-PKH ( Child Labor } \\
\text { Reduction-Conditional Cash } \\
\text { Transfer ) }\end{array}$ & $\begin{array}{l}\text { 1. Yes } \\
\text { 3. No } \downarrow\end{array}$ & $\begin{array}{l}\text { 1. } \sqcup \sqcup / \sqcup \sqcup \perp \perp \perp \text { / } \sqcup \text { Mear } \\
\text { Month Year } \\
\text { 8. DON'T KNOW }\end{array}$ & $\begin{array}{l}\text { 1. Yes } \rightarrow \text { PAP8 } \\
\text { 3. No } \\
\text { 8. DK }\end{array}$ & 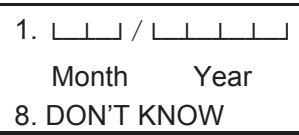 & & & \\
\hline 13. & $\begin{array}{l}\text { JSPACA/JSODK ( Disabled } \\
\text { Social Insurance/Severe- } \\
\text { Disabled Social Insurance ) }\end{array}$ & $\begin{array}{l}\text { 1. Yes } \\
\text { 3. No } \downarrow\end{array}$ & 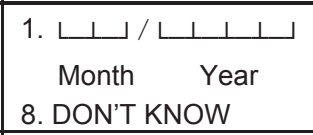 & $\begin{array}{l}\text { 1. Yes } \rightarrow \text { PAP8 } \\
\text { 3. No } \\
\text { 8. DK }\end{array}$ & 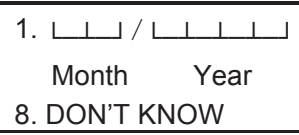 & & & \\
\hline 14. & $\begin{array}{l}\text { JSLU/ASLUT ( Elderly Social } \\
\text { Insurance ) }\end{array}$ & $\begin{array}{l}\text { 1. Yes } \\
\text { 3. No } \downarrow\end{array}$ & 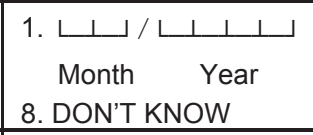 & $\begin{array}{l}\text { 1. Yes } \rightarrow \text { PAP8 } \\
\text { 3. No } \\
\text { 8. DK }\end{array}$ & 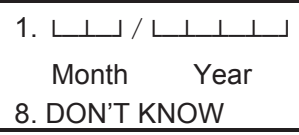 & & & \\
\hline 15. & $\begin{array}{l}\text { KUBE/UEP ( Joint Enterprise } \\
\text { Group/Productive Economic } \\
\text { Enterprise ) }\end{array}$ & $\begin{array}{l}\text { 1. Yes } \\
\text { 3. No } \downarrow\end{array}$ & 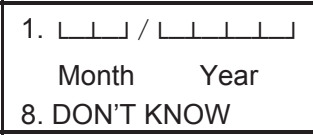 & $\begin{array}{l}\text { 1. Yes } \rightarrow \text { PAP8 } \\
\text { 3. No } \\
\text { 8. DK }\end{array}$ & 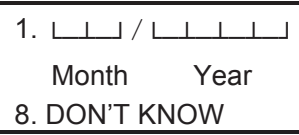 & & & \\
\hline 16. & $\begin{array}{l}\text { RTLH ( Renovation program for } \\
\text { home unfit for habitation/house } \\
\text { renovation) }\end{array}$ & $\begin{array}{l}\text { 1. Yes } \\
\text { 3. No } \downarrow\end{array}$ & 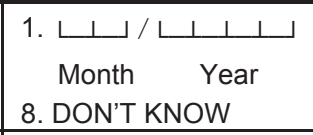 & $\begin{array}{l}\text { 1. Yes } \rightarrow \text { PAP8 } \\
\text { 3. No } \\
\text { 8. DK }\end{array}$ & 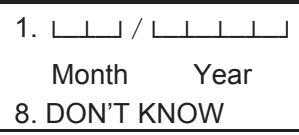 & & & \\
\hline 17. & $\begin{array}{l}\text { PKSA ( Children social welfare } \\
\text { program ) }\end{array}$ & $\begin{array}{l}\text { 1. Yes } \\
\text { 3. No } \downarrow\end{array}$ & 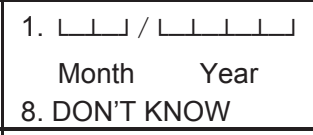 & $\begin{array}{l}\text { 1. Yes } \rightarrow \text { PAP8 } \\
\text { 3. No } \\
\text { 8. DK }\end{array}$ & 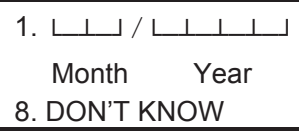 & & & \\
\hline 18. & KPS ( Social Security Card ) & $\begin{array}{l}\text { 1. Yes } \\
\text { 3. No } \downarrow\end{array}$ & 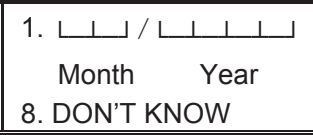 & $\begin{array}{l}\text { 1. Yes } \rightarrow \text { PAP8 } \\
\text { 3. No } \\
\text { 8. DK }\end{array}$ & 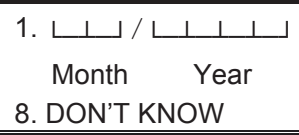 & & & \\
\hline
\end{tabular}




\section{SECTION PAP : POVERTY ALLEVIATION PROGRAM}

\begin{tabular}{|c|c|c|}
\hline$\overline{\text { PAP17 }}$ & $\begin{array}{l}\text { Which program } \\
\text { received the most } \\
\text { complaints/protests } \\
\text { from the community? }\end{array}$ & $\begin{array}{l}\text { 01. Jamkesda } \\
\text { 02. Jamkesmas } \\
\text { 03. Jampersal } \\
\text { 04. Raskin } \\
\text { 05. Rice Market operation } \\
\text { 06. Others Market Operation } \\
\text { 07. PKPS BBM - SLT (UCT) } \\
\text { 08. Keluarga Harapan (CCT) } \\
\text { 09. PNPM MANDIRI } \\
\text { 10. BLSM 2013 } \\
\text { 11. BSM ( Cash transfer for poor student ) } \\
\text { 12. Askesos ( Social Welfare Insurance ) } \\
\text { 13. PPA-PKH ( Child Labor Reduction-Conditional Cash Transfer ) } \\
\text { 14. JSPACA/JSODK ( Disabled Social Insurance/Severe-Disabled Social } \\
\text { Insurance ) } \\
\text { 15. JSLU/ASLUT ( Elderly Social Insurance ) } \\
\text { 16. KUBE/UEP ( Joint Enterprise Group/Productive Economic Enterprise ) } \\
\text { 17. RTLH ( Renovation program for home unfit for habitation/house } \\
\text { renovation) } \\
\text { 18. PKSA ( Children social welfare program ) } \\
\text { 19. KPS ( Social Security Card ) } \\
\text { 20. JKN (National Health Insurance) } \\
\text { 96. DON'T COMPAINTS/PROTESTS }\end{array}$ \\
\hline PAP18 & $\begin{array}{l}\text { Which of the program } \\
\text { was the most accepted } \\
\text { by the community? }\end{array}$ & $\begin{array}{l}\text { 01. Jamkesda } \\
\text { 02. Jamkesmas } \\
\text { 03. Jampersal } \\
\text { 04. Raskin } \\
\text { 05. Rice Market operation } \\
\text { 06. Others Market Operation } \\
\text { 07. PKPS BBM - SLT (UCT) } \\
\text { 08. Keluarga Harapan (CCT) } \\
\text { 09. PNPM MANDIRI } \\
\text { 10. BLSM } 2013 \\
\text { 11. BSM ( Cash transfer for poor student ) } \\
\text { 12. Askesos ( Social Welfare Insurance ) } \\
\text { 13. PPA-PKH ( Child Labor Reduction-Conditional Cash Transfer ) } \\
\text { 14. JSPACA/JSODK ( Disabled Social Insurance/Severe-Disabled Social } \\
\text { Insurance ) } \\
\text { 15. JSLU/ASLUT ( Elderly Social Insurance ) } \\
\text { 16. KUBE/UEP ( Joint Enterprise Group/Productive Economic Enterprise ) } \\
\text { 17. RTLH ( Renovation program for home unfit for habitation/house } \\
\text { renovation) } \\
\text { 18. PKSA ( Children social welfare program ) } \\
\text { 19. KPS ( Social Security Card ) } \\
\text { 20. JKN (National Health Insurance) }\end{array}$ \\
\hline
\end{tabular}

PAP19 Why do you think the program was accepted by the community?

A. The program helped the poor

B. Low leakage in the program executio

C. Program did not create dependency

D. Easy access for community monitoring of the program

E. Program was not a burden to village officials

F. Program was sustainable

G. Most fair

V. Other

\begin{tabular}{|c|c|c|}
\hline PAP20a & $\begin{array}{l}\text { Was there Regional health insurance } \\
\text { program(jamkesda) at yours Kabupaten? }\end{array}$ & $\begin{array}{l}\text { 3. No } \rightarrow \text { PAP24a } \\
\text { 1. Yes }\end{array}$ \\
\hline PAP20 & $\begin{array}{l}\text { Was there Regional health insurance } \\
\text { program ( jamkesda) in the last } 12 \text { months? }\end{array}$ & $\begin{array}{l}\text { 3. No } \rightarrow \text { PAP24a } \\
\text { 1. Yes }\end{array}$ \\
\hline PAP21 & $\begin{array}{l}\text { From which facilities is someone who has a } \\
\text { Regional health insurance program } \\
\text { jamkesda ) able to obtain services? } \\
\text { CIRCLE ALL THAT APPLY }\end{array}$ & $\begin{array}{l}\text { A. Puskesmas } \\
\text { B. Puskesmas Pembantu } \\
\text { C. Village Midwife } \\
\text { D. Private Practice } \\
\text { E. Public Hospital } \\
\text { F. Private Hospital }\end{array}$ \\
\hline PAP22. & $\begin{array}{l}\text { Have you ever heard of any case where a } \\
\text { patient being denied services when using a } \\
\text { Jamkesda? }\end{array}$ & $\begin{array}{l}\text { 1. Yes } \\
\text { A. Puskesmas } \\
\text { B. Puskesmas Pembantu } \\
\text { C. Village Midwife } \\
\text { D. Private Practice } \\
\text { E. Public Hospital } \\
\text { F. Private Hospital } \\
\text { 3. No } \\
\text { 8. DON'T KNOW }\end{array}$ \\
\hline
\end{tabular}




\section{SECTION PAP : POVERTY ALLEVIATION PROGRAM}

Now we would like to inquire about the program Jampersal ( Delivery insurance )

\begin{tabular}{|c|c|c|}
\hline PAP24a & $\begin{array}{l}\text { Was there care and childbirth insurance } \\
\text { program ( Jampersal) at yours } \\
\text { kabupaten? }\end{array}$ & $\begin{array}{l}\text { No } \\
\text { Yes }\end{array}$ \\
\hline PAP25a & $\begin{array}{l}\text { Was there care and childbirth insurance } \\
\text { program ( Jampersal) in the last } 12 \\
\text { months? }\end{array}$ & 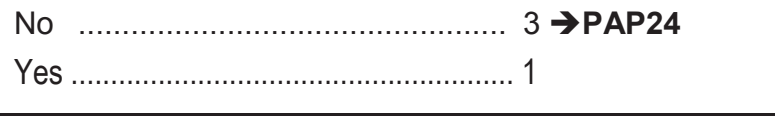 \\
\hline PAP26a & Who is eligible for jampersal? & $\begin{array}{l}\text { A. Pregnant Women } \\
\text { B. Mother Maternity } \\
\text { C. Postpartum women (up to } 42 \text { days postpartum) } \\
\text { D. Newborns (up to age } 28 \text { days) } \\
\text { V. Others }\end{array}$ \\
\hline PAP27a & $\begin{array}{l}\text { From which facilities is someone who } \\
\text { has a jampersal able to obtain services? } \\
\text { CIRCLE ALL THAT APPLY }\end{array}$ & $\begin{array}{l}\text { A. Puskesmas } \\
\text { B. Puskesmas Pembantu } \\
\text { C. Village Midwife } \\
\text { D. Private Practice } \\
\text { E. Public Hospital } \\
\text { F. Private Hospital }\end{array}$ \\
\hline PAP28a & $\begin{array}{l}\text { What kind of treatment obtained } \\
\text { Jampersal cardholder? }\end{array}$ & $\begin{array}{l}\text { A. Pregnancy care } \\
\text { B. maternity services } \\
\text { C. Mother Postpartum Care (up to } 42 \text { days postpartum } \\
\text { D. Newborn Care (up to age } 28 \text { days) } \\
\text { E. Postpartum family planning } \\
\text { V. Others }\end{array}$ \\
\hline PAP29a & $\begin{array}{l}\text { Are there people who are eligible but do } \\
\text { not get Jampersal? }\end{array}$ & 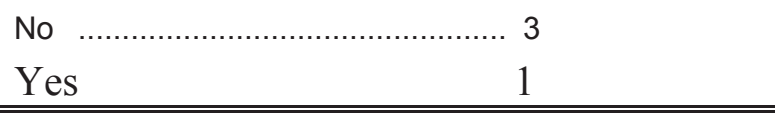 \\
\hline
\end{tabular}

Now we would like to inquire about the JAMKESMAS program (Public Health Insurance)

\begin{tabular}{|c|c|c|}
\hline PAP24. & $\begin{array}{l}\text { JAMKESMAS program in the last } 12 \\
\text { months? }\end{array}$ & $\begin{array}{l}\text { 3. No } \rightarrow \text { PAP28 } \\
\text { 1. Yes }\end{array}$ \\
\hline PAP25. & $\begin{array}{l}\text { From which facilities is someone who } \\
\text { has JAMKESMAS able to obtain } \\
\text { services? } \\
\text { CIRCLE ALL THAT APPLY }\end{array}$ & $\begin{array}{l}\text { A. Puskesmas } \\
\text { B. Puskesmas Pembantu } \\
\text { C. Village Midwife } \\
\text { D. Private Practice } \\
\text { E. Public Hospital } \\
\text { F. Private Hospital }\end{array}$ \\
\hline PAP26. & $\begin{array}{l}\text { For how long is the JAMKESMAS } \\
\text { valid? }\end{array}$ & $\begin{array}{l}\text { 1. } ـ \_ \text {months } \\
\text { 6. No expiration } \\
\text { 8. DON'T KNOW }\end{array}$ \\
\hline
\end{tabular}

PAP27. Have you ever heard of any case where a patient being $d$ when using JAMKESMAS?

1. Yes

A. Puskesmas

B. Puskesmas Pembantu

C. Village Midwife

D. Private Practice

E. Public Hospital

3. No

F. Private Hospital

8. DON'T KNOW

Now we would like to inquire about the JKN program (National Health Insurance)

\begin{tabular}{|c|c|c|}
\hline PAP24b. & JKN program in the last 12 months? & $\begin{array}{l}\text { 3. No } \rightarrow \text { PAP28 } \\
\text { 1. Yes }\end{array}$ \\
\hline PAP25b. & $\begin{array}{l}\text { From which facilities is someone who } \\
\text { has JKN able to obtain services? } \\
\text { CIRCLE ALL THAT APPLY }\end{array}$ & $\begin{array}{l}\text { A. Puskesmas } \\
\text { B. Puskesmas Pembantu } \\
\text { C. Village Midwife } \\
\text { D. Private Practice } \\
\text { E. Public Hospital } \\
\text { F. Private Hospital }\end{array}$ \\
\hline PAP26b. & For how long is the JKN valid? & $\begin{array}{l}\text { 1.ـــ months } \\
\text { 6. No expiration } \\
\text { 8. DON'T KNOW }\end{array}$ \\
\hline PAP27b. & $\begin{array}{l}\text { Have you ever heard of any case } \\
\text { where a patient being denied services } \\
\text { when using JKN? }\end{array}$ & $\begin{array}{l}\text { 1. Yes } \\
\text { A. Puskesmas } \\
\text { B. Puskesmas Pembantu } \\
\text { C. Village Midwife } \\
\text { D. Private Practice } \\
\text { E. Public Hospital } \\
\text { F. Private Hospital } \\
\text { 3. No } \\
\text { 8. DON'T KNOW }\end{array}$ \\
\hline
\end{tabular}


SECTION PAP : POVERTY ALLEVIATION PROGRAM

\begin{tabular}{|c|c|c|}
\hline PAP28. & $\begin{array}{l}\text { Was there Raskin program in the last } \\
12 \text { months? }\end{array}$ & $\begin{array}{l}\text { 3. NO } \rightarrow \text { PAP46 } \\
\text { 1. YES }\end{array}$ \\
\hline PAP29. & $\begin{array}{l}\text { In the last } 12 \text { months, how many times } \\
\text { has RASKIN rice been distributed in } \\
\text { this village? }\end{array}$ & $\begin{array}{l}\text { 1. } \sqcup \sqcup \text { times } \\
\text { 8. DON'T KNOW }\end{array}$ \\
\hline PAP30. & $\begin{array}{l}\text { How many tonnes of RASKIN rice were } \\
\text { delivered to this village to be } \\
\text { distributed? }\end{array}$ & $\begin{array}{l}\text { 1. } \sqcup \perp \text { tonnes } \\
\text { 8. DON'T KNOW }\end{array}$ \\
\hline PAP31. & $\begin{array}{l}\text { On average, in the last } 12 \text { months was } \\
\text { the percentage of households that } \\
\text { received RASKIN rice? }\end{array}$ & $\begin{array}{l}\text { 1. } \downarrow \perp \perp \text { percent } \\
\text { 8. DON'T KNOW }\end{array}$ \\
\hline
\end{tabular}

Now we would like to ask you about the last time RASKIN was distributed in this village in the last 12 months.

\begin{tabular}{|c|c|c|}
\hline PAP32. & $\begin{array}{l}\text { When was the last time RASKIN rice was } \\
\text { distributed in the last } 12 \text { months? }\end{array}$ & 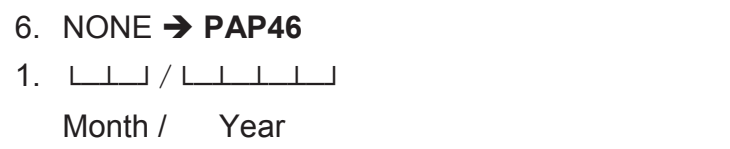 \\
\hline PAP34. & $\begin{array}{l}\text { How many tonnes was delivered to this } \\
\text { village to be distributed? }\end{array}$ & $\begin{array}{l}\text { 1. ᄂ」 tonnes } \\
\text { 8. DON'T KNOW }\end{array}$ \\
\hline PAP35. & $\begin{array}{l}\text { What was the percentage of households } \\
\text { that received RASKIN rice? }\end{array}$ & $\begin{array}{l}\text { 1. } \perp \perp \text { percent } \\
\text { 8. DON'T KNOW }\end{array}$ \\
\hline PAP36. & $\begin{array}{l}\text { What was the average amount of rice } \\
\text { received by all RASKIN household } \\
\text { recipients? }\end{array}$ & $\begin{array}{lll}\perp & \mathrm{kg}\end{array}$ \\
\hline PAP37. & $\begin{array}{l}\text { What was the price of each kilogram of } \\
\text { RASKIN rice (include other costs) paid by } \\
\text { each household? }\end{array}$ & 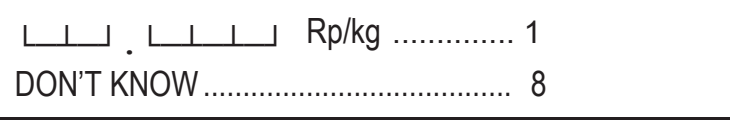 \\
\hline PAP38. & $\begin{array}{l}\text { What was the market price of each } \\
\text { kilogram of the rice in that time? }\end{array}$ & 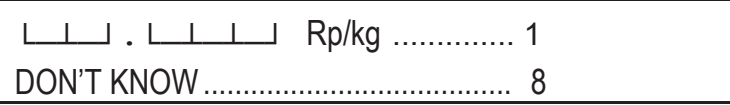 \\
\hline PAP39a & $\begin{array}{l}\text { How to decide the household to receive } \\
\text { RASKIN? }\end{array}$ & $\begin{array}{l}\text { 1. Based on list name which is decided by } \\
\text { government (DPM) } \rightarrow \text { PAP39 } \\
\text { 2. Based on list name from government (DPM) and } \\
\text { modified } \\
\text { 3. Do not use DPM }\end{array}$ \\
\hline PAP39b & $\begin{array}{l}\text { Based on what decision for the receiver } \\
\text { of RASKIN? }\end{array}$ & $\begin{array}{l}\text { A. Floor type of low quality (e.g ground, no tiles) } \\
\text { B. Wall of low quality (e.g bamboo, boards) } \\
\text { C. No toilet/latrines for defecation } \\
\text { D. Source of drinking water not piped from PAM } \\
\text { E. Source of lighting is not electricity } \\
\text { F. Use wood/charcoal for cooking fuel } \\
\text { G. Head of household didn't go to school/finish } \\
\text { elementary school } \\
\text { H. Elderly }\end{array}$ \\
\hline
\end{tabular}

\begin{tabular}{|c|c|c|}
\hline & & $\begin{array}{l}\text { I. Widowed } \\
\text { J. Widow } \\
\text { K. Don't have permanent job } \\
\text { L. Considered as poor by community } \\
\text { V. Other }\end{array}$ \\
\hline PAP39. & $\begin{array}{l}\text { Did all households that received RASKIN } \\
\text { rice received equal amount? }\end{array}$ & 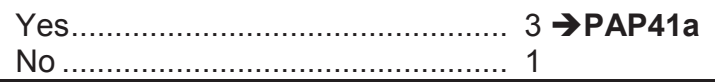 \\
\hline PAP40. & $\begin{array}{l}\text { How did you determine the amount of } \\
\text { rice that a household can buy/receive? }\end{array}$ & $\begin{array}{l}\text { A. Based on the number of household members } \\
\text { B. Based on the poverty level of the household } \\
\text { C. Based on the number of children } \\
\text { V. Other } \\
\rightarrow \text { PAP45aa }\end{array}$ \\
\hline PAP41a & How was it distributed equally?: & $\begin{array}{l}\text { A. Distributed equally among the poor households } \\
\text { B. Distributed equally between rich and poor } \\
\text { C. Distributed equally to rich and poor but in shifts } \\
\text { D. Given to the poor, if can not buy sale to the } \\
\text { rich. }\end{array}$ \\
\hline PAP45aa & $\begin{array}{l}\text { How was the quality of the rice } \\
\text { purchased from the Raskin program? }\end{array}$ & 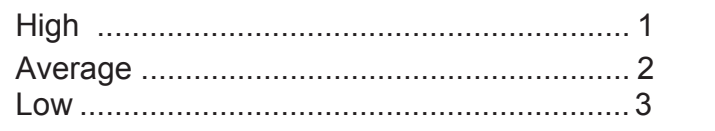 \\
\hline
\end{tabular}

MARKET OPERATION FOR RICE

Now we would like to ask you about the last time market operation for rice was conducted in this village in the last 12 months.

\begin{tabular}{|c|c|c|}
\hline PAP46. & $\begin{array}{l}\text { Was there market operation for rice in the } \\
\text { last } 12 \text { months? }\end{array}$ & $\begin{array}{l}\text { 3. No } \rightarrow \text { PAP56 } \\
\text { 1. Yes }\end{array}$ \\
\hline PAP47. & $\begin{array}{l}\text { In the last } 12 \text { months, how many times was } \\
\text { there market operation for rice in this } \\
\text { village? }\end{array}$ & $\begin{array}{l}\text { 1. ــ L times } \\
\text { 8. DON'T KNOW }\end{array}$ \\
\hline PAP48. & $\begin{array}{l}\text { How many tons of market operation rice } \\
\text { were delivered to this village to be } \\
\text { distributed? }\end{array}$ & $\begin{array}{l}\text { 1. } \longleftarrow \perp \perp \_. \sqcup \text { tons } \\
\text { 8. DON'T KNOW }\end{array}$ \\
\hline PAP49. & $\begin{array}{l}\text { On average, in the last } 12 \text { months was the } \\
\text { percentage of households that received } \\
\text { market operation rice? }\end{array}$ & $\begin{array}{l}\text { 1. } ـ \perp \perp \text { percent } \\
\text { 8. DON'T KNOW }\end{array}$ \\
\hline PAP50. & $\begin{array}{l}\text { When was the last time market operation } \\
\text { for rice was conducted in this village in the } \\
\text { last } 12 \text { months? }\end{array}$ & $\begin{array}{l}\text { 1.レ」 times } \\
\text { 8. DON'T KNOW }\end{array}$ \\
\hline PAP50a. & Who distributed/sold the rice? & $\begin{array}{l}\text { Central government } \\
\text { Regional government } \\
\text { Private/Non-government } \\
\text { DON'T KNOW }\end{array}$ \\
\hline
\end{tabular}




\section{SECTION PAP : POVERTY ALLEVIATION PROGRAM}

\begin{tabular}{|c|c|c|}
\hline PAP51. & $\begin{array}{l}\text { How many tons was delivered to this } \\
\text { village to be sold? }\end{array}$ & $\begin{array}{l}\text { 1. } \sqcup \perp \cdot \sqcup \text { tons } \\
\text { 8. DON'T KNOW }\end{array}$ \\
\hline PAP52. & $\begin{array}{l}\text { What was the percentage of } \\
\text { households that bought rice from the } \\
\text { market operation? }\end{array}$ & $\begin{array}{l}\text { 1. } ـ \perp \perp \text { percent } \\
\text { 8. DON'T KNOW }\end{array}$ \\
\hline PAP53. & $\begin{array}{l}\text { What was the maximum amount of rice } \\
\text { (in } \mathrm{kg} \text { ) that a household can purchase? }\end{array}$ & 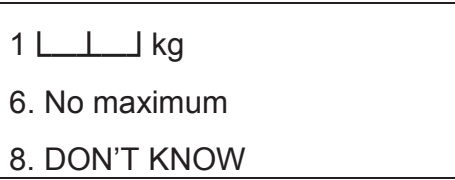 \\
\hline PAP54. & $\begin{array}{l}\text { What was the price per } \mathrm{kg} \text { of rice paid } \\
\text { by each household in the market } \\
\text { operation? }\end{array}$ & 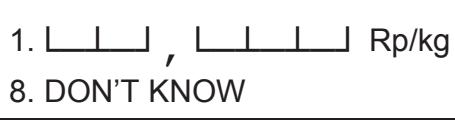 \\
\hline PAP55. & $\begin{array}{l}\text { What was the market price of rice per } \\
\mathrm{kg} \text { at that time? }\end{array}$ & 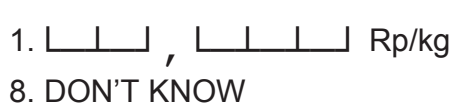 \\
\hline
\end{tabular}

Now we would like to ask about the PKPS BBM-SLT (Unconditional Cash Transfer Program).

\begin{tabular}{|c|c|c|c|c|}
\hline PAP56. & $\begin{array}{l}\text { Was there ever BLSM program in this } \\
\text { village since } 2012 \text { ? }\end{array}$ & $\begin{array}{l}\text { 3. No } \rightarrow P \\
\text { 1. Yes } \\
2014 \\
2013 \\
2012\end{array}$ & $\begin{array}{l}\text { 1. Yes, BLSM } \sqcup \text { times } \\
\text { 1. Yes, BLSM } \sqcup \text { times } \\
\text { 1. Yes, BLSM } \sqcup \text { times }\end{array}$ & $\begin{array}{l}\text { 3. No } \\
\text { 3. No } \\
\text { 3. No }\end{array}$ \\
\hline PAP57. & $\begin{array}{l}\text { Who did the field registration of poor } \\
\text { households who were candidates to } \\
\text { receive the BLSM funds? }\end{array}$ & \multicolumn{3}{|c|}{$\begin{array}{l}\text { A. Village/township officials } \\
\text { B. PKK/Posyandu cadres } \\
\text { C. PUSKESMAS staff/PLKB staff/Midwives } \\
\text { D. Community figure } \\
\text { E. BPS officials/BPS partners/ Mantri Statistik } \\
\text { V. Other }\end{array}$} \\
\hline PAP58. & $\begin{array}{l}\text { When was the last time household in } \\
\text { this village received the BLSM funds? }\end{array}$ & \multicolumn{3}{|c|}{ 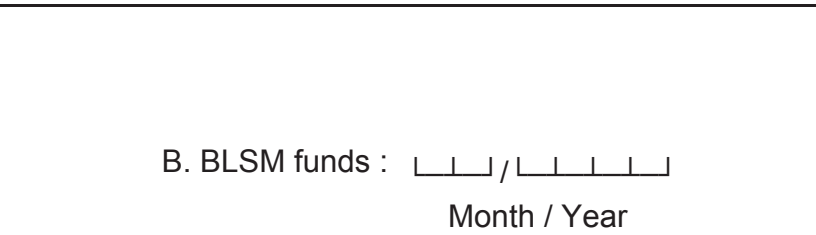 } \\
\hline
\end{tabular}

\begin{tabular}{|c|c|c|}
\hline PAP59. & $\begin{array}{l}\text { How many percent household in this } \\
\text { village received the SLT funds / BLSM } \\
\text { funds? }\end{array}$ & B. BLSM funds : : الــلــــent \\
\hline PAP60. & $\begin{array}{l}\text { How many rupiah did each household } \\
\text { at the last time receive the SLT funds / } \\
\text { BLSM funds? }\end{array}$ & 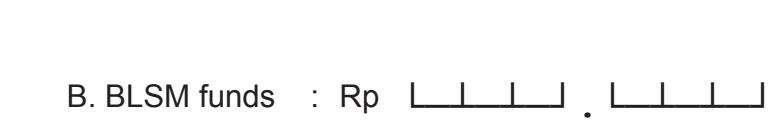 \\
\hline PAP61. & $\begin{array}{l}\text { If a household was not able to collect } \\
\text { the fund at the designated time, can } \\
\text { they came at a different time? }\end{array}$ & 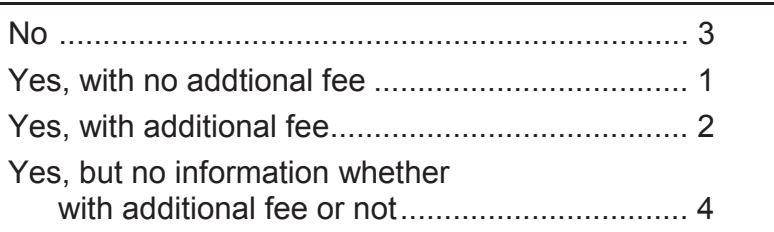 \\
\hline PAP62. & $\begin{array}{l}\text { Can someone else pick up the funds } \\
\text { so long as they show the BLT cards? }\end{array}$ & 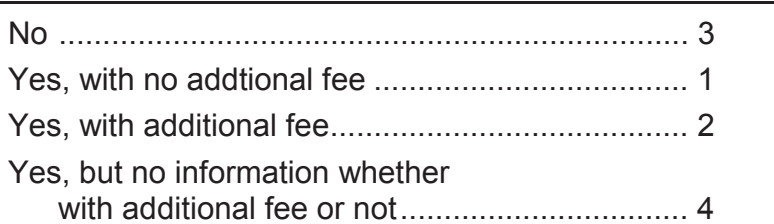 \\
\hline
\end{tabular}

Now we would like to ask about the Keluarga Harapan (Conditional Cash Transfer Program).

\begin{tabular}{|ll|l||}
\hline PAP63a & $\begin{array}{l}\text { Was there Keluarga Harapan Program } \\
\text { has been started in this village? }\end{array}$ & $\begin{array}{l}\text { 3. No } \rightarrow \text { PAP65a } \\
\text { 1. Yes }\end{array}$ \\
\hline PAP64a & $\begin{array}{l}\text { Is Keluarga Harapan Programme } \\
\text { implemented in the last 12 months? }\end{array}$ & $\begin{array}{l}\text { 3. No } \rightarrow \text { PAP65a } \\
\text { 1. Yes }\end{array}$ \\
\hline PAP64b & $\begin{array}{l}\text { Are there a family who should receive } \\
\text { assistance PKH but did not get? }\end{array}$ & $\begin{array}{l}\text { 3. No } \\
\text { 1. Yes }\end{array}$ \\
\hline
\end{tabular}

Now we would like to inquire about PNPM Mandiri

\begin{tabular}{|c|c|c|}
\hline PAP65a. & $\begin{array}{l}\text { Whether in the last } 12 \text { months there } \\
\text { PNPM Mandiri program? }\end{array}$ & $\begin{array}{l}\text { No } \\
\text { Yes }\end{array}$ \\
\hline PAP66a. & $\begin{array}{l}\text { What PNPM Mandiri program exist in } \\
\text { this vilage? }\end{array}$ & $\begin{array}{l}\text { A. PNPM Urban } \\
\text { B. PNPM Rural } \\
\text { C. PNPM PISEW } \\
\text { D. PNPM PUAP } \\
\text { E. PNPM Poor and Disadvantaged Areas } \\
\text { F. PNPM Generation } \\
\text { G. PNPM Mandiri Respect }\end{array}$ \\
\hline
\end{tabular}




\section{SECTION PAP : POVERTY ALLEVIATION PROGRAM}

\begin{tabular}{|c|c|c|}
\hline PAP67a. & $\begin{array}{l}\text { Who are the beneficiaries of PNPM } \\
\text { Mandiri program? }\end{array}$ & $\begin{array}{l}\text { A. Very poor households } \\
\text { B. Poor households } \\
\text { C. Non-poor households } \\
\text { D. Female-headed households }\end{array}$ \\
\hline & & $\begin{array}{l}\text { E. woman } \\
\text { F. schoolchild } \\
\text { G. Residents in nearby activities } \\
\text { V. Other: } \\
\text { W. All people in the village } \\
\text { Y. DO NOT KNOW }\end{array}$ \\
\hline PAP68a. & $\begin{array}{l}\text { Do you think the PNPM activities was } \\
\text { appropriate with the needs of the } \\
\text { people in this village? }\end{array}$ & 1. Yes 3. No $\rightarrow$ PAP70a \\
\hline PAP69a. & $\begin{array}{l}\text { Why PNPM Mandiri activities in } \\
\text { accordance with the people needs in } \\
\text { this village? }\end{array}$ & $\begin{array}{l}\text { A. Opening up economic opportunities } \\
\text { B. easier access } \\
\text { C. The main of requirement } \\
\text { D. Help reduce poverty } \\
\text { V. Other } \\
\text { Y. DO NOT KNOW } \quad \rightarrow \text { PAP71a }\end{array}$ \\
\hline PAP70a. & $\begin{array}{l}\text { Why the PNPM Mandiri program } \\
\text { does not appropriate with the needs } \\
\text { of the people in this village? }\end{array}$ & $\begin{array}{l}\text { A. Not the main needs } \\
\text { B. Can not be enjoyed by communities } \\
\text { C. Already there are other similar programs } \\
\text { D. Can only be enjoyed by certain people } \\
\text { E. Must pay to enjoy the facilities } \\
\text { V. Others, } \\
\text { Y. DO NOT KNOW }\end{array}$ \\
\hline
\end{tabular}

\begin{tabular}{||l|l||}
\hline PAP74a. What assistance programs are the & A. RASKIN \\
social security card used ? & B. BLSM \\
& C. BSM ( Bantuan Siswa Miskin ) \\
& D. PKH ( Program Keluarga Harapan ) \\
\hline
\end{tabular}

Now i would like to ask about KIS (Kartu Indonesia Sehat) Program

\begin{tabular}{|c|c|c|}
\hline PAP75. & $\begin{array}{l}\text { Do you have KIS ( Kartu Indonesia Sehat ) } \\
\text { program in this village/kelurahan? }\end{array}$ & $\begin{array}{l}\text { No } 1 \ldots 1.1 \\
\text { Yes }\end{array}$ \\
\hline PAP76. & $\begin{array}{l}\text { When was }[\ldots] \text { first initiated in this } \\
\text { village/kelurahan? }\end{array}$ & 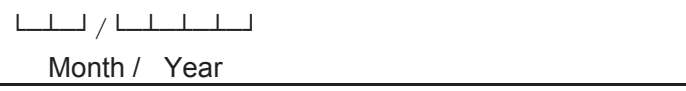 \\
\hline PAP77. & Who benefit from KIS program? & $\begin{array}{l}\text { A. Very poor household } \\
\text { B. Poor household } \\
\text { C. Non poor household } \\
\text { D. Homeless } \\
\text { E. Disable } \\
\text { V. Other: } \\
\text { Y. DON'T KNOW }\end{array}$ \\
\hline PAP78. & $\begin{array}{l}\text { Who conducted listing for household } \\
\text { beneficiaries of KIS program? } \\
\text { (CIRCLE ALL THAT APPLY }\end{array}$ & $\begin{array}{l}\text { A. Village/kelurhan officials } \\
\text { B. Kader PKK/Posyandu } \\
\text { C. PUSKESMAS Official/PLKB /Midwife } \\
\text { D. Community figure } \\
\text { E. BPS Official /Mitra BPS/Mantri Statistik } \\
\text { F. DPM from Government } \\
\text { V. Other }\end{array}$ \\
\hline
\end{tabular}

Now we would like to ask you about the KPS ( Social Security Card)

\begin{tabular}{|c|c|c|c|}
\hline PAP71a. & $\begin{array}{l}\text { Was there KPS ( Social Security } \\
\text { Card ) program in this village? }\end{array}$ & $\begin{array}{l}\text { No } \\
\text { Yes }\end{array}$ & $\begin{array}{l}3 \rightarrow \text { PAP75 } \\
1\end{array}$ \\
\hline PAP72a. & $\begin{array}{l}\text { When was the first time the program } \\
\text { begin? }\end{array}$ & 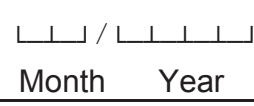 & \\
\hline PAP73a. & For how long is the KPS valid? & $\begin{array}{l}\text { 1. لــ months } \\
\text { 6. No expiration } \\
\text { 8. DON'T KNOW }\end{array}$ & \\
\hline
\end{tabular}

Now i would like to ask about KIP ( Kartu Indonesia Pintar

\begin{tabular}{|c|c|c|}
\hline PAP79. & $\begin{array}{l}\text { Do you have KIP ( Kartu Indonesia } \\
\text { Pintar ) program in this } \\
\text { village/kelurahan? }\end{array}$ & 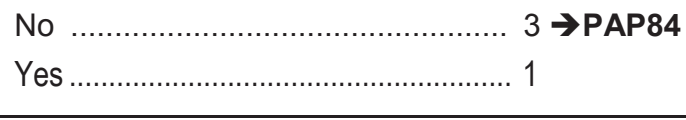 \\
\hline PAP80. & $\begin{array}{l}\text { When was }[\ldots] \text { first initiated in this } \\
\text { village/kelurahan ? }\end{array}$ & 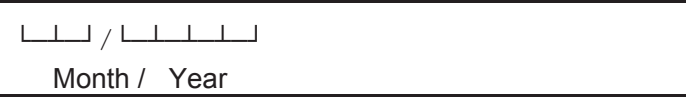 \\
\hline PAP81. & Who benefit from KIP program? & $\begin{array}{ll}\text { A. Very poor household } \\
\text { B. Poor household } \\
\text { C. Non poor household } \\
\text { D. Homeless } \\
\text { E. Disable } \\
\text { V. Other: } \\
\text { Y. DON'T KNOW }\end{array}$ \\
\hline
\end{tabular}




\section{SECTION PAP : POVERTY ALLEVIATION PROGRAM}

\begin{tabular}{|c|c|c|}
\hline PAP82. & $\begin{array}{l}\text { Who conducted listing for } \\
\text { household beneficiaries of KIP } \\
\text { program? } \\
\text { (CIRCLE ALL THAT APPLY) }\end{array}$ & $\begin{array}{l}\text { A. Village/kelurahan officials } \\
\text { B. Kader PKK/Posyandu } \\
\text { C. PUSKESMAS Official/PLKB /Midwife } \\
\text { D. Community figure } \\
\text { E. BPS Official /Mitra BPS/Mantri Statistik } \\
\text { F. DPM from Government } \\
\text { V. Other }\end{array}$ \\
\hline \multirow[t]{2}{*}{ PAP83 } & How much amount is given? & \\
\hline & 1.SD & 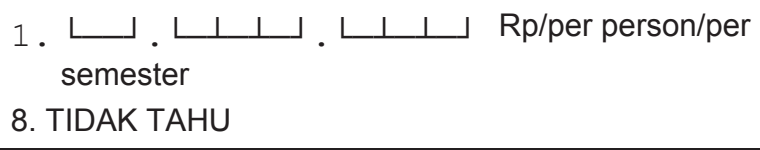 \\
\hline & 2. SMP & 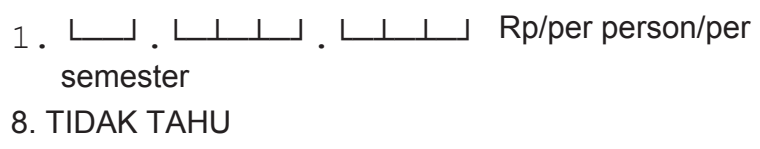 \\
\hline & 3. SMA & 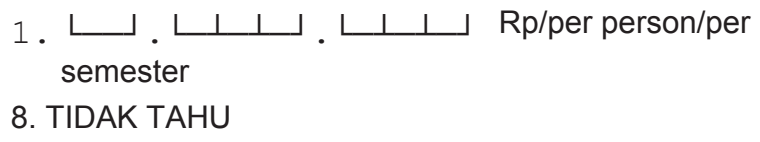 \\
\hline
\end{tabular}

\begin{tabular}{||l|l||}
\hline PAP88 How much amount is given? & $\begin{array}{l}\text { 1. } \perp \perp \perp . \sqcup \perp \perp \perp \text { Rp/per person/per month } \\
\text { 8. DON'T KNOW }\end{array}$ \\
\hline
\end{tabular}

Now I would like to know about KKS ( Kartu Keluarga Sejahtera ) Program

\begin{tabular}{|c|c|c|}
\hline PAP84. & $\begin{array}{l}\text { Do you have KKS ( Kartu Keluarga } \\
\text { Sejahtera ) program in this } \\
\text { village/kelurahan? }\end{array}$ & $\begin{array}{l}\text { No } \\
\text { Yes } \ldots \ldots \ldots \ldots \ldots \ldots \ldots \ldots \ldots \ldots \ldots \ldots \\
3\end{array}$ \\
\hline PAP85. & $\begin{array}{l}\text { When was }[\ldots] \text { first initiated in this } \\
\text { village/kelurahan ? }\end{array}$ & 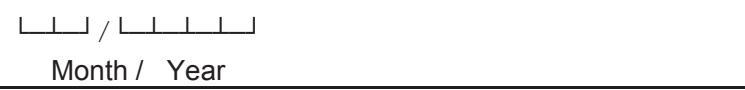 \\
\hline PAP86. & Who benefit from KKS program? & $\begin{array}{l}\text { A. Very poor household } \\
\text { B. Poor household } \\
\text { C. Non poor household } \\
\text { D. Homeless } \\
\text { E. Disable } \\
\text { V. Other: } \\
\text { Y. DON'T KNOW }\end{array}$ \\
\hline PAP87. & $\begin{array}{l}\text { Who conducted listing for } \\
\text { household beneficiaries of KKS } \\
\text { program? } \\
\text { (CIRCLE ALL THAT APPLY) }\end{array}$ & 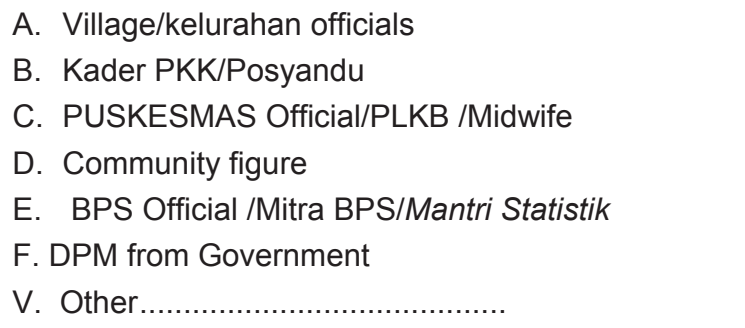 \\
\hline
\end{tabular}




\section{SECTION PPS : PERCEPTION ON PUBLIC SERVICES AND INFRASTRUCTURE}

Section PPS Respondent:

\begin{tabular}{|c|c|}
\hline 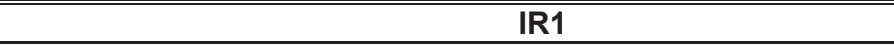 & IR2 \\
\hline $\begin{array}{l}\text { 1_PPS. Name of Respondent: } \\
\text { CAPI WILL SKIP TO PPS1 IF THE SAME NAME } \\
\text { HAS ALREADY BEEN ENTERED EARLIER }\end{array}$ & \\
\hline Position in the village : & $\begin{array}{ccccc}01 & 02 & 03 & 04 & 05 \\
06 & 07 & 95 & \ldots \ldots \ldots \ldots \ldots . .\end{array}$ \\
\hline 2_PPS. Age: & $\downarrow$ Years \\
\hline 2a_PPS. Length of tenure in positions: & 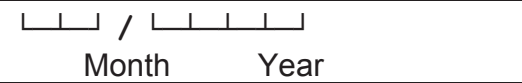 \\
\hline $\begin{array}{ll}\text { 3_PPS. } & \text { INTERVIEWER CHECK : } \\
& \text { SEX OF RESPONDENT }\end{array}$ & $\begin{array}{l}\text { MALE } \ldots \ldots \ldots \ldots \ldots \ldots \ldots \ldots . . .1 \\
\text { FEMALE ………....... } 3\end{array}$ \\
\hline 3a_PPS. Highest level of education attended: & 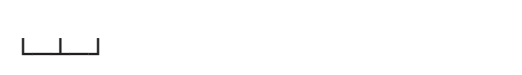 \\
\hline 4_PPS. Highest class completed: & $\begin{array}{lllll}00 & 01 & 02 & 03 & 04 \\
05 & 06 & 07 & 98 & 96 \\
\end{array}$ \\
\hline Length of time lived in the village : & 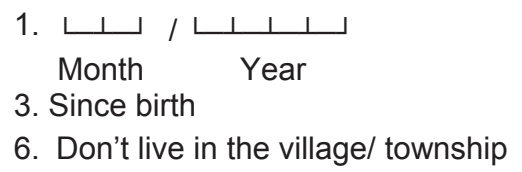 \\
\hline 6_PPS. How did $[\ldots]$ come to assume to the position & $\begin{array}{l}\text { 1. Elected } \\
\text { 2. Appointed by residence } \\
\text { 3. Appointed by camat } \\
\text { 4. Appointed by Bupati/Walikota } \\
\text { 5. Other }\end{array}$ \\
\hline
\end{tabular}

\section{Code for position in village (1a_PPS):}

01. Village/township heads

02. Village secretary

overnment Administration

04. Head of Village Development

55. Head of Welfare

66. Head of Financial Matters

07. Head of General Affairs

\section{Codes for highest level of education (3a_PPS):}

01. No school/not yet in school

02. Elementary school

04. Junior High - Vocational

05. High School - General

06. High School - Vocational

60. D1, D2, D3 (Junior College)

61. University $S 1$ (Bachelor)

62. University S2 (Masters)
63. University S3 (Ph.D.)
1. Kejar Paket A (Adult Education A)

12. Kejar Paket $B$ (Adult Education $B$ )

13. Open Univeristy

4. Islamic School (Pondok Pensantren)

5. Kejar Paket C (Adult Education C)

17. School for the disabled

2. Islamic Elementary School (Madrasah Ibtidaiyah)

73. Islamic Junior High School (Madrasah Tsanawiyah)

74. Islamic Senior High School (Madrasah Alliyah)

98. DON'T KNOW

95. Other
Codes for highest grade (4 PPS):

00. Never completed class 1

01. 1

03.3

04. 4

05. 5

06. 6

07. Graduated

96. No school
98. DON'T KNOW 
SECTION PPS : PERCEPTION ON PUBLIC SERVICES AND INFRASTRUCTURE

We would like to ask you about the public infrastructure and facilities in this village/township.

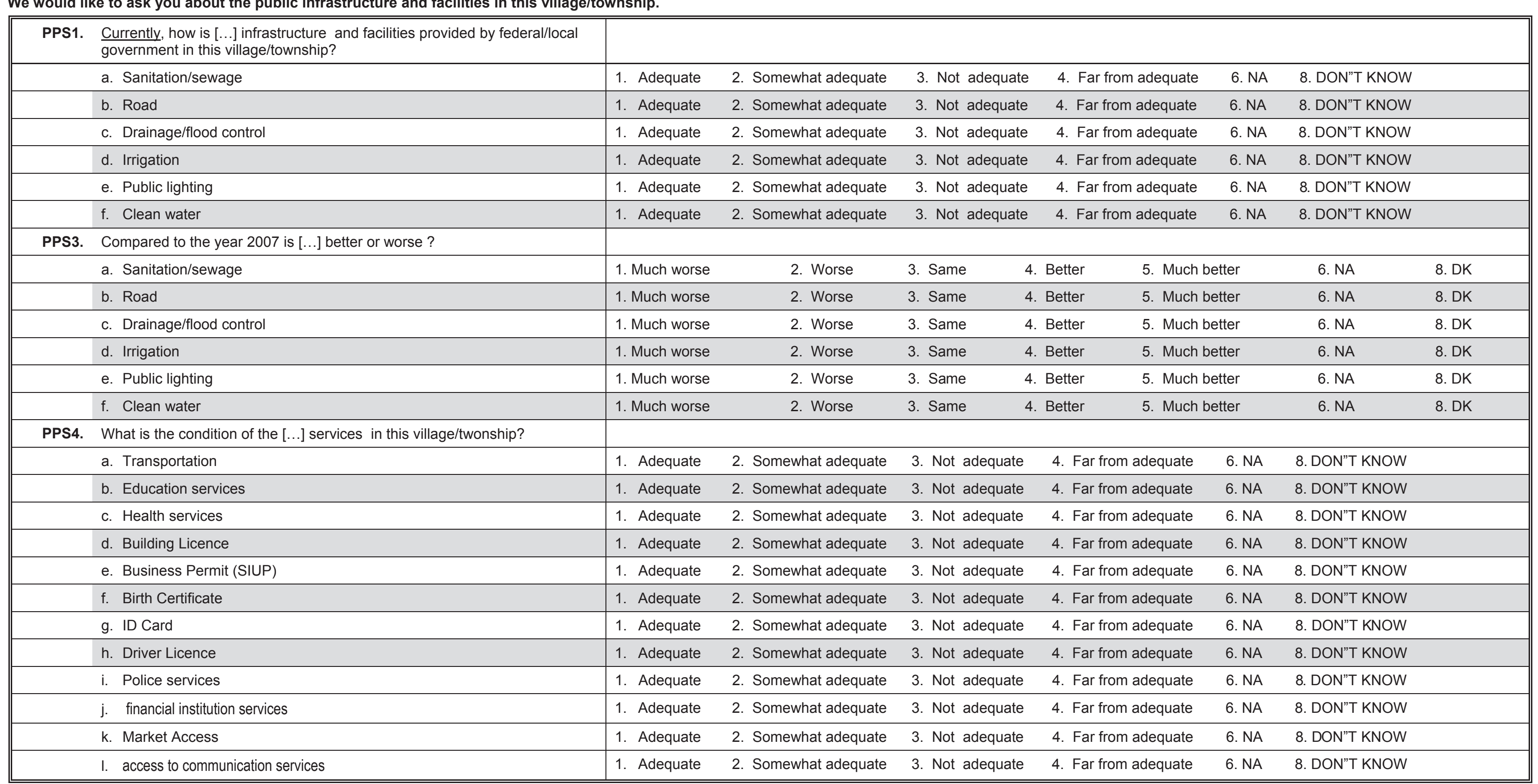




\section{SECTION PPS : PERCEPTION ON PUBLIC SERVICES AND INFRASTRUCTURE}

\begin{tabular}{|c|c|c|c|c|c|c|c|c|}
\hline & a. Transportation & 1. Much worse & 2. Worse & 3. Same & 4. Better & 5. Much better & 6. NA & 8. DK \\
\hline & b. Education services & 1. Much worse & 2. Worse & 3. Same & 4. Better & 5. Much better & 6. NA & 8. DK \\
\hline & c. Health services & 1. Much worse & 2. Worse & 3. Same & 4. Better & 5. Much better & 6. NA & 8. DK \\
\hline & d. Building Licence & 1. Much worse & 2. Worse & 3. Same & 4. Better & 5. Much better & 6. NA & 8. DK \\
\hline & e. Business Permit (SIUP) & 1. Much worse & 2. Worse & 3. Same & 4. Better & 5. Much better & 6. NA & 8. DK \\
\hline & f. Birth Certificate & 1. Much worse & 2. Worse & 3. Same & 4. Better & 5. Much better & 6. NA & 8. DK \\
\hline & g. ID Card & 1. Much worse & 2. Worse & 3. Same & 4. Better & 5. Much better & 6. NA & 8. DK \\
\hline & h. Driver Licence & 1. Much worse & 2. Worse & 3. Same & 4. Better & 5. Much better & 6. NA & 8. DK \\
\hline & i. Police services & 1. Much worse & 2. Worse & 3. Same & 4. Better & 5. Much better & 6. NA & 8. DK \\
\hline & j. financial institution services & 1. Much worse & 2. Worse & 3. Same & 4. Better & 5. Much better & 6. NA & 8. DK \\
\hline & k. Market Access & 1. Much worse & 2. Worse & 3. Same & 4. Better & 5. Much better & 6. NA & 8. DK \\
\hline & I. access to communication services & 1. Much worse & 2. Worse & 3. Same & 4. Better & 5. Much better & 6. NA & 8. DK \\
\hline
\end{tabular}




\section{SECTION GD : GOVERNMENT AND DECENTRALIZATION}

Section GD Respondent:

\begin{tabular}{|c|c|c|}
\hline ב- & IR1 & IR2 \\
\hline 1_GD. & $\begin{array}{l}\text { Name of Respondent: } \\
\text { CAPI WILL SKIP TO GD2 IF THE SAME NAME } \\
\text { HAS ALREADY BEEN ENTERED EARLIER }\end{array}$ & \\
\hline $1 a_{-}$GD. & Position in the village : & $\begin{array}{rrrrr}01 & 02 & 03 & 04 & 05 \\
06 & 07 & 95 & \ldots \ldots \ldots \ldots \ldots \ldots\end{array}$ \\
\hline 2_GD. & Age : & $\downarrow$ Y Years \\
\hline $2 a_{-} G D$. & Length of tenure in positions: & 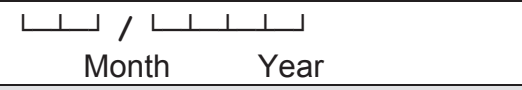 \\
\hline 3_GD. & $\begin{array}{l}\text { INTERVIEWER CHECK : } \\
\text { SEX OF RESPONDENT }\end{array}$ & $\begin{array}{l}\text { MALE } \ldots \ldots \ldots \ldots \ldots \ldots . . . .1 \\
\text { FEMALE .................... } 3\end{array}$ \\
\hline $3 a_{-} G D$. & Highest level of education attended: & 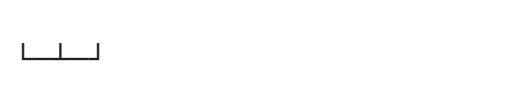 \\
\hline 4_GD. & Highest class completed : & $\begin{array}{lllll}00 & 01 & 02 & 03 & 04 \\
05 & 06 & 07 & 98 & 96 \\
\end{array}$ \\
\hline 5_GD. & Length of time lived in the village : & 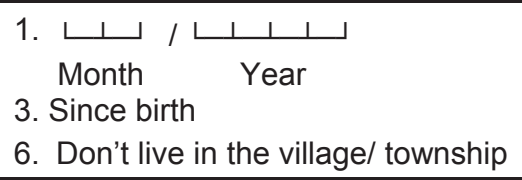 \\
\hline 6_GD. & How did $[\ldots]$ come to assume to the position & $\begin{array}{l}\text { 1. Elected } \\
\text { 2. Appointed by residence } \\
\text { 3. Appointed by camat } \\
\text { 4. Appointed by Bupati/Walikota } \\
\text { 5. Other }\end{array}$ \\
\hline
\end{tabular}

\section{Code for position in village (1a_GD):}

\section{Village/township head}

03. Head of Government Administration

04. Head of Village Development

05. Head of Welfare

06. Head of Financial Matters

97. Other

\section{Codes for highest level of education (3a_GD)}
01. No school/not yet in school
02. Elementary school
03. Junior High - General
04. Junior High - Vocational
05. High School - General
06. High School - Vocational
61. University $\mathrm{S} 1$ (Bio College)
62. University $S 2$ (Masters)
63. University S3 (Ph.D.)

11. Kejar Paket A (Adult Education A)

2. Kejar Paket B (Adult Education B)

13. Open Univeristy

(Pondok Pensantren)

15. Kejar Paket C (Adult Education C)

72. Islamic Elementary School (Madrasah Ibtidaiyah)

73. Islamic Junior High

74. Islamic Senior High School (Madrasah Alliyah)

90. Kindergarten

95. Other
Codes for highest grade (4_GD)

00. Never completed class 1

01. 1

02.

03. 3

04. 4

07. Graduated

96. No school 


\section{SECTION GD : GOVERNMENT AND DECENTRALIZATION}

\section{Now, we would like to ask your opinion on regional autonomy.}

\begin{tabular}{|c|c|c|}
\hline GD2. & $\begin{array}{l}\text { Was the village office involved in the } \\
\text { construction of facilities and } \\
\text { infrastructures by the district local } \\
\text { government? }\end{array}$ & $\begin{array}{l}\text { 3. No } \rightarrow \text { GD4 } \\
\text { 1. Yes } \\
\text { 8. DON'T KNOW } \rightarrow \text { GD4 }\end{array}$ \\
\hline GD3. & $\begin{array}{l}\text { On what level was the village office } \\
\text { involved? }\end{array}$ & $\begin{array}{l}\text { 1. Planning } \\
\text { 2. Construction } \\
\text { 3. Control/Supervision } \\
\text { 4. Maintainance } \\
\text { 5. Other } \\
\text { 8. DON'T KNOW }\end{array}$ \\
\hline GD4. & $\begin{array}{l}\text { If this village need new infrasctructure or } \\
\text { if the existing infrastructure need repair, } \\
\text { how can this village ask for assistance } \\
\text { from the government? }\end{array}$ & $\begin{array}{l}\text { 01. Request through the sub district office } \\
\text { 02. Request through the district office } \\
\text { 03. Request through the province office } \\
\text { 06. No mechanism } \\
\text { 95. Other }\end{array}$ \\
\hline GD5. & $\begin{array}{l}\text { Who determine the village budget in the } \\
\text { current budget year? }\end{array}$ & 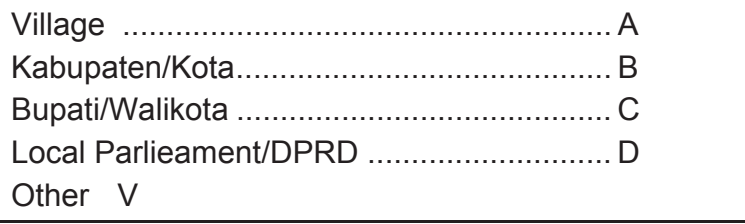 \\
\hline GD6. & $\begin{array}{l}\text { Does the village has the auhtority to } \\
\text { reallocate budget between budeget } \\
\text { posts/categories without approval from } \\
\text { Kabupaten/Kota government or other } \\
\text { parties? }\end{array}$ & Yes \\
\hline GD7. & $\begin{array}{l}\text { Compared to } 2007 \text {, in your view, is the } \\
\text { current budget system better, worse, or } \\
\text { the same? }\end{array}$ & $\begin{array}{l}\text { Much better } \\
\text { Better } \\
\text { Same } \\
\text { NA } \\
\text { Worse }\end{array}$ \\
\hline
\end{tabular}

\begin{tabular}{|c|c|c|}
\hline GD8. & $\begin{array}{l}\text { Why do you think the current system is } \\
\text { worse? }\end{array}$ & 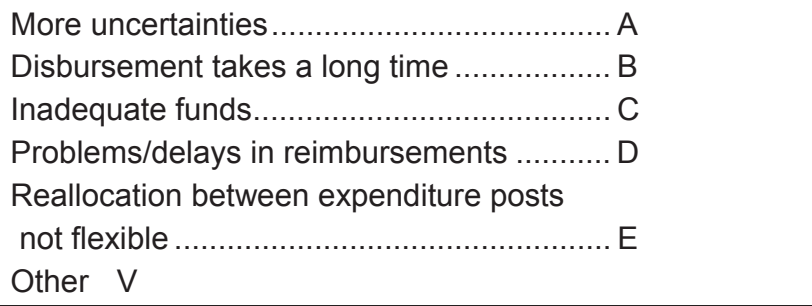 \\
\hline GD9 & $\begin{array}{l}\text { Does the village have BUMDes (village- } \\
\text { owned enterprises)? }\end{array}$ & $\begin{array}{l}\text { Yes } \\
\text { No.. }\end{array}$ \\
\hline GD10 & When BUMDes is was founded? & 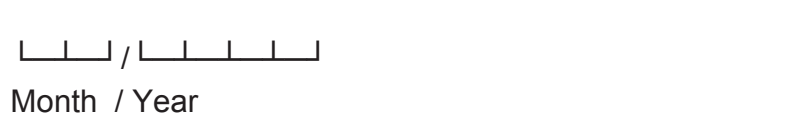 \\
\hline GD11 & $\begin{array}{l}\text { How does the process of BUMDes } \\
\text { establishment? }\end{array}$ & $\begin{array}{l}\text { Community meetings and village governments } \ldots \ldots \ldots \ldots . . . \mathrm{A} \\
\text { Formed by the Village Head } \\
\text { Formed by BPD } \\
\text { Others }\end{array}$ \\
\hline GD12 & In what bussines BUMDes operate? & 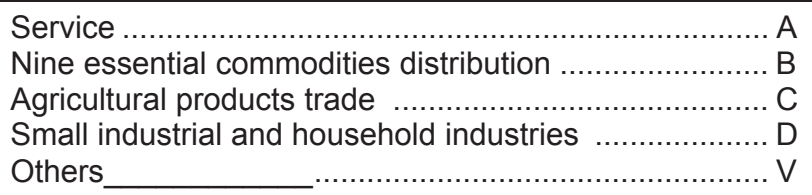 \\
\hline
\end{tabular}


SECTION TR: TRUST

Section TR Respondent:

\begin{tabular}{|c|c|c|}
\hline 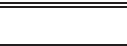 & ( & IR2 \\
\hline 1_TR. & $\begin{array}{l}\text { Name of Respondent: } \\
\text { CAPI WILL SKIP TO TR01 IF THE SAME NAME } \\
\text { HAS ALREADY BEEN ENTERED EARLIER }\end{array}$ & \\
\hline 1a_TR. & Position in the village: & $\begin{array}{ccccc}01 & 02 & 03 & 04 & 05 \\
06 & 07 & 95 & \ldots \ldots \ldots \ldots \ldots . .\end{array}$ \\
\hline 2_TR. & Age : & $\perp$ Years \\
\hline 2a_TR. & Length of tenure in positions: & 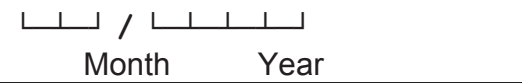 \\
\hline 3_TR. & $\begin{array}{l}\text { INTERVIEWER CHECK: } \\
\text { SEX OF RESPONDENT }\end{array}$ & 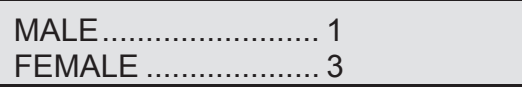 \\
\hline 3a_TR. & Highest level of education attended: & $\sqcup$ \\
\hline 4_TR. & Highest class completed : & $\begin{array}{lllll}00 & 01 & 02 & 03 & 04 \\
05 & 06 & 07 & 98 & 96 \\
\end{array}$ \\
\hline 5_TR. & Length of time lived in the village : & 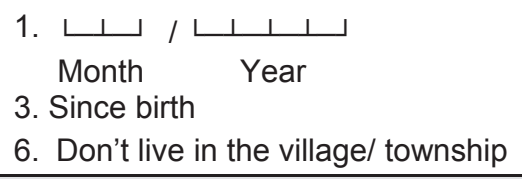 \\
\hline 6_TR. & How did $[\ldots]$ come to assume to the position & $\begin{array}{l}\text { 1. Elected } \\
\text { 2. Appointed by residence } \\
\text { 3. Appointed by camat } \\
\text { 4. Appointed by Bupati/Walikota } \\
\text { 5. Other }\end{array}$ \\
\hline
\end{tabular}

\section{Code for position in village (1a_TR)}

01. Village/township heads

02. Head of Government Administration

04. Head of Village Development

05. Head of Welfare

06. Head of Financial Matter

07. Head of General Affairs

\section{Codes for highest level of education (3a_ TR):}

01. No school/not yet in school

02. Elementary school

04. Junior High - Vocational

05. High School - General

06. High School - Vocational

60. D1, D2, D3 (Junior College)

62. University $\mathrm{S} 2$ (Masters)

62. University S2 (Masters)
11. Kejar Paket $A$ (Adult Education $A$ )

12. Kejar Paket $B$ (Adult Education $B$ )

14. Open Univeristy

15. Kejor Paket (Adul Edunsantren)

17. School for the disabled

72. Islamic Elementary School (Madrasah Ibtidaiyah)

73. Islamic Junior High School (Madrasah Tsanawiyah)

74. Islamic Senior High School (Madrasah Alliyah)

90. Kindergarten

95. Other

\section{Codes for highest grade (4_TR)}

00. Never completed class 1

01. 1

03. 3

04. 4

05. 5

06.

Graduated

96. No school 


\section{SECTION TR: TRUST}

Now we want to ask you about conflicts that have occurred in this village/township since 2007.

\begin{tabular}{|c|c|c|c|c|c|c|c|c|c|c|c|c|c|c|c|c|c|c|}
\hline & \multicolumn{3}{|c|}{ TR01. } & TR02. & TR02a & \multicolumn{10}{|c|}{ TR03. } & \multicolumn{3}{|c|}{ TR04. } \\
\hline Type of conflicts & $\begin{array}{r}\mathrm{Ha} \\
\text { village }\end{array}$ & $\begin{array}{l}\text {...] occl } \\
\text { wnshop }\end{array}$ & $\begin{array}{l}\text { in this } \\
\text { nce } 2007 ?\end{array}$ & $\begin{array}{c}\text { Was there violence in the } \\
{[[\ldots . .] \text { ? }}\end{array}$ & $\begin{array}{l}\text { Was there anyone dead in } \\
\text { (...) }\end{array}$ & & Whe & sual & vere & volv & in th & effo & to re & Plve t & e conflict? & $\begin{array}{r}\text { Sinc } \\
\text { numb } \\
\text { incre } \\
\end{array}$ & $\begin{array}{l}\text { of th } \\
\text { flict } \\
\text { ng, t } \\
\text { crea }\end{array}$ & $\begin{array}{l}\text { the } \\
\text { pe of } \\
\text { ame, } \\
\text { ? }\end{array}$ \\
\hline $\begin{array}{l}\text { a. Conflicts on land/building } \\
\text { between citizen and } \\
\text { government }\end{array}$ & 3. No & 8. DK & 1. Yes $\rightarrow$ & 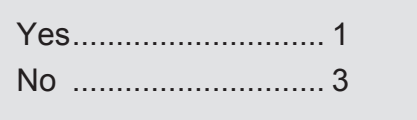 & 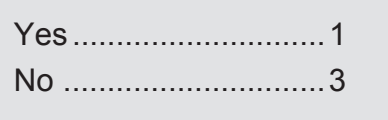 & $\mathrm{Y}$ & $\begin{array}{l}\text { A } \\
K\end{array}$ & $\begin{array}{l}\mathrm{B} \\
\mathrm{V}\end{array}$ & C & D & E & $\mathrm{F}$ & G & $\mathrm{H}$ & $\mathrm{J}$ & 1 & 2 & 3 \\
\hline $\begin{array}{l}\text { b. Conflicts on land building } \\
\text { between citizens }\end{array}$ & 3. No & 8. DK & 1. Yes $\rightarrow$ & 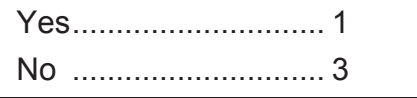 & Yes $\ldots \ldots \ldots \ldots \ldots \ldots \ldots \ldots \ldots . . .1$ & Y & $\begin{array}{l}\mathrm{A} \\
\mathrm{K}\end{array}$ & $\begin{array}{l}\mathrm{B} \\
\mathrm{V}\end{array}$ & C & D & E & $F$ & G & $\mathrm{H}$ & $J$ & 1 & 2 & 3 \\
\hline $\begin{array}{l}\text { c. Conflicts arising from abuses } \\
\text { of power/authority }\end{array}$ & 3. No & 8. DK & 1. Yes $\rightarrow$ & 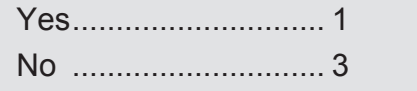 & 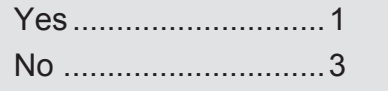 & Y & $\begin{array}{l}\text { A } \\
K\end{array}$ & $\begin{array}{l}\mathrm{B} \\
\mathrm{V}\end{array}$ & C & D & E & $\mathrm{F}$ & G & $\mathrm{H}$ & $J$ & 1 & 2 & 3 \\
\hline $\begin{array}{l}\text { d. Conflicts related to election of } \\
\text { public officials or general } \\
\text { election }\end{array}$ & 3. No & $\stackrel{\text { 8. DK }}{\downarrow}$ & 1. Yes $\rightarrow$ & 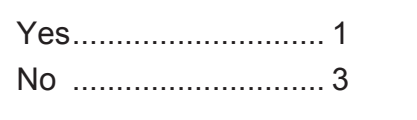 & 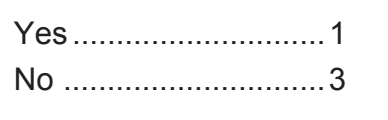 & Y & $\begin{array}{l}\mathrm{A} \\
\mathrm{K}\end{array}$ & $\begin{array}{l}\mathrm{B} \\
\mathrm{V}\end{array}$ & C & D & E & $\mathrm{F}$ & G & $\mathrm{H}$ & $J$ & 1 & 2 & 3 \\
\hline $\begin{array}{l}\text { e. Conflicts between members } \\
\text { of different ethnic groups }\end{array}$ & 3. No & 8. DK & 1. Yes $\rightarrow$ & 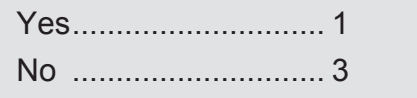 & 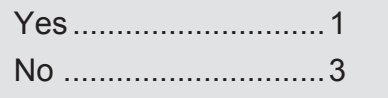 & Y & $\begin{array}{l}\mathrm{A} \\
\mathrm{K}\end{array}$ & $\begin{array}{l}B \\
V\end{array}$ & C & D & E & $\mathrm{F}$ & G & $\mathrm{H}$ & $J$ & 1 & 2 & 3 \\
\hline $\begin{array}{l}\text { f. Conflicts between members } \\
\text { of different religions/faiths }\end{array}$ & 3. No & 8. DK & 1. Yes $\rightarrow$ & $\begin{array}{l}\text { Yes } \\
\text { No } \ldots \ldots \ldots \ldots \ldots \ldots \ldots \ldots \ldots \ldots \ldots \ldots \\
\end{array}$ & 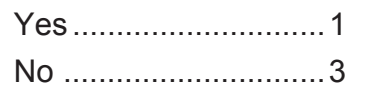 & Y & $\begin{array}{l}\mathrm{A} \\
\mathrm{K}\end{array}$ & $\begin{array}{l}\mathrm{B} \\
\mathrm{V}\end{array}$ & C & D & $\mathrm{E}$ & $\mathrm{F}$ & G & $\mathrm{H}$ & $J$ & 1 & 2 & 3 \\
\hline $\begin{array}{l}\text { g. Conflict between community and } \\
\text { private business/corporation }\end{array}$ & $\begin{array}{l}\text { 3. No } \\
\downarrow \\
\text { TR05 } \\
\end{array}$ & $\begin{array}{l}\text { 8. DK } \\
\downarrow \\
\text { TR05 } \\
\end{array}$ & 1. Yes $\rightarrow$ & 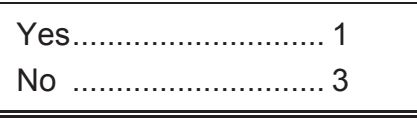 & 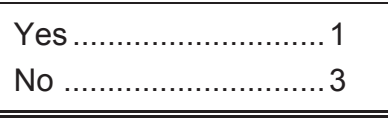 & Y & $\begin{array}{l}\mathrm{A} \\
\mathrm{K}\end{array}$ & $\begin{array}{l}B \\
V\end{array}$ & C & D & $\mathrm{E}$ & $\mathrm{F}$ & G & $\mathrm{H}$ & $\begin{array}{ll} \\
\end{array}$ & 1 & 2 & 3 \\
\hline
\end{tabular}




\section{SECTION TR: TRUST}

Now we want to ask you about crime in this village.

\begin{tabular}{|c|c|c|}
\hline TR05. & How safe do you consider this village? & 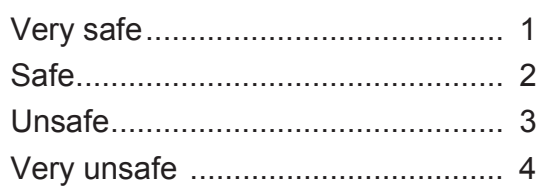 \\
\hline TR06. & $\begin{array}{l}\text { Compared to } 2007 \text {, how safe do you } \\
\text { consider this village is? }\end{array}$ & 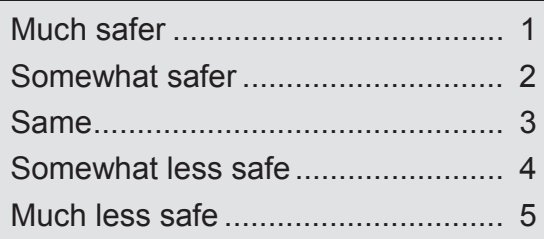 \\
\hline TR07. & $\begin{array}{l}\text { In most part of the village, is it safe to walk } \\
\text { at night? }\end{array}$ & $\begin{array}{l}\text { Very safe } \\
\text { Safe } \\
\text { Unsafe } \\
\text { Very unsafe }\end{array}$ \\
\hline TR07a & $\begin{array}{l}\text { In most part of the village, is it safe for a } \\
\text { woman to walk at night? }\end{array}$ & 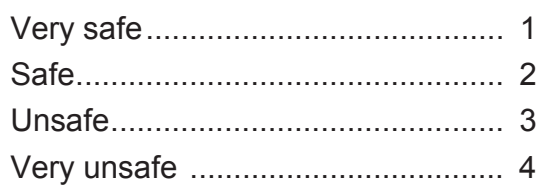 \\
\hline TR08. & $\begin{array}{l}\text { In the last } 12 \text { months has any household } \\
\text { in this village been a victim of theft? }\end{array}$ & Yes \\
\hline TR09. & $\begin{array}{l}\text { In the last } 12 \text { months has any household } \\
\text { in this village been a victim of } \\
\text { crop/livestock theft? }\end{array}$ & 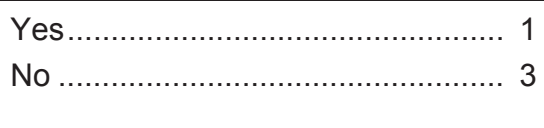 \\
\hline TR10. & $\begin{array}{l}\text { In the last } 12 \text { months has any household } \\
\text { in this village been a victim of robbery? }\end{array}$ & Yes \\
\hline TR11. & $\begin{array}{l}\text { In the last } 12 \text { months, has there been any } \\
\text { incident of sexual assaults? }\end{array}$ & 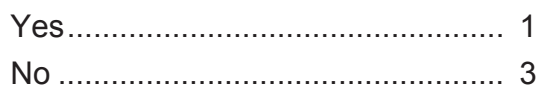 \\
\hline TR12. & $\begin{array}{l}\text { In the last } 12 \text { months, has there been any } \\
\text { incident of domestic assaults? }\end{array}$ & 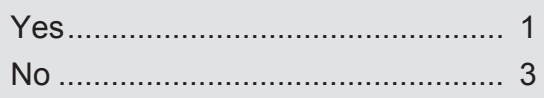 \\
\hline TR13. & $\begin{array}{l}\text { In the last } 12 \text { months, has there been any } \\
\text { other violences/assaults, include civil } \\
\text { strife? }\end{array}$ & 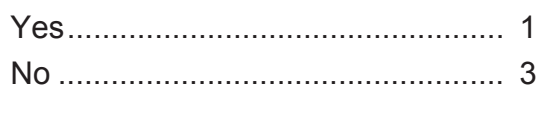 \\
\hline
\end{tabular}

TRUST

Now we want to ask you about the sense of trust within this community.

\begin{tabular}{|c|c|c|}
\hline TR14. & $\begin{array}{l}\text { People in this village are always looking } \\
\text { out for each other }\end{array}$ & 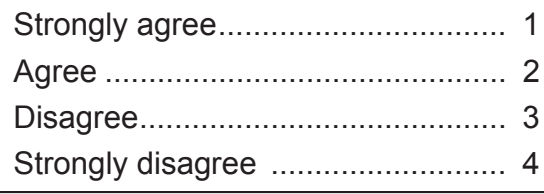 \\
\hline TR15. & $\begin{array}{l}\text { Most people in the village are willing to } \\
\text { help if you need it. }\end{array}$ & 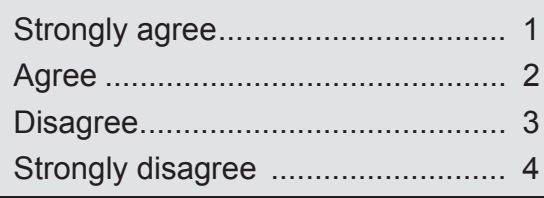 \\
\hline TR16. & $\begin{array}{l}\text { In this village one has to be alert or } \\
\text { someone is likely to take advantage of } \\
\text { you. }\end{array}$ & 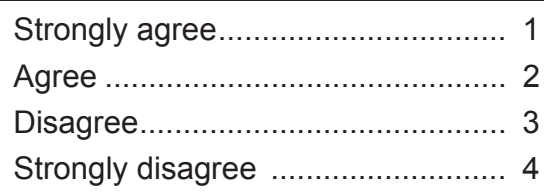 \\
\hline TR17. & $\begin{array}{l}\text { In this village, residents from the same } \\
\text { ethnicity trust each other more than they } \\
\text { trust those with different ethnicity. }\end{array}$ & 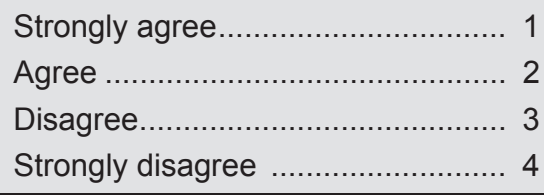 \\
\hline TR18. & $\begin{array}{l}\text { In this village, residents from the same } \\
\text { religion trust each ither more than they } \\
\text { trust those with different religion. }\end{array}$ & 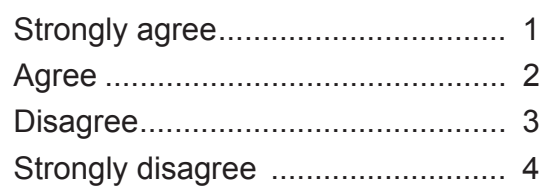 \\
\hline TR19. & $\begin{array}{l}\text { Would people in this village be willing to } \\
\text { leave their children with their neighbors } \\
\text { for a few hours if they cannot bring their } \\
\text { children with them? }\end{array}$ & 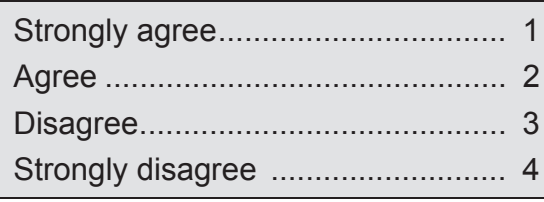 \\
\hline TR20. & $\begin{array}{l}\text { Would people in this village be willing to } \\
\text { ask their neighbors to look after their } \\
\text { house if they leave for a few days? }\end{array}$ & $\begin{array}{l}\text { Strongly agree } \\
\text { Agree } \\
\text { Disagree } \\
\text { Strongly disagree }\end{array}$ \\
\hline
\end{tabular}




\section{SECTION CP: INTERVIEWER NOTES}

\begin{tabular}{|c|c|c|c|c|c|c|c|}
\hline LANGMAIN (CK1). & \multicolumn{2}{|l|}{ Interview was entirely/mostly conducted in what language? } & \multicolumn{5}{|l|}{ ـــ other } \\
\hline LANGOTHR (CK2). & Other languaged used (if any): & & \multicolumn{5}{|l|}{ 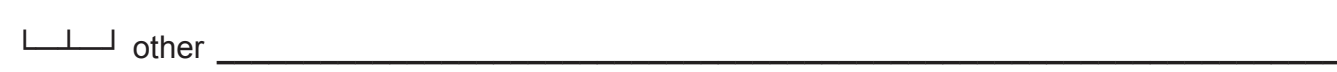 } \\
\hline \multicolumn{8}{|l|}{ LANGUAGE CODE: } \\
\hline 00. Bahasa Indonesia & 04. Batak & Sasak & 12. Makassar & 16. & Toraja & & Lampung \\
\hline 01. Java & 05. Bugis & 09. Minang & 13. Nias & 17. & Lahat & 95. & Other, \\
\hline 02. Sunda & 06. Cina & Banjar & 14. Palembang & 18. & Other South Sumatera & 96. & NA \\
\hline 03. Bali & 07. Madura & 11. Bima & 15. Sumbawa & 19. & Betawi & & \\
\hline
\end{tabular}

\begin{tabular}{|c|c|c|c|}
\hline RESULT (FP3). RESULTS OF INTERVIEW & REASON (FP4). REASON FOR ANSWERING “2” I "3" IN RESULT. & FP6. MONITORING BY SUPERVISOR & \\
\hline $\begin{array}{l}\text { 1. Completed } \rightarrow \text { FP6 } \\
\text { 2. Partly completed } \\
\text { 3. Not completed } \\
\text { 4. Twin EA with EA } \downarrow \perp \text { LPP6 }\end{array}$ & $\begin{array}{l}\text { 1. Respondent was travelling/not in location } \\
\text { 2. Respondent was too busy } \\
\text { 3. Respondent refused }\end{array}$ & $\begin{array}{ll}\text { a. } & \text { Observed (sup_obs) .......... } 1 \\
\text { b. } & \text { Checked (sup_edit)........... } 1 \\
\text { c. } & \text { Verified (sup_veri).............. } 1\end{array}$ & $\begin{array}{l}\text { No } \\
3 \\
3 \\
3\end{array}$ \\
\hline
\end{tabular}

\section{INTERVIEWER NOTE:}




\section{INDONESIAN FAMILY LIFE SURVEY 2014 \\ COMMUNITY AND FACILITY \\ PEOPLE CHARACTERISTICS BOOK II}

SECTIONS : LK, KA, PL, ST, PR, LU, KD, SD, OL, CP EA / BOOK TYPE

NAME OF DESA/KELURAHAN :

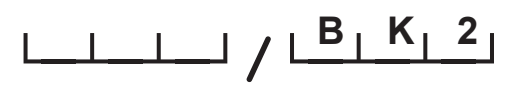

COV1X. CAPI CHECK: IS THIS A TWIN EA?

1. Yes $\rightarrow$ CK1

3. No 


\section{SECTION LK (CONTROL SHEET)}

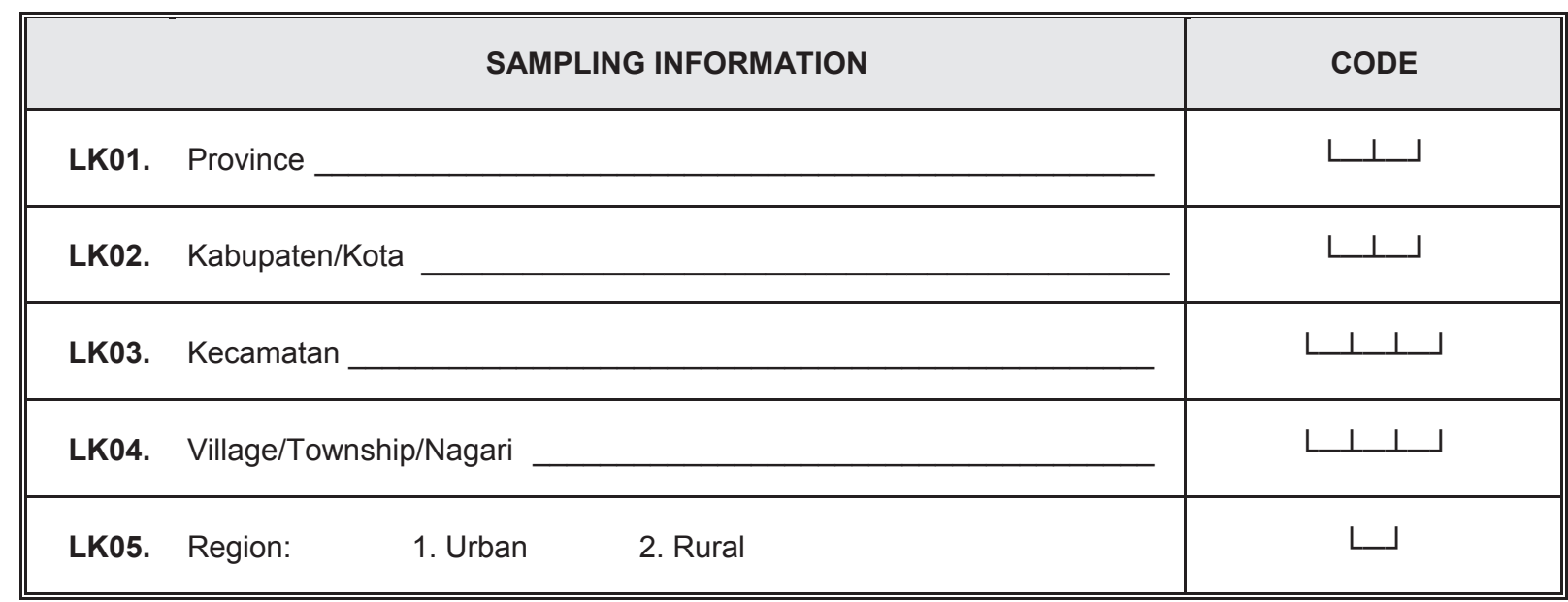

\begin{tabular}{|c|c|}
\hline SUPERVISION & CODE \\
\hline LK15. Name of Interviewer: & 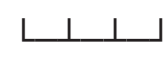 \\
\hline LK17. Name of Local Supervisor: & 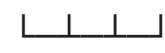 \\
\hline LK19. Name of Field Coordinator: & 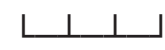 \\
\hline
\end{tabular}


SECTION KA (ENVIRONMENTAL CONDITIONS)

ENVIRONMENTAL CONDITIONS AND LAND USE

\begin{tabular}{|c|c|c|}
\hline so1. & Size of village/township & $\begin{array}{l}\text { 1. } \sqcup \sqcup, \sqcup \sqcup \sqcup \cdot \sqcup \sqcup \mathrm{Ha} \\
\text { 8. } \mathrm{DK}\end{array}$ \\
\hline SA1 & $\begin{array}{l}\text { Altitude Location } \\
\text { (village/Township) }\end{array}$ & 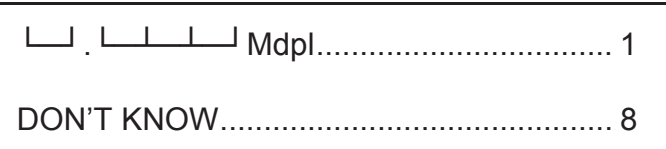 \\
\hline SA2 & $\begin{array}{l}\text { The condition of the Village / } \\
\text { Township area }\end{array}$ & flat $\begin{array}{l} \\
\text { ramps } \\
\text { wavy } \\
\text { hilly } \\
\text { mountainous }\end{array}$ \\
\hline SA3 & Average temperatures & $\begin{array}{c}\sqcup{ }^{\circ} \mathrm{C} \\
\text { DON'T KNOW }\end{array}$ \\
\hline
\end{tabular}




\section{SECTION KA (ENVIRONMENTAL CONDITIONS)}

S01a. Is there forest cover in this village 5 years ago?

$3 \rightarrow$ S01b

S02a. What percentage of the village area was forest land 5 years ago?

1. $\longleftarrow \perp \%$

8. DK

\begin{tabular}{|c|c|c|c|c|c|c|c|}
\hline & PURPOSE OF FOREST & s03a. & S04a. & & & 55 a. & s06a. \\
\hline & $\begin{array}{l}\text { CLEARING } \\
\text { (KA3TYPE) }\end{array}$ & $\begin{array}{l}\text { Has there been any clearing of forest for } \\
\text { the purpose of }[\ldots] \text { in the past } 5 \text { years? }\end{array}$ & $\begin{array}{l}\text { In the last } 5 \text { years, what percentage of the } \\
\text { forest was cleared for the purpose of }[\ldots] \text { ? }\end{array}$ & $\begin{array}{c}\text { From whon } \\
\text { clear }\end{array}$ & $\begin{array}{l}\text { hey } r \\
\text { rest } \mathrm{f}\end{array}$ & $\begin{array}{l}\text { to obtain permission to } \\
\text { e purpose of }[\ldots] \text { ? }\end{array}$ & $\begin{array}{l}\text { Since } 2007 \text {, has there been an increase or } \\
\text { decrease in the clearing of the forest for }[\ldots] \text { ? }\end{array}$ \\
\hline A. & Housing & 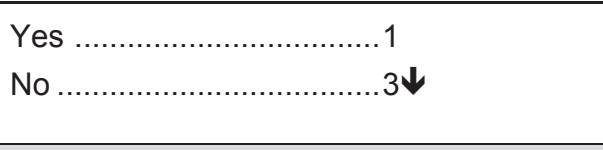 & $\begin{array}{l}\text { 1. } \sqcup \perp \vdash \text { \% } \\
\text { 8. } \mathrm{DK}\end{array}$ & $\begin{array}{ll}\text { A } & \text { B } \\
\text { E } & F\end{array}$ & $\begin{array}{l}C \\
W\end{array}$ & $\begin{array}{l}\mathrm{D} \\
\mathrm{V}\end{array}$ & 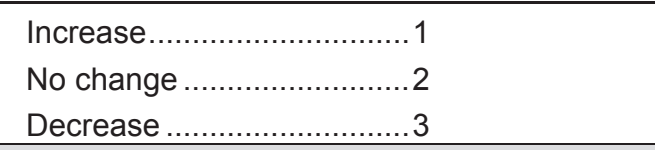 \\
\hline B. & Cultivation & 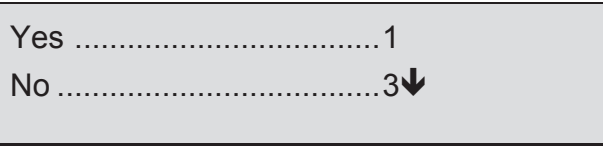 & 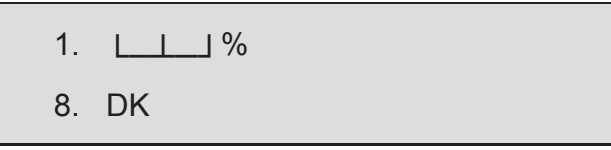 & $\begin{array}{l}\mathrm{B} \\
\mathrm{F}\end{array}$ & $\begin{array}{l}C \\
W\end{array}$ & $\begin{array}{l}D \\
V_{-}\end{array}$ & 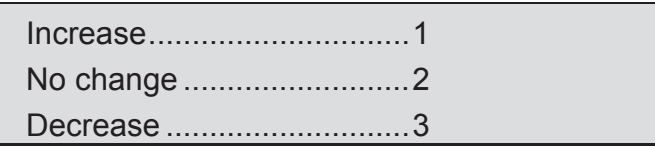 \\
\hline C. & Plantation & 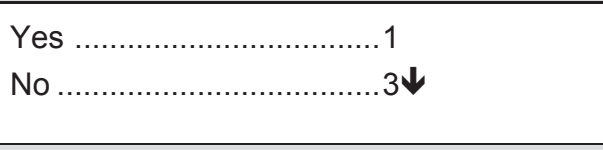 & 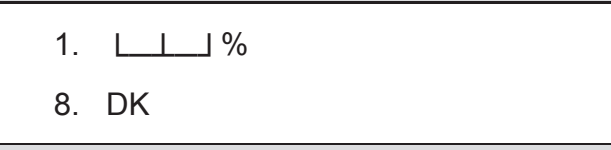 & $\begin{array}{l}\text { A } \\
E\end{array}$ & $\begin{array}{l}\mathrm{C} \\
\mathrm{W}\end{array}$ & $\begin{array}{l}D \\
V_{-}\end{array}$ & 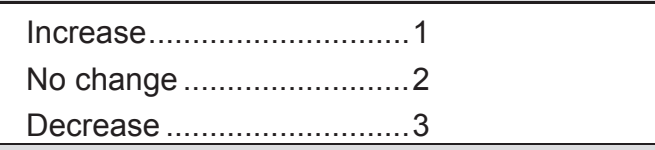 \\
\hline D. & Roads/infrastructure & 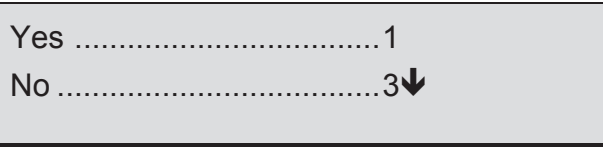 & 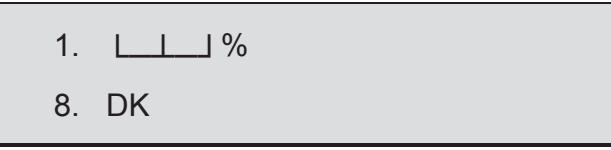 & $\begin{array}{l}\text { A } \\
E\end{array}$ & $\begin{array}{l}\text { C } \\
W\end{array}$ & $\begin{array}{l}D \\
V_{-}\end{array}$ & 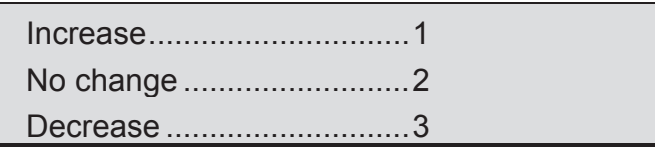 \\
\hline E. & Factory & 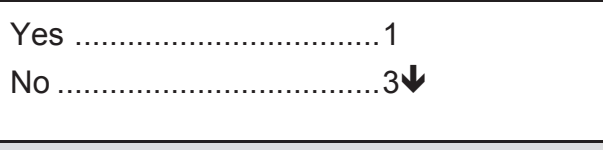 & 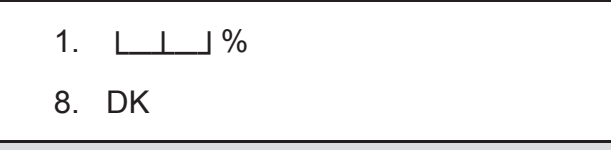 & $\begin{array}{l}A \\
E\end{array}$ & $\begin{array}{l}C \\
W\end{array}$ & $\begin{array}{l}\mathrm{D} \\
\mathrm{V}\end{array}$ & 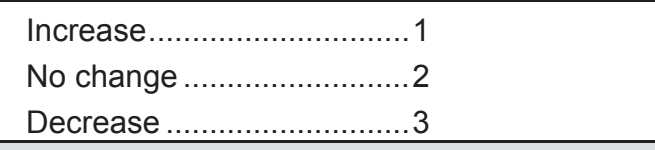 \\
\hline v. & Other & 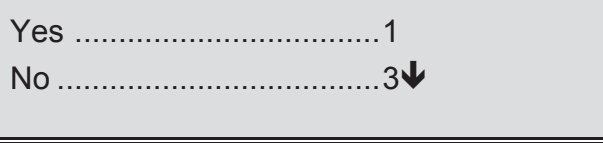 & 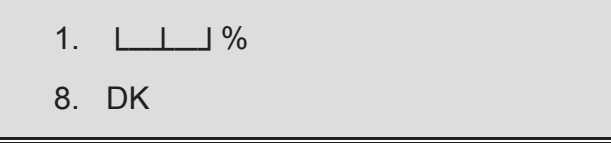 & $\begin{array}{l}A \\
E\end{array}$ & $\begin{array}{l}\text { C } \\
W\end{array}$ & $\begin{array}{l}D \\
V_{-}\end{array}$ & $\begin{array}{l}\text { Increase } \\
\text { No change } \ldots \ldots \ldots \\
\text { Decrease } \ldots \ldots \ldots \ldots \ldots . . .2 \\
\end{array}$ \\
\hline
\end{tabular}




\section{SECTION KA (ENVIRONMENTAL CONDITIONS)}

\begin{tabular}{|c|c|}
\hline S01b. Is there farm land in this village 5 years ago? & $\begin{array}{l}\text { No } \\
\text { Yes }\end{array}$ \\
\hline S02b. What percentage of the village area was farm land 5 years ago? & $\begin{array}{l}\text { 1. } \sqcup \downarrow \% \\
\text { 8. DK }\end{array}$ \\
\hline
\end{tabular}

\begin{tabular}{|c|c|c|c|c|c|c|c|}
\hline & \multirow{2}{*}{$\begin{array}{l}\text { PURPOSE OF FARM LAND } \\
\text { CONVERTION } \\
\text { (KA4TYPE) }\end{array}$} & \multirow{2}{*}{$\begin{array}{l}\text { S03b. } \\
\text { Has there been any convertion of farm land } \\
\text { for the purpose of }[\ldots] \text { in the past } 5 \text { years? }\end{array}$} & \multirow{2}{*}{\begin{tabular}{|l|}
\multicolumn{1}{|c}{ S04b. } \\
In the last 5 years, what percentage of the \\
farm land was converted for the purpose of \\
{$[\ldots]$ ? } \\
\end{tabular}} & \multicolumn{3}{|c|}{ S05b. } & S06b. \\
\hline & & & & \multicolumn{3}{|c|}{$\begin{array}{l}\text { From whom do they need to obtain permission to } \\
\text { convert land for the purpose of }[\ldots] \text { ? }\end{array}$} & $\begin{array}{l}\text { Since 2007, has there been an increase or } \\
\text { decrease in the clearing of the forest for }[\ldots] \text { ? }\end{array}$ \\
\hline A. & Housing & 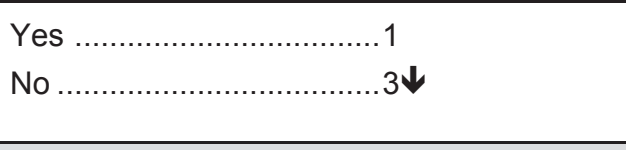 & $\begin{array}{l}\text { 1. } \downarrow \perp \perp \% \\
\text { 8. } \mathrm{DK}\end{array}$ & $\begin{array}{l}B \\
F\end{array}$ & $\begin{array}{l}C \\
W\end{array}$ & $\begin{array}{l}\mathrm{D} \\
\mathrm{V}\end{array}$ & 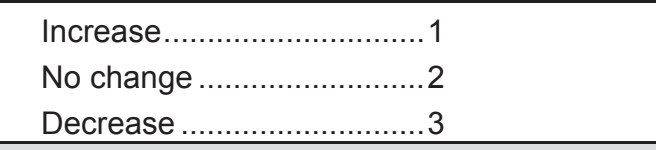 \\
\hline D. & Roads/infrastructure & 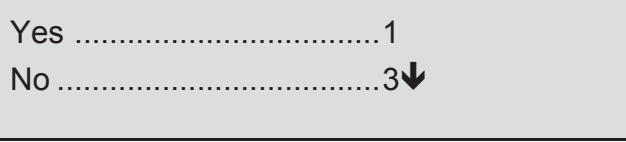 & 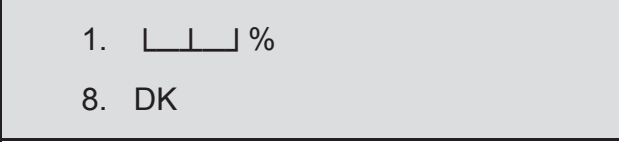 & $\begin{array}{l}A \\
E\end{array}$ & $\begin{array}{l}\mathrm{C} \\
\mathrm{W}\end{array}$ & $\begin{array}{l}\mathrm{D} \\
\mathrm{V}\end{array}$ & $\begin{array}{l}\text { Increase } \\
\text { No change } \ldots \ldots \ldots \ldots . .1 \\
\text { Decrease } \ldots \ldots \ldots \ldots \ldots \ldots \ldots . .1\end{array}$ \\
\hline E. & Factory & 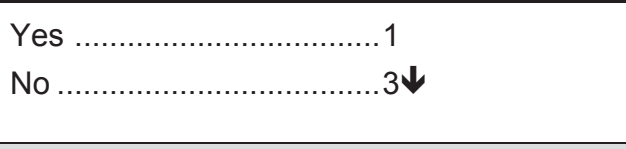 & 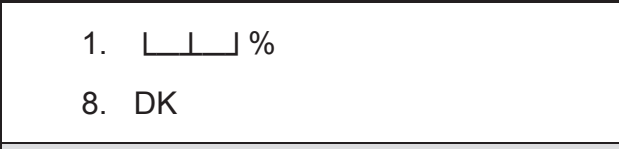 & $\begin{array}{l}A \\
E\end{array}$ & $\begin{array}{l}\mathrm{C} \\
\mathrm{W}\end{array}$ & $\begin{array}{l}\mathrm{D} \\
\mathrm{V}\end{array}$ & $\begin{array}{l}\text { Increase } \ldots \ldots \ldots \ldots \ldots \ldots \ldots \ldots \ldots \ldots \\
\text { No change } \ldots \ldots \ldots \ldots \ldots \ldots \ldots \ldots \ldots \ldots \\
\text { Decrease } \ldots \ldots \ldots \ldots \ldots \ldots \ldots \ldots \ldots . . . \\
\end{array}$ \\
\hline v. & Other & 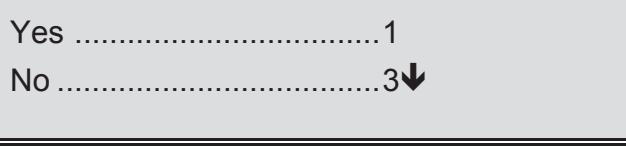 & $\begin{array}{l}\text { 1. } \downarrow \perp \text { \% } \\
\text { 8. DK }\end{array}$ & $\begin{array}{l}\mathrm{A} \\
\mathrm{E}\end{array}$ & $\begin{array}{l}\mathrm{C} \\
\mathrm{W}\end{array}$ & $\begin{array}{l}\mathrm{D} \\
\mathrm{V}\end{array}$ & $\begin{array}{l}\text { Increase } \ldots \ldots \ldots \ldots \ldots \ldots \ldots \ldots \ldots . . .1 \\
\text { No change } \ldots \ldots \ldots \ldots \ldots \ldots \ldots \ldots \ldots . . . \\
\text { Decrease } \ldots \ldots \ldots \ldots \ldots \ldots \ldots \ldots . . . \\
\end{array}$ \\
\hline & & & & $\begin{array}{l}\text { Code for So: } \\
\text { F. Central g } \\
\text { G. Province } \\
\text { H. District g } \\
\text { I. Subdistri } \\
\end{array}$ & $\begin{array}{l}\text { ment } \\
\text { nmer } \\
\text { nent } \\
\text { ennm }\end{array}$ & & $\begin{array}{l}\text { E. Village office } \\
\text { F. Community leader } \\
\text { W. No permission needed } \\
\text { V. Other }\end{array}$ \\
\hline
\end{tabular}




\section{SECTION PL (ENVIRONMENTAL POLLUTION)}

\section{ENVIRONMENTAL POLLUTION}

\begin{tabular}{|c|c|c|c|c|c|c|c|c|}
\hline & (KA1TYPE) & S08. & so9. & S10a. & S11. & S12. & S12a & S12b \\
\hline & POLLUTION & $\begin{array}{l}\text { In the last year, was there a } \\
{[\ldots] \text { pollution in the village? }}\end{array}$ & $\begin{array}{l}\text { Where did the }[\ldots] \text { pollution } \\
\text { originate from? }\end{array}$ & $\begin{array}{l}\text { What kinds of } \\
\text { pollutants were } \\
\text { there in the }[\ldots] \\
\text { pollution? }\end{array}$ & $\begin{array}{c}\text { Was the source of }[\ldots] \\
\text { pollution inside or outside this } \\
\text { village? }\end{array}$ & $\begin{array}{l}\text { Was there any complaint } \\
\text { about the }[\ldots . .] \text { pollution to the } \\
\text { office of the Head of the } \\
\text { Village/Township? }\end{array}$ & $\begin{array}{c}\text { When was the }[\ldots] \text { pollution the } \\
\text { most severe? }\end{array}$ & $\begin{array}{c}\text { How long did the } \\
\text { most severe [...] } \\
\text { pollution occurred? }\end{array}$ \\
\hline 1. & Water & Yes & $\begin{array}{l}\text { A. Households } \\
\text { B. Factories } \\
\text { C. Vehicles } \\
\text { D. Businesses } \\
\text { V. Other }\end{array}$ & $\begin{array}{l}\text { A. Solid } \\
\text { B. Liquid } \\
\text { C. Gas }\end{array}$ & $\begin{array}{l}\text { 1. Inside the village } \\
\text { 2. Outside the village } \\
\text { 3. Inside and outside the } \\
\text { village }\end{array}$ & 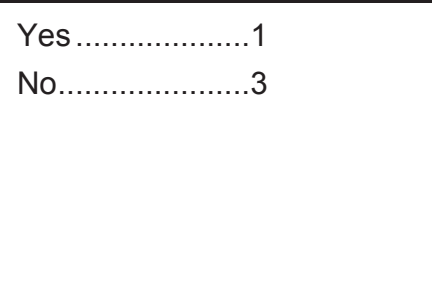 & 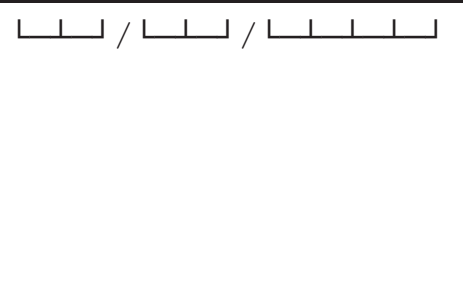 & $\begin{array}{l}\text { 1. } \downarrow \perp \perp ـ \text { day } \\
\text { 3. day } \\
\text { 4. week } \\
\text { 5. month } \\
\text { 8. Don't Know }\end{array}$ \\
\hline 2. & Soil & Yes & $\begin{array}{l}\text { A. Households } \\
\text { B. Factories } \\
\text { C. Vehicles } \\
\text { D. Businesses } \\
\text { V. Other }\end{array}$ & $\begin{array}{l}\text { A. Solid } \\
\text { B. Liquid } \\
\text { C. Gas }\end{array}$ & $\begin{array}{l}\text { 1. Inside the village } \\
\text { 2. Outside the village } \\
\text { 3. Inside and outside the } \\
\text { village }\end{array}$ & 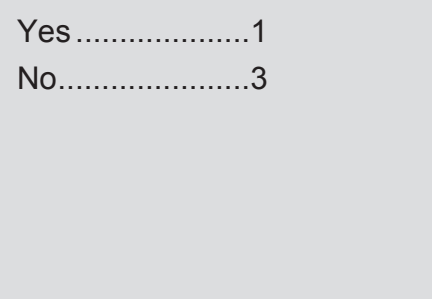 & 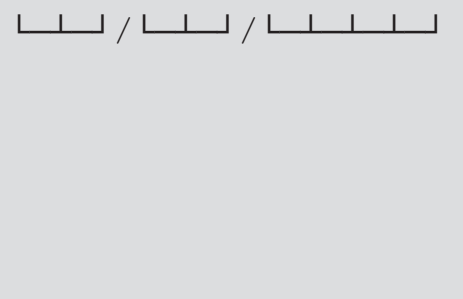 & $\begin{array}{l}\text { 1. } \downarrow \text {.ـ day } \\
\text { 4. week } \\
\text { 5. month } \\
\text { 8. Don't Know }\end{array}$ \\
\hline 3. & Air & 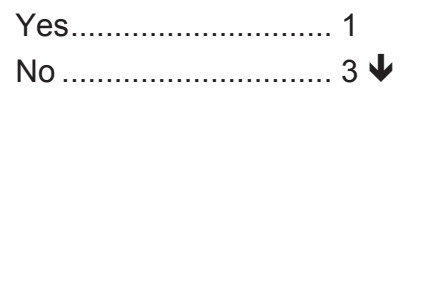 & $\begin{array}{l}\text { A. Households } \\
\text { B. Factories } \\
\text { C. Vehicles } \\
\text { D. Businesses } \\
\text { V. Other }\end{array}$ & $\begin{array}{l}\text { A. Solid } \\
\text { B. Liquid } \\
\text { C. Gas }\end{array}$ & $\begin{array}{l}\text { 1. Inside the village } \\
\text { 2. Outside the village } \\
\text { 3. Inside and outside the } \\
\text { village }\end{array}$ & 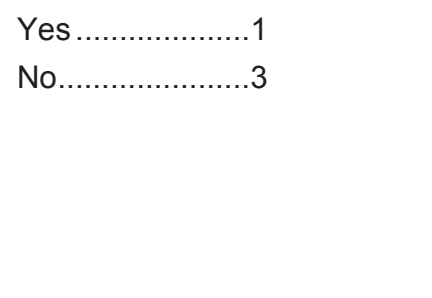 & 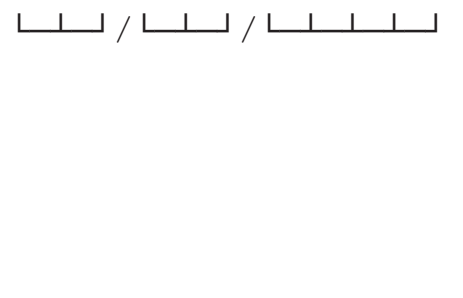 & $\begin{array}{l}\text { 1. } \downarrow \text {.ـ day } \\
\text { 3. deek } \\
\text { 5. month } \\
\text { 8. Don't Know }\end{array}$ \\
\hline 4. & $\begin{array}{l}\text { Noise/Vibrati } \\
\text { on }\end{array}$ & $\begin{array}{l}\text { Yes } \\
\text { No } \ldots \ldots \ldots \ldots \ldots \ldots \ldots \ldots \ldots . . . . .1 \\
\\
\text { S14a }\end{array}$ & $\begin{array}{l}\text { A. Households } \\
\text { B. Factories } \\
\text { C. Vehicles } \\
\text { D. Businesses } \\
\text { V. Other }\end{array}$ & & $\begin{array}{l}\text { 1. Inside the village } \\
\text { 2. Outside the village } \\
\text { 3. Inside and outside the } \\
\text { village }\end{array}$ & 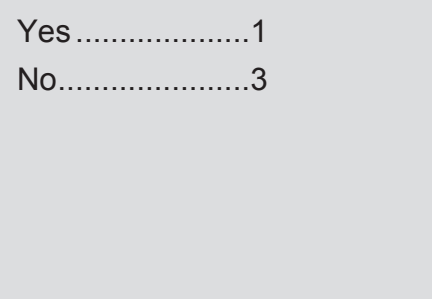 & 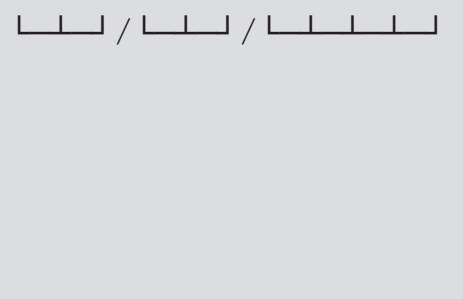 & $\begin{array}{l}\text { 1. } \downarrow \text { 3. day } \\
\text { 4. week } \\
\text { 5. month } \\
\text { 8. Don't Know }\end{array}$ \\
\hline
\end{tabular}




\section{SECTION ST (LAND CERTIFICATE)}

LAND CERTIFICATION

\begin{tabular}{|c|c|c|c|}
\hline \multirow{2}{*}{\multicolumn{2}{|c|}{$\begin{array}{l}\text { TYPES OF LAND CERTIFICATE } \\
\text { (S1TYPE) }\end{array}$}} & \multicolumn{2}{|c|}{$\begin{array}{l}\text { S14a. } \\
\end{array}$} \\
\hline & & \multicolumn{2}{|c|}{$\begin{array}{l}\text { Is there any piece of land in this village with } \\
\text { the following }[\ldots . .] \text { certification status: }\end{array}$} \\
\hline 01. & Ownership certificate (SHM) & 1. Yes & 3. No \\
\hline 02. & Commercial Use Certificate (HGU) & 1. Yes & 3. No \\
\hline 03. & Conditional Use Certificate(HGB) & 1. Yes & 3. No \\
\hline 04. & Village Treasury Land & 1. Yes & 3. No \\
\hline & $\begin{array}{l}\text { 04a. Land for use of village employee in place of salary } \\
\text { (Tanah Bengkok) }\end{array}$ & 1. Yes & 3. No \\
\hline & 04b. Titisara Land (Land ownership of the village) & 1. Yes & 3. No \\
\hline & 04c. Pasture (Tanah Pangonan/Penggembalaan) & 1. Yes & 3. No \\
\hline 05. & A certificate from PRONA & 1. Yes & 3. No \\
\hline 06. & Land without certificate & 1. Yes & 3. No \\
\hline 07. & Letter C & 1. Yes & 3. No \\
\hline 08. & Patok D & 1. Yes & 3. No \\
\hline
\end{tabular}




\section{SECTION PR (HOUSING SECTION)}

\begin{tabular}{|c|c|c|}
\hline S16. & $\begin{array}{l}\text { Are there village/township residents } \\
\text { that live along the edge of a river? }\end{array}$ & Yes \\
\hline S19. & $\begin{array}{l}\text { Are there any slums in this } \\
\text { village/township? }\end{array}$ & Yes $\begin{array}{l}\text { Y } \\
\text { No }\end{array}$ \\
\hline S23. & $\begin{array}{l}\text { Are there any residents in this } \\
\text { village/township that live below high } \\
\text { voltage electrical wires? }\end{array}$ & $\begin{array}{l}\text { Yes } \\
\text { No }\end{array}$ \\
\hline S26. & $\begin{array}{l}\text { In the last five years has there been } \\
\text { any new houses built by local } \\
\text { residents in this village/township? }\end{array}$ & $\begin{array}{l}\text { Yes } \\
\text { No }\end{array}$ \\
\hline S26a. & $\begin{array}{l}\text { In the last five years, were there any } \\
\text { new houses built as a result of natural } \\
\text { disasters? }\end{array}$ & $\begin{array}{l}\text { Yes } \\
\text { No }\end{array}$ \\
\hline S28. & $\begin{array}{l}\text { In the last five years has there been } \\
\text { any new houses built by developers in } \\
\text { this village/township? }\end{array}$ & $\begin{array}{l}\text { Yes } \\
\text { No }\end{array}$ \\
\hline S30. & $\begin{array}{l}\text { How many RT, RW, } \\
\text { dusun/lingkungan are in this } \\
\text { village/township? }\end{array}$ & 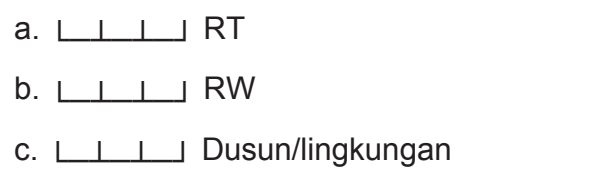 \\
\hline S31. & $\begin{array}{l}\text { What is the total population in this } \\
\text { village/township? }\end{array}$ & 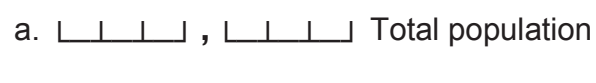 \\
\hline S32. & $\begin{array}{l}\text { What is the total number of } \\
\text { households in this village/township? }\end{array}$ & 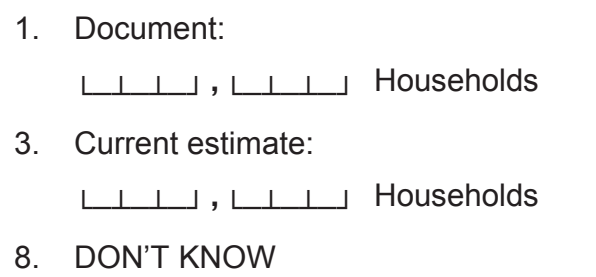 \\
\hline
\end{tabular}

S32b What religions are followed by villagers in this village/kelurahan? What are the percentages?

A. Islam $\longleftarrow \perp \_\%$

B. Catholic $\llcorner\perp \perp$ \%

C. Protestant $\left\llcorner\perp \_\right.$\%

D. Hindu $\sqcup, \downarrow$ \%

E. Budha $\llcorner\perp \perp$ \%

F. Confucius $\downarrow \perp$ \&

V. Others $\downarrow \perp \perp \%$

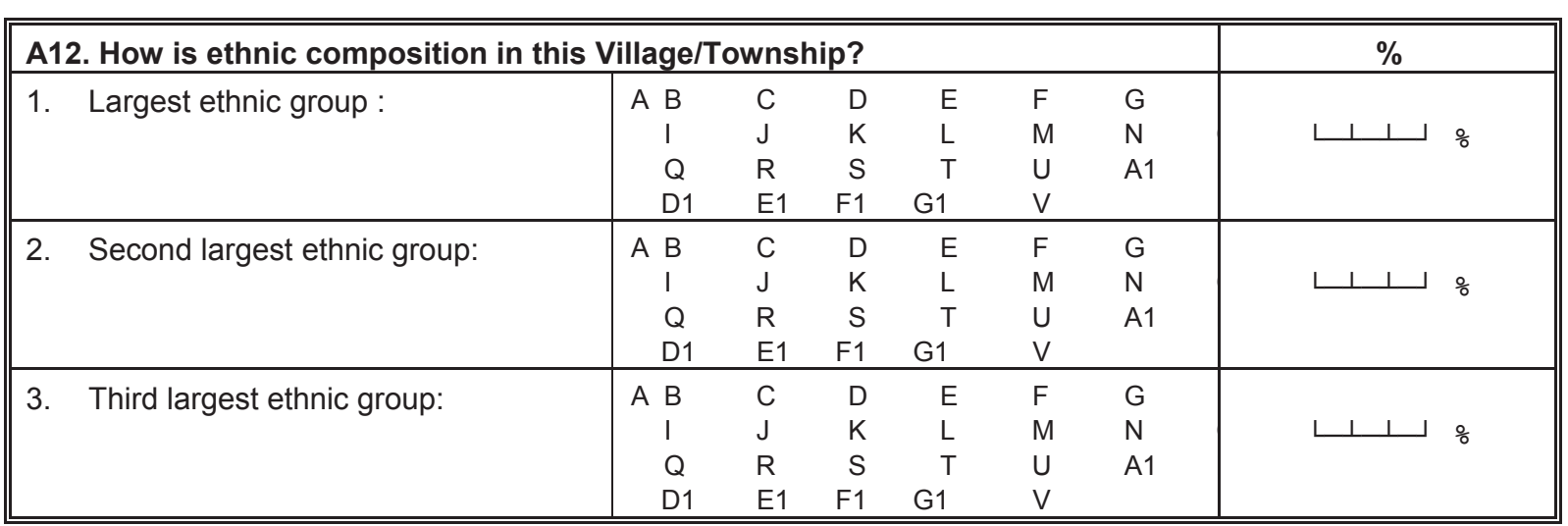

\section{Code for $\mathbf{A} 12$}

Java..

Sund

Bali..........

Bugis.

Tiongho

Madura

Minang

Baniar

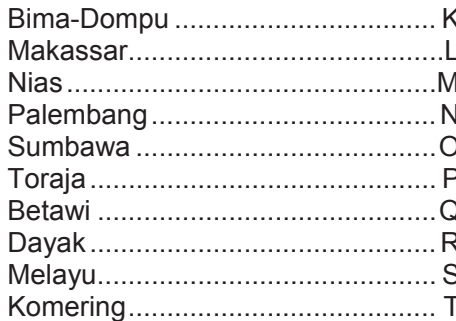




\section{SECTION PR (HOUSING SECTION)}

\begin{tabular}{||l|l|l|}
\hline S37b. & $\begin{array}{l}\text { INTERVIEWER CHECK: DATA } \\
\text { SOURCES OF THE NUMBER OF } \\
\text { POOR FAMILY IN THIS } \\
\text { VILLAGE/TOWNSHIP)? }\end{array}$ & 1. DOCUMENT $\rightarrow$ S37d \\
\hline S37c. ESTIMATION \\
$\begin{array}{l}\text { How many poor households are in } \\
\text { this village/township? }\end{array}$
\end{tabular}




\section{SECTION LU (TYPE OF EMPLOYMENT)}

\section{TYPE OF EMPLOYMENT}

\begin{tabular}{|c|c|c|c|c|}
\hline & \multirow[b]{2}{*}{$\begin{array}{l}\text { TYPE OF EMPLOYMENT } \\
\text { (S38TYPE) }\end{array}$} & \multicolumn{2}{|c|}{$\begin{array}{l}\text { S39. } \\
\text {. }\end{array}$} & $\overline{~ S 40 . ~}$ \\
\hline & & \multicolumn{2}{|c|}{$\begin{array}{l}\text { What are the enterprises of the village/township } \\
\text { population? }\end{array}$} & $\begin{array}{l}\text { Which are the three most predominant sources of income for } \\
\text { the village/township population? } \\
\text { (ONLY THREE ANSWER) }\end{array}$ \\
\hline 01. & Farming (including forestry, hunting and fishing) & 1. Yes & 3. No & \multirow{10}{*}{ a. First $\downarrow$} \\
\hline 02. & 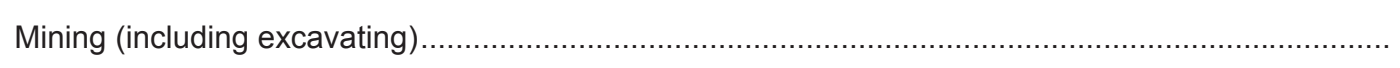 & 1. Yes & 3. No & \\
\hline 03. & Manufacturing industry & 1. Yes & 3. No & \\
\hline 04. & Electricity, gas and water & 1. Yes & 3. No & \\
\hline 05. & Building construction ........................................... & 1. Yes & 3. No & \\
\hline 06. & Large trade, retail trade, restaurants and hotels & 1. Yes & 3. No & \\
\hline 07. & Transportation, warehousing and communications & 1. Yes & 3. No & \\
\hline 08. & 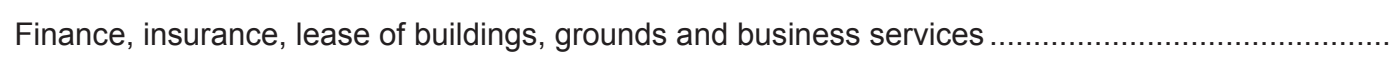 & 1. Yes & 3. No & \\
\hline 09. & Social services …1-1. & 1. Yes & 3. No & \\
\hline 95. & Other & 1. Yes & 3. No & \\
\hline
\end{tabular}




\section{SECTION KD (VILLAGE FINANCE)}

\begin{tabular}{|c|c|c|}
\hline & & 2014 \\
\hline S45. & $\begin{array}{l}\text { What was the total Village Revenue and Expenditure Budget } \\
\text { (APPKD)/Village Budget Management (PAK) in your village in } \\
\text { the fiscal year [...]? }\end{array}$ & 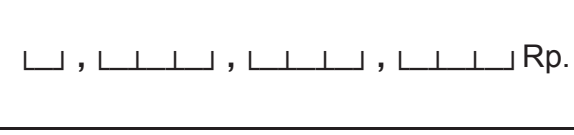 \\
\hline \multirow[t]{9}{*}{ S46. } & $\begin{array}{l}\text { How much was the revenue from the sources below in the } \\
\text { budget year }[\ldots] \text { : }\end{array}$ & \\
\hline & a. Balance from previous year & 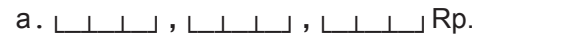 \\
\hline & b. Revenue originated from the village (Pendapatan Asli Desa)..... & b. $\sqcup \perp, \sqcup, \sqcup \perp, \sqcup, \perp \_$Rp. \\
\hline & c. Central government contribution .................... & 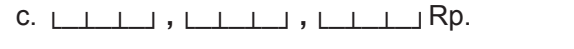 \\
\hline & d. Provincial government contribution .......... & 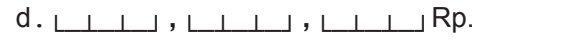 \\
\hline & 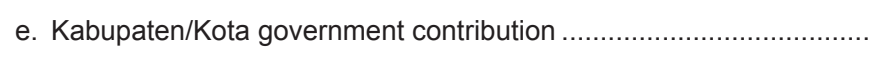 & 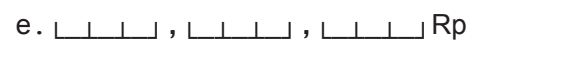 \\
\hline & $\begin{array}{l}\text { f. Third party contribution (Local State Enterprises/NGO/other s local } \\
\text { government, etc). }\end{array}$ & 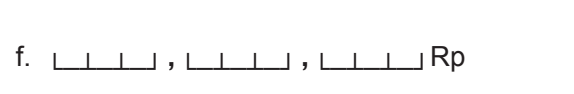 \\
\hline & g. Village/Township Loan & 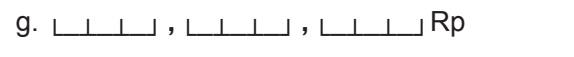 \\
\hline & $\begin{array}{l}\text { h. Kecamatan Development Program (KDP)/Urban Poverty } \\
\text { Program(UPP) - PPK/P2KP }\end{array}$ & 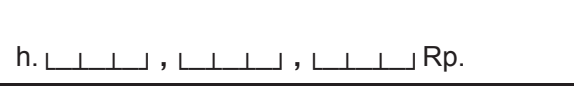 \\
\hline \multirow[t]{14}{*}{ S48. } & $\begin{array}{l}\text { How much was the revenue originated from the village } \\
\text { (Pendapatan Asli Desa) in the fiscal year [...] from each of } \\
\text { these sources: }\end{array}$ & \\
\hline & 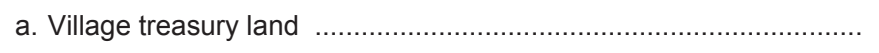 & 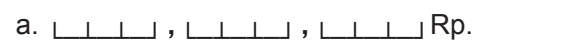 \\
\hline & b. Village markets/kiosks . & 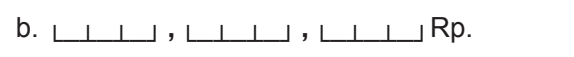 \\
\hline & c. Village facilities ............... & 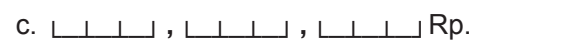 \\
\hline & d. Recreation sites .......... & 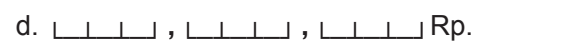 \\
\hline & e. Village buildings ......... & 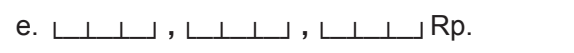 \\
\hline & 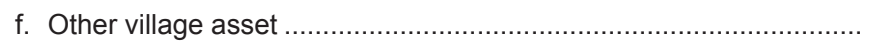 & 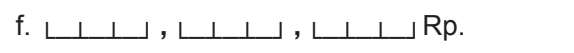 \\
\hline & g. Self-reliant efforts or other form of participation.......... & g. $\sqcup \perp, \sqcup, \sqcup \perp, \sqcup, \perp \perp$ Rp. \\
\hline & h. Community joint efforts (gotong royong)...... & 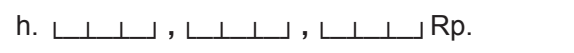 \\
\hline & i. Contribution based on occupation .................. & i. $\sqcup \perp \perp, \sqcup \perp \perp, \sqcup \sqcup \_$Rp. \\
\hline & 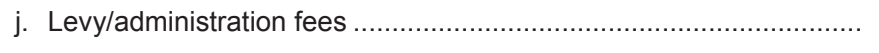 & j. $\sqcup \perp, \sqcup, \sqcup \perp, \sqcup \sqcup \_$Rp. \\
\hline & k. Tax on land produce (Pajak Hasil Bumi) .......................................... & 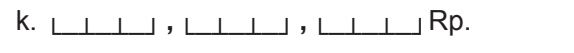 \\
\hline & I. Village enterprise & I. $\sqcup \perp \perp, \sqcup \perp \perp, \sqcup, \perp \perp$ Rp. \\
\hline & v. Other_ & 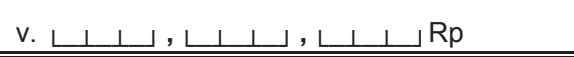 \\
\hline S48x. & $\begin{array}{l}\text { From the Total amount of Pendapatan Asli Desa (Village } \\
\text { Original Revenue), how much of the village received? }\end{array}$ & 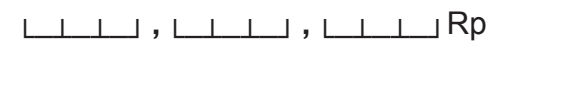 \\
\hline S49. & $\begin{array}{l}\text { From funds originating from self-reliant effortst in the fiscal } \\
\text { year }[\ldots] \text {, how much was received in the following form: }\end{array}$ & \\
\hline
\end{tabular}

\begin{tabular}{|c|c|c|}
\hline & & 2014 \\
\hline & 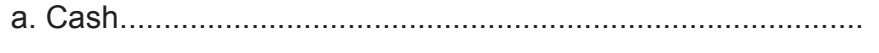 & a. $\sqcup \perp \perp,\llcorner\perp \perp, \sqcup, \perp \perp \operatorname{Rp}$. \\
\hline & b. Goods . & 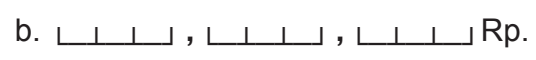 \\
\hline S50. & $\begin{array}{l}\text { What was the amount of routine expenditure and development } \\
\text { expenditure in the fiscal year }[\ldots] \text { ? }\end{array}$ & \\
\hline & a. Routine expenditure ....................... & 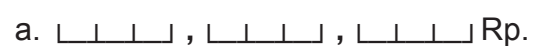 \\
\hline & b. Development expenditure ........... & 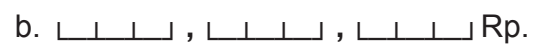 \\
\hline S54. & What were the salaries of the village officials? & \\
\hline & a. Head of Village........ & a. $\sqcup \perp \perp \_,\left\llcorner\perp \perp \_,\left\llcorner\perp \perp \_ \text {Rp. }\right.\right.$ \\
\hline & 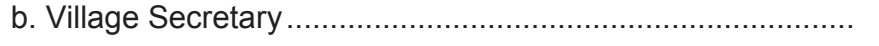 & b. $\llcorner\perp \perp \neg,\llcorner\perp \perp \neg,\llcorner\perp \perp \neg$ Rp. \\
\hline & c. Head of Sections & C. $\left\llcorner\perp \perp \neg,\left\llcorner\perp \perp,\left\llcorner\perp \perp \_\right.\right.\right.$Rp. \\
\hline & d. Head of Dusun & d. $\llcorner\perp \perp \neg,\llcorner\perp \perp \neg,\llcorner\perp \perp \neg$ Rp. \\
\hline & 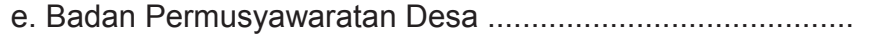 & 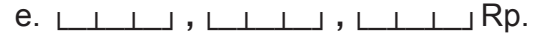 \\
\hline
\end{tabular}




\section{SECTION KD (VILLAGE FINANCE)}

\begin{tabular}{|c|c|c|}
\hline S53. & INTERVIEWER CHECK & $\begin{array}{l}\text { URBAN (TOWNSHIP) } \\
\text { RURAL (VILLAGE) }\end{array}$ \\
\hline
\end{tabular}

S55. What is the size of the village treasury land in this village?

$\llcorner\perp \perp \cdot\llcorner\perp \perp \downarrow$ hectares

S56. What is the total amount generated by the village treasury land over the past year?

$\sqcup \perp, \sqcup, \perp \perp,\llcorner\perp \perp$ Rp.

S57. What is the size of bengkok land allocated for:
b. Head of village ...........................

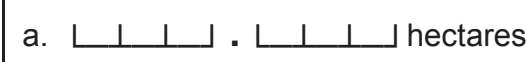

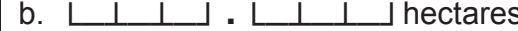
c. Head of development section
d. Head of general section...................................................

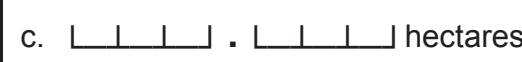
e. Head of finance section

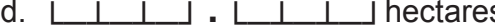
f. Head of welfare section

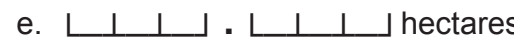

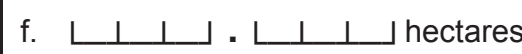
v. Head of other sections

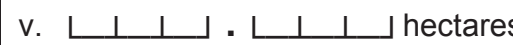

S58. How many dusun (sub-village) are there in this village?

S59. What is the size of bengkok land allocated for each head of dusun? $\llcorner\perp$ dusun

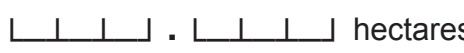

\begin{tabular}{|l|l|l|l|}
\hline S60. How was the Village Development funds allocated \\
in the fiscal year [...]?
\end{tabular}

S61. Has this village ever received village Infrastructure Funds from PKPS-BBM Rural Infrastructure?

S61a When the program was first implemented / accepted?

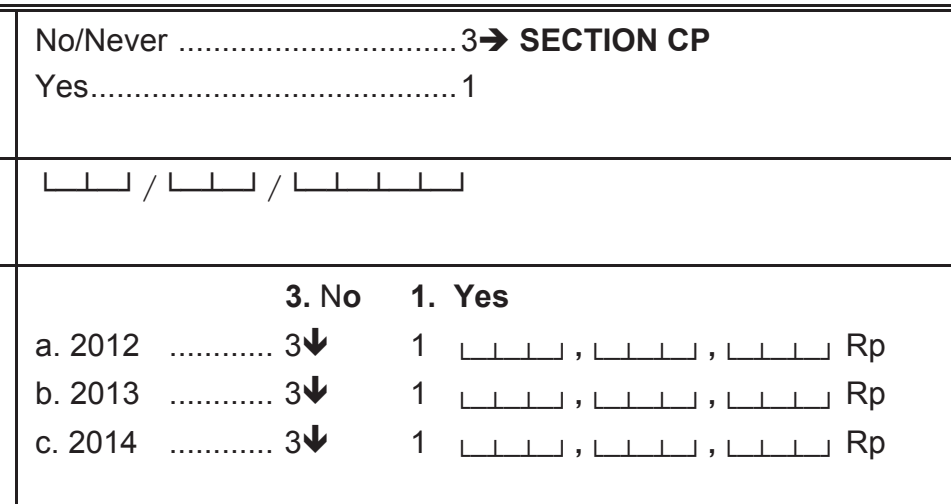




\section{SECTION SD (DATA SOURCE)}

DATA SOURCES : (TRY TO USE THE MOST RECENT SOURCES AND WRITE DOWN THE DATE)

01.

02

03.

04.

05.

06.

07

08.

09.

10. 


\section{SECTION OL (DIRECT OBSERVATION)}

NOTE FOR INTERVIEWER: DIRECT OBSERVATION OF THE REGION TO BE CONDUCTED BETWEEN THE ENUMERATION AREA AND THE VILLAGE/TOWNSHIP/TOWNSHIP OFFICE.

\begin{tabular}{|c|c|c|}
\hline OL01. & $\begin{array}{l}\text { IS THE AIR IN THIS VILLAGE/TOWNSHIP } \\
\text { GENERALLY POLLUTED (FOR WHATEVER } \\
\text { REASON)? }\end{array}$ & YES \\
\hline OL02. & $\begin{array}{l}\text { IS THERE GENERALLY ANY EXPOSED } \\
\text { GARBAGE PILE VISIBLE ALONG THE } \\
\text { ROADS? }\end{array}$ & YES \\
\hline OL03. & $\begin{array}{l}\text { IS THERE GENERALLY ANY EXPOSED } \\
\text { CATTLE MANURE PILE IN THIS } \\
\text { VILLAGE/TOWNSHIP? }\end{array}$ & YES \\
\hline OL04. & $\begin{array}{l}\text { IS THERE IN GENERAL ANY BLOCKED } \\
\text { WATER DUCT OR OBSTRUCTED } \\
\text { RIVER/GUTTER IN THIS } \\
\text { VILLAGE/TOWNSHIP? }\end{array}$ & YES \\
\hline OL05. & $\begin{array}{l}\text { ARE THERE STANDING POOLS OF } \\
\text { WATER? (NOT INCLUDING MARSHES, } \\
\text { LAKES) IN THIS VILLAGE/TOWNSHIP? }\end{array}$ & YES \\
\hline OL06. & $\begin{array}{l}\text { ARE THERE GENERALLY ANY CATTLE } \\
\text { VISIBLE IN THIS VILLAGE/TOWNSHIP (NO } \\
\text { FOWL) THAT ARE ROAMING AROUND IN } \\
\text { THE SETTLEMENTS OF THE } \\
\text { POPULATION? }\end{array}$ & YES \\
\hline OL07. & $\begin{array}{l}\text { ARE THE HOUSE YARDS IN THIS } \\
\text { VILLAGE/TOWNSHIP GENERALLY SWEPT } \\
\text { CLEAN? }\end{array}$ & YES \\
\hline OL08. & $\begin{array}{l}\text { IS THE GRASS GROWING IN PUBLIC } \\
\text { SQUARES IN THIS VILLAGE/TOWNSHIP } \\
\text { GENERALLY WELL CARED FOR? }\end{array}$ & YES \\
\hline OL09. & $\begin{array}{l}\text { ARE THERE IN GENERAL MANY FLIES } \\
\text { VISIBLE (NEAR THE FOOD VENDORS)? }\end{array}$ & YES \\
\hline
\end{tabular}

VILLAGE/TOWNSHIP/TOWNSHIP WELFARE

\begin{tabular}{|c|c|c|}
\hline OL22. & $\begin{array}{l}\text { IN THIS VILLAGE/TOWNSHIP IS THERE } \\
\text { AN NEIGHBORHOOD SECURITY POST } \\
\text { (POSKAMLING)? }\end{array}$ & $\begin{array}{l}\text { YES } \\
\text { NO }\end{array}$ \\
\hline OL23. & $\begin{array}{l}\text { IN THIS VILLAGE/TOWNSHIP ARE } \\
\text { THERE ANY SIGNS/SIGN BOARDS } \\
\text { WITH PUBLIC FACILITY DIRECTIONS? }\end{array}$ & $\begin{array}{l}\text { YES } \\
\text { NO }\end{array}$ \\
\hline OL24. & $\begin{array}{l}\text { DOES THIS VILLAGE/TOWNSHIP HAVE } \\
\text { A VILLAGE/TOWNSHIP HALL OR } \\
\text { CONVENTION HALL? }\end{array}$ & $\begin{array}{l}\text { YES } \\
\text { NO }\end{array}$ \\
\hline OL25. & $\begin{array}{l}\text { IN THIS VILLAGE/TOWNSHIP DOES } \\
\text { ONE SEE ANY BOARDS WITH } 10 \text { MAIN } \\
\text { ASPECTS OF THE ASSOCIATION OF } \\
\text { FAMILY WELFARE (PKK)? }\end{array}$ & $\begin{array}{l}\text { YES } \\
\text { NO }\end{array}$ \\
\hline OL26. & $\begin{array}{l}\text { IN THIS SHOPPING DISTRICT OF THIS } \\
\text { VILLAGE/TOWNSHIP DOES ONE SEE } \\
\text { SHOPS OR BUILDINGS THAT HAVE } \\
\text { BEEN DAMAGED OR BURNED? }\end{array}$ & $\begin{array}{l}\text { YES } \\
\text { NO } \\
\text { NO SHOPPING DISTRICT }\end{array}$ \\
\hline OL27. & $\begin{array}{l}\text { IN THIS SHOPPING DISTRICT OF THIS } \\
\text { VILLAGE/TOWNSHIP DOES ONE SEE } \\
\text { SHOPS THAT HAVE CLOSED/GONE } \\
\text { OUT OF BUSINESS }\end{array}$ & YES \\
\hline
\end{tabular}




\section{SECTION CP (INTERVIEW SESSION NOTES)}

\begin{tabular}{|c|c|c|c|c|c|c|c|}
\hline LANGMAIN (CK1). & Interview was entirely/mostly conducted in what language? & & $\perp \_$other & & & & \\
\hline LANGOTHR (CK2). & Other languaged used (if any): & & $\llcorner\perp$ other. & & & & \\
\hline LANGUAGE CODE: & & & & & & & \\
\hline 00. Bahasa Indonesia & 04. Batak & 08. Sasak & 12. Makassar & 16. & Toraja & 20. & Lampung \\
\hline 01. Java & 05. Bugis & 09. Minang & 13. Nias & 17. & Lahat & 95. & Other, \\
\hline 02. Sunda & 06. Cina & 10. Banjar & 14. Palembang & 18. & Other South Sumatera & 96. & NA \\
\hline 03. Bali & 07. Madura & 11. Bima & 15. Sumbawa & 19. & Betawi & & \\
\hline
\end{tabular}

\begin{tabular}{|c|c|c|c|}
\hline RESULT (FP3). RESULTS OF INTERVIEW & REASON (FP4). REASON FOR ANSWERING “2” / “3” IN RESULT. & FP6. MONITORING BY SUPERVISOR & \\
\hline $\begin{array}{l}\text { 1. Completed } \rightarrow \text { FP6 } \\
\text { 2. Partly completed } \\
\text { 3. Not completed } \\
\text { 4. Twin EA with EA } \downarrow \perp \neg \text { LP6 }\end{array}$ & $\begin{array}{l}\text { 1. Respondent was travelling/not in location } \\
\text { 2. Respondent was too busy } \\
\text { 3. Respondent refused }\end{array}$ & $\begin{array}{ll}\text { a. } & \text { Observed (sup_obs) .......... } 1 \\
\text { b. } & \text { Checked (sup_edit)............ } 1 \\
\text { c. } & \text { Verified (sup_veri)............. } 1\end{array}$ & $\begin{array}{l}\text { No } \\
3 \\
3 \\
3\end{array}$ \\
\hline
\end{tabular}

INTERVIEWER NOTE: 


\section{INDONESIAN FAMILY LIFE SURVEY 2014 \\ COMMUNITY AND FACILITY SURVEY \\ DESA/KELURAHAN WOMEN'S GROUP (PKK)}

SECTIONS : LK, I, J, PM, PAP, KSR, CP 


\section{SECTION LK: CONTROL SHEET}

\begin{tabular}{|c|c|c|}
\hline \multicolumn{2}{|r|}{ SAMPLING INFORMATION } & CODE \\
\hline LK01 & Province & \llcorner \\
\hline LK02 & Kabupaten/Kota & 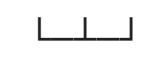 \\
\hline LK03 & Kecamatan & 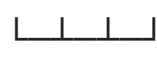 \\
\hline LK04 & Village/Township/Nagari & 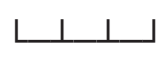 \\
\hline LK05 & 1. Urban & $\sqcup$ \\
\hline
\end{tabular}

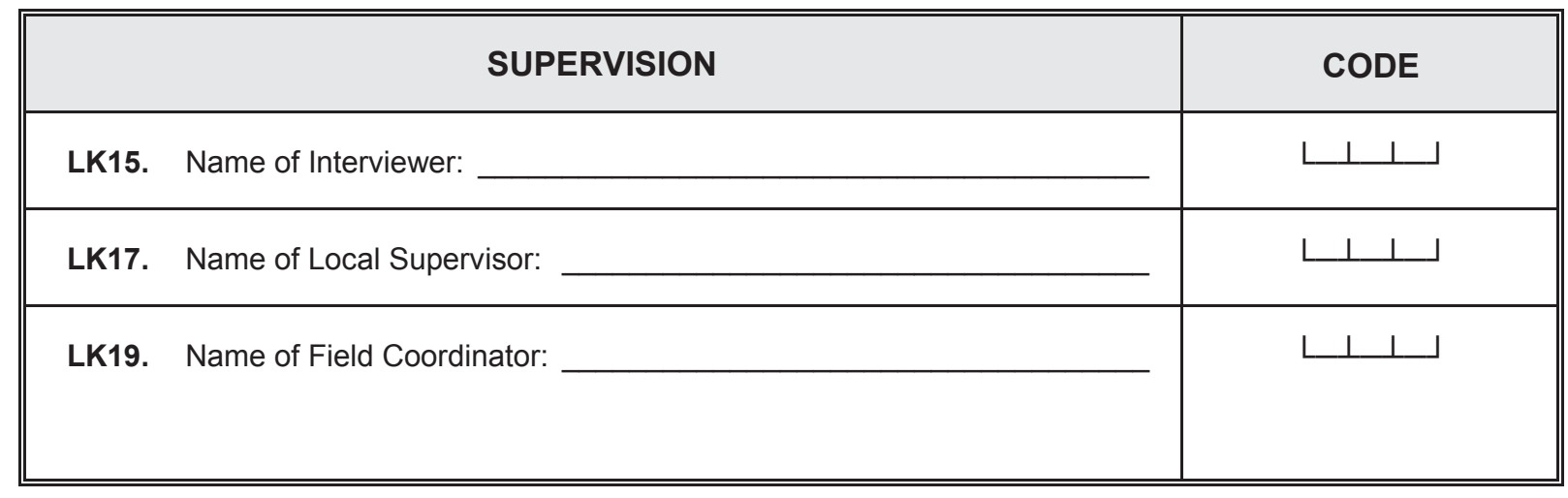




\section{SECTION I: THE PRESENCE OF SCHOOLS}

SECTION I RESPONDENT

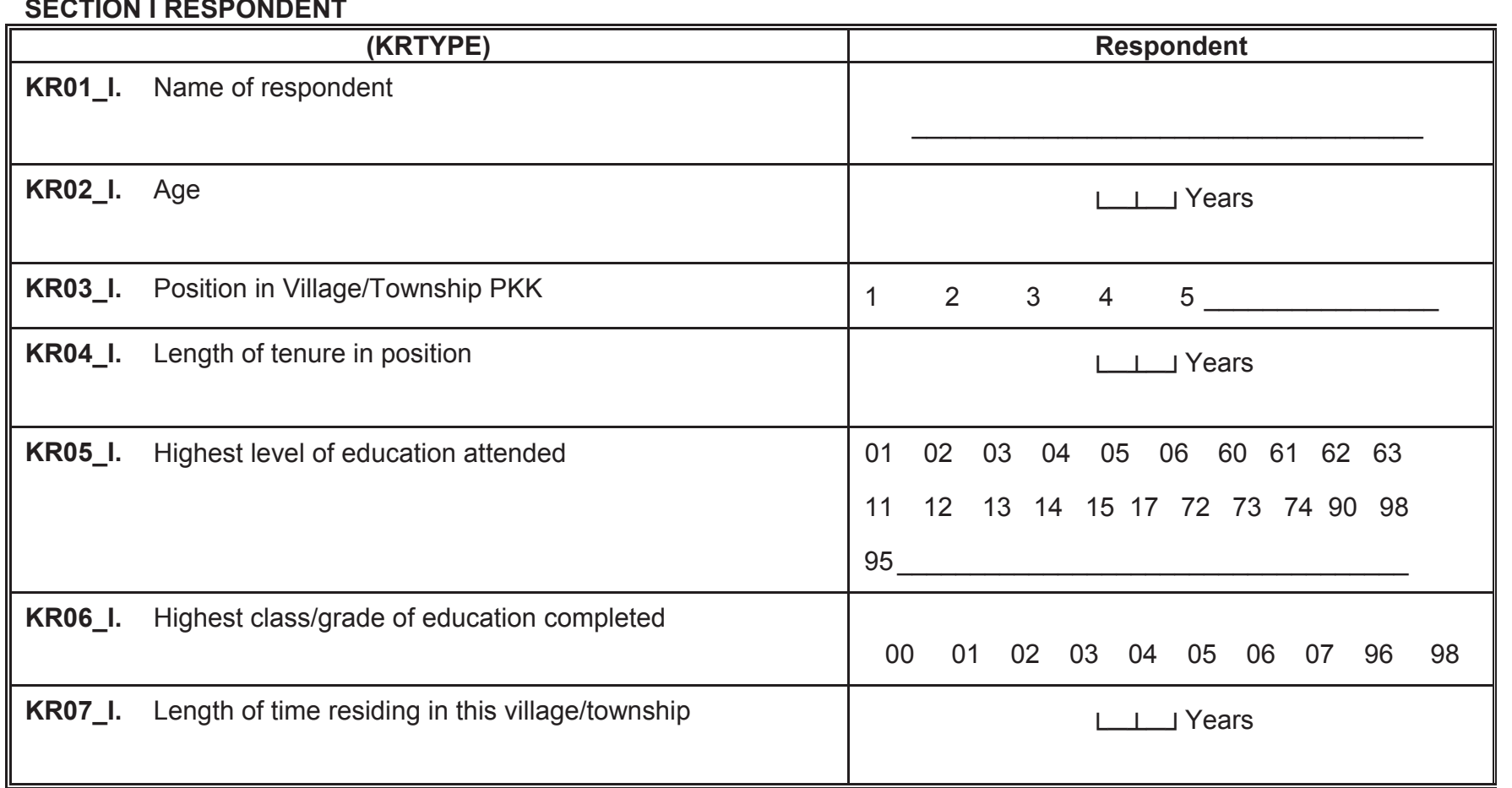

\begin{tabular}{|c|c|c|c|c|}
\hline $\begin{array}{l}\text { Code KR03_I: } \\
\text { 1. Head of PKK } \\
\text { 2. Organizer of PKK } \\
\text { 3. Cadre of PKK } \\
\text { 4. Ex organizer of PKK } \\
\text { 5. Other }\end{array}$ & $\begin{array}{l}\text { Code KR05_I: } \\
\text { 01. No school } \\
\text { 02. Elementary School (SD) } \\
\text { 03. Junior High School - General } \\
\text { 04. Junior High School - Vocational } \\
\text { 05. Senior High School - General } \\
\text { 06. Senior High School - Vocational } \\
\text { 11. Kejar Paket A (Adult Educ. A) } \\
\text { 12. Kejar Paket B (Adult Educ. B) }\end{array}$ & $\begin{array}{l}\text { 13. Open University } \\
\text { 14. Islamic School (Pesantren) } \\
\text { 15. Kejar Paket C (Adult Educ. C) } \\
\text { 17. School for the disabled } \\
\text { 60. D1, D2, D3 (Junior College) } \\
\text { 61. University S1 (Bachelor) } \\
\text { 62. University S2 (Masters) } \\
\text { 63. University S3 (Doctorate) }\end{array}$ & $\begin{array}{l}\text { 72. Islamic Elementary School } \\
\text { (Madrasah Ibtidaiyah) } \\
\text { 73. Islamic Junior High School } \\
\text { (Madrasah Tsanawiyah) } \\
\text { 74. Islamic Senior High School (Madrasah Aaliyah) } \\
\text { 90. Kindergarten } \\
\text { 98. DON'T KNOW } \\
\text { 95. Other }\end{array}$ & $\begin{array}{l}\text { Code KR06_I: } \\
\text { 00. Never completed } 1^{\text {st }} \text { class/grade } \\
\text { 01. } 1 \\
\text { 02. } 2 \\
\text { 03. } 3 \\
\text { 04. } 4 \\
\text { 05. } 5 \\
\text { 06. } 6 \\
\text { 07. Graduated } \\
\text { 96. No school } \\
\text { 98. DON'T KNOW }\end{array}$ \\
\hline
\end{tabular}


SECTION I: THE PRESENCE OF SCHOOLS

Now, we would like to ask about the history of the presence of education facilities in this village.

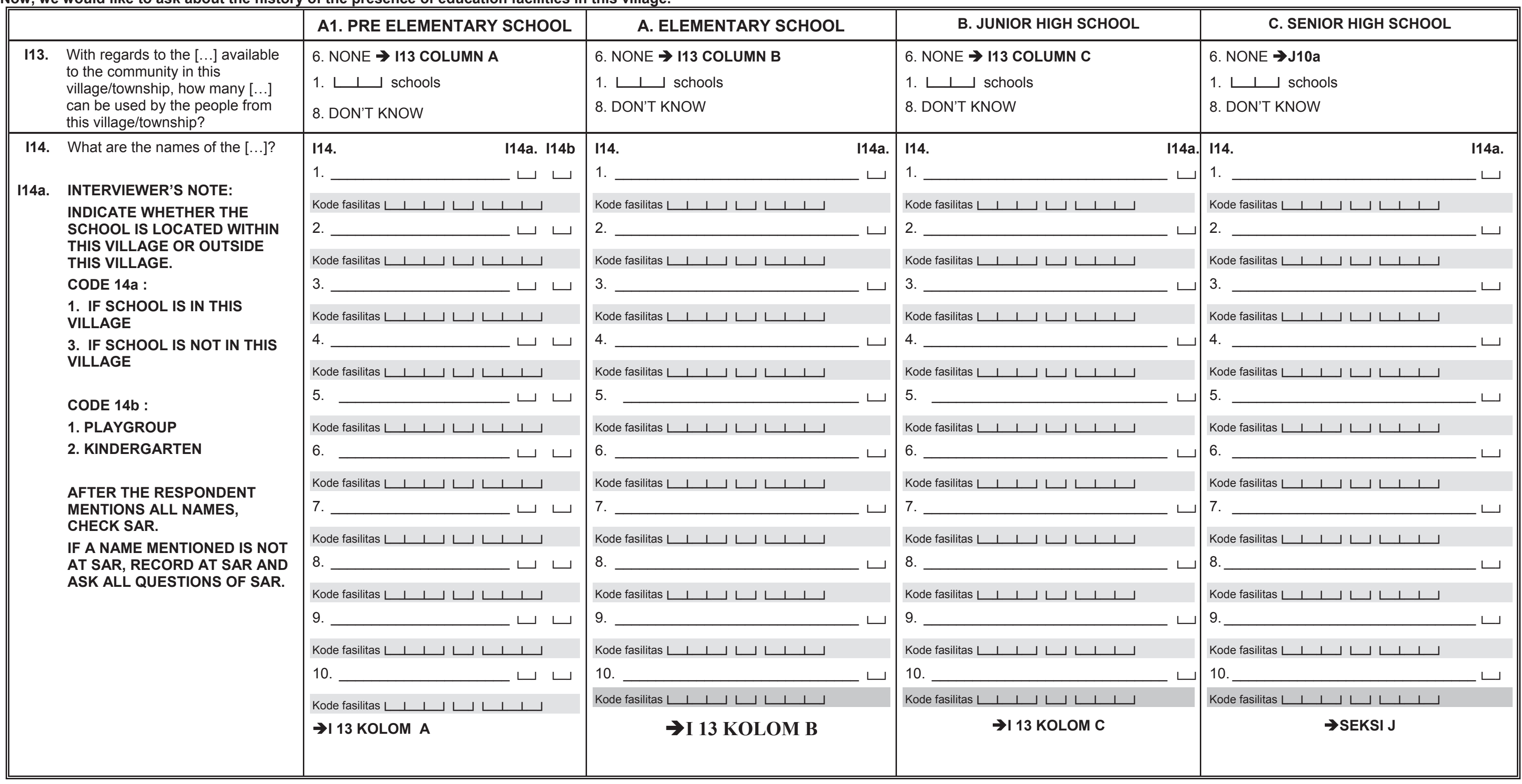


SECTION J: HISTORY OF THE PRESENCE OF HEALTH FACILITIES

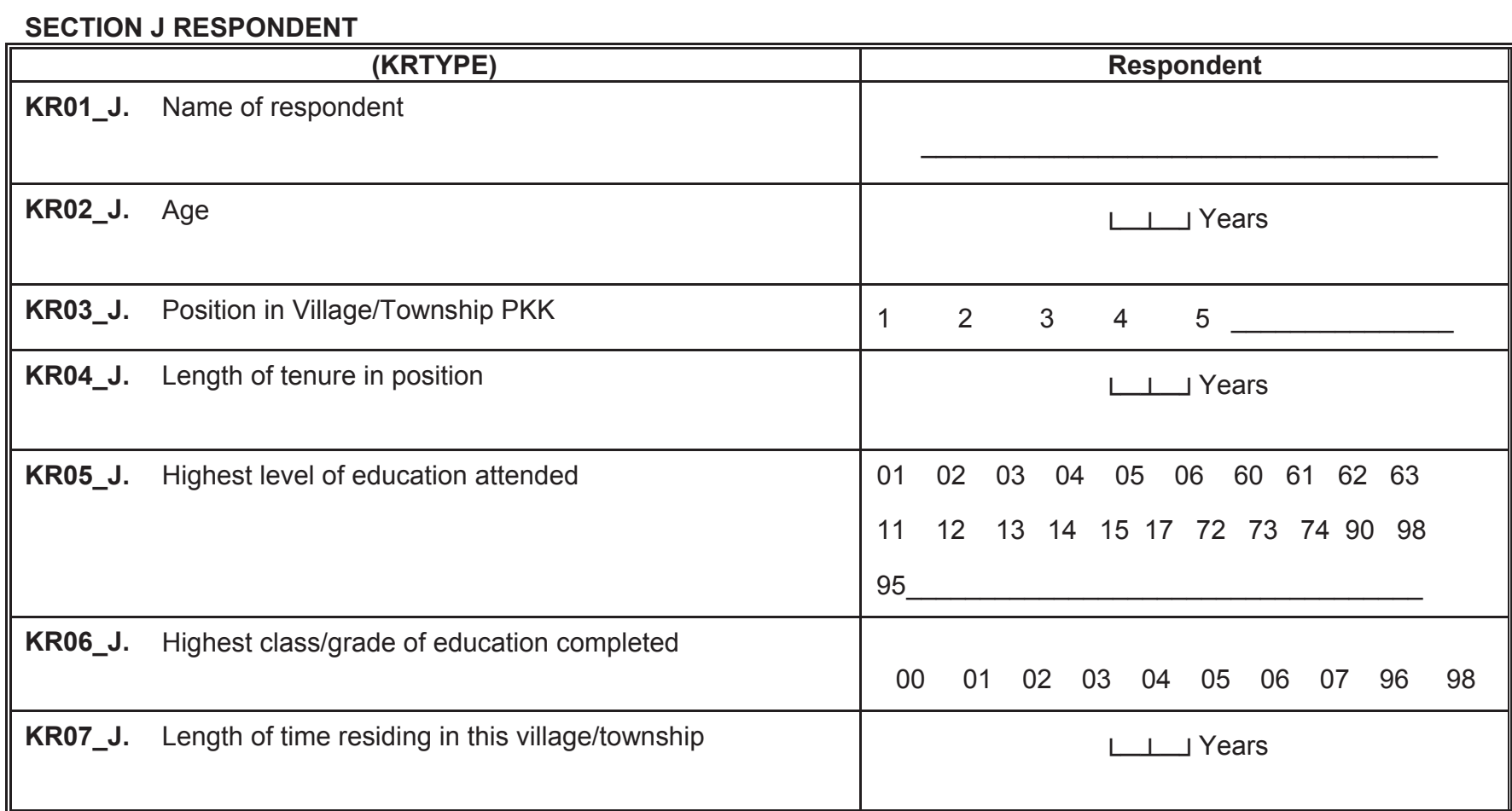

\begin{tabular}{|c|c|c|c|c|}
\hline $\begin{array}{l}\text { Code KR03_J : } \\
\text { 1. Head of PKK } \\
\text { 2. Organizer of PKK } \\
\text { 3. Cadre of PKK } \\
\text { 4. Ex organizer of PKK } \\
\text { 5. Other }\end{array}$ & $\begin{array}{l}\text { Code KR05_J: } \\
\text { 01. No school } \\
\text { 02. Elementary School (SD) } \\
\text { 03. Junior High School - General } \\
\text { 04. Junior High School - Vocational } \\
\text { 05. Senior High School - General } \\
\text { 06. Senior High School - Vocational } \\
\text { 11. Kejar Paket A (Adult Educ. A) } \\
\text { 12. Kejar Paket B (Adult Educ. B) }\end{array}$ & $\begin{array}{l}\text { 13. Open University } \\
\text { 14. Islamic School (Pesantren) } \\
\text { 15. Kejar Paket C (Adult Educ. C) } \\
\text { 17. School for the disabled } \\
\text { 60. D1, D2, D3 (Junior College) } \\
\text { 61. University S1 (Bachelor) } \\
\text { 62. University S2 (Masters) } \\
\text { 63. University S3 (Doctorate) }\end{array}$ & $\begin{array}{l}\text { 72. Islamic Elementary School } \\
\text { (Madrasah Ibtidaiyah) } \\
\text { 73. Islamic Junior High School } \\
\text { (Madrasah Tsanawiyah) } \\
\text { 74. Islamic Senior High School (Madrasah Aaliyah) } \\
\text { 90. Kindergarten } \\
\text { 98. DON'T KNOW } \\
\text { 95. Other }\end{array}$ & $\begin{array}{l}\text { Code KR06_J: } \\
\text { 00. Never completed } 1^{\text {st }} \text { class/grade } \\
01.1 \\
\text { 02. } 2 \\
\text { 03. } 3 \\
\text { 04. } 4 \\
\text { 05. } 5 \\
\text { 06. } 6 \\
\text { 07. Graduated } \\
\text { 96. No school } \\
\text { 98. DON'T KNOW }\end{array}$ \\
\hline
\end{tabular}


SECTION J: HISTORY OF THE PRESENCE OF HEALTH FACILITIES

Now, we would like to ask about history of the existence of health facilities in this village.

\begin{tabular}{|c|c|c|}
\hline J10a. & $\begin{array}{l}\text { Is there a Village Midwife (Bidan Desa) } \\
\text { located in this village? }\end{array}$ & Yes \\
\hline J10a1. & $\begin{array}{l}\text { Has there ever been a Village Midwife in } \\
\text { this village? }\end{array}$ & $\begin{array}{l}\text { No } \\
\text { Yes }\end{array}$ \\
\hline J10a2. & $\begin{array}{l}\text { When did the Village Midwife leave this } \\
\text { village? }\end{array}$ & 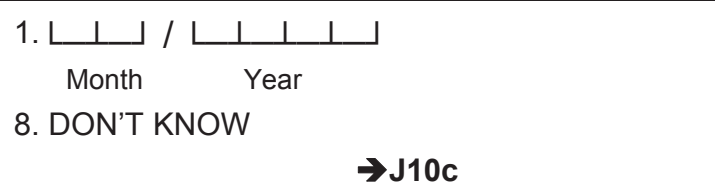 \\
\hline J10b. & $\begin{array}{l}\text { How many Village Midwives are located } \\
\text { in this village? }\end{array}$ & $\begin{array}{l}\text { 1. } ـ \perp \text { people } \\
\text { 8. DON'T KNOW }\end{array}$ \\
\hline J10c. & $\begin{array}{l}\text { In what year did the first Village Midwife } \\
\text { begin to practice in this village? }\end{array}$ & 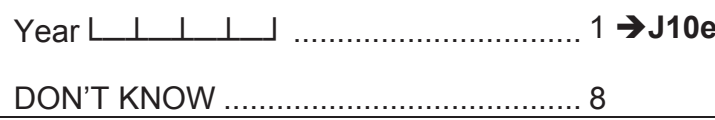 \\
\hline J10d. & $\begin{array}{l}\text { How many years has the Village Midwife } \\
\text { practiced in this village? }\end{array}$ & $\begin{array}{c}\perp \\
\mathrm{D} \\
\mathrm{DON} T \text { KNOW }\end{array}$ \\
\hline J10e. & $\begin{array}{l}\text { What type of support does/did the Village } \\
\text { Midwife (the first one) receive from the } \\
\text { local people or the village office? }\end{array}$ & $\begin{array}{l}\text { NONE } \\
\text { Housing } \\
\text { Food } \\
\text { A place to practice } \\
\text { Salary } / \text { cash }\end{array}$ \\
\hline J19. & $\begin{array}{l}\text { In this village is there a Delivery Post } \\
\text { (Pos Bersalin Desa/Polindes)? }\end{array}$ & 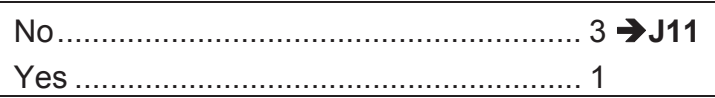 \\
\hline J19a. & $\begin{array}{l}\text { In what year was the first Polindes } \\
\text { established in this village? }\end{array}$ & 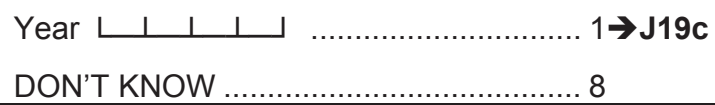 \\
\hline J19b. & $\begin{array}{l}\text { How many years has the Polindes } \\
\text { operated in this village? }\end{array}$ & $\begin{array}{l}\square \\
\text { DON'T KNOW }\end{array}$ \\
\hline J19c. & $\begin{array}{l}\text { How many beds does the Polindes } \\
\text { have? }\end{array}$ & 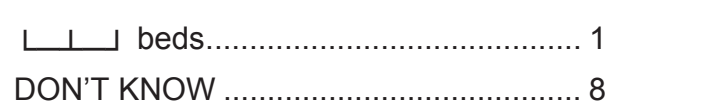 \\
\hline J11. & $\begin{array}{l}\text { How many Health Posts (Posyandu) } \\
\text { exist in this village? }\end{array}$ & DON'T KNOW \\
\hline
\end{tabular}

\begin{tabular}{|c|c|c|}
\hline J13. & $\begin{array}{l}\text { How many times have staff from Health } \\
\text { Centers (Puskesmas) visited the Posyandu } \\
\text { in the last year? }\end{array}$ & 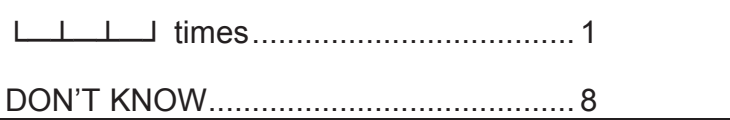 \\
\hline J21a. & $\begin{array}{l}\text { Is there any Posyandu Lansia in this } \\
\text { village? }\end{array}$ & 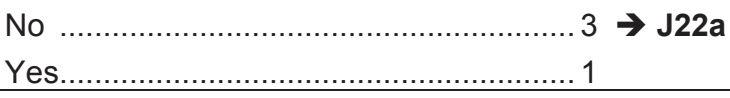 \\
\hline J21b. & $\begin{array}{l}\text { How many Posyandu Lansia are located in } \\
\text { this village? }\end{array}$ & 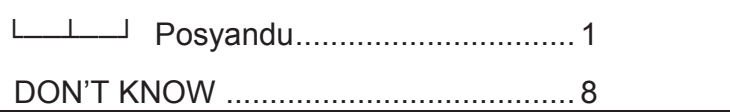 \\
\hline J21c. & $\begin{array}{l}\text { In what year did the first Posyandu Lansia } \\
\text { begin to practice in this village? }\end{array}$ & 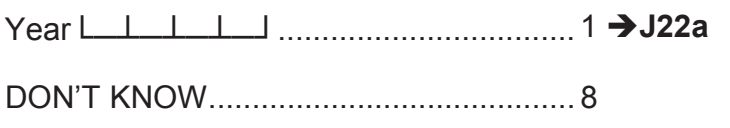 \\
\hline J21d. & $\begin{array}{l}\text { How many years has Posyandu Lansia } \\
\text { begun to practice in this village? }\end{array}$ & DON'T KNOW \\
\hline J22a. & Is there any Bidan Delima in this village? & $\begin{array}{l}\text { No } \\
\text { Yes }\end{array}$ \\
\hline J22b. & $\begin{array}{l}\text { How many Bidan Delima are located in this } \\
\text { village? }\end{array}$ & 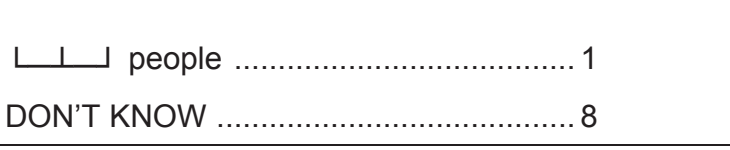 \\
\hline J22c. & $\begin{array}{l}\text { In what year did the first many Bidan Delima } \\
\text { begin to practice in this village? }\end{array}$ & 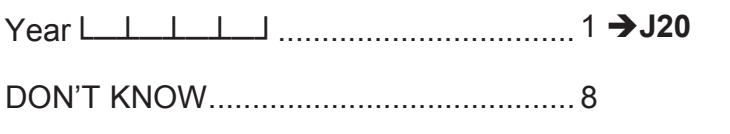 \\
\hline J22d. & $\begin{array}{l}\text { How many years has the Bidan Delima } \\
\text { begin to practice in this village? }\end{array}$ & $\begin{array}{l}\perp \\
\\
\text { DON'T KNOW }\end{array}$ \\
\hline J20. & $\begin{array}{l}\text { Is there a Medicine Post (Pos Obat Desa) in } \\
\text { this village? }\end{array}$ & No \\
\hline J24. & $\begin{array}{l}\text { Since } 2007 \text {, have there ever been any mass } \\
\text { immunization/vaccination for the people in } \\
\text { the village? }\end{array}$ & No \\
\hline
\end{tabular}




\section{SECTION J: HISTORY OF THE PRESENCE OF HEALTH FACILITIES}

\begin{tabular}{|c|c|c|c|c|c|c|c|c|c|}
\hline \multicolumn{10}{|c|}{ J25. } \\
\hline \multicolumn{10}{|c|}{ In what year were mass immunization/vaccination and what were the types of mass immunization/vaccination conducted since $2007 ?$} \\
\hline & YEAR & J25a. & \multicolumn{6}{|c|}{$\begin{array}{l}\text { J25. Types of mass immunization/vaccination. } \\
\text { [SEE CODE J.25, CIRCLE THE APPROPRIATE ANSWER CODE, THERE CAN BE MORE THAN ONE ANSWER] }\end{array}$} & Code J.25: \\
\hline 1. & 2014 & $\begin{array}{l}\text { 1. Yes } \\
\text { 3. None } \\
\text { 8. DON'T KNOW } \downarrow\end{array}$ & A1 & A2 & B & C & D & $\mathrm{F}$ & A1. Measles \\
\hline 2. & 2013 & $\begin{array}{l}\text { 1. Yes } \\
\text { 3. None } \\
\text { 8. DON'T KNOW } \downarrow\end{array}$ & A1 & A2 & B & C & D & E & A2. Smallpox \\
\hline 3. & 2012 & $\begin{array}{l}\text { 1. Yes } \\
\text { 3. None } \\
\text { 8. DON'T KNOW } \downarrow\end{array}$ & A1 & A2 & B & C & D & $E$ & B. BCG \\
\hline 4. & 2011 & $\begin{array}{l}\text { 1. Yes } \\
\text { 3. None } \\
\text { 8. DON'T KNOW } \downarrow\end{array}$ & A1 & A2 & B & C & D & E & C. Polio \\
\hline 5. & 2010 & $\begin{array}{l}\text { 1. Yes } \\
\text { 3. None } \\
\text { 8. DON'T KNOW } \downarrow\end{array}$ & A1 & A2 & B & C & D & $\mathrm{F}$ & D. DPT \\
\hline 6. & 2009 & $\begin{array}{l}\text { 1. Yes } \\
\text { 3. None } \\
\text { 8. DON'T KNOW } \downarrow\end{array}$ & A1 & A2 & B & C & D & $E$ & E. Tetanus Toxoid \\
\hline 7. & 2008 & $\begin{array}{l}\text { 1. Yes } \\
\text { 3. None } \\
\text { 8. DON'T KNOW } \downarrow\end{array}$ & A1 & A2 & B & C & D & $E$ & F. Hepatitis B \\
\hline 8. & 2007 & $\begin{array}{l}\text { 1. Yes } \\
\text { 3. None } \\
\text { 8. DON'T KNOW } \downarrow \\
\end{array}$ & A1 & A2 & B & $\mathrm{C}$ & D & $E$ & \\
\hline
\end{tabular}




\begin{tabular}{|c|c|c|c|c|c|c|c|c|}
\hline & (JTYPE) & $\begin{array}{l}\text { Aa. Integrated Community } \\
\text { Health } \\
\text { (Posyandu) }\end{array}$ & $\begin{array}{l}\text { Ab. Integrated } \\
\text { Health Pommunity } \\
\text { elderly for the } \\
\text { Lansia) }\end{array}$ & $\begin{array}{l}\text { A. Health Centers } \\
\text { (Puskesmas) and } \\
\text { Health Sub-centers } \\
\text { (Puskesmas } \\
\text { Pembantu/Pustu) }\end{array}$ & $\begin{array}{l}\text { B. Private health } \\
\text { practitioner such } \\
\text { as doctors, clinics, } \\
\text { medical posts, } \\
\text { maternity clinics, } \\
\text { nurses, midwives, } \\
\text { medical } \\
\text { assistants, and } \\
\text { other paramedical } \\
\text { workers }\end{array}$ & $\begin{array}{l}\text { C. Traditional health } \\
\text { workers, such as: } \\
\text { midwives, Chinese } \\
\text { healer (sinshe), } \\
\text { shaman, etc. }\end{array}$ & $\begin{array}{l}\text { D. Public or private } \\
\text { hospital }\end{array}$ & Ba. Pharmacy \\
\hline J26. & $\begin{array}{l}\text { With regards to the }[\ldots] \\
\text { available to the community } \\
\text { in this village/township, } \\
\text { how many [...] can be } \\
\text { used by the people from } \\
\text { this village/township? }\end{array}$ & $\begin{array}{l}\text { 1. L ل } 1 \text { facilities } \\
\text { 8. DON'T KNOW } \\
\text { 6. NONE } \rightarrow \text { J26Ab }\end{array}$ & $\begin{array}{l}\text { 1. } ـ ل \text { facilities } \\
\text { 8. DON'T KNOW } \\
\text { 6. NONE } \rightarrow \text { J26A }\end{array}$ & 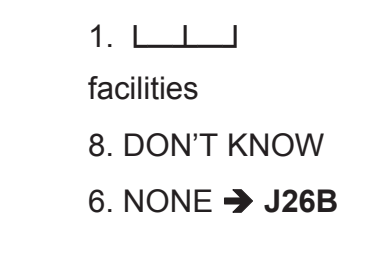 & $\begin{array}{l}\text { 1. } \downarrow \perp 1 \_ \\
\text {facilities } \\
\text { 8. DON'T KNOW } \\
\text { 6. NONE } \rightarrow \text { J26C }\end{array}$ & 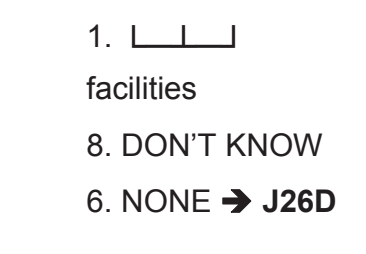 & $\begin{array}{l}\text { 1. } \downarrow \text { ــ لـ } \\
\text { facilities } \\
\text { 8. DON'T KNOW } \\
\text { 6. NONE } \rightarrow \\
\text { J26Ba }\end{array}$ & $\begin{array}{l}\text { 1. } ـ \perp \text { facilities } \\
\text { 8. DON'T KNOW } \\
\text { 6.NONE } \rightarrow \text { SECTION } \\
\text { PMKD }\end{array}$ \\
\hline $\begin{array}{l}\text { J27. } \\
\text { J27a }\end{array}$ & $\begin{array}{l}\text { What are the names of the } \\
\text { facilities? } \\
\text { INTERVIEWER'S NOTE: } \\
\text { INDICATE WHETHER } \\
\text { FACILITY IS LOCATED } \\
\text { WITHIN THIS VILLAGE } \\
\text { OR OUTSIDE THIS } \\
\text { VILLAGE, } \\
\text { CODE FOR J27a: } \\
\text { 1. IF FACILITY IS IN } \\
\text { THIS VILLAGE } \\
\text { 3. IF FACILITY IS NOT } \\
\text { IN THIS VILLAGE }\end{array}$ & 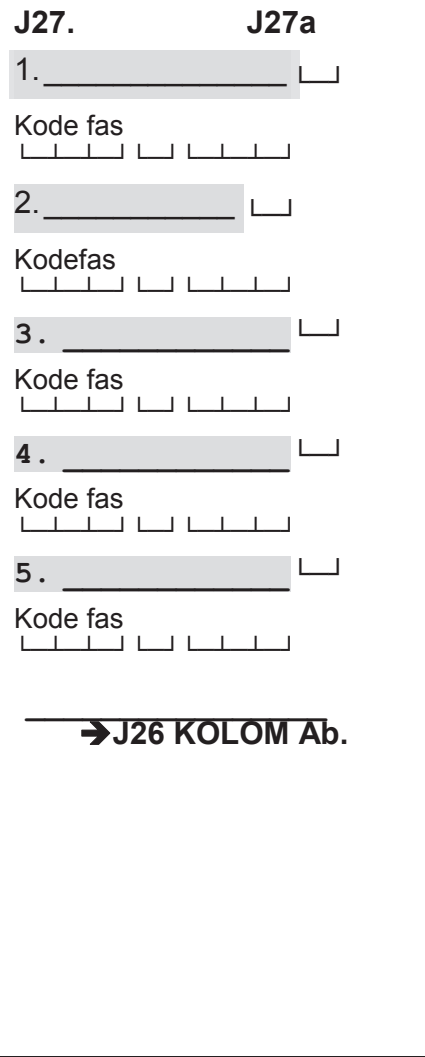 & 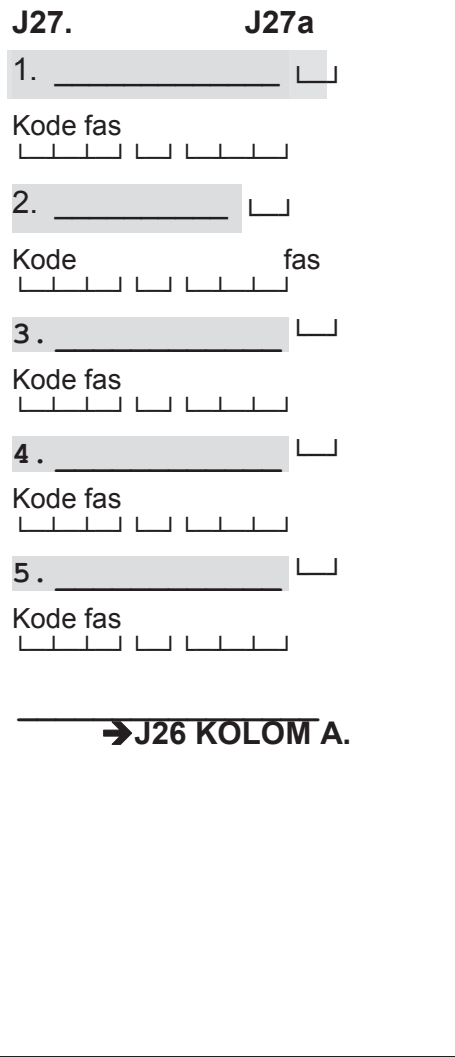 & 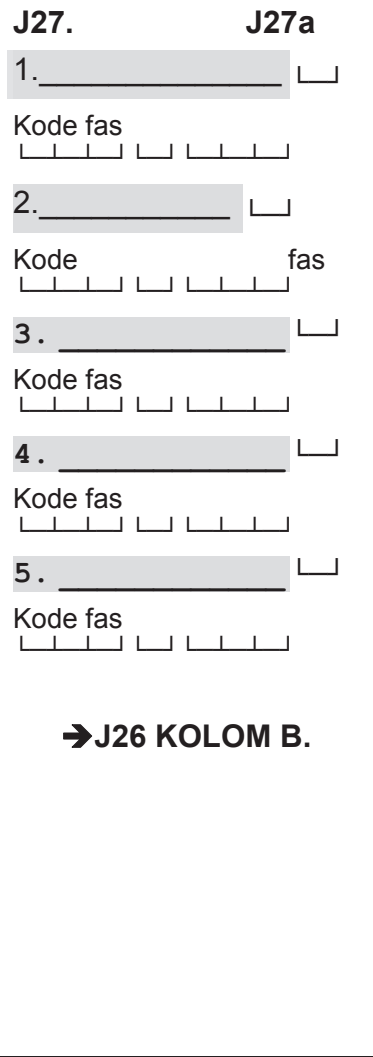 & 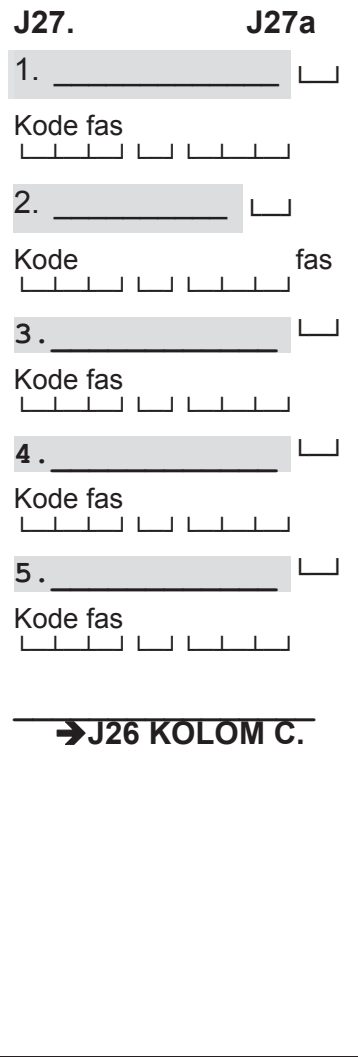 & 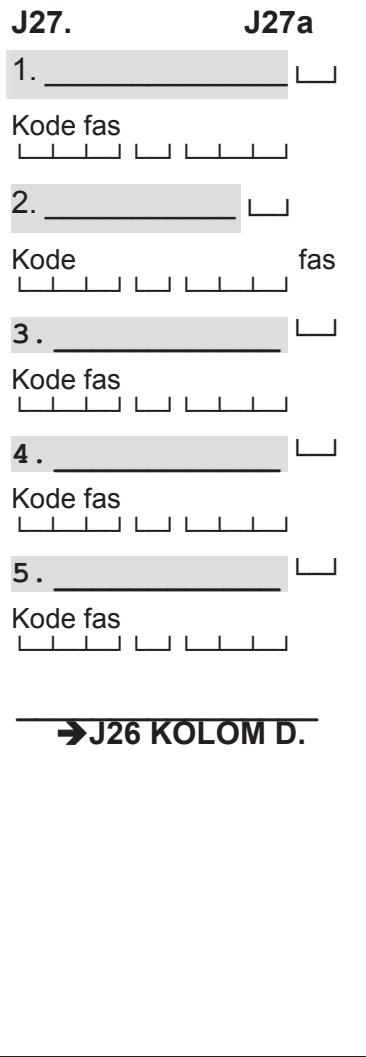 & 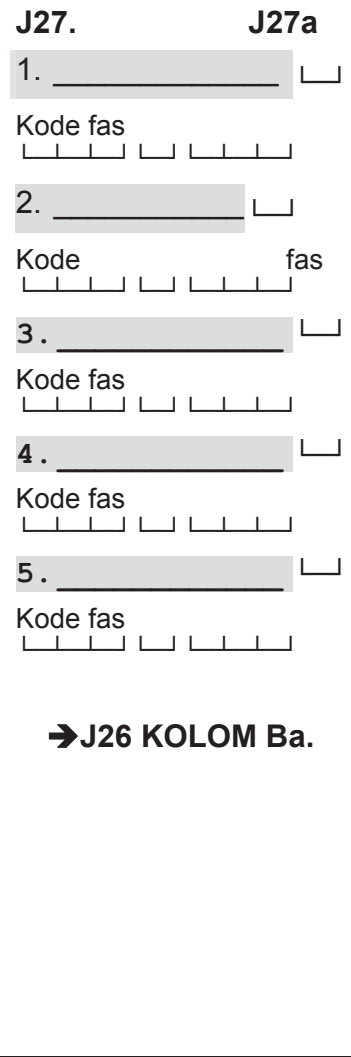 & 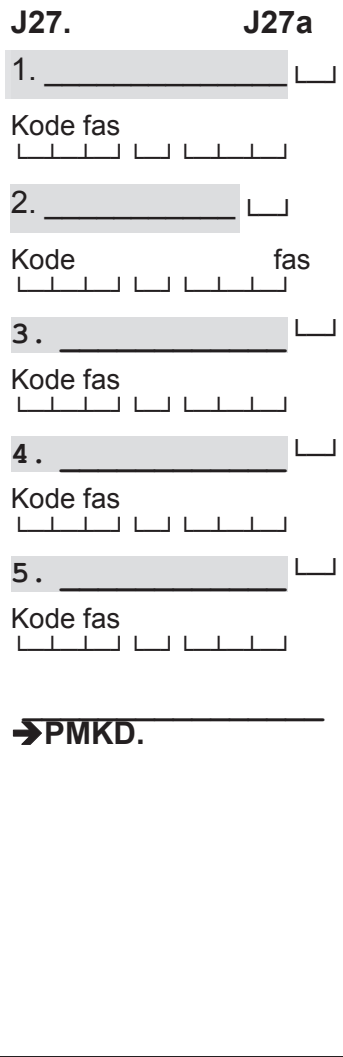 \\
\hline
\end{tabular}




\section{SECTION PM: CITIZEN PARTICIPATION}

\begin{tabular}{|c|c|}
\hline (KRTYPE) & Respondent \\
\hline KR01_PM. Name of respondent & \\
\hline KR02_PM. Age & 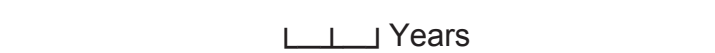 \\
\hline KR03_PM. Position in Village/Township PKK & 2 \\
\hline KR04_PM. Length of tenure in position & ــ Y Years \\
\hline KR05_PM. Highest level of education attended & 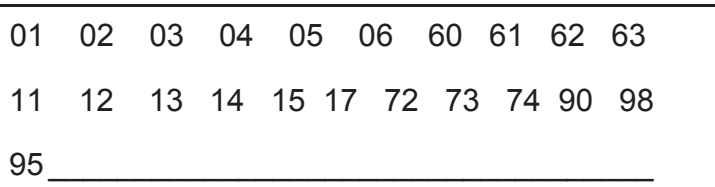 \\
\hline Highest class/grade of education completed & $\begin{array}{lllllllll}00 & 01 & 02 & 03 & 04 & 05 & 06 & 07 & 96\end{array}$ \\
\hline KR07_PM. Length of time residing in this village/township & ــ Y Years \\
\hline
\end{tabular}

\begin{tabular}{|c|c|c|c|c|}
\hline $\begin{array}{l}\text { Code KR03_PM : } \\
\text { 1. Head of PKK } \\
\text { 2. Organizer of PKK } \\
\text { 3. Cadre of PKK } \\
\text { 4. Ex organizer of PKK } \\
\text { 5. Other }\end{array}$ & $\begin{array}{l}\text { Code KR05_PM: } \\
\text { 01. No school } \\
\text { 02. Elementary School (SD) } \\
\text { 03. Junior High School - General } \\
\text { 04. Junior High School - Vocational } \\
\text { 05. Senior High School - General } \\
\text { 06. Senior High School - Vocational } \\
\text { 11. Kejar Paket A (Adult Educ. A) } \\
\text { 12. Kejar Paket B (Adult Educ. B) }\end{array}$ & $\begin{array}{l}\text { 13. Open University } \\
\text { 14. Islamic School (Pesantren) } \\
\text { 15. Kejar Paket C (Adult Educ. C) } \\
\text { 17. School for the disabled } \\
\text { 60. D1, D2, D3 (Junior College) } \\
\text { 61. University S1 (Bachelor) } \\
\text { 62. University S2 (Masters) } \\
\text { 63. University S3 (Doctorate) }\end{array}$ & $\begin{array}{l}\text { 72. Islamic Elementary School } \\
\text { (Madrasah Ibtidaiyah) } \\
\text { 73. Islamic Junior High School } \\
\text { (Madrasah Tsanawiyah) } \\
\text { 74. Islamic Senior High School (Madrasah Aaliyah) } \\
\text { 90. Kindergarten } \\
\text { 98. DON'T KNOW } \\
\text { 95. Other }\end{array}$ & $\begin{array}{l}\text { Code KR06_PM: } \\
\text { 00. Never completed } 1^{\text {st }} \text { class/grade } \\
\text { 01. } 1 \\
\text { 02. } 2 \\
\text { 03. } 3 \\
\text { 04. } 4 \\
\text { 05. } 5 \\
\text { 06. } 6 \\
\text { 07. Graduated } \\
\text { 96. No school } \\
\text { 98. DON'T KNOW }\end{array}$ \\
\hline
\end{tabular}


SECTION PM: CITIZEN PARTICIPATION

Now, we would like to ask about people's programs/activities in this village/Township

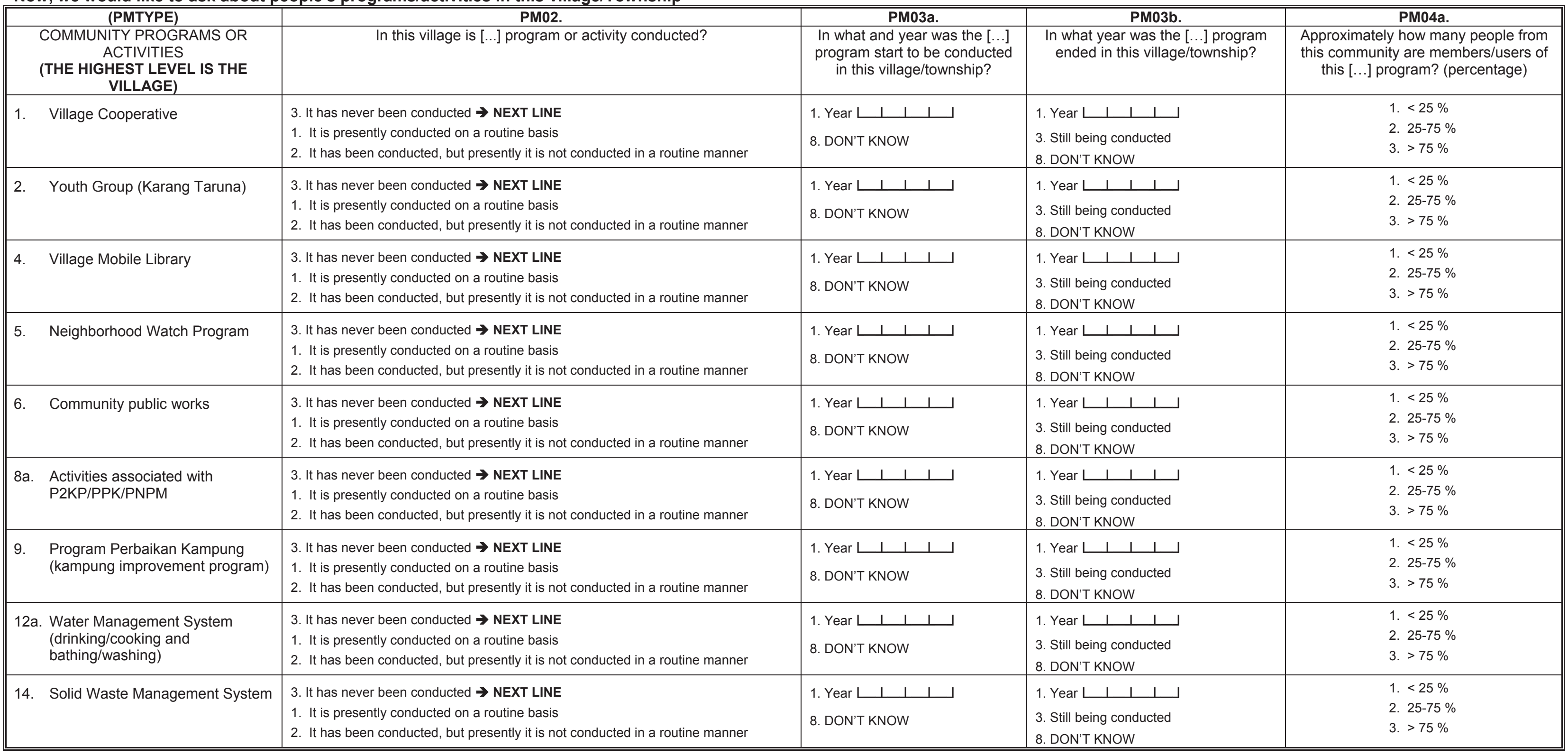




\section{SECTION PM: CITIZEN PARTICIPATION}

\begin{tabular}{|c|c|c|c|c|}
\hline (PMTYPE) & $\begin{array}{l}\text { PM02. } \\
\end{array}$ & PM03a. & PM03b. & PM04a. \\
\hline $\begin{array}{c}\text { COMMUNITY PROGRAMS OR } \\
\text { ACTIVITIES } \\
\text { (THE HIGHEST LEVEL IS THE } \\
\text { VILLAGE) }\end{array}$ & In this village is $[\ldots]$ program or activity conducted? & $\begin{array}{l}\text { In what and year was the }[\ldots] \\
\text { program start to be conducted } \\
\text { in this village/township? }\end{array}$ & $\begin{array}{l}\text { In what year was the }[\ldots] \text { program } \\
\text { ended in this village/township? }\end{array}$ & $\begin{array}{l}\text { Approximately how many people from } \\
\text { this community are members/users of } \\
\text { this [...] program? (percentage) }\end{array}$ \\
\hline $\begin{array}{l}\text { 15. Infrastructure Development } \\
\text { Program for Underdeveloped } \\
\text { Village (P3DT) }\end{array}$ & $\begin{array}{l}\text { 3. It has never been conducted } \rightarrow \text { NEXT LINE } \\
\text { 1. It is presently conducted on a routine basis } \\
\text { 2. It has been conducted, but presently it is not conducted in a routine manner }\end{array}$ & 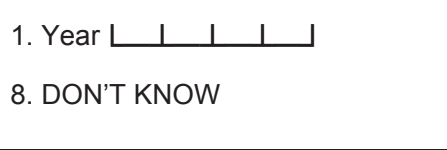 & 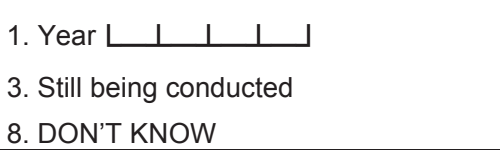 & $\begin{array}{l}\text { 1. }<25 \% \\
\text { 2. } 25-75 \% \\
\text { 3. }>75 \%\end{array}$ \\
\hline 18. Religious Activities & $\begin{array}{l}\text { 3. It has never been conducted } \rightarrow \text { NEXT LINE } \\
\text { 1. It is presently conducted on a routine basis } \\
\text { 2. It has been conducted, but presently it is not conducted in a routine manner }\end{array}$ & 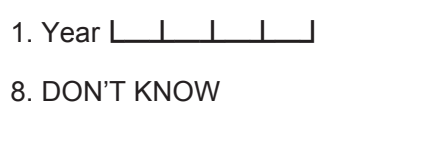 & 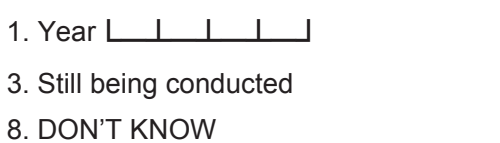 & $\begin{array}{l}\text { 1. }<25 \% \\
\text { 2. } 25-75 \% \\
\text { 3. }>75 \%\end{array}$ \\
\hline $\begin{array}{l}\text { 19. Medicinal Herb Garden (Apotik } \\
\text { Hidup) }\end{array}$ & $\begin{array}{l}\text { 3. It has never been conducted } \rightarrow \text { NEXT LINE } \\
\text { 1. It is presently conducted on a routine basis } \\
\text { 2. It has been conducted, but presently it is not conducted in a routine manner }\end{array}$ & 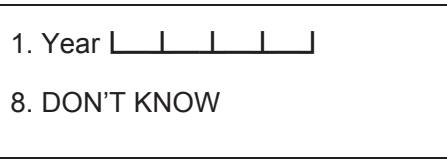 & 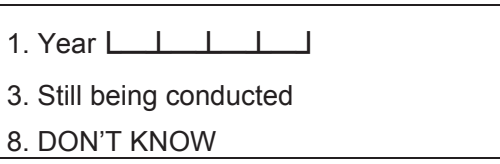 & $\begin{array}{l}\text { 1. }<25 \% \\
\text { 2. } 25-75 \% \\
\text { 3. }>75 \%\end{array}$ \\
\hline $\begin{array}{l}\text { 20. Bina Keluarga Balita (Child } \\
\text { Development Program) }\end{array}$ & $\begin{array}{l}\text { 3. It has never been conducted } \rightarrow \text { NEXT LINE } \\
\text { 1. It is presently conducted on a routine basis } \\
\text { 2. It has been conducted, but presently it is not conducted in a routine manner }\end{array}$ & 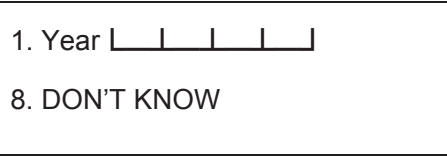 & 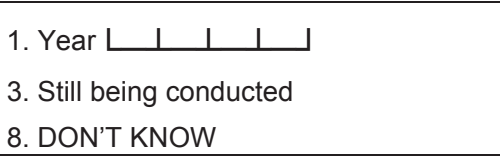 & $\begin{array}{l}\text { 1. }<25 \% \\
\text { 2. } 25-75 \% \\
\text { 3. }>75 \%\end{array}$ \\
\hline $\begin{array}{l}\text { 21. Bina Keluarga Remaja } \\
\text { (Youth/Teen Development } \\
\text { Program) }\end{array}$ & $\begin{array}{l}\text { 3. It has never been conducted } \rightarrow \text { NEXT LINE } \\
\text { 1. It is presently conducted on a routine basis } \\
\text { 2. It has been conducted, but presently it is not conducted in a routine manner }\end{array}$ & 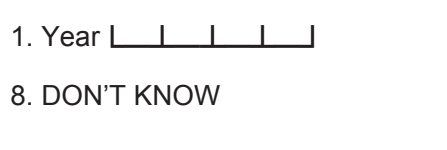 & 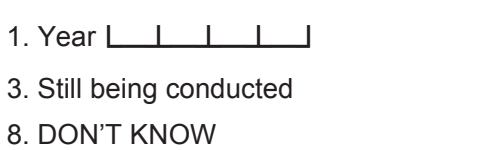 & $\begin{array}{l}\text { 1. }<25 \% \\
\text { 2. } 25-75 \% \\
\text { 3. }>75 \%\end{array}$ \\
\hline $\begin{array}{l}\text { 22. Bina Keluarga Manula (Program } \\
\text { Lansia) (Program for Elderly) }\end{array}$ & $\begin{array}{l}\text { 3. It has never been conducted } \rightarrow \text { NEXT LINE } \\
\text { 1. It is presently conducted on a routine basis } \\
\text { 2. It has been conducted, but presently it is not conducted in a routine manner }\end{array}$ & 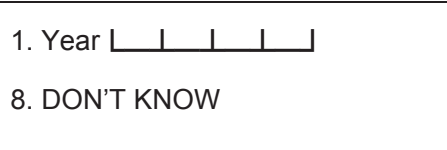 & 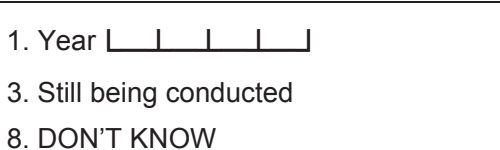 & $\begin{array}{l}\text { 1. }<25 \% \\
\text { 2. } 25-75 \% \\
\text { 3. }>75 \%\end{array}$ \\
\hline 23. Dana Sehat (Health Fund) & $\begin{array}{l}\text { 3. It has never been conducted } \rightarrow \text { NEXT LINE } \\
\text { 1. It is presently conducted on a routine basis } \\
\text { 2. It has been conducted, but presently it is not conducted in a routine manner }\end{array}$ & 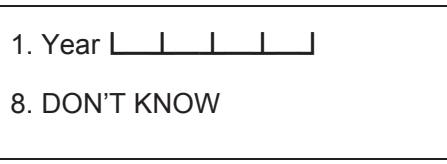 & 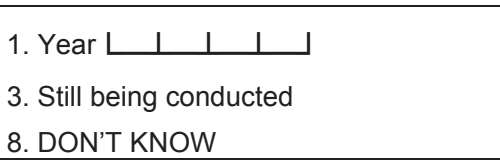 & $\begin{array}{l}\text { 1. }<25 \% \\
\text { 2. } 25-75 \% \\
\text { 3. }>75 \%\end{array}$ \\
\hline 24. Dasa Wisma (Family Group) & $\begin{array}{l}\text { 3. It has never been conducted } \rightarrow \text { NEXT LINE } \\
\text { 1. It is presently conducted on a routine basis } \\
\text { 2. It has been conducted, but presently it is not conducted in a routine manner }\end{array}$ & 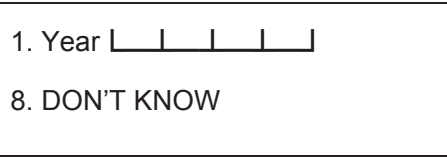 & 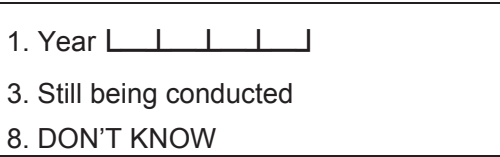 & $\begin{array}{l}\text { 1. }<25 \% \\
\text { 2. } 25-75 \% \\
\text { 3. }>75 \%\end{array}$ \\
\hline 25. Village Savings/Loan & $\begin{array}{l}\text { 3. It has never been conducted } \rightarrow \text { PAP28 } \\
\text { 1. It is presently conducted on a routine basis } \\
\text { 2. It has been conducted, but presently it is not conducted in a routine manner }\end{array}$ & 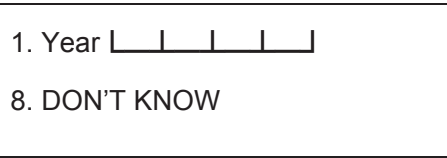 & 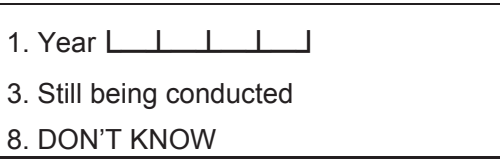 & $\begin{array}{l}\text { 1. }<25 \% \\
\text { 2. } 25-75 \% \\
\text { 3. }>75 \%\end{array}$ \\
\hline
\end{tabular}




\section{SECTION PAP: POVERTY ALLEVIATION PROGRAM}

SECTION PAP RESPONDENT

\begin{tabular}{|c|c|}
\hline $\begin{array}{l}\text { (KRTYPE) } \\
\end{array}$ & Respondent \\
\hline KR01_PAP. Name of respondent & \\
\hline KR02_PAP Age & ــ Y Years \\
\hline KR03_PAP. Position in Village/Township PKK & 4 \\
\hline KR04_PAP. Length of tenure in position & ـــ Y Years \\
\hline KR05_PAP. Highest level of education attended & 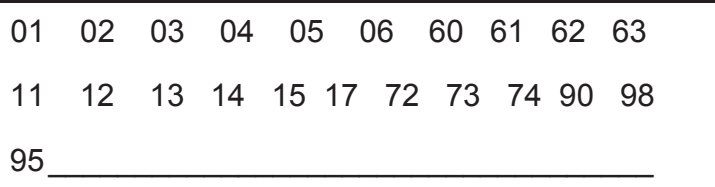 \\
\hline Highest class/grade of education completed & $\begin{array}{llllllllll}00 & 01 & 02 & 03 & 04 & 05 & 06 & 07 & 96 & 98\end{array}$ \\
\hline KR07_PAP. Length of time residing in this village/township & $\downarrow \perp$ Years \\
\hline
\end{tabular}

\begin{tabular}{|c|c|c|c|c|}
\hline $\begin{array}{l}\text { Code KR03_PAP : } \\
\text { 1. Head of PKK } \\
\text { 2. Organizer of PKK } \\
\text { 3. Cadre of PKK } \\
\text { 4. Ex organizer of PKK } \\
\text { 5. Other }\end{array}$ & $\begin{array}{l}\text { Code KR05_PAP: } \\
\text { 01. No school } \\
\text { 02. Elementary School (SD) } \\
\text { 03. Junior High School - General } \\
\text { 04. Junior High School - Vocational } \\
\text { 05. Senior High School - General } \\
\text { 06. Senior High School - Vocational } \\
\text { 11. Kejar Paket A (Adult Educ. A) } \\
\text { 12. Kejar Paket B (Adult Educ. B) }\end{array}$ & $\begin{array}{l}\text { 13. Open University } \\
\text { 14. Islamic School (Pesantren) } \\
\text { 15. Kejar Paket C (Adult Educ. C) } \\
\text { 17. School for the disabled } \\
\text { 60. D1, D2, D3 (Junior College) } \\
\text { 61. University S1 (Bachelor) } \\
\text { 62. University S2 (Masters) } \\
\text { 63. University S3 (Doctorate) }\end{array}$ & $\begin{array}{l}\text { 72. Islamic Elementary School } \\
\text { (Madrasah Ibtidaiyah) } \\
\text { 73. Islamic Junior High School } \\
\text { (Madrasah Tsanawiyah) } \\
\text { 74. Islamic Senior High School (Madrasah Aaliyah) } \\
\text { 90. Kindergarten } \\
\text { 98. DON'T KNOW } \\
\text { 95. Other }\end{array}$ & $\begin{array}{l}\text { Code KR06_PAP: } \\
\text { 00. Never completed } 1^{\text {st }} \text { class/grade } \\
01 . \quad 1 \\
\text { 02. } 2 \\
\text { 03. } 3 \\
\text { 04. } 4 \\
\text { 05. } 5 \\
\text { 06. } 6 \\
\text { 07. Graduated } \\
\text { 96. No school } \\
\text { 98. DON'T KNOW }\end{array}$ \\
\hline
\end{tabular}


SECTION PAP: POVERTY ALLEVIATION PROGRAM

Now, we would like to know about the distribution of Raskin

\begin{tabular}{|c|c|c|}
\hline PAP28. & $\begin{array}{l}\text { Was there Raskin program in the last } \\
12 \text { months? }\end{array}$ & $\begin{array}{l}\text { 3. NO } \rightarrow \text { PAP75 } \\
\text { 1. YES }\end{array}$ \\
\hline PAP29. & $\begin{array}{l}\text { In the last } 12 \text { months, how many times } \\
\text { has RASKIN rice been distributed in } \\
\text { this village? }\end{array}$ & $\begin{array}{l}\text { 1. } ـ \perp \text { times } \\
\text { 8. DON'T KNOW }\end{array}$ \\
\hline PAP30. & $\begin{array}{l}\text { How many tonnes of RASKIN rice were } \\
\text { delivered to this village to be } \\
\text { distributed? }\end{array}$ & $\begin{array}{l}\text { 1. ـــ tonnes } \\
\text { 8. DON'T KNOW }\end{array}$ \\
\hline PAP31. & $\begin{array}{l}\text { On average, in the last } 12 \text { months was } \\
\text { the percentage of households that } \\
\text { received RASKIN rice? }\end{array}$ & 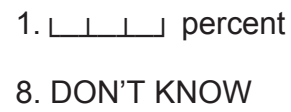 \\
\hline
\end{tabular}

Now we would like to ask you about the last time RASKIN was distributed in this village in the last 12 months.

\begin{tabular}{|c|c|c|}
\hline PAP32. & $\begin{array}{l}\text { When was the last time RASKIN rice } \\
\text { was distributed in the last } 12 \text { months? }\end{array}$ & 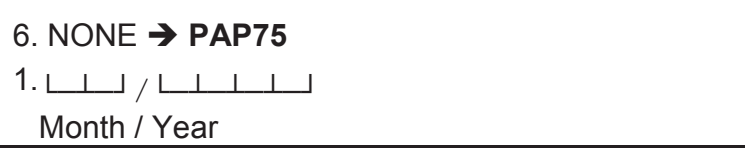 \\
\hline PAP36. & $\begin{array}{l}\text { What was the average amount of rice } \\
\text { received by all RASKIN household } \\
\text { recipients? }\end{array}$ & 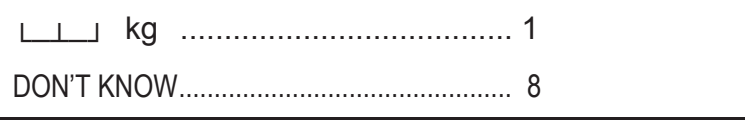 \\
\hline PAP37. & $\begin{array}{l}\text { What was the price of each kilogram of } \\
\text { RASKIN rice (include other costs) paid } \\
\text { by each household? }\end{array}$ & 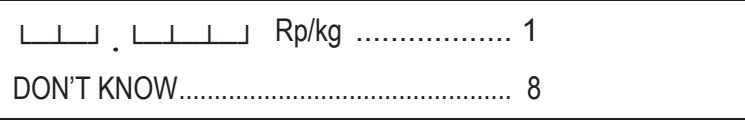 \\
\hline PAP38. & $\begin{array}{l}\text { What was the market price of each } \\
\text { kilogram of the rice in that time? }\end{array}$ & 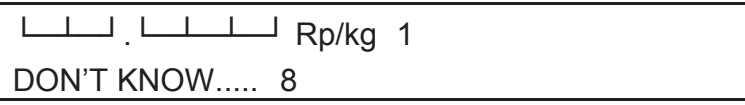 \\
\hline PAP39a & $\begin{array}{l}\text { How to decide the household to } \\
\text { receive RASKIN? }\end{array}$ & $\begin{array}{l}\text { 1. Based on list name which is decided by } \\
\text { government (DPM) } \rightarrow \text { PAP39 } \\
\text { 2. Based on list name from government (DPM) and } \\
\text { modified } \\
\text { 3. Do not use DPM }\end{array}$ \\
\hline PAP39b & $\begin{array}{l}\text { Based on what decision for the } \\
\text { receiver of RASKIN? }\end{array}$ & $\begin{array}{l}\text { A. Floor type of low quality (e.g ground, no tiles) } \\
\text { B. Wall of low quality (e.g bamboo, boards) } \\
\text { C. No toilet/latrines for defecation } \\
\text { D. Source of drinking water not piped from PAM } \\
\text { E. Source of lighting is not electricity } \\
\text { F. Use wood/charcoal for cooking fuel } \\
\text { G. Head of household didn't go to school/finish } \\
\text { elementary school } \\
\text { H. Elderly } \\
\text { I. Widowed }\end{array}$ \\
\hline
\end{tabular}

\begin{tabular}{|c|c|c|}
\hline & & $\begin{array}{l}\text { J. Widow } \\
\text { K. Don't have permanent job } \\
\text { L. Considered as poor by community } \\
\text { V. Other }\end{array}$ \\
\hline PAP39. & $\begin{array}{l}\text { Did all households that received } \\
\text { RASKIN rice receive equal amounts? }\end{array}$ & 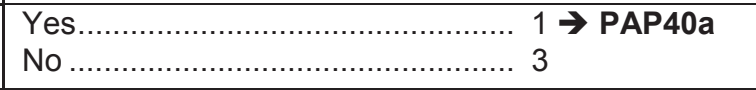 \\
\hline PAP40. & $\begin{array}{l}\text { How did you determine the amount of } \\
\text { rice that a household can buy/receive? }\end{array}$ & $\begin{array}{l}\text { A. Based on the number of household members } \\
\text { B. Based on the poverty level of the household } \\
\text { C. Based on the number of children } \\
\text { V. Other } \\
\rightarrow \text { PAP41 }\end{array}$ \\
\hline PAP40a. & $\begin{array}{l}\text { If yes how the system is distributed } \\
\text { equally? }\end{array}$ & $\begin{array}{l}\text { A. Distributed equally among the poor households } \\
\text { B. Distributed equally between rich and poor } \\
\text { C. Distributed equally to rich and poor but in shifts } \\
\text { D. Given to the poor, if can not buy sale to the rich. } \\
\rightarrow \text { PAP45aa }\end{array}$ \\
\hline PAP45aa & $\begin{array}{l}\text { How was the quality of the rice } \\
\text { purchased from the Raskin program? }\end{array}$ & 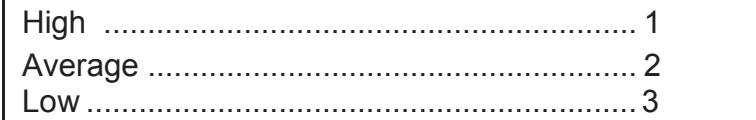 \\
\hline
\end{tabular}

Now i would like to ask about KIS (Kartu Indonesia Sehat) Program

\begin{tabular}{|c|c|c|}
\hline PAP75. & $\begin{array}{l}\text { Do you have KIS ( Kartu Indonesia Sehat ) } \\
\text { program in this village/kelurahan? }\end{array}$ & $\begin{array}{l}\text { No } \\
\text { Yes }\end{array}$ \\
\hline PAP76. & $\begin{array}{l}\text { When was }[\ldots] \text { first initiated in this } \\
\text { village/kelurahan? }\end{array}$ & 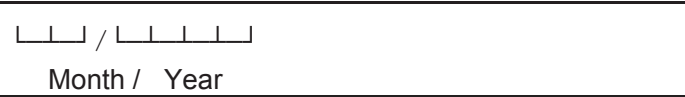 \\
\hline PAP77. & Who benefit from KIS program? & $\begin{array}{l}\text { A. Very poor household } \\
\text { B. Poor household } \\
\text { C. Non poor household } \\
\text { D. Homeless } \\
\text { E. Disable } \\
\text { V. Other: } \\
\text { Y. DON'T KNOW }\end{array}$ \\
\hline PAP78. & $\begin{array}{l}\text { Who conducted listing for household } \\
\text { beneficiaries of KIS program? } \\
\text { (CIRCLE ALL THAT APPLY }\end{array}$ & $\begin{array}{l}\text { A. Village/kelurhan officials } \\
\text { B. Kader PKK/Posyandu } \\
\text { C. PUSKESMAS Official/PLKB /Midwife } \\
\text { D. Community figure } \\
\text { E. BPS Official /Mitra BPS/Mantri Statistik } \\
\text { F. DPM from Government } \\
\text { V. Other }\end{array}$ \\
\hline
\end{tabular}

COMFAS 2014 


\section{SECTION PAP: POVERTY ALLEVIATION PROGRAM}

Now i would like to ask about KIP (Kartu Indonesia Pintar )

\begin{tabular}{|c|c|c|}
\hline PAP79. & $\begin{array}{l}\text { Do you have KIP ( Kartu Indonesia } \\
\text { Pintar ) program in this } \\
\text { village/kelurahan? }\end{array}$ & $\begin{array}{l}\text { No } \\
\text { Yes }\end{array}$ \\
\hline PAP80. & $\begin{array}{l}\text { When was }[\ldots] \text { first initiated in this } \\
\text { village/kelurahan? }\end{array}$ & 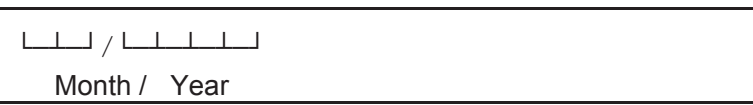 \\
\hline PAP81. & Who benefit from KIP program? & $\begin{array}{l}\text { A. Very poor household } \\
\text { B. Poor household } \\
\text { C. Non poor household } \\
\text { D. Homeless } \\
\text { E. Disable } \\
\text { V. Other: } \\
\text { Y. DON'T KNOW }\end{array}$ \\
\hline PAP82. & $\begin{array}{l}\text { Who conducted listing for } \\
\text { household beneficiaries of KIP } \\
\text { program? } \\
\text { (CIRCLE ALL THAT APPLY) }\end{array}$ & $\begin{array}{l}\text { A. Village/kelurahan officials } \\
\text { B. Kader PKK/Posyandu } \\
\text { C. PUSKESMAS Official/PLKB /Midwife } \\
\text { D. Community figure } \\
\text { E. BPS Official /Mitra BPS/Mantri Statistik } \\
\text { F. DPM from Government } \\
\text { V. Other }\end{array}$ \\
\hline PAP83 & How much amount is given? & \\
\hline & 1.SD & 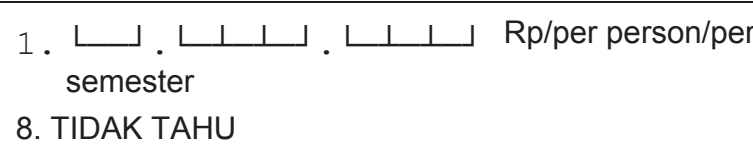 \\
\hline & 2. SMP & 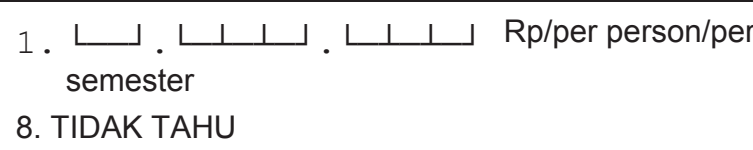 \\
\hline & 3. SMA & 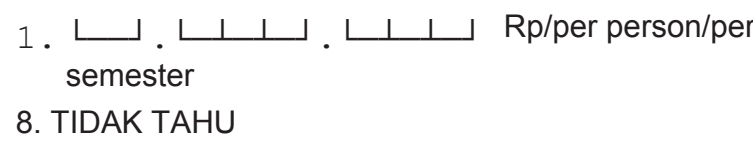 \\
\hline
\end{tabular}

Now I would like to know about KKS ( Kartu Keluarga Sejahtera) Program

\begin{tabular}{|c|c|c|}
\hline PAP84. & $\begin{array}{l}\text { Do you have KKS ( Kartu Keluarga } \\
\text { Sejahtera ) program in this } \\
\text { village/kelurahan? }\end{array}$ & 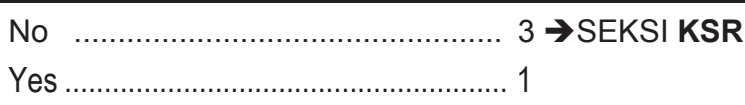 \\
\hline PAP85. & $\begin{array}{l}\text { When was }[\ldots] \text { first initiated in this } \\
\text { village/kelurahan? }\end{array}$ & 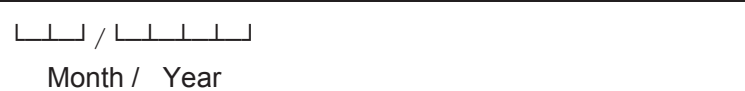 \\
\hline
\end{tabular}

\begin{tabular}{|c|c|c|}
\hline PAP86. & Who benefit from KKS program? & $\begin{array}{l}\text { A. Very poor household } \\
\text { B. Poor household } \\
\text { C. Non poor household } \\
\text { D. Homeless } \\
\text { E. Disable } \\
\text { V. Other: } \\
\text { Y. DON'T KNOW }\end{array}$ \\
\hline PAP87. & $\begin{array}{l}\text { Who conducted listing for } \\
\text { household beneficiaries of KKS } \\
\text { program? } \\
\text { (CIRCLE ALL THAT APPLY) }\end{array}$ & 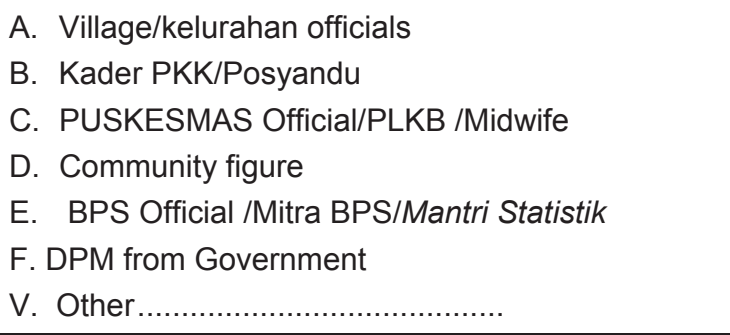 \\
\hline PAP88 & How much amount is given? & 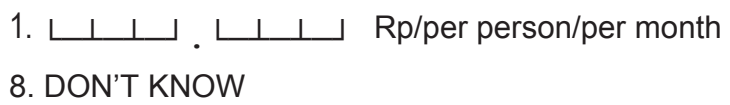 \\
\hline
\end{tabular}




\section{SECTION KSR: WELFARE}

\section{SECTION KSR RESPONDENT}

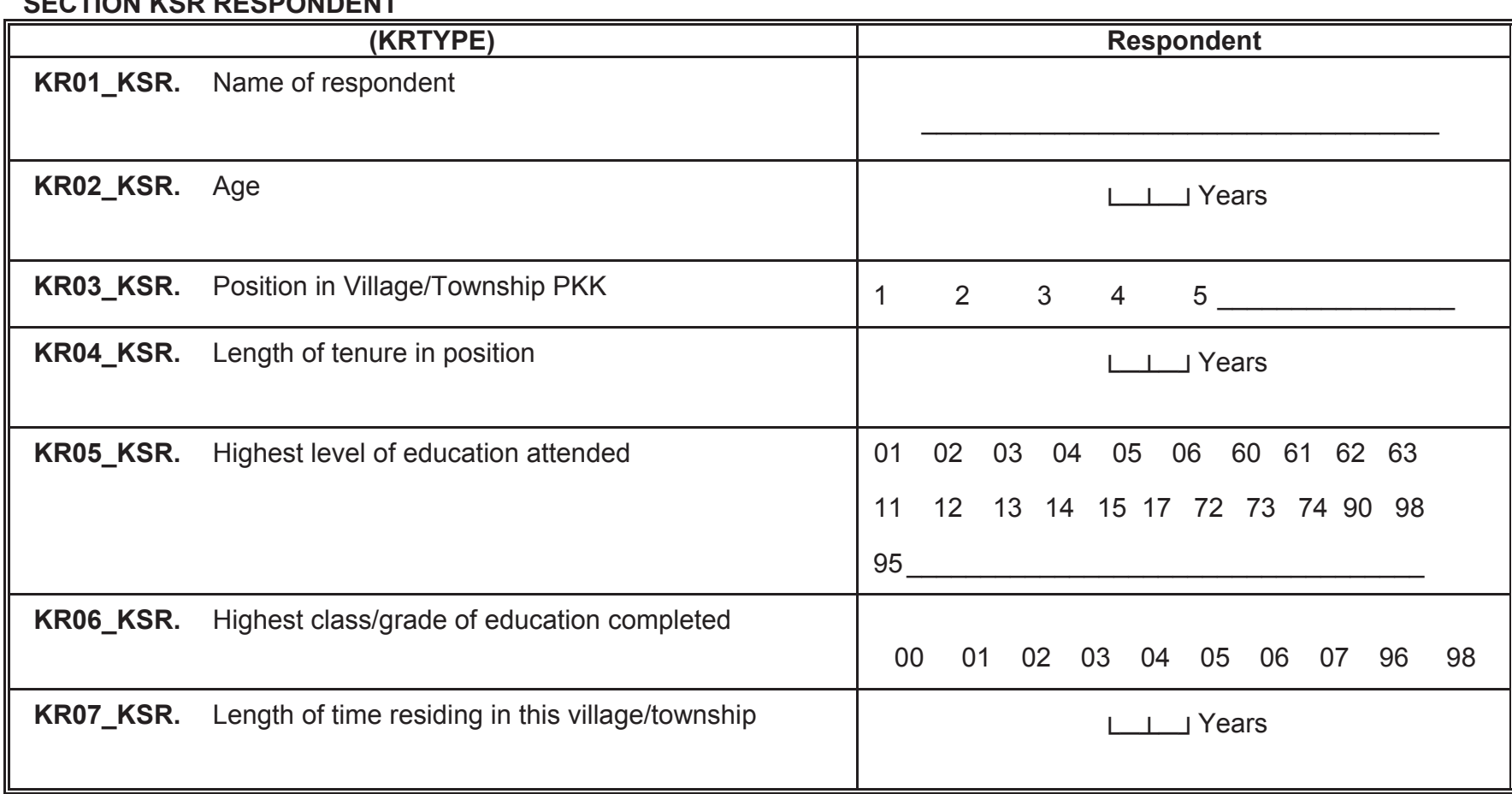

\begin{tabular}{|c|c|c|c|c|}
\hline $\begin{array}{l}\text { Code KR03_KSR: } \\
\text { 1. Head of PKK } \\
\text { 2. Organizer of PKK } \\
\text { 3. Cadre of PKK } \\
\text { 4. Ex organizer of PKK } \\
\text { 5. Other }\end{array}$ & $\begin{array}{l}\text { Code KR05_KSR: } \\
\text { 01. No school } \\
\text { 02. Elementary School (SD) } \\
\text { 03. Junior High School - General } \\
\text { 04. Junior High School - Vocational } \\
\text { 05. Senior High School - General } \\
\text { 06. Senior High School - Vocational } \\
\text { 11. Kejar Paket A (Adult Educ. A) } \\
\text { 12. Kejar Paket B (Adult Educ. B) }\end{array}$ & $\begin{array}{l}\text { 13. Open University } \\
\text { 14. Islamic School (Pesantren) } \\
\text { 15. Kejar Paket C (Adult Educ. C) } \\
\text { 17. School for the disabled } \\
\text { 60. D1, D2, D3 (Junior College) } \\
\text { 61. University S1 (Bachelor) } \\
\text { 62. University S2 (Masters) } \\
\text { 63. University S3 (Doctorate) }\end{array}$ & $\begin{array}{l}\text { 72. Islamic Elementary School } \\
\text { (Madrasah Ibtidaiyah) } \\
\text { 73. Islamic Junior High School } \\
\text { (Madrasah Tsanawiyah) } \\
\text { 74. Islamic Senior High School (Madrasah Aaliyah) } \\
\text { 90. Kindergarten } \\
\text { 98. DON'T KNOW } \\
\text { 95. Other }\end{array}$ & $\begin{array}{l}\text { Code KR06_KSR: } \\
\text { 00. Never completed } 1^{\text {st }} \text { class/grade } \\
\text { 01. } 1 \\
\text { 02. } 2 \\
\text { 03. } 3 \\
\text { 04. } 4 \\
\text { 05. } 5 \\
\text { 06. } 6 \\
\text { 07. Graduated } \\
\text { 96. No school } \\
\text { 98. DON'T KNOW }\end{array}$ \\
\hline
\end{tabular}




\section{SECTION KSR: WELFARE}

Now, we would like to know about the Market Operation programs of kerosene, sugar, and cooking oil in the last 12 months.

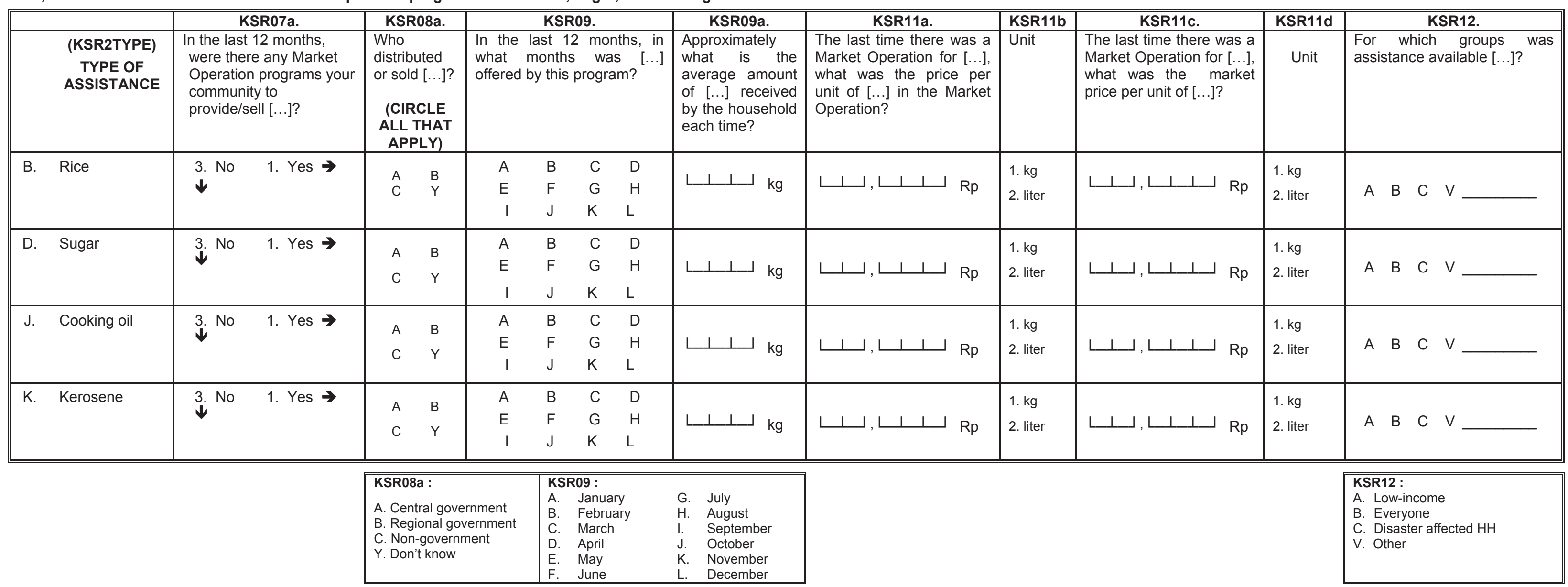




\section{SECTION CP: INTERVIEW SESSION NOTES}

\begin{tabular}{|c|c|c|c|c|c|c|c|}
\hline \multirow{2}{*}{$\begin{array}{l}\text { LANGMAIN (CK1). } \\
\text { LANGOTHR (CK2). }\end{array}$} & \multicolumn{2}{|l|}{ Interview was entirely/mostly conducted in what language? } & \multicolumn{5}{|l|}{$ـ$ other } \\
\hline & Other languaged used (if any): & & ــ other & & & & \\
\hline \multicolumn{8}{|l|}{ LANGUAGE CODE: } \\
\hline 00. Bahasa Indonesia & 04. Batak & 08. Sasak & 12. Makassar & 16. & Toraja & 20. & Lampung \\
\hline 01. Java & 05. Bugis & 09. Minang & 13. Nias & 17. & Lahat & 95. & Other, \\
\hline 02. Sunda & 06. Cina & 10. Banjar & 14. Palembang & 18. & Other South Sumatera & 96. & NA \\
\hline 03. Bali & 07. Madura & 11. Bima & 15. Sumbawa & 19. & Betawi & & \\
\hline
\end{tabular}

\begin{tabular}{|c|c|c|c|}
\hline RESULT (FP3). RESULTS OF INTERVIEW & $\begin{array}{l}\text { REASON (FP4). REASON FOR ANSWERING “2” / “3” IN } \\
\text { RESULT. }\end{array}$ & FP6. MONITORING BY SUPERVISOR & \\
\hline $\begin{array}{l}\text { 1. Completed } \rightarrow \text { FP6 } \\
\text { 2. Partly completed } \\
\text { 3. Not completed } \\
\text { 4. Twin EA with EA Lـ } \downarrow \rightarrow F P 6\end{array}$ & $\begin{array}{l}\text { 1. Respondent was travelling/not in location } \\
\text { 2. Respondent was too busy } \\
\text { 3. Respondent refused }\end{array}$ & $\begin{array}{ll} & \\
\text { a. } & \text { Observed (sup_obs) .......... } 1 \\
\text { b. } & \text { Checked (sup_edit)........... } 1 \\
\text { c. } & \text { Verified (sup_veri)............. } 1\end{array}$ & $\begin{array}{l}\text { No } \\
3 \\
3 \\
3\end{array}$ \\
\hline
\end{tabular}

\section{INTERVIEWER NOTE:}




\section{INDONESIAN FAMILY LIFE SURVEY 2014 \\ PUBLIC PERCEPTION ON GOVERNMENT PROGRAMS AND PUBLIC SERVICES (INFORMANT BOOK)}

SECTIONS : LK, K, PAP, PPS, GD, TR, CP

Cov1: Respondent is:

1. School principal/senior teacher

4. Religious Leader

2. Health professional

5. Local Political Party Activist

3. Youth Activist

6. Local Business leader

NAME OF DESA/KELURAHAN :

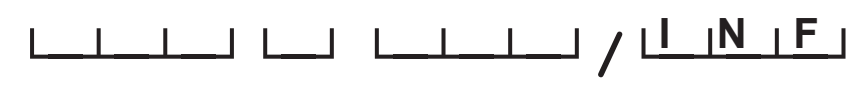

1. Yes $\rightarrow \mathrm{CK} 1$

3. No 


\section{SECTION LK : CONTROL SHEET}

\begin{tabular}{||c|c||}
\hline \multicolumn{1}{|c|}{ SAMPLING INFORMATION } & CODE \\
\hline LK01. Province & $\sqcup$ \\
\hline LK02. Kabupaten/Kota & $\sqcup$ \\
\hline LK03. Kecamatan & $\llcorner\perp 1$ \\
\hline LK04. Village/Urban Township & \llcorner \\
\hline LK05. Region: 1. Urban 2. Rural & \\
\hline
\end{tabular}

\begin{tabular}{||c|c||}
\hline \multicolumn{1}{|c|}{ SUPERVISION: } & CODE \\
\hline LK15. Name of Interviewer: & \\
\hline LK17. Name of Local Supervisor: & \\
\hline LK19. Name of Field Coordinator: & \\
\hline
\end{tabular}

\begin{tabular}{|c|c|c|}
\hline \multicolumn{3}{|r|}{ VILLAGE/TOWNSHIP ADDRESS } \\
\hline LK23. & Name of respondent: & \\
\hline \multirow[t]{3}{*}{ LK08. } & a. Address & \\
\hline & \multicolumn{2}{|c|}{ b. Description of location } \\
\hline & c. Postal code & $:$ : \\
\hline \multirow[t]{4}{*}{ LK09. } & Telephone number: & 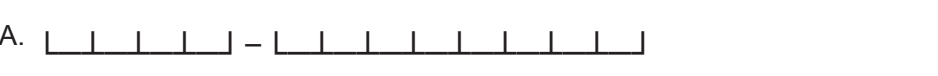 \\
\hline & & $\begin{array}{ll}\text { a. code } & \text { b. number }\end{array}$ \\
\hline & & 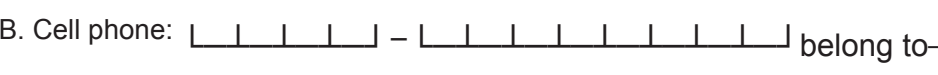 \\
\hline & 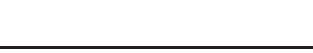 & Y. DON'T KNOW $\quad$ W. NOT APPLICABLE \\
\hline LK09a & E-mail address & \\
\hline
\end{tabular}




\section{SECTION K : RESPONDENT IDENTITY}

\begin{tabular}{|c|c|}
\hline & RESPONDENT A \\
\hline & $\begin{array}{c}\text { IR1. } \\
\end{array}$ \\
\hline 1. Name of Respondent: & \\
\hline 2. Age : & 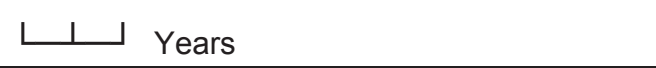 \\
\hline 3. INVERVIEWER CHECK: & 1. MALE 3. FEMALE \\
\hline 4. Position in the village/community & $\begin{array}{l}\text { 1. SCHOOL PRINCIPAL/SENIOR TEACHER } \\
\text { 2. HEALTH PROFESSIONAL } \\
\text { 3. YOUTH ACTIVIST } \\
\text { 4. RELIGIOUS LEADER } \\
\text { 5. LOCAL POLITICAL PARTY ACTIVIST } \\
\text { 6. LOCAL BUSINESS LEADER }\end{array}$ \\
\hline 5. Length of tenure in the position: & 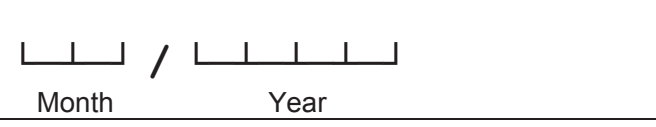 \\
\hline 6. Highest level of education attended : & 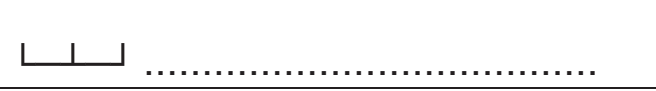 \\
\hline 7. Highest class completed : & $\begin{array}{llllllll}00 & 01 & 02 & 03 & 04 & 05 & 06 & 07 \\
96 & 98 & & & & & & \end{array}$ \\
\hline 8. Length of time lived in the village : & 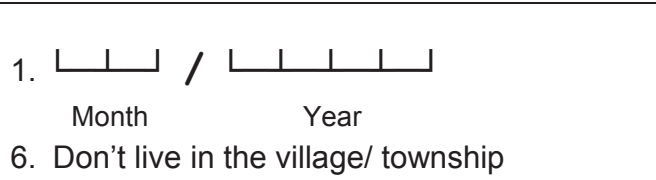 \\
\hline
\end{tabular}

Codes for last level of education (6):
01. No school/not yet in school
11. Kejar Paket A (Adult Educ. A)
02. Elementary school
03. Junior High - General
04. Junior High - Vocationa
05. High School - General
60. D1 D2, D3 (Junior College)
60. D1, D2, D3 (Junior College)
62. University $\mathrm{S}$ (Masters)
63. University S3 (Doctorate)
13. Open Univeristy
14. Islamic School (Pondok Pensantren)
2. Islamic Elementary School (Madrasah Ibtidaiyah)
3. Islamic Junior/Senior High School (Madrasah Tsanawiyah)
8. Kindergarten
95. Other

Codes for last level of education (7):

00 . Not completed $1^{\text {st }}$ grade/class

01. 1

02. 2

03. 3

04. 4

06.6

07. Graduated

96. No school

98. DON'T KNOW 


\section{SECTION PAP : POVERTY ALLEVIATION PROGRAM}

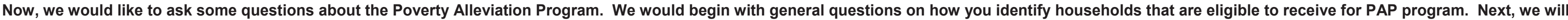
continue with questions on specific programs

\begin{tabular}{|c|c|c|}
\hline PAP01. & $\begin{array}{l}\text { Does the community have a system } \\
\text { to identify the households as Poor } \\
\text { Households (GAKIN) that are } \\
\text { eligible for a poverty alleviation } \\
\text { program? }\end{array}$ & $\begin{array}{l}\text { No PAP program ............. } 6 \text { } \rightarrow \text { SECTION PPS } \\
\text { No } \\
\text { Yes }\end{array}$ \\
\hline PAP06. & $\begin{array}{l}\text { If it was felt that a household was } \\
\text { eligible to receive PAP program, but } \\
\text { household was not on the GAKIN } \\
\text { list, is there any mechanism in which } \\
\text { the household can apply to be a } \\
\text { recipient of the program? }\end{array}$ & $\begin{array}{l}\text { 1. Report to RT/RW head } \\
\text { 2. Report to village } \\
\text { 3. Report to kecamatan } \\
\text { 4 Depends on type of PAP program } \\
\text { 6. No mechanism } \\
\text { 8. DON'T KNOW }\end{array}$ \\
\hline
\end{tabular}


SECTION PAP : POVERTY ALLEVIATION PROGRAM

\begin{tabular}{|c|c|c|c|c|c|c|c|c|c|c|}
\hline \multirow{2}{*}{\multicolumn{2}{|c|}{$\begin{array}{c}\text { TYPE OF POVERTY } \\
\text { PROGRAM } \\
\text { (PAP1TYPE) }\end{array}$}} & \multirow[b]{2}{*}{$\begin{array}{c}\text { PAP7. } \\
\text { Was there } \\
{[\ldots]} \\
\text { program in } \\
\text { this village } \\
\text { since } \\
2007 ?\end{array}$} & \multirow{2}{*}{\begin{tabular}{l}
\multicolumn{1}{c}{ PAP7a. } \\
When was the first \\
time the program \\
begin?
\end{tabular}} & \multirow{2}{*}{$\begin{array}{l}\text { PAP7c. } \\
\text { Is the program still } \\
\text { running? }\end{array}$} & \multirow{2}{*}{\begin{tabular}{l}
\multicolumn{1}{c}{ PAP7d. } \\
When did the program \\
stop?
\end{tabular}} & \multirow[b]{2}{*}{\begin{tabular}{l}
\multicolumn{1}{c}{ PAP14. } \\
Before the [...] \\
program was \\
implemented, \\
has there been \\
information \\
campaign of the \\
program to the \\
community in this \\
village?
\end{tabular}} & \multirow[b]{2}{*}{\begin{tabular}{l}
\multicolumn{1}{c}{ PAP17a. } \\
Has there been \\
a \\
complaint/prote \\
st from the \\
community with \\
respect to the \\
selection of \\
those eligible to \\
receive [....]?
\end{tabular}} & PAP66. & PAP67. & PAP68. \\
\hline & & & & & & & & $\begin{array}{l}\begin{array}{l}\text { According to } \\
\text { opinion, was } \\
\text { program the } \\
\text { implemented } \\
\text { transparently? }\end{array} \\
\end{array}$ & 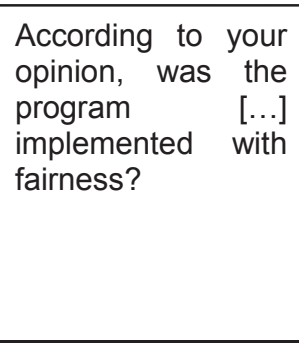 & $\begin{array}{l}\text { According to your } \\
\text { opinion, was there } \\
\text { any corruption, } \\
\text { collusion or } \\
\text { nepotism practice } \\
\text { during the program } \\
{[\ldots]} \\
\text { implementation? }\end{array}$ \\
\hline 1. & $\begin{array}{l}\text { Jamkesda ( Regional } \\
\text { health insurance ) }\end{array}$ & $\begin{array}{l}\text { 1. Yes } \\
\text { 3. No } \downarrow\end{array}$ & 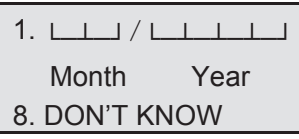 & $\begin{array}{l}\text { 1. Yes } \rightarrow \text { PAP14 } \\
\text { 3. No } \\
\text { 8. DK }\end{array}$ & 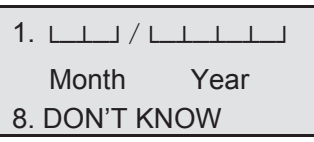 & $\begin{array}{l}\text { 1. Yes } \\
\text { 3. No }\end{array}$ & $\begin{array}{l}\text { 1. Yes } \\
\text { 3. No }\end{array}$ & & & \\
\hline 2. & $\begin{array}{l}\text { Jamkesmas ( Public } \\
\text { health insurance ) }\end{array}$ & $\begin{array}{l}\text { 1. Yes } \\
\text { 3. No } \downarrow\end{array}$ & 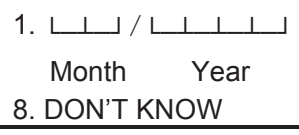 & $\begin{array}{l}\text { 1. Yes } \rightarrow \text { PAP14 } \\
\text { 3. No } \\
\text { 8. DK }\end{array}$ & 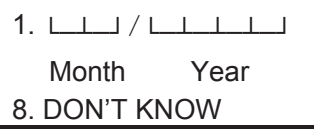 & $\begin{array}{l}\text { 1. Yes } \\
\text { 3. No }\end{array}$ & $\begin{array}{l}\text { 1. Yes } \\
\text { 3. No }\end{array}$ & & & \\
\hline $2 a$ & $\begin{array}{l}\text { Jampersal ( Delivery } \\
\text { insurance ) }\end{array}$ & $\begin{array}{l}\text { 1. Yes } \\
\text { 3. No } \downarrow\end{array}$ & 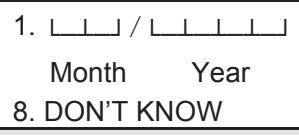 & $\begin{array}{l}\text { 1. Yes } \rightarrow \text { PAP14 } \\
\text { 3. No } \\
\text { 8. DK }\end{array}$ & 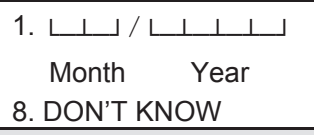 & $\begin{array}{l}\text { 1. Yes } \\
\text { 3. No }\end{array}$ & $\begin{array}{l}\text { 1. Yes } \\
\text { 3. No }\end{array}$ & & & \\
\hline $2 b$. & $\begin{array}{l}\text { JKN (National Health } \\
\text { Insurance) }\end{array}$ & $\begin{array}{l}\text { 1. Yes } \\
\text { 3. No } \downarrow\end{array}$ & 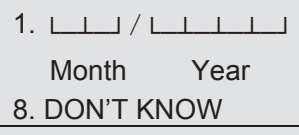 & $\begin{array}{l}\text { 1. Yes } \rightarrow \text { PAP14 } \\
\text { 3. No } \\
\text { 8. DK }\end{array}$ & 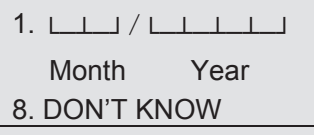 & $\begin{array}{l}\text { 1. Yes } \\
\text { 3. No }\end{array}$ & $\begin{array}{l}\text { 1. Yes } \\
\text { 3. No }\end{array}$ & & & \\
\hline 3. & $\begin{array}{l}\text { Raskin (Rice for the } \\
\text { poor) }\end{array}$ & $\begin{array}{l}\text { 1. Yes } \\
\text { 3. No } \downarrow\end{array}$ & 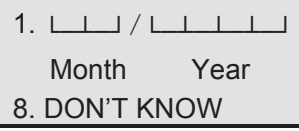 & $\begin{array}{l}\text { 1. Yes } \rightarrow \text { PAP14 } \\
\text { 3. No } \\
\text { 8. DK }\end{array}$ & 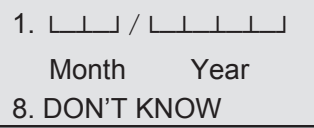 & $\begin{array}{l}\text { 1. Yes } \\
\text { 3. No }\end{array}$ & $\begin{array}{l}\text { 1. Yes } \\
\text { 3. No }\end{array}$ & $\begin{array}{l}\text { 1. Yes } \\
\text { 3. No }\end{array}$ & $\begin{array}{l}\text { 1. Yes } \\
\text { 3. No }\end{array}$ & $\begin{array}{l}\text { 1. Yes } \\
\text { 3. No }\end{array}$ \\
\hline & $\begin{array}{l}\text { Market Operation for } \\
\text { Rice }\end{array}$ & $\begin{array}{l}\text { 1. Yes } \\
\text { 3. No } \downarrow\end{array}$ & 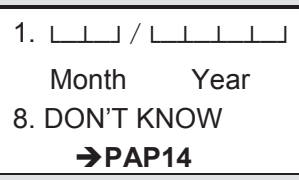 & & & $\begin{array}{l}\text { 1. Yes } \\
\text { 3. No }\end{array}$ & $\begin{array}{l}\text { 1. Yes } \\
\text { 3. No }\end{array}$ & & & \\
\hline 5. & $\begin{array}{l}\text { Market Operation for } \\
\text { Other goods }\end{array}$ & $\begin{array}{l}\text { 1. Yes } \\
\text { 3. No } \downarrow\end{array}$ & 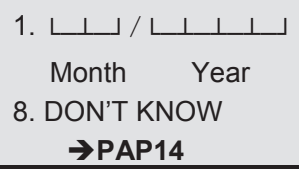 & & & $\begin{array}{l}\text { 1. Yes } \\
\text { 3. No }\end{array}$ & $\begin{array}{l}\text { 1. Yes } \\
\text { 3. No }\end{array}$ & & & \\
\hline 6. & $\begin{array}{l}\text { PKPS BBM - SLT } \\
\text { (Unconditional Cash } \\
\text { Transfer) }\end{array}$ & $\begin{array}{l}\text { 1. Yes } \\
\text { 3. No } \downarrow\end{array}$ & 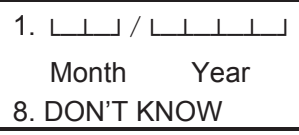 & $\begin{array}{l}\text { 1. Yes } \rightarrow \text { PAP14 } \\
\text { 3. No } \\
\text { 8. DK }\end{array}$ & 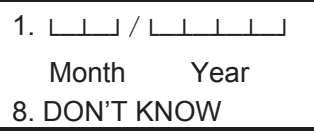 & $\begin{array}{l}\text { 1. Yes } \\
\text { 3. No }\end{array}$ & $\begin{array}{l}\text { 1. Yes } \\
\text { 3. No }\end{array}$ & $\begin{array}{l}\text { 1. Yes } \\
\text { 3. No }\end{array}$ & $\begin{array}{l}\text { 1. Yes } \\
\text { 3. No }\end{array}$ & $\begin{array}{l}\text { 1. Yes } \\
\text { 3. No }\end{array}$ \\
\hline 7. & $\begin{array}{l}\text { Keluarga Harapan } \\
\text { (Conditional Cash } \\
\text { Transfer) } \\
\end{array}$ & $\begin{array}{l}\text { 1. Yes } \\
\text { 3. No } \downarrow\end{array}$ & 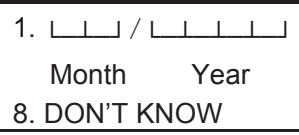 & $\begin{array}{l}\text { 1. Yes } \rightarrow \text { PAP14 } \\
\text { 3. No } \\
\text { 8. DK }\end{array}$ & 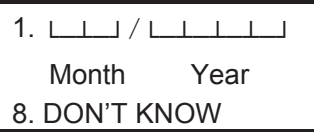 & $\begin{array}{l}\text { 1. Yes } \\
\text { 3. No }\end{array}$ & $\begin{array}{l}\text { 1. Yes } \\
\text { 3. No }\end{array}$ & $\begin{array}{l}\text { 1. Yes } \\
\text { 3. No }\end{array}$ & $\begin{array}{l}\text { 1. Yes } \\
\text { 3. No }\end{array}$ & $\begin{array}{l}\text { 1. Yes } \\
\text { 3. No }\end{array}$ \\
\hline & PNPM Mandiri & $\begin{array}{l}\text { 1. Yes } \\
\text { 3. No } \downarrow\end{array}$ & 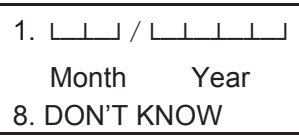 & $\begin{array}{l}\text { 1. Yes } \rightarrow \text { PAP14 } \\
\text { 3. No } \\
\text { 8. DK }\end{array}$ & 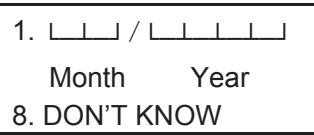 & $\begin{array}{l}\text { 1. Yes } \\
\text { 3. No }\end{array}$ & $\begin{array}{l}\text { 1. Yes } \\
\text { 3. No }\end{array}$ & $\begin{array}{l}\text { 1. Yes } \\
\text { 3. No }\end{array}$ & $\begin{array}{l}\text { 1. Yes } \\
\text { 3. No }\end{array}$ & $\begin{array}{l}\text { 1. Yes } \\
\text { 3. No }\end{array}$ \\
\hline
\end{tabular}


SECTION PAP : POVERTY ALLEVIATION PROGRAM

\begin{tabular}{|c|c|c|c|c|c|c|c|c|c|c|}
\hline 9. & $\begin{array}{l}\text { BLSM ( Direct Cash } \\
\text { Assistance for the } \\
\text { People ) }\end{array}$ & $\begin{array}{l}\text { 1. Yes } \\
\text { 3. No } \downarrow\end{array}$ & 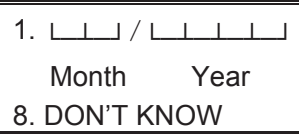 & $\begin{array}{l}\text { 1. Yes } \rightarrow \text { PAP14 } \\
\text { 3. No } \\
\text { 8. DK }\end{array}$ & 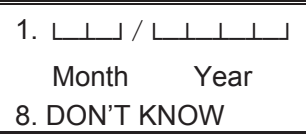 & $\begin{array}{l}\text { 1. Yes } \\
\text { 3. No }\end{array}$ & $\begin{array}{l}\text { 1. Yes } \\
\text { 3. No }\end{array}$ & $\begin{array}{l}\text { 1. Yes } \\
\text { 3. No }\end{array}$ & $\begin{array}{l}\text { 1. Yes } \\
\text { 3. No }\end{array}$ & $\begin{array}{l}\text { 1. Yes } \\
\text { 3. No }\end{array}$ \\
\hline 10. & $\begin{array}{l}\text { BSM ( Cash transfer } \\
\text { for poor student) }\end{array}$ & $\begin{array}{l}\text { 1. Yes } \\
\text { 3. No } \downarrow\end{array}$ & 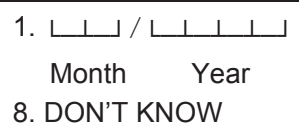 & $\begin{array}{l}\text { 1. Yes } \rightarrow \text { PAP14 } \\
\text { 3. No } \\
\text { 8. DK }\end{array}$ & 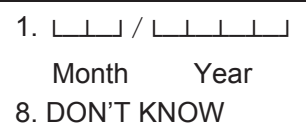 & $\begin{array}{l}\text { 1. Yes } \\
\text { 3. No }\end{array}$ & $\begin{array}{l}\text { 1. Yes } \\
\text { 3. No }\end{array}$ & $\begin{array}{l}\text { 1. Yes } \\
\text { 3. No }\end{array}$ & $\begin{array}{l}\text { 1. Yes } \\
\text { 3. No }\end{array}$ & $\begin{array}{l}\text { 1. Yes } \\
\text { 3. No }\end{array}$ \\
\hline 11. & $\begin{array}{l}\text { Askesos ( Social } \\
\text { Welfare Insurance ) }\end{array}$ & $\begin{array}{l}\text { 1. Yes } \\
\text { 3. No } \downarrow\end{array}$ & 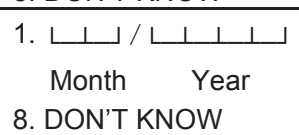 & $\begin{array}{l}\text { 1. Yes } \downarrow \\
\text { 3. No } \\
\text { 8. DK }\end{array}$ & 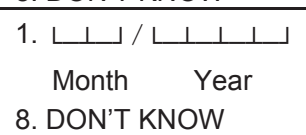 & & & & & \\
\hline 12. & $\begin{array}{l}\text { PPA-PKH ( Child } \\
\text { Labor Reduction- } \\
\text { Conditional Cash } \\
\text { Transfer ) }\end{array}$ & $\begin{array}{l}\text { 1. Yes } \\
\text { 3. No } \downarrow\end{array}$ & 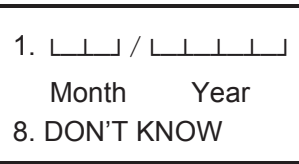 & $\begin{array}{l}\text { 1. Yes } \downarrow \\
\text { 3. No } \\
\text { 8. DK }\end{array}$ & 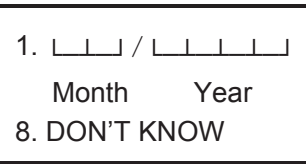 & & & & & \\
\hline 13. & $\begin{array}{l}\text { JSPACA/JSODK ( } \\
\text { Disabled Social } \\
\text { Insurance/Severe- } \\
\text { Disabled Social } \\
\text { Insurance ) } \\
\end{array}$ & $\begin{array}{ll}\text { 1. Yes } \\
\text { 3. No } \downarrow\end{array}$ & 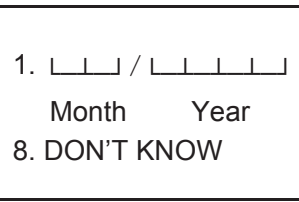 & $\begin{array}{l}\text { 1. Yes } \downarrow \\
\text { 3. No } \\
\text { 8. DK }\end{array}$ & 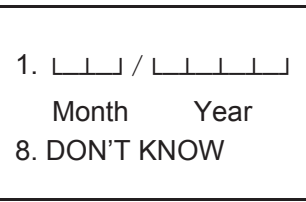 & & & & & \\
\hline 14. & $\begin{array}{l}\text { JSLU/ASLUT ( } \\
\text { Elderly Social } \\
\text { Insurance ) }\end{array}$ & $\begin{array}{l}\text { 1. Yes } \\
\text { 3. No } \downarrow\end{array}$ & 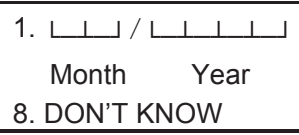 & $\begin{array}{l}\text { 1. Yes } \downarrow \\
\text { 3. No } \\
\text { 8. DK }\end{array}$ & 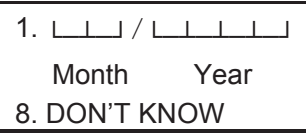 & & & & & \\
\hline 15. & $\begin{array}{l}\text { KUBE/UEP ( Joint } \\
\text { Enterprise } \\
\text { Group/Productive } \\
\text { Economic Enterprise } \\
\text { ) }\end{array}$ & $\begin{array}{l}\text { 1. Yes } \\
\text { 3. No } \downarrow\end{array}$ & 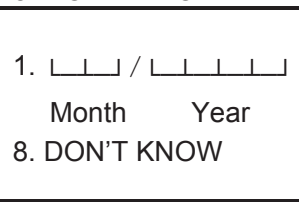 & $\begin{array}{l}\text { 1. Yes } \downarrow \\
\text { 3. No } \\
\text { 8. DK }\end{array}$ & 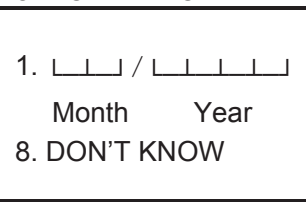 & & & & & \\
\hline 16. & $\begin{array}{l}\text { RTLH ( Renovation } \\
\text { program for home } \\
\text { unfit for } \\
\text { habitation/house } \\
\text { renovation) }\end{array}$ & $\begin{array}{l}\text { 1. Yes } \\
\text { 3. No } \downarrow\end{array}$ & 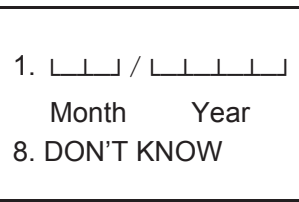 & $\begin{array}{l}\text { 1. Yes } \downarrow \\
\text { 3. No } \\
\text { 8. DK }\end{array}$ & 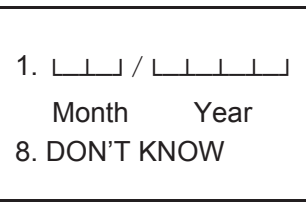 & & & & & \\
\hline 17. & $\begin{array}{l}\text { PKSA ( Children } \\
\text { social welfare } \\
\text { program ) }\end{array}$ & $\begin{array}{l}\text { 1. Yes } \\
\text { 3. No } \downarrow\end{array}$ & 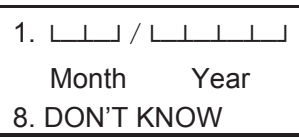 & $\begin{array}{l}\text { 1. Yes } \downarrow \\
\text { 3. No } \\
\text { 8. DK }\end{array}$ & 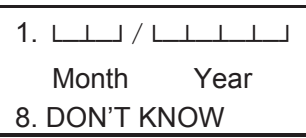 & & & & & \\
\hline 18. & $\begin{array}{l}\text { KPS ( Social Security } \\
\text { Card) }\end{array}$ & $\begin{array}{l}\text { 1. Yes } \\
\text { 3. No } \downarrow\end{array}$ & 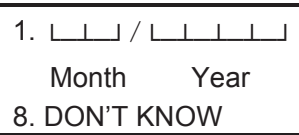 & $\begin{array}{l}\text { 1. Yes } \downarrow \\
\text { 3. No } \\
\text { 8. DK }\end{array}$ & 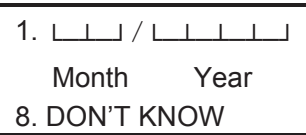 & & & & & \\
\hline
\end{tabular}




\section{SECTION PAP : POVERTY ALLEVIATION PROGRAM}

\begin{tabular}{|c|c|c|}
\hline PAP17. & $\begin{array}{l}\text { Which program received the } \\
\text { most complaints/protests } \\
\text { from the community? }\end{array}$ & $\begin{array}{l}\text { 01. Jamkesda } \\
\text { 02. Jamkesmas } \\
\text { 03. Jampersal } \\
\text { 04. Raskin } \\
\text { 05. Rice Market operation } \\
\text { 06. Others Market Operation } \\
\text { 07. PKPS BBM - SLT (UCT) } \\
\text { 08. Keluarga Harapan (CCT) } \\
\text { 09. PNPM MANDIRI } \\
\text { 10. BLSM } 2013 \\
\text { 11. BSM ( Cash transfer for poor student ) } \\
\text { 12. Askesos ( Social Welfare Insurance ) } \\
\text { 13. PPA-PKH ( Child Labor Reduction-Conditional Cash Transfer } \\
\text { ) } \\
\text { 14. JSPACA/JSODK ( Disabled Social Insurance/Severe- } \\
\text { Disabled Social Insurance) } \\
\text { 15. JSLU/ASLUT ( Elderly Social Insurance ) } \\
\text { 16. KUBE/UEP ( Joint Enterprise Group/Productive Economic } \\
\text { Enterprise ) } \\
\text { 17. RTLH ( Renovation program for home unfit for } \\
\text { habitation/house renovation) } \\
\text { 18. PKSA ( Children social welfare program ) } \\
\text { 19. KPS ( Social Security Card ) } \\
\text { 20. JKN (National Health Insurace) }\end{array}$ \\
\hline PAP18. & $\begin{array}{l}\text { Which of the program was } \\
\text { the most accepted by the } \\
\text { community? }\end{array}$ & $\begin{array}{l}\text { 01. Jamkesda } \\
\text { 02. Jamkesmas } \\
\text { 03. Jampersal } \\
\text { 04. Raskin } \\
\text { 05. Rice Market operation } \\
\text { 06. Others Market Operation } \\
\text { 07. PKPS BBM - SLT (UCT) } \\
\text { 08. Keluarga Harapan (CCT) } \\
\text { 09. PNPM MANDIRI } \\
\text { 10. BLSM } 2013 \\
\text { 11. BSM ( Cash transfer for poor student ) } \\
\text { 12. Askesos ( Social Welfare Insurance ) } \\
\text { 13. PPA-PKH ( Child Labor Reduction-Conditional Cash Transfer } \\
\text { ) } \\
\text { 14. JSPACA/JSODK ( Disabled Social Insurance/Severe- } \\
\text { Disabled Social Insurance ) } \\
\text { 15. JSLU/ASLUT ( Elderly Social Insurance ) } \\
\text { 16. KUBE/UEP ( Joint Enterprise Group/Productive Economic } \\
\text { Enterprise ) } \\
\text { 17. RTLH ( Renovation program for home unfit for } \\
\text { habitation/house renovation) } \\
\text { 18. PKSA ( Children social welfare program ) }\end{array}$ \\
\hline
\end{tabular}

\begin{tabular}{||l|l||}
\hline \multicolumn{1}{||}{} & 19. KPS ( Social Security Card ) \\
20. JKN (National Health Insurace)
\end{tabular}

Now we would like to inquire about the program JAMKESDA

\begin{tabular}{|c|c|c|}
\hline PAP20a & $\begin{array}{l}\text { Was there Regional health insurance } \\
\text { program(jamkesda) at yours Kabupaten? }\end{array}$ & $\begin{array}{l}\text { 3. No } \rightarrow \text { PAP24a } \\
\text { 1. Yes }\end{array}$ \\
\hline PAP20 & $\begin{array}{l}\text { Was there Regional health insurance } \\
\text { program ( jamkesda )in the last } 12 \\
\text { months? }\end{array}$ & $\begin{array}{l}\text { 3. No } \rightarrow \text { PAP24a } \\
\text { 1. Yes }\end{array}$ \\
\hline PAP21 & $\begin{array}{l}\text { From which facilities is someone who has } \\
\text { a Regional health insurance program ( } \\
\text { jamkesda ) able to obtain services? } \\
\text { CIRCLE ALL THAT APPLY }\end{array}$ & $\begin{array}{l}\text { A. Puskesmas } \\
\text { B. Puskesmas Pembantu } \\
\text { C. Village Midwife } \\
\text { D. Private Practice } \\
\text { E. Public Hospital } \\
\text { F. Private Hospital }\end{array}$ \\
\hline PAP23. & $\begin{array}{l}\text { Have you ever heard of any case where a } \\
\text { patient being denied services when using } \\
\text { a Health Card? }\end{array}$ & $\begin{array}{l}\text { 1. Yes } \\
\text { A. Puskesmas } \\
\text { B. Puskesmas Pembantu } \\
\text { C. Village Midwife } \\
\text { D. Private Practice } \\
\text { E. Public Hospital } \\
\text { F. Private Hospital } \\
\text { 3. No } \\
\text { 8. DON'T KNOW }\end{array}$ \\
\hline
\end{tabular}




\section{SECTION PAP: POVERTY ALLEVIATION PROGAM}

Now we would like to inquire about the program Jampersal (Delivery insurance)

\begin{tabular}{|c|c|c|}
\hline PAP24a & $\begin{array}{l}\text { Was there care and childbirth insurance } \\
\text { program ( Jampersal) at yours } \\
\text { kabupaten? }\end{array}$ & 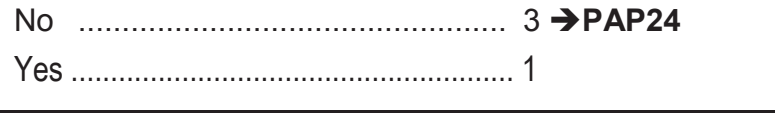 \\
\hline PAP25a & $\begin{array}{l}\text { Was there care and childbirth insurance } \\
\text { program ( Jampersal) in the last } 12 \\
\text { months? }\end{array}$ & 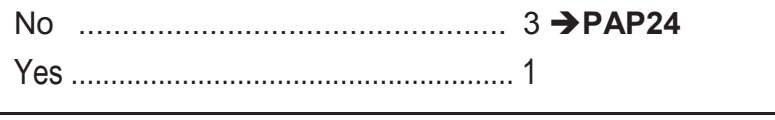 \\
\hline PAP26a & Who is eligible for jampersal? & $\begin{array}{l}\text { A. Pregnant Women } \\
\text { B. Mother Maternity } \\
\text { C. Postpartum women (up to } 42 \text { days postpartum) } \\
\text { D. Newborns (up to age } 28 \text { days) } \\
\text { V. Others }\end{array}$ \\
\hline PAP27a & $\begin{array}{l}\text { From which facilities is someone who } \\
\text { has a jampersal able to obtain services? } \\
\text { CIRCLE ALL THAT APPLY }\end{array}$ & $\begin{array}{l}\text { A. Puskesmas } \\
\text { B. Puskesmas Pembantu } \\
\text { C. Village Midwife } \\
\text { D. Private Practice } \\
\text { E. Public Hospital } \\
\text { A.11F. Private Hospital }\end{array}$ \\
\hline PAP28a & $\begin{array}{l}\text { What kind of treatment obtained } \\
\text { Jampersal cardholder? }\end{array}$ & $\begin{array}{l}\text { A. Pregnancy care } \\
\text { B. maternity services } \\
\text { C. Mother Postpartum Care (up to } 42 \text { days postpartum } \\
\text { D. Newborn Care (up to age } 28 \text { days) } \\
\text { E. Postpartum family planning } \\
\text { V. Others }\end{array}$ \\
\hline PAP29a & $\begin{array}{l}\text { Are there people who are eligible but do } \\
\text { not get Jampersal? }\end{array}$ & 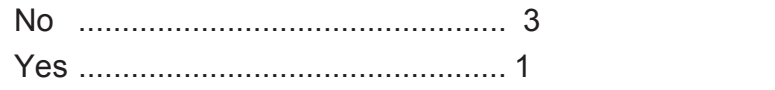 \\
\hline
\end{tabular}

Now we would like to inquire about the JAMKESMAS program (Public Health Insurance)

PAP24. JAMKESMAS program in the last 12 months?

PAP25. From which facilities is someone who has JAMKESMAS able to obtain services?

CIRCLE ALL THAT APPLY
3. No $\rightarrow$ PAP28

1. Yes

A. Puskesmas

B. Puskesmas Pembantu

C. Village Midwife

D. Private Practice

E. Public Hospital

F. Private Hospital

\begin{tabular}{|c|c|c|}
\hline PAP26. & $\begin{array}{l}\text { For how long is the JAMKESMAS } \\
\text { valid? }\end{array}$ & $\begin{array}{l}\text { 1. ـــ months } \\
\text { 6. No expiration } \\
\text { 8. DON'T KNOW }\end{array}$ \\
\hline PAP27. & $\begin{array}{l}\text { Have you ever heard of any case } \\
\text { where a patient being denied services } \\
\text { when using JAMKESMAS? }\end{array}$ & $\begin{array}{l}\text { 1. Yes } \\
\text { A. Puskesmas } \\
\text { B. Puskesmas Pembantu } \\
\text { C. Village Midwife } \\
\text { D. Private Practice } \\
\text { E. Public Hospital } \\
\text { F. Private Hospital } \\
\text { 3. No } \\
\text { 8. DON'T KNOW }\end{array}$ \\
\hline
\end{tabular}

Now we would like to inquire about the JKN program (National Health Insurance)

PAP24b. JKN program in the last 12 months?

3. No $\rightarrow$ PAP28

1. Yes

PAP25b. From which facilities is someone who A. Puskesmas has JKN able to oblain services? CIRCLE ALL THAT APPLY

B. Puskesmas Pembantu

C. Village Midwife

D. Private Practice

E. Public Hospital

F. Private Hospital

PAP26b. For how long is the JKN valid?

1 months

6. No expiration

8. DON'T KNOW

PAP27b. Have you ever heard of any case where a patient being denied services when using JKN?
1. Yes

A. Puskesmas

B. Puskesmas Pembantu

C. Village Midwife

D. Private Practice

E. Public Hospital

F. Private Hospital

3. No

8. DON'T KNOW 


\section{SECTION PAP: POVERTY ALLEVIATION PROGAM}

\section{RASKIN}

\begin{tabular}{|c|c|c|}
\hline PAP28. & $\begin{array}{l}\text { Was there Raskin program in the last } \\
12 \text { months? }\end{array}$ & $\begin{array}{l}\text { 3. NO } \rightarrow \text { PAP56 } \\
\text { 1. YES }\end{array}$ \\
\hline PAP29. & $\begin{array}{l}\text { In the last } 12 \text { months, how many times } \\
\text { has RASKIN rice been distributed in this } \\
\text { village? }\end{array}$ & $\begin{array}{l}\text { 1. }\llcorner\perp \text { times } \\
\text { 8. DON'T KNOW }\end{array}$ \\
\hline PAP30. & $\begin{array}{l}\text { How many tonnes of RASKIN rice were } \\
\text { delivered to this village to be } \\
\text { distributed? }\end{array}$ & $\begin{array}{l}\text { 1. }\llcorner\perp \text { tonnes } \\
\text { 8. DON'T KNOW }\end{array}$ \\
\hline PAP31. & $\begin{array}{l}\text { On average, in the last } 12 \text { months was } \\
\text { the percentage of households that } \\
\text { received RASKIN rice? }\end{array}$ & $\begin{array}{l}\text { 1. } \perp \perp \perp \text { percent } \\
\text { 8. DON'T KNOW }\end{array}$ \\
\hline
\end{tabular}

Now we would like to ask you about the last time RASKIN was distributed in this village in the last 12

months.

\begin{tabular}{|c|c|c|}
\hline PAP32. & $\begin{array}{l}\text { When was the last time RASKIN rice } \\
\text { was distributed in the last } 12 \text { months? }\end{array}$ & 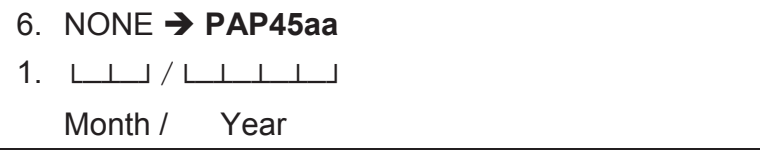 \\
\hline PAP34. & $\begin{array}{l}\text { How many tonnes was delivered to this } \\
\text { village to be distributed? }\end{array}$ & $\begin{array}{l}\text { 1. } \sqcup \sqcup \text { tonnes } \\
\text { 8. DON'T KNOW }\end{array}$ \\
\hline PAP35. & $\begin{array}{l}\text { What was the percentage of } \\
\text { households that received RASKIN } \\
\text { rice? }\end{array}$ & $\begin{array}{l}\text { 1. } \sqcup \perp \perp \text { percent } \\
\text { 8. DON'T KNOW }\end{array}$ \\
\hline PAP36. & $\begin{array}{l}\text { What was the average amount of rice } \\
\text { received by all RASKIN household } \\
\text { recipients? }\end{array}$ & 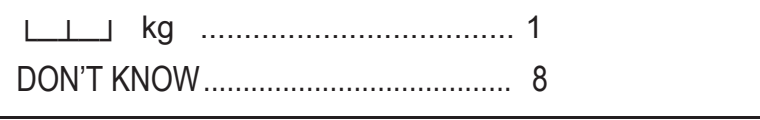 \\
\hline PAP37. & $\begin{array}{l}\text { What was the price of each kilogram of } \\
\text { RASKIN rice (include other costs) paid } \\
\text { by each household? }\end{array}$ & 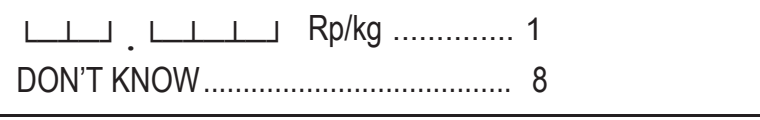 \\
\hline PAP38. & $\begin{array}{l}\text { What was the market price of each } \\
\text { kilogram of the rice in that time? }\end{array}$ & 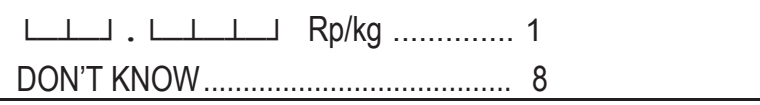 \\
\hline PAP39a & $\begin{array}{l}\text { How to decide the household to receive } \\
\text { RASKIN? }\end{array}$ & $\begin{array}{l}\text { 1. Based on list name which is decided by } \\
\text { government (DPM) } \rightarrow \text { PAP39 } \\
\text { 2. Based on list name from government (DPM) and } \\
\text { modified } \\
\text { 3. Do not use DPM }\end{array}$ \\
\hline
\end{tabular}

\begin{tabular}{|c|c|c|}
\hline PAP39b & $\begin{array}{l}\text { Based on what decision for the } \\
\text { receiver of RASKIN? }\end{array}$ & $\begin{array}{l}\text { A. Floor type of low quality (e.g ground, no tiles) } \\
\text { B. Wall of low quality (e.g bamboo, boards) } \\
\text { C. No toilet/latrines for defecation } \\
\text { D. Source of drinking water not piped from PAM } \\
\text { E. Source of lighting is not electricity } \\
\text { F. Use wood/charcoal for cooking fuel } \\
\text { G. Head of household didn't go to school/finish } \\
\text { elementary school } \\
\text { H. Elderly } \\
\text { I. Widowed } \\
\text { J. Widow } \\
\text { K. Don't have permanent job } \\
\text { L. Considered as poor by community } \\
\text { V. Other }\end{array}$ \\
\hline PAP39. & $\begin{array}{l}\text { Did all households that received } \\
\text { RASKIN rice received equal amount? }\end{array}$ & 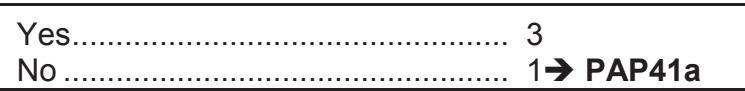 \\
\hline PAP40. & $\begin{array}{l}\text { How did you determine the amount of } \\
\text { rice that a household can buy/receive? }\end{array}$ & $\begin{array}{l}\text { A. Based on the number of household members } \\
\text { B. Based on the poverty level of the household } \\
\text { C. Based on the number of children } \\
\text { V. Other } \\
\rightarrow \text { PAP41 }\end{array}$ \\
\hline PAP41a & $\begin{array}{l}\text { If yes how the system is distributed } \\
\text { equally? }\end{array}$ & $\begin{array}{l}\text { A. Distributed equally among the poor households } \\
\text { B. Distributed equally between rich and poor } \\
\text { C. Distributed equally to rich and poor but in shifts } \\
\text { D. Given to the poor, if can not buy sale to the rich. } \\
\rightarrow \text { PAP45aa }\end{array}$ \\
\hline PAP45aa & $\begin{array}{l}\text { How the quality of the rice purchased } \\
\text { from the Raskin program? }\end{array}$ & 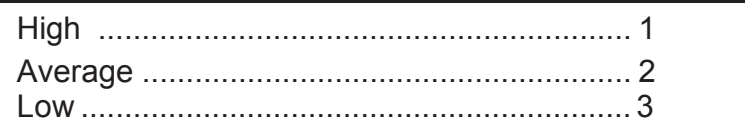 \\
\hline
\end{tabular}




\section{SECTION PAP: POVERTY ALLEVIATION PROGAM}

\section{Now we would like to ask about the BLSM.}

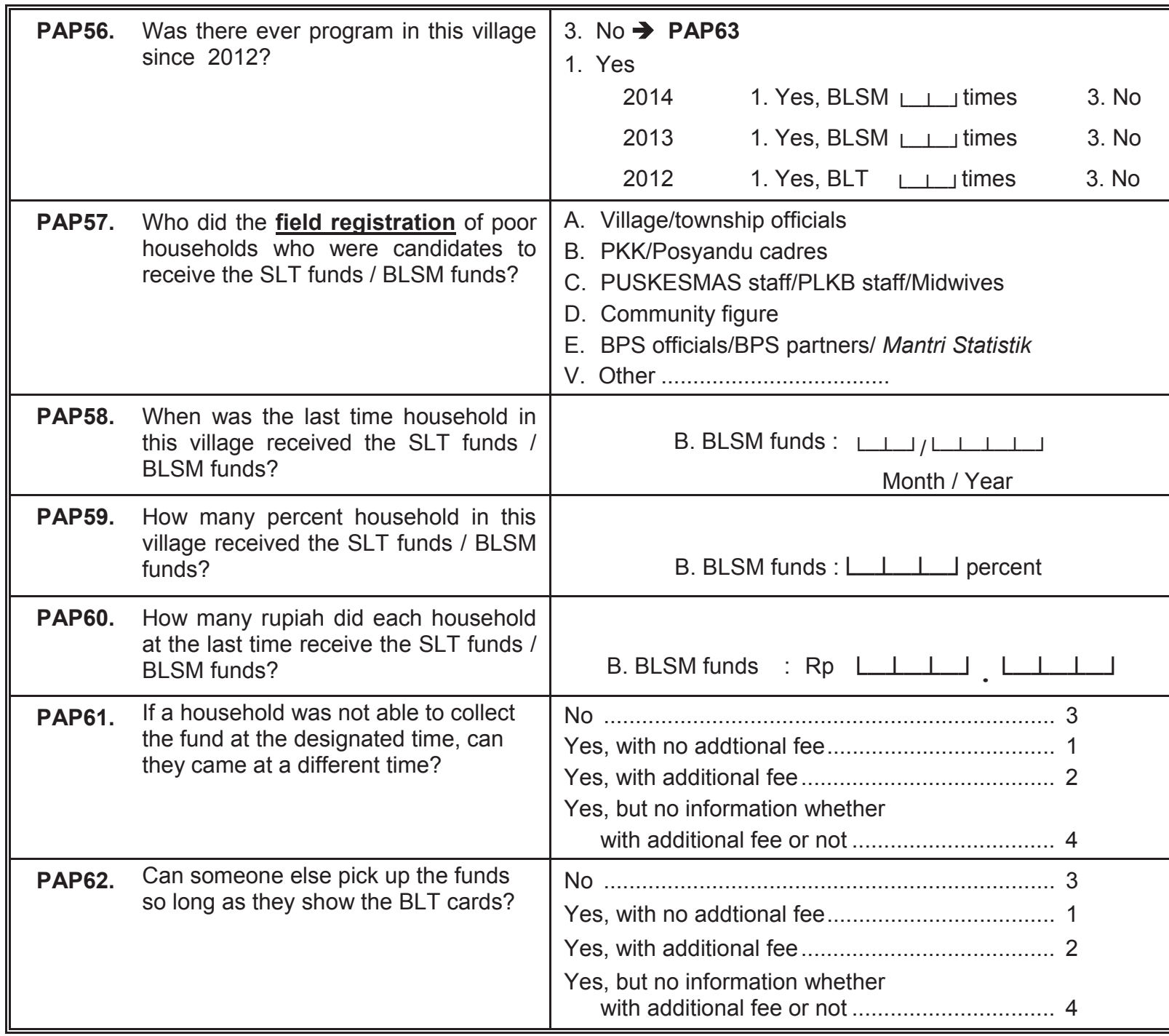

Now we would like to ask about the Keluarga Harapan (Conditional Cash Transfer Program).

\begin{tabular}{|c|c|c|}
\hline PAP63. & $\begin{array}{l}\text { Has the Keluarga Harapan Program } \\
\text { started in this village/township? }\end{array}$ & $\begin{array}{l}\text { 3. No } \rightarrow \text { PAP65 } \\
\text { 1. Yes, it has } \\
\text { 2. Yes, it will }\end{array}$ \\
\hline
\end{tabular}

\begin{tabular}{||ll|l||}
\hline PAP64a & $\begin{array}{l}\text { Is Keluarga Harapan Programme } \\
\text { implemented in the last 12 months? }\end{array}$ & $\begin{array}{l}\text { 3. No } \rightarrow \text { PAP65a } \\
\text { 1. Yes }\end{array}$ \\
\hline PAP64b & $\begin{array}{l}\text { Are there a family who should receive } \\
\text { assistance PKH but did not get? }\end{array}$ & $\begin{array}{l}\text { 3. No } \\
\text { 1. Yes }\end{array}$ \\
\hline
\end{tabular}

\begin{tabular}{|c|c|c|}
\hline PAP65a. & $\begin{array}{l}\text { Whether in the last } 12 \text { months there } \\
\text { PNPM Mandiri program? }\end{array}$ & $\begin{array}{l}\text { No } \\
\text { Yes }\end{array}$ \\
\hline PAP66a. & $\begin{array}{l}\text { What PNPM Mandiri program exist in } \\
\text { this vilage? }\end{array}$ & $\begin{array}{l}\text { A. PNPM Urban } \\
\text { B. PNPM Rural } \\
\text { C. PNPM PISEW } \\
\text { D. PNPM PUAP } \\
\text { E. PNPM Poor and Disadvantaged Areas } \\
\text { F. PNPM Generation } \\
\text { G. PNPM Mandiri Respect }\end{array}$ \\
\hline PAP67a. & $\begin{array}{l}\text { Who are the beneficiaries of PNPM } \\
\text { Mandiri program? }\end{array}$ & $\begin{array}{l}\text { A. Very poor households } \\
\text { B. Poor households } \\
\text { C. Non-poor households } \\
\text { D. Female-headed households } \\
\text { E. woman } \\
\text { F. schoolchild } \\
\text { G. Residents in nearby activities } \\
\text { V. Other: } \\
\text { W. All people in the village } \\
\text { Y. DO NOT KNOW }\end{array}$ \\
\hline PAP68a. & $\begin{array}{l}\text { Do you think the PNPM activities was } \\
\text { appropriate with the needs of the } \\
\text { people in this village? }\end{array}$ & 1. Yes 3 3. No $\rightarrow$ PAP70a \\
\hline PAP69a. & $\begin{array}{l}\text { Why PNPM Mandiri activities in } \\
\text { accordance with the people needs in } \\
\text { this village? }\end{array}$ & $\begin{array}{l}\text { A. Opening up economic opportunities } \\
\text { B. easier access } \\
\text { C. The main of requirement } \\
\text { D. Help reduce poverty } \\
\text { V. Others, } \\
\text { Y. DO NOT KNOW } \quad \rightarrow \text { PAP71a }\end{array}$ \\
\hline
\end{tabular}




\section{SECTION PAP: POVERTY ALLEVIATION PROGAM}

PAP70a. Why the PNPM Mandiri program does $A$. Not the main needs

not appropriate with the needs of the

B. Can not be enjoyed by communities

C. Already there are other similar programs

D. Can only be enjoyed by certain people

E. Must pay to enjoy the facilities

V. Others

Y. DO NOT KNOW

PAP78. Who conducted listing for household

beneficiaries of KIS program ?

(CIRCLE ALL THAT APPLY
A. Village/kelurhan officials

B. Kader PKK/Posyandu

C. PUSKESMAS Official/PLKB /Midwife

D. Community figure

E. BPS Official /Mitra BPS/Mantri Statistik

F. DPM from Government

V. Other
Now we would like to inquire about KPS ( Social Security Card)

\begin{tabular}{|c|c|c|}
\hline PAP71a. & $\begin{array}{l}\text { Was there KPS ( Social Security } \\
\text { Card ) program in this village? }\end{array}$ & $\begin{array}{l}\text { No } \\
\text { Yes }\end{array}$ \\
\hline PAP72a. & $\begin{array}{l}\text { When was the first time the program } \\
\text { begin? }\end{array}$ & 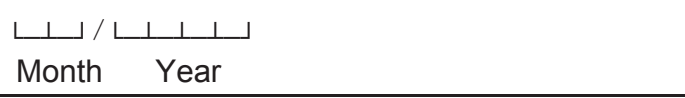 \\
\hline PAP73a. & For how long is the KPS valid? & $\begin{array}{l}\text { 1. ــ ـ months } \\
\text { 6. No expiration } \\
\text { 8. DON'T KNOW }\end{array}$ \\
\hline PAP74a. & $\begin{array}{l}\text { What assistance programs are the } \\
\text { social security card used for? }\end{array}$ & $\begin{array}{l}\text { A. RASKIN } \\
\text { B. BLSM } \\
\text { C. BSM ( Bantuan Siswa Miskin ) } \\
\text { D. PKH ( Program Keluarga Harapan ) } \\
\text { V. Others }\end{array}$ \\
\hline
\end{tabular}

Now i would like to ask about KIS (Kartu Indonesia Sehat) Program

\begin{tabular}{|c|c|c|}
\hline PAP75. & $\begin{array}{l}\text { Do you have KIS ( Kartu Indonesia Sehat ) } \\
\text { program in this village/kelurahan? }\end{array}$ & $\begin{array}{l}\text { No } 1 \ldots 1.1 \\
\text { Yes }\end{array}$ \\
\hline PAP76. & $\begin{array}{l}\text { When was }[\ldots] \text { first initiated in this } \\
\text { village/kelurahan? }\end{array}$ & 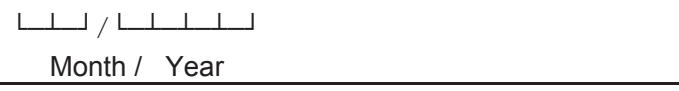 \\
\hline PAP77. & Who benefit from KIS program? & $\begin{array}{l}\text { A. Very poor household } \\
\text { B. Poor household } \\
\text { C. Non poor household } \\
\text { D. Homeless } \\
\text { E. Disable } \\
\text { V. Other: }\end{array}$ \\
\hline
\end{tabular}

Now i would like to ask about KIP (Kartu Indonesia Pintar)

\begin{tabular}{|c|c|c|}
\hline PAP79. & $\begin{array}{l}\text { Do you have KIP ( Kartu } \\
\text { Indonesia Pintar ) program in this } \\
\text { village/kelurahan? }\end{array}$ & 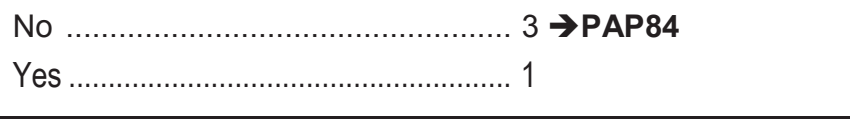 \\
\hline PAP80. & $\begin{array}{l}\text { When was }[\ldots] \text { first initiated in this } \\
\text { village/kelurahan? }\end{array}$ & 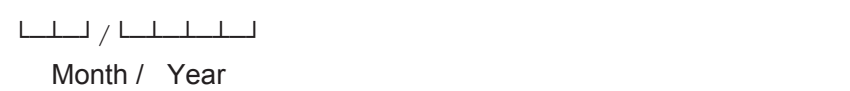 \\
\hline PAP81. & Who benefit from KIP program? & $\begin{array}{ll}\text { A. } & \text { Very poor household } \\
\text { B. Poor household } \\
\text { C. Non poor household } \\
\text { D. Homeless } \\
\text { E. Disable } \\
\text { V. Other: } \\
\text { Y. DON'T KNOW }\end{array}$ \\
\hline PAP82. & $\begin{array}{l}\text { Who conducted listing for } \\
\text { household beneficiaries of KIP } \\
\text { program? } \\
\text { (CIRCLE ALL THAT APPLY) }\end{array}$ & 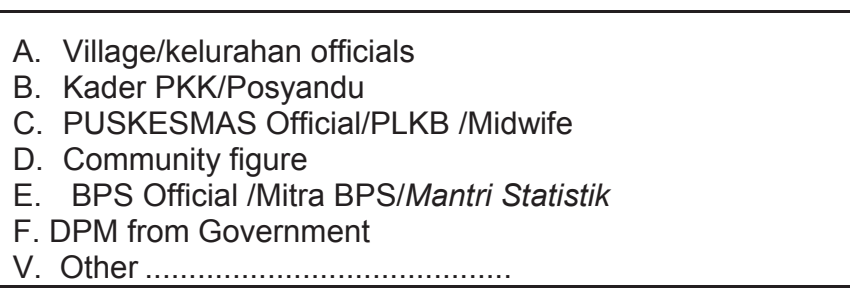 \\
\hline PAP83. & How much amount was given? & \\
\hline & 1.SD & 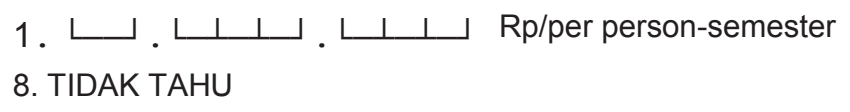 \\
\hline & 2. SMP & 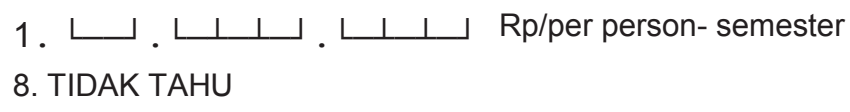 \\
\hline & 3. SMA & 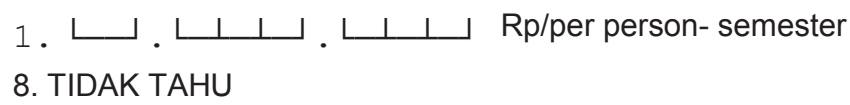 \\
\hline
\end{tabular}

COMFAS 2014 


\section{SECTION PAP: POVERTY ALLEVIATION PROGAM}

Now I would like to know about KKS ( Kartu Keluarga Sejahtera ) Program

\begin{tabular}{|c|c|c|}
\hline PAP84. & $\begin{array}{l}\text { Do you have KKS ( Kartu Keluarga } \\
\text { Sejahtera ) program in this } \\
\text { village/kelurahan? }\end{array}$ & $\begin{array}{l}\text { No } \\
\text { Yes }\end{array}$ \\
\hline PAP85. & $\begin{array}{l}\text { When was }[\ldots] \text { first initiated in this } \\
\text { village/kelurahan? }\end{array}$ & 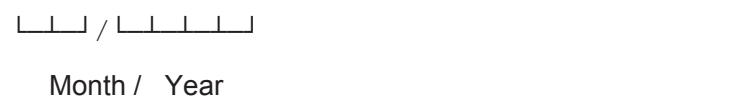 \\
\hline PAP86. & Who benefit from KKS program? & $\begin{array}{l}\text { A. Very poor household } \\
\text { B. Poor household } \\
\text { C. Non poor household } \\
\text { D. Homeless } \\
\text { E. Disable } \\
\text { V. Other: } \\
\text { Y. DON'T KNOW }\end{array}$ \\
\hline PAP87. & $\begin{array}{l}\text { Who conducted listing for } \\
\text { household beneficiaries of KKS } \\
\text { program? } \\
\text { (CIRCLE ALL THAT APPLY) }\end{array}$ & $\begin{array}{l}\text { A. Village/kelurahan officials } \\
\text { B. Kader PKK/Posyandu } \\
\text { C. PUSKESMAS Official/PLKB /Midwife } \\
\text { D. Community figure } \\
\text { E. BPS Official /Mitra BPS/Mantri Statistik } \\
\text { F. DPM from Government } \\
\text { V. Other................................................ }\end{array}$ \\
\hline PAP88 & How much amount is given? & 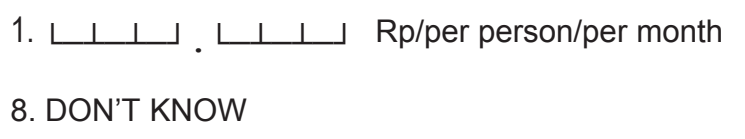 \\
\hline
\end{tabular}




\section{SECTION PPS: PERCEPTION ON PUBLIC INFRASTRUCTURE AND SERVICES}

We would like to ask you about the public infrastructure and facilities in this village/township.

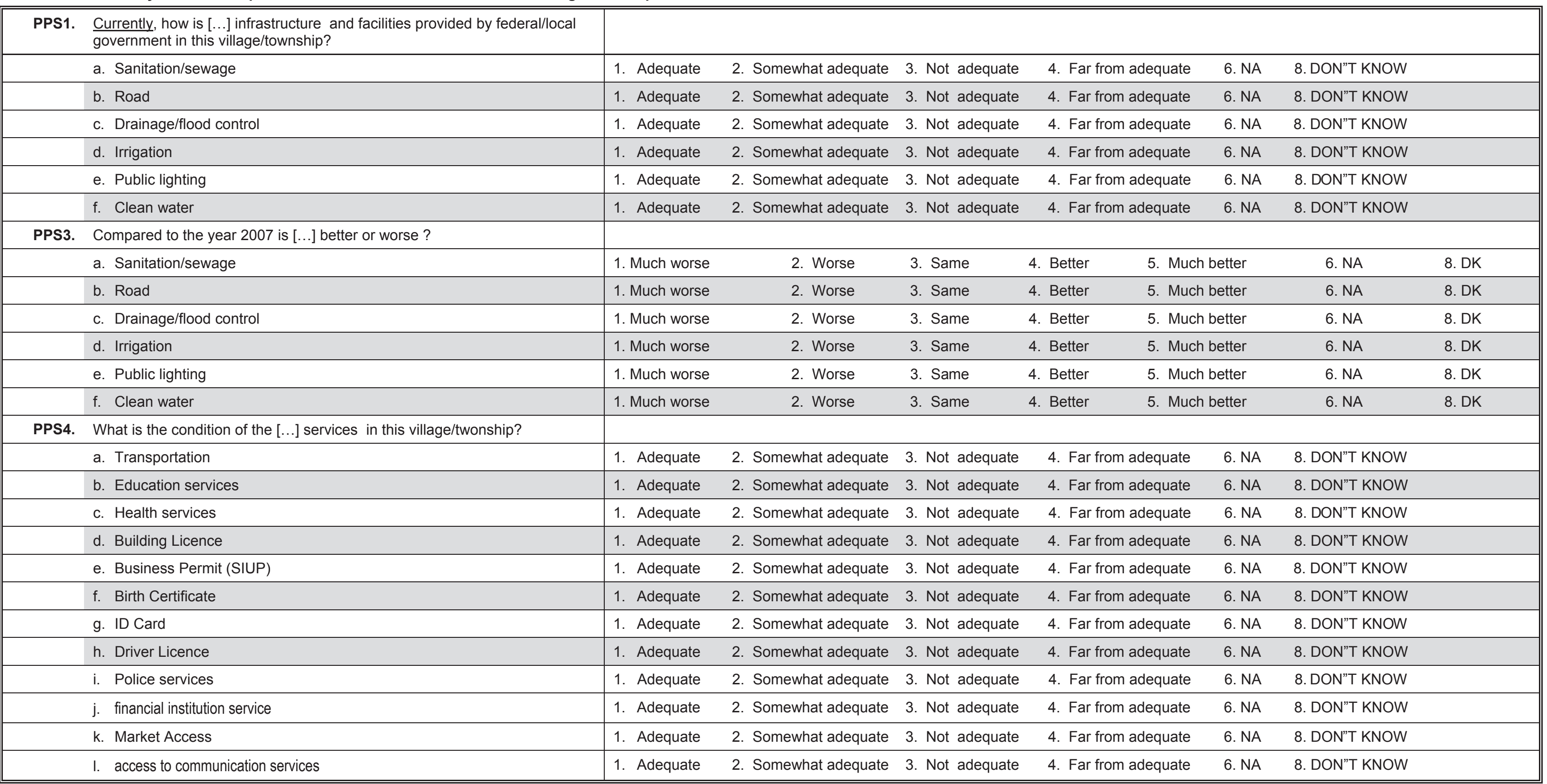




\section{SECTION PPS: PERCEPTION ON PUBLIC INFRASTRUCTURE AND SERVICES}

\begin{tabular}{|c|c|c|c|c|c|c|c|c|}
\hline PPS10. & Compared to the year 2007 is services[...] better or worse ? & & & & & & & \\
\hline & a. Transportation & 1. Much worse & 2. Worse & 3. Same & 4. Better & 5. Much better & 6. NA & 8. DK \\
\hline & b. Education services & 1. Much worse & 2. Worse & 3. Same & 4. Better & 5. Much better & 6. NA & 8. DK \\
\hline & c. Health services & 1. Much worse & 2. Worse & 3. Same & 4. Better & 5. Much better & 6. NA & 8. DK \\
\hline & d. Building Licence & 1. Much worse & 2. Worse & 3. Same & 4. Better & 5. Much better & 6. NA & 8. DK \\
\hline & e. Business Permit (SIUP) & 1. Much worse & 2. Worse & 3. Same & 4. Better & 5. Much better & 6. NA & 8. DK \\
\hline & f. Birth Certificate & 1. Much worse & 2. Worse & 3. Same & 4. Better & 5. Much better & 6. NA & 8. DK \\
\hline & g. ID Card & 1. Much worse & 2. Worse & 3. Same & 4. Better & 5. Much better & 6. NA & 8. DK \\
\hline & h. Driver Licence & 1. Much worse & 2. Worse & 3. Same & 4. Better & 5. Much better & 6. NA & 8. DK \\
\hline & i. Police services & 1. Much worse & 2. Worse & 3. Same & 4. Better & 5. Much better & 6. NA & 8. DK \\
\hline & j. financial institution service & 1. Much worse & 2. Worse & 3. Same & 4. Better & 5. Much better & 6. NA & 8. DK \\
\hline & k. Market Access & 1. Much worse & 2. Worse & 3. Same & 4. Better & 5. Much better & 6. NA & 8. DK \\
\hline & I. access to communication services & 1. Much worse & 2. Worse & 3. Same & 4. Better & 5. Much better & 6. NA & 8. DK \\
\hline
\end{tabular}




\section{SECTION GD: GOVERNANCE AND DECENTRALIZATION}

Now, we would like to ask your opinion on regional autonomy.

GD05. According to your opinion, how is the governance in this village/township?

1. Very good

2. Good

3. Bad

4. Very bad

GD06. According to your opinion, comparing to 2007 , how is the governance in this village/township?

1. Much better

2. Better

3. Worst

4. Much worst

AD07. According to your opinion, how is the govenance in this kabupathota?

1. Very good

2. Good

3. Bad

GD08. According to your opinion, comparing to 2007 , how is the governance in this kabupaten/kota?

4. Very bad

GD08. According to your opinion, comparing to 2007, how is the governance in this kabupaten/kota?

GD09. According to your opinion, are there any cases of corruption, collusion, and nepotism (KKN) in [...] now?

$$
\begin{aligned}
& \text { 2. Better } \\
& \text { 3. Worst } \\
& \text { 4. Much worst }
\end{aligned}
$$

$$
[\ldots] \text { now? }
$$

\begin{tabular}{|l}
\hline a. Village government office \\
b. Kecamatan office \\
c. Kabupaten/Kota government office \\
d. Local Parliament (DPRD) of Kabupaten/Kota \\
e. District Police Headquarter \\
f. Hospital / Puskesmas / Pustu \\
g. State Schools
\end{tabular}

GD10. Since 2007, how has the incidence of corruption, collusion, and nepotism (KKN) in [...] changed?

a. Village government office
b. Kecamatan office
c. Kabupaten/Kota government office
d. Local Parliament (DPRD) of Kabupaten/Kota
e. District Police Headquarter
f. Hospital / Puskesmas / Pustu
g. State Schools

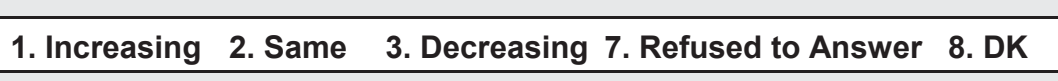

\begin{tabular}{lllll||}
1 & 2 & 3 & 7 & 8 \\
1 & 2 & 3 & 7 & 8 \\
1 & 2 & 3 & 7 & 8 \\
1 & 2 & 3 & 7 & 8 \\
1 & 2 & 3 & 7 & 8 \\
1 & 2 & 3 & 7 & 8 \\
1 & 2 & 3 & 7 & 8 \\
\hline
\end{tabular}




\section{SECTION GD: GOVERNANCE AND DECENTRALIZATION}

\begin{tabular}{|c|c|c|}
\hline GD11. & What kind of decision making process was used for the village head election? & Consensus among all villagers \\
\hline GD12. & Who is most often involved in resolving conflict between relatives or conflict between citizens? & Community leader \\
\hline GD13. & What arethe qualifications that make someone a leader in the community? & $\begin{array}{l}\text { Knowledge and expertise } \\
\text { Voting } \\
\text { Wealth } \\
\text { Social spirit } \\
\text { Inherited } \\
\text { Character/leadership/charisma }\end{array}$ \\
\hline
\end{tabular}




\section{SECTION TR: TRUST}

CONFLICTS

Now we want to ask you about conflicts that have occurred in this village/township since 2007.

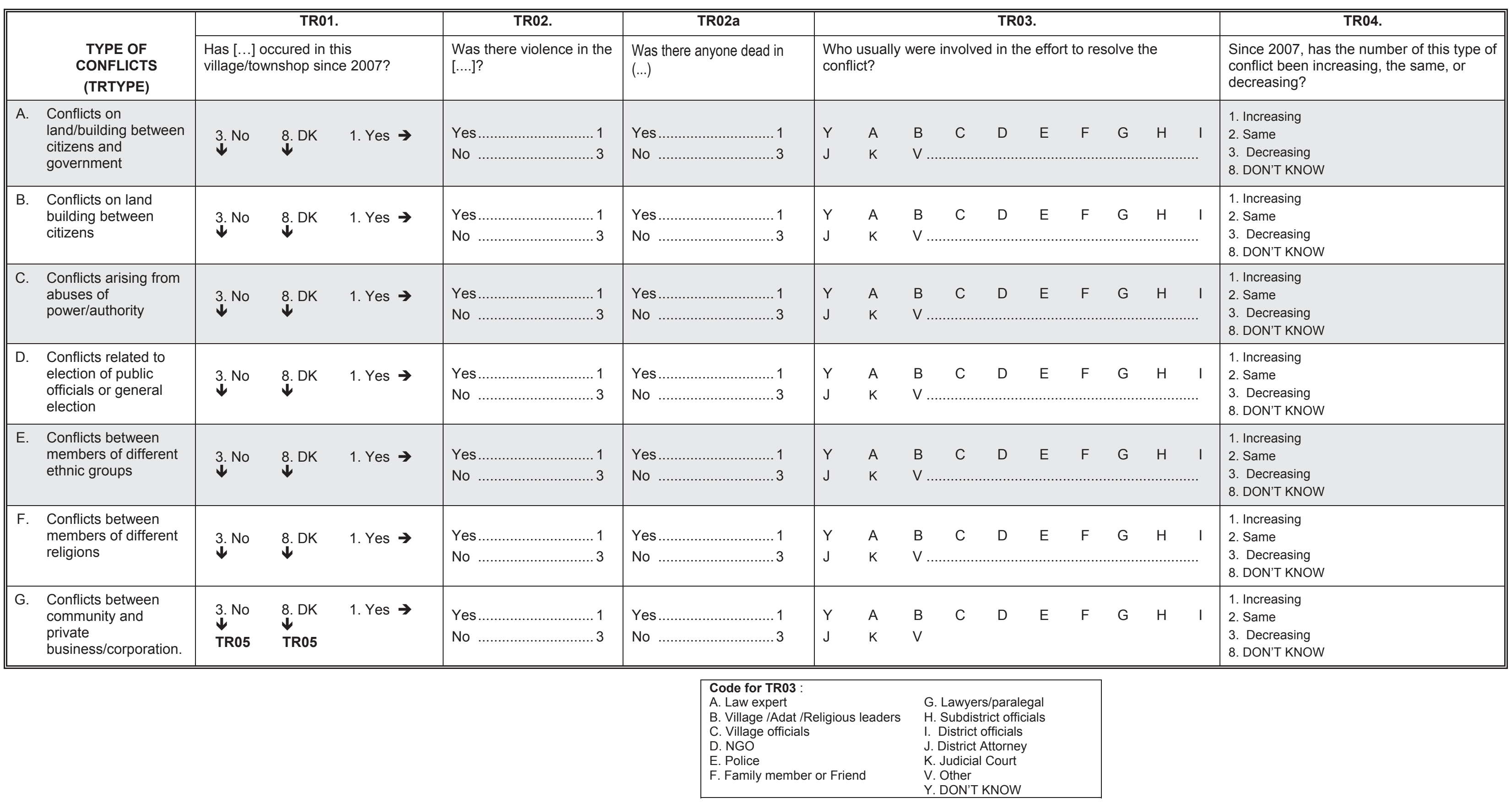




\section{SECTION TR: TRUST}

Now we want to ask you about crime in this village.

\begin{tabular}{|c|c|c|}
\hline TR05. & How safe do you consider this village? & 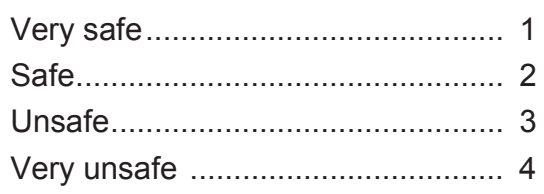 \\
\hline TR06. & $\begin{array}{l}\text { Compared to } 2007 \text {, how safe do you } \\
\text { consider this village is? }\end{array}$ & 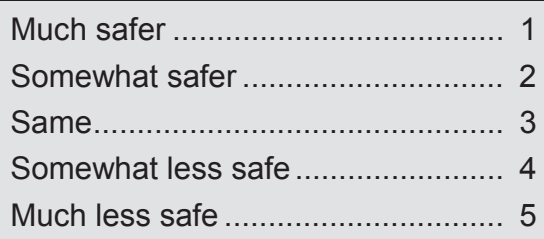 \\
\hline TR07. & $\begin{array}{l}\text { In most part of the village, is it safe to walk } \\
\text { at night? }\end{array}$ & $\begin{array}{l}\text { Very safe } \\
\text { Safe } \\
\text { Unsafe } \\
\text { Very unsafe }\end{array}$ \\
\hline TR07a & $\begin{array}{l}\text { In most part of the village, is it safe for a } \\
\text { woman to walk at night? }\end{array}$ & 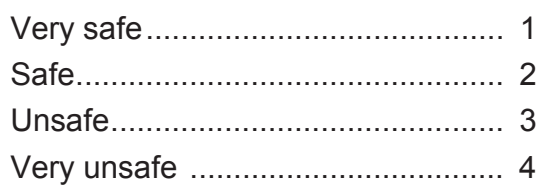 \\
\hline TR08. & $\begin{array}{l}\text { In the last } 12 \text { months has any household } \\
\text { in this village been a victim of theft? }\end{array}$ & Yes \\
\hline TR09. & $\begin{array}{l}\text { In the last } 12 \text { months has any household } \\
\text { in this village been a victim of } \\
\text { crop/livestock theft? }\end{array}$ & 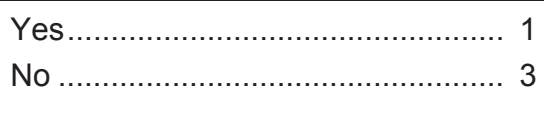 \\
\hline TR10. & $\begin{array}{l}\text { In the last } 12 \text { months has any household } \\
\text { in this village been a victim of robbery? }\end{array}$ & Yes \\
\hline TR11. & $\begin{array}{l}\text { In the last } 12 \text { months, has there been any } \\
\text { incident of sexual assaults? }\end{array}$ & 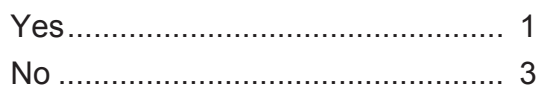 \\
\hline TR12. & $\begin{array}{l}\text { In the last } 12 \text { months, has there been any } \\
\text { incident of domestic assaults? }\end{array}$ & 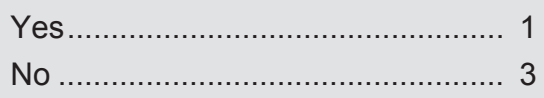 \\
\hline TR13. & $\begin{array}{l}\text { In the last } 12 \text { months, has there been any } \\
\text { other violences/assaults, include civil } \\
\text { strife? }\end{array}$ & 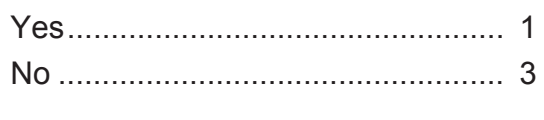 \\
\hline
\end{tabular}

TRUST

Now we want to ask you about the sense of trust within this community.

\begin{tabular}{|c|c|c|}
\hline TR14. & $\begin{array}{l}\text { People in this village are always looking } \\
\text { out for each other }\end{array}$ & 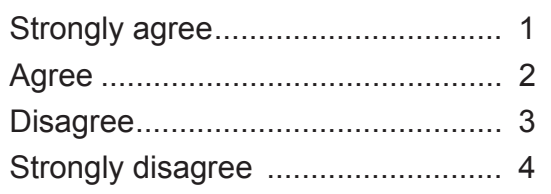 \\
\hline TR15. & $\begin{array}{l}\text { Most people in the village are willing to } \\
\text { help if you need it. }\end{array}$ & 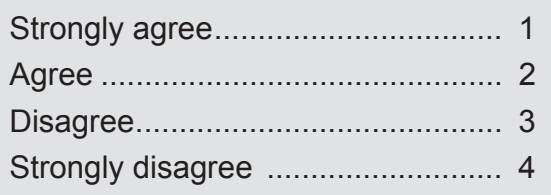 \\
\hline TR16. & $\begin{array}{l}\text { In this village one has to be alert or } \\
\text { someone is likely to take advantage of } \\
\text { you. }\end{array}$ & 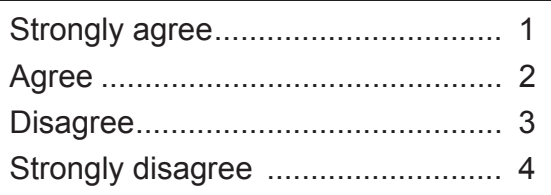 \\
\hline TR17. & $\begin{array}{l}\text { In this village, residents from the same } \\
\text { ethnicity trust each other more than they } \\
\text { trust those with different ethnicity. }\end{array}$ & 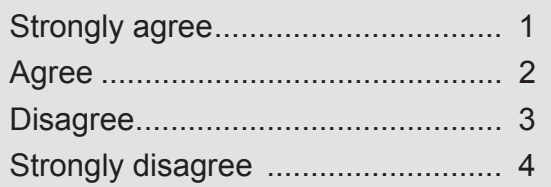 \\
\hline TR18. & $\begin{array}{l}\text { In this village, residents from the same } \\
\text { religion trust each ither more than they } \\
\text { trust those with different religion. }\end{array}$ & 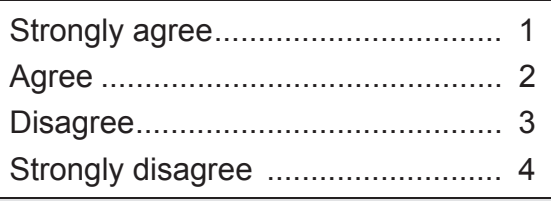 \\
\hline TR19. & $\begin{array}{l}\text { Would people in this village be willing to } \\
\text { leave their children with their neighbors } \\
\text { for a few hours if they cannot bring their } \\
\text { children with them? }\end{array}$ & 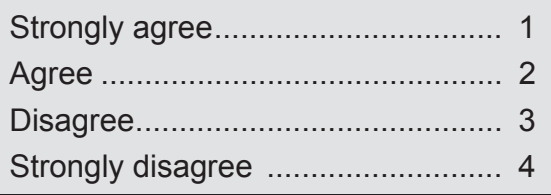 \\
\hline TR20. & $\begin{array}{l}\text { Would people in this village be willing to } \\
\text { ask their neighbors to look after their } \\
\text { house if they leave for a few days? }\end{array}$ & 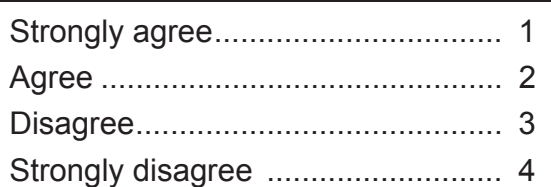 \\
\hline
\end{tabular}




\section{SECTION CP: INTERVIEWER NOTES}

\begin{tabular}{|c|c|c|c|c|c|c|c|}
\hline LANGMAIN (CK1). & \multicolumn{2}{|l|}{ Interview was entirely/mostly conducted in what language? } & \multicolumn{5}{|l|}{ ـــ other } \\
\hline LANGOTHR (CK2). & Other languaged used (if any): & & \multicolumn{5}{|l|}{ 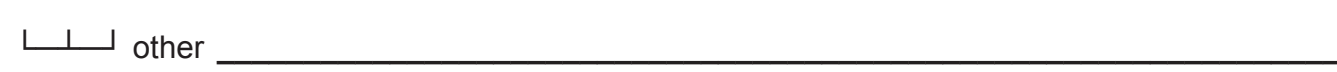 } \\
\hline \multicolumn{8}{|l|}{ LANGUAGE CODE: } \\
\hline 00. Bahasa Indonesia & 04. Batak & Sasak & 12. Makassar & 16. & Toraja & & Lampung \\
\hline 01. Java & 05. Bugis & 09. Minang & 13. Nias & 17. & Lahat & 95. & Other, \\
\hline 02. Sunda & 06. Cina & Banjar & 14. Palembang & 18. & Other South Sumatera & 96. & NA \\
\hline 03. Bali & 07. Madura & 11. Bima & 15. Sumbawa & 19. & Betawi & & \\
\hline
\end{tabular}

\begin{tabular}{|c|c|c|c|}
\hline RESULT (FP3). RESULTS OF INTERVIEW & REASON (FP4). REASON FOR ANSWERING “2” I "3" IN RESULT. & FP6. MONITORING BY SUPERVISOR & \\
\hline $\begin{array}{l}\text { 1. Completed } \rightarrow \text { FP6 } \\
\text { 2. Partly completed } \\
\text { 3. Not completed } \\
\text { 4. Twin EA with EA } \downarrow \perp \text { LPP6 }\end{array}$ & $\begin{array}{l}\text { 1. Respondent was travelling/not in location } \\
\text { 2. Respondent was too busy } \\
\text { 3. Respondent refused }\end{array}$ & $\begin{array}{ll}\text { a. } & \text { Observed (sup_obs) .......... } 1 \\
\text { b. } & \text { Checked (sup_edit)........... } 1 \\
\text { c. } & \text { Verified (sup_veri).............. } 1\end{array}$ & $\begin{array}{l}\text { No } \\
3 \\
3 \\
3\end{array}$ \\
\hline
\end{tabular}

\section{INTERVIEWER NOTE:}




\begin{tabular}{|c|c|c|c|}
\hline $\begin{array}{l}\text { INTERVIEWER: } \\
\text { EDITOR: }\end{array}$ & 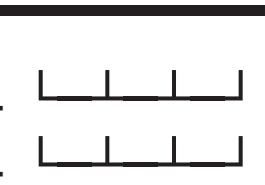 & CONFIDENTIAL & IDW: $\downarrow$ \\
\hline
\end{tabular}

\section{INDONESIAN FAMILY LIFE SURVEY 2014 HEALTH FACILITY COMMUNITY HEALTH CENTER/SUB-CENTER (PUSKESMAS / PUSKESMAS PEMBANTU) \\ BOOK A}

SECTIONS : LK, A, SDP, AKM, DM, CP

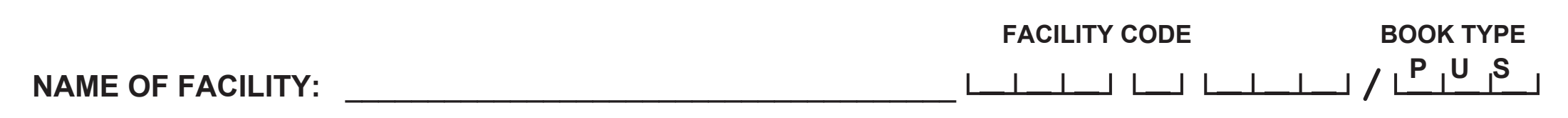




\section{SECTION LK: CONTROL SHEET}

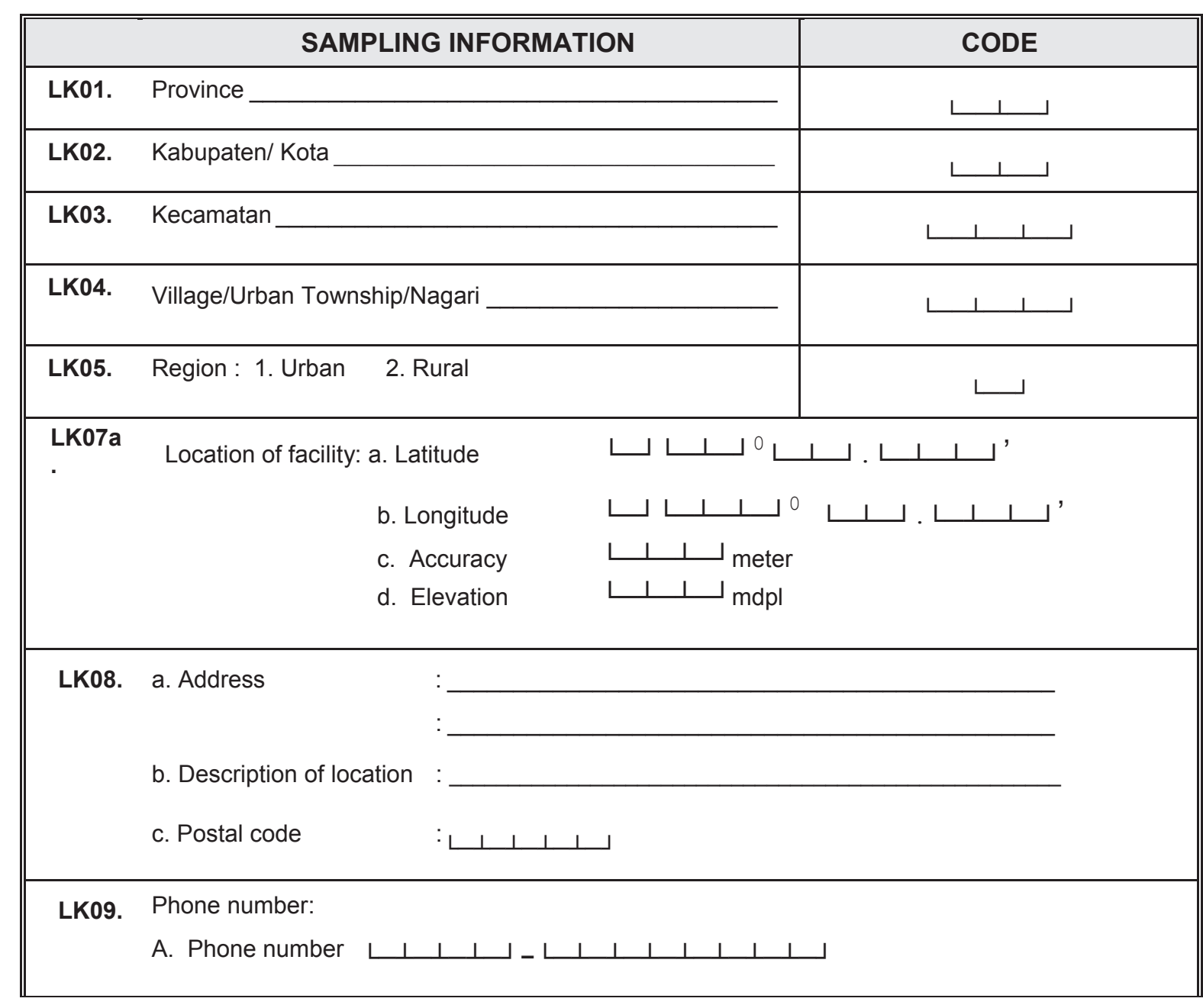

\begin{tabular}{|c|c|c|}
\hline & \multicolumn{2}{|c|}{ 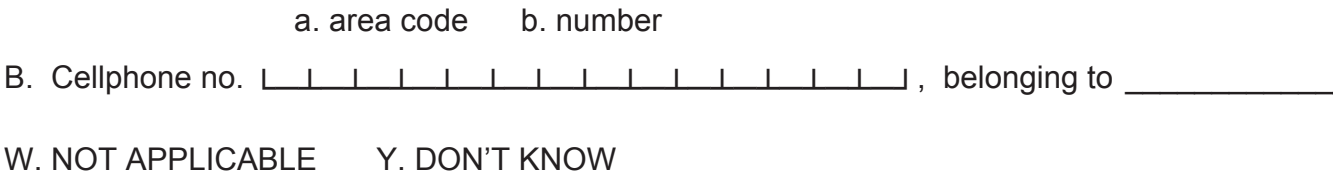 } \\
\hline LK09a. & Email: & \\
\hline LK11. & $\begin{array}{ll}\text { Facility: } & \text { 1. Puskesmas } \\
& \text { 2. PuskesmasPembantu }\end{array}$ & $ـ$ \\
\hline LK12. & Number of villages/townships covered by this facility & $\downarrow ـ$ _ـ villages/townships \\
\hline
\end{tabular}

\begin{tabular}{||c|c|}
\hline \hline \multicolumn{1}{|c|}{ SUPERVISION } & CODE \\
\hline LK15. Name of Interviewer & \\
\hline LK17. Name of Local Supervisor & \\
\hline LK19. Name of Field Coordinator & \\
\hline
\end{tabular}

\begin{tabular}{||ll|l||}
\hline \hline LK20 & Was this Puskesmas/Puskesmas Pembantu interviewed in 1993? & $\begin{array}{r}\text { Yes.............. } 1 \\
\text { No .............. } 3\end{array}$ \\
\hline LK21 & Was this Puskesmas/Puskesmas Pembantu interviewed in 1997? & $\begin{array}{r}\text { Yes.............. } 1 \\
\text { No .............. } 3\end{array}$ \\
\hline LK22 & Was this Puskesmas/Puskesmas Pembantu inteviewed in 1998? & $\begin{array}{r}\text { Yes.............. } 1 \\
\text { No ............... } 3\end{array}$ \\
\hline LK23 & Was this Puskesmas/Puskesmas Pembantu inteviewed in 2000? & $\begin{array}{r}\text { Yes............... } 1 \\
\text { No ............. } 3\end{array}$ \\
\hline LK24 & Was this Puskesmas/Puskesmas Pembantu inteviewed in 2007? & Yes............. 1 \\
No ............. 3
\end{tabular}




\section{SECTION A: HEAD OF PUSKESMAS/PUSKESMAS PEMBANTU}

A26. In this study, we would like to gather some information about this facility. Please give us the names of people, we can ask questions to about the following topics:

\begin{tabular}{|c|c|c|}
\hline SECTIONS & TOPICS: & Name of Potential Respondent \\
\hline A. & Head of Puskesmas / PuskesmasPembantu & \\
\hline B. & Development of Puskesmas / PuskesmasPembantu & \\
\hline C. & Acitivities of Puskesmas / PuskesmasPembantu & \\
\hline D. & Puskesmas/ Pustu Employees & \\
\hline E. & Health Instruments & \\
\hline SDP. & Resources of Puskesmas & \\
\hline AKM. & Program of Askeskin/JPKMM (Health Insurance for the Poor) & \\
\hline DM. & Decision making & \\
\hline F. & Direct observation & \\
\hline G. & Family Planning services & \\
\hline
\end{tabular}




\begin{tabular}{|c|c|c|}
\hline Aa. & Name of head of the Puskesmas/Pustu & 1. Head of Puskesmas 3. Official Head of Puskesmas \\
\hline Aa1. & Sex & 3. Female \\
\hline Ab. & Profession of the Facility Head & Doctor \\
\hline A1a. & $\begin{array}{l}\text { What is the highest level of education } \\
\text { completed by }[\ldots] \text { ? }\end{array}$ & 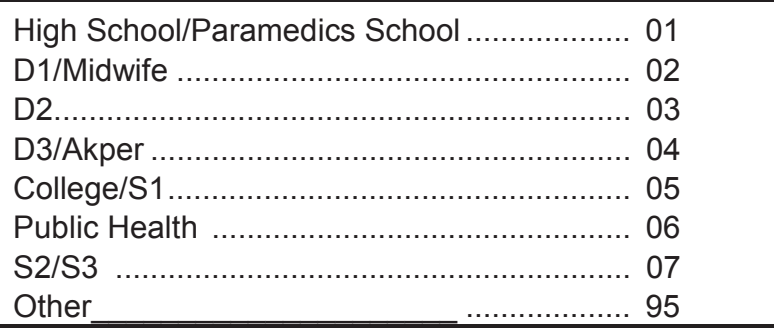 \\
\hline A1. & $\begin{array}{l}\text { Where did the head of the } \\
\text { Puskesmas/Pustu complete his/her } \\
\text { education? }\end{array}$ & $\begin{array}{l}\text { a. University of Indonesia } \ldots \ldots \ldots \ldots \ldots \ldots \ldots \ldots \ldots \ldots . . . \\
\text { University of GadjahMada }\end{array}$ \\
\hline A1b & What Accreditation ofuniversitiesyou? & $\begin{array}{l}\text { 01. accreditation } A \\
\text { 02. accreditation } B \\
\text { 03. accreditation } C \\
\text { 04. Stillin the processwaitedforthe results } \\
\text { 05. Notaccredited } \\
\text { 06. have notbeen registered } \\
\text { 98. Dont Know }\end{array}$ \\
\hline A2. & $\begin{array}{l}\text { When did the head of the } \\
\text { Puskesmas/Pustu start working in this } \\
\text { facility? }\end{array}$ & 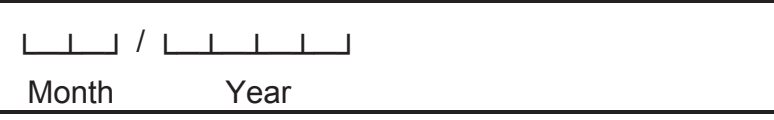 \\
\hline A3. & $\begin{array}{l}\text { Is the head of the Puskesmas/Pustu able } \\
\text { to speak the local language? }\end{array}$ & 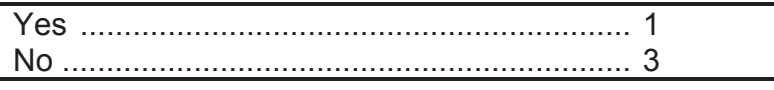 \\
\hline A4. & $\begin{array}{l}\text { Last month, how many hours per week } \\
\text { did the head of Puskesmas/Pustu work in } \\
\text { the building? }\end{array}$ & $\begin{array}{l}\text { 1. } \longleftarrow \_ \text {hours per week } \\
\text { 8. DK }\end{array}$ \\
\hline
\end{tabular}

\begin{tabular}{|c|c|c|}
\hline A5. & $\begin{array}{l}\text { In rendering services in the building, on the } \\
\text { average, how many hours per week does } \\
\text { the head of Puskesmas/Pustu offer his/her } \\
\text { services directly to the patients? [examine } \\
\text { the patients] }\end{array}$ & $\begin{array}{l}\text { 1. } \downarrow \downarrow \downarrow \text { L } \_ \text {hours per week } \\
\text { 6. NONE } \\
\text { 8. DK }\end{array}$ \\
\hline A5a. & $\begin{array}{l}\text { Approximately, what is the amount of basic } \\
\text { salary you received as the head of } \\
\text { Puskesmas? }\end{array}$ & 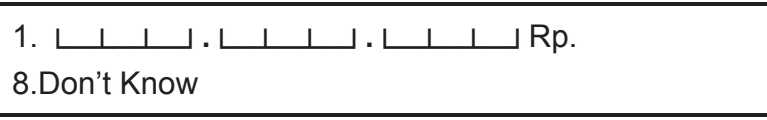 \\
\hline A6. & $\begin{array}{l}\text { In general, are patients here directly } \\
\text { examined by doctors? }\end{array}$ & 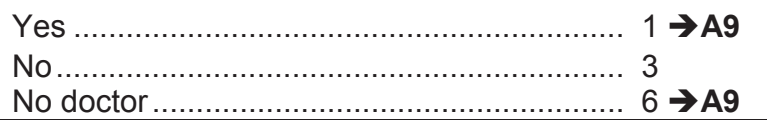 \\
\hline A7. & $\begin{array}{l}\text { If not, which patients does the doctor } \\
\text { examine? } \\
\text { [CIRCLE ALL THAT APPLY] }\end{array}$ & $\begin{array}{l}\text { Patients referred by paramedics/midwives ......... A } \\
\text { Patients with special arrangements ................... B } \\
\text { Patients with difficult cases................................ C } \\
\text { Other }\end{array}$ \\
\hline A9. & $\begin{array}{l}\text { Last month, on the average, how many } \\
\text { hours does the head of Puskesmas/Pustu } \\
\text { performs his/her duties outside the } \\
\text { Puskesmas/Pustu building? } \\
\text { a. For administrative activities such as } \\
\text { meetings, seminars, etc......................... } \\
\text { b. For field activities such as counseling, }\end{array}$ & $\begin{array}{l}\text { 6. NONE } \\
\text { 8. DK } \\
\text { 1. } \\
\text { a. } \longleftarrow \perp \text { hours per week } \\
\text { b. } \longleftarrow \perp \perp \text { hours per week }\end{array}$ \\
\hline A8. & $\begin{array}{l}\text { On average, how many patients per week } \\
\text { examined by the head of the } \\
\text { Puskesmas/Pustu? }\end{array}$ & $\begin{array}{l}\text { 1. } \downarrow \perp \_\_ \text {patients per week } \\
\text { 6. NONE } \\
\text { 8. DK }\end{array}$ \\
\hline A10. & $\begin{array}{l}\text { Does the head of Puskesmas/Pustu have a } \\
\text { private practice? }\end{array}$ & $\begin{array}{l}\text { No } \\
\text { Yes }\end{array}$ \\
\hline A10a & $\begin{array}{l}\text { Approximately, what is the amount of } \\
\text { revenue per month you received as the } \\
\text { head of Puskesmas? }\end{array}$ & 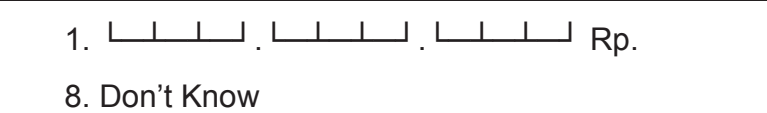 \\
\hline A11. & $\begin{array}{l}\text { How far is the place of the private practice } \\
\text { from the Puskesmas? } \\
\text { [IF THE PRACTICE IS AT THE } \\
\text { PUSKESMAS OR IN THE YARD OF THE } \\
\text { PUSKESMAS, WRITE "0" (ZERO)!] }\end{array}$ & 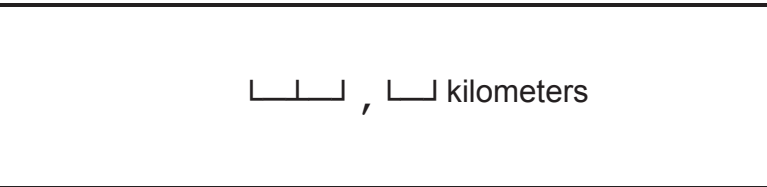 \\
\hline
\end{tabular}




\section{SECTION A: HEAD OF PUSKESMAS/PUSKESMAS PEMBANTU}

Opinion of head of puskesmas/pustu / Respondent :

A25a. Are you aware of the Minimum Service Standard on health?

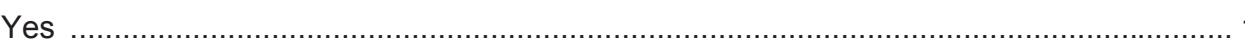

A25. Now, we would like to know three main problems you face at this Puskesmas:

Lack of funds.

Lack of medical staff

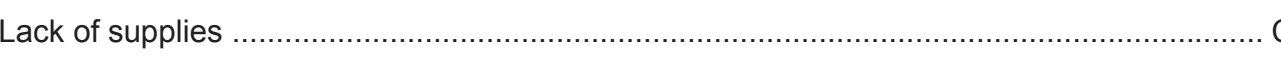

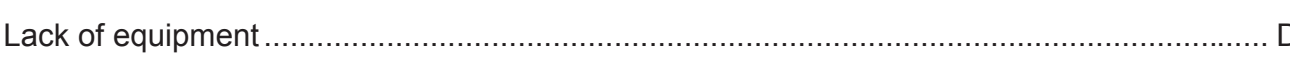

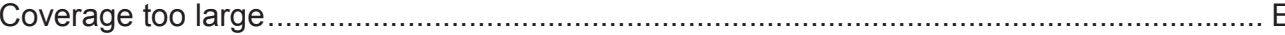

Lack of support from government

Official (village, regionl, central)

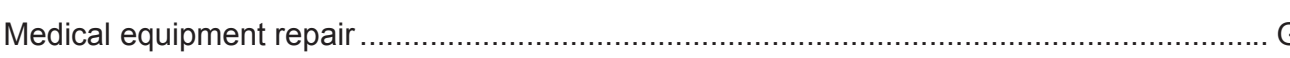

Other

... V 


\section{SECTION A: HEAD OF PUSKESMAS/PUSKESMAS PEMBANTU}

Now, we would like to know the changes experienced since 2007.

\begin{tabular}{|c|c|c|c|}
\hline \multirow[b]{2}{*}{ (ATYPE) } & \multirow{2}{*}{\multicolumn{2}{|c|}{$\begin{array}{c}\text { A27bb. } \\
\text { Since 2007, has there been change } \\
\text { in }[\ldots . .] \text { ? }\end{array}$}} & \multirow{2}{*}{$\begin{array}{c}\text { A27ab. } \\
\text { How was this facility affected? }\end{array}$} \\
\hline & & & \\
\hline a. Availability of drugs & $\begin{array}{l}\text { 3. No } \\
\downarrow\end{array}$ & 1. Yes $\rightarrow$ & $\begin{array}{l}\text { Yes became better ............ } 1 \\
\text { No change ..................... } 2 \\
\text { Yes became worse ........... } 3\end{array}$ \\
\hline b. Availability of supplies & $\begin{array}{l}\text { 3. No } \\
\downarrow\end{array}$ & 1. Yes $\rightarrow$ & $\begin{array}{l}\text { Yes became better ........... } 1 \\
\text { No change ..................... } 2 \\
\text { Yes became worse ........... } 3 \\
\end{array}$ \\
\hline c. Availability of water & $\begin{array}{l}\text { 3. No } \\
\downarrow\end{array}$ & 1. Yes $\rightarrow$ & $\begin{array}{l}\text { Yes became better ........... } 1 \\
\text { No change ..................... } 2 \\
\text { Yes became worse ........... } 3 \\
\end{array}$ \\
\hline d. Price of drugs & $\begin{array}{l}\text { 3. No } \\
\downarrow\end{array}$ & 1. Yes $\rightarrow$ & $\begin{array}{l}\text { Yes became better ............ } 1 \\
\text { No change ..................... } 2 \\
\text { Yes became worse ........... } 3\end{array}$ \\
\hline e. Price of supplies & $\begin{array}{l}\text { 3. No } \\
\downarrow\end{array}$ & 1. Yes $\rightarrow$ & $\begin{array}{l}\text { Yes became better ............ } 1 \\
\text { No change ..................... } 2 \\
\text { Yes became worse ........... } 3 \\
\end{array}$ \\
\hline f. Price of fuel & $\begin{array}{l}\text { 3. No } \\
\downarrow\end{array}$ & 1. Yes $\rightarrow$ & $\begin{array}{l}\text { Yes became better ............. } 1 \\
\text { No change ..................... } 2 \\
\text { Yes became worse ........... } 3\end{array}$ \\
\hline
\end{tabular}

\begin{tabular}{|c|c|c|}
\hline \multirow{2}{*}{ (ATYPE) } & A27bb. & A27ab. \\
\hline & $\begin{array}{l}\text { Since 2007, has their been change } \\
\text { in [...]? }\end{array}$ & How was this facility affected? \\
\hline g. Price of other goods & $\begin{array}{l}\text { 3. No } \quad \text { 1. Yes } \rightarrow \\
\downarrow\end{array}$ & $\begin{array}{l}\text { Yes became better ........... } 1 \\
\text { No change ..................... } 2 \\
\text { Yes became worse ........... } 3\end{array}$ \\
\hline h. Number of patients & 1. Yes $\rightarrow$ & $\begin{array}{l}\text { Yes became better ............ } 1 \\
\text { No change ..................... } 2 \\
\text { Yes became worse ........... } 3\end{array}$ \\
\hline i. Staff size & 1. Yes $\rightarrow$ & $\begin{array}{l}\text { Yes became better ............ } 1 \\
\text { No change ....................... } 2 \\
\text { Yes became worse ........... } 3\end{array}$ \\
\hline j. Supply of contraceptives & $\begin{array}{l}\text { 3. No } \\
\downarrow\end{array}$ & $\begin{array}{l}\text { Yes became better ........... } 1 \\
\text { No change .................... } 2 \\
\text { Yes became worse ........... } 3\end{array}$ \\
\hline $\begin{array}{l}\text { k. Air quality (as the result of } \\
\text { forest fire) }\end{array}$ & $\begin{array}{l}\text { 3. No } \quad \text { 1. Yes } \rightarrow \\
\downarrow \\
\text { SECTION SDP }\end{array}$ & $\begin{array}{l}\text { Yes became better ........... } 1 \\
\text { No change ..................... } 2 \\
\text { Yes became worse ........... } 3 \\
\end{array}$ \\
\hline
\end{tabular}


SECTION SDP: RESOURCES OF PUSKESMAS

Now, we would like to ask about the budget of and source of revenue for the Puskesmas.

\begin{tabular}{|c|c|c|}
\hline SDP00a & $\begin{array}{l}\text { Does this puskesmas/pustu } \\
\text { receive Health Operational } \\
\text { Assistance (BOK) program? }\end{array}$ & 3. No $\rightarrow$ SDP0O \\
\hline SDP00b & $\begin{array}{l}\text { Since when have this } \\
\text { Puskesmas/Pustu been } \\
\text { receiving BOK program? }\end{array}$ & 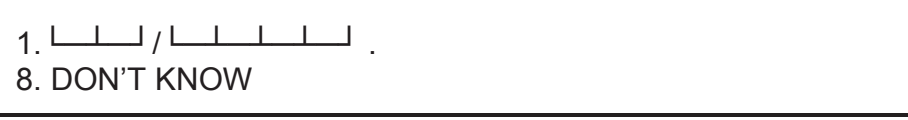 \\
\hline SDP00c & $\begin{array}{l}\text { In the past budget year and } \\
\text { the budget now how much } \\
\text { budget / sources of income } \\
\text { derived from: HEALTH } \\
\text { CARE OPERATIONS } \\
\text { (BOK)? }\end{array}$ & 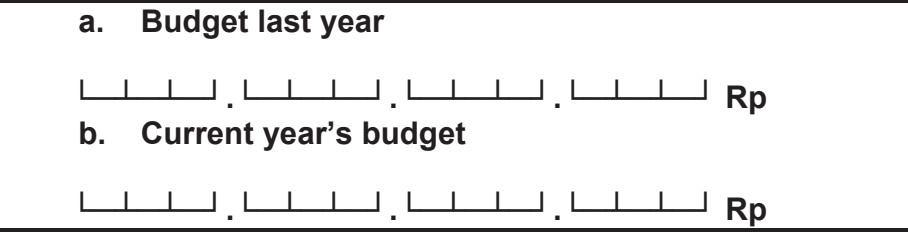 \\
\hline SDP00d & $\begin{array}{l}\text { What did the Puskesmas } \\
\text { /Pustu do with the fund from } \\
\text { BOK? }\end{array}$ & $\begin{array}{l}\text { A. Transportation cost for health officers to carry out outdoor } \\
\text { activities. } \\
\text { B. Transportation cost for health care in supporting Puskesmas } \\
\text { activities and its networks, Poskesdes andPosyandu. } \\
\text { C. Transportation cost for indigenous medical practitioner } \\
\text { (dukunberanak) as partner of midwife and other practitioner. } \\
\text { D. Purchase of materials/food for } \\
\text { PMT activities (counseling and/or malnutrition treatment of } \\
\text { babies aged 6-59 months. } \\
\text { E. Curative and rehabilitation effort } \\
\text { F. Salary, work overtime fee, incentives } \\
\text { G. Building maintenance (minor and major) } \\
\text { H. Vehicle maintenance (minor and major) } \\
\text { I. Phone, electricity, and water bills. } \\
\text { J. Provisions of medicines, vaccines, health appliance } \\
\text { K. Transportation cost for referenced patients }\end{array}$ \\
\hline SDP00. & $\begin{array}{l}\text { What Budget Year that has } \\
\text { been finished recently? }\end{array}$ & $\begin{array}{l}\text { 1. } 2014 \\
\text { 2. } 2013 / 2014\end{array}$ \\
\hline SDP01a. & $\begin{array}{l}\text { What was the budget } \\
\text { proposed by Puskesmas to } \\
\text { Local Government/Dinas for } \\
\text { the past budget year? }\end{array}$ & 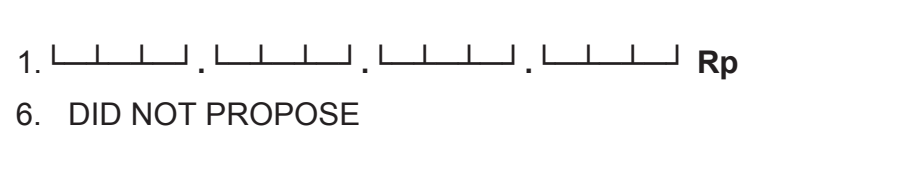 \\
\hline SDP01b. & $\begin{array}{l}\text { What was the budget of the } \\
\text { Puskesmas for the past } \\
\text { budget year? }\end{array}$ & 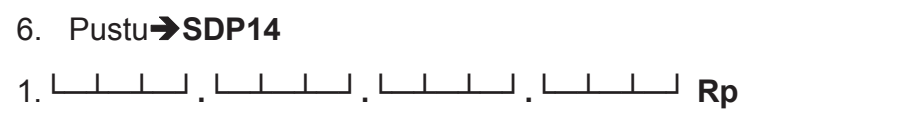 \\
\hline
\end{tabular}

\begin{tabular}{|c|c|c|}
\hline \multirow[t]{8}{*}{ SDP02a. } & $\begin{array}{l}\text { For the past budget year, } \\
\text { how much comprise from: }\end{array}$ & \\
\hline & $\begin{array}{l}\text { a. Assistance from regional } \\
\text { government }\end{array}$ & 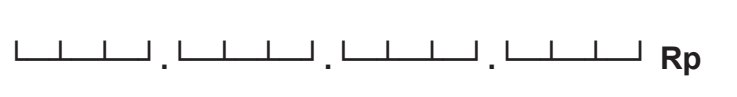 \\
\hline & $\begin{array}{l}\text { b. Assistance from central } \\
\text { government }\end{array}$ & 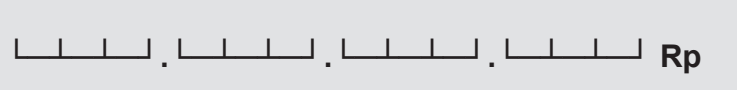 \\
\hline & $\begin{array}{l}\text { c. Claim from Jamkesmas/ } \\
\text { Jamkesda/Jampersal }\end{array}$ & 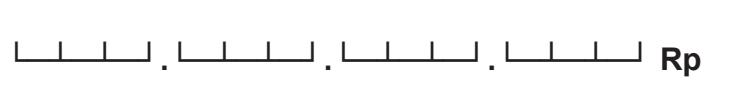 \\
\hline & c1. BPJS capitation & 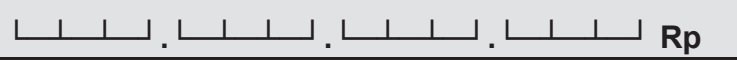 \\
\hline & d. Patients & 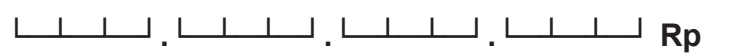 \\
\hline & e. Askes & 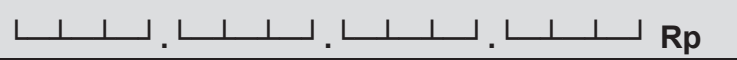 \\
\hline & f. Other assistance & 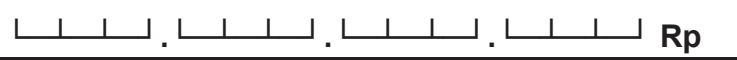 \\
\hline SDP03a. & $\begin{array}{l}\text { For the past budget year, } \\
\text { what was the target of } \\
\text { revenue from patients set by } \\
\text { regional government/Dinas? }\end{array}$ & 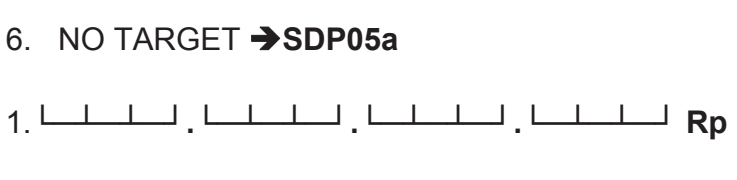 \\
\hline SDP04a. & $\begin{array}{l}\text { What was the realization of } \\
\text { the target? }\end{array}$ & 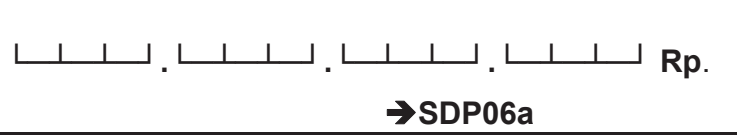 \\
\hline SDP05a. & $\begin{array}{l}\text { What was the revenue from } \\
\text { patients in the last budget } \\
\text { year? }\end{array}$ & 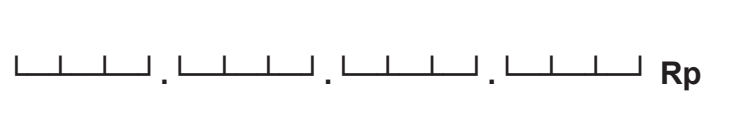 \\
\hline SDP06a. & $\begin{array}{l}\text { What was the percentage of } \\
\text { patients revenue directly } \\
\text { given back to DInas? }\end{array}$ & 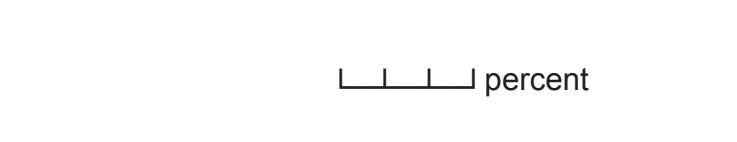 \\
\hline SDP07a. & $\begin{array}{l}\text { At the last budget year2014, } \\
\text { how many percent the } \\
\text { recieving from patient that } \\
\text { given to the Dinas directly? }\end{array}$ & $\downarrow \perp \perp \_$percent \\
\hline
\end{tabular}




\section{SECTION SDP: RESOURCES OF PUSKESMAS}

\begin{tabular}{|c|c|c|}
\hline SDP08. & $\begin{array}{l}\text { For the present budget year, what is } \\
\text { the target of revenue from patients set } \\
\text { by regional government/Dinas? }\end{array}$ & 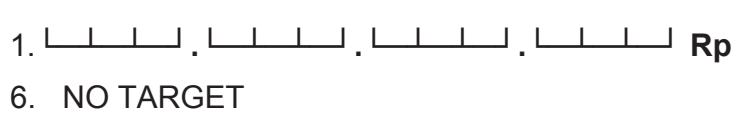 \\
\hline SDP09a. & $\begin{array}{l}\text { How much was this Puskesmas/Pustu } \\
\text { expenditure in the past budget year? }\end{array}$ & 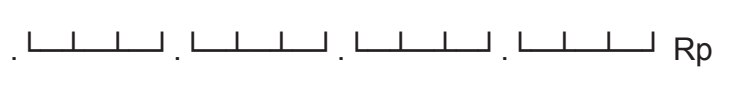 \\
\hline \multirow[t]{9}{*}{ SDP09b. } & $\begin{array}{l}\text { For the past budget year how much of } \\
\text { the expenditures was comprised of: }\end{array}$ & \\
\hline & a. Health services & 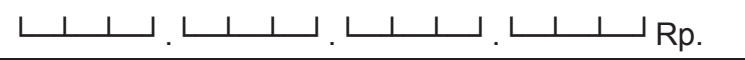 \\
\hline & b. Salary for civil servant & 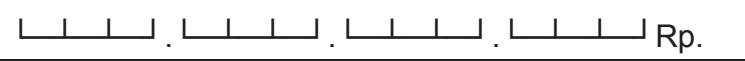 \\
\hline & c. Salary for non-civil servant & 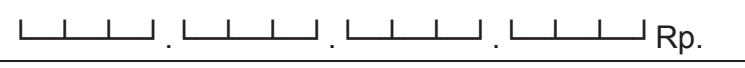 \\
\hline & d. Equipment and supplies & 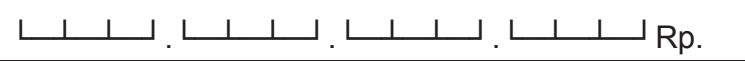 \\
\hline & e. Drugs and medicine & 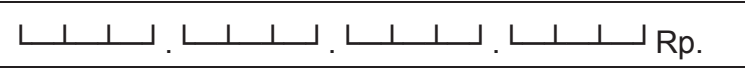 \\
\hline & f. Health communication & 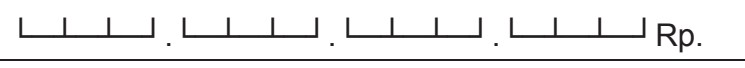 \\
\hline & $\begin{array}{l}\text { g. Posyandu support (including } \\
\text { Posyandu Revitalization Program) }\end{array}$ & 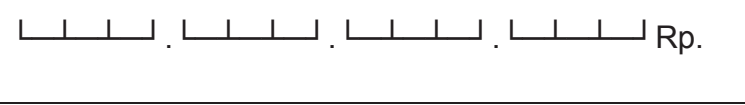 \\
\hline & v. Other & 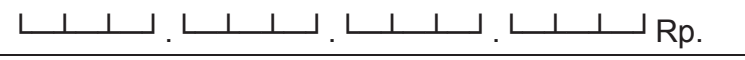 \\
\hline SDP09. & $\begin{array}{l}\text { For the past budget year, what was the } \\
\text { Puskesmas expenditure for Posyandu } \\
\text { Revitalization Program? }\end{array}$ & 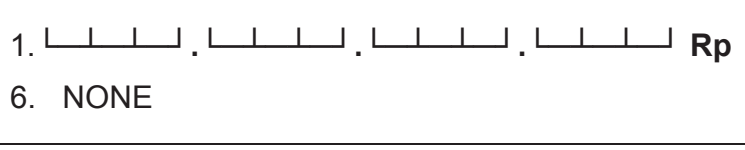 \\
\hline SDP10. & Who currently determines the budget? & 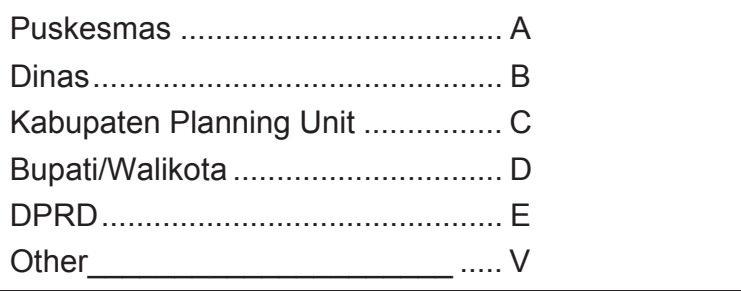 \\
\hline SDP11. & $\begin{array}{l}\text { Do you have the authority to reallocate } \\
\text { between posts of expenditure without } \\
\text { approval from the Dinas or any other } \\
\text { parties? }\end{array}$ & Yes \\
\hline SDP12. & $\begin{array}{l}\text { Compared to the year } 2007 \text {, is the } \\
\text { Puskesmas budget better or worse? }\end{array}$ & 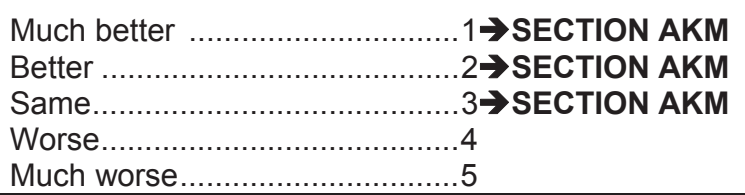 \\
\hline
\end{tabular}




\section{SECTION AKM :HEALTH INSURANCE FOR THE POOR}

We would like to know about the Health Insurance for the Poor (Jamkesmas) program in this fasilities.

\begin{tabular}{|c|c|c|}
\hline AKM01. & $\begin{array}{l}\text { Does this Puskesmas/Pustu } \\
\text { provide health service for } \\
\text { Jamkesmasholders? }\end{array}$ & $\begin{array}{l}\text { No } \\
\text { Yes }\end{array}$ \\
\hline AKM02. & $\begin{array}{l}\text { Since when did this } \\
\text { Puskesmas/Pustu provide this kind } \\
\text { of service? }\end{array}$ & 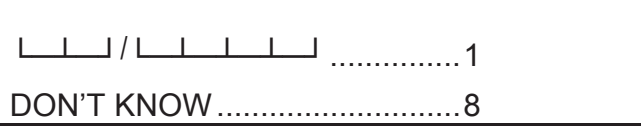 \\
\hline AKM03. & $\begin{array}{l}\text { What should one do to get free of } \\
\text { charge or subsidized } \\
\text { medication/service in this } \\
\text { Puskesmas/Pustu if he/she did not } \\
\text { possess any Jamkesmasand that } \\
\text { one could not afford the } \\
\text { medication? }\end{array}$ & $\begin{array}{l}\text { No } \\
\text { Yes }\end{array}$ \\
\hline AKM04. & $\begin{array}{l}\text { What should one do to get service } \\
\text { in this Puskesmas/Pustu if he/she } \\
\text { did not possess any Jamkesmas? }\end{array}$ & $\begin{array}{l}\text { Showing Letter Confirming } \\
\text { Poverty Status (SKTM) ............. } 1 \\
\text { Showing Health Card ............... } 2 \\
\text { Other }\end{array}$ \\
\hline AKM04a. & $\begin{array}{l}\text { How much should one pay in the } \\
\text { counter for Jamkesmas card } \\
\text { holders? }\end{array}$ & 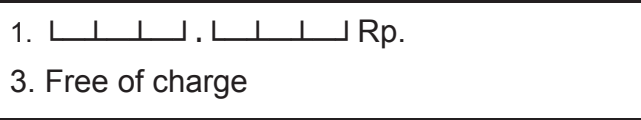 \\
\hline
\end{tabular}

Now, we want to ask about service fees charged to JAMKESMAS Card holders.

\begin{tabular}{|c|c|c|}
\hline & \multicolumn{2}{|c|}{ AKM05. } \\
\hline SERVICES & \multicolumn{2}{|c|}{$\begin{array}{l}\text { How much is additional cost of [...]for someone with } \\
\text { JAMKESMAS Card? (excluding cost paid in the counter) }\end{array}$} \\
\hline $\begin{array}{l}\text { A. General examination }+ \\
\text { medicine/injection for adult }\end{array}$ & 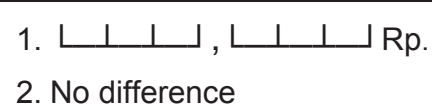 & $\begin{array}{l}\text { 3. Free of charge } \\
\text { 6. Service not offered }\end{array}$ \\
\hline $\begin{array}{l}\text { J. Check-up+injections and medicine for } \\
\text { child }\end{array}$ & 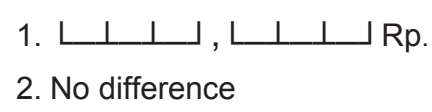 & $\begin{array}{l}\text { 3. Free of charge } \\
6 . \text { Service not offered }\end{array}$ \\
\hline K. Pregnancy check & 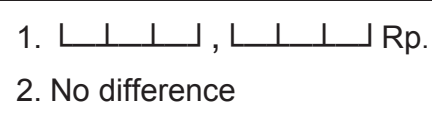 & $\begin{array}{l}\text { 3. Free of charge } \\
\text { 6. Service not offered }\end{array}$ \\
\hline L .Ordinari Labor/childbirth & 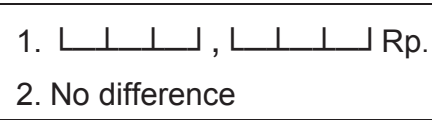 & $\begin{array}{l}\text { 3. Free of charge } \\
\text { 6. Service not offered }\end{array}$ \\
\hline $\begin{array}{l}\text { M. Neonatuscheck up (newborn aged 0-28 } \\
\text { days) }\end{array}$ & 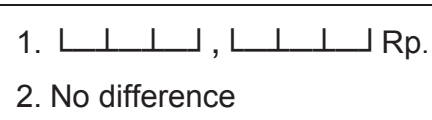 & $\begin{array}{l}\text { 3. Free of charge } \\
\text { 6. Service not offered }\end{array}$ \\
\hline N. Children Imunization & 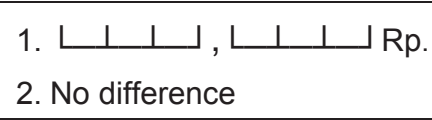 & $\begin{array}{l}\text { 3. Free of charge } \\
\text { 6. Service not offered }\end{array}$ \\
\hline H. Contraceptive Pills & $\begin{array}{l}\text { 1. } \downarrow \perp \perp \perp, \downarrow \perp \perp \_ \text {Rp. } \\
\text { 2. No difference }\end{array}$ & $\begin{array}{l}\text { 3. Free of charge } \\
\text { 6. Service not offered }\end{array}$ \\
\hline I. Family Planning Injection & 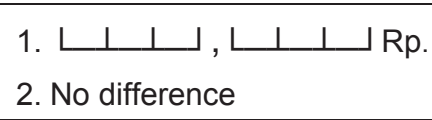 & $\begin{array}{l}\text { 3. Free of charge } \\
\text { 6. Service not offered }\end{array}$ \\
\hline
\end{tabular}


SECTION AKM :HEALTH INSURANCE FOR THE POOR

We would like to know about the Health Insurance for the Poor (JKN/KIS) program in this fasilities.

\begin{tabular}{|c|c|c|}
\hline AKM01a. & $\begin{array}{l}\text { Does this Puskesmas/Pustu provide } \\
\text { health service for JKN/KIS holders? }\end{array}$ & 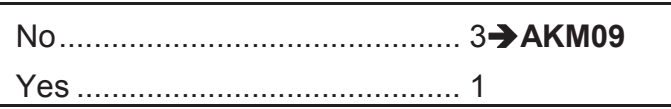 \\
\hline AKM02a. & $\begin{array}{l}\text { Since when did this } \\
\text { Puskesmas/Pustu provide this kind of } \\
\text { service? }\end{array}$ & 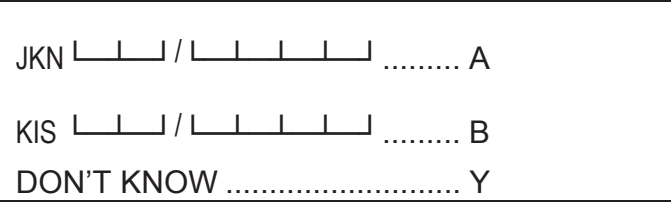 \\
\hline АКМ03а. & $\begin{array}{l}\text { What should one do to get free of } \\
\text { charge or subsidized } \\
\text { medication/service in this } \\
\text { Puskesmas/Pustu if he/she did not } \\
\text { possess any JKN/KIS and that one } \\
\text { could not afford the medication? }\end{array}$ & 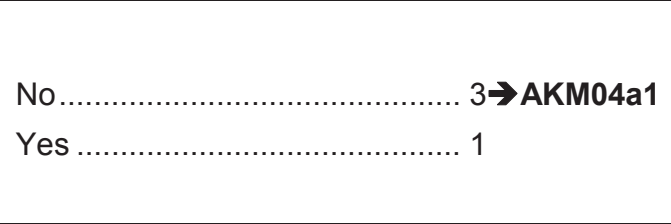 \\
\hline AKM04a. & $\begin{array}{l}\text { What should one do to get service in } \\
\text { this Puskesmas/Pustu if he/she did } \\
\text { not possess any JKN/KIS? }\end{array}$ & $\begin{array}{l}\text { Showing Letter Confirming } \\
\text { Poverty Status (SKTM) ............ } 1 \\
\text { Showing Health Card (Jamkesmas/, } \\
\text { askeskin/jempersal ...................... } 2 \\
\text { Other }\end{array}$ \\
\hline AKM04a1. & $\begin{array}{l}\text { How much should one pay in the } \\
\text { counter for JKN/KIS card holders? }\end{array}$ & $\begin{array}{l}\text { 1. } \longleftarrow \perp \perp \neg . \sqcup \perp \perp \neg R \text { R. } \\
\text { 3. Free of charge }\end{array}$ \\
\hline
\end{tabular}

Now, we want to ask about service fees charged to JKN/KIS Card holders.

\begin{tabular}{|c|c|c|}
\hline & \multicolumn{2}{|c|}{ AKM05a. } \\
\hline SERVICES & \multicolumn{2}{|c|}{$\begin{array}{l}\text { How much is additional cost of [...]for someone with JKN/KIS } \\
\text { Card? (excluding cost paid in the counter) }\end{array}$} \\
\hline $\begin{array}{l}\text { A. General examination }+ \\
\text { medicine/injection for adult }\end{array}$ & 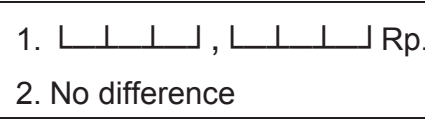 & $\begin{array}{l}\text { 3. Free of charge } \\
6 \text {. Service not offered }\end{array}$ \\
\hline $\begin{array}{l}\text { J. Check-up+injections and medicine for } \\
\text { child }\end{array}$ & 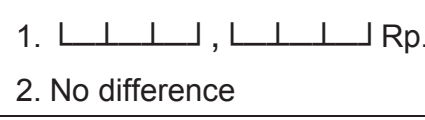 & $\begin{array}{l}\text { 3. Free of charge } \\
\text { 6. Service not offered }\end{array}$ \\
\hline K. Pregnancy check & 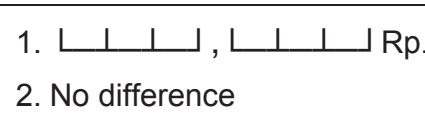 & $\begin{array}{l}\text { 3. Free of charge } \\
6 . \text { Service not offered }\end{array}$ \\
\hline L .Ordinari Labor/childbirth & 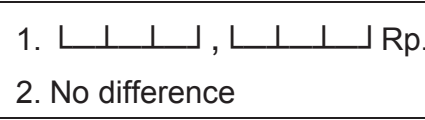 & $\begin{array}{l}\text { 3. Free of charge } \\
\text { 6. Service not offered }\end{array}$ \\
\hline $\begin{array}{l}\text { M. Neonatuscheck up (newborn aged 0-28 } \\
\text { days) }\end{array}$ & 1. $\downarrow$ & 3. Free of charge \\
\hline
\end{tabular}




\section{SECTION DM :DECISION MAKING IN PUSKESMAS}

Now we want to know about decision making in Puskesmas/pustu

\begin{tabular}{|c|c|c|c|c|c|c|c|}
\hline \multirow{3}{*}{\multicolumn{2}{|c|}{ (DMTYPE) }} & \multicolumn{6}{|c|}{ DM1. What level of authority makes the decision on: } \\
\hline & & \multirow{2}{*}{\multicolumn{3}{|c|}{$\begin{array}{c}\text { A } \\
\text { Puskesmas }\end{array}$}} & \multirow{2}{*}{\begin{tabular}{|c|} 
Babupaten \\
$\begin{array}{c}\text { Health Unit } \\
\text { (Dinas) }\end{array}$ \\
\end{tabular}} & \multirow{2}{*}{$\begin{array}{c}\text { C } \\
\text { Kabupaten } \\
\text { Planning Board } \\
\end{array}$} & \multirow{2}{*}{$\begin{array}{c}\text { D } \\
\text { Central } \\
\text { Government } \\
\text { (Health Ministry) }\end{array}$} \\
\hline & & & & & & & \\
\hline a. & Hiring and firing of staff (PNS) & 1 & 2 & 3 & $\begin{array}{l}\text { Yes........ } 1 \\
\text { No ........ } 3\end{array}$ & $\begin{array}{l}\text { Yes....... } 1 \\
\text { No ....... } 3\end{array}$ & $\begin{array}{l}\text { Yes....... } 1 \\
\text { No ....... } 3\end{array}$ \\
\hline a1 & $\begin{array}{l}\text { Recruitment and dismissal of } \\
\text { honorary staff }\end{array}$ & 1 & 2 & 3 & $\begin{array}{l}\text { Yes........ } 1 \\
\text { No ........ } 3\end{array}$ & $\begin{array}{l}\text { Yes........ } 1 \\
\text { No ....... } 3\end{array}$ & $\begin{array}{l}\text { Yes........ } 1 \\
\text { No ........ } 3\end{array}$ \\
\hline b. & $\begin{array}{l}\text { Levels and procedure for payroll } \\
\text { (PNS) }\end{array}$ & 1 & 2 & 3 & $\begin{array}{l}\text { Yes....... } 1 \\
\text { No ........ } 3\end{array}$ & $\begin{array}{l}\text { Yes....... } 1 \\
\text { No ....... } 3\end{array}$ & $\begin{array}{l}\text { Yes....... } 1 \\
\text { No ....... } 3\end{array}$ \\
\hline b1 & $\begin{array}{l}\text { Levels and procedure of salary } \\
\text { for honorary staff }\end{array}$ & 1 & 2 & 3 & $\begin{array}{l}\text { Yes } \ldots . . . .1 \\
\text { No } \ldots \ldots . . .3\end{array}$ & $\begin{array}{l}\text { Yes........ } 1 \\
\text { No ........ } 3\end{array}$ & $\begin{array}{l}\text { Yes........ } 1 \\
\text { No ........ } 3\end{array}$ \\
\hline c. & Service provision & 1 & 2 & 3 & $\begin{array}{l}\text { Yes........ } 1 \\
\text { No ........ } 3\end{array}$ & $\begin{array}{l}\text { Yes........ } 1 \\
\text { No ....... } 3\end{array}$ & $\begin{array}{l}\text { Yes........ } 1 \\
\text { No ........ } 3\end{array}$ \\
\hline d. & $\begin{array}{l}\text { Kinds of medicine to be } \\
\text { purchased and time of purchase }\end{array}$ & 1 & 2 & 3 & $\begin{array}{l}\text { Yes........ } 1 \\
\text { No ......... } 3\end{array}$ & $\begin{array}{l}\text { Yes........ } 1 \\
\text { No ......... } 3\end{array}$ & $\begin{array}{l}\text { Yes........ } 1 \\
\text { No ........ } 3\end{array}$ \\
\hline e. & $\begin{array}{l}\text { Kinds of medical equipment to } \\
\text { be purchased and time of } \\
\text { purchase }\end{array}$ & 1 & 2 & 3 & $\begin{array}{l}\text { Yes } \ldots . . .11 \\
\text { No ........ } 3\end{array}$ & $\begin{array}{l}\text { Yes....... } 1 \\
\text { No ........ } 3\end{array}$ & $\begin{array}{l}\text { Yes ....... } 1 \\
\text { No ........ } 3\end{array}$ \\
\hline f. & Charge of services & 1 & 2 & 3 & $\begin{array}{l}\text { Yes....... } 1 \\
\text { No ........ } 3\end{array}$ & $\begin{array}{l}\text { Yes....... } 1 \\
\text { No ........ } 3\end{array}$ & $\begin{array}{l}\text { Yes....... } 1 \\
\text { No ........ } 3\end{array}$ \\
\hline g. & Price of medicine & 1 & 2 & 3 & $\begin{array}{l}\text { Yes........ } 1 \\
\text { No ........ } 3\end{array}$ & $\begin{array}{l}\text { Yes....... } 1 \\
\text { No ........ } 3\end{array}$ & $\begin{array}{l}\text { Yes........ } 1 \\
\text { No ........ } 3\end{array}$ \\
\hline h. & $\begin{array}{l}\text { Building maintenance and } \\
\text { expansion }\end{array}$ & 1 & 2 & 3 & $\begin{array}{l}\text { Yes ........ } 1 \\
\text { No ........ } 3\end{array}$ & $\begin{array}{l}\text { Yes........ } 1 \\
\text { No ........ } 3\end{array}$ & $\begin{array}{l}\text { Yes ........ } 1 \\
\text { No ........ } 3\end{array}$ \\
\hline
\end{tabular}

CODE FOR DM1 COLUMN A:

\begin{tabular}{|c|c|c|c|}
\hline & \multirow[t]{2}{*}{ (DMTYPE) } & \multicolumn{2}{|c|}{$\begin{array}{l}\text { DM2. In the previous years, has this } \\
\text { Puskesmas ever submitted } \\
\text { suggestions to the Dinas or Central } \\
\text { Government on: }\end{array}$} \\
\hline & & $\begin{array}{l}\text { A. Kabupaten Health } \\
\text { Unit (Dinas) }\end{array}$ & $\begin{array}{l}\text { B. Central Government } \\
\text { (Health Ministry) }\end{array}$ \\
\hline & Hiring and firing of staff & $\begin{array}{l}\text { Yes ........ } 1 \\
\text { No......... } 3\end{array}$ & $\begin{array}{l}\text { Yes } \ldots \ldots . .1 \\
\text { No } \ldots \ldots \ldots . . .3\end{array}$ \\
\hline b. & Levels and procedures for payroll & $\begin{array}{l}\text { Yes } \ldots . . . .1 \\
\text { No......... }\end{array}$ & $\begin{array}{l}\text { Yes } \ldots \ldots . .1 \\
\text { No } \ldots \ldots \ldots . . . .3 \\
\end{array}$ \\
\hline c. & Service provision & $\begin{array}{l}\text { Yes ........ } 1 \\
\text { No......... }\end{array}$ & $\begin{array}{l}\text { Yes } \ldots . . . .1 \\
\text { No } \ldots . . . . .3\end{array}$ \\
\hline d. & $\begin{array}{l}\text { Kinds of medicine to be purchased and time of } \\
\text { purchase }\end{array}$ & $\begin{array}{l}\text { Yes........ } 1 \\
\text { No........ } 3\end{array}$ & $\begin{array}{l}\text { Yes } \ldots \ldots . .1 \\
\text { No } \ldots . . . . .3\end{array}$ \\
\hline e. & $\begin{array}{l}\text { Kinds of medical equipment to be purchased and time } \\
\text { of purchase }\end{array}$ & $\begin{array}{l}\text { Yes } \ldots \ldots . .1 \\
\text { No.......... }\end{array}$ & $\begin{array}{l}\text { Yes } \ldots \ldots . .1 \\
\text { No.......... }\end{array}$ \\
\hline f. & Charge of services & $\begin{array}{l}\text { Yes } \ldots . . . .1 \\
\text { No......... }\end{array}$ & $\begin{array}{l}\text { Yes } \ldots . . . .1 \\
\text { No } \ldots . . . . . .3\end{array}$ \\
\hline g. & Price of medicine & $\begin{array}{l}\text { Yes ........ } 1 \\
\text { No.......... }\end{array}$ & $\begin{array}{l}\text { Yes } \ldots . . . .1 \\
\text { No.......... }\end{array}$ \\
\hline h. & Building maintenance and expansion & $\begin{array}{l}\text { Yes ........ } 1 \\
\text { No......... } 3 \\
\end{array}$ & $\begin{array}{l}\text { Yes } \ldots \ldots . .1 \\
\text { No } \ldots \ldots \ldots .3 \\
\end{array}$ \\
\hline
\end{tabular}

1. Give suggestions

2. Making decisions

3. Abide by decision from higher authority 


\section{SECTION CP: INTERVIEWER NOTES}

\begin{tabular}{|c|c|c|c|c|c|c|c|}
\hline LANGMAIN (CK1). & \multicolumn{2}{|l|}{ Interview was entirely/mostly conducted in what language? } & \multicolumn{5}{|l|}{$ـ$ other } \\
\hline & Other languaged used (if any): & & \multicolumn{5}{|l|}{$\downarrow \downarrow$ L other } \\
\hline LANGUAGE CODE: & & & & & & & \\
\hline 00. Bahasa Indonesia & 04. Batak & 08. Sasak & 12. Makassar & 16. & Toraja & 20. & Lampung \\
\hline 01. Java & 05. Bugis & 09. Minang & 13. Nias & 17. & Lahat & 95. & Other, \\
\hline 02. Sunda & 06. Cina & 10. Banjar & 14. Palembang & 18. & Other South Sumatera & 96. & NA \\
\hline 03. Bali & 07. Madura & 11. Bima & 15. Sumbawa & 19. & Betawi & & \\
\hline
\end{tabular}

\begin{tabular}{|c|c|c|c|}
\hline RESULT (FP3). RESULTS OF INTERVIEW & REASON (FP4). REASON FOR ANSWERING "2" I "3" IN RESULT. & FP6. MONITORING BY SUPERVISOR & \\
\hline $\begin{array}{l}\text { 1. Completed } \rightarrow \text { FP6 } \\
\text { 2. Partly completed } \\
\text { 3. Not completed } \\
\text { 4. Twin EA with EA } \downarrow \perp \rightarrow F P 6\end{array}$ & $\begin{array}{l}\text { 1. Respondent was travelling/not in location } \\
\text { 2. Respondent was too busy } \\
\text { 3. Respondent refused }\end{array}$ & $\begin{array}{ll}\text { a. } & \text { Observed (sup_obs) .......... } 1 \\
\text { b. } & \text { Checked (sup_edit)............ } 1 \\
\text { c. } & \text { Verified (sup_veri).............. } 1\end{array}$ & $\begin{array}{l}\text { No } \\
3 \\
3 \\
3\end{array}$ \\
\hline
\end{tabular}

INTERVIEWER NOTE: 


\begin{tabular}{|c|c|c|c|}
\hline INTERVIEWER: & 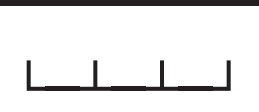 & CONFIDENTIAL & IDW: \\
\hline
\end{tabular}

\section{INDONESIAN FAMILY LIFE SURVEY 2014 HEALTH FACILITY COMMUNITY HEALTH CENTER/SUB-CENTER (PUSKESMAS / PUSKESMAS PEMBANTU) BOOK B}

SECTIONS : D, H, CP 
SECTION D: PUSKESMAS/PUSTU EMPLOYEES

\begin{tabular}{|c|c|}
\hline Name: & Position \\
\hline
\end{tabular}

Now, we would like to inquire about staff at the Puskesmas/Pustu

D01. How many employees are there working at this facility? [Including Government Employee and those paid by honoraria]

$\llcorner\llcorner$ employees

Specifically for Government Employee, please specify those according to types of employees

\begin{tabular}{|c|c|c|}
\hline D02. & D03. & D04. \\
\hline Type of employee & Number of full time employees & Number of part-time employees \\
\hline I. Obsetrician/gynecologist & $\llcorner\perp$ employees & $\llcorner\perp$ employees \\
\hline m. Specialized in elderly & $\downarrow \downarrow$ ـ employees & $\downarrow \downarrow$ ـ لmployees \\
\hline j. Public Health & $\sqcup \downarrow$ ـ employees & $\llcorner\perp$ employees \\
\hline f. Assistant Nutrition Expert & $\llcorner\perp$ employees & $\llcorner\perp$ employees \\
\hline
\end{tabular}

\section{INTERVIEWER CHECK:}

Total employees $[1+\mathrm{m}]$ full and part-time $=(\mathrm{D} 03+\mathrm{D} 04)$

\begin{tabular}{|c|c|c|}
\hline D05. & $\begin{array}{l}\text { Are there any employees on } \\
\text { honoraria? }\end{array}$ & $\begin{array}{l}\text { No } \\
\text { Yes }\end{array}$ \\
\hline
\end{tabular}

For honoraria employees, specify according to types:

\begin{tabular}{|c|c|c|}
\hline D06. & D07. & D08. \\
\hline Types of Employees & Number of full time employees & Number of part-time employees \\
\hline a. Nurses & $\llcorner\perp$ employees & $\llcorner\downarrow$ employees \\
\hline b. Midwives & $\llcorner\perp$ employees & $\llcorner\sqcup$ • employees \\
\hline c. Bidan Desa (Village Midwive) & $\llcorner\perp$ ــmployees & $\llcorner\downarrow$ employees \\
\hline d. Paramedics, other & $\llcorner\perp$ employees & $\llcorner\perp$ ـ employees \\
\hline e. Staff & $\llcorner\llcorner$ employees & $\llcorner\sqcup$ employees \\
\hline f. Administrative employees & $\llcorner\perp$ ـ employees & $\llcorner\perp$ ـ employees \\
\hline v. Other & $\llcorner\llcorner$ employees & $\llcorner\sqcup$ employees \\
\hline \multicolumn{2}{|c|}{$\begin{array}{l}\text { INTERVIEWER CHECK: } \\
\text { Total employees [a through d] full and part-time }=(\text { D07 + D08) }\end{array}$} & $\downarrow ـ$ ــ employees \\
\hline
\end{tabular}


SECTION D: PUSKESMAS/PUSTU EMPLOYEES

Only for doctors, nurses, paramedics, midwives, and mental health experts:

\begin{tabular}{|c|c|c|c|c|c|c|c|c|c|c|c|c|c|c|}
\hline \multicolumn{4}{|c|}{ D10. } & D15. & D16. & D17. & D11. & D12. & D13. & \multicolumn{5}{|c|}{ D14. } \\
\hline Em & loy & e C & & Male/female & $\begin{array}{l}\text { How many years has } \\
{[\ldots] \text { been working in }} \\
\text { this facility? }\end{array}$ & $\begin{array}{l}\text { How many hours per } \\
\text { week does }[\ldots . .] \text { work } \\
\text { in this facility? }\end{array}$ & $\begin{array}{l}\text { How many hours per } \\
\text { week does [...] provide } \\
\text { services to patients? }\end{array}$ & $\begin{array}{l}\text { Is }[\ldots] \text { able to } \\
\text { speak in the } \\
\text { local } \\
\text { language? }\end{array}$ & $\begin{array}{c}\text { Does }[\ldots] \text { have a private } \\
\text { practice? }\end{array}$ & $\begin{array}{l}\text { If yes } \\
\text { of the }\end{array}$ & $\begin{array}{l}\text { hou } \\
\text { prive } \\
\text { e P }\end{array}$ & far & & $\begin{array}{l}\text { place } \\
\text { from }\end{array}$ \\
\hline $\begin{array}{l}1 \\
5\end{array}$ & $\begin{array}{l}2 \\
6\end{array}$ & $\begin{array}{l}3 \\
7\end{array}$ & $\begin{array}{l}4 \\
8\end{array}$ & $\begin{array}{l}\text { Male ................... } 1 \\
\text { Female ................ }\end{array}$ & $\llcorner\perp$ years & $\left\llcorner \_\right.$hour/week & $\llcorner\perp$ hour/week & $\begin{array}{l}\text { Yes.................. } 1 \\
\text { No ................ } 3\end{array}$ & $\begin{array}{l}\text { Yes ................ } 1 \rightarrow \\
\text { No } \ldots \ldots \ldots \ldots . . . .3 \rightarrow \text { D18 }\end{array}$ & 0 & 1 & 2 & 3 & 4 \\
\hline $\begin{array}{l}1 \\
5\end{array}$ & $\begin{array}{l}2 \\
6\end{array}$ & $\begin{array}{l}3 \\
7\end{array}$ & $\begin{array}{l}4 \\
8\end{array}$ & $\begin{array}{l}\text { Male ................... } 1 \\
\text { Female ................ }\end{array}$ & $\llcorner\perp$ years & $\left\llcorner \_\right.$h hour/week & $\llcorner\perp$ hour/week & $\begin{array}{l}\text { Yes................. } 1 \\
\text { No ............... } 3.3\end{array}$ & 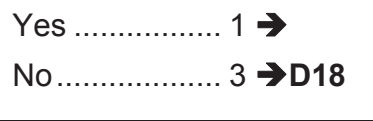 & 0 & 1 & 2 & 3 & 4 \\
\hline $\begin{array}{l}1 \\
5\end{array}$ & $\begin{array}{l}2 \\
6\end{array}$ & $\begin{array}{l}3 \\
7\end{array}$ & $\begin{array}{l}4 \\
8\end{array}$ & $\begin{array}{l}\text { Male ........................ } \\
\text { Female _............... }\end{array}$ & $\llcorner\perp$ years & $\left\llcorner \_\right.$hour/week & $\left\llcorner \_\right.$hour/week & $\begin{array}{l}\text { Yes................. } 1 \\
\text { No .............. } 3\end{array}$ & 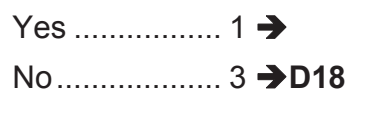 & 0 & 1 & 2 & 3 & 4 \\
\hline $\begin{array}{l}1 \\
5\end{array}$ & $\begin{array}{l}2 \\
6\end{array}$ & $\begin{array}{l}3 \\
7\end{array}$ & $\begin{array}{l}4 \\
8\end{array}$ & $\begin{array}{l}\text { Male .................... } 1 \\
\text { Female ................ }\end{array}$ & $\llcorner\perp$ years & $\left\llcorner \_\right.$h hour/week & $\llcorner\perp$ hour/week & $\begin{array}{l}\text { Yes................ } 1 \\
\text { No ................ }\end{array}$ & 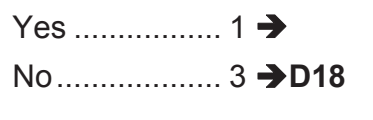 & 0 & 1 & 2 & 3 & 4 \\
\hline $\begin{array}{l}1 \\
5\end{array}$ & $\begin{array}{l}2 \\
6\end{array}$ & $\begin{array}{l}3 \\
7\end{array}$ & $\begin{array}{l}4 \\
8\end{array}$ & $\begin{array}{l}\text { Male ................... } 1 \\
\text { Female ................ }\end{array}$ & $\llcorner\perp$ years & $\left\llcorner \_\right.$hour/week & $\llcorner\perp$ hour/week & $\begin{array}{l}\text { Yes.................. } 1 \\
\text { No ................ }\end{array}$ & $\begin{array}{l}\text { Yes ............... } 1 \rightarrow \\
\text { No ................. } 3 \rightarrow \text { D18 }\end{array}$ & 0 & 1 & 2 & 3 & 4 \\
\hline $\begin{array}{l}1 \\
5\end{array}$ & $\begin{array}{l}2 \\
6\end{array}$ & $\begin{array}{l}3 \\
7\end{array}$ & $\begin{array}{l}4 \\
8\end{array}$ & $\begin{array}{l}\text { Male ......................... } \\
\text { Female _............... }\end{array}$ & $\llcorner\perp$ years & $\left\llcorner \_\right.$hour/week & $\left\llcorner \_\right.$hour/week & $\begin{array}{l}\text { Yes................. } 1 \\
\text { No ................. }\end{array}$ & 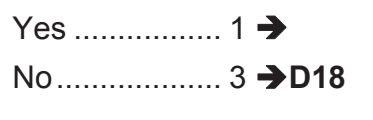 & 0 & 1 & 2 & 3 & 4 \\
\hline $\begin{array}{l}1 \\
5\end{array}$ & $\begin{array}{l}2 \\
6\end{array}$ & $\begin{array}{l}3 \\
7\end{array}$ & $\begin{array}{l}4 \\
8\end{array}$ & 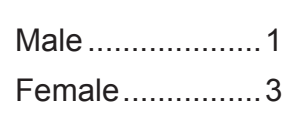 & $\llcorner\perp \neg$ years & $\left\llcorner \_\right.$hour/week & $\llcorner\perp$ hour/week & $\begin{array}{l}\text { Yes................ } 1 \\
\text { No ............... }\end{array}$ & 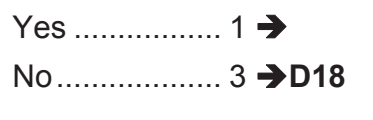 & 0 & 1 & 2 & 3 & 4 \\
\hline
\end{tabular}

Code D10 :

1. Doctor (general)

2. Dentist

3. Nurse

4. Midwife

5. Village Midwife

7. Dartor specialst

8. Mental health experts 
SECTION D: PUSKESMAS/PUSTU EMPLOYEES

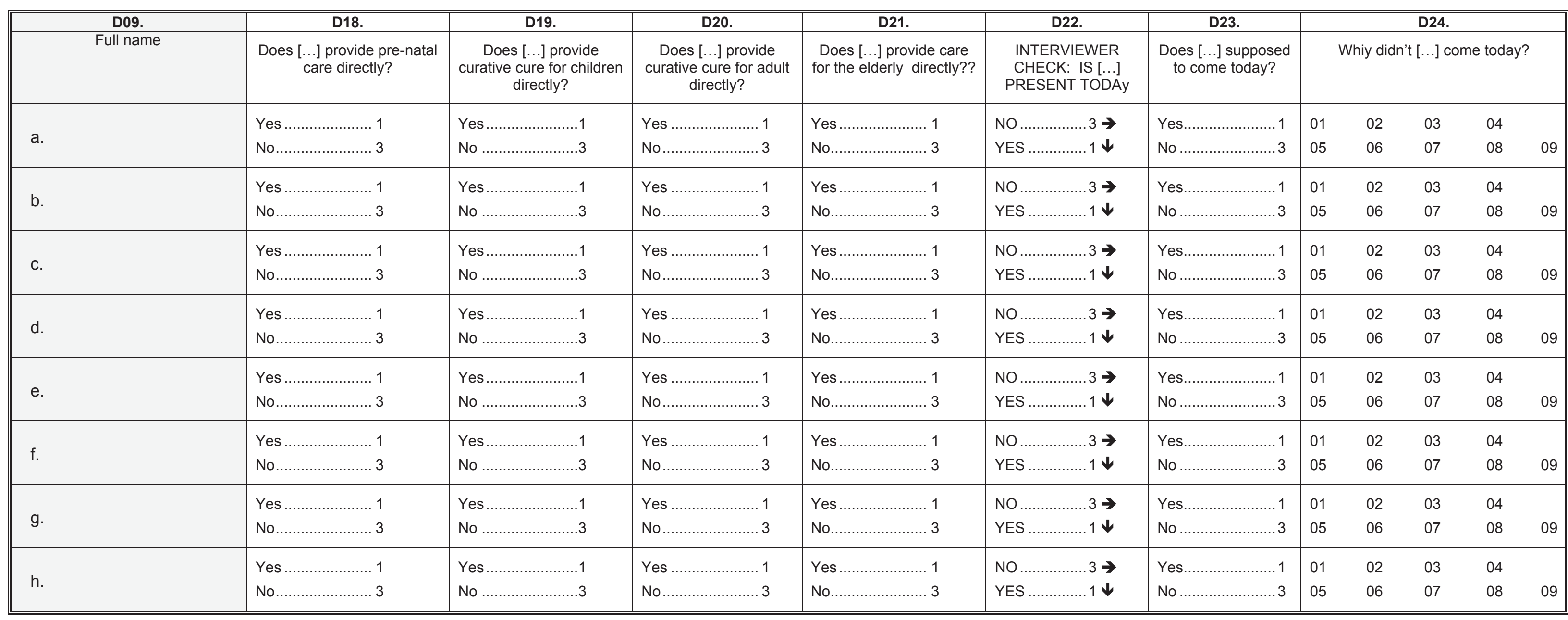

Code D24

01. Off duty
02 . Vacation

03. No longer work at the facility

04. Sick

05. Family , member is sick

06. Other authorized absence

08. Unauthorized absence

09. Have a break 
SECTION H: FACILITY VIGNETTES

\section{VIGNETTE FOR PRIVATE PRACTICE}

$*$ Curative Care for Adult

* Curative Care for Adult with Diabetes

* Curative care for children

* Prenatal Care 
Curative Care for Adult

\begin{tabular}{|c|c|c|}
\hline H1. & Does this health facility provide curative care for adults? & 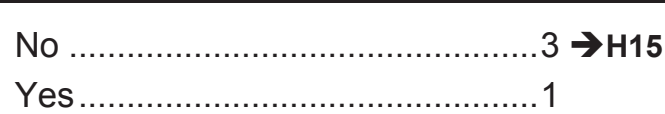 \\
\hline H2. & Name of Respondent : & \\
\hline H3. & Can you please tell me your qualifications? & 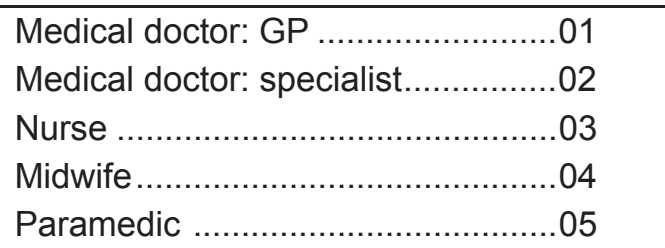 \\
\hline $\mathrm{H3a}$ & Where did you complete your studies? & 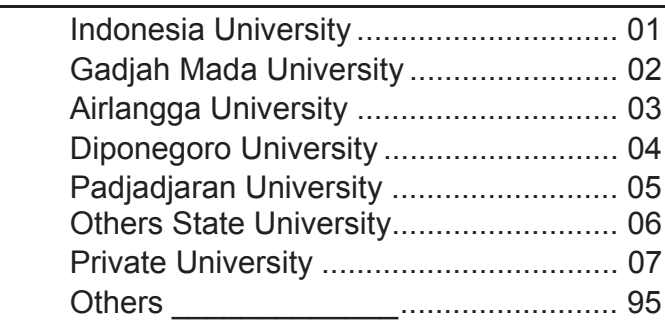 \\
\hline H4. & In what year did you complete your studies? & 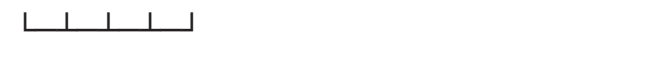 \\
\hline H5. & Have you received additional training since you graduated? & 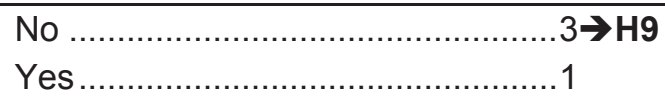 \\
\hline
\end{tabular}

Can you tell me, for each of the following areas, whether you received additional training and, if so, when this training occurred?

\begin{tabular}{|c|c|c|c|c|}
\hline & & H6. & H7. & H8. \\
\hline & & $\begin{array}{l}\text { Have you ever received } \\
\text { training of }[. . .] \text { after you } \\
\text { finished the study? }\end{array}$ & $\begin{array}{l}\text { In the last } 12 \\
\text { months? }\end{array}$ & $\begin{array}{c}\text { In the last } 5 \\
\text { years? }\end{array}$ \\
\hline 1. & $\begin{array}{l}\text { Diagnostic algorithm for adult } \\
\text { diseases }\end{array}$ & $\begin{array}{l}\text { 1. Yes } \\
\text { 3. No } \downarrow\end{array}$ & $\begin{array}{l}\text { 3. No } \\
\text { 1. Yes } \downarrow\end{array}$ & $\begin{array}{l}\text { 3. No } \\
\text { 1. Yes }\end{array}$ \\
\hline 2. & Non-communicable disease & $\begin{array}{l}\text { 1. Yes } \\
\text { 3. No } \downarrow\end{array}$ & $\begin{array}{l}\text { 3. No } \\
\text { 1. Yes } \downarrow\end{array}$ & $\begin{array}{l}\text { 3. No } \\
\text { 1. Yes }\end{array}$ \\
\hline 3. & Respiratory disease & $\begin{array}{l}\text { 1. Yes } \\
\text { 3. No } \downarrow\end{array}$ & $\begin{array}{l}\text { 3. No } \\
\text { 1. Yes } \downarrow\end{array}$ & $\begin{array}{l}\text { 3. No } \\
\text { 1. Yes }\end{array}$ \\
\hline & Antibiotic for respiratory disease & $\begin{array}{l}\text { 1. Yes } \\
\text { 3. No } \downarrow\end{array}$ & $\begin{array}{l}\text { 3. No } \\
\text { 1. Yes } \downarrow\end{array}$ & $\begin{array}{l}\text { 3. No } \\
\text { 1. Yes }\end{array}$ \\
\hline
\end{tabular}

H9. For the rest of the interview, we would like to understand the process by which you examine an adult person suffering from cough and fever. We would like to know everything you do, beginning with the arrival of the patient, waiting upon the patient and ending when he/she goes home. I shall describe the patient, and I will ask you a series of questions about activities you perform regularly. Once a section is complete, we are unable to go back and change answers. Now I will read out the case.

\section{INSTRUCTIONS TO INTERVIEWER:}

1. READ OUT CASE 2 TIMES, AND THEN ASK QUESTIONS H11 - H14.

2. FIRST LISTEN TO THE RESPONDENT. ANY RESPONSE THAT HE OR SHE MENTIONS SPONTANEOUSLY SHOULD BE MARKED WITH CODE 1.

3. AFTER THE RESPONDENT IS FINISHED WITH THE WHOLE QUESTIONNAIRE, TELL THE RESPONDENT THAT YOU ARE GOING TO REVIEW THE CASE AGAIN.

4. START FROM THE BEGINNING AND READ THE CASE A SECOND TIME. ASK QUESTIONS H11 - H14.

5. READ OUT ANY RESPONSES THAT HE/SHE DID NOT MENTION SPONTANEOUSLY THE FIRST TIME. CODE THE APPROPRIATE RESPONSE.

H10. Mr.Widyono came to this facility with a complaint of coughing and a fever. Now I would like to ask you exactly what you would do for this patient.

H11. What questions do you ask the patient about his cough and fever, and current health?
a. How long have you suffered from this condition?
b. Any shortness of breath?
c. Is there any blood when you cough?
d. What was the color of the sputum?
e. Do you have any pain in the chest?
f. Any weight loss?
g. Is cough productive?
h. Any contact with others with respiratory problems/TB?

\begin{tabular}{|c|cc||}
\hline $\begin{array}{c}\text { Mentioned } \\
\text { spontaneously }\end{array}$ & \multicolumn{2}{|c|}{ Prompted } \\
\hline 1 & 2 & 3 \\
1 & 2 & 3 \\
1 & 2 & 3 \\
1 & 2 & 3 \\
1 & 2 & 3 \\
1 & 2 & 3 \\
1 & 2 & 3 \\
1 & 2 & 3 \\
\hline
\end{tabular}




\begin{tabular}{|c|c|c|c|c|}
\hline H11. & $\begin{array}{l}\text { What questions do you ask the patient about his } \\
\text { cough and fever, and current health? }\end{array}$ & $\begin{array}{l}\text { Mentioned } \\
\text { spontaneously }\end{array}$ & \multicolumn{2}{|c|}{ Prompted } \\
\hline & i. Any night sweats? & 1 & 2 & 3 \\
\hline & j. What medicine have been taken? & 1 & 2 & 3 \\
\hline & k. Any fever? & 1 & 2 & 3 \\
\hline & I. Feeling weak? & 1 & 2 & 3 \\
\hline & m. Any headache & 1 & 2 & 3 \\
\hline & n. Losing appetite? & 1 & 2 & 3 \\
\hline & o. Nauseous? & 1 & 2 & 3 \\
\hline \multirow[t]{15}{*}{ H12. } & $\begin{array}{l}\text { What questions do you ask the patient about his } \\
\text { medical history and behavior? }\end{array}$ & $\begin{array}{c}\text { Mentioned } \\
\text { spontaneously }\end{array}$ & \multicolumn{2}{|c|}{ Prompted } \\
\hline & a. Previous TB case or took TB medicine? & 1 & 2 & 3 \\
\hline & $\begin{array}{l}\text { b. BCG immunization or ever positive PPD? } \\
{ }^{*} \text { Note: } P P D=\text { Purified Protein Derivative or Mantoux, } \\
\text { examination of TBC }\end{array}$ & 1 & 2 & 3 \\
\hline & $\begin{array}{l}\text { c. History of asthma or COPD? } \\
{ }^{*} \text { Note: } \mathrm{COPD}=\text { Chronic Obstructive Pulmonary } \\
\text { Disease, chronic lungs disease }\end{array}$ & 1 & 2 & 3 \\
\hline & d. History of cardiac problems? & 1 & 2 & 3 \\
\hline & e. History of malignancy or gastric surgery? & 1 & 2 & 3 \\
\hline & f. Medications recently or currently taking? & 1 & 2 & 3 \\
\hline & g. Drug allergies? & 1 & 2 & 3 \\
\hline & h. Smoking history? & 1 & 2 & 3 \\
\hline & i. Number of packages/quantity of smoking? & 1 & 2 & 3 \\
\hline & j. Alcohol use? & 1 & 2 & 3 \\
\hline & k. Live alone or with others? & 1 & 2 & 3 \\
\hline & I. Employment? & 1 & 2 & 3 \\
\hline & m. Family health history? & 1 & 2 & 3 \\
\hline & n. Sanitation, ventilation at home? & 1 & 2 & 3 \\
\hline \multirow[t]{10}{*}{ H13. } & $\begin{array}{l}\text { What do you do when you conduct a physical } \\
\text { examination of the patient? }\end{array}$ & $\begin{array}{c}\text { Mentioned } \\
\text { spontaneously }\end{array}$ & \multicolumn{2}{|c|}{ Prompted } \\
\hline & a. Examine general appearance? & 1 & 2 & 3 \\
\hline & b. Take temperature? & 1 & 2 & 3 \\
\hline & c. Listen to respiration? & 1 & 2 & 3 \\
\hline & d. Check for sore throat? & 1 & 2 & 3 \\
\hline & e. Palpitate / feel throat / lymph nodes? & 1 & 2 & 3 \\
\hline & f. Is chest indrawing? & 1 & 2 & 3 \\
\hline & $\begin{array}{l}\text { g. Palpate abdomen? } \\
{ }^{*} \text { Note: palpation = examination by palpating and } \\
\text { pressing }\end{array}$ & 1 & 2 & 3 \\
\hline & $\begin{array}{l}\text { h. Pulse } \\
{ }^{*} \text { Note: vital signs = breath, pulse } \\
{ }^{*} \text { Note: IPPA = Inspection, Palpation, Percussion, } \\
\text { Auscultation }\end{array}$ & 1 & 2 & 3 \\
\hline & i. Blood pressure & 1 & 2 & 3 \\
\hline H14. & What laboratory examinations would you conduct? & $\begin{array}{c}\text { Mentioned } \\
\text { spontaneously }\end{array}$ & \multicolumn{2}{|c|}{ Prompted } \\
\hline & a. Chest x-ray & 1 & 2 & 3 \\
\hline & b. PPD ormantoux test & 1 & 2 & 3 \\
\hline & c. Sputum exam for TB & 1 & 2 & 3 \\
\hline & d. Routine bloodwork & 1 & 2 & 3 \\
\hline & e. Liver function & 1 & 2 & 3 \\
\hline & $\begin{array}{l}\text { f. CD4/cell count } \\
\text { *Note: blood test to see the immune system }\end{array}$ & 1 & 2 & 3 \\
\hline & g. Urinalysis & 1 & 2 & 3 \\
\hline
\end{tabular}


Curative Care for Adult with Diabetes

\begin{tabular}{|c|c|c|}
\hline H15. & $\begin{array}{l}\text { Does this health facility provide curative care for adults with } \\
\text { diabetes? }\end{array}$ & 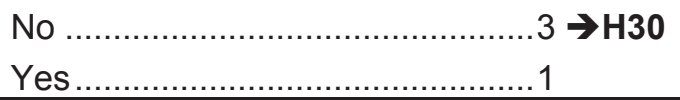 \\
\hline H16. & Name of respondent & \\
\hline H17. & Can you please tell me your qualifications? & 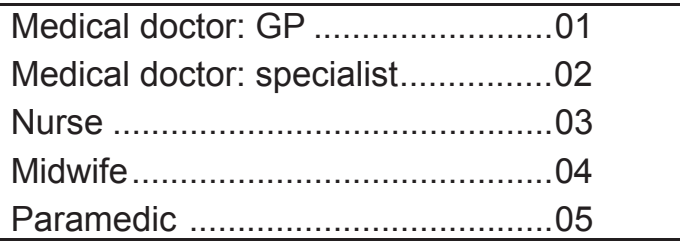 \\
\hline H17a & Where did you complete your studies? & 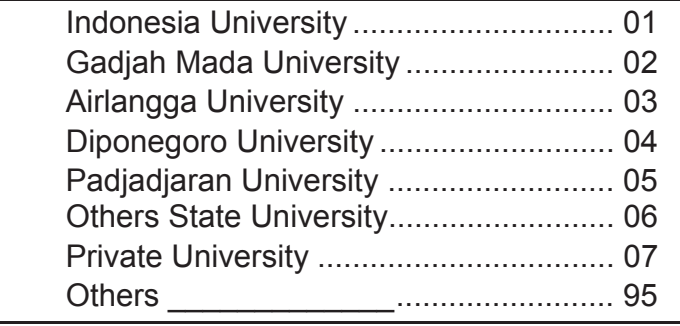 \\
\hline H18. & In what year did you complete your studies? & 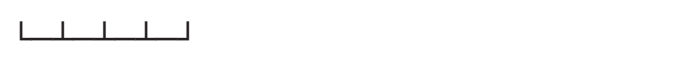 \\
\hline H19. & Have you received additional training since you graduated? & 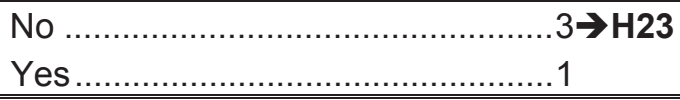 \\
\hline
\end{tabular}

\begin{tabular}{|c|c|c|c|c|}
\hline & & H2O. & H21. & H22. \\
\hline & & $\begin{array}{c}\text { Have you ever } \\
\text { received training of } \\
{[\ldots . .] \text { after you }} \\
\text { finished the study? }\end{array}$ & $\begin{array}{c}\text { In the last } 12 \\
\text { months? }\end{array}$ & In the last 5 years? \\
\hline 1. & $\begin{array}{l}\text { Diagnostic algorithm for adult } \\
\text { diseases }\end{array}$ & $\begin{array}{l}\text { 1. Yes } \\
\text { 3. No } \downarrow\end{array}$ & $\begin{array}{l}\text { 3. No } \\
\text { 1. Yes } \downarrow\end{array}$ & $\begin{array}{l}\text { 3. No } \\
\text { 1. Yes }\end{array}$ \\
\hline 2. & Non-communicable disease & $\begin{array}{l}\text { 1. Yes } \\
\text { 3. No } \downarrow\end{array}$ & $\begin{array}{l}\text { 3. No } \\
\text { 1. Yes } \downarrow\end{array}$ & $\begin{array}{l}\text { 3. No } \\
\text { 1. Yes }\end{array}$ \\
\hline 3. & Mengenaipenyakit diabetes & $\begin{array}{l}\text { 1. Yes } \\
\text { 3. No } \downarrow\end{array}$ & $\begin{array}{l}\text { 3. No } \\
\text { 1. Yes } \downarrow\end{array}$ & $\begin{array}{l}\text { 3. No } \\
\text { 1. Yes }\end{array}$ \\
\hline 4. & $\begin{array}{l}\text { Mengenai obat untuk penyakit } \\
\text { diabetes }\end{array}$ & $\begin{array}{l}\text { 1. Yes } \\
\text { 3. No } \downarrow\end{array}$ & $\begin{array}{l}\text { 3. No } \\
\text { 1. Yes } \downarrow\end{array}$ & $\begin{array}{l}\text { 3. No } \\
\text { 1. Yes }\end{array}$ \\
\hline
\end{tabular}

H23. For the rest of the interview, we would like to understand the process by which you examine an adult person suffering from diabetes. We would like to know everything you do, beginning with the arrival of the patient, waiting upon the patient and ending when he/she goes home. I shall describe the patient, and I will ask you a series of questions about activities you perform regularly. Once a section is complete, we are unable to go back and change answers. Now I will read out the case

INSTRUCTIONS TO INTERVIEWER:

1. READ OUT CASE 2 TIMES, AND THEN ASK QUESTIONS H25 - H29.

2. FIRST LISTEN TO THE RESPONDENT. ANY RESPONSE THAT HE OR SHE MENTIONS SPONTANEOUSLY SHOULD BE MARKED WITH CODE 1.

3. AFTER THE RESPONDENT IS FINISHED WITH THE WHOLE QUESTIONNAIRE, TELL THE RESPONDENT THAT YOU ARE GOING TO REVIEW THE CASE AGAIN.

4. START FROM THE BEGINNING AND READ THE CASE A SECOND TIME. ASK QUESTIONS H25 - H29.

5. READ OUT ANY RESPONSES THAT HE/SHE DID NOT MENTION SPONTANEOUSLY THE FIRST TIME. CODE THE APPROPRIATE RESPONSE.

H24. Mr. Widyono came to this facility, and presents to you "to get my sugar checked." He has just moved to the community and has never visited the facility. Now I would like to ask you exactly what you would do for this patient.

\begin{tabular}{|c|c|c|c|c|}
\hline \multicolumn{2}{|c|}{$\begin{array}{l}\text { H25 What questions do you ask the patient about his } \\
\text { present physical condition, high blood sugar, and } \\
\text { medications? }\end{array}$} & \multirow{2}{*}{$\begin{array}{c}\begin{array}{c}\text { Mentioned } \\
\text { spontaneously }\end{array} \\
1\end{array}$} & \multicolumn{2}{|c|}{ Prompted } \\
\hline & a. How long have you suffered from this condition? & & 2 & 3 \\
\hline & b. Medications recently or currently taking? & 1 & 2 & 3 \\
\hline & c. Do you have to urinate frequently? & 1 & 2 & 3 \\
\hline & d. Frequent thirst? & 1 & 2 & 3 \\
\hline & e. Any weight loss? & 1 & 2 & 3 \\
\hline & f. Any sweating? & 1 & 2 & 3 \\
\hline & g. Any anxiety or heart palpitations? & 1 & 2 & 3 \\
\hline & h. Abdominal fullness prematurely after meals? & 1 & 2 & 3 \\
\hline & i. Edema or weight retention? & 1 & 2 & 3 \\
\hline & j. Current treatment for hypertension? & 1 & 2 & 3 \\
\hline
\end{tabular}




\begin{tabular}{|c|c|c|c|c|}
\hline $\mathrm{H} 25$ & $\begin{array}{l}\text { What questions do you ask the patient about his } \\
\text { present physical condition, high blood sugar, and } \\
\text { medications? }\end{array}$ & $\begin{array}{l}\text { Mentioned } \\
\text { spontaneously }\end{array}$ & \multicolumn{2}{|c|}{ Prompted } \\
\hline & k. Often feel tingling? & 1 & 2 & 3 \\
\hline & I. Wound that stays? & 1 & 2 & 3 \\
\hline & m. Often have ulcer? & 1 & 2 & 3 \\
\hline & n. Family history & 1 & 2 & 3 \\
\hline & o. Feel weary & 1 & 2 & 3 \\
\hline & p. Have check blood sugar? & 1 & 2 & 3 \\
\hline H26 & $\begin{array}{l}\text { What questions do you ask Mr. Widyono about his } \\
\text { medical history and behavior? }\end{array}$ & $\begin{array}{c}\text { Mentioned } \\
\text { spontaneously }\end{array}$ & \multicolumn{2}{|c|}{ Prompted } \\
\hline & a. History of hypertension? & 1 & 2 & 3 \\
\hline & b. History of high cholesterol? & 1 & 2 & 3 \\
\hline & c. Co-existing or prior heart condition? & 1 & 2 & 3 \\
\hline & d. Prior eye examination? & 1 & 2 & 3 \\
\hline & e. Prior hospitalization? & 1 & 2 & 3 \\
\hline & f. Prior diabetic coma? & 1 & 2 & 3 \\
\hline & g. Prior renal failure? & 1 & 2 & 3 \\
\hline & h. Does he smoke regularly? & 1 & 2 & 3 \\
\hline & Number of packages/quantity of smoking? & 1 & 2 & 3 \\
\hline & j. $\quad$ Alcohol use? & 1 & 2 & 3 \\
\hline & k. Immunization history? & 1 & 2 & 3 \\
\hline & I. Regular exercise? & 1 & 2 & 3 \\
\hline & m. Questions about nutrion/eating habits? & 1 & 2 & 3 \\
\hline & $\mathrm{n} . \quad$ Is there any family member with this disease? & 1 & 2 & 3 \\
\hline $\mathrm{H} 27$ & $\begin{array}{l}\text { What do you do when you conduct a physical } \\
\text { examination of the patient? }\end{array}$ & $\begin{array}{c}\text { Mentioned } \\
\text { spontaneously }\end{array}$ & \multicolumn{2}{|c|}{ Prompted } \\
\hline & a. Blood pressure in one arm & 1 & 2 & 3 \\
\hline & b. Blood pressure in both arms & 1 & 2 & 3 \\
\hline & c. Listen to chest/heart? & 1 & 2 & 3 \\
\hline & d. Listen to abdomen? & 1 & 2 & 3 \\
\hline & e. Examine the feet? & 1 & 2 & 3 \\
\hline & f. Examine peripheral vascular system? & 1 & 2 & 3 \\
\hline & g. $\quad$ Check for edema? & 1 & 2 & 3 \\
\hline & h. Examine prostate? & 1 & 2 & 3 \\
\hline & i. $\quad$ Pulse & 1 & 2 & 3 \\
\hline & j. $\quad$ Respiration & 1 & 2 & 3 \\
\hline $\mathrm{H} 28$ & What laboratory examinations would you conduct? & $\begin{array}{l}\text { Mentioned } \\
\text { spontaneously }\end{array}$ & \multicolumn{2}{|c|}{ Prompted } \\
\hline & a. Chest x-ray? & 1 & 2 & 3 \\
\hline & b. Blood chemistry: creatinine, glucose? & 1 & 2 & 3 \\
\hline & c. Sputum exam? & 1 & 2 & 3 \\
\hline & $\begin{array}{l}\text { d. } \quad \text { CBC (Complete Blood Count)? } \\
{ }^{*} \text { Note: blood examination to count the red blood cells, } \\
\text { white blood cells, and blood platelet }\end{array}$ & 1 & 2 & 3 \\
\hline & $\begin{array}{l}\text { e. Test for triglycerides? } \\
\text { *Note: examination to check the lipid excess in the } \\
\text { blood }\end{array}$ & 1 & 2 & 3 \\
\hline & Ultrasound? & 1 & 2 & 3 \\
\hline & g. Liver function? & 1 & 2 & 3 \\
\hline & $\begin{array}{l}\text { h. HgbA1c? } \\
\text { *Note: examination to check the glucose amount in the } \\
\text { haemoglobyn }\end{array}$ & 1 & 2 & 3 \\
\hline & Hepatic enzymes? & 1 & 2 & 3 \\
\hline
\end{tabular}




\begin{tabular}{|c|c|c|c|c|c|}
\hline \multirow{2}{*}{$\begin{array}{r}\mathrm{H} 29 \\
\end{array}$} & \multicolumn{2}{|r|}{$\begin{array}{l}\text { What advice or future examinatios would you offer for the } \\
\text { patient? }\end{array}$} & \multirow{2}{*}{$\begin{array}{c}\begin{array}{c}\text { Mentioned } \\
\text { spontaneously }\end{array} \\
1\end{array}$} & \multicolumn{2}{|c|}{ Prompted } \\
\hline & a. & Recommend stop smoking? & & 2 & 3 \\
\hline & b. & Nutritional advice? & 1 & 2 & 3 \\
\hline & c. & Advice about exercise? & 1 & 2 & 3 \\
\hline & d. & Examine the feet? & 1 & 2 & 3 \\
\hline & e. & Refer to other specialist (eye,foot, or heart)? & 1 & 2 & 3 \\
\hline & f. & $\begin{array}{l}\text { Prescribe anti-hypertensives? } \\
{ }^{*} \text { Note: medicine to control high blood pressure }\end{array}$ & 1 & 2 & 3 \\
\hline & g. & $\begin{array}{l}\text { Prescribe Metformin? } \\
{ }^{*} \text { Note: medicine for diabetes }\end{array}$ & 1 & 2 & 3 \\
\hline & h. & Make an appointment for the next visit? & 1 & 2 & 3 \\
\hline
\end{tabular}


Curative care for children

\begin{tabular}{|c|c|c|}
\hline H30 & Does this health facility provide curative care for children? & 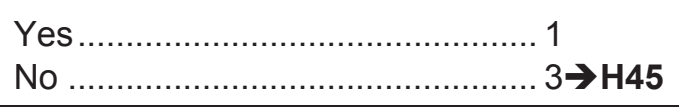 \\
\hline H31 & Name of respondent: & \\
\hline H32 & Can you please tell me your qualifications? & 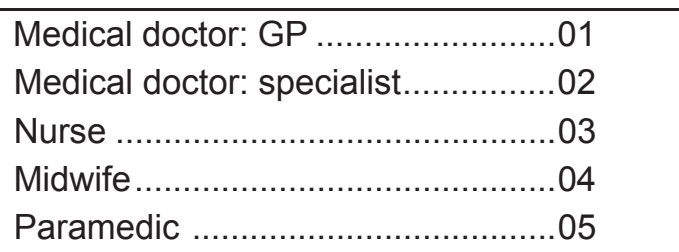 \\
\hline $\mathrm{H32a}$ & Where did you complete your studies? & 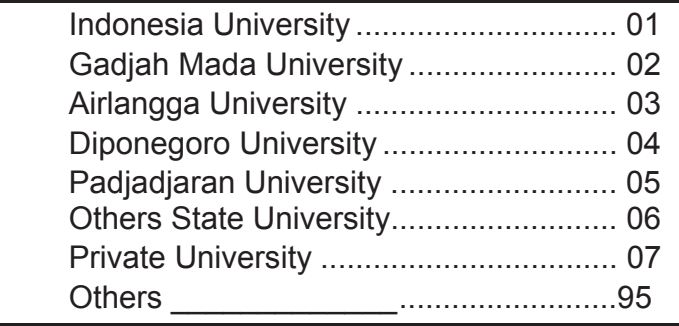 \\
\hline H33 & In what year did you complete your studies? & 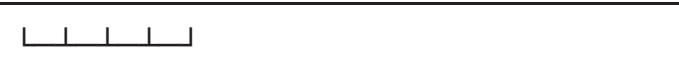 \\
\hline H34 & Have you received additional training since you graduated? & 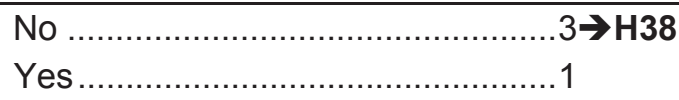 \\
\hline
\end{tabular}

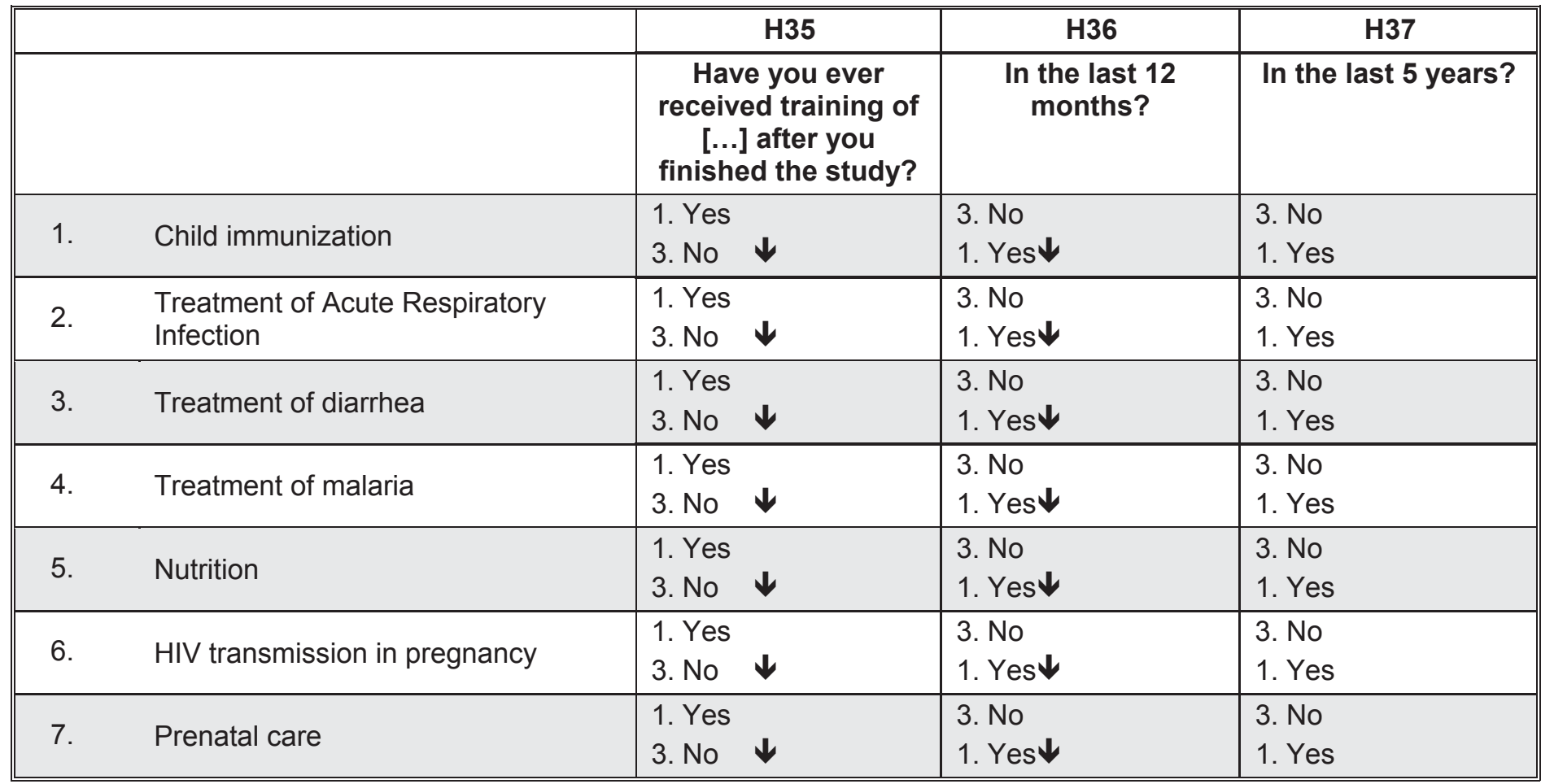

For the rest of the interview, we would like to understand the process by which you provide curative care for children. We would like to know everything you do, beginning with the arrival of the patient, waiting upon the patient and ending when he/she goes home. I shall describe the patient, and I will ask you a series of questions about activities you perform regularly. Once a section is complete, we are unable to go back and change answers. Now I will read out the case

\section{INSTRUCTIONS TO INTERVIEWER:}

1. READ OUT CASE 2 TIMES, AND THEN ASK QUESTIONS H40 - H44.

2. FIRST LISTEN TO THE RESPONDENT. ANY RESPONSE THAT HE OR SHE MENTIONS SPONTANEOUSLY SHOULD BE MARKED WITH CODE 1.

3. AFTER THE RESPONDENT IS FINISHED WITH THE WHOLE QUESTIONNAIRE, TELL THE RESPONDENT THAT YOU ARE GOING TO REVIEW THE CASE AGAIN.

4. START FROM THE BEGINNING AND READ THE CASE A SECOND TIME. ASK QUESTIONS H40 - H44.

5. READ OUT ANY RESPONSES THAT HE/SHE DID NOT MENTION SPONTANEOUSLY THE FIRST TIME. CODE THE APPROPRIATE RESPONSE:.

H39 Mrs. Nani comes to this facility with her daughter, an 8 month old baby. She says that her daughter has had diarrhea for 2 days with vomiting. 


\begin{tabular}{|l|c|c|c|}
\hline H40 What are the 13-14 most important questions you & $\begin{array}{c}\text { Mentioned } \\
\text { spontaneously } \\
\text { ask about the diarrhea and vomiting? }\end{array}$ & 1 & Prompted \\
\hline a. When did the diarrhea start? & 1 & 2 & 3 \\
b. How frequently does diarrhea occur? & 1 & 2 & 3 \\
c. What do the feces/vomit look like of smell like & 1 & 2 & 3 \\
d. Any blood in vomit? & 1 & 2 & 3 \\
e. Any blood in stools? & 1 & 2 & 3 \\
f. Any fever?
\end{tabular}

\begin{tabular}{|c|c|c|c|c|}
\hline H40. & $\begin{array}{l}\text { What are the } 13-14 \text { most important questions you } \\
\text { ask about the diarrhea and vomiting? }\end{array}$ & $\begin{array}{c}\text { Mentioned } \\
\text { spontaneously }\end{array}$ & \multicolumn{2}{|c|}{ Prompted } \\
\hline & g. Level of activity (active vs listless)? & 1 & 2 & 3 \\
\hline & h. Is the child feeding and drinking? & 1 & 2 & 3 \\
\hline & i. Given any medication already? & 1 & 2 & 3 \\
\hline & j. Any evidence of dehydration? & 1 & 2 & 3 \\
\hline & k. Vomits everything? & 1 & 2 & 3 \\
\hline & I. Has convulsions? & 1 & 2 & 3 \\
\hline & m. Eaten anything unusual? & 1 & 2 & 3 \\
\hline & n. Any ill contacts? & 1 & 2 & 3 \\
\hline & o. Urinating? & 1 & 2 & 3 \\
\hline H41 & $\begin{array}{l}\text { What do you ask about the baby's medical history } \\
\text { and environment? }\end{array}$ & $\begin{array}{c}\text { Mentioned } \\
\text { spontaneously }\end{array}$ & \multicolumn{2}{|c|}{ Prompted } \\
\hline & a. History of similar disease? & 1 & 2 & 3 \\
\hline & b. Drug allergies? & 1 & 2 & 3 \\
\hline & c. Any other medical or surgical problems or HIV? & 1 & 2 & 3 \\
\hline & d. Any complications at delivery or prematurity? & 1 & 2 & 3 \\
\hline & e. Access to water or sanitation? & 1 & 2 & 3 \\
\hline & f. Immunization history? & 1 & 2 & 3 \\
\hline & g. Breastfeeding/other fluids? & 1 & 2 & 3 \\
\hline & h. Digestive system normal? & 1 & 2 & 3 \\
\hline & i. Ever had surgery on digestive organs? & 1 & 2 & 3 \\
\hline & k. Eating & 1 & 2 & 3 \\
\hline & I. Baby care & 1 & 2 & 3 \\
\hline H42 & $\begin{array}{l}\text { What do you do when you conduct a physical } \\
\text { examination of the child? }\end{array}$ & $\begin{array}{c}\text { Mentioned } \\
\text { spontaneously }\end{array}$ & \multicolumn{2}{|c|}{ Prompted } \\
\hline & a. Check appearance / alertness? & 1 & 2 & 3 \\
\hline & b. Take her temperature? & 1 & 2 & 3 \\
\hline & $\begin{array}{l}\text { c. Examine the crown of the head? } \\
\text { *Note: is it concave? }\end{array}$ & 1 & 2 & 3 \\
\hline & d. Check pulse? & 1 & 2 & 3 \\
\hline & e. Weigh? & 1 & 2 & 3 \\
\hline & f. Check height? & 1 & 2 & 3 \\
\hline & g. Determine capillary refill time/check nailbeds? & 1 & 2 & 3 \\
\hline & h. Examine eyes? & 1 & 2 & 3 \\
\hline & i. Check skin turgor/elasticity? & 1 & 2 & 3 \\
\hline & j. Auscultate abdomen for bowel sounds? & 1 & 2 & 3 \\
\hline & $\begin{array}{l}\text { k. Palpitate abdomen? } \\
\text { *Note: examination of stomach by palpating and } \\
\text { pressing }\end{array}$ & 1 & 2 & 3 \\
\hline & I. Check feces for blood or mucous & 1 & 2 & 3 \\
\hline & m. Check palms of hands? & 1 & 2 & 3 \\
\hline & n. Check for edema in feet? & 1 & 2 & 3 \\
\hline & o. Breathing normally? & 1 & 2 & 3 \\
\hline & p. Blood pressure? & 1 & 2 & 3 \\
\hline
\end{tabular}


SECTION H: FACILITY VIGNETTES

\begin{tabular}{|c|c|c|c|c|}
\hline \multirow[t]{4}{*}{ H43 } & What laboratory examinations would you conduct? & \multirow{2}{*}{$\begin{array}{c}\begin{array}{c}\text { Mentioned } \\
\text { spontaneously }\end{array} \\
1\end{array}$} & \multicolumn{2}{|c|}{ Prompted } \\
\hline & $\begin{array}{l}\text { a. Routine bloodwork/CBC? } \\
{ }^{*} \text { Note: } C B C=\text { Complete Blood Count }\end{array}$ & & 2 & 3 \\
\hline & b. Stool culture? & 1 & 2 & 3 \\
\hline & $\begin{array}{l}\text { c. Blood smear/dipstick for malaria? } \\
{ }^{*} \text { Note: quick test for malaria }\end{array}$ & 1 & 2 & 3 \\
\hline H44 & $\begin{array}{l}\text { If this child has mild dehydration of viral etiology, } \\
\text { what would you do? }\end{array}$ & $\begin{array}{c}\text { Mentioned } \\
\text { spontaneously }\end{array}$ & \multicolumn{2}{|c|}{ Prompted } \\
\hline & a. Recommend to increase fluids? & 1 & 2 & 3 \\
\hline & b. Provide rehydration solution in clinic? & 1 & 2 & 3 \\
\hline & $\begin{array}{l}\text { c. Show how/recommend rehydration solution for } \\
\text { home? }\end{array}$ & 1 & 2 & 3 \\
\hline & d. Recommend vitamin supplements? & 1 & 2 & 3 \\
\hline & e. Recommend medicine for fever? & 1 & 2 & 3 \\
\hline & f. Instruct about returning to clinic if health worsens? & 1 & 2 & 3 \\
\hline & g. Update immunizations? & 1 & 2 & 3 \\
\hline & h. Administrate IV fluids? & 1 & 2 & 3 \\
\hline & i. Recommend antibiotics? & 1 & 2 & 3 \\
\hline & j. Hospitalize? & 1 & 2 & 3 \\
\hline & k. Continue to breastfeed? & 1 & 2 & 3 \\
\hline
\end{tabular}


Prenatal Care

\begin{tabular}{|c|c|c|}
\hline H45 & Does this health facility provide prenatal care? & 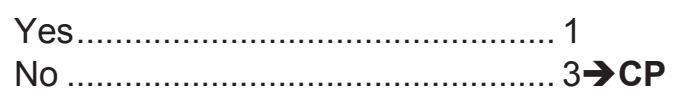 \\
\hline H46 & \multicolumn{2}{|l|}{ Name of respondent : } \\
\hline H47 & Can you please tell me your qualifications? & 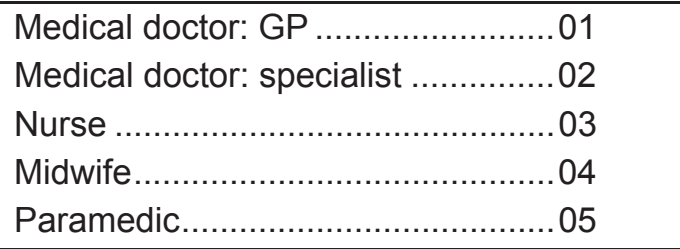 \\
\hline $\mathrm{H} 47 \mathrm{a}$ & Where did you complete your studies? & 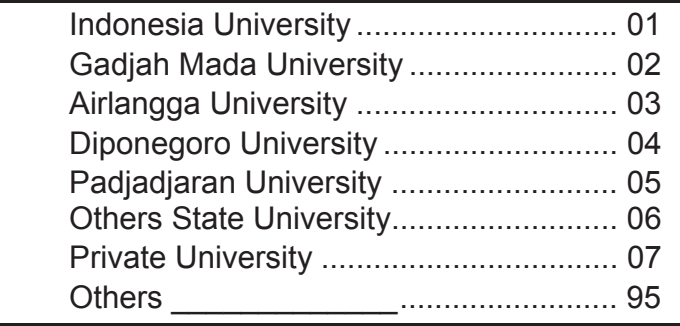 \\
\hline H48 & In what year did you complete your studies? & 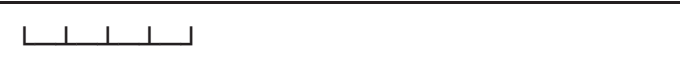 \\
\hline H49 & Have you received additional training since you graduated? & 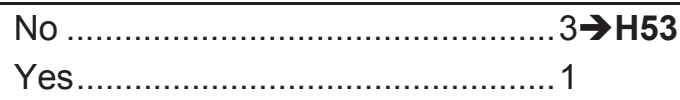 \\
\hline
\end{tabular}

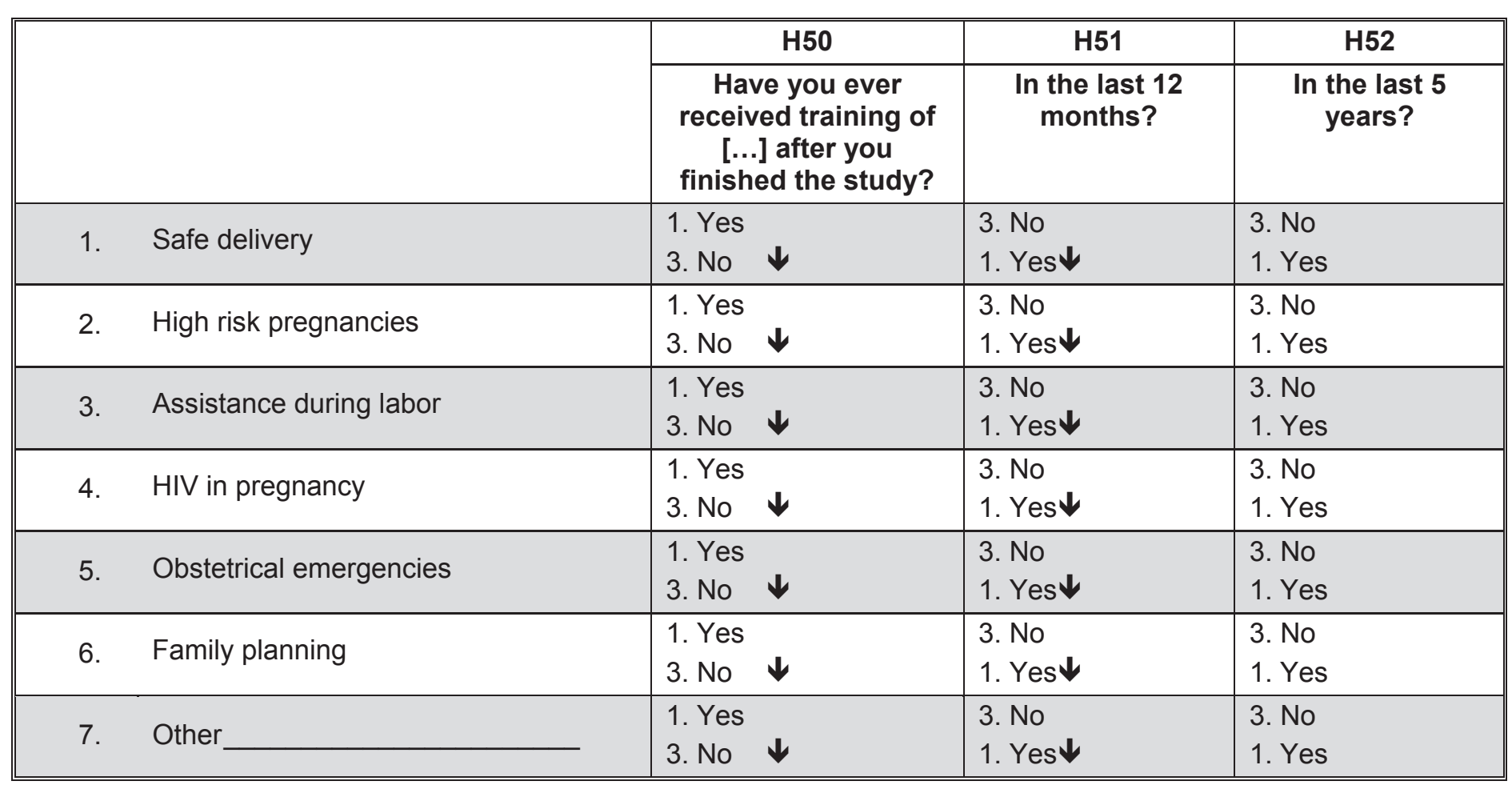

For the rest of the interview, we would like to understand the process by which you provide a pregnancy examination. We would like to know everything you do, beginning with the arrival of the patient, waiting upon the patient and ending when she goes home. I shall describe the patient, and I will ask you a series of questions about activities you perform regularly. Once a section is complete, we are unable to go back and change answers. Now I will read out the case

\section{INSTRUCTIONS TO INTERVIEWER:}

1. READ OUT CASE 2 TIMES, AND THEN ASK QUESTIONS H55 - H60.

2. FIRST LISTEN TO THE RESPONDENT. ANY RESPONSE THAT HE OR SHE MENTIONS SPONTANEOUSLY SHOULD BE MARKED WITH CODE 1.

3. AFTER THE RESPONDENT IS FINISHED WITH THE WHOLE QUESTIONNAIRE, TELL THE RESPONDENT THAT YOU ARE GOING TO REVIEW THE CASE AGAIN.

4. START FROM THE BEGINNING AND READ THE CASE A SECOND TIME. ASK QUESTIONS H55 - H60.

5. READ OUT ANY RESPONSES THAT HE/SHE DID NOT MENTION SPONTANEOUSLY THE FIRST TIME. CODE THE APPROPRIATE RESPONSE:

H54 Mrs. Ani, a married woman of 26, has not had her period for 3 months. She has come to you for a pregancy examination. This is her first visit. Please recount everything you would do during the pregnancy examination.. 


\begin{tabular}{|c|c|c|c|c|}
\hline H55 & $\begin{array}{l}\text { What are most important questions you would ask Mrs. } \\
\text { Ani about her previous pregnancies and labor? }\end{array}$ & $\begin{array}{l}\text { Mentioned } \\
\text { spontaneously }\end{array}$ & \multicolumn{2}{|c|}{ Prompted } \\
\hline & a. Number of prior pregnancies? & 1 & 2 & 3 \\
\hline & b. Number of living children & 1 & 2 & 3 \\
\hline & c. Number of miscarriages/abortions/stillbirths? & 1 & 2 & 3 \\
\hline & d. Any bleeding during previous labor? & 1 & 2 & 3 \\
\hline
\end{tabular}

\begin{tabular}{|c|c|c|c|c|}
\hline H55 & $\begin{array}{l}\text { What are most important questions you would ask } \\
\text { Mrs. Ani about her previous pregnancies and labor? }\end{array}$ & $\begin{array}{c}\text { Mentioned } \\
\text { spontaneously }\end{array}$ & \multicolumn{2}{|c|}{ Prompted } \\
\hline & e. How the last child was delivered? & 1 & 2 & 3 \\
\hline & f. Birth weight of previous child? & 1 & 2 & 3 \\
\hline & g. History of genetic anamolies? & 1 & 2 & 3 \\
\hline & $\begin{array}{l}\text { h. Gynecological history (STIs, pap smear, } \\
\text { contraceptive use, etc.) }\end{array}$ & 1 & 2 & 3 \\
\hline \multirow[t]{6}{*}{ H56 } & $\begin{array}{l}\text { What are themost important questions you ask Mrs. } \\
\text { Ani about her current pregnancy? }\end{array}$ & $\begin{array}{l}\text { Mentioned } \\
\text { spontaneously }\end{array}$ & \multicolumn{2}{|c|}{ Prompted } \\
\hline & a. Last menstrual date? & 1 & 2 & 3 \\
\hline & b. Any health problems now? & 1 & 2 & 3 \\
\hline & $\begin{array}{l}\text { c. Any obstetric symptoms (contractions, vaginal } \\
\text { bleeding, etc)? }\end{array}$ & 1 & 2 & 3 \\
\hline & d. Any weight loss/gain, nausea, vomiting? & 1 & 2 & 3 \\
\hline & e. Taking any medications now? & 1 & 2 & 3 \\
\hline \multirow[t]{15}{*}{ H57 } & $\begin{array}{l}\text { What are the most important questions you want to } \\
\text { ask about her medical and social/behavioral history? }\end{array}$ & $\begin{array}{c}\text { Mentioned } \\
\text { spontaneously }\end{array}$ & \multicolumn{2}{|c|}{ Prompted } \\
\hline & a. Any history of high blood pressure? & 1 & 2 & 3 \\
\hline & b. Any history of diabetes? & 1 & 2 & 3 \\
\hline & c. Any previous STI, including HIV+? & 1 & 2 & 3 \\
\hline & d. Any previous IUD or contraceptive use? & 1 & 2 & 3 \\
\hline & e. Tetanus shot in previous pregnancy? & 1 & 2 & 3 \\
\hline & f. Any previous heart disease? & 1 & 2 & 3 \\
\hline & g. Family history of hereditary disease? & 1 & 2 & 3 \\
\hline & h. Ever had malaria? & 1 & 2 & 3 \\
\hline & i. Present or previous smoker? & 1 & 2 & 3 \\
\hline & j. Any history of alcohol use? & 1 & 2 & 3 \\
\hline & k. Assess whether pregnancy is high risk? & 1 & 2 & 3 \\
\hline & I. Ever had surgery? & 1 & 2 & 3 \\
\hline & m. Any history of asthmatism? & 1 & 2 & 3 \\
\hline & n. Any history of kidney disease? & 1 & 2 & 3 \\
\hline H58 & $\begin{array}{l}\text { What would you do when you conduct a physical } \\
\text { examination of Mrs. Ani? }\end{array}$ & $\begin{array}{c}\text { Mentioned } \\
\text { spontaneously }\end{array}$ & \multicolumn{2}{|c|}{ Prompted } \\
\hline & a. Body height? & 1 & 2 & 3 \\
\hline & b. Body weight? & 1 & 2 & 3 \\
\hline & c. Take blood pressure? & 1 & 2 & 3 \\
\hline & d. Palpitate abdomen/measure uterine height? & 1 & 2 & 3 \\
\hline & e. Listen to fetal heartbeat? & 1 & 2 & 3 \\
\hline & $\begin{array}{l}\text { f. Pelvic examination? } \\
\text { *Note: internal examination }\end{array}$ & 1 & 2 & 3 \\
\hline & $\begin{array}{l}\text { g. Check for edema? } \\
\text { 'Note: swelling or 'odim' }\end{array}$ & 1 & 2 & 3 \\
\hline & h. Upper arm measurement & 1 & 2 & 3 \\
\hline & i. Facial appearance, paleornot & 1 & 2 & 3 \\
\hline
\end{tabular}




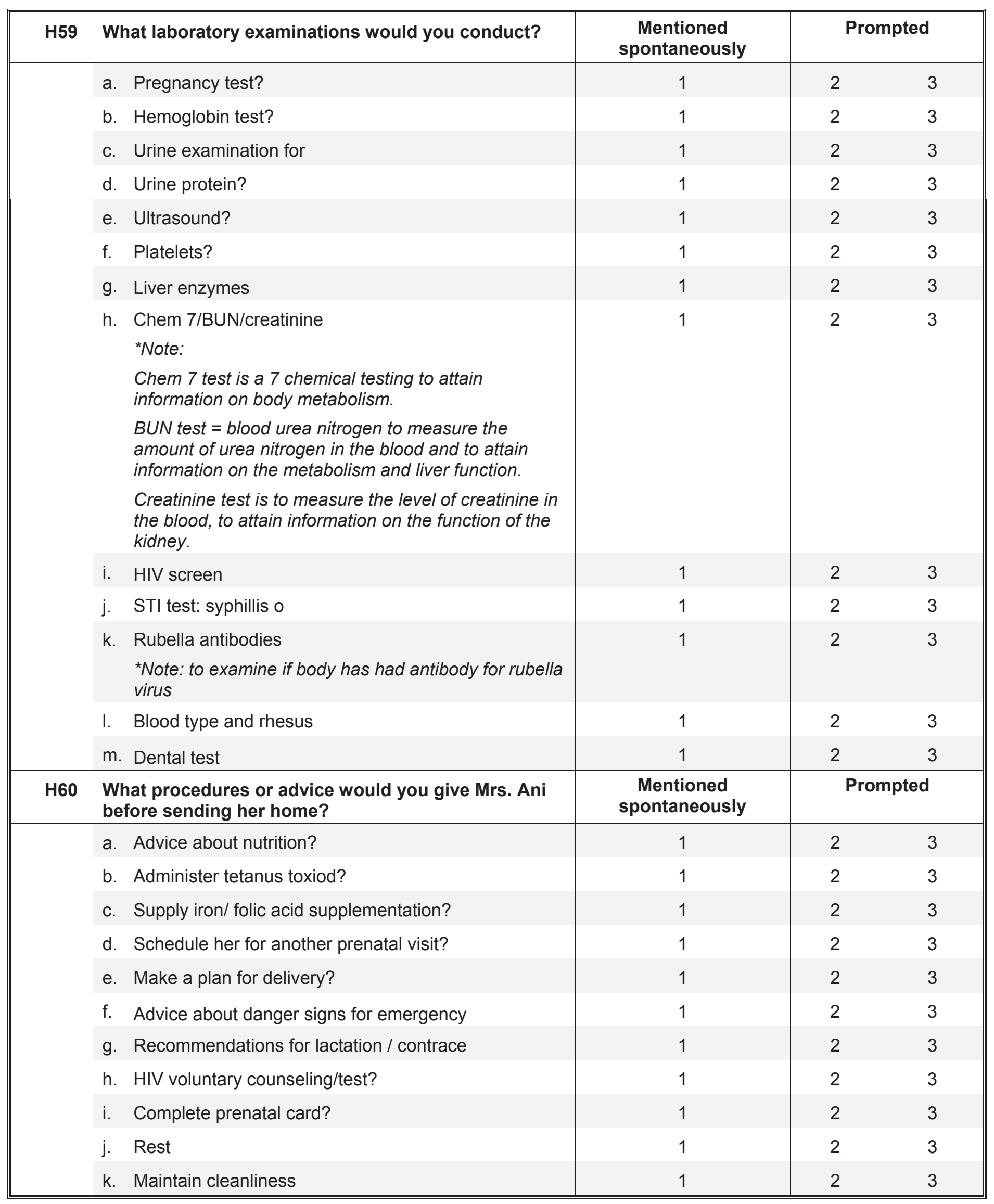




\section{SECTION H: FACILITY VIGNETTES}

\begin{tabular}{|c|c|c|c|c|c|c|c|}
\hline LANGMAIN (CK1). & \multicolumn{2}{|l|}{ Interview was entirely/mostly conducted in what language? } & \multicolumn{5}{|l|}{$\llcorner$ ـ other. } \\
\hline & Other languaged used (if any): & & \multicolumn{5}{|l|}{$\downarrow \downarrow \downarrow \downarrow \downarrow$ other } \\
\hline \multicolumn{8}{|l|}{ LANGUAGE CODE: } \\
\hline 00. Bahasa Indonesia & 04. Batak & 08. Sasak & 12. Makassar & 16. & Toraja & 20. & Lampung \\
\hline 01. Java & 05. Bugis & 09. Minang & 13. Nias & 17. & Lahat & 95. & Other, \\
\hline 02. Sunda & 06. Cina & 10. Banjar & 14. Palembang & 18. & Other South Sumatera & 96. & NA \\
\hline 03. Bali & 07. Madura & 11. Bima & 15. Sumbawa & 19. & Betawi & & \\
\hline
\end{tabular}

\begin{tabular}{|c|c|c|c|}
\hline RESULT (FP3). RESULTS OF INTERVIEW & $\begin{array}{l}\text { REASON (FP4). REASON FOR ANSWERING “2” / “3” IN } \\
\text { RESULT. }\end{array}$ & FP6. MONITORING BY SUPERVISOR & \\
\hline $\begin{array}{l}\text { 1. Completed } \rightarrow \text { FP6 } \\
\text { 2. Partly completed } \\
\text { 3. Not completed } \\
\text { 4. Twin EA with EA } \downarrow \perp \neg \rightarrow \text { LP6 }\end{array}$ & $\begin{array}{l}\text { 1. Respondent was travelling/not in location } \\
\text { 2. Respondent was too busy } \\
\text { 3. Respondent refused }\end{array}$ & $\begin{array}{ll} & \\
\text { a. } & \text { Observed (sup_obs) ......... } 1 \\
\text { b. } & \text { Checked (sup_edit)........... } 1 \\
\text { c. } & \text { Verified (sup_veri).............. } 1\end{array}$ & $\begin{array}{c}\text { No } \\
3 \\
3 \\
3\end{array}$ \\
\hline
\end{tabular}

INTERVIEWER NOTE: 


\begin{tabular}{|c|c|c|c|}
\hline INTERVIEWER: & 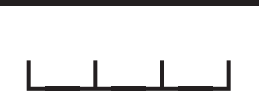 & CONFIDENTIAL & IDW: \\
\hline
\end{tabular}

\section{INDONESIAN FAMILY LIFE SURVEY 2014 HEALTH FACILITY COMMUNITY HEALTH CENTER/SUB-CENTER (PUSKESMAS / PUSKESMAS PEMBANTU) BOOK B}

SECTIONS : B, C, E, F, G, CP

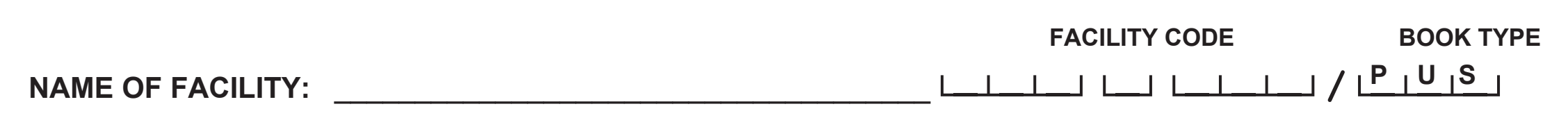


SECTION B : DEVELOPMENT OF PUSKESMAS/PUSKESMAS PEMBANTU

Now, we would like to ask about the development of the puskesmas/puskesmas pembantu RESPONDENT IS A PUSKESMAS EMPLOYEE WHO HAS WORKED AT THIS PUSKESMAS FOR A LONG TIME

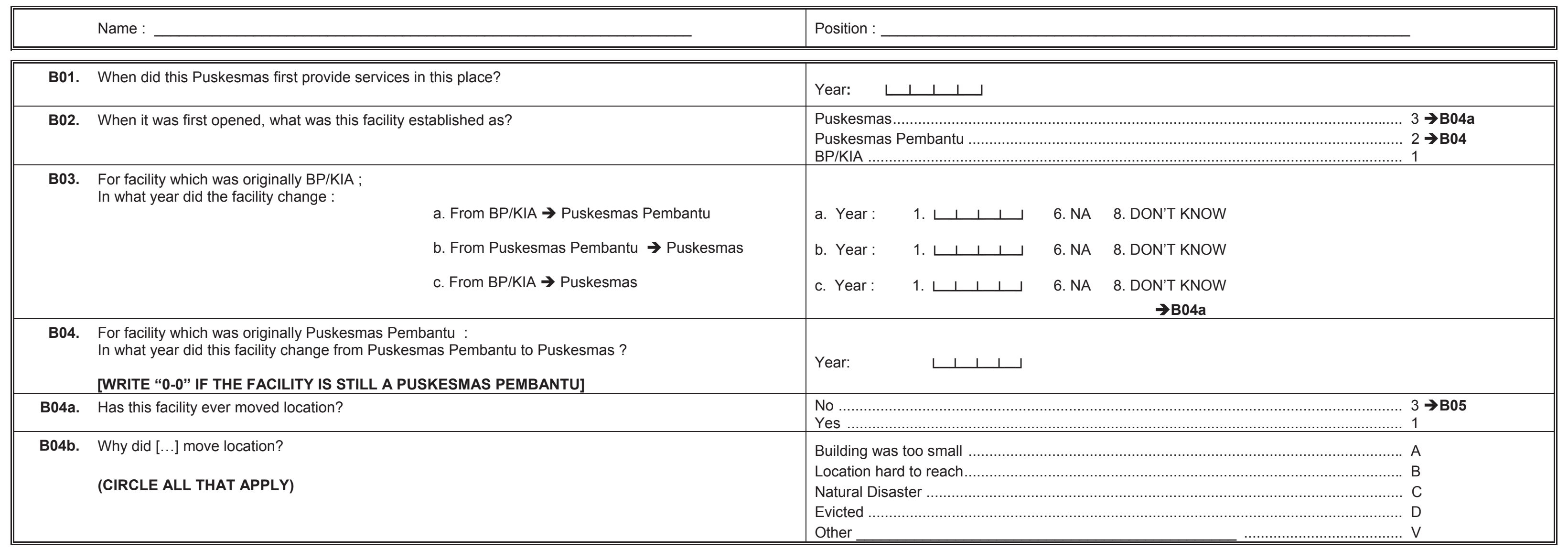


SECTION B : DEVELOPMENT OF PUSKESMAS/PUSKESMAS PEMBANTU

Now, we would like to ask about the development of the puskesmas/puskesmas pembantu

\begin{tabular}{|c|c|c|c|c|c|c|c|c|c|c|c|c|c|c|c|c|}
\hline $\begin{array}{l}\text { B05. In the year } \\
{[\ldots] \text {, do you }} \\
\text { have }[\ldots . .] \text { for } \\
\text { more than } 6 \\
\text { months? }\end{array}$ & B06. & B07. & B08a. & B08b. & B09. & B9a. & B10. & B10a. & B11. & B11a. & B12. & B13. & B14. & B14a. & B14b. & B14c. \\
\hline (BTYPE) & Laboratory & $\begin{array}{l}\text { Medicine } \\
\text { room }\end{array}$ & $\begin{array}{l}\text { General } \\
\text { practitioner }\end{array}$ & $\begin{array}{l}\text { Specialist } \\
\text { doctor }\end{array}$ & Dentist & Midwife & $\begin{array}{l}\text { Puskesmas } \\
\text { Pembantu }\end{array}$ & Polindes & Posyandu & $\begin{array}{l}\text { Posyandu } \\
\text { for elderly } \\
\text { (Posyandu } \\
\text { Lansia) }\end{array}$ & $\begin{array}{c}\text { Mobile } \\
\text { Puskesmas }\end{array}$ & $\begin{array}{l}\text { Inpatient } \\
\text { Facility }\end{array}$ & $\begin{array}{c}\text { Birth- } \\
\text { Delivery }\end{array}$ & $\begin{array}{l}\text { Sterilization } \\
\text { Service for } \\
\text { Male }\end{array}$ & $\begin{array}{c}\text { Sterilization } \\
\text { Service for } \\
\text { Female }\end{array}$ & $\begin{array}{c}\text { Mass } \\
\text { Immunization } \\
\text { for TT }\end{array}$ \\
\hline c. 2014 & $\begin{array}{l}\text { 1. Yes } \\
\text { 3. No }\end{array}$ & $\begin{array}{l}\text { 1. Yes } \\
\text { 3. No }\end{array}$ & $\begin{array}{l}\text { 1. Yes } \\
\text { 3. No }\end{array}$ & $\begin{array}{l}\text { 1. Yes } \\
\text { 3. No }\end{array}$ & $\begin{array}{l}\text { 1. Yes } \\
\text { 3. No }\end{array}$ & $\begin{array}{l}\text { 1. Yes } \\
\text { 3. No }\end{array}$ & $\begin{array}{l}\text { 1. Yes } \\
\text { 3. No } \\
\text { 6. NA }\end{array}$ & $\begin{array}{l}\text { 1. Yes } \\
\text { 3. No }\end{array}$ & $\begin{array}{l}\text { 1. Yes } \\
\text { 3. No }\end{array}$ & $\begin{array}{l}\text { 1. Yes } \\
\text { 3. No }\end{array}$ & $\begin{array}{l}\text { 1. Yes } \\
\text { 3. No }\end{array}$ & $\begin{array}{l}\text { 1. Yes } \\
\text { 3. No }\end{array}$ & $\begin{array}{l}\text { 1. Yes } \\
\text { 3. No }\end{array}$ & $\begin{array}{l}\text { 1. Yes } \\
\text { 3. No }\end{array}$ & $\begin{array}{l}\text { 1. Yes } \\
\text { 3. No }\end{array}$ & $\begin{array}{l}\text { 1. Yes } \\
\text { 3. No }\end{array}$ \\
\hline
\end{tabular}

\begin{tabular}{|c|c|c|}
\hline B15. & $\begin{array}{l}\text { Is there electricity at this } \\
\text { Puskesmas? }\end{array}$ & $\begin{array}{l}\text { No } \\
\text { Yes }\end{array}$ \\
\hline B16. & $\begin{array}{l}\text { If there is, please state the electricity } \\
\text { source used: }\end{array}$ & 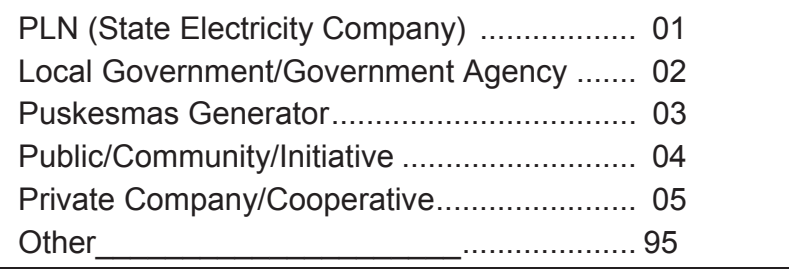 \\
\hline B17. & $\begin{array}{l}\text { Mention the main water sources } \\
\text { used: }\end{array}$ & 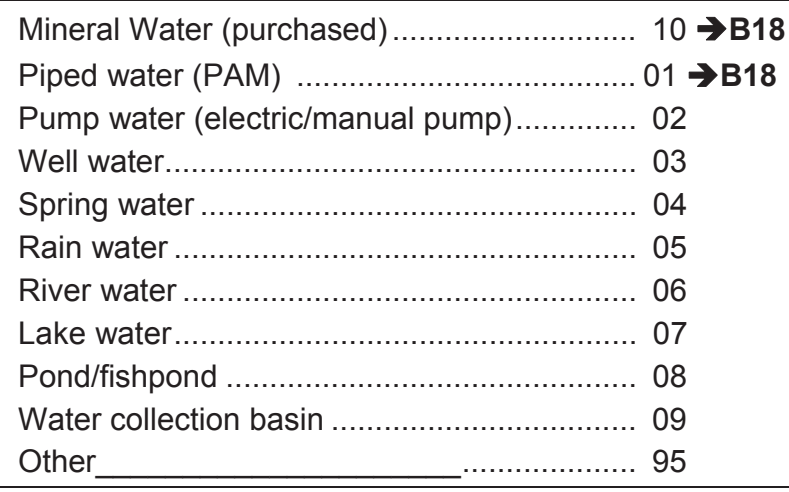 \\
\hline B17a. & $\begin{array}{l}\text { Is this }[\ldots] \text { water transported by } \\
\text { pipes? }\end{array}$ & $\begin{array}{l}\text { No } \\
\text { Yes }\end{array}$ \\
\hline B18. & $\begin{array}{l}\text { Is this water source in the } \\
\text { Puskesmas building? }\end{array}$ & $\begin{array}{l}\text { Yes } \\
\text { No }\end{array}$ \\
\hline B19. & $\begin{array}{l}\text { If it is not inside, how far is it from the } \\
\text { Puskesmas? }\end{array}$ & $\begin{array}{l}\text { Less than } 10 \text { meters } \\
10-30 \text { meters } \\
\text { more than } 30 \text { meters }\end{array}$ \\
\hline
\end{tabular}

\begin{tabular}{|c|c|c|}
\hline B19a. & Does this facility have a toilet? & $\begin{array}{l}\text { No } \\
\text { Yes }\end{array}$ \\
\hline B20. & Mention the toilet facilities used: & 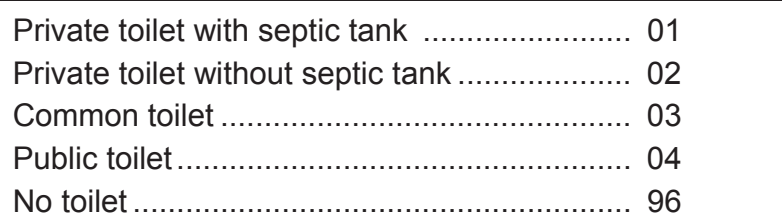 \\
\hline B20a. & $\begin{array}{l}\text { What is the waste water disposal } \\
\text { system at this facility? }\end{array}$ & 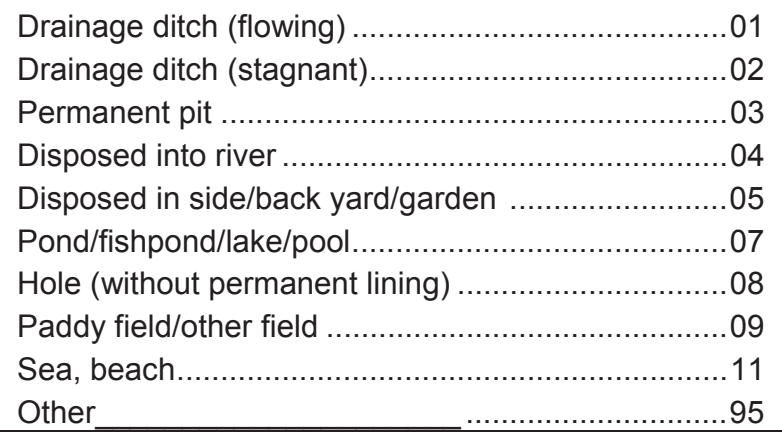 \\
\hline B20b. & $\begin{array}{l}\text { What is the solid waste disposal } \\
\text { system at this facility? }\end{array}$ & $\begin{array}{l}\text { Disposed in trash can, collected by } \\
\text { sanitation service } \\
\text { Burned } \\
\text { Disposed into river/creek/sewer } \\
\text { Disposed in yard and let decompose } \\
\text { Disposed in pit } \\
\text { Forest, mountain } \\
\text { Sea, lake, beach } \\
\text { Paddy field/other field } \\
\text { Other }\end{array}$ \\
\hline
\end{tabular}


SECTION B : DEVELOPMENT OF PUSKESMAS/PUSKESMAS PEMBANTU

\begin{tabular}{|c|c|c|}
\hline \multirow[t]{11}{*}{ B20c. Does this facility have $[\ldots]$ ? } & 1.Yes & 3. No \\
\hline & a. Registration table/booth .................. 1 & 3 \\
\hline & b. Patient waiting room & 3 \\
\hline & c. Check up room ……………….... & 3 \\
\hline & d. Injection room & 3 \\
\hline & e. FP/MCH consultation room ............. 1 & 3 \\
\hline & f. FP service room …………........ 1 & 3 \\
\hline & g. Delivery room & 3 \\
\hline & h. Inpatient room & 3 \\
\hline & 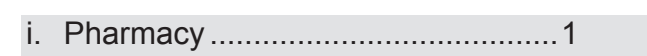 & 3 \\
\hline & j. Laboratory ……………………....... & 3 \\
\hline
\end{tabular}


SECTION C: ACTIVITIES OF PUSKESMAS/PUSKESMAS PEMBANTU

\begin{tabular}{|c|c|c|c|c|}
\hline C01. & C02a. & C03a. & C02b. & C03b. \\
\hline When is the Puskesmas open? On [...] days & Opening Hours Counter & Closing Hours Counter & Opening Hours of service & Closing Hours of service \\
\hline 1. Monday.. & 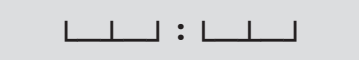 & 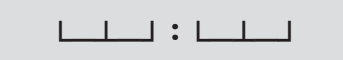 & 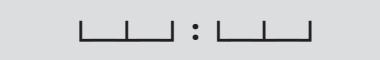 & 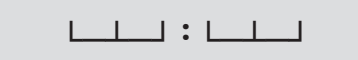 \\
\hline 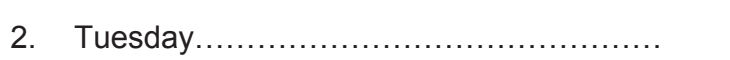 & 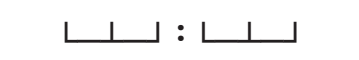 & 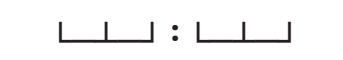 & لـــــــــــــــــــ & 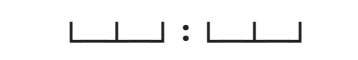 \\
\hline 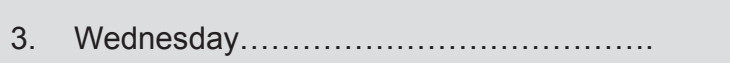 & $\downarrow$ & 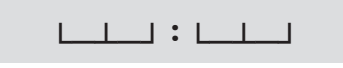 & $\downarrow$ & $\downarrow$ \\
\hline 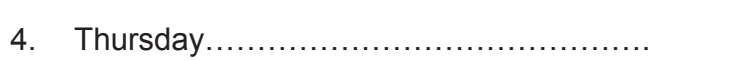 & 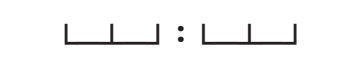 & 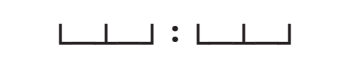 & 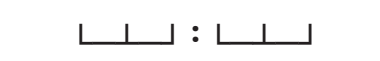 & 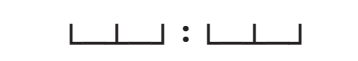 \\
\hline 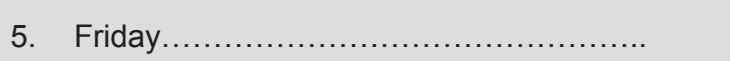 & 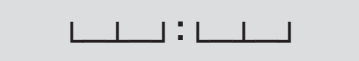 & 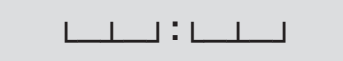 & 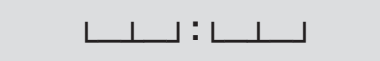 & 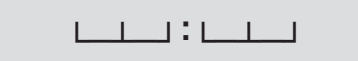 \\
\hline
\end{tabular}

C04. What is the registration fee?

C04a What is the registration fee for patients with jamkesmas card?

\begin{tabular}{|c|c|}
\hline $\begin{array}{l}\text { First visits....... } \\
\text { Repeat visits .. }\end{array}$ & 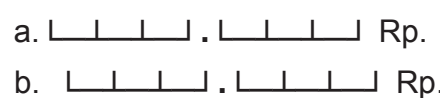 \\
\hline First visits ......... & 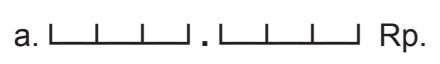 \\
\hline Repeat visits .. & 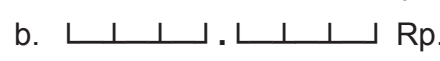 \\
\hline
\end{tabular}

Service in the building

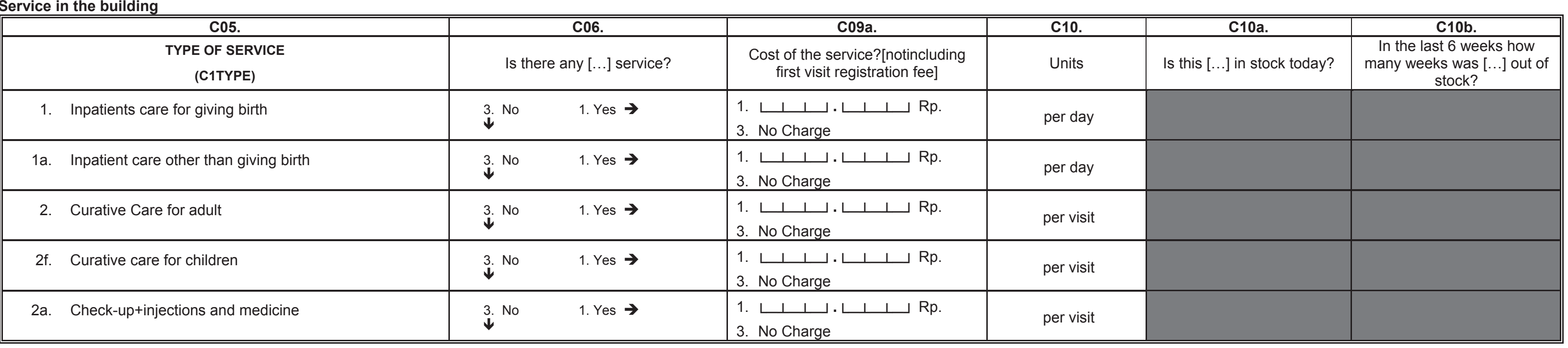


SECTION C: ACTIVITIES OF PUSKESMAS/PUSKESMAS PEMBANTU

\begin{tabular}{|c|c|c|c|c|c|}
\hline C05. & C06. & C09a. & C10. & C10a. & C10b. \\
\hline $\begin{array}{l}\text { TYPE OF SERVICE } \\
\text { (C1TYPE) }\end{array}$ & Is there any $[\ldots]$ service? & $\begin{array}{l}\text { Cost of the service?[notincluding } \\
\text { first visit registration fee] }\end{array}$ & Units & Is this $[\ldots]$ in stock today? & $\begin{array}{c}\text { In the last } 6 \text { weeks how } \\
\text { many weeks was }[\ldots] \text { out of } \\
\text { stock? }\end{array}$ \\
\hline \multicolumn{6}{|l|}{ 3. Stitching of wounds: } \\
\hline a. First stitch & $\begin{array}{l}\text { 3. No } 1 \text {. Yes } \rightarrow \\
\downarrow\end{array}$ & 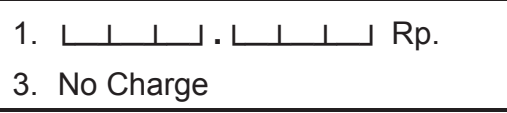 & per stitch & & \\
\hline b. Additional stitches & 1. Yes $\rightarrow$ & 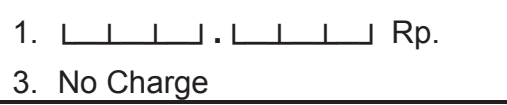 & per stitch & & \\
\hline 4. Changing of wound dressing & 1. Yes $\rightarrow$ & 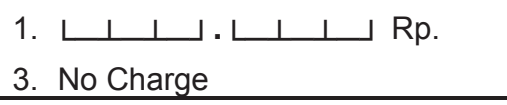 & per visit & & \\
\hline 5. Incision of abscess/piercing of boils & 1. Yes $\rightarrow$ & 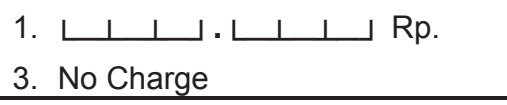 & per action & & \\
\hline 6. Circumcisions & 1. Yes $\rightarrow$ & $\begin{array}{l}\text { 1. } \downarrow \perp \perp \_. \downarrow \perp \_\_ \text {Rp. } \\
\text { 3. No Charge }\end{array}$ & per time & & \\
\hline 7. Medical treatment for tuberculoses [TBC] & 1. Yes $\rightarrow$ & 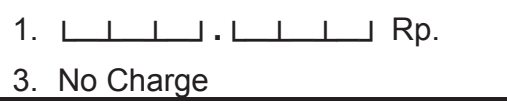 & per visit & & \\
\hline 8. Check up/health examination & 1. Yes $\rightarrow$ & 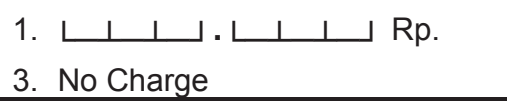 & per visit & & \\
\hline 9. Dental exam & 1. Yes $\rightarrow$ & 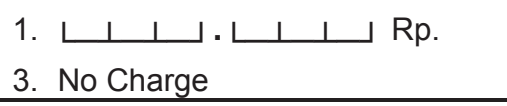 & per exam & & \\
\hline 10. Prenatal care & 1. Yes $\rightarrow$ & 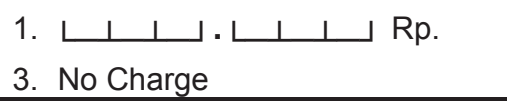 & per exam & & \\
\hline 11. Aid for childbirth & 1. Yes $\rightarrow$ & 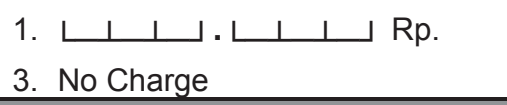 & per delivery & & \\
\hline 42. Complication of pregnancy & 1. Yes & & & & \\
\hline 43. Complication of childbirth & 1. Yes & & & & \\
\hline 44. Complication of postnatal & 1. Yes & & & & \\
\hline 45. Postnatal care (post childbirth) for at least 3 times & 1. Yes & & & & \\
\hline
\end{tabular}


SECTION C: ACTIVITIES OF PUSKESMAS/PUSKESMAS PEMBANTU

\begin{tabular}{|c|c|c|c|c|c|c|c|}
\hline \multicolumn{2}{|r|}{ C05. } & \multicolumn{2}{|r|}{ 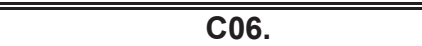 } & \multirow{2}{*}{$\begin{array}{c}\text { C09a. } \\
\begin{array}{l}\text { Cost of the service?[notincluding } \\
\text { first visit registration fee] }\end{array}\end{array}$} & \multirow{2}{*}{$\begin{array}{l}\text { C10. } \\
\text { Units }\end{array}$} & \multirow{2}{*}{$\begin{array}{c}\text { C10a. } \\
\text { Is this }[\ldots] \text { in stock today? }\end{array}$} & \multirow{2}{*}{$\begin{array}{c}\text { C10b. } \\
\text { In the last } 6 \text { weeks how } \\
\text { many weeks was [...] out of } \\
\text { stock? }\end{array}$} \\
\hline & $\begin{array}{c}\text { TYPE OF SERVICE } \\
\text { (C1TYPE) }\end{array}$ & \multicolumn{2}{|c|}{ Is there any $[\ldots]$ service? } & & & & \\
\hline 46. & $\begin{array}{l}\text { Neonatal check up (newborn } 0 \text { to } 28 \text { days old) with } \\
\text { complication }\end{array}$ & 3. No & 1. Yes & & & & \\
\hline 47. & Post-natal service ( 29 days to 11 months old) & 3. No & 1. Yes & & & & \\
\hline 48. & $\begin{array}{l}\text { Growth and development monitoring for for babies } \\
\text { under } 5 \text { years old }\end{array}$ & 3. No & 1. Yes & & & & \\
\hline 49. & $\begin{array}{l}\text { Additional nutrition aside from breast milk distribution } \\
\text { for babies aged } 6-24 \text { months of poor families }\end{array}$ & 3. No & 1. Yes & & & & \\
\hline 50. & Treatment for malnutrition of under-five children & 3. No & 1. Yes & & & & \\
\hline \multicolumn{8}{|c|}{ 15. Supply of Oral Contraceptives: } \\
\hline & a. Microgynon30 [PT Schering] & 3. No & 1. Yes $\rightarrow$ & 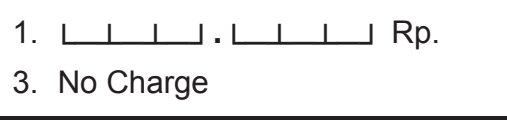 & Per Strip & $\begin{array}{l}\text { Yes } \ldots \ldots \ldots \ldots \ldots \ldots \ldots \ldots \ldots \ldots \ldots \ldots \ldots \ldots \ldots \\
\text { No }\end{array}$ & 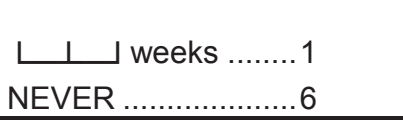 \\
\hline & b. Marvelon 28 & 3. No & 1. Yes $\rightarrow$ & 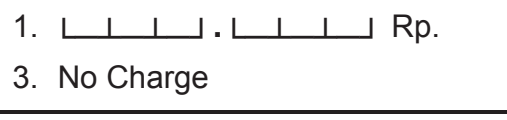 & Per Strip & 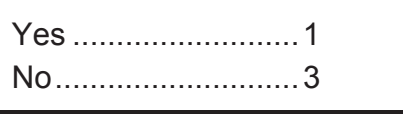 & 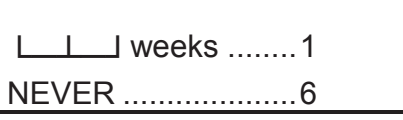 \\
\hline & c. Excluton 28 & 3. No & 1. Yes $\rightarrow$ & 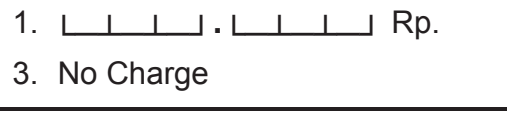 & Per Strip & $\begin{array}{l}\text { Yes } \ldots \ldots \ldots \ldots \ldots \ldots \ldots \ldots \ldots \ldots \ldots \ldots \ldots \ldots \ldots \\
\text { No }\end{array}$ & 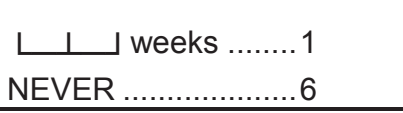 \\
\hline & f. Pil KB Andalan/BKKBN & 3. No & 1. Yes $\rightarrow$ & 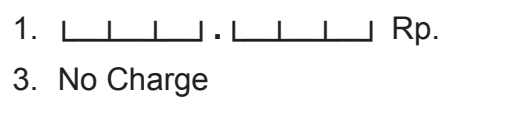 & Per Strip & $\begin{array}{l}\text { Yes } \ldots \ldots \ldots \ldots \ldots \ldots \ldots \ldots \ldots \ldots \ldots \ldots \ldots \ldots \ldots \\
\text { No }\end{array}$ & 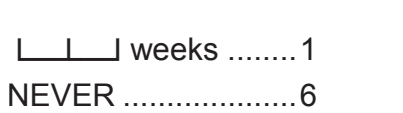 \\
\hline
\end{tabular}


SECTION C: ACTIVITIES OF PUSKESMAS/PUSKESMAS PEMBANTU

\begin{tabular}{|c|c|c|c|c|c|}
\hline 005. & C06. & C09a. & C10. & C10a. & C10b. \\
\hline $\begin{array}{c}\text { TYPE OF SERVICE } \\
\text { (C1TYPE) }\end{array}$ & Is there any $[\ldots]$ service? & $\begin{array}{l}\text { Cost of the service?[notincluding } \\
\text { first visit registration fee] }\end{array}$ & Units & Is this $[\ldots]$ in stock today? & $\begin{array}{c}\text { In the last } 6 \text { weeks how } \\
\text { many weeks was }[\ldots] \text { out of } \\
\text { stock? }\end{array}$ \\
\hline \multicolumn{6}{|l|}{ 17. IUD Copper T } \\
\hline a. Insertion & 1. Yes $\rightarrow$ & 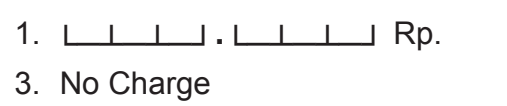 & $\begin{array}{l}\text { Per visit for one time } \\
\text { insertion }\end{array}$ & 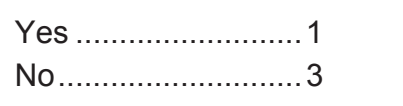 & 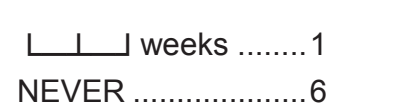 \\
\hline b. Removal & 1. Yes $\rightarrow$ & 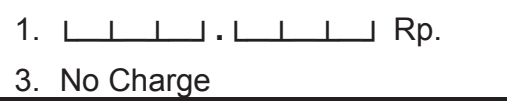 & $\begin{array}{l}\text { Per visit for one time } \\
\text { removal }\end{array}$ & & \\
\hline \multicolumn{6}{|l|}{ 18. Contraceptive injection } \\
\hline a. Depo-Provera & 3. No & $\begin{array}{l}\text { 1. } \downarrow \perp \perp \_. \perp \perp \perp \_ \text {Rp. } \\
\text { 3. No Charge } \\
\text { 6. Bring their own }\end{array}$ & Per injection & 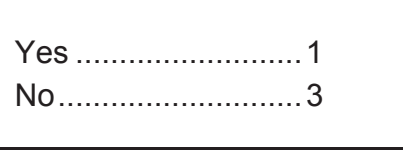 & 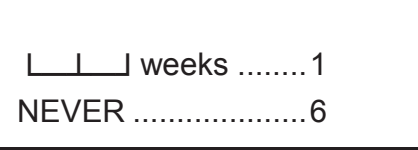 \\
\hline b. Depo- Progestin/Andalan & 3. No & $\begin{array}{l}\text { 1. } \perp \perp \perp \perp . \perp \perp \perp \perp \text { Rp. } \\
\text { 3. No Charge } \\
\text { 6. Bring their own }\end{array}$ & Per injection & 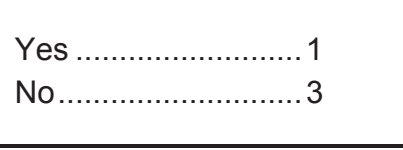 & 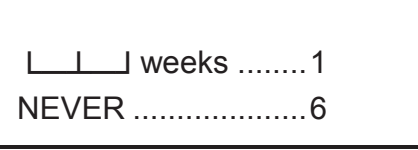 \\
\hline d. Cyclofeem & 3. No & 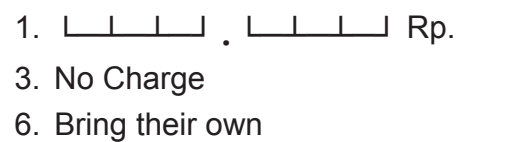 & Per injection & 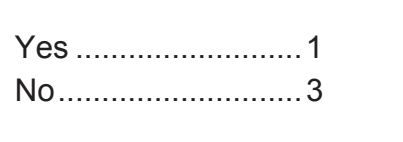 & 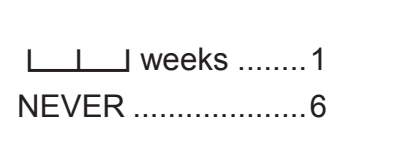 \\
\hline
\end{tabular}

\begin{tabular}{|c|c|c|c|c|c|c|c|}
\hline \multirow{2}{*}{\multicolumn{2}{|c|}{$\begin{array}{c}05 . \\
\text { TYPE OF SERVICE } \\
\text { (C1TYPE) }\end{array}$}} & \multirow{2}{*}{\multicolumn{2}{|c|}{ Is there any $[\ldots]$ service? }} & \multirow{2}{*}{$\begin{array}{l}\text { C09a. } \\
\text { Cost of the service?[notincluding } \\
\text { first visit registration fee] }\end{array}$} & \multirow{2}{*}{$\begin{array}{l}\text { C10. } \\
\text { Units }\end{array}$} & C10a. & $\begin{array}{l}\text { C10b. } \\
\end{array}$ \\
\hline & & & & & & Is this $[\ldots]$ in stock today? & $\begin{array}{c}\text { In the last } 6 \text { weeks how } \\
\text { many weeks was }[\ldots] \text { out of } \\
\text { stock? }\end{array}$ \\
\hline \multicolumn{8}{|c|}{ 19a. Norplant } \\
\hline & a. Insertion & 3. No & 1. Yes $\rightarrow$ & $\begin{array}{l}\text { 1. } \downarrow \perp \perp \_. \sqcup \perp \perp \perp \_ \text {Rp. } \\
\text { 3. No Charge }\end{array}$ & $\begin{array}{l}\text { Per vist for one time } \\
\text { insertion }\end{array}$ & $\begin{array}{l}\text { Yes } \ldots \ldots \ldots \ldots \ldots \ldots \ldots \ldots \ldots \ldots \\
\text { No } \ldots \ldots \ldots \ldots \ldots \ldots \ldots \ldots \ldots \ldots\end{array}$ & 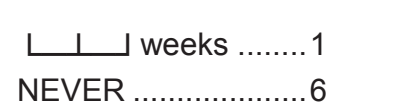 \\
\hline & b. Removal & 3. No & 1. Yes $\rightarrow$ & $\begin{array}{l}\text { 1. } \downarrow \perp \perp \_. \downarrow \perp \perp \_ \text {Rp. } \\
\text { 3. No Charge }\end{array}$ & $\begin{array}{l}\text { Per visit for one time } \\
\text { removal }\end{array}$ & & \\
\hline & c. Insertion of Implanon & 3. No & 1. Yes $\rightarrow$ & $\begin{array}{l}\text { 1. } \downarrow \perp \perp \_\cdot \downarrow \perp \_\_ \text {Rp. } \\
\text { 3. No Charge }\end{array}$ & $\begin{array}{l}\text { Per visit for one time } \\
\text { insertion }\end{array}$ & $\begin{array}{l}\text { Yes } \\
\text { No } \ldots \ldots \ldots \ldots \ldots \ldots \ldots \ldots \ldots \ldots \ldots \\
\end{array}$ & $\underset{\text { NEVER }}{\downarrow}$. \\
\hline & d. Removal of Implanon & 3. No & 1. Yes $\rightarrow$ & 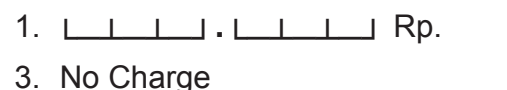 & $\begin{array}{l}\text { Per visit for one time } \\
\text { removal }\end{array}$ & & \\
\hline
\end{tabular}


SECTION C: ACTIVITIES OF PUSKESMAS/PUSKESMAS PEMBANTU

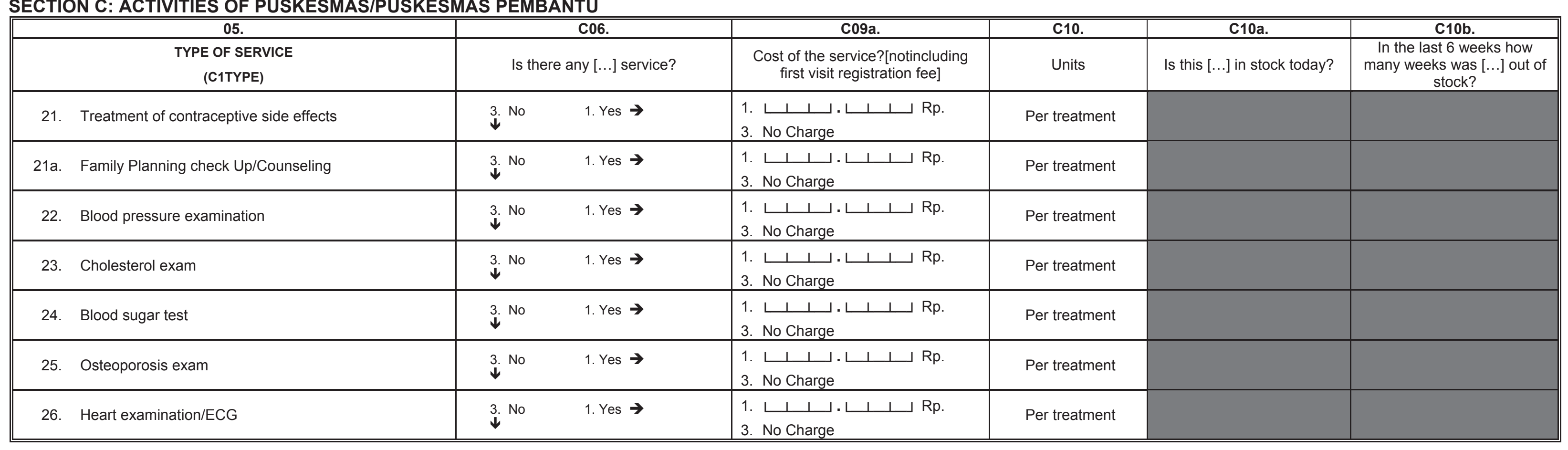


SECTION C: ACTIVITIES OF PUSKESMAS/PUSKESMAS PEMBANTU

\begin{tabular}{|c|c|c|c|c|c|c|c|}
\hline \multicolumn{2}{|r|}{ C05. } & \multicolumn{2}{|r|}{ C06. } & \multirow{2}{*}{$\begin{array}{c}\text { C09. } \\
\text { Cost of the service? } \\
\text { [including first visit registration fee] }\end{array}$} & C10. & C10a. & \multirow{2}{*}{$\begin{array}{c}\text { C10b. } \\
\text { In the last } 6 \text { weeks how } \\
\text { many weeks was }[\ldots] \text { out of } \\
\text { stock? }\end{array}$} \\
\hline & $\begin{array}{l}\text { TYPE OF SERVICE } \\
\text { (C1TYPE) } \\
\end{array}$ & & ny $[\ldots]$ service? & & Units & Is this $[\ldots]$ in stock today? & \\
\hline 27. & $\begin{array}{l}\text { Antibiotic } \\
\text { a. Oral antibiotic (most commonly prescribed) }\end{array}$ & 3. No & 1. Yes $\rightarrow$ & 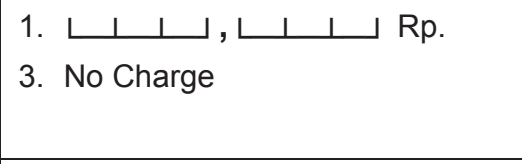 & $\begin{array}{l}\text { 1. Strip } \\
\text { 2. Bottle } \\
\text { 3. Ampule } \\
\text { 4. Seeds/Grains } \\
\end{array}$ & 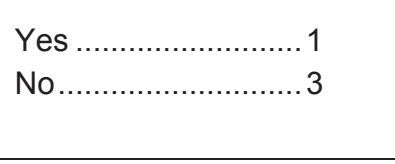 & 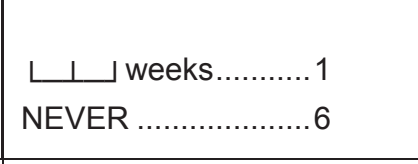 \\
\hline & o. Eye oinment Antibiotics & $\stackrel{3 .}{\downarrow}$ No & 1. Yes $\rightarrow$ & 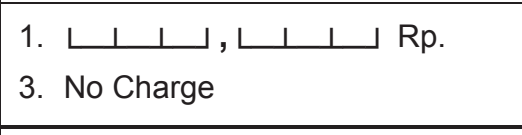 & Tube & 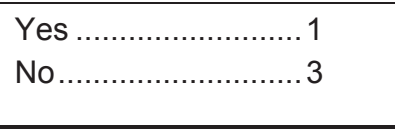 & $\begin{array}{c}\sqcup \downarrow \text { weeks............ } 1 \\
\text { NEVER ................... }\end{array}$ \\
\hline & Analgetic (e.g. ibuprofen) & 3. No & 1. Yes $\rightarrow$ & $\begin{array}{l}\text { 1. } \downarrow \perp \perp \_, \downarrow \perp \perp \perp \_ \text {Rp. } \\
\text { 3. No Charge }\end{array}$ & $\begin{array}{l}\text { 1. Strip } \\
\text { 2. Bottle } \\
\text { 3. Ampule } \\
\text { 4. Seeds/Grains }\end{array}$ & 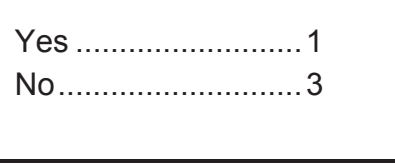 & $\begin{array}{c}\sqcup \sqcup \text { weeks............ } \\
\text { NEVER } \ldots \ldots \ldots \ldots \ldots \ldots . . .6\end{array}$ \\
\hline 29. & Antipyretic (e.g. acetosal, paracetamol) & 3. No & 1. Yes $\rightarrow$ & 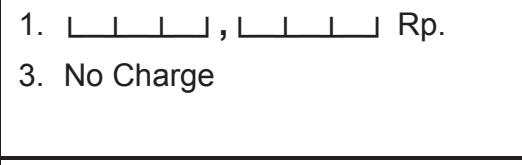 & $\begin{array}{l}\text { 1. Strip } \\
\text { 2. Bottle } \\
\text { 3. Ampule } \\
\text { 4. Seeds/Grains } \\
\end{array}$ & 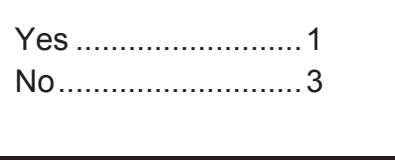 & $\begin{array}{c}\sqcup \sqcup \text { weeks........... } 1 \\
\text { NEVER } \ldots \ldots \ldots \ldots \ldots \ldots \ldots . . .6\end{array}$ \\
\hline & Anti fungi (e.g Nystatin) & 3. No & 1. Yes $\rightarrow$ & 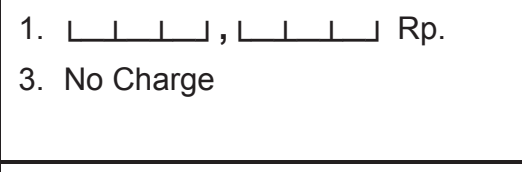 & $\begin{array}{l}\text { 1. Strip } \\
\text { 2. Bottle } \\
\text { 3. Ampule } \\
\text { 4. Seeds/Grains } \\
\end{array}$ & 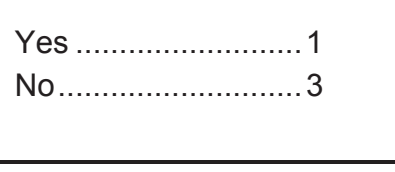 & $\begin{array}{c}\sqcup \sqcup \text { weeks........... } 1 \\
\text { NEVER } \ldots \ldots \ldots \ldots \ldots \ldots \ldots . . .6\end{array}$ \\
\hline 31. & Antihelminth (e.g. Mebendazole) & 3. No & 1. Yes $\rightarrow$ & 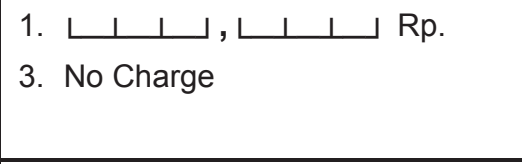 & $\begin{array}{l}\text { 1. Strip } \\
\text { 2. Bottle } \\
\text { 3. Ampule } \\
\text { 4. Seeds/Grains } \\
\end{array}$ & 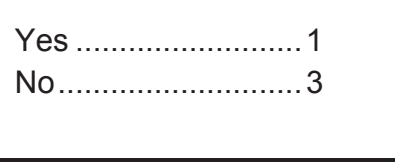 & 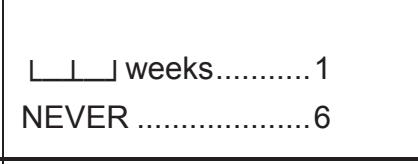 \\
\hline 32. & $\begin{array}{l}\text { Anti -TBC (short-term, e.g. Rifampicin, Ethambutol, } \\
\text { Isoniazid/INH }\end{array}$ & 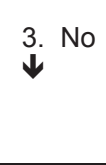 & 1. Yes $\rightarrow$ & 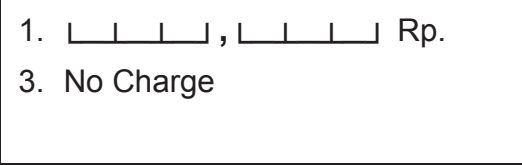 & $\begin{array}{l}\text { 1. Strip } \\
\text { 2. Bottle } \\
\text { 3. Ampule } \\
\text { 4. Seeds/Grains }\end{array}$ & $\begin{array}{l}\text { Yes } \ldots \ldots \ldots \ldots \ldots \ldots \ldots \ldots \ldots \ldots \\
\text { No } \ldots \ldots \ldots \ldots \ldots \ldots \ldots \ldots \ldots\end{array}$ & 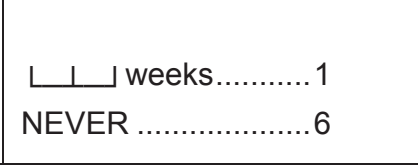 \\
\hline
\end{tabular}


SECTION C: ACTIVITIES OF PUSKESMAS/PUSKESMAS PEMBANTU

\begin{tabular}{|c|c|c|c|c|c|c|}
\hline C05. & & C06. & C09. & C10. & C10a. & C10b. \\
\hline $\begin{array}{l}\text { TYPE OF SERVICE } \\
\text { (C1TYPE) }\end{array}$ & & ny $[\ldots]$ service? & $\begin{array}{l}\text { Cost of the service? } \\
\text { [including first visit registration fee] }\end{array}$ & Units & Is this $[\ldots]$ in stock today? & $\begin{array}{c}\text { In the last } 6 \text { weeks how } \\
\text { many weeks was }[\ldots] \text { out of } \\
\text { stock? }\end{array}$ \\
\hline 33. Anti malaria (e.g Chloroquine, Sulfadoxine) & 3. No & 1. Yes $\rightarrow$ & $\begin{array}{l}\text { 1. } \sqcup \perp \perp \perp, \downarrow \perp \perp \perp \_ \text {Rp. } \\
\text { 3. No Charge }\end{array}$ & $\begin{array}{l}\text { 1. Strip } \\
\text { 2. Bottle } \\
\text { 3. Ampule } \\
\text { 4. Seeds/Grains }\end{array}$ & 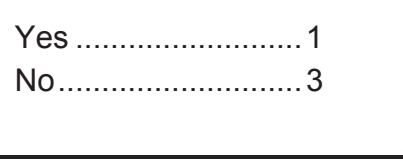 & 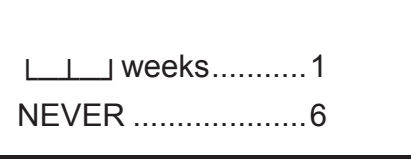 \\
\hline 34. Oralit & 3. No & 1. Yes $\rightarrow$ & $\begin{array}{l}\text { 1. } \downarrow \perp \perp \_, \downarrow \perp \perp \_\_ \text {Rp. } \\
\text { 3. No Charge }\end{array}$ & Per Sachet & 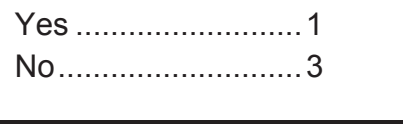 & $\begin{array}{l}\sqcup \dashv \text { weeks............ } 1 \\
\text { NEVER ..................6 }\end{array}$ \\
\hline 35. Iron tablets / FESO4 & 3. No & 1. Yes $\rightarrow$ & 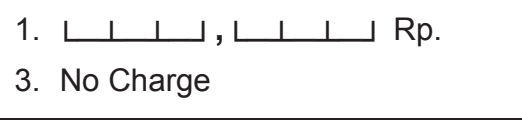 & Per Strip & 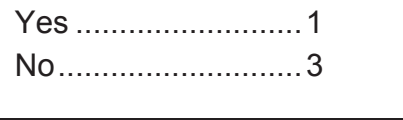 & $\begin{array}{l}\sqcup ل \text { weeks............. } 1 \\
\text { NEVER .................. } 6\end{array}$ \\
\hline 36. Vitamin A & 3. No & 1. Yes $\rightarrow$ & 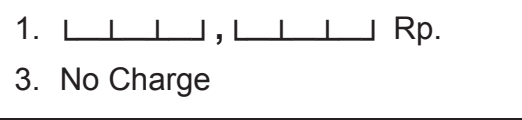 & Per Seeds & 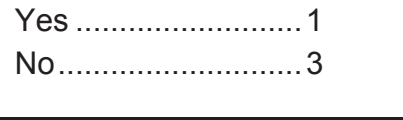 & $\begin{array}{l}\sqcup \sqcup \text { weeks.............. } 1 \\
\text { NEVER .................6 }\end{array}$ \\
\hline 37. Medicine to control blood pressure (e.g. Captopril) & 3. No & 1. Yes $\rightarrow$ & $\begin{array}{l}\text { 1. } \downarrow \perp \perp \_, \downarrow \perp \perp \perp \_ \text {Rp. } \\
\text { 3. No Charge }\end{array}$ & $\begin{array}{l}\text { 1. Strip } \\
\text { 2. Bottle } \\
\text { 3. Ampule } \\
\text { 4. Seeds/Grains }\end{array}$ & 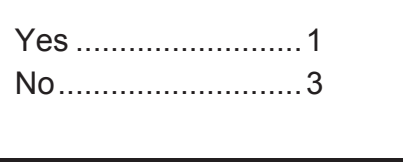 & 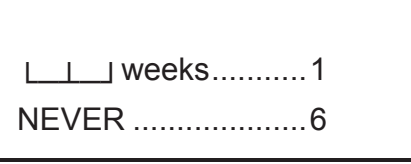 \\
\hline 38. Anesthetic (e.g. Oxytocin-ergometrine) & 3. No & 1. Yes $\rightarrow$ & $\begin{array}{l}\text { 1. } \downarrow \perp \perp \perp, \downarrow \perp \_\_ \text {Rp. } \\
\text { 3. No Charge }\end{array}$ & $\begin{array}{l}\text { 1. Strip } \\
\text { 2. Bottle } \\
\text { 3. Ampule } \\
\text { 4. Seeds/Grains }\end{array}$ & 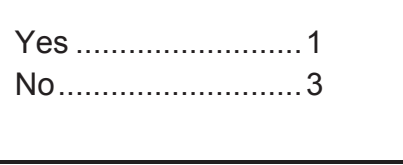 & 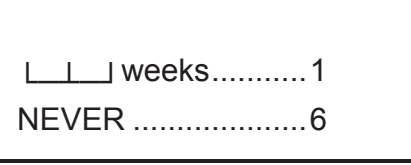 \\
\hline 39. Medicine to lower cholesterol,(e.g. simvastatin)) & 3. No & 1. Yes $\rightarrow$ & 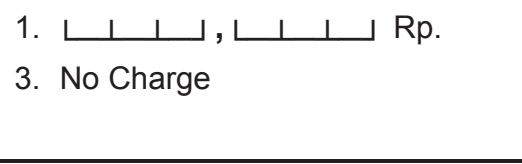 & $\begin{array}{l}\text { 1. Strip } \\
\text { 2. Bottle } \\
\text { 3. Ampule } \\
\text { 4. Seeds/Grains }\end{array}$ & 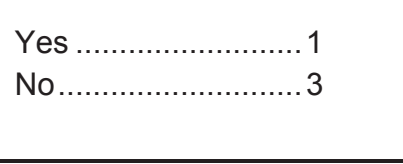 & $\begin{array}{c}\sqcup \sqcup \text { weeks............ } 1 \\
\text { NEVER .................... }\end{array}$ \\
\hline 40. Medicine to control blood sugar, (e.g. metformin) & 3. No & 1. Yes $\rightarrow$ & 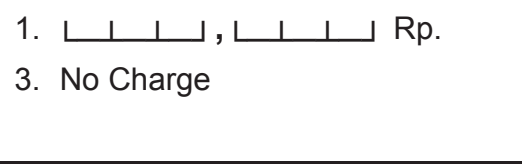 & $\begin{array}{l}\text { 1. Strip } \\
\text { 2. Bottle } \\
\text { 3. Ampule } \\
\text { 4. Seeds/Grains }\end{array}$ & 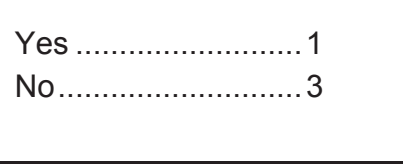 & $\begin{array}{c}\sqcup \sqcup \text { weeks............ } 1 \\
\text { NEVER ..................... }\end{array}$ \\
\hline
\end{tabular}


SECTION C: ACTIVITIES OF PUSKESMAS/PUSKESMAS PEMBANTU

\begin{tabular}{|c|c|c|c|c|c|}
\hline C05. & C06. & C09a. & C10. & C10a. & C10b. \\
\hline $\begin{array}{l}\text { TYPE OF SERVICE } \\
\text { (C1TYPE) }\end{array}$ & Is there any $[\ldots]$ service? & $\begin{array}{l}\text { Cost of the service?[notincluding } \\
\text { first visit registration fee] }\end{array}$ & Units & Is this $[\ldots]$ in stock today? & $\begin{array}{c}\text { In the last } 6 \text { weeks how } \\
\text { many weeks was }[\ldots] \text { out of } \\
\text { stock? }\end{array}$ \\
\hline \multicolumn{6}{|l|}{ 41. Vaccines } \\
\hline a. BCG & 1. Yes $\rightarrow$ & 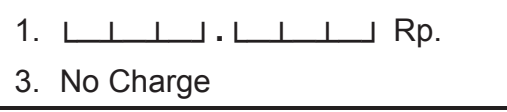 & Per treatment & 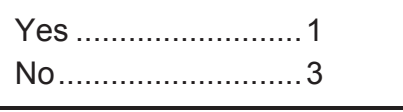 & $\begin{array}{l}\perp \_ \text {weeks .......... } 1 \\
\text { NEVER .................6 }\end{array}$ \\
\hline b. DPT (Combo) & 1. Yes $\rightarrow$ & $\begin{array}{l}\text { 1. } \downarrow \perp \perp \_. \downarrow \perp \_\_ \text {Rp. } \\
\text { 3. No Charge }\end{array}$ & Per treatment & 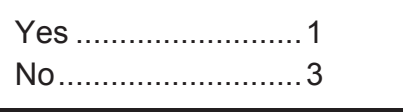 & 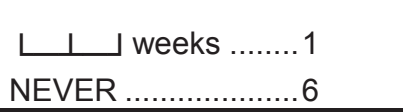 \\
\hline c. Anti polio & 1. Yes $\rightarrow$ & $\begin{array}{l}\text { 1. } \downarrow \perp \perp \_. \downarrow \perp \_\_ \text {Rp. } \\
\text { 3. No Charge }\end{array}$ & Per treatment & 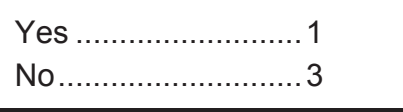 & 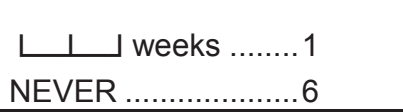 \\
\hline d. Measles & 1. Yes $\rightarrow$ & 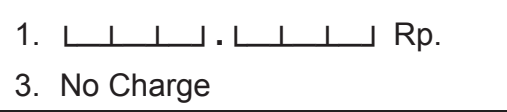 & Per treatment & 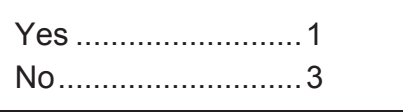 & $\begin{array}{l}\left\llcorner \_ \text {weeks ......... } 1\right. \\
\text { NEVER .................6 }\end{array}$ \\
\hline e. Tetanus Toxoid & 3. No & $\begin{array}{l}\text { 1. } \downarrow \perp \perp \_. \downarrow \perp \_\_ \text {Rp. } \\
\text { 3. No Charge }\end{array}$ & Per treatment & $\begin{array}{l}\text { Yes } \ldots \ldots \ldots \ldots \ldots \ldots \ldots \ldots \ldots \ldots \\
\text { No } \ldots \ldots \ldots \ldots \ldots \ldots \ldots \ldots \ldots\end{array}$ & 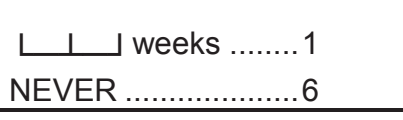 \\
\hline f. Hepatitis B, for infants & 1. Yes $\rightarrow$ & $\begin{array}{l}\text { 1. } \downarrow \perp \perp \_\_\_\_\_\_ \text {Rp. } \\
\text { 3. No Charge }\end{array}$ & Per treatment & $\begin{array}{l}\text { Yes } \ldots \ldots \ldots \ldots \ldots \ldots \ldots \ldots \ldots \\
\text { No }\end{array}$ & $\begin{array}{l}\perp ل \text { weeks .......... } 1 \\
\text { NEVER ..................6 }\end{array}$ \\
\hline g. Hepatitis B, for adult & 1. Yes $\rightarrow$ & $\begin{array}{l}\text { 1. } \downarrow \perp \perp \_. \downarrow \perp \_\_ \text {Rp. } \\
\text { 3. No Charge }\end{array}$ & Per treatment & 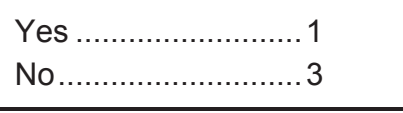 & 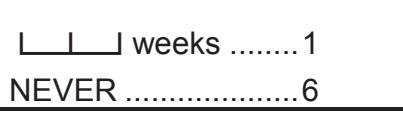 \\
\hline h. Shingles & 1. Yes $\rightarrow$ & $\begin{array}{l}\text { 1. } \downarrow \perp \perp \_. \downarrow \perp \_\_ \text {Rp. } \\
\text { 3. No Charge }\end{array}$ & Per treatment & $\begin{array}{l}\text { Yes } \\
\text { No } \ldots \ldots \ldots \ldots \ldots \ldots \ldots \ldots \ldots \ldots \\
\end{array}$ & 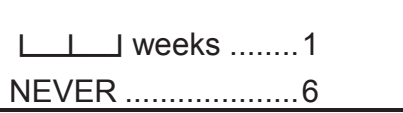 \\
\hline i. Influenza & 1. Yes $\rightarrow$ & 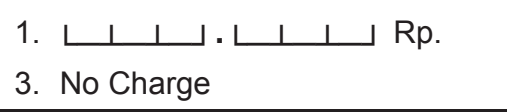 & Per treatment & 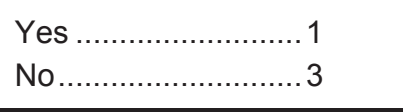 & 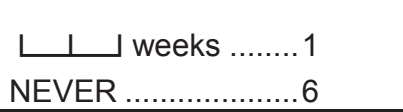 \\
\hline j. Pneumonia & 1. Yes $\rightarrow$ & $\begin{array}{l}\text { 1. } \downarrow \perp \perp \_. \downarrow \perp \_\_ \text {Rp. } \\
\text { 3. No Charge }\end{array}$ & Per treatment & 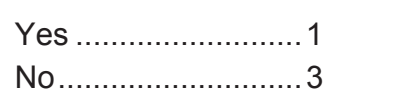 & 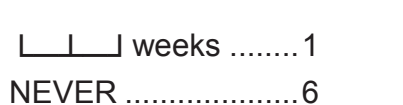 \\
\hline
\end{tabular}


SECTION C: ACTIVITIES OF PUSKESMAS/PUSKESMAS PEMBANTU

\begin{tabular}{|c|c|c|c|}
\hline C39a & $\begin{array}{l}\text { Are there any finding of new patients of } \\
\text { positive TB BTA in this puskesmas/pustu } \\
\text { in the last } 12 \text { months? }\end{array}$ & 1. Yes,,$\perp \perp \_$cases & 3. No \\
\hline $\mathrm{C} 40 \mathrm{a}$ & $\begin{array}{l}\text { Has this puskesmas/pustu treated } \\
\text { patients with dengue fever in the last } 12 \\
\text { months? }\end{array}$ & 1. Yes,,$\perp \perp \_$cases & 3. No \\
\hline C41a & $\begin{array}{l}\text { Has this puskesmas/pustu ever treated } \\
\text { HIVIAIDS patients in the last } 12 \text { months? }\end{array}$ & 1. Yes, $\downarrow \perp \perp \_$cases & 3. No \\
\hline
\end{tabular}

\begin{tabular}{|c|c|c|c|}
\hline \multicolumn{2}{|r|}{ DISEASES } & \multicolumn{2}{|c|}{$\begin{array}{l}\text { C42ab. Has this puskesmas/pustu ever carried the } \\
\text { STD tests using }[\ldots] \text { ? }\end{array}$} \\
\hline A & Vaginal mucous & 1. Yes, $\downarrow \perp \perp \_$cases & 3. No \\
\hline B & Penis mucous & 1. Yes, $\downarrow \perp \perp \perp$ cases & 3. No \\
\hline C & Blood & 1. Yes, $\downarrow \perp \perp \_$c لـ & 3. No \\
\hline
\end{tabular}

\begin{tabular}{|c|c|c|}
\hline C43a. & $\begin{array}{l}\text { Has this Puskesmas applied age-friendly } \\
\text { program (Prilaku Santun Lansia)? }\end{array}$ & 3. $\mathrm{No} \rightarrow \mathrm{C} 11$ \\
\hline C44a & $\begin{array}{l}\text { What kind of services provided in the } \\
\text { program? }\end{array}$ & $\begin{array}{l}\text { A. Special registration for the elderly } \\
\text { B. Special check up for the elderly } \\
\text { C. Special drug Service for the elderly } \\
\text { D. One stop service (from registration to receiving } \\
\text { medicines attained in one room) }\end{array}$ \\
\hline C45a & $\begin{array}{l}\text { Since when did this Puskesmas/Pustu } \\
\text { provide special service for the elderly? }\end{array}$ & 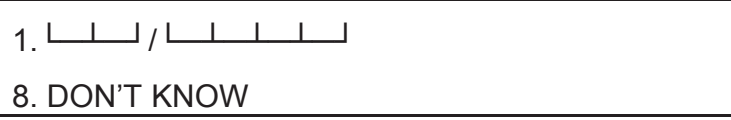 \\
\hline C46a & $\begin{array}{l}\text { What kind of program for the elderly } \\
\text { carried out outside this } \\
\text { Puskesmas/pustu? }\end{array}$ & $\begin{array}{l}\text { A. Posyandu for elderly } \\
\text { B. Counseling through karang taruna (old folk's } \\
\text { home) } \\
\text { C. Counseling through Karang werda forum in } \\
\text { kecamatan/sub-district } \\
\text { D. Counseling through old folk's home } \\
\text { E. Home visit } \\
\text { F. Special event for elderly; talk show, gymnastic } \\
\text { competition for elderly, having stroll } \\
\end{array}$ \\
\hline
\end{tabular}

Service outside the building

C11. On an average day, how many staff On an average day how many steride the building for outreach activitiessuch Posyandu, mobile Puskesmas, etc? Posyandu (Integrated Service Post)

C14. How many Posyandu are there in the work region of this Puskesmas?

C14a. How many Posyandu are active?

C15a. In one month, how many times Puskesmas staff go to the Posyandu?

$\downarrow \downarrow$ staff ................................... 1

No activities................................... 6

C16b. Since 2007, has the number of Posyandus in the work region of this

$$
\text { Puskesmas changed? }
$$

No Posyandu .................................. $6 \rightarrow$ C14a1

$\perp$. Posyandu .................... 1 $\left\llcorner \_\right.$Posyandu

$\downarrow \downarrow$ times per month .................. 1 $\downarrow \downarrow \downarrow$ times per year ....................... 4 DON'T KNOW ............................... 8

Increased a lot ............................... 1

Increased some ............................... 2

No change....................................... 3

Decreased some.

Decreased a lot.............................. 5

Posyandu for Elderly

C14a1. How many Posyandu for Elderly are $\quad$ No Posyandu for Rlderly ............... $6 \rightarrow$ C17 there in the work region of this Puskesmas?

C14a2. When did this Posyandu for the Elderly start its activities? $\left\llcorner \_\right.$Posyandu .............................. 1

C14aa. How many Posyandu for elderly are activein the work region of this Puskesmas?

C15aa. In one month, how many times Puskesmas staff go to the Posyandu for Elderly?

C16ba. Since 2007, has the number of Posyandu for Elderly in the work region of this Puskesmas changed?
1. Year ᄂ $\perp \perp \perp$

8. DON'T KNOW

$\left\llcorner \_\right.$Posyandu

$\longleftarrow \_$times per month .................. 1

$1+1$ times per year

DON'T KNOW .................................. 8

Increased a lot .................................. 01

Increased some .................................... 02

No change........................................... 03

Decreased some .............................. 04

Decreased a lot................................ 05 
SECTION C: ACTIVITIES OF PUSKESMAS/PUSKESMAS PEMBANTU

Puskesmas Pembantu

C17. How many Puskesmas Pembantu are there in the work region of this Puskesmas?

C19b. Since 2007, have any Puskesmas Pembantu in the work region of this Puskesmas closed?

Mobile Puskesmas

C20a. How many times in a month does the Puskesmas staff go on duty trip of the Mobile Puskesmas?

C21b. Since 2007, has the number of trips changed?

\begin{tabular}{|c|c|}
\hline 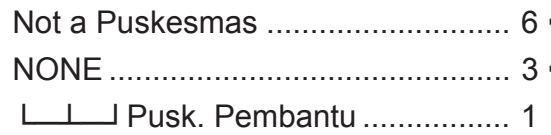 & $\begin{array}{l}6 \rightarrow \mathrm{C20a} \\
3 \rightarrow \mathrm{C20a} \\
1\end{array}$ \\
\hline$ل$ لـ number closed ..................... 1 & \\
\hline NONE & \\
\hline
\end{tabular}

NONE ................................................ 6 $\rightarrow$ C22a

$\downarrow \downarrow$ times per month .................. 1

$\longleftarrow$ times per year .................... 4

Increased a lot ................................. 01

Increased some ….............................. 02

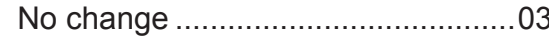

Decreased some ................................ 04

Decreased a lot .............................. 05

UKS /UKGS

C22a. How many times a month does the

for the UKS/UKGS program?

C23b. Since 2007, has the number of UKS/UKGS visits per month changed?

$\downarrow \downarrow$ times per month .................. 1

$\downarrow \downarrow$ times per year ...................... 4

Increased a lot ................................... 01

Increased some ................................02

No change ........................................

Decreased some

Decreased a lot ............................ 05

Pondok Bersalin Desa [Polindes]

C24. Is there a childbirth post (Polindes) program / Poskesdes in the work region of this Puskesmas?

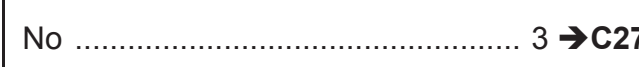

Yes …............................................ 1

C25. How many Polindes program are there in the work region of this Puskesmas?

C25b. How many Polindes are still active?

C25c. Since 2007, have any Polindes program in the work region of this Puskesmas closed?

$\llcorner\sim$ Polindes

$\llcorner\sim$ Polindes

$\downarrow \downarrow$ Number closed .................... 1

NONE
Medicine Post (Pos Obat Desa)

C27 Is there a Medicine Post Program in the work region of this Puskesmas?

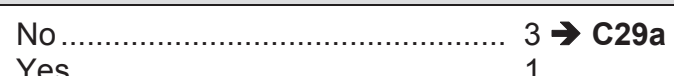

C28 How many Medicine Posts program are there in the work region of this Puskesmas?

C28a How many Medicine Posts are still active?

C29 In what year was the first Medicine Post started?

C29ab Since 2007, has the number of Medicine Post in the work region of this Puskesmas changed?

$\left\llcorner \_\right.$Medicine Posts

$\downarrow \downarrow$ Medicine Posts

Year $\llcorner\perp \perp \perp$ DON'T KNOW ............................... 8

Increased a lot ................................... 01

Increased some ................................ 02

No change ............................................ 03

Decreased some.............................. 04

Decreased a lot.......................... 05

Village Midwife (Bidan Desa)

C29a How many Village Midwives work in the region of this Puskesmas?

C29b In what year did the first Village Midwife start working?

C29c What type of support is usually given to the Village Midwives?

(CIRCLE ALL THAT APPLY)

C29db Since 2007, have any village midwives in the work region of this Puskesmas quit working?

NONE ………........................... $3 \rightarrow$ C30

$\downarrow \downarrow$ people ............................... 1

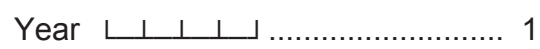

DON'T KNOW ................................ 8

Medical supplies ............................. A

Health supplies ................................. B

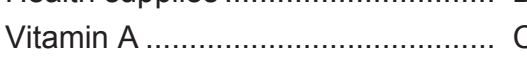

Iron tablets .....................................

Other

$\llcorner\perp$ Number who quit ............... 1

NONE ..................................... 3

DON'T KNOW ……………………..... 8

\section{Poskesdes}

C29dc Does this Puskesmas/pustu give counseling for Poskesdes as part of desa siaga program
1.Yes $\llcorner$ unit

3.No Poskesdes 


\section{SECTION C: ACTIVITIES OF PUSKESMAS/PUSKESMAS PEMBANTU}

We would like to ask about the referrals that are usually provided by this facility

\begin{tabular}{|c|c|c|c|c|}
\hline \multirow{2}{*}{\multicolumn{2}{|c|}{ (C2 TYPE) }} & \multicolumn{3}{|c|}{ FACILITIES TO WHICH PATIENTS ARE REFERRED } \\
\hline & & Hospital & Other Puskesmas & Private Practice \\
\hline C30. & $\begin{array}{l}\text { If a patient is referred to another facility, } \\
\text { where do you usually send them? }\end{array}$ & $\begin{array}{l}\text { No............. } 3 \rightarrow \text { NEXT COLUMN } \\
\text { DON'T KNOW .... } 8 \rightarrow \text { NEXT COLUMN } \\
\text { Yes................ } 1 \\
\text { Name: } \\
\text { Address: } \\
\text { Loc: } \\
\text { Vill: } \\
\text { Kec.: } \\
\text { Kab.: }\end{array}$ & 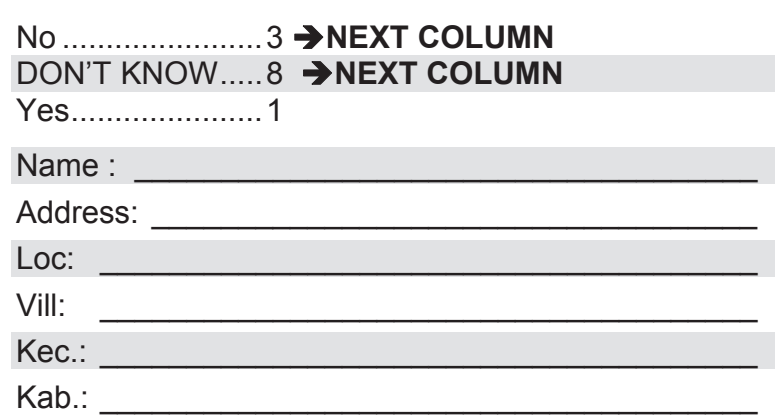 & 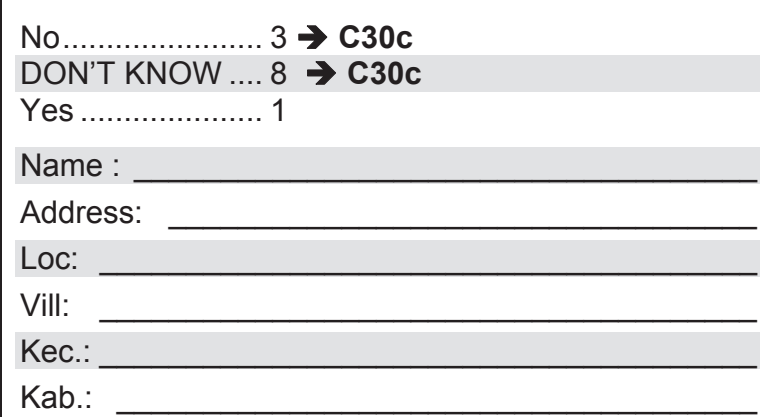 \\
\hline C30a. & $\begin{array}{l}\text { What is the distance that must be traveled } \\
\text { from your facility to the referenced facility? }\end{array}$ & 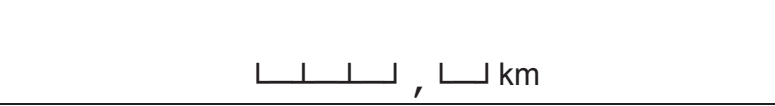 & 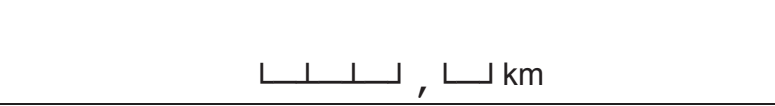 & 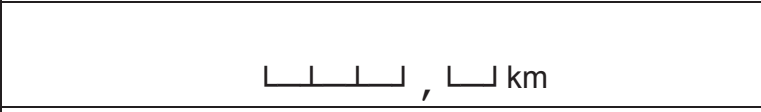 \\
\hline C30b. & $\begin{array}{l}\text { What type of transportation is used to refer a } \\
\text { patient? }\end{array}$ & 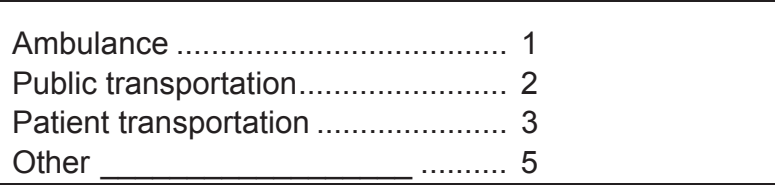 & 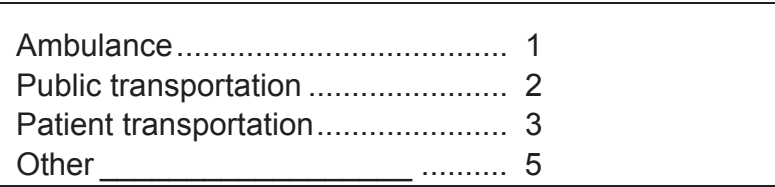 & 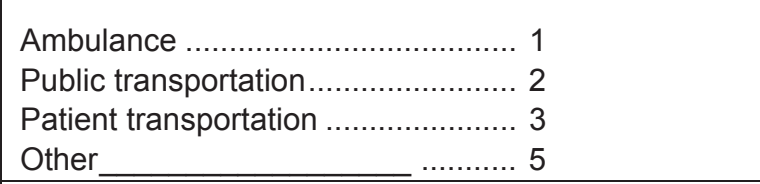 \\
\hline C30g. & $\begin{array}{l}\text { If a poor patient is referred to }[\ldots . .] \text {, is he/she } \\
\text { provided with transportation to the facility? }\end{array}$ & $\begin{array}{l}\text { 1. Yes, transportation is provided using ambulance } \\
\text { free of charge } \\
\text { 2. Yes, patient is provided with money to travel to the } \\
\text { referred facility } \\
\text { 3. No, neither transportation nor money is provided } \rightarrow \\
\text { NEXT COLUMN }\end{array}$ & $\begin{array}{l}\text { 1. Yes, transportation is provided using ambulance } \\
\text { free of charge } \\
\text { 2. Yes, patient is provided with money to travel to the } \\
\text { referred facility } \\
\text { 3. No, neither transportation nor money is provided } \rightarrow \\
\text { NEXT COLUMN }\end{array}$ & $\begin{array}{l}\text { 1. Yes, transportation is provided using ambulance } \\
\text { free of charge } \\
\text { 2. Yes, patient is provided with money to travel to the } \\
\text { referred facility } \\
\text { 3. No, neither transportation nor money is provided } \\
\rightarrow \text { C } 30 \text { c }\end{array}$ \\
\hline C30h. & $\begin{array}{l}\text { What is the market value of the transportation } \\
\text { provided? }\end{array}$ & 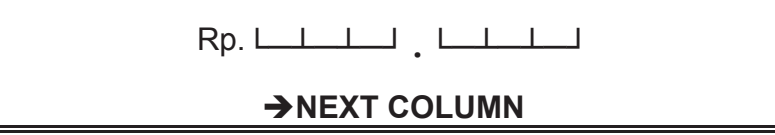 & 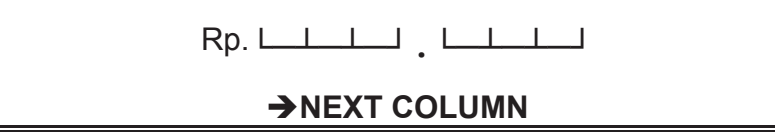 & 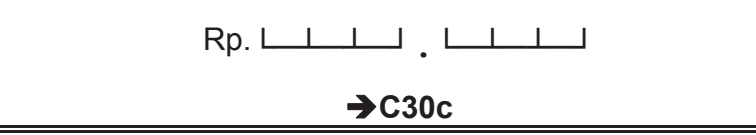 \\
\hline \multirow[t]{2}{*}{ C30c. } & $\begin{array}{l}\text { If a patient is in critical or serious condition } \\
\text { when referred to another facility, does the staff } \\
\text { from this facility accompany the patient? }\end{array}$ & 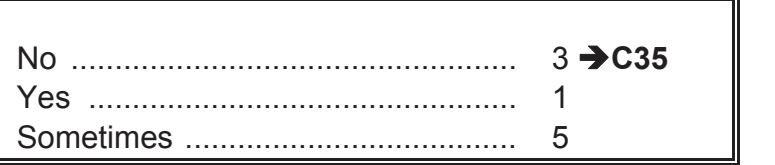 & \multirow[t]{2}{*}{ C30d. Who accompanies the patient? } & 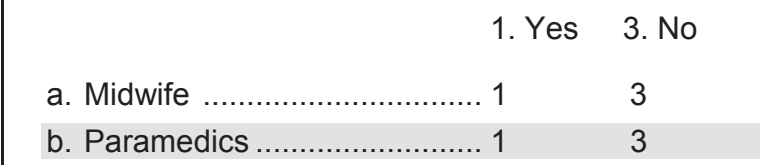 \\
\hline & & & & 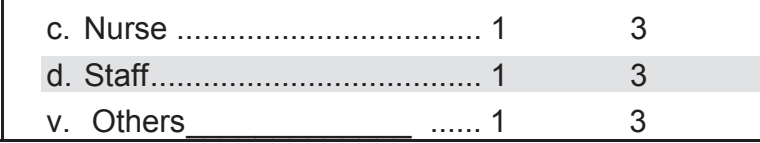 \\
\hline
\end{tabular}


SECTION C: ACTIVITIES OF PUSKESMAS/PUSKESMAS PEMBANTU

Laboratory Examination

\begin{tabular}{|c|c|c|c|c|c|}
\hline & C35. & C36. & \multicolumn{2}{|c|}{ C37. } & C38. \\
\hline $\begin{array}{c}\text { TYPE OF EXAMINATION } \\
\text { (C3TYPE) }\end{array}$ & $\begin{array}{c}\text { Can this lab work be done in the } \\
\text { Puskesmas? }\end{array}$ & How much is the charge to the patient? & $\begin{array}{r}\text { For lab wor } \\
\text { patient }\end{array}$ & $\begin{array}{l}\text { done here, is the } \\
\text { ed outside? }\end{array}$ & $\begin{array}{c}\text { How far is this facility from the } \\
\text { Puskesmas? }\end{array}$ \\
\hline a. Hemoglobin $(\mathrm{Hb})$ & $\begin{array}{l}\text { No ..................... } 3 \rightarrow \text { C37 } \\
\text { Yes ................. } 1\end{array}$ & 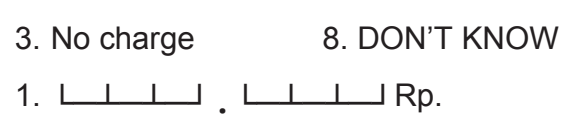 & $\begin{array}{l}\text { 3. } \\
\Downarrow\end{array}$ & 1. Yes $\rightarrow$ & 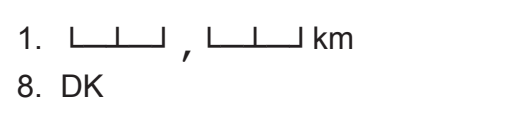 \\
\hline b. Leucocyte calculation & 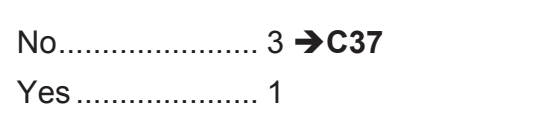 & 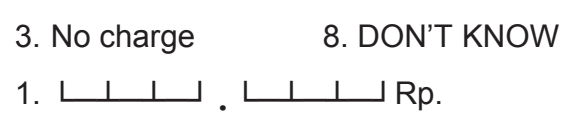 & $\begin{array}{l}\text { 3. } \\
\Downarrow\end{array}$ & 1. Yes $\rightarrow$ & 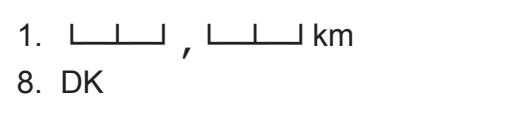 \\
\hline c. Blood type calculation & 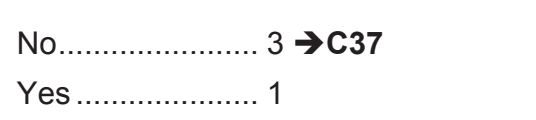 & 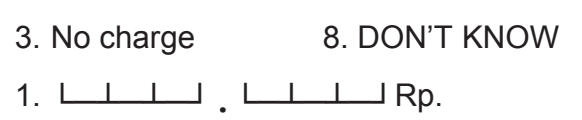 & $\begin{array}{l}\text { 3. } \\
\Downarrow\end{array}$ & 1. Yes $\rightarrow$ & 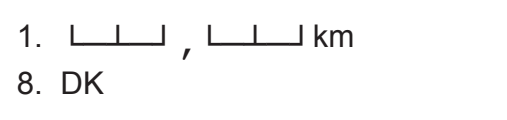 \\
\hline d. Erythrocyte calculation & $\begin{array}{l}\text { No } \ldots \ldots \ldots \ldots . . .13 \rightarrow \mathrm{C} 37 \\
\text { Yes } \ldots \ldots \ldots \ldots \ldots \ldots . . .11\end{array}$ & 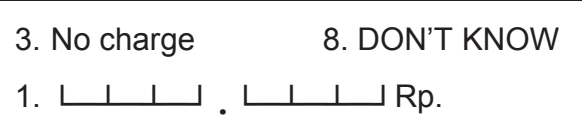 & $\begin{array}{l}\text { 3. } \\
\Downarrow\end{array}$ & 1. Yes $\rightarrow$ & 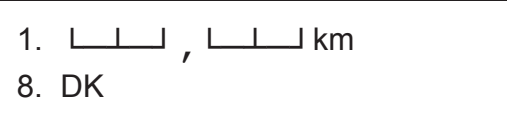 \\
\hline e. Urinalysis & $\begin{array}{l}\text { No } \ldots \ldots \ldots \ldots \ldots \ldots \ldots . . . .3 \rightarrow \text { C37 } \\
\text { Yes ..................... } 1\end{array}$ & $\begin{array}{l}\text { 3. No charge } \\
\text { 1. DON'T KNOW } \\
\end{array}$ & $\begin{array}{l}\text { 3. } \\
\Downarrow\end{array}$ & 1. Yes $\rightarrow$ & 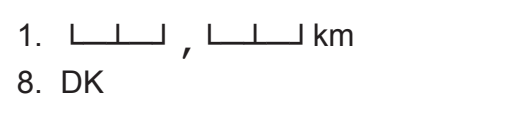 \\
\hline f. Pregnancy test & $\begin{array}{l}\text { No..................... } 3 \rightarrow \text { C37 } \\
\text { Yes ................. } 1\end{array}$ & 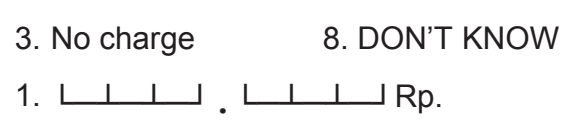 & $\begin{array}{l}\text { 3. } \\
\Downarrow\end{array}$ & 1. Yes $\rightarrow$ & 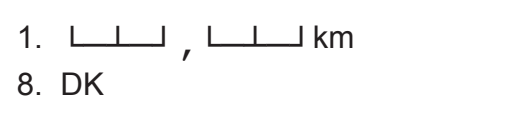 \\
\hline h. Sputum examination & 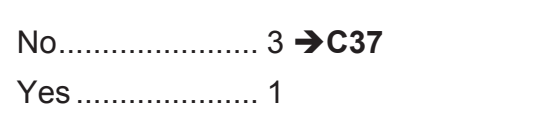 & 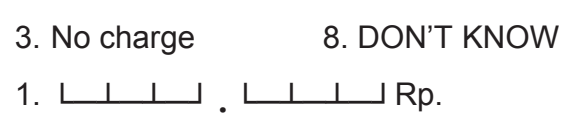 & $\begin{array}{l}\text { 3. } \quad \mathrm{N} \\
\Downarrow\end{array}$ & 1. Yes $\rightarrow$ & 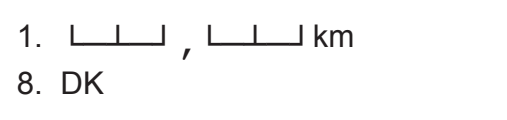 \\
\hline j. Cholesterol test & $\begin{array}{l}\text { No } \ldots \ldots \ldots \ldots \ldots \ldots \ldots . . . .3 \rightarrow \text { C37 } \\
\text { Yes .................... } 1\end{array}$ & 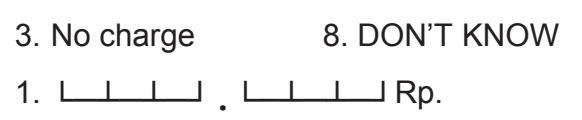 & $\begin{array}{l}3 . \\
\Downarrow\end{array}$ & 1. Yes $\rightarrow$ & 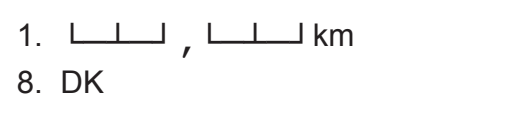 \\
\hline k. Blood Sugar test & 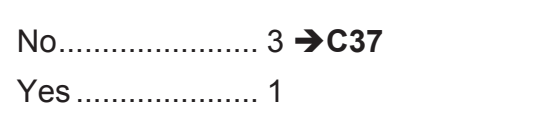 & 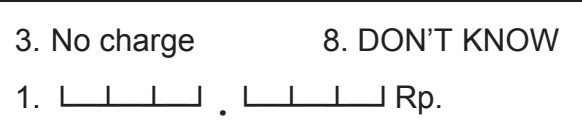 & $\begin{array}{l}\text { 3. } \\
\Downarrow\end{array}$ & 1. Yes $\rightarrow$ & 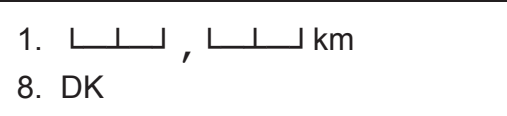 \\
\hline
\end{tabular}


SECTION C: ACTIVITIES OF PUSKESMAS/PUSKESMAS PEMBANTU

\begin{tabular}{|c|c|c|c|c|}
\hline & C35. & C36. & C37. & C38. \\
\hline $\begin{array}{c}\text { TYPE OF EXAMINATION } \\
\text { (С3TYPE) }\end{array}$ & $\begin{array}{c}\text { Can this lab work be done in the } \\
\text { Puskesmas? }\end{array}$ & How much is the charge to the patient? & $\begin{array}{c}\text { For lab work not done here, is the } \\
\text { patient referred outside? }\end{array}$ & $\begin{array}{c}\text { How far is this facility from the } \\
\text { Puskesmas? }\end{array}$ \\
\hline I. Osteoporosis / bone density examination & 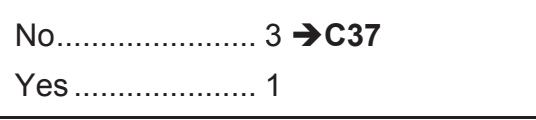 & 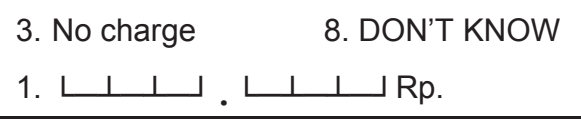 & 1. Yes $\rightarrow$ & 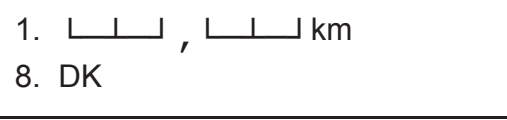 \\
\hline m. Lung examination & 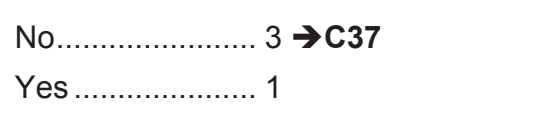 & 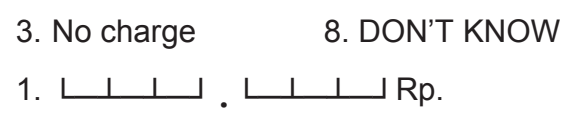 & 1. Yes $\rightarrow$ & 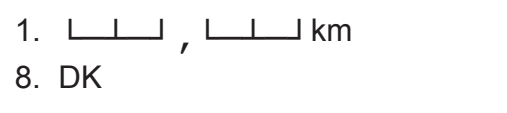 \\
\hline n. Heart examination (ECG) & 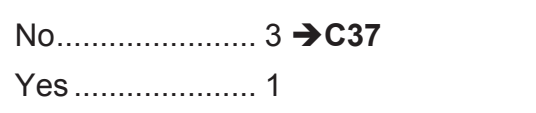 & 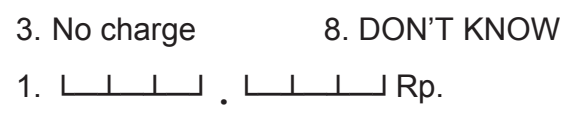 & 1. Yes $\rightarrow$ & 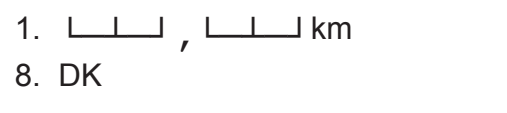 \\
\hline
\end{tabular}

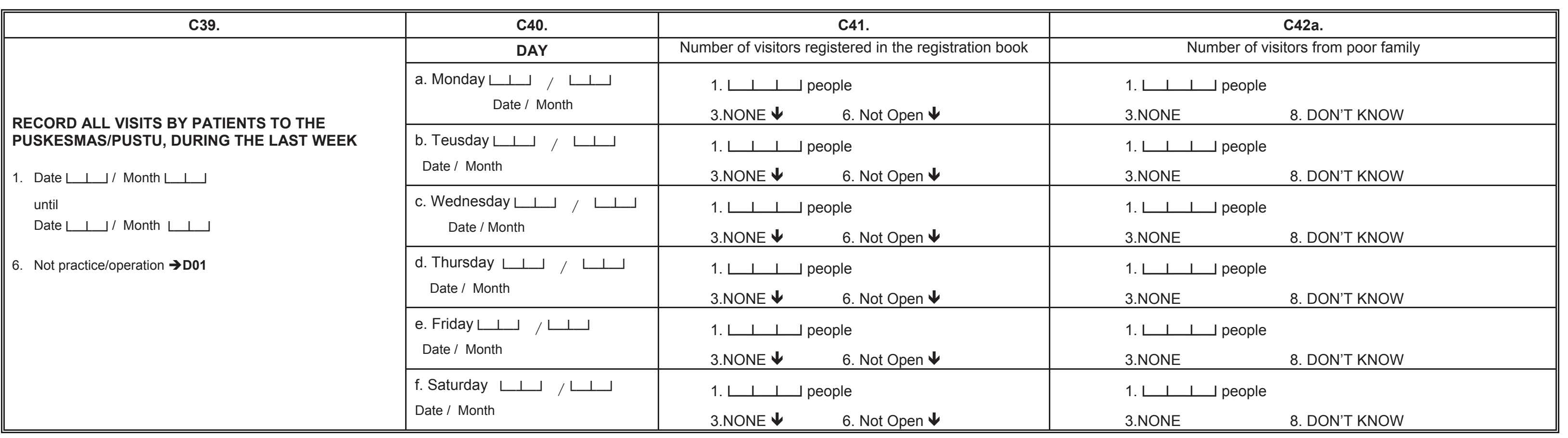


SECTION E: HEALTH INSTRUMENTS

Name: Position:

Please give information about various medical instruments found at this Puskesmas.

\begin{tabular}{|c|c|c|}
\hline E01. & E03. & " \\
\hline $\begin{array}{l}\text { TYPE OF INSTRUMENTS } \\
\text { (E1TYPE) }\end{array}$ & $\begin{array}{l}\text { The number of instruments } \\
\text { owned by this Puskesmas that } \\
\text { are in good repair? }\end{array}$ & $\begin{array}{l}\text { The number of privately owned } \\
\text { instruments used here? }\end{array}$ \\
\hline a. Regular stethoscope & \llcorner & $\sqcup$ \\
\hline b. Stethoscope for pregnant mothers & $\downarrow$ & $\sqcup$ \\
\hline c. Blood pressure meter & \llcorner & $\sqcup$ \\
\hline d. Sterilization/autoclaves & 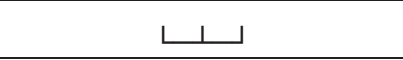 & $\sqcup$ \\
\hline e. Scales for adults & 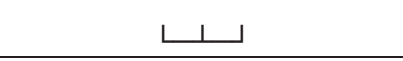 & $\sqcup$ \\
\hline f. Scales for infants & \llcorner & $\sqcup$ \\
\hline g. Measures for body height & 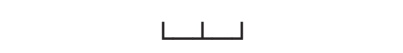 & $\sqcup$ \\
\hline h. Thermometer & ــ & \\
\hline i. Beds & \llcorner & \\
\hline ia.Inpatient bed & \llcorner & \\
\hline j. Delivery kit & $\downarrow$ & $\sqcup$ \\
\hline k. Forceps & $\sqcup$ & $\sqcup$ \\
\hline I. Vaginal Speculum & $\downarrow$ & 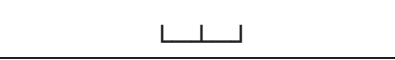 \\
\hline m. Sahli Set & $\downarrow$ & 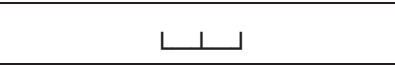 \\
\hline n. Tenaculum & 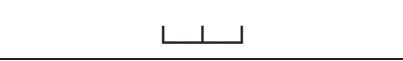 & 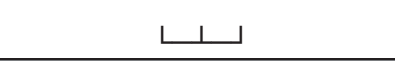 \\
\hline o. Uterus Sound & \llcorner & \\
\hline p. Gynecology Table & 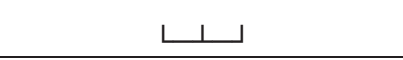 & \\
\hline q. Bone-setting equipment & 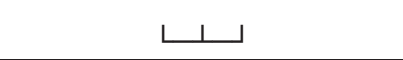 & \\
\hline r. Oxygen Tank & 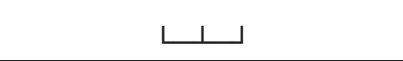 & \\
\hline s. Incubators & \llcorner & \\
\hline t. Minor surgical instruments & $\downarrow$ & \\
\hline $\begin{array}{l}\text { u. Communication equipments (SSB Radio, } \\
\text { phone) }\end{array}$ & 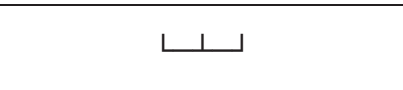 & \\
\hline v. Scissors & 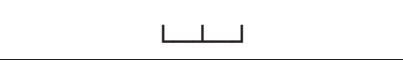 & \\
\hline w. Electrocardiogram & $\sqcup$ & \\
\hline
\end{tabular}

\begin{tabular}{||l|c|}
\hline aa. Microscopes & \llcorner \\
\hline ba. centrifuges & \llcorner \\
\hline ca. Syringes & \llcorner\lrcorner \\
\hline da.Cholesterol test kit & \llcorner\lrcorner \\
\hline ea. Blood sugar test kit & $\llcorner\llcorner$ \\
\hline
\end{tabular}

\begin{tabular}{|c|c|c|c|c|}
\hline E05. & \multicolumn{2}{|c|}{ E06. } & \multicolumn{2}{|c|}{ E07. } \\
\hline $\begin{array}{c}\text { TYPE OF INSTRUMENTS } \\
\text { (E2TYPE) }\end{array}$ & Does this & lave $[\ldots]$ ? & Is there & h stock? \\
\hline 1. Alcohol & 3. No $\downarrow$ & 1. Yes & 3. No & 1. Yes \\
\hline 2. Betadine & 3. No $\downarrow$ & 1. Yes & 3. No & 1. Yes \\
\hline 3. Whitfield cream & 3. No $\downarrow$ & 1. Yes & 3. No & 1. Yes \\
\hline b. Bandages & 3. No $\downarrow$ & 1. Yes & 3. No & 1. Yes \\
\hline c. Gloves & 3. No $\downarrow$ & 1. Yes & 3. No & 1. Yes \\
\hline d. Infuse instruments and needles & 3. No $\downarrow$ & 1. Yes & 3. No & 1. Yes \\
\hline d1. Disposable needles & 3. No $\downarrow$ & 1. Yes & 3. No & 1. Yes \\
\hline d2. Cotton & 3. No $\downarrow$ & 1. Yes & 3. No & 1. Yes \\
\hline e. Giemsa dyeing solutions & 3. No $\downarrow$ & 1. Yes & 3. No & 1. Yes \\
\hline f. Benedict solutions & 3. No $\downarrow$ & 1. Yes & 3. No & 1. Yes \\
\hline g. Wright solutions & 3. No $\downarrow$ & 1. Yes & 3. No & 1. Yes \\
\hline h. Pregnancy test (strip) & 3. No $\downarrow$ & 1. Yes & 3. No & 1. Yes \\
\hline i. Protein urine tests (strip) & 3. No & 1. Yes & & \\
\hline j. Glucose urine tests (strip) & 3. No & 1. Yes & & \\
\hline m. Cholesterol test kit & 3. No & 1. Yes & & \\
\hline n. Blood sugar test kit & 3. No & 1. Yes & & \\
\hline
\end{tabular}




\section{SECTION G: FAMILY PLANNING SERVICES}

RESPONDENT: PERSON RESPONSIBLE FOR FAMILY PLANNING SERVICE UNIT

\begin{tabular}{|c|c|c|}
\hline G0 & Name & \\
\hline G0x & Position & \\
\hline G1. & $\begin{array}{l}\text { How many staff members of the } \\
\text { Puskesmas are involved in } \\
\text { providing family planning } \\
\text { services? }\end{array}$ & $\begin{array}{l}\text { 1. } \sqcup \sqcup \sqcup \text { 8. DON'T KNOW } \\
\text { 8. }\end{array}$ \\
\hline G2. & $\begin{array}{l}\text { Mention the number of } \\
\text { employees according to the } \\
\text { category here below: }\end{array}$ & 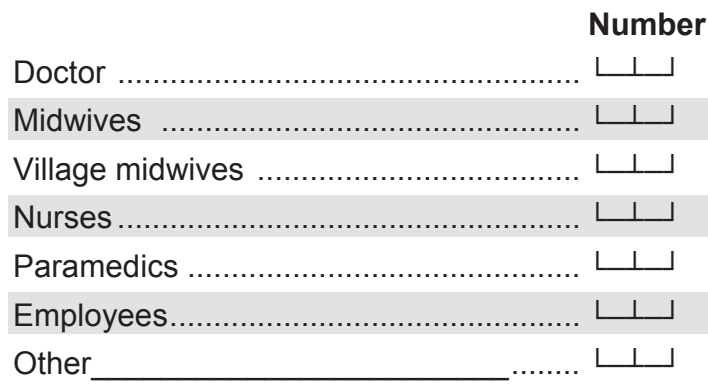 \\
\hline
\end{tabular}

If client desires a certain method that is not available here, where is the patient referred to?

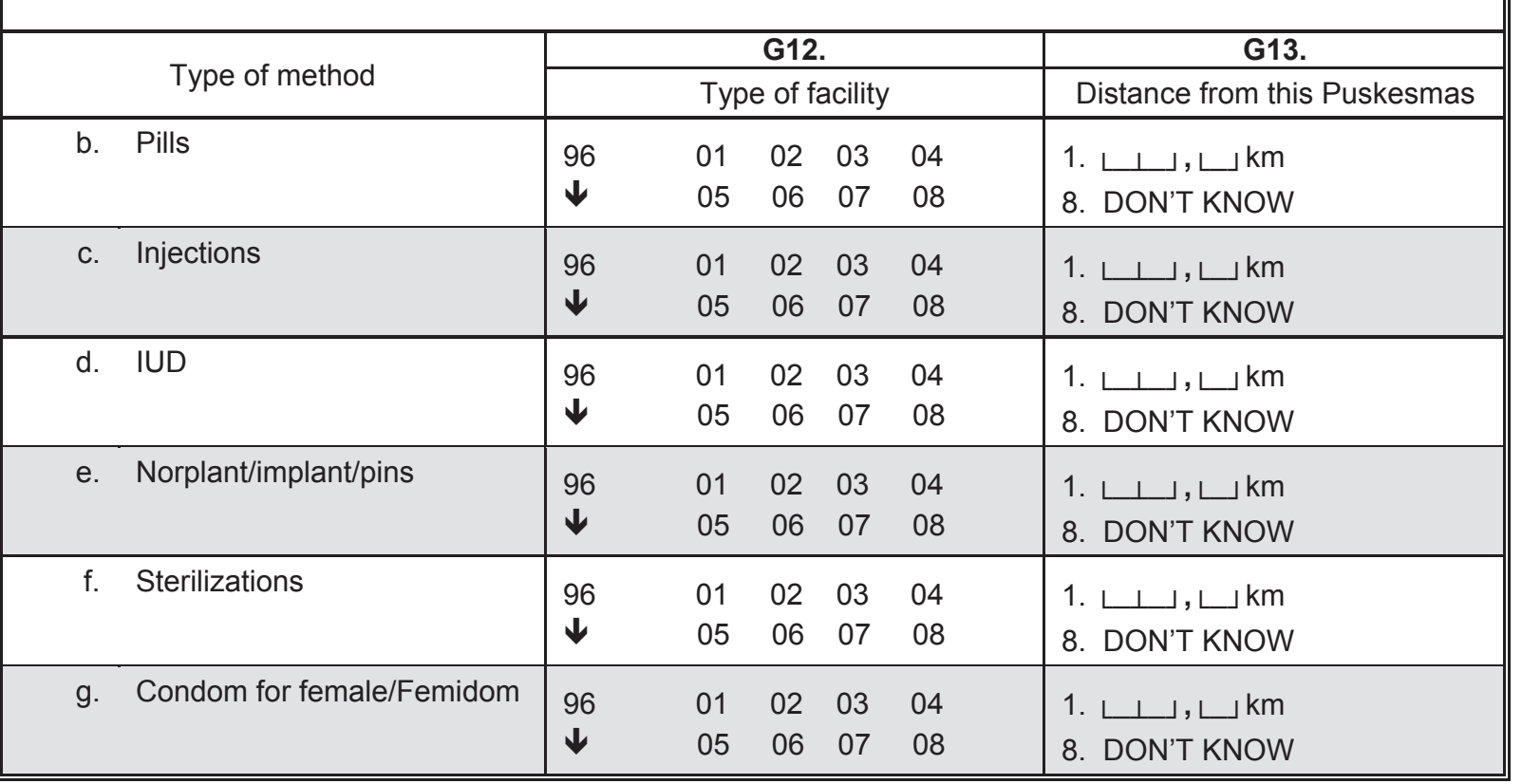

G12:

01. Government Hospitals

02. Private Hospital

04. Auxillary Puskesmas

5. Private Clinic

66. Doctors with private practice

07. Midwives/nurses/practicing

paramedics

96. NO REFERRAL 


\section{SECTION G: FAMILY PLANNING SERVICES}

\begin{tabular}{|c|c|c|c|}
\hline G14. & $\begin{array}{l}\text { In what year was the low dose oral } \\
\text { contraceptive introduced at this } \\
\text { facility? }\end{array}$ & 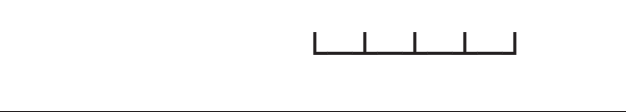 & \\
\hline G15a. & $\begin{array}{l}\text { If a mother is breastfeeding her child } \\
\text { but wishes to contracept, what } \\
\text { methods do you recommend? }\end{array}$ & 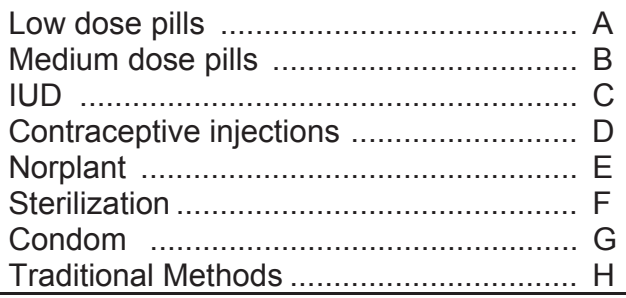 & \\
\hline G15b. & $\begin{array}{l}\text { If a mother is breastfeeding her child } \\
\text { but wishes to contracept, what } \\
\text { methods do you recommend the most? }\end{array}$ & 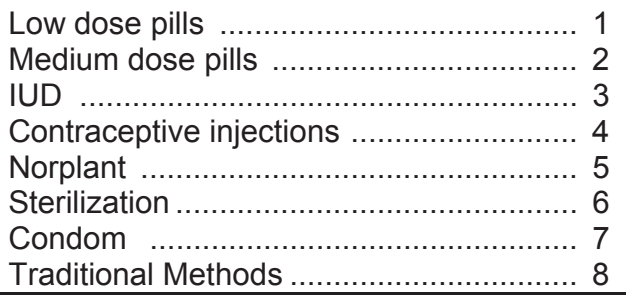 & \\
\hline G16. & $\begin{array}{l}\text { In the last year, what complaints have } \\
\text { you received from birth control pill } \\
\text { users regarding complications/side } \\
\text { effects? }\end{array}$ & 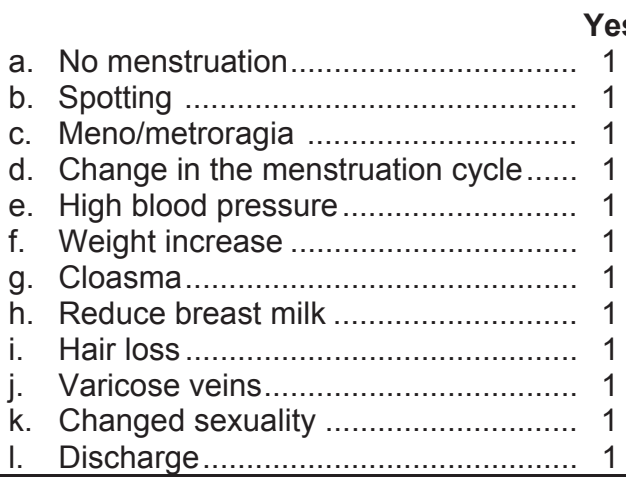 & $\begin{array}{l}\text { No } \\
3 \\
3 \\
3 \\
3 \\
3 \\
3 \\
3 \\
3 \\
3 \\
3 \\
3 \\
3 \\
\end{array}$ \\
\hline G17. & $\begin{array}{l}\text { In the last year, what complaints have } \\
\text { you received from patients receiving } \\
\text { contraceptive injections? }\end{array}$ & 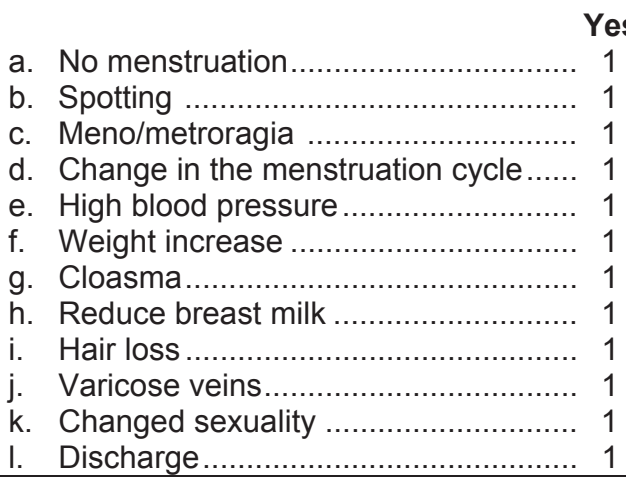 & $\begin{array}{l}\text { No } \\
3 \\
3 \\
3 \\
3 \\
3 \\
3 \\
3 \\
3 \\
3 \\
3 \\
3 \\
3\end{array}$ \\
\hline
\end{tabular}

\section{COMPLICATIONS/SIDE EFFECTS}

\begin{tabular}{|c|c|c|c|c|}
\hline G18. & $\begin{array}{l}\text { In the last year, what complaints have } \\
\text { you received for side } \\
\text { effects/complications from Cooper T } \\
\text { IUD users? }\end{array}$ & 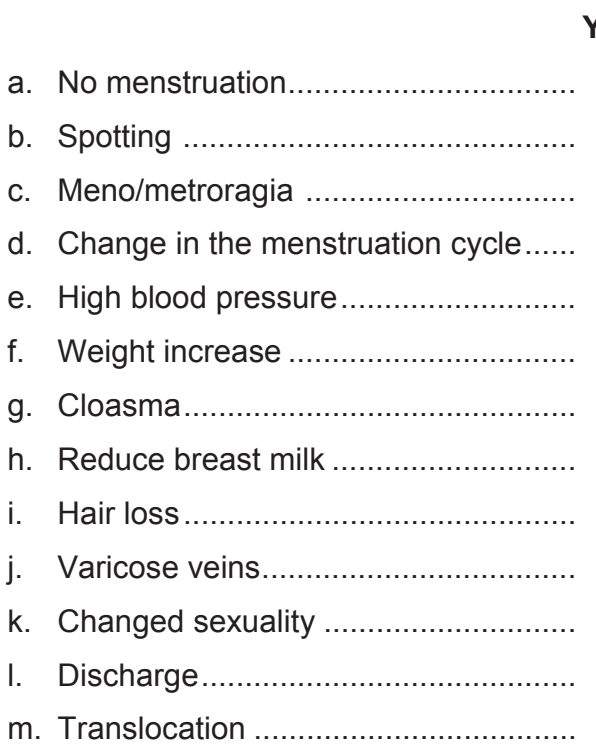 & $\begin{array}{l}\text { Yes } \\
1 \\
1 \\
1 \\
1 \\
1 \\
1 \\
1 \\
1 \\
1 \\
1 \\
1 \\
1 \\
1\end{array}$ & $\begin{array}{l}3 \\
3 \\
3 \\
3 \\
3 \\
3 \\
3 \\
3 \\
3 \\
3 \\
3 \\
3\end{array}$ \\
\hline G19. & $\begin{array}{l}\text { In the last year, what complaints have } \\
\text { you received regarding } \\
\text { complications/side effects from Implant } \\
\text { users? }\end{array}$ & 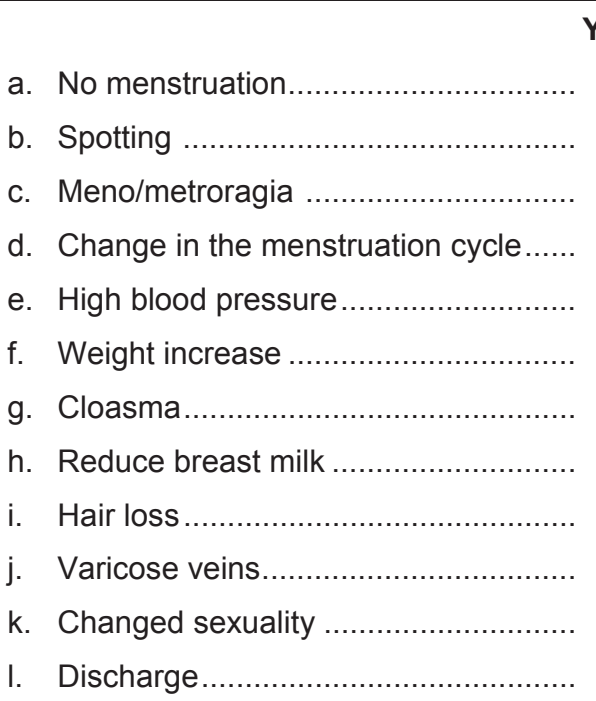 & $\begin{array}{l}\text { Yes } \\
1 \\
1 \\
1 \\
1 \\
1 \\
1 \\
1 \\
1 \\
1 \\
1 \\
1 \\
1\end{array}$ & $\begin{array}{l}3 \\
3 \\
3 \\
3 \\
3 \\
3\end{array}$ \\
\hline
\end{tabular}




\section{SECTION F: DIRECT OBSERVATION}

\section{REGISTRATION AND WAITING ROOM}

\begin{tabular}{|c|c|c|}
\hline FT1. & THE PLACE TO REGISTER IS: & 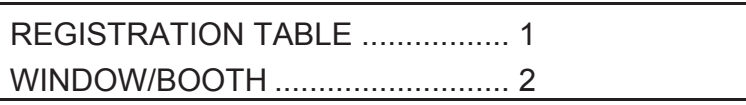 \\
\hline FT2. & DOES THIS ROOM HAVE: & 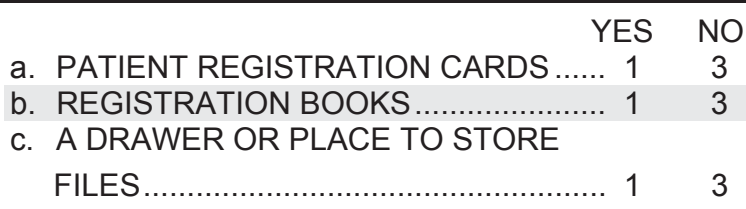 \\
\hline FT3. & IS THE WAITING ROOM CLOSED? & 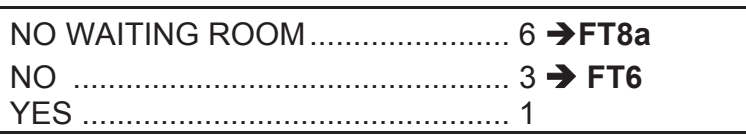 \\
\hline FT4. & DOES THE ROOM HAVE: & 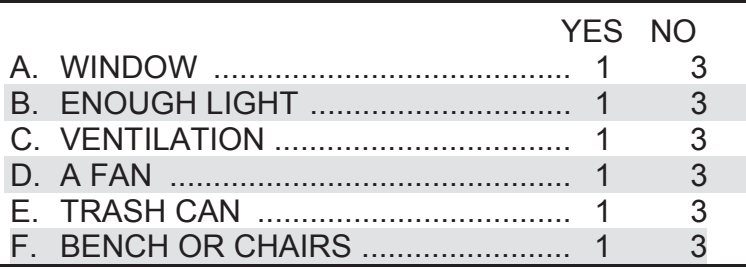 \\
\hline FT5. & $\begin{array}{l}\text { IS THE FLOOR CLEAN? } \\
\text { DIRTY = DUST, FOOD REMNANTS, AND } \\
\text { GARBAGE. }\end{array}$ & $\begin{array}{l}\text { DIRTY } \\
\text { CLEAN }\end{array}$ \\
\hline FT6. & $\begin{array}{l}\text { HOW CLEAN ARE THE WALLS IN THIS } \\
\text { ROOM? } \\
\text { DIRTY = IF THERE ARE MANY SPIDER } \\
\text { WEBS, SCRIBBLINGS, MOISTURE OR } \\
\text { PAINT PEELING. }\end{array}$ & $\begin{array}{l}\text { DIRTY } \\
\text { CLEAN }\end{array}$ \\
\hline FT7. & $\begin{array}{l}\text { WHAT IS THE CONDITION/CLEANLINESS } \\
\text { OF THE CEILING IN THIS ROOM? } \\
\text { DIRTY = IF THERE ARE MANY SPIDER } \\
\text { WEBS, DAMPNESS, ETC. }\end{array}$ & $\begin{array}{l}\text { DIRTY } \\
\text { CLEAN } \\
\text { BROKEN }\end{array}$ \\
\hline FT8. & $\begin{array}{l}\text { When it is the rainy season does this room } \\
\text { experience }[\ldots] \text { ? }\end{array}$ & 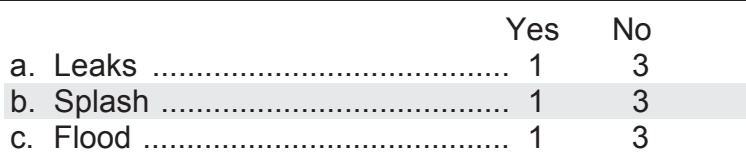 \\
\hline
\end{tabular}

\begin{tabular}{|c|c|c|}
\hline FT8a. & THE STAIRS IS NOT TOO STEEP & Yes No \\
\hline FT8b. & $\begin{array}{l}\text { SITTING TOILET IS AVAILABLE TO AVOID } \\
\text { THE ELDERLY FROM SQUATTING }\end{array}$ & 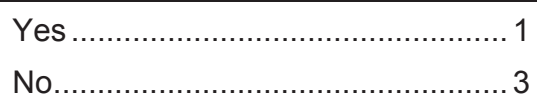 \\
\hline FT8c. & $\begin{array}{l}\text { CREEPING OF HANDLING ON THE STAIRS } \\
\text { AND TOILET }\end{array}$ & Yes \\
\hline FT8d. & $\begin{array}{l}\text { SPECIAL COUNTER/ REGISTRATION TABLE } \\
\text { FOR ELDERLY }\end{array}$ & $\begin{array}{ll} \\
\text { Yes } \\
\text { No }\end{array}$ \\
\hline
\end{tabular}




\section{SECTION F: DIRECT OBSERVATION}

EXAMINATION ROOM

\begin{tabular}{|c|c|c|}
\hline F1. & $\begin{array}{l}\text { HOW CLEAN IS THE FLOOR IN THIS } \\
\text { ROOM? } \\
\text { DIRTY = IF A LOT OF, FOOD REMNANTS, } \\
\text { SCATTERED GARBAGE IS FOUND. }\end{array}$ & $\begin{array}{l}\text { DIRTY } \\
\text { CLEAN }\end{array}$ \\
\hline F2. & $\begin{array}{l}\text { HOW CLEAN ARE THE WALLS IN THIS } \\
\text { ROOM? } \\
\text { DIRTY = IF LOTS OF SPIDER WEBS, } \\
\text { SCRIBBLINGS, MOISTURE OR PAINT } \\
\text { PEELING OFF }\end{array}$ & $\begin{array}{l}\text { DIRTY } \\
\text { CLEAN }\end{array}$ \\
\hline F2a. & $\begin{array}{l}\text { WHAT IS THE CONDITION/CLEANLINESS } \\
\text { OF THE CEILING IN THIS ROOM ? } \\
\text { DIRTY = IF THERE ARE LOTS OF SPIDER } \\
\text { WEBS, DAMPNESS, ETC. }\end{array}$ & 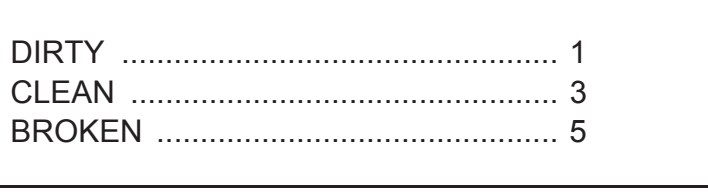 \\
\hline F2b. & DOES THE ROOM HAVE: & 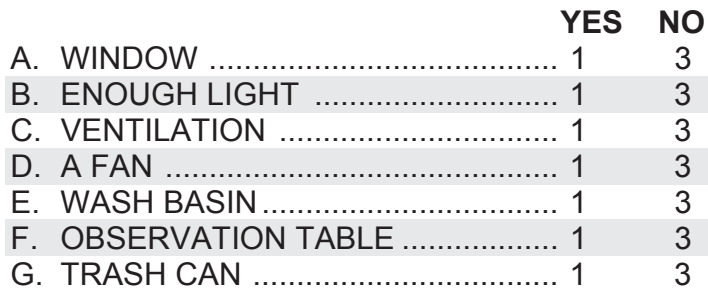 \\
\hline F3. & $\begin{array}{l}\text { ARE THERE CURTAINS SEPARATING THE } \\
\text { EXAMINATION ROOMS? }\end{array}$ & 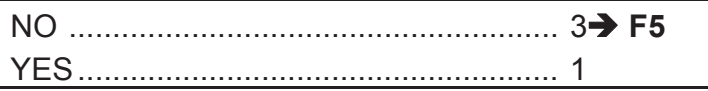 \\
\hline F4. & $\begin{array}{l}\text { WHAT IS THE CONDITION OF THE } \\
\text { CURTAINS? } \\
\text { DIRTY = NOT BEEN WASHED FOR A } \\
\text { LONG TIME, IF THEY HAVE BLOOD, DIRT, } \\
\text { ETC. ON THEM }\end{array}$ & $\begin{array}{l}\text { DIRTY } \\
\text { CLEAN }\end{array}$ \\
\hline
\end{tabular}

\begin{tabular}{|c|c|c|}
\hline F5. & $\begin{array}{l}\text { WHAT PROVISIONS ARE MADE FOR } \\
\text { WASHING HANDS IN THIS ROOM? }\end{array}$ & $\begin{array}{l}\text { WASH STAND WITH RUNNING WATER …….. } 1 \\
\text { WASH BASIN WITH CLEAN WATER } \ldots \ldots \ldots \ldots \ldots . . . \\
\text { NOT AVAILABLE }\end{array}$ \\
\hline F8. & $\begin{array}{l}\text { CHECK: } \\
\text { IS THERE A SPECIAL INJECTION } \\
\text { ROOM? }\end{array}$ & $\begin{array}{l}\text { NO } \\
\text { YES }\end{array}$ \\
\hline F9. & $\begin{array}{l}\text { HOW CLEAN IS THE FLOOR IN THIS } \\
\text { ROOM? } \\
\text { DIRTY = IF THERE IS LOTS OF DUST, } \\
\text { FOOD REMNANTS, SCATTERED } \\
\text { GARBAGE. }\end{array}$ & $\begin{array}{l}\text { DIRTY } \\
\text { CLEAN }\end{array}$ \\
\hline F10. & $\begin{array}{l}\text { HOW CLEAN ARE THE WALLS IN THIS } \\
\text { ROOM? } \\
\text { DIRTY = IF A LOT OF SPIDER WEBS, } \\
\text { SCRIBBLINGS, MOISTURE, PAINT } \\
\text { PEELING OFF }\end{array}$ & 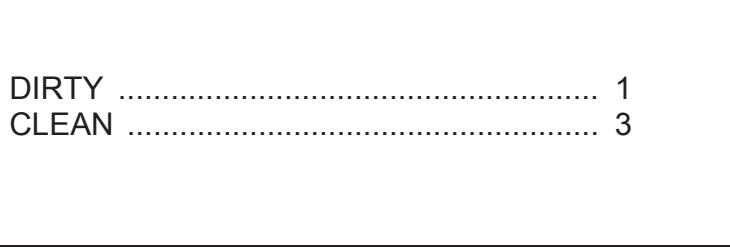 \\
\hline F10a. & $\begin{array}{l}\text { WHAT IS THE } \\
\text { CONDITION/CLEANLINESS OF THE } \\
\text { CEILING IN THIS ROOM? } \\
\text { DIRTY = IF THERE ARE MANY SPIDER } \\
\text { WEBS, DAMPNESS, ETC. }\end{array}$ & 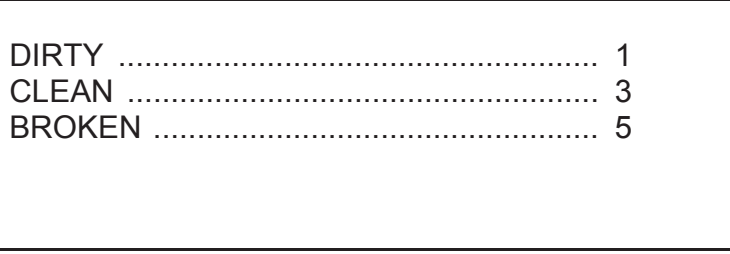 \\
\hline F10b. & DOES THE ROOM HAVE: & 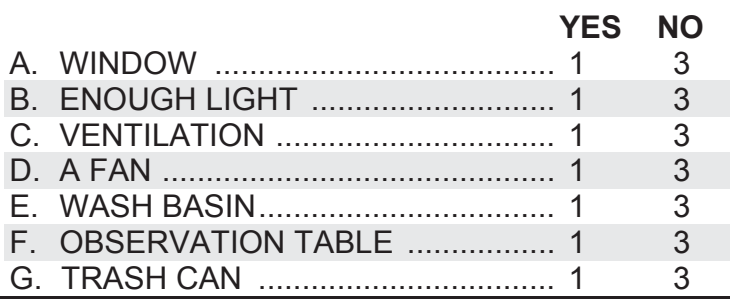 \\
\hline F10c. & $\begin{array}{l}\text { ARE THERE ANY CURTAINS } \\
\text { SEPARATING INJECTION ROOM? }\end{array}$ & 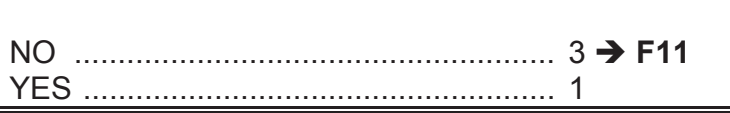 \\
\hline
\end{tabular}




\begin{tabular}{|c|c|c|}
\hline F10d. & $\begin{array}{l}\text { WHAT IS THE CONDITION OF THE } \\
\text { CURTAINS? } \\
\text { DIRTY = NOT BEEN WASHED FOR A } \\
\text { LONG TIME, IF THEY HAVE BLOOD, } \\
\text { DIRT, ETC. ON THEM. }\end{array}$ & 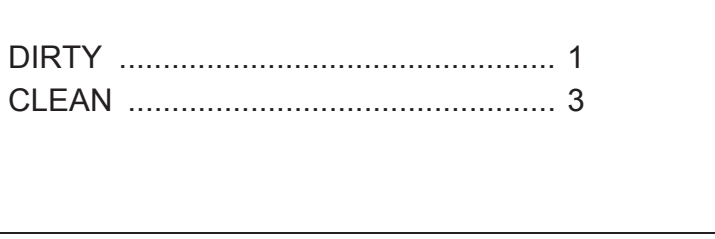 \\
\hline F11. & $\begin{array}{l}\text { WHAT PROVISIONS ARE MADE FOR } \\
\text { WASHING HANDS IN THIS ROOM? }\end{array}$ & $\begin{array}{l}\text { WASH STAND WITH RUNNING WATER } \ldots \ldots \ldots . .1 \\
\text { WASH BASIN WITH CLEAN WATER } \ldots \ldots \ldots \ldots . . . \\
\text { NOT AVAILABLE }\end{array}$ \\
\hline F13. & $\begin{array}{l}\text { For injecting patients, what kind of needle is } \\
\text { used? }\end{array}$ & $\begin{array}{l}\text { Disposable } \\
\text { Non disposable } \\
\text { Both }\end{array}$ \\
\hline F14. & $\begin{array}{l}\text { How are the needles used for injecting } \\
\text { patients sterilized? } \\
\text { THERE CAN BE MORE THAN ONE } \\
\text { ANSWER }\end{array}$ & 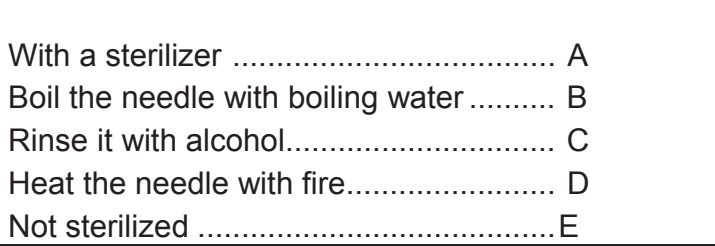 \\
\hline
\end{tabular}

\section{FP - MCH ROOMS}

\begin{tabular}{|c|c|c|}
\hline F15. & $\begin{array}{l}\text { CHECK POINT: } \\
\text { ARE THERE SPECIAL ROOMS FOR } \\
\text { FP/MCH? }\end{array}$ & $\begin{array}{l}\text { NO } \\
\text { YES }\end{array}$ \\
\hline F16. & $\begin{array}{l}\text { HOW CLEAN ARE THE FLOORS IN THIS } \\
\text { ROOM? } \\
\text { DIRTY - IF LOTS OF DUST, FOOD } \\
\text { REMNANTS, SCATTERED GARBAGE. }\end{array}$ & 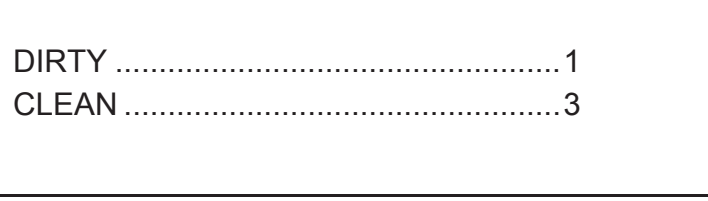 \\
\hline F17a. & $\begin{array}{l}\text { HOW CLEAN ARE THE CEILINGS IN THIS } \\
\text { ROOM? } \\
\text { DIRTY - IF THERE ARE LOTS OF SPIDER } \\
\text { WEBS, SCRIBBLINGS, MOISTURE, } \\
\text { PAINT PEELING OFF. }\end{array}$ & 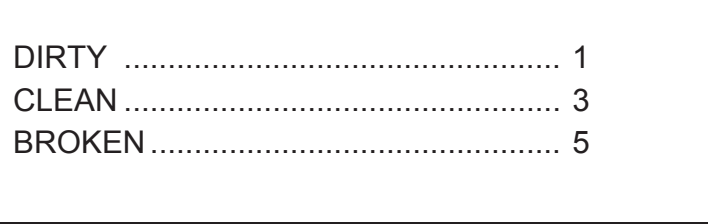 \\
\hline
\end{tabular}

\begin{tabular}{|c|c|c|c|c|}
\hline F17b. & DOES THE ROOM HAVE: & 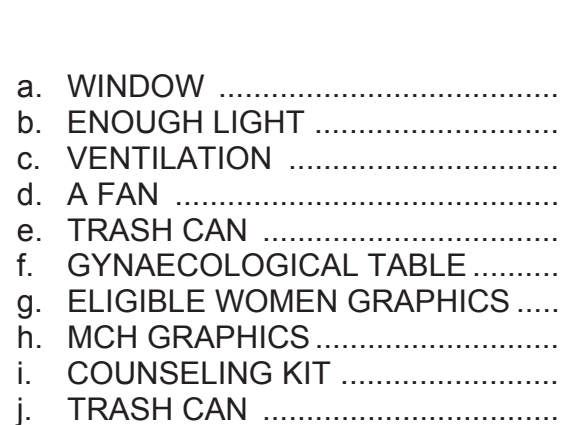 & $\begin{array}{l}\text { YES } \\
1 \\
1 \\
1 \\
1 \\
1 \\
1 \\
1 \\
1 \\
1 \\
1\end{array}$ & $\begin{array}{l}\text { NO } \\
3 \\
3 \\
3 \\
3 \\
3 \\
3 \\
3 \\
3 \\
3 \\
3\end{array}$ \\
\hline F18. & $\begin{array}{l}\text { ARE THERE CURTAINS THAT SHUT } \\
\text { OFF THE EXAMINATIONS ROOMS? }\end{array}$ & \multicolumn{3}{|c|}{ 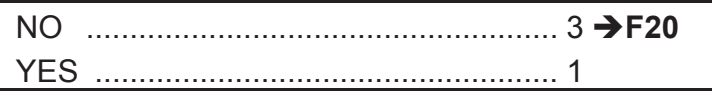 } \\
\hline F19. & $\begin{array}{l}\text { HOW ARE THE CONDITIONS OF THESE } \\
\text { CURTAINS? } \\
\text { DIRTY = NOT BEEN WASHED FOR A } \\
\text { LONG TIME, THEY HAVE BLOOD, DIRT, } \\
\text { ETC. ON THEM. }\end{array}$ & \multicolumn{3}{|l|}{ 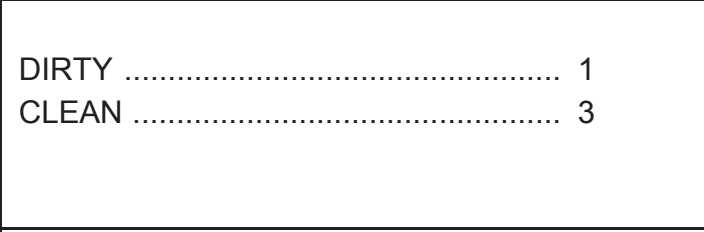 } \\
\hline F20. & $\begin{array}{l}\text { WHAT ARE THE PROVISIONS FOR } \\
\text { WASHING HANDS THAT ARE FOUND IN } \\
\text { THIS ROOM? }\end{array}$ & \multicolumn{3}{|c|}{$\begin{array}{l}\text { WASH STAND WITH RUNNING WATER } \ldots \ldots . .1 \\
\text { WASH BASIN WITH CLEAN WATER } \\
\text { NOT AVAILABLE }\end{array}$} \\
\hline
\end{tabular}

\section{VACCINE ROOMS}

\begin{tabular}{|c|c|c|}
\hline F30a. & $\begin{array}{l}\text { Does this Puskesmas/Pustu store have } \\
\text { vaccines? }\end{array}$ & No \\
\hline F30. & $\begin{array}{l}\text { Where are vaccines stored at this } \\
\text { Puskesmas? }\end{array}$ & $\begin{array}{l}\text { No storing place for vaccines available } \ldots . . .6 \rightarrow \mathrm{F} 33 \\
\text { Refrigerator/freezer/special vaccine } \\
\text { Cooling box } \\
\text { Regular refrigerator }\end{array}$ \\
\hline
\end{tabular}




\section{SECTION F: DIRECT OBSERVATION}

\section{VACCINE STORAGE ROOM}

\begin{tabular}{|c|c|c|}
\hline F31. & $\begin{array}{l}\text { Is there any graphic/record on the freezer's } \\
\text { temperature? }\end{array}$ & 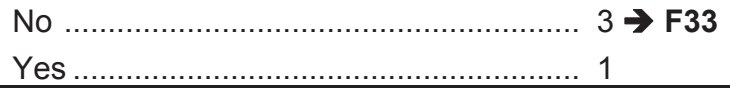 \\
\hline F32. & $\begin{array}{l}\text { If there is one, state the the latest record. } \\
\text { a. When was the latest record taken } \\
\text { b. Freezer's temperature at last record }\end{array}$ & 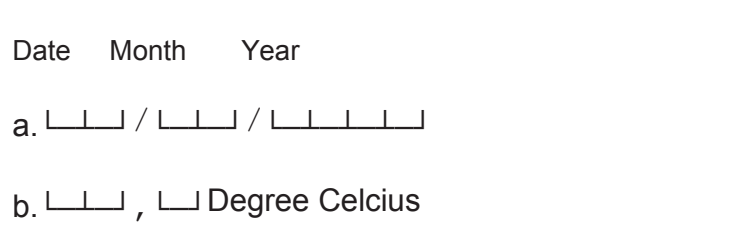 \\
\hline F33. & $\begin{array}{l}\text { How many thermos flasks are used for } \\
\text { carrying vaccines to the field? }\end{array}$ & 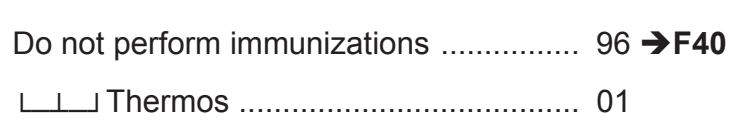 \\
\hline F34. & $\begin{array}{l}\text { For vaccinations, what kind of needle is } \\
\text { used? }\end{array}$ & 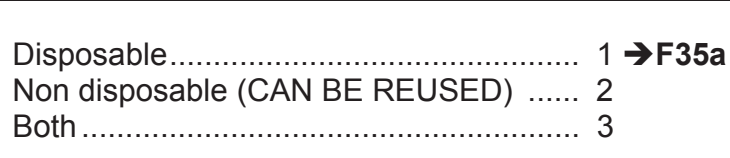 \\
\hline F35. & $\begin{array}{l}\text { How are the needles used for injecting } \\
\text { patients sterilized? } \\
\text { THERE CAN BE MORE THAN ONE } \\
\text { ANSWER }\end{array}$ & 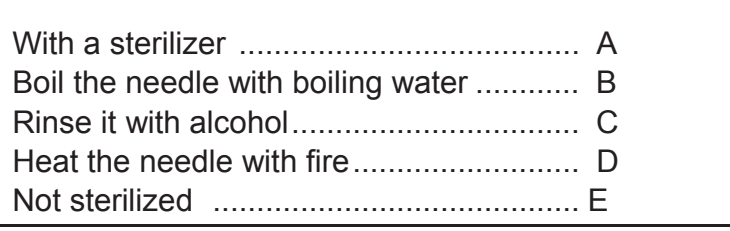 \\
\hline F35a. & Do you have needles in stock today? & 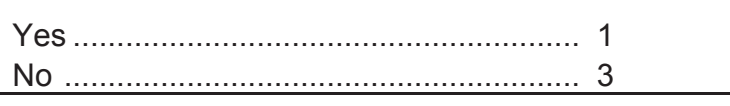 \\
\hline F35b. & $\begin{array}{l}\text { In the last } 6 \text { months, how many weeks } \\
\text { were you out of stock of needles? }\end{array}$ & 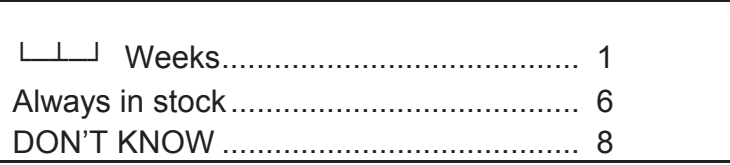 \\
\hline
\end{tabular}

LABORATORY

\begin{tabular}{|c|c|c|}
\hline F40. & Is there a laboratory at the Puskesmas? & $\begin{array}{l}\text { No } \\
\text { Yes } \ldots \ldots \ldots \ldots \ldots \ldots \ldots \ldots \\
\end{array}$ \\
\hline F41. & $\begin{array}{l}\text { HOW CLEAN IS THE FLOOR IN THIS } \\
\text { ROOM? } \\
\text { DIRTY = IF A LOT OF DUST, FOOD } \\
\text { REMNANTS, SCATTERED GARBAGE }\end{array}$ & 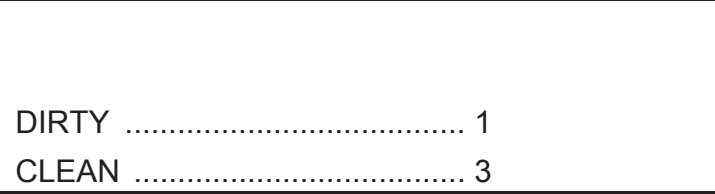 \\
\hline F42. & $\begin{array}{l}\text { HOW CLEAN ARE THE WALLS IN THIS } \\
\text { ROOM? } \\
\text { DIRTY = IF A LOT OF SPIDER WEBS, } \\
\text { SCIBBLINGS, MOISTURE, PAINT } \\
\text { PEELING OFF. }\end{array}$ & 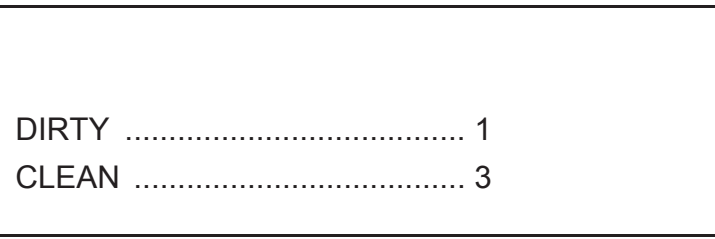 \\
\hline F43. & $\begin{array}{l}\text { WHAT PROVISIONS IS MADE FOR HAND } \\
\text { WASHING IN THIS ROOM? }\end{array}$ & $\begin{array}{l}\text { WASH STAND WITH RUNNING WATER } \ldots . . .1 \\
\text { WASHBASIN WITH CLEAN WATER } \ldots \ldots \ldots \ldots . . .3 \\
\text { NONE }\end{array}$ \\
\hline F44. & $\begin{array}{l}\text { IS THERE A GARBAGE CAN IN THIS } \\
\text { ROOM? }\end{array}$ & YES \\
\hline F45. & Does this lab have these instruments? & 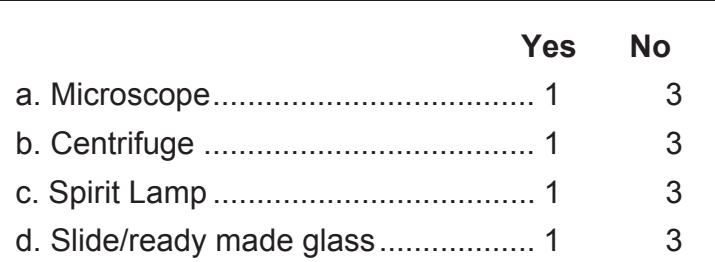 \\
\hline
\end{tabular}




\section{SECTION CP: INTERVIEW SESSION NOTES}

\begin{tabular}{|c|c|c|c|c|c|c|c|}
\hline \multirow{2}{*}{$\begin{array}{l}\text { LANGMAIN (CK1). } \\
\text { LANGOTHR (CK2). }\end{array}$} & \multicolumn{2}{|l|}{ Interview was entirely/mostly conducted in what language? } & \multicolumn{5}{|l|}{$\perp \_$other } \\
\hline & Other languaged used (if any): & & \multicolumn{5}{|l|}{ ــ other } \\
\hline \multicolumn{8}{|l|}{ LANGUAGE CODE: } \\
\hline 00. Bahasa Indonesia & 04. Batak & 08. Sasak & 12. Makassar & 16. & Toraja & & Lampung \\
\hline 01. Java & 05. Bugis & 09. Minang & 13. Nias & 17. & Lahat & 95. & Other, \\
\hline 02. Sunda & 06. Cina & 10. Banjar & 14. Palembang & 18. & Other South Sumatera & 96. & NA \\
\hline 03. Bali & 07. Madura & 11. Bima & 15. Sumbawa & 19. & Betawi & & \\
\hline
\end{tabular}

\begin{tabular}{|c|c|c|c|}
\hline RESULT (FP3). RESULTS OF INTERVIEW & $\begin{array}{l}\text { REASON (FP4). REASON FOR ANSWERING "2" / “3” IN } \\
\text { RESULT. }\end{array}$ & FP6. MONITORING BY SUPERVISOR & \\
\hline $\begin{array}{l}\text { 1. Completed } \rightarrow \text { FP6 } \\
\text { 2. Partly completed } \\
\text { 3. Not completed } \\
\text { 4. Twin EA with EA } \downarrow ـ \rightarrow \text { FP6 }\end{array}$ & $\begin{array}{l}\text { 1. Respondent was travelling/not in location } \\
\text { 2. Respondent was too busy } \\
\text { 3. Respondent refused }\end{array}$ & $\begin{array}{ll}\text { a. } & \text { Observed (sup_obs) .......... } 1 \\
\text { b. } & \text { Checked (sup_edit)........... } 1 \\
\text { c. } & \text { Verified (sup_veri).............1 }\end{array}$ & $\begin{array}{l}\text { No } \\
3 \\
3 \\
3\end{array}$ \\
\hline
\end{tabular}

\section{INTERVIEWER NOTE:}


INDONESIAN FAMILY LIFE SURVEY 2014 HEALTH FACILITY

DOCTOR / CLINIC / MIDWIFE / PARAMEDIC / NURSE / VILLAGE MIDWIFE SECTIONS: LK, PB, A, B, PH, C, BD, E, H, CP

NAME OF FACILITY: 


\section{SECTION LK : CONTROL SHEET}

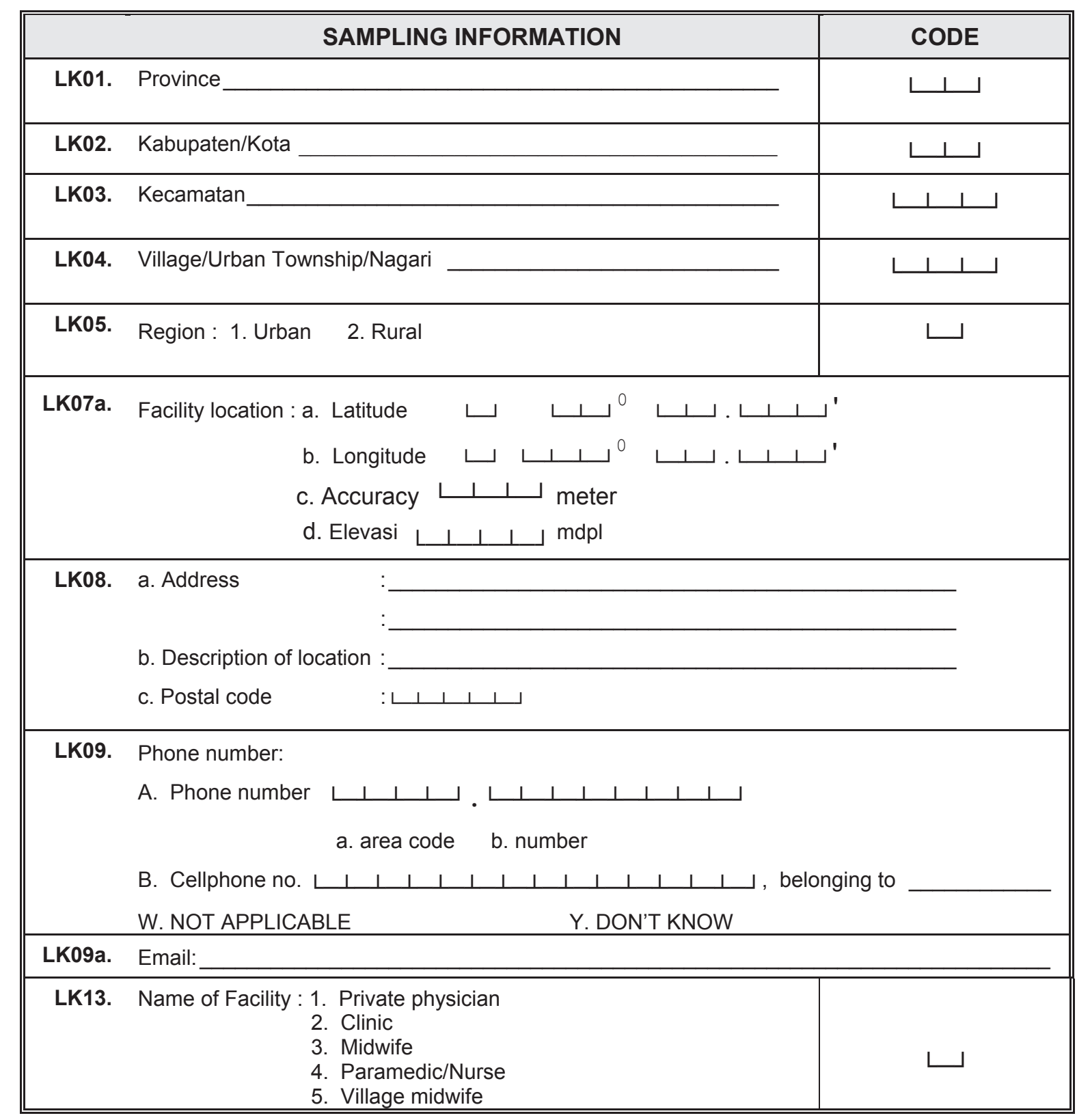

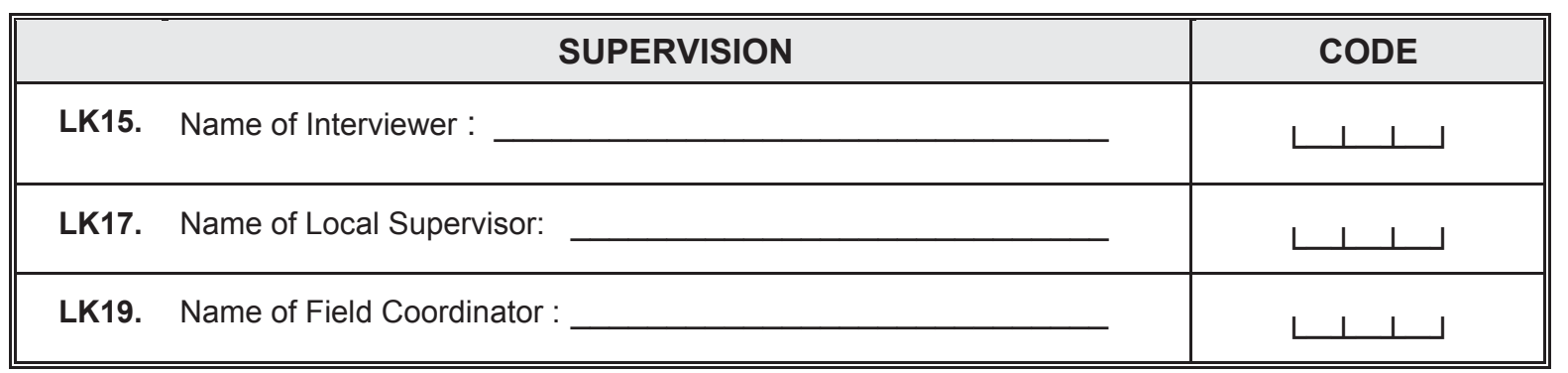

\begin{tabular}{|c|c|c|c|}
\hline LK20. & Did this facility have an interview in $1993 ?$ & 1. Yes & 3. No \\
\hline LK21. & Did this facility have an interview in $1997 ?$ & 1. Yes & 3. No \\
\hline LK22. & Did this facility have an interview in $1998 ?$ & 1. Yes & 3. No \\
\hline LK23. & Did this facility have an interview in $2000 ?$ & 1. Yes & 3. No \\
\hline LK24. & Did this facility have an interview in 2007 ? & 1. Yes & 3. No \\
\hline
\end{tabular}




\section{SECTION PB : JOINT PRACTICE}

Now, we would like to ask you some information about your place of practice.

\begin{tabular}{|c|c|c|}
\hline PB1. & Does this facility have more than one medical workers? & No \\
\hline PB2. & How many medical workers practice at this place? & $\downarrow \downarrow ـ$ medical workers \\
\hline \multirow[t]{9}{*}{ PB3. } & How many $[\ldots]$ practice at this place: & \\
\hline & A. General practitioner & A. $\left\llcorner\perp \_\right.$people \\
\hline & B. Pediatrician & B. $\llcorner\perp$ ل people \\
\hline & C. Obstetrician & C. Lـــ لـ people \\
\hline & E. Ear, nose, and throat specialist & E. $\left\llcorner\perp \_\right.$people \\
\hline & F. Dentist & F. Lــــــ people \\
\hline & G. Midwife & G. $\left\llcorner\perp \_\right.$people \\
\hline & H. Nurse & H. Lــ L p people \\
\hline & I. Other specialist & I. $L \perp$ لـ people \\
\hline PB4. & Do the medical workers in this place of practice share the same medical equipment? & Yes $\mathrm{C}$ \\
\hline PB5. & In this place of practice, how is the financial matters managed? & $\begin{array}{l}\text { Jointly managed } \\
\text { Individually managed } \\
\text { Other }\end{array}$ \\
\hline
\end{tabular}


SECTION A : GENERAL

Now, we would like to ask about your history.
A1. Name
A2. Age : 1 Years
8. DON'T KNOW

\begin{tabular}{|c|c|c|}
\hline$A 2 x$ & Sex & 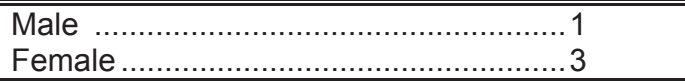 \\
\hline A2a. & Are you a doctor? & Yes \\
\hline A2b. & $\begin{array}{l}\text { What is the highest level of education } \\
\text { you have completed? }\end{array}$ & 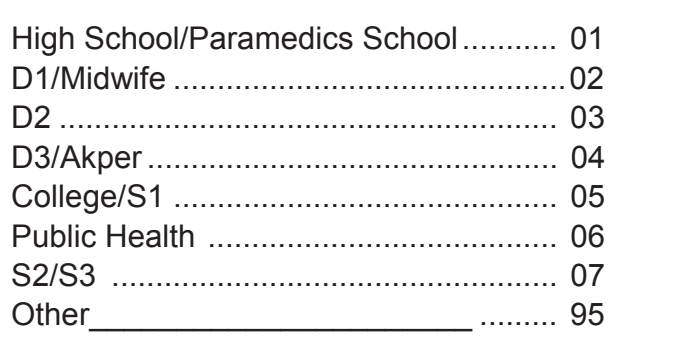 \\
\hline A2bb & $\begin{array}{l}\text { What is the accreditation status of your } \\
\text { university? }\end{array}$ & $\begin{array}{l}\text { A. Accreditation A } \\
\text { B. Accreditation B } \\
\text { C. Accreditation C } \\
\text { D. Accreditation process ongoing } \\
\text { E. Not accredited } \\
\text { F. Have not been registered } \\
\text { Y. DON'T KNOW }\end{array}$ \\
\hline A2c. & Are you a Bidan Delima? & 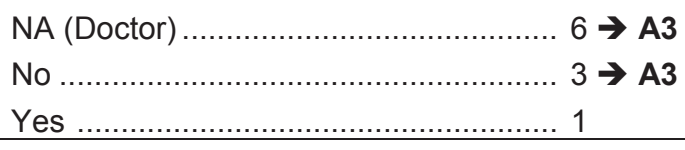 \\
\hline A2d. & $\begin{array}{l}\text { How long was the additional training to } \\
\text { be a Bidan Delima? }\end{array}$ & $\begin{array}{l}\text { 6. No additional training } \rightarrow \mathbf{A} \mathbf{3} \\
\text { 1. Training } \\
\text { 1. Weeks } \\
\text { 2. Months }\end{array}$ \\
\hline A2e. & $\begin{array}{l}\text { How much is the cost of training and } \\
\text { certication to be a Bidan Delima? }\end{array}$ & 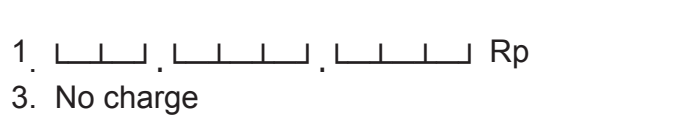 \\
\hline
\end{tabular}

\begin{tabular}{|ll|}
\hline A3. At what university did you graduate as \\
physician: \\
a. Name of University? \\
b. Date graduated? \\
\hline A3a. In what year did you first start your \\
practice?
\end{tabular}

A4. How long have you been practicing here?

A5. What is the status of the place where you practice?

\begin{tabular}{||cl|}
\hline A5a & $\begin{array}{l}\text { Usually, what is the approximate total } \\
\text { number of hours you work here in a } \\
\text { week? }\end{array}$ \\
\hline A5b & $\begin{array}{l}\text { What is your estimated monthly income } \\
\text { from working in this place? }\end{array}$ \\
\hline A9. & Do you speak the local language? \\
\hline A10. & Do you originate from this province? \\
\hline A17. & $\begin{array}{l}\text { Do you have electricity at this place of } \\
\text { practice? }\end{array}$ \\
\hline
\end{tabular}

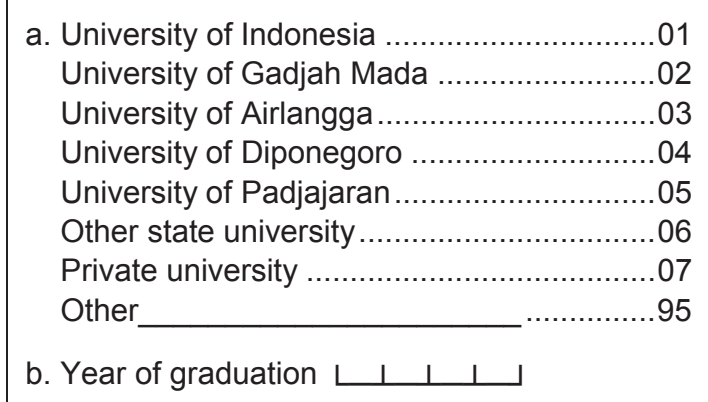

a. University of Indonesia

University of Gadah Mada ......................01

University of Airlangga ....................................... 03

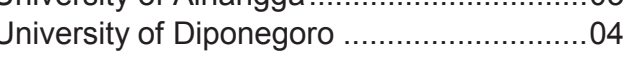

University of Padjajaran .................................05

Other state university ....................................06

Private university ........................................ 07

Other _ _................95

b. Year of graduation $\llcorner\perp \perp$

Year $\perp \perp \perp$

a. ......................................... $\sqcup \sqcup$ years

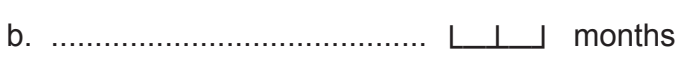

Own house ……………………………..... 01

Government house ............ 02

Other place, rented/contracted/

inco sharing /.....

.

........................ 04

Puskesmas/Pustu ............................................... 05

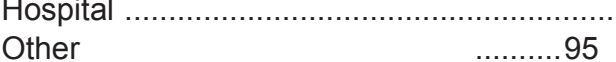

$\downarrow \perp$ Hours

$\sqcup \perp . \sqcup \perp \perp . \sqcup \perp \perp$ Rp

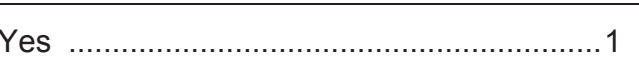
No ………………………......................

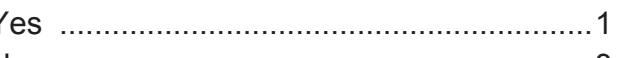
No

No

2
3
4
6
7
5





01
02
03
04
05
06




\section{SEKSI A :UMUM}

\begin{tabular}{|c|c|c|}
\hline A18. & If yes, mention the electricity source used: & 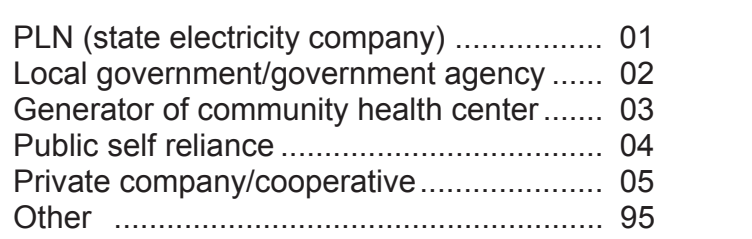 \\
\hline A19. & Mention the main water source used: & 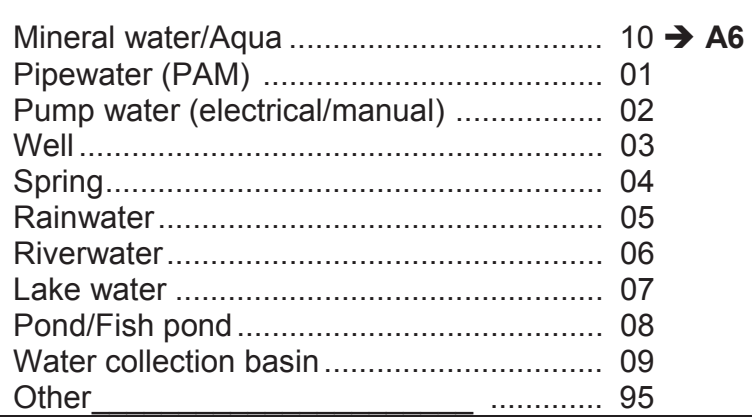 \\
\hline A20. & $\begin{array}{l}\text { Is this water source located in the } \\
\text { building? }\end{array}$ & Yes \\
\hline A21. & $\begin{array}{l}\text { If not, how far is the water source from the } \\
\text { practice? }\end{array}$ & 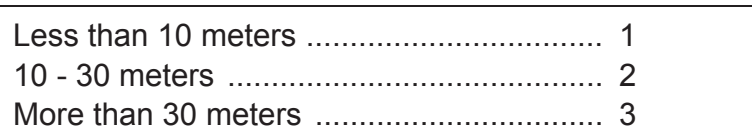 \\
\hline A6. & $\begin{array}{l}\text { Before practicing here, were you ever a } \\
\text { practicing physician at: } \\
\text { CAPI CEK: JIKA a dan b JAWABAN }=3 \\
\text { MAKA } \rightarrow \text { A } 7\end{array}$ & 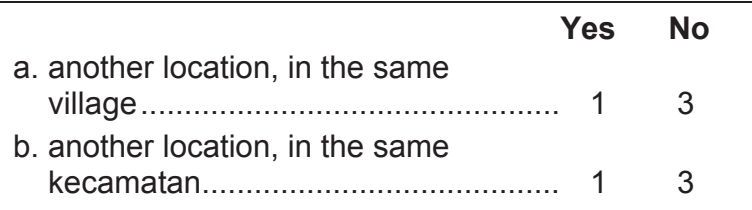 \\
\hline A6a. & Does that facility belong to Puskesmas? & 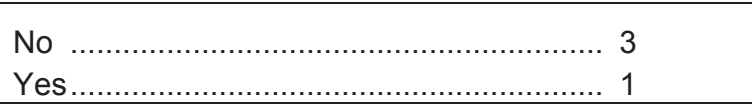 \\
\hline A7. & $\begin{array}{l}\text { Do you as a physician also have another } \\
\text { place of practice? }\end{array}$ & 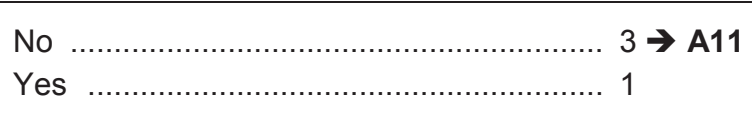 \\
\hline A7a. & How many practice place do you have? & $\left\llcorner\perp \_\right.$practice place \\
\hline A8. & $\begin{array}{l}\text { How far is this practice place from here? } \\
\text { [IF THERE IS MORE THAN ONE PLACE } \\
\text { TO PRACTICE MENTION THE MOST } \\
\text { IMPORTANT] }\end{array}$ & $\begin{array}{l}\text { Less than } 5 \mathrm{~km} \ldots \ldots \ldots \ldots \ldots \ldots \ldots \ldots \ldots \\
\text { Between } 5 \text { and } 10 \mathrm{kms} \\
\text { More than } 10 \mathrm{kms}\end{array}$ \\
\hline
\end{tabular}

\begin{tabular}{|c|c|c|c|}
\hline A11. & Where is your primary place of work? & 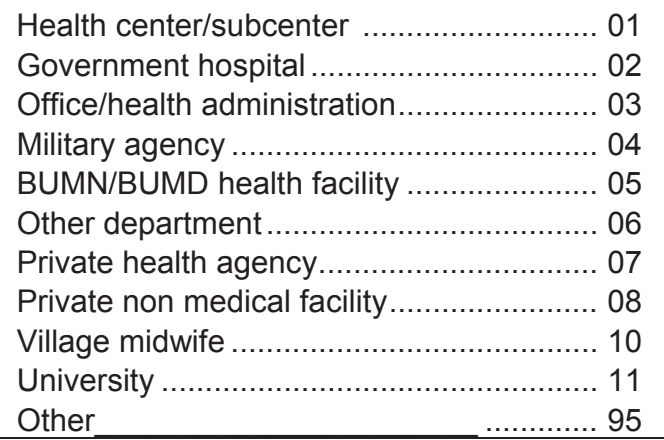 & \\
\hline A12. & $\begin{array}{l}\text { Please give us the name and address of } \\
\text { your primary place of work: } \\
\text { (IF ADDRESS IS THE SAME AS IN LK, } \\
\text { CIRCLE "3") }\end{array}$ & \begin{tabular}{lll} 
a. Institution & \\
\cline { 2 - 2 } b. Address & \\
c. Village & 1. & 3. Same \\
d. Kecamatan 1. & 3. Same \\
e. Kabupaten 1. & 3. Same \\
f. Province & 1. & 3. Same \\
\end{tabular} & $\begin{array}{l}\text { 8. DK } \\
\text { 8. DK } \\
\text { 8. DK } \\
\text { 8. DK } \\
\text { 8. DK }\end{array}$ \\
\hline A26. & $\begin{array}{l}\text { Which category best describes the work } \\
\text { you did in your primary job? }\end{array}$ & 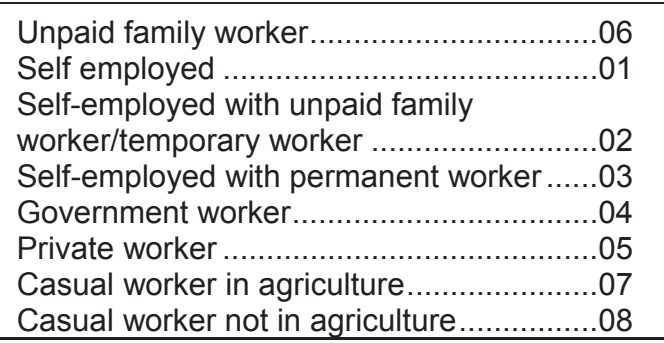 & \\
\hline A21a. & $\begin{array}{l}\text { What is your estimated monthly income } \\
\text { from your primary place of work? }\end{array}$ & 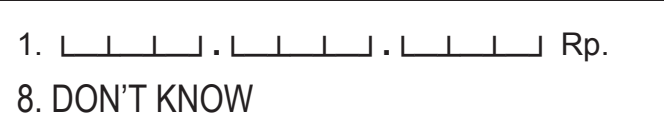 & \\
\hline A21b. & $\begin{array}{l}\text { What is your overall montly income from } \\
\text { all practices you have? }\end{array}$ & 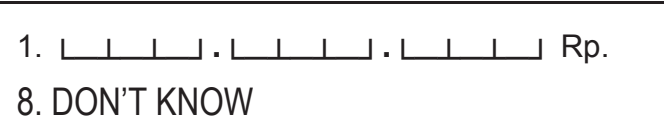 & \\
\hline
\end{tabular}


SEKSI A :UMUM

\begin{tabular}{|c|c|c|c|c|}
\hline \multirow[b]{2}{*}{ (ATYPE) } & \multicolumn{3}{|c|}{ A23. } & \multirow{2}{*}{ How did the change in $[\ldots]$ affect services at this facility? } \\
\hline & Since 2007 have & een dist & & \\
\hline A. Availability of drugs & $\begin{array}{l}\text { 6. NA } \\
\downarrow\end{array}$ & $\begin{array}{l}\text { 3. No } \\
\downarrow\end{array}$ & 1. Yes $\rightarrow$ & $\begin{array}{l}\text { Yes, became better } \\
\text { No change } \\
\text { Yes, became worse }\end{array}$ \\
\hline B. Availability of equipment & $\begin{array}{l}\text { 6. NA } \\
\downarrow\end{array}$ & $\begin{array}{l}\text { 3. No } \\
\downarrow\end{array}$ & 1. Yes $\rightarrow$ & $\begin{array}{l}\text { Yes, became better } \\
\text { No change } \\
\text { Yes, became worse }\end{array}$ \\
\hline C. Availability of water & $\begin{array}{l}\text { 6. NA } \\
\downarrow\end{array}$ & $\begin{array}{l}\text { 3. No } \\
\downarrow\end{array}$ & 1. Yes $\rightarrow$ & $\begin{array}{l}\text { Yes, became better } \\
\text { No change } \\
\text { Yes, became worse }\end{array}$ \\
\hline D. Price of drugs & $\begin{array}{l}\text { 6. NA } \\
\downarrow\end{array}$ & $\begin{array}{l}\text { 3. No } \\
\downarrow\end{array}$ & 1. Yes $\rightarrow$ & Yes, became better \\
\hline E. Price of equipment & $\begin{array}{l}\text { 6. NA } \\
\downarrow\end{array}$ & $\begin{array}{l}\text { 3. No } \\
\downarrow\end{array}$ & 1. Yes $\rightarrow$ & $\begin{array}{l}\text { Yes, became better } \\
\text { No change } \\
\text { Yes, became worse }\end{array}$ \\
\hline F. Price of fuels & $\begin{array}{l}\text { 6. NA } \\
\downarrow\end{array}$ & $\begin{array}{l}\text { 3. No } \\
\downarrow\end{array}$ & 1. Yes $\rightarrow$ & 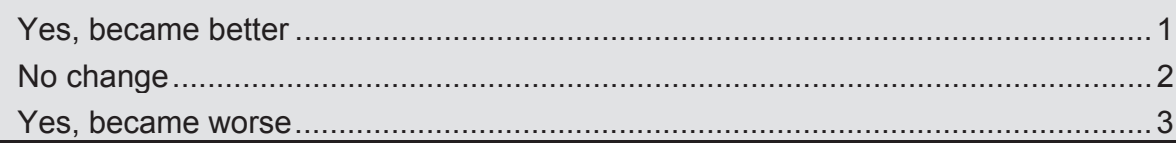 \\
\hline H. Number of patients & $\begin{array}{l}\text { 6. NA } \\
\downarrow\end{array}$ & $\begin{array}{l}\text { 3. No } \\
\downarrow\end{array}$ & 1. Yes $\rightarrow$ & $\begin{array}{l}\text { Yes, became better } \\
\text { No change } \\
\text { Yes, became worse }\end{array}$ \\
\hline I. Number of staff & $\begin{array}{l}\text { 6. NA } \\
\downarrow\end{array}$ & $\begin{array}{l}\text { 3. No } \\
\downarrow\end{array}$ & 1. Yes $\rightarrow$ & 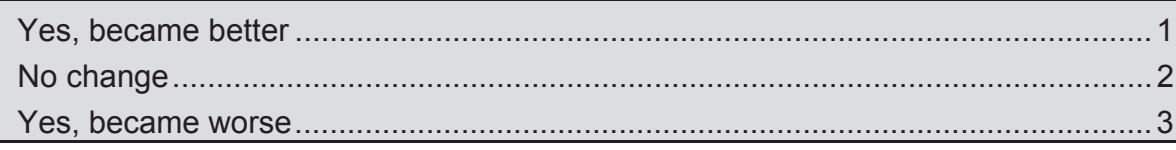 \\
\hline J. Family planning supplies & $\begin{array}{l}\text { 6. NA } \\
\downarrow\end{array}$ & $\begin{array}{l}\text { 3. No } \\
\downarrow\end{array}$ & 1. Yes $\rightarrow$ & $\begin{array}{l}\text { Yes, became better } \\
\text { No change } \\
\text { Yes, became worse }\end{array}$ \\
\hline K. Air Quality (Smoke from forest fires) & $\begin{array}{l}\text { 6. NA } \\
\downarrow \\
\text { SECTION B }\end{array}$ & $\begin{array}{l}\text { 3. No } \\
\downarrow \\
\text { SECTI }\end{array}$ & 1. Yes $\rightarrow$ & $\begin{array}{l}\text { Yes, became better } \\
\text { No change } \\
\text { Yes, became worse }\end{array}$ \\
\hline
\end{tabular}


SECTION B : SERVICE ABILITY

Now, we would like to ask about the time and the types of service in this facility.

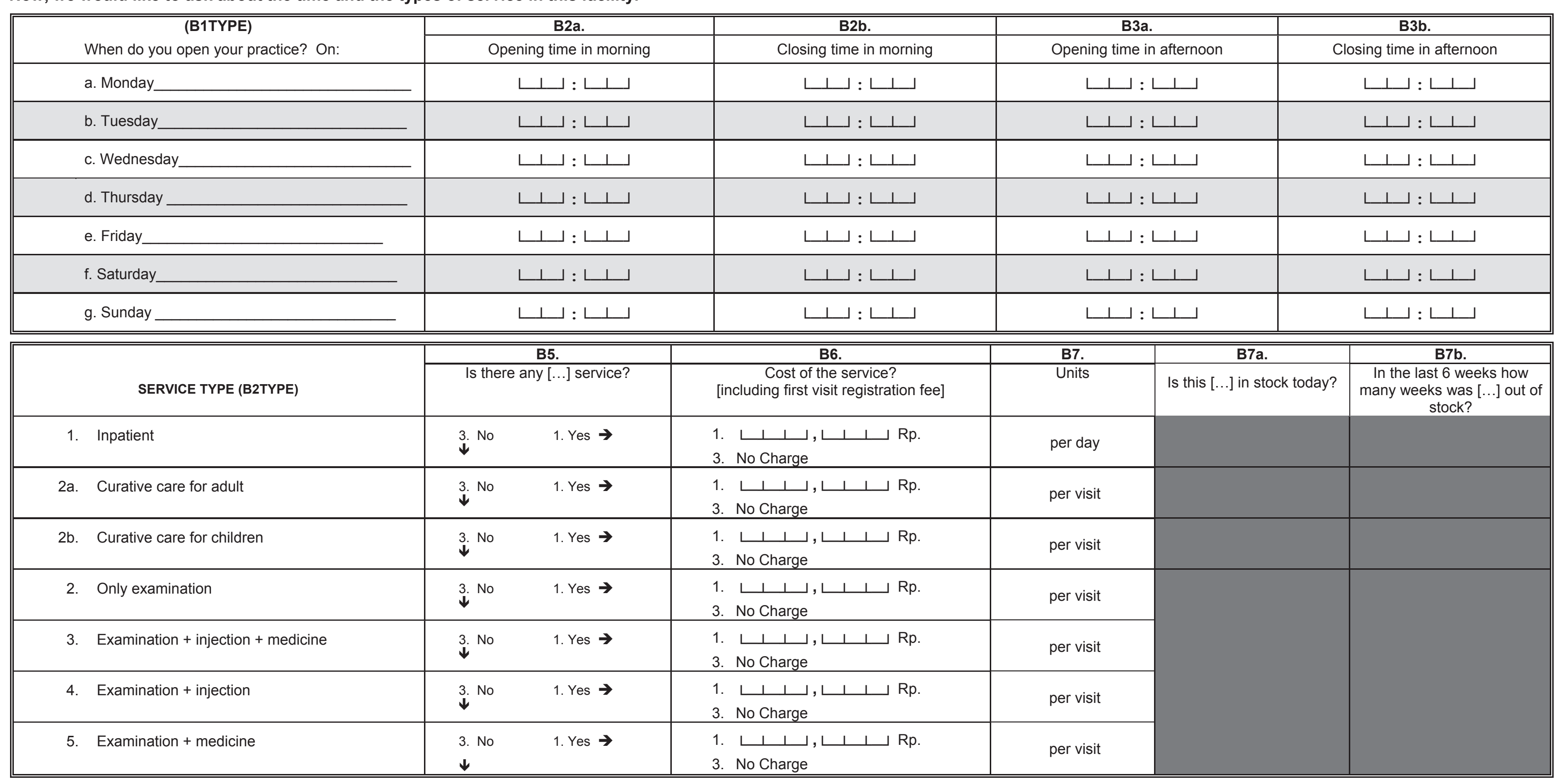




\section{SECTION B : SERVICE ABILITY}

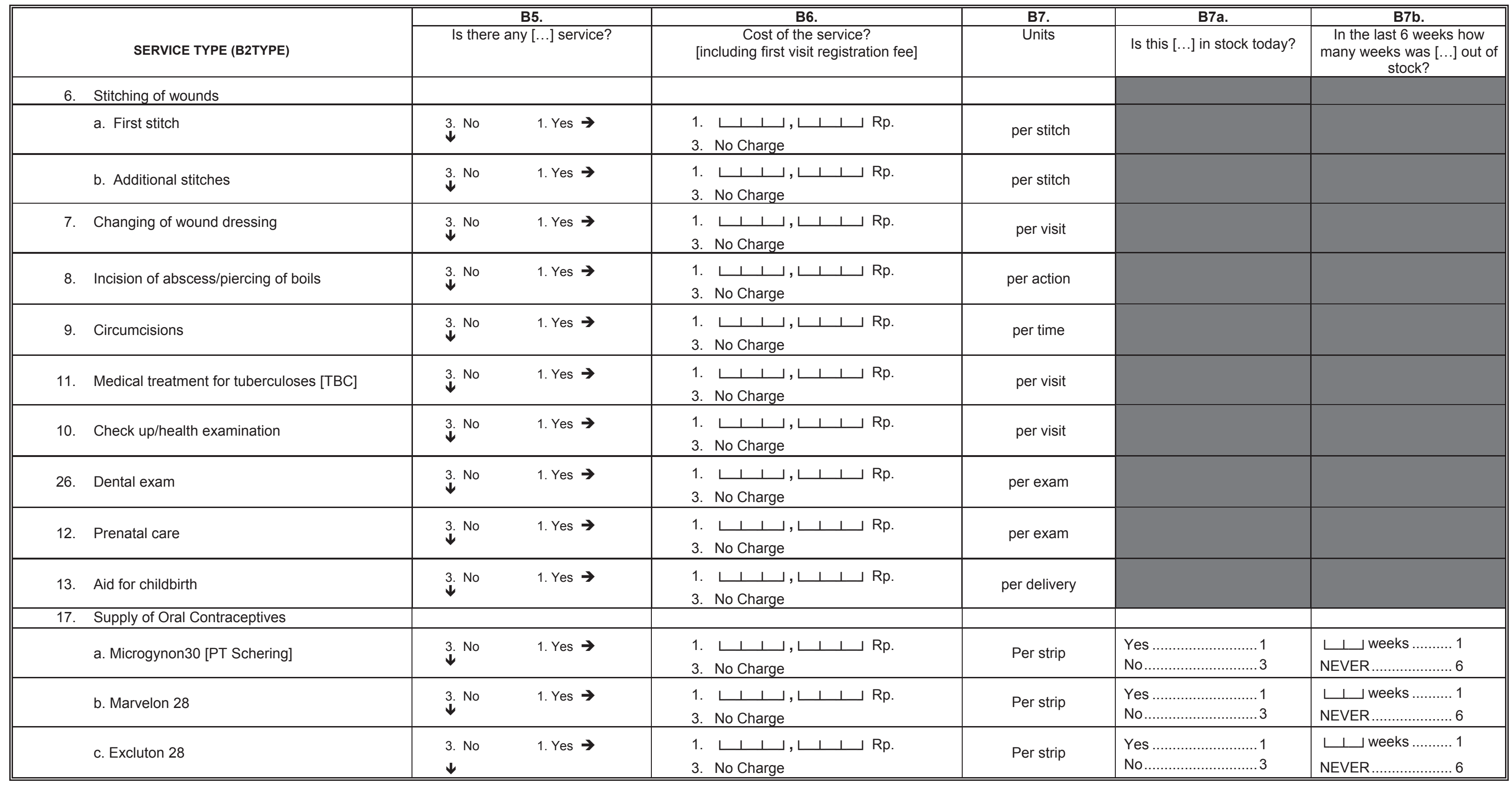




\section{SECTION B : SERVICE ABILITY}

\begin{tabular}{|c|c|c|c|c|c|c|c|}
\hline \multirow{2}{*}{\multicolumn{2}{|c|}{ SERVICE TYPE (B2TYPE) }} & \multirow{2}{*}{\multicolumn{2}{|c|}{$\begin{array}{l}\text { B5. } \\
\text { Is there any [...] service? }\end{array}$}} & \multirow{2}{*}{$\begin{array}{c}\text { B6. } \\
\text { Cost of the service? } \\
\text { [including first visit registration fee] }\end{array}$} & \multirow{2}{*}{$\begin{array}{l}\text { B7. } \\
\text { Units }\end{array}$} & B7a. & \multirow{2}{*}{$\begin{array}{c}\text { B7b. } \\
\text { In the last } 6 \text { weeks how } \\
\text { many weeks was }[\ldots] \text { out of } \\
\text { stock? }\end{array}$} \\
\hline & & & & & & Is this $[\ldots]$ in stock today? & \\
\hline & f. Pil KB Andalan/BKKBN & 3. No & 1. Yes $\rightarrow$ & 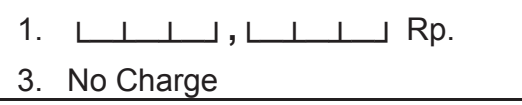 & Per strip & 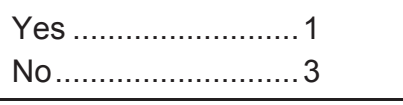 & $\begin{array}{l}\perp \_ \text {weeks ............ } 1 \\
\text { NEVER ...................... } 6\end{array}$ \\
\hline & g. Cycloginon & $\begin{array}{l}\text { 3. No } \\
\downarrow\end{array}$ & 1. Yes $\rightarrow$ & 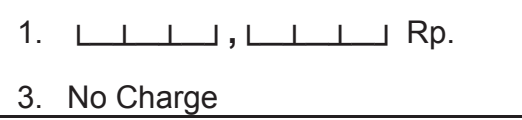 & Per strip & 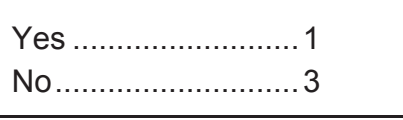 & 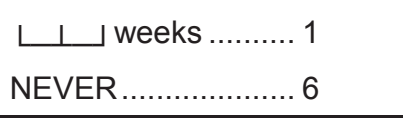 \\
\hline \multirow[t]{3}{*}{19.} & IUD Copper T & & & & & & \\
\hline & a. Insertion & $\begin{array}{l}\text { 3. No } \\
\Downarrow\end{array}$ & 1. Yes $\rightarrow$ & 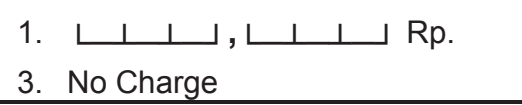 & $\begin{array}{l}\text { Per treatment for } \\
\text { one time insertion }\end{array}$ & 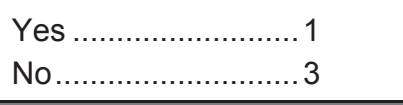 & 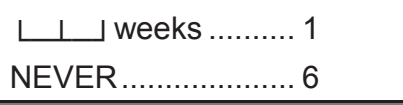 \\
\hline & b. Removal & 3. No & 1. Yes $\rightarrow$ & 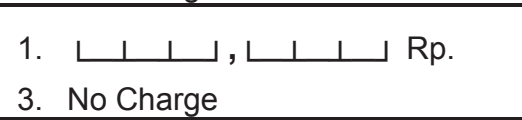 & $\begin{array}{l}\text { Pre treatment for } \\
\text { one time removal }\end{array}$ & & \\
\hline \multirow[t]{2}{*}{20.} & Contraceptive injection & & & & & & \\
\hline & a. Depo-Provera & 3. No & 1. Yes $\rightarrow$ & 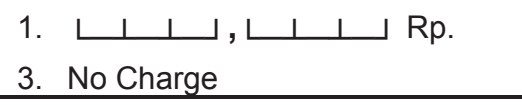 & per injection & $\begin{array}{l}\text { Yes } \ldots \ldots \ldots \ldots \ldots \ldots \ldots \ldots \ldots \ldots . . .1 \\
\text { No } \ldots \ldots \ldots \ldots \ldots \ldots \ldots \ldots \ldots \ldots \ldots \ldots \ldots \ldots \ldots \ldots \ldots \\
\end{array}$ & 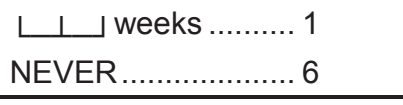 \\
\hline & b. Depo- Progestin/Andalan & 3. No & 1. Yes $\rightarrow$ & $\begin{array}{l}\text { 1. } \downarrow \perp \perp \perp, \perp \perp \perp \perp \perp \text { Rp. } \\
\text { 3. No Charge }\end{array}$ & per injection & 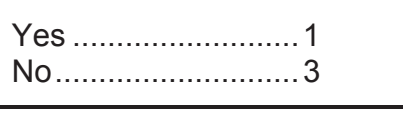 & 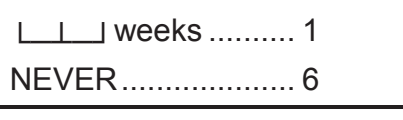 \\
\hline
\end{tabular}




\section{SECTION B : SERVICE ABILITY}

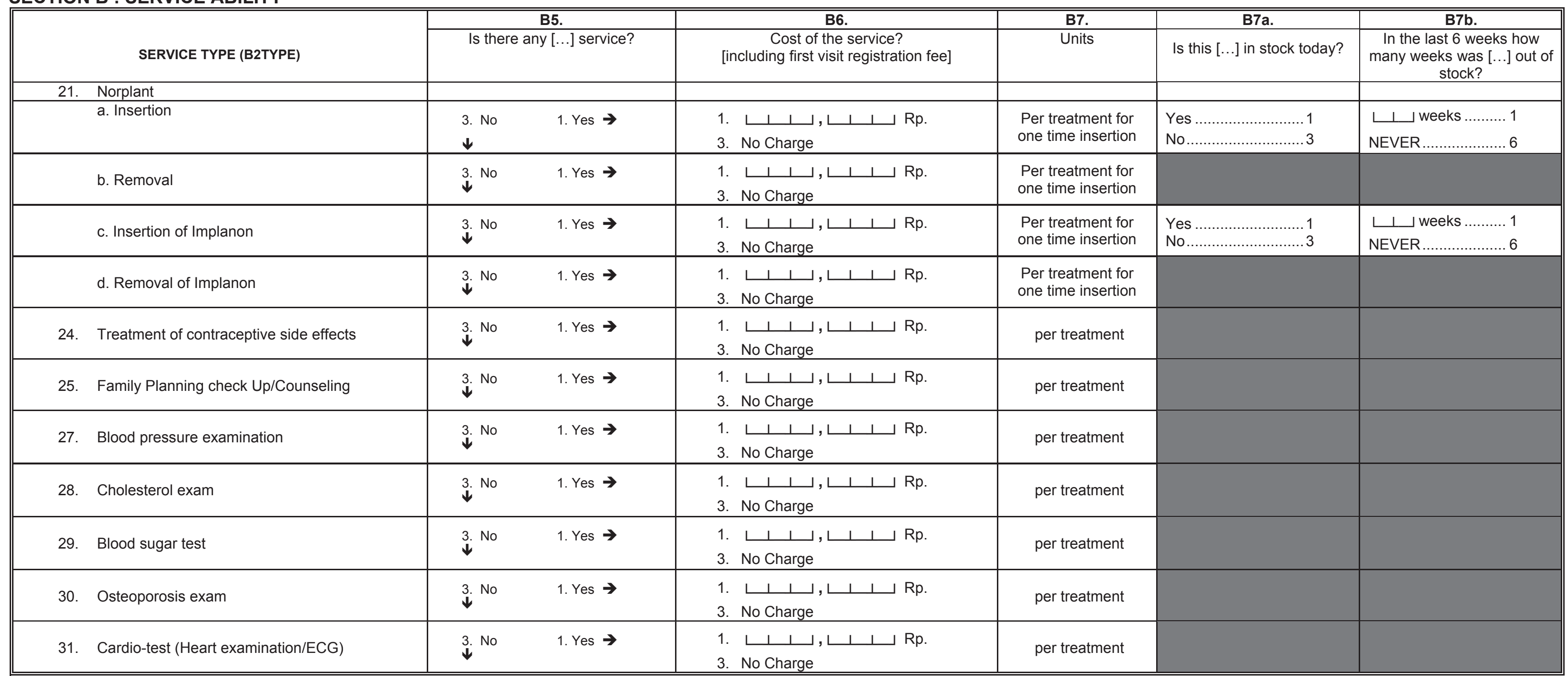




\section{SECTION B : SERVICE ABILITY}

\begin{tabular}{|c|c|c|c|c|c|c|c|}
\hline \multirow{2}{*}{\multicolumn{2}{|c|}{ SERVICE TYPE (B2TYPE) }} & \multirow{2}{*}{\multicolumn{2}{|c|}{$\begin{array}{c}\text { B5. } \\
\text { Is there any }[\ldots] \text { service? }\end{array}$}} & \multirow{3}{*}{$\begin{array}{c}\text { B6. } \\
\text { Cost of the service? } \\
\text { [including first visit registration fee] }\end{array}$} & \multirow{2}{*}{$\begin{array}{l}\text { B7. } \\
\text { Units }\end{array}$} & \multirow{2}{*}{$\begin{array}{c}\text { B7a. } \\
\text { Is this }[\ldots] \text { in stock today? }\end{array}$} & \multirow{2}{*}{$\begin{array}{c}\text { B7b. } \\
\text { In the last } 6 \text { weeks how } \\
\text { many weeks was }[\ldots] \text { out of } \\
\text { stock? }\end{array}$} \\
\hline & & & & & & & \\
\hline \multicolumn{7}{|c|}{ stoch! } & \\
\hline & a. BCG & 3. No & 1. Yes $\rightarrow$ & 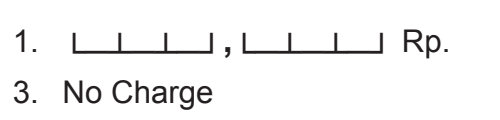 & per injection & 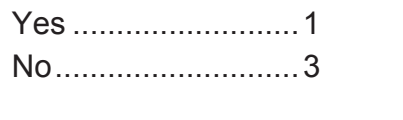 & 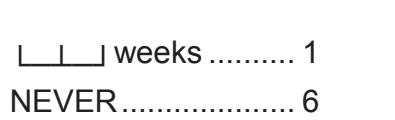 \\
\hline & b. DPT & 3. No & 1. Yes $\rightarrow$ & 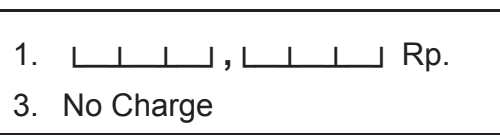 & per injection & 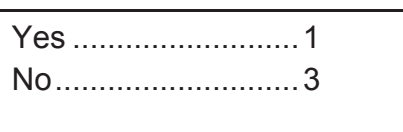 & $\begin{array}{l}\sqcup \sqcup \text { weeks ............. } 1 \\
\text { NEVER } \ldots \ldots \ldots \ldots \ldots \ldots \ldots . . .6\end{array}$ \\
\hline & c. Anti polio & 3. No & 1. Yes $\rightarrow$ & $\begin{array}{l}\text { 1. } \downarrow \perp \perp \perp, \perp \perp \perp \perp \_ \text {Rp. } \\
\text { 3. No Charge }\end{array}$ & Per treatment & 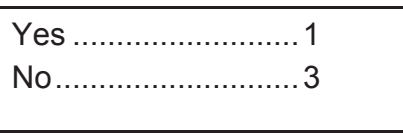 & $\begin{array}{l}\sqcup \perp \text { weeks ............. } 1 \\
\text { NEVER .................. } 6\end{array}$ \\
\hline & d. Measles & 3. No & 1. Yes $\rightarrow$ & 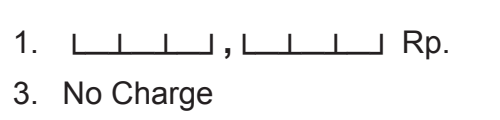 & per injection & 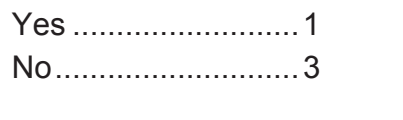 & $\begin{array}{l}\sqcup \perp \text { weeks ............ } 1 \\
\text { NEVER } \ldots \ldots \ldots \ldots \ldots \ldots \ldots \ldots . .6\end{array}$ \\
\hline & e. Tetanus Toxoid & 3. No & 1. Yes $\rightarrow$ & 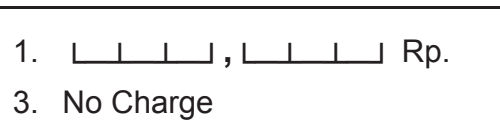 & per injection & 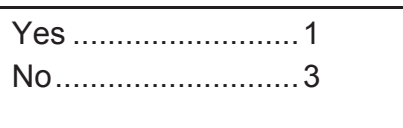 & 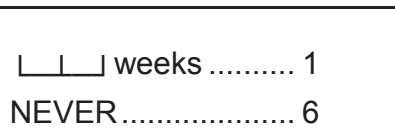 \\
\hline & f. Hepatitis B, for infants & 3. No & 1. Yes $\rightarrow$ & 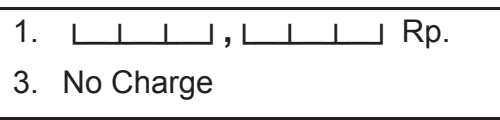 & per injection & 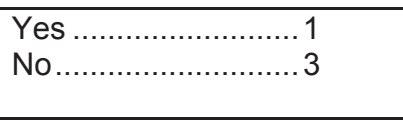 & $\begin{array}{l}\sqcup \perp \text { weeks ............ } 1 \\
\text { NEVER } \ldots \ldots \ldots \ldots \ldots \ldots \ldots . .6\end{array}$ \\
\hline & g. Hepatitis B, for adult & 3. No & 1. Yes $\rightarrow$ & $\begin{array}{l}\text { 1. }\left\llcorner\perp \perp \_, \downarrow \perp \perp \_\_ \text {Rp. }\right. \\
\text { 3. No Charge }\end{array}$ & per injection & 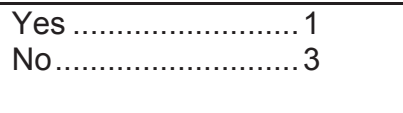 & 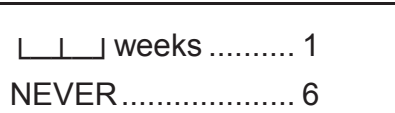 \\
\hline & h. Chickenpox & 3. No & 1. Yes $\rightarrow$ & 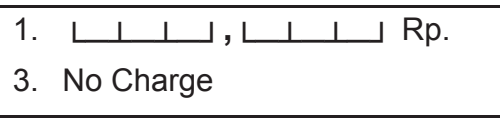 & per injection & $\begin{array}{l}\text { Yes } \ldots \ldots \ldots \ldots \ldots \ldots \ldots \ldots . .1 \\
\text { No } \ldots \ldots \ldots \ldots \ldots \ldots \ldots \ldots \ldots \ldots \ldots\end{array}$ & 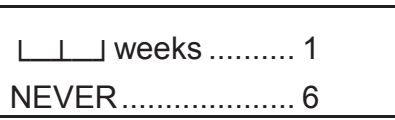 \\
\hline & i. Influenza & 3. No & 1. Yes $\rightarrow$ & 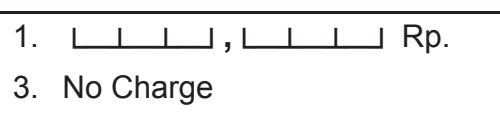 & per injection & 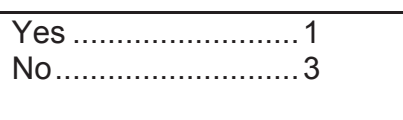 & 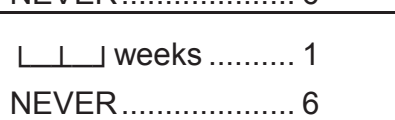 \\
\hline & j. Pneumonia & 3. No & 1. Yes $\rightarrow$ & 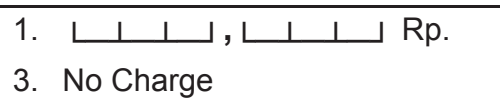 & per injection & 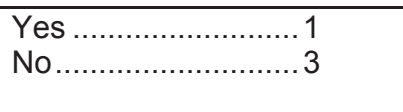 & 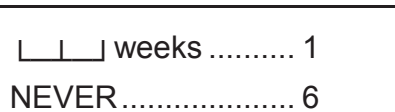 \\
\hline 33 & Treatment of Malaria & 3. No & 1. Yes $\rightarrow$ & $\begin{array}{l}\text { 1. } \sqcup \perp \perp \perp,\llcorner\perp \perp \perp \text { Rp. } \\
\text { 3. No Charge }\end{array}$ & Per treatment & & \\
\hline 34 & Dengue treatment & 3. No & 1. Yes $\rightarrow$ & 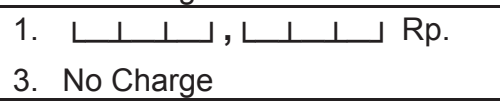 & Per treatment & & \\
\hline 35 & Treatment of HIV-AIDS & 3. No & 1. Yes $\rightarrow$ & 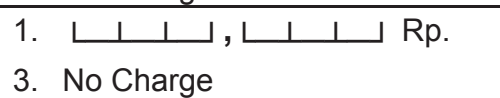 & Per treatment & & \\
\hline & Treatment of diarrhea & 3. No & 1. Yes $\rightarrow$ & 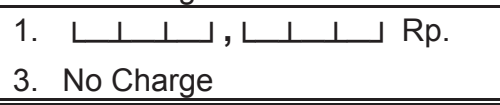 & Per treatment & & \\
\hline
\end{tabular}




\section{SECTION B : SERVICE ABILITY}

Now, we would like to ask about patients that cannot be treated and need to be referred to another facility.

\begin{tabular}{|c|c|c|c|c|c|}
\hline & (B3TYPE) & A. Hospital & B. Puskesmas & C. Laboratory & D. Other Private Practice \\
\hline \multirow[t]{8}{*}{ B10. } & \multirow[t]{8}{*}{$\begin{array}{l}\text { If patient must be referred to } \\
\text { another facility, do you send the } \\
\text { patient to }[\ldots] \text { ? }\end{array}$} & 1. Yes & 1. Yes & 1. Yes & 1. Yes \\
\hline & & 1. Name & 1. Name & 1. Name & 1. Name \\
\hline & & 1. Address & 1. Address & 1. Address & 1. Address \\
\hline & & 1. Location & 1. Location & 1. Location & 1. Location \\
\hline & & $\begin{array}{l}\text { Vill: } 1 . \overline{\text { Same }} \\
\quad \text { 3. }\end{array}$ & $\begin{array}{ll}\text { Vill: } & 1 . \\
& \text { 3. Same }\end{array}$ & $\begin{array}{ll}\text { Vill: } & 1 . \\
& \text { 3. Same }\end{array}$ & $\begin{array}{ll}\text { Vill: } & \text { 1. } \\
& \text { 3. Same }\end{array}$ \\
\hline & & $\begin{array}{ll}\text { Kec: } & \text { 1. } \\
& \text { 3. Same }\end{array}$ & $\begin{array}{ll}\text { Kec: } & \text { 1. } \\
& \text { 3. Same }\end{array}$ & $\begin{array}{ll}\text { Kec: } & \text { 1. } \\
& \text { 3. Same }\end{array}$ & $\begin{array}{ll}\text { Kec: } & \text { 1. } \\
& \text { 3. Same }\end{array}$ \\
\hline & & Kab: $\begin{array}{l}\text { 1. } \\
\text { 3. Same }\end{array}$ & $\begin{array}{l}\text { Kab: } 1 . \\
\text { 3. Same }\end{array}$ & Kab: $1 . \overline{ }$ 3. Same & $\begin{array}{ll}\text { Kab: } & \text { 1. } \\
\text { 3. Same }\end{array}$ \\
\hline & & $\begin{aligned} \text { Prov: } & 1 . \\
& \text { 3. Same }\end{aligned}$ & $\begin{aligned} \text { Prov: } & 1 . \\
& \text { 3. Same }\end{aligned}$ & $\begin{aligned} \text { Prov: } & 1 . \\
& \text { 3. Same }\end{aligned}$ & $\begin{aligned} \text { Prov: } & 1 . \\
& \text { 3. Same }\end{aligned}$ \\
\hline B11. & $\begin{array}{l}\text { What is the distance that must be } \\
\text { traveled from your facility to the } \\
\text { referred facility? }\end{array}$ & 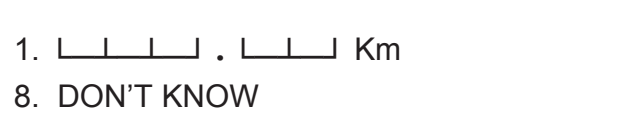 & 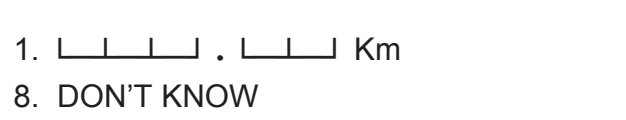 & 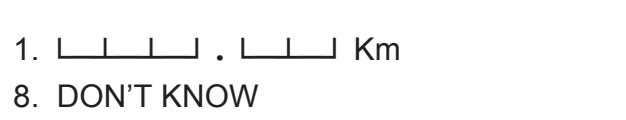 & 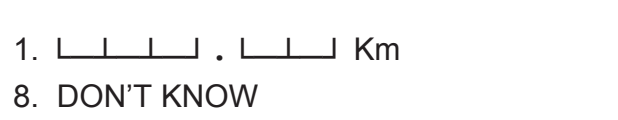 \\
\hline B13. & $\begin{array}{l}\text { Approximately how much does it } \\
\text { cost the referred patient to travel } \\
\text { one way to the referred facility? }\end{array}$ & 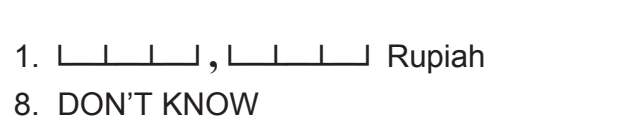 & 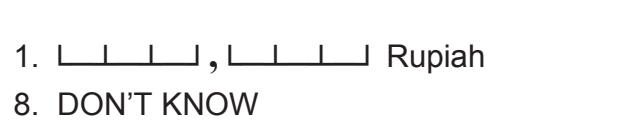 & 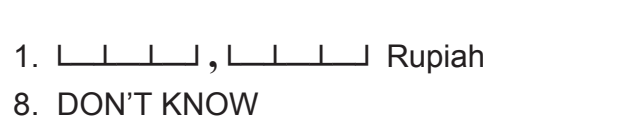 & 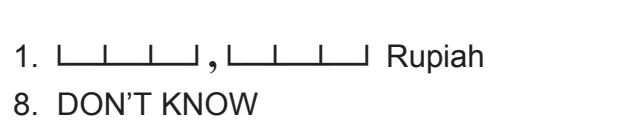 \\
\hline B13a. & $\begin{array}{l}\text { If a poor patient is referred to }[\ldots] \text {, } \\
\text { is he/she provided with } \\
\text { transportation to the facility? }\end{array}$ & $\begin{array}{l}\text { 3. No, neither transportation nor money is } \\
\text { provided } \rightarrow \text { B10 NEXT COLUMN } \\
\text { 1. Yes, transportation is provided using } \\
\text { ambulance free of charge } \\
\text { 2. Yes, patient is provided with money to } \\
\text { travel to the referred facility }\end{array}$ & $\begin{array}{l}\text { 3. No, neither transportation nor money is } \\
\text { provided } \rightarrow \text { B10 NEXT COLUMN } \\
\text { 1. Yes, transportation is provided using } \\
\text { ambulance free of charge } \\
\text { 2. Yes, patient is provided with money to } \\
\text { travel to the referred facility }\end{array}$ & $\begin{array}{l}\text { 3. No, neither transportation nor money is } \\
\text { provided } \rightarrow \text { B10 NEXT COLUMN } \\
\text { 1. Yes, transportation is provided using } \\
\text { ambulance free of charge } \\
\text { 2. Yes, patient is provided with money to } \\
\text { travel to the referred facility }\end{array}$ & $\begin{array}{l}\text { 3. No, neither transportation nor money is } \\
\text { provided } \rightarrow \text { B14 } \\
\text { 1. Yes, transportation is provided using } \\
\text { ambulance free of charge } \\
\text { 2. Yes, patient is provided with money to } \\
\text { travel to the referred facility }\end{array}$ \\
\hline B13b. & $\begin{array}{l}\text { What is the market value of the } \\
\text { transportation provided? }\end{array}$ & 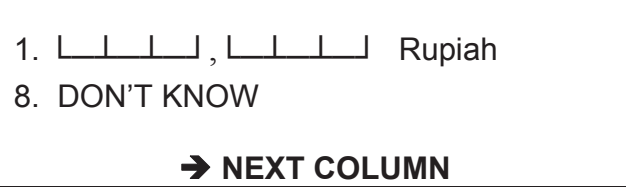 & $\begin{array}{l}\text { 1. } \downarrow \perp \perp \perp, \downarrow \perp \perp \perp \text { Rupiah } \\
\text { 8. DON'T KNOW } \\
\quad \rightarrow \text { NEXT COLUMN }\end{array}$ & $\begin{array}{l}\text { 1. } \downarrow \perp \perp \perp, \downarrow \perp \_\_ \text {Rupiah } \\
\text { 8. DON'T KNOW } \\
\quad \rightarrow \text { NEXT COLUMN }\end{array}$ & $\begin{array}{l}\text { 1. } \downarrow \perp \perp \perp, \downarrow \perp \perp \perp \text { Rupiah } \\
\text { 8. DON'T KNOW } \\
\Rightarrow \text { B14 }\end{array}$ \\
\hline
\end{tabular}


SECTION B : SERVICE ABILITY

Number of patient visits:

\begin{tabular}{|c|c|c|c|c|c|}
\hline \multirow{3}{*}{$\begin{array}{l}\text { WRITE ALL PATIENT VISITS IN } \\
\text { THIS PRACTICE PLACE DURING } \\
\text { THE LAST WEEK, BEGINNING } \\
\text { SUNDAY THROUGH SATURDAY! }\end{array}$} & B15 (B6TYPE) & \multicolumn{4}{|c|}{ B16. } \\
\hline & Hari & \multicolumn{4}{|c|}{$\begin{array}{c}\text { Number of patient }[\ldots] \\
\text { Total }\end{array}$} \\
\hline & A. Sunday & 1. ᄂ & $\begin{array}{l}\text { 3. NONE } \\
\downarrow\end{array}$ & $\begin{array}{l}\text { 6. Not Open } \\
\downarrow\end{array}$ & 8. DK \\
\hline \multirow{2}{*}{ 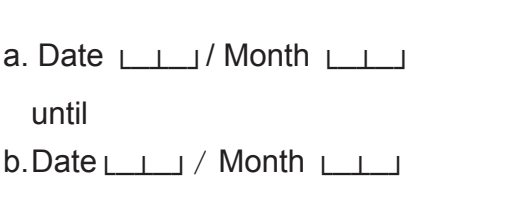 } & B. Monday & 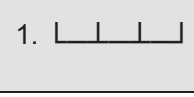 & $\begin{array}{l}\text { 3. NONE } \\
\Downarrow\end{array}$ & $\begin{array}{l}\text { 6. Not Open } \\
\downarrow\end{array}$ & $\begin{array}{l}\text { 8. DK } \\
\downarrow\end{array}$ \\
\hline & C. Tuesday & 1. ᄂ & $\begin{array}{l}\text { 3. NONE } \\
\downarrow\end{array}$ & $\begin{array}{l}\text { 6. Not Open } \\
\downarrow\end{array}$ & $\begin{array}{c}\text { 8. DK } \\
\downarrow\end{array}$ \\
\hline \multirow[t]{4}{*}{ c. Not practicing $\rightarrow$ B16ba } & D. Wednesday & 1. ᄂ & $\begin{array}{l}\text { 3. NONE } \\
\downarrow\end{array}$ & $\begin{array}{l}\text { 6. Not Open } \\
\downarrow\end{array}$ & $\begin{array}{l}\text { 8. DK } \\
\downarrow\end{array}$ \\
\hline & E. Thursday & 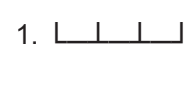 & $\begin{array}{l}\text { 3. NONE } \\
\downarrow\end{array}$ & $\begin{array}{l}\text { 6. Not Open } \\
\Downarrow\end{array}$ & $\begin{array}{l}\text { 8. DK } \\
\downarrow\end{array}$ \\
\hline & F. Friday & 1. ᄂ لــ & $\begin{array}{l}\text { 3. NONE } \\
\downarrow\end{array}$ & $\begin{array}{l}\text { 6. Not Open } \\
\downarrow\end{array}$ & $\begin{array}{l}\text { 8. DK } \\
\downarrow\end{array}$ \\
\hline & G. Saturday & 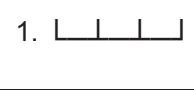 & $\begin{array}{l}\text { 3. NONE } \\
\Downarrow\end{array}$ & $\begin{array}{l}\text { 6. Not Open } \\
\downarrow\end{array}$ & 8. DK \\
\hline
\end{tabular}

B16ba. Do you provide services free of charge or at

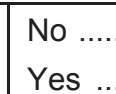

$3 \rightarrow$ B16aa

discount for the poor?

$\ldots 1$

\begin{tabular}{|c|c|}
\hline \multirow{2}{*}{ B5TYPE } & B16da. \\
\hline & How much is the service charge for $[\ldots]$ for the poor? \\
\hline A. Basic examination + medicine/injection & 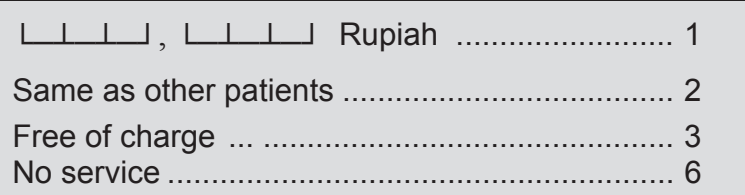 \\
\hline B. Antenatal services & 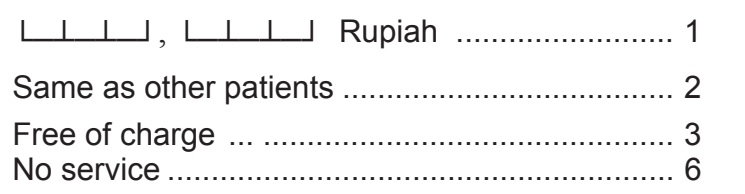 \\
\hline C. Delivery & 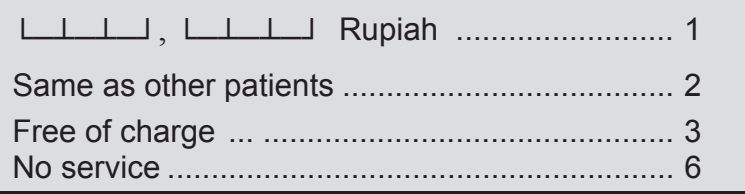 \\
\hline E. Child Immunization & 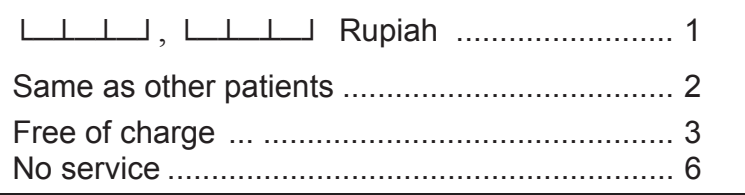 \\
\hline F. Contraceptive pill & 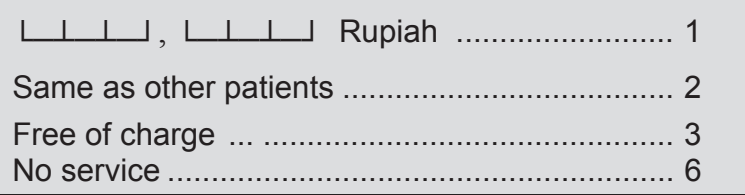 \\
\hline G. Contraceptive injection & 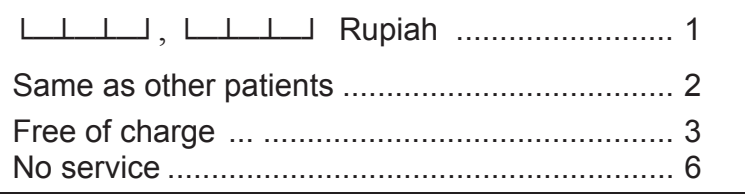 \\
\hline
\end{tabular}




\section{SECTION B : SERVICE ABILITY}

\begin{tabular}{|c|c|c|}
\hline B16aa & $\begin{array}{l}\text { Does private practice/facility } \\
\text { provide service for JKN/KIS } \\
\text { holders? }\end{array}$ & 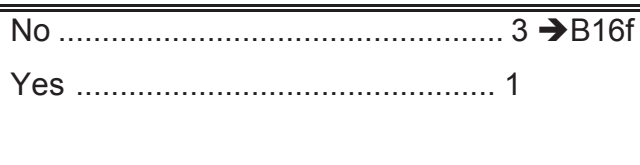 \\
\hline B16ab & $\begin{array}{l}\text { Since when did the private } \\
\text { practice/facility provide this } \\
\text { service? }\end{array}$ & 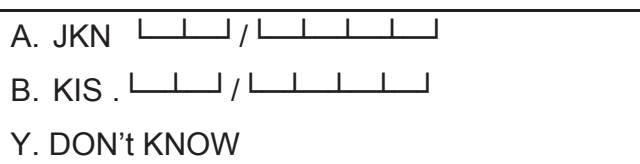 \\
\hline
\end{tabular}

\begin{tabular}{|c|c|}
\hline \multirow[t]{2}{*}{ (B7TYPE) } & B16ac. \\
\hline & How much is cost of service of $[\ldots .$.$] for JKN/KIS holders?$ \\
\hline $\begin{array}{l}\text { Basic examination + } \\
\text { medicine/injection }\end{array}$ & 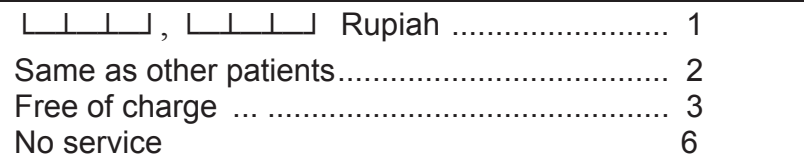 \\
\hline B. Antenatal services & 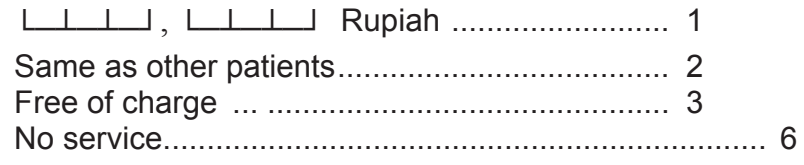 \\
\hline C. Delivery & 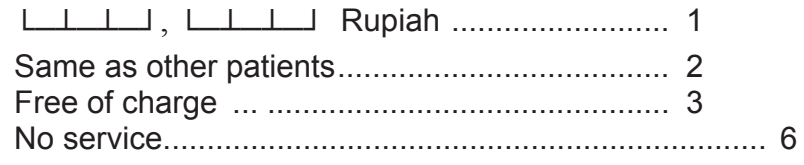 \\
\hline E. Child Immunization & 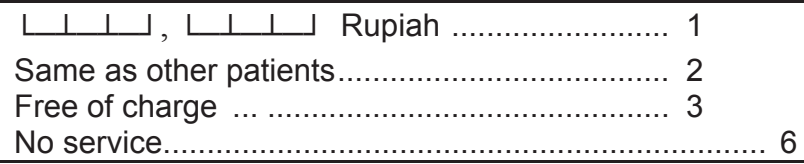 \\
\hline F. Contraceptive pill & 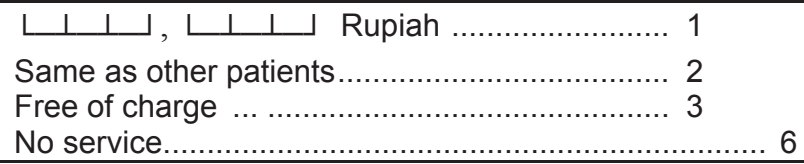 \\
\hline G. Contraceptive injection & 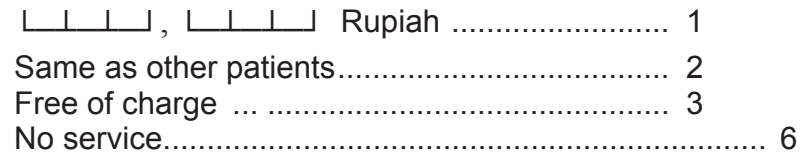 \\
\hline
\end{tabular}

\begin{tabular}{|c|c|c|}
\hline B16f. & $\begin{array}{l}\text { Compared to } 2007 \text {, is there a } \\
\text { change in the number of patients } \\
\text { visiting this facility per week? }\end{array}$ & 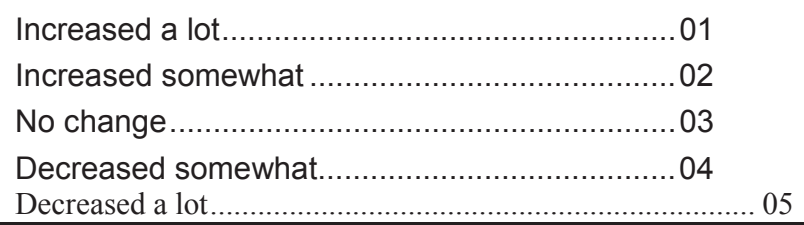 \\
\hline
\end{tabular}


SECTION B : SERVICE ABILITY

Laboratory examination

\begin{tabular}{|c|c|c|c|c|}
\hline & B17. & B18. & B19. & B20. \\
\hline & $\begin{array}{c}\text { KINDS OF TESTS } \\
\text { (B4TYPE) }\end{array}$ & Can lab work be done in this practice? & How much are the patients charged? & $\begin{array}{l}\text { When patients are referred to an outside testing site for lab work, } \\
\text { what is the distance from this practice to the extended site? }\end{array}$ \\
\hline a. & Hemoglobin $(\mathrm{Hb})$ & 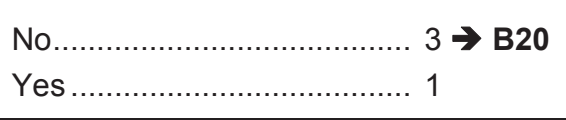 & 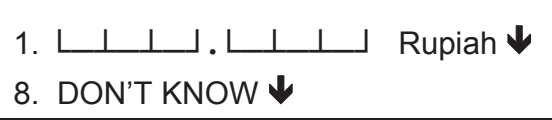 & $\begin{array}{l}\text { 1. } \sqcup \perp \perp\lrcorner, \sqcup \text { km } \\
\text { 8. DON'T KNOW }\end{array}$ \\
\hline b. & Leukocyte count & 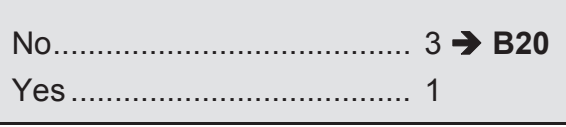 & $\begin{array}{l}\text { 1. } \downarrow \perp \perp \_. \sqcup \perp \_\_ \text {Rupiah } \downarrow \\
\text { 8. DON'T KNOW } \downarrow\end{array}$ & $\begin{array}{l}\text { 1. } \sqcup \perp \perp\lrcorner, \sqcup \text { km } \\
\text { 8. DON'T KNOW }\end{array}$ \\
\hline c. & Blood typing & 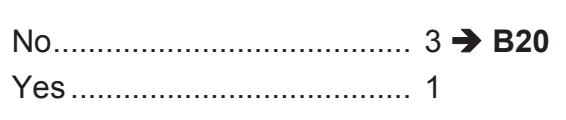 & $\begin{array}{l}\text { 1. } \longleftarrow \perp \perp \_. \sqcup \downarrow \perp \_ \text {Rupiah } \downarrow \\
\text { 8. DON'T KNOW } \downarrow\end{array}$ & $\begin{array}{l}\text { 1. } \sqcup \perp \perp, \sqcup \sqcup \text { km } \\
\text { 8. DON'T KNOW }\end{array}$ \\
\hline d. & Erythrocyte count & 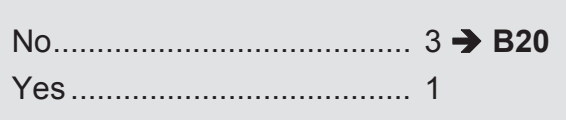 & $\begin{array}{l}\text { 1. } \downarrow|\downarrow| \downarrow|\downarrow| \downarrow \text { Rupiah } \downarrow \\
\text { 8. DON'T KNOW } \downarrow\end{array}$ & $\begin{array}{l}\text { 1. } \sqcup \perp \perp, \sqcup \sqcup \mathrm{km} \\
\text { 8. DON'T KNOW }\end{array}$ \\
\hline e. & Urinalisis & 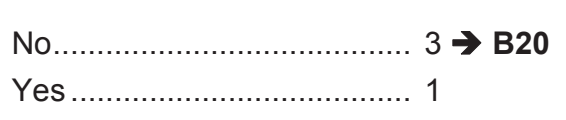 & 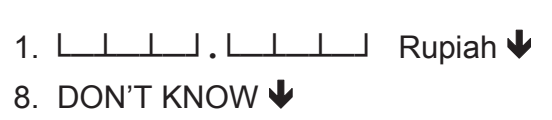 & $\begin{array}{l}\text { 1. } \sqcup \perp 」, \sqcup \sqcup \mathrm{km} \\
\text { 8. DON'T KNOW }\end{array}$ \\
\hline f. & Pregnancy Test & 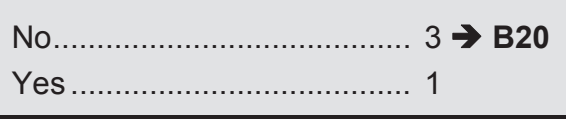 & 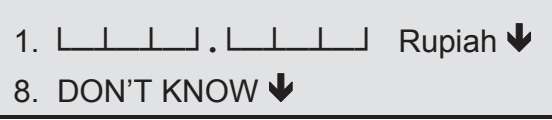 & $\begin{array}{l}\text { 1. } \sqcup \perp \perp, \sqcup \text { ㄴ } \sqcup \text { km } \\
\text { 8. DON'T KNOW }\end{array}$ \\
\hline g. & Faeces examination & 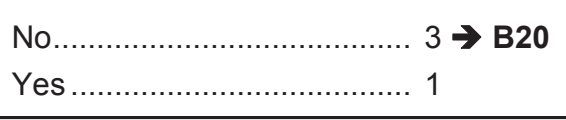 & 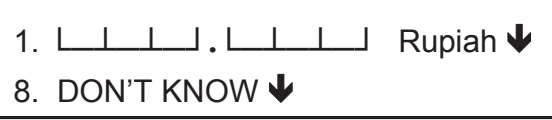 & $\begin{array}{l}\text { 1. } \sqcup \perp \perp\lrcorner, \sqcup \sqcup \mathrm{km} \\
\text { 8. DON'T KNOW }\end{array}$ \\
\hline h. & Sputum examination & $\begin{array}{l}\text { No } \\
\text { Yes } \ldots \ldots \ldots \ldots \ldots \ldots \ldots \ldots \ldots \ldots \ldots \ldots \\
\text { 3 }\end{array}$ & $\begin{array}{l}\text { 1. } \downarrow \perp \perp \_. \downarrow \perp \_\_ \text {Rupiah } \downarrow \\
\text { 8. DON'T KNOW } \downarrow\end{array}$ & $\begin{array}{l}\text { 1. } \sqcup \perp \perp\lrcorner, \sqcup \text { km } \\
\text { 8. DON'T KNOW }\end{array}$ \\
\hline i. & Cholesterol examination & 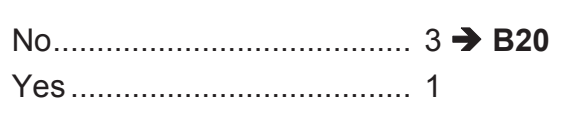 & 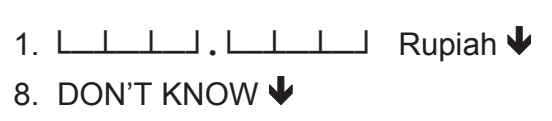 & $\begin{array}{l}\text { 1. } \sqcup \perp \perp, \sqcup \sqcup \mathrm{km} \\
\text { 8. DON'T KNOW }\end{array}$ \\
\hline j. & Blood sugar test & 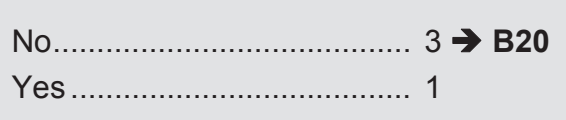 & $\begin{array}{l}\text { 1. } \downarrow \perp \perp \downarrow . \sqcup \downarrow \mid \downarrow \text { Rupiah } \downarrow \\
\text { 8. DON'T KNOW } \downarrow\end{array}$ & $\begin{array}{l}\text { 1. } \sqcup \perp \perp\lrcorner, \sqcup \text { km } \\
\text { 8. DON'T KNOW }\end{array}$ \\
\hline k. & Osteoporosis / bone density examination & 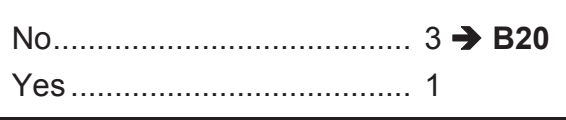 & $\begin{array}{l}\text { 1. } \sqcup \downarrow \downarrow \downarrow . \sqcup \downarrow \mid \downarrow \text { Rupiah } \downarrow \\
\text { 8. DON'T KNOW } \downarrow\end{array}$ & $\begin{array}{l}\text { 1. } \sqcup \perp \perp\lrcorner, \sqcup \text { km } \\
\text { 8. DON'T KNOW }\end{array}$ \\
\hline I. & Lung examination & 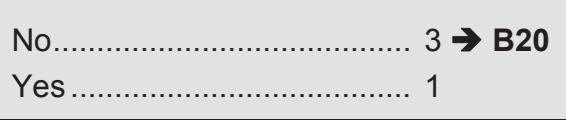 & $\begin{array}{l}\text { 1. } \downarrow \perp \perp 」 . \sqcup \mid \perp \_ \text {Rupiah } \downarrow \\
\text { 8. DON'T KNOW } \downarrow\end{array}$ & $\begin{array}{l}\text { 1. } \sqcup \perp \perp, \sqcup \sqcup \mathrm{km} \\
\text { 8. DON'T KNOW }\end{array}$ \\
\hline
\end{tabular}




\section{SECTION B : SERVICE ABILITY}

\begin{tabular}{|c|c|c|}
\hline & \multicolumn{2}{|c|}{ B21 } \\
\hline \multirow[t]{2}{*}{$\begin{array}{c}\text { Types of Conditions } \\
\text { (B8TYPE) }\end{array}$} & \multicolumn{2}{|c|}{ Do you ever deal with cases of patients with $[\ldots]$} \\
\hline & 3. No $\downarrow$ & 1. Yes \\
\hline B. high risk neonatal & 3. No $\downarrow$ & 1. Yes \\
\hline C. babies born with Low Birth Weight & 3. No $\downarrow$ & 1. Yes \\
\hline D. diarrhea & 3. No $\downarrow$ & 1. Yes \\
\hline E. dengue hemorrhagic fever & 3. No $\downarrow$ & 1. Yes \\
\hline F. malaria & 3. No $\downarrow$ & 1. Yes \\
\hline G. malnutrition & 3. No $\downarrow$ & 1. Yes \\
\hline H. TB & 3. No $\downarrow$ & 1. Yes \\
\hline I. HIVIAIDS & 3. No $\downarrow$ & 1. Yes \\
\hline
\end{tabular}




\section{SECTION PH: PHARMACY}

\begin{tabular}{|c|c|c|}
\hline PHOO. & Do you provide prescription for the patients? & $\begin{array}{l}\text { No } \\
\text { Yes }\end{array}$ \\
\hline PH01. & Where do you refer the patients for the medicine? & 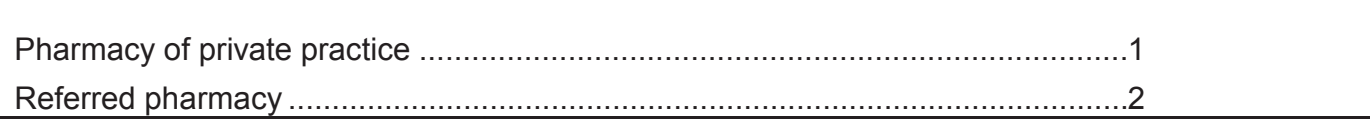 \\
\hline PH02. & $\begin{array}{l}\text { Kindly mention the name and address of pharmacy that you usually recommend the patients } \\
\text { INTERVIEWER CHECK: WRITE DOWN THE NAME, ADDRES, LOCATION AND } \\
\text { INTERVIEW THE PHARMACY }\end{array}$ & $\begin{array}{ll}\text { Pharmacy } & : \\
\text { Address } & : \\
\text { Description of location } & : \\
\text { Postal code } & :\end{array}$ \\
\hline PH02x & INTERVIEWER'S NOTE: HAS INTERVIEW BEEN CONDUCTED IN DRUG STORE? & 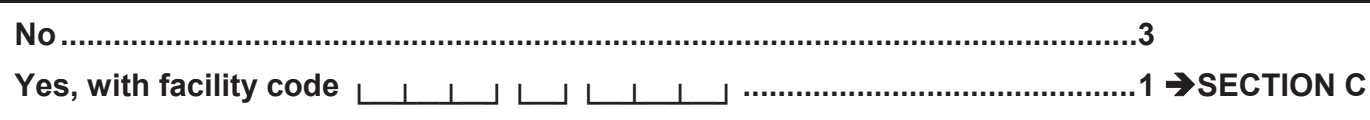 \\
\hline
\end{tabular}


SECTION PH: PHARMACY

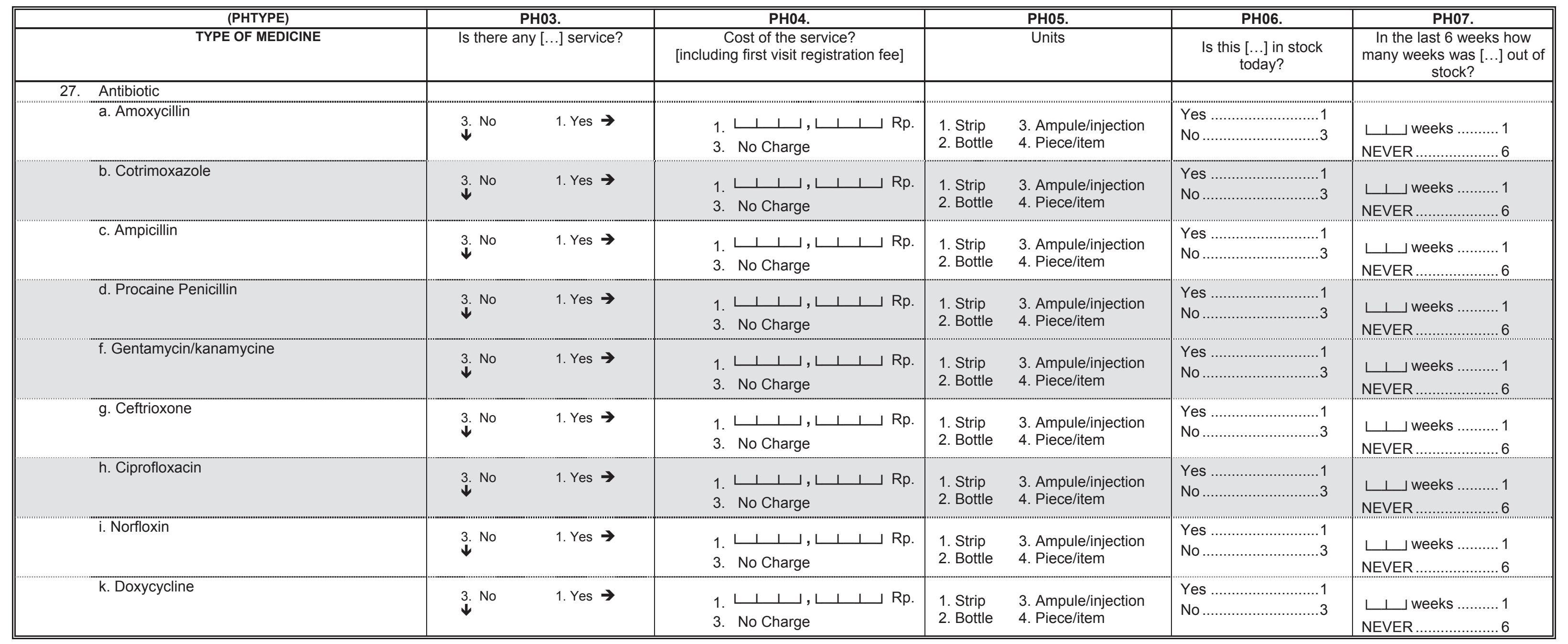




\section{SECTION PH: PHARMACY}

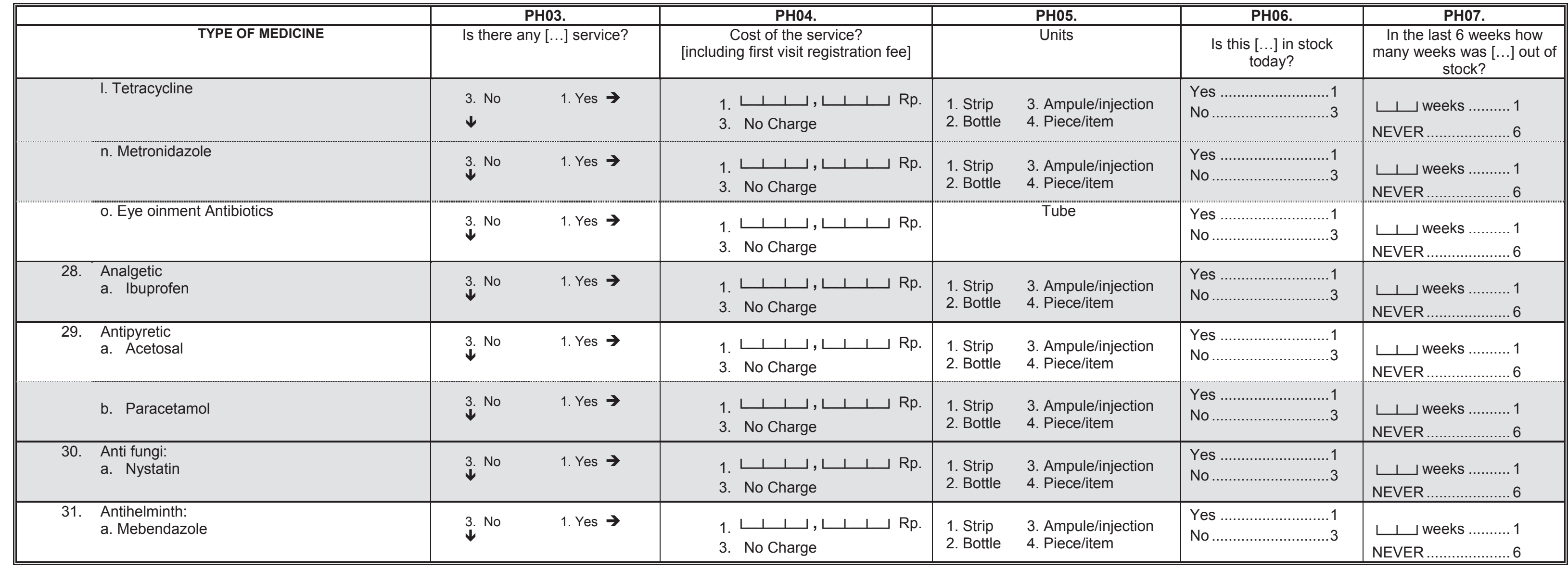




\section{SECTION PH: PHARMACY}

\begin{tabular}{|c|c|c|c|c|c|c|c|c|}
\hline \multirow{2}{*}{\multicolumn{2}{|c|}{ TYPE OF MEDICINE }} & \multicolumn{2}{|c|}{ PH03. } & \multirow{2}{*}{$\begin{array}{c}\text { PH04. } \\
\text { Cost of the service? } \\
\text { [including first visit registration fee] }\end{array}$} & \multicolumn{2}{|r|}{$\begin{array}{l}\text { PH05. } \\
\text { Units }\end{array}$} & $\begin{array}{l}\text { PH06. } \\
\end{array}$ & \multirow{2}{*}{$\begin{array}{c}\text { PH07. } \\
\text { In the last } 6 \text { weeks how } \\
\text { many weeks was }[\ldots . .] \text { out of } \\
\text { stock? }\end{array}$} \\
\hline & & Is ther & ...] service? & & & Units & $\begin{array}{l}\text { Is this }[\ldots] \text { in stock } \\
\text { today? }\end{array}$ & \\
\hline \multicolumn{9}{|c|}{ 32. $\quad$ Anti -TBC (short-term): } \\
\hline & c. Rifampicin & 3. No & 1. Yes $\rightarrow$ & 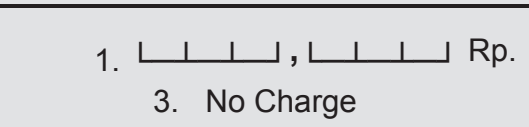 & $\begin{array}{l}\text { 1. Strip } \\
\text { 2. Bottle }\end{array}$ & $\begin{array}{l}\text { 3. Ampule/injection } \\
\text { 4. Piece/item }\end{array}$ & 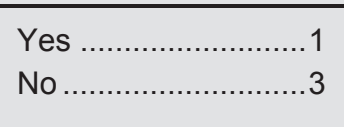 & $\begin{array}{l}\sqcup \perp \text { weeks ............. } 1 \\
\text { NEVER .................... } 6\end{array}$ \\
\hline & d. Ethambutol & 3. No & 1. Yes $\rightarrow$ & 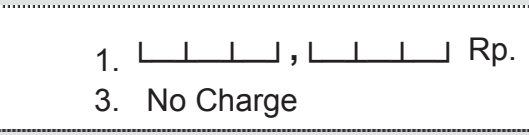 & $\begin{array}{l}\text { 1. Strip } \\
\text { 2. Bottle }\end{array}$ & $\begin{array}{l}\text { 3. Ampule/injection } \\
\text { 4. Piece/item }\end{array}$ & 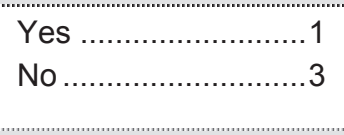 & $\begin{array}{l}\sqcup \sqcup \text { weeks ............ } 1 \\
\text { NEVER ....................6 }\end{array}$ \\
\hline & e. Isoniazid/INH & 3. No & 1. Yes $\rightarrow$ & 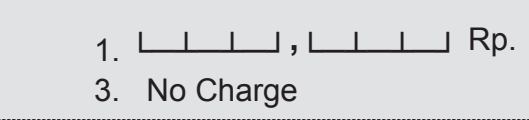 & $\begin{array}{l}\text { 1. Strip } \\
\text { 2. Bottle }\end{array}$ & $\begin{array}{l}\text { 3. Ampule/injection } \\
\text { 4. Piece/item }\end{array}$ & 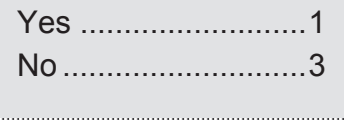 & $\begin{array}{l}\sqcup \perp \text { weeks ............. } 1 \\
\text { NEVER } \ldots \ldots \ldots \ldots \ldots \ldots \ldots . . .6\end{array}$ \\
\hline & h. Rifater (Rif+Iso+Pyran) & 3. No & 1. Yes $\rightarrow$ & 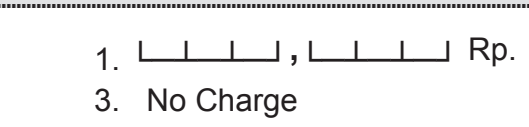 & $\begin{array}{l}\text { 1. Strip } \\
\text { 2. Bottle }\end{array}$ & $\begin{array}{l}\text { 3. Ampule/injection } \\
\text { 4. Piece/item }\end{array}$ & 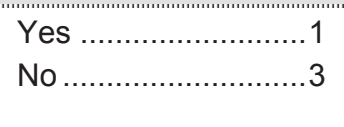 & $\begin{array}{l}\sqcup \perp \text { weeks ............. } 1 \\
\text { NEVER .................... } 6\end{array}$ \\
\hline \multicolumn{9}{|c|}{ 33. Anti malaria } \\
\hline & a. Chloroquine & 3. No & 1. Yes $\rightarrow$ & 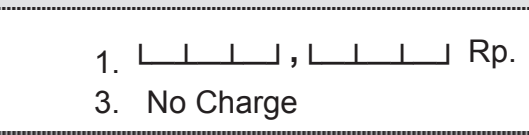 & $\begin{array}{l}\text { 1. Strip } \\
\text { 2. Bottle }\end{array}$ & $\begin{array}{l}\text { 3. Ampule/injection } \\
\text { 4. Piece/item }\end{array}$ & 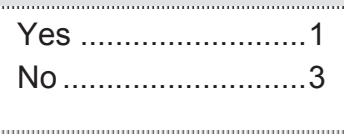 & $\begin{array}{l}\sqcup \dashv \text { weeks ............ } 1 \\
\text { NEVER ...................6 }\end{array}$ \\
\hline & d. Sulfadoxine & 3. No & 1. Yes $\rightarrow$ & 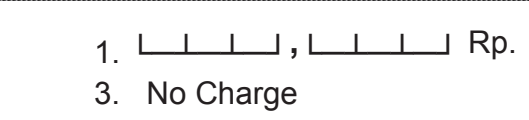 & $\begin{array}{l}\text { 1. Strip } \\
\text { 2. Bottle }\end{array}$ & $\begin{array}{l}\text { 3. Ampule/injection } \\
\text { 4. Piece/item }\end{array}$ & 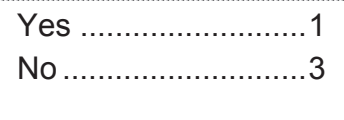 & $\begin{array}{l}\sqcup \perp \text { weeks ............. } 1 \\
\text { NEVER .................... } 6\end{array}$ \\
\hline 34. & Oralit & 3. No & 1. Yes $\rightarrow$ & 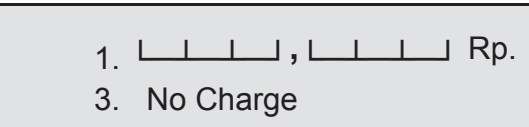 & & Per sachet & 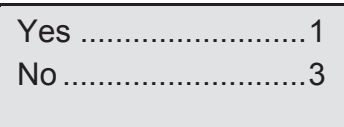 & $\begin{array}{l}\sqcup \perp \text { weeks ............. } 1 \\
\text { NEVER } \ldots \ldots \ldots \ldots \ldots \ldots \ldots . .6\end{array}$ \\
\hline
\end{tabular}




\section{SECTION PH: PHARMACY}

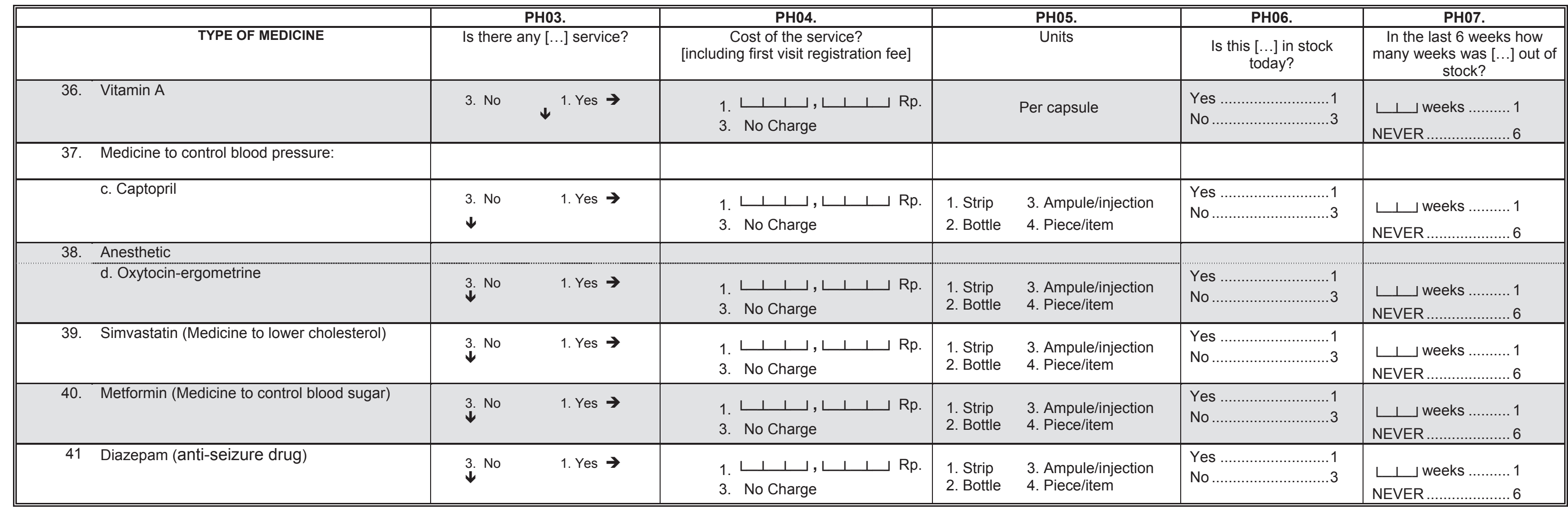


SECTION C : HEALTH INSTRUMENTS

Now, we want to ask about medical instruments used in this place.

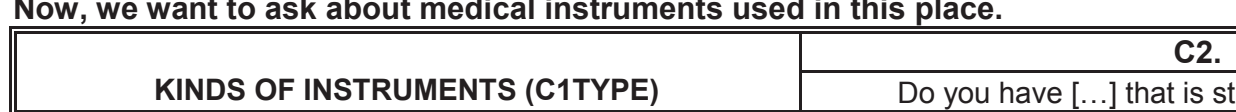

\begin{tabular}{|c|c|c|}
\hline \multirow{2}{*}{ KINDS OF INSTRUMENTS (C1TYPE) } & \\
\hline & Do you have & on properly? \\
\hline a. Regular stethoscope & 3. No & 1. Yes \\
\hline b. Stethoscope to examine pregnancy & 3. No & 1. Yes \\
\hline c. Blood pressure monitor & 3. No & 1. Yes \\
\hline d. Sterilisatir.aytickave & 3. No & 1. Yes \\
\hline e. Adult scales & 3. No & 1. Yes \\
\hline f. Baby scales & 3. No & 1. Yes \\
\hline g. Measurers for body height & 3. No & 1. Yes \\
\hline h. Thermometer & 3. No & 1. Yes \\
\hline i. Beds & 3. No & 1. Yes \\
\hline j. $\quad$ Normal delivery set & 3. No & 1. Yes \\
\hline k. Forceps & 3. No & 1. Yes \\
\hline I. Vaginal Speculum & 3. No & 1. Yes \\
\hline m. Sahli Set & 3. No & 1. Yes \\
\hline n. Scalpel & 3. No & 1. Yes \\
\hline o. Hammer for reflexes & 3. No & 1. Yes \\
\hline p. Flash light & 3. No & 1. Yes \\
\hline q. Disposable needles & 3. No & 1. Yes \\
\hline r. Sterile table & 3. No & 1. Yes \\
\hline s. Pinset & 3. No & 1. Yes \\
\hline t. $\quad$ Tongue depressor & 3. No & 1. Yes \\
\hline u. Uteriane sound & 3. No & 1. Yes \\
\hline v. Electrocardiogram (ECG) machine & 3. No & 1. Yes \\
\hline aa. Microscopes & 3. No & 1. Yes \\
\hline ba. Centrifuges & 3. No & 1. Yes \\
\hline ca. Syringes & 3. No & 1. Yes \\
\hline da. Cholesterol test kit & 3. No & 1. Yes \\
\hline ea. Blood sugar test kit & 3. No & 1. Yes \\
\hline fa. Gynecological Table & 3. No & 1. Yes \\
\hline ga. Spotlight & 3. No & 1. Yes \\
\hline ha. Refrigerator/cold storage & 3. No & 1. Yes \\
\hline ia. suction mucus & 3. No & 1. Yes \\
\hline ja. mask & 3. No & 1. Yes \\
\hline
\end{tabular}

\begin{tabular}{|c|c|c|c|c|}
\hline & \multicolumn{2}{|c|}{ C5. } & \multicolumn{2}{|c|}{ C6. } \\
\hline $\begin{array}{c}\text { KINDS OF INSTRUMENTS } \\
\text { (C2TYPE) }\end{array}$ & Does th & place have a & Does the $[.$. & properly? \\
\hline $\begin{array}{l}\text { a. Antiseptic: } \\
\text { 1. Alcohol }\end{array}$ & 3. No & 1. Yes & & \\
\hline 2. Betadine & 3. No & 1. Yes & & \\
\hline 3. Whitfield cream & 3. No & 1. Yes & & \\
\hline b. Bandages & 3. No & 1. Yes & & \\
\hline c. Oxygen tank & $\begin{array}{l}\text { 3. No } \\
\downarrow\end{array}$ & 1. Yes & 1. Yes & 3. No \\
\hline d. Incubator & $\begin{array}{ll}\text { 3. } & \text { No } \\
\downarrow & \end{array}$ & 1. Yes & 1. Yes & 3. No \\
\hline d1. Cotton & 3. No & 1. Yes & & \\
\hline e. Minor surgical instruments & $\begin{array}{l}\text { 3. No } \\
\downarrow\end{array}$ & 1. Yes & 1. Yes & 3. No \\
\hline f. Infuse instruments and needles & $\begin{array}{l}\text { 3. No } \\
\downarrow\end{array}$ & 1. Yes & 1. Yes & 3. No \\
\hline g. Gloves & 3. No & 1. Yes & & \\
\hline h. Scissors & $\begin{array}{ll}\text { 3. } & \text { No } \\
\downarrow & \end{array}$ & 1. Yes & 1. Yes & 3. No \\
\hline Giemsa solution & 3. No & 1. Yes & & \\
\hline j. Benedict solution & 3. No & 1. Yes & & \\
\hline k. Wright solution & 3. No & 1. Yes & & \\
\hline I. Pregnancy test (strip) & 3. No & 1. Yes & & \\
\hline m. Protein test (strip) & 3. No & 1. Yes & & \\
\hline n. Glucose test (Strip) & 3. No & 1. Yes & & \\
\hline t. Cholesterol test kit & $\begin{array}{l}\text { 3. No } \\
\downarrow\end{array}$ & 1. Yes & 1. Yes & 3. No \\
\hline u. Blood sugar test kit & 3. No & 1. Yes & 1. Yes & 3. No \\
\hline
\end{tabular}




\section{SECTION BD: VILLAGE MIDWIFE}

Now, we want to ask about the activities of village midwife

\begin{tabular}{|c|c|c|}
\hline BD00a. & $\begin{array}{l}\text { INTERVIEWER CHECK : LK13 } \\
\text { IS RESPONDENT A VILLAGE MIDWIFE IN THIS VILLAGE? } \\
\text { LK13 = } 5 \text { (VILLAGE MIDWIFE) ? }\end{array}$ & $\begin{array}{l}\text { NO............ } \rightarrow \text { SECTION E } \\
\text { YES ..........1 }\end{array}$ \\
\hline BD01a. & 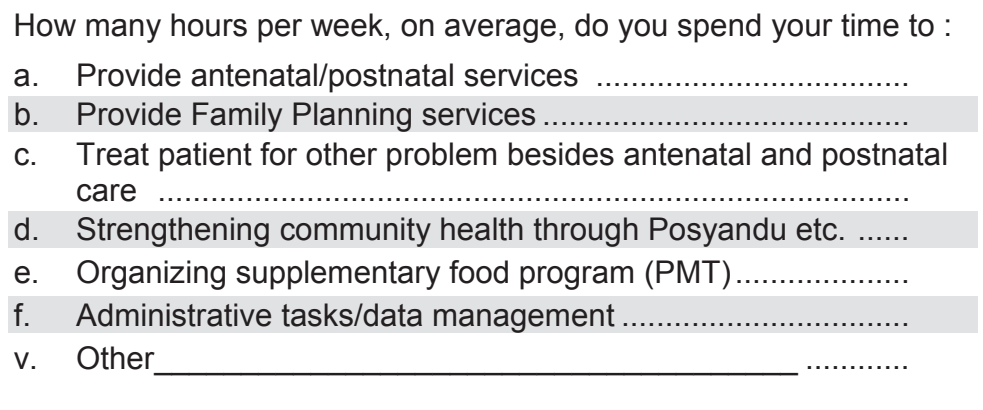 & $\begin{array}{l}\text { a. } \sqcup \text { hours } \\
\text { b. } \sqcup \text { hours } \\
\text { c. } \sqcup \text { hours } \\
\text { d. } \sqcup \text { hours } \\
\text { e. } \sqcup \text { hours } \\
\text { f. } \sqcup \text { hours } \\
\text { v. } \sqcup \text { hours }\end{array}$ \\
\hline BD01aa. & $\begin{array}{l}\text { How many hours in a week do you spend your time performing duties } \\
\text { as the Village Midwive? }\end{array}$ & hـلـ hours/week \\
\hline BD01ba. & $\begin{array}{l}\text { On average, the percentage of your patients in a week which are }[\ldots] \\
\text { is: } \\
\text { a. Female (15 years or more) } \\
\text { b. Male }(15 \text { years or more) } \\
\text { c. Children } 5-14 \text { years } \\
\text { d. Children less than } 5 \text { years. }\end{array}$ & 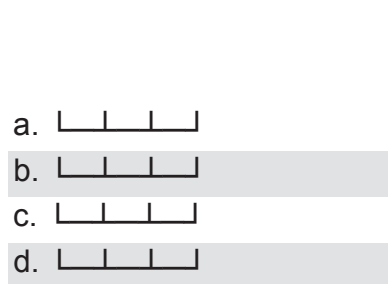 \\
\hline BD02a. & $\begin{array}{l}\text { Of the medical equipment that you used in providing health } \\
\text { services, what is the percentage that you privately purchased? }\end{array}$ & $\downarrow \downarrow ل \_$لـ \\
\hline BD03b. & $\begin{array}{l}\text { Of the medicine that you used in providing health services, what is } \\
\text { the percentage that you privately purchased? }\end{array}$ & 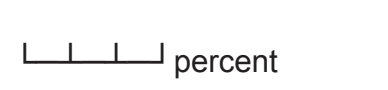 \\
\hline BD04b. & $\begin{array}{l}\text { Of the contraceptives that you used in providing health services, } \\
\text { what is the percentage that you privately purchased? }\end{array}$ & $\llcorner\perp \perp$ ـ \\
\hline
\end{tabular}

\begin{tabular}{|c|c|c|}
\hline BD04a. & Where is your place of practice? & 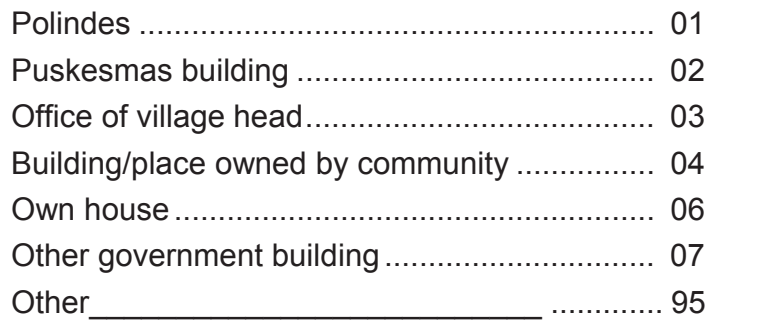 \\
\hline BD08. & $\begin{array}{l}\text { Are you in communication with traditional } \\
\text { midwives in this village? }\end{array}$ & 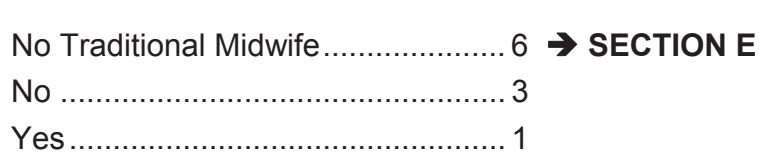 \\
\hline BD09. & $\begin{array}{l}\text { Do you consult with the traditional } \\
\text { midwives in this village? }\end{array}$ & Yes \\
\hline BD10. & $\begin{array}{l}\text { Have you ever worked cooperatively with } \\
\text { a traditional midwife in performing } \\
\text { childbirth? }\end{array}$ & Yes \\
\hline
\end{tabular}




\section{EXAMINATION ROOM}

\begin{tabular}{|c|c|c|}
\hline E1. & $\begin{array}{l}\text { HOW CLEAN IS THE FLOOR IN THIS } \\
\text { ROOM? } \\
\text { (DIRTY=IF A LOT OF DUST, FOOD } \\
\text { REMNANTS, SCATTERED GARBAGE ARE } \\
\text { FOUND) }\end{array}$ & DIRTY \\
\hline E2. & $\begin{array}{l}\text { HOW CLEAN ARE THE WALLS IN THIS } \\
\text { ROOM? } \\
\text { (DIRTY=IF MANY SPIDER WEBS, } \\
\text { SCRIBBLING, DUST, MOISTURE, PAINT } \\
\text { PEELING OFF ARE FOUND) }\end{array}$ & 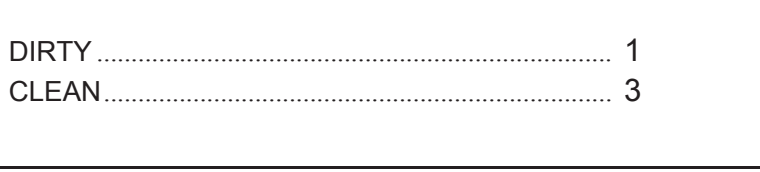 \\
\hline E3. & $\begin{array}{l}\text { ARE THERE CURTAINS THAT SEPARATE } \\
\text { THE EXAMINATION ROOM? }\end{array}$ & 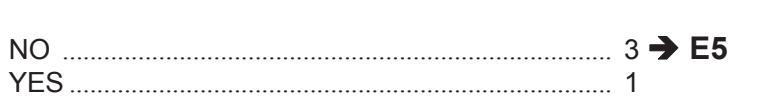 \\
\hline E4. & $\begin{array}{l}\text { HOW CLEAN ARE THESE CURTAINS? } \\
\text { (DIRTY=WHEN IT LOOKS UNWASHED, } \\
\text { THERE ARE BLOOD STAINS, OR OTHER } \\
\text { DIRT STICKING TO IT) }\end{array}$ & DIRTY \\
\hline E5. & $\begin{array}{l}\text { WHAT PROVISIONS ARE MADE FOR } \\
\text { WASHING HANDS IN THIS ROOM? }\end{array}$ & 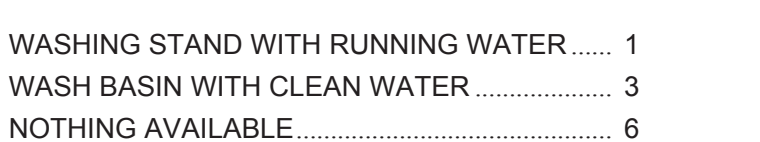 \\
\hline E6. & $\begin{array}{l}\text { IS THERE A WASTE BASKET IN THE } \\
\text { ROOM? }\end{array}$ & YES $\ldots$ \\
\hline E7. & $\begin{array}{l}\text { IS THERE AN EXAMINATION TABLE IN THE } \\
\text { ROOM? }\end{array}$ & 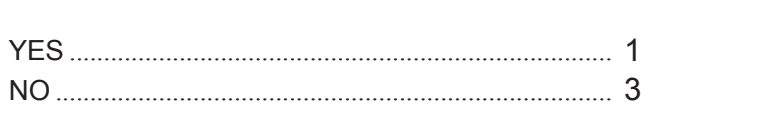 \\
\hline E8. & $\begin{array}{l}\text { What kind of needles are used for } \\
\text { injections? }\end{array}$ & 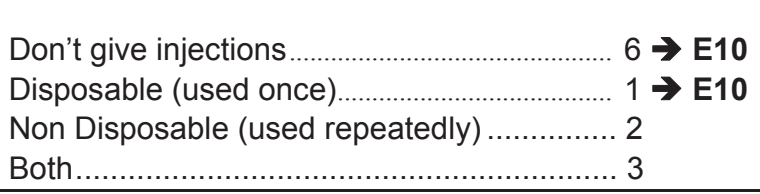 \\
\hline E9. & $\begin{array}{l}\text { How are needles sterilized? } \\
\text { MORE THAN ONE ANSWER POSSIBLE }\end{array}$ & $\begin{array}{l}\text { With a sterilizer ........................................ A } \\
\text { Boiling the needle in boiling water................ B } \\
\text { Rinsing in alcohol .............................. C } \\
\text { By heating the needle with fire .................. D } \\
\text { No sterilization................................ E } \\
\text { Other }\end{array}$ \\
\hline
\end{tabular}

KIA - KB (MCH-FP) ROOM

\begin{tabular}{|c|c|c|}
\hline E10. & $\begin{array}{l}\text { CHECK POINT: } \\
\text { IS THERE A SPECIAL ROOM FOR MCH-FP } \\
\text { ACTIVITIES? }\end{array}$ & 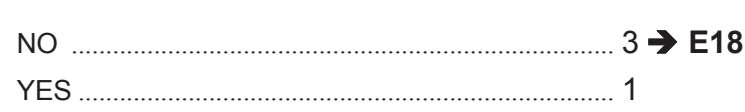 \\
\hline E11. & $\begin{array}{l}\text { HOW CLEAN ARE THE FLOORS IN THIS } \\
\text { ROOM? } \\
\text { (DIRTY=IF A LOT OF DUST, FOOD REMNANTS, } \\
\text { SCATTERED GARBAGE ARE FOUND) }\end{array}$ & 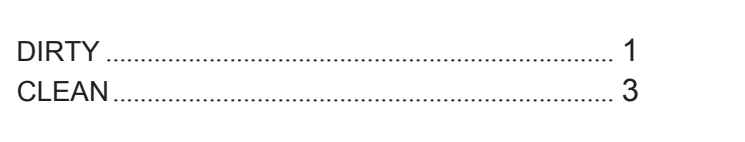 \\
\hline E12. & $\begin{array}{l}\text { HOW CLEAN ARE THE WALLS IN THIS ROOM? } \\
\text { (DIRTY=IF MANY SPIDER WEBS, SCRIBBLING, } \\
\text { DUST, MOISTURE, PAINT PEELING OFF ARE } \\
\text { FOUND) }\end{array}$ & 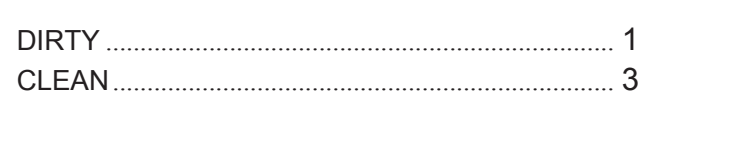 \\
\hline E13. & $\begin{array}{l}\text { ARE THERE CURTAINS THAT SEPARATE THE } \\
\text { EXAMINATION ROOM? }\end{array}$ & 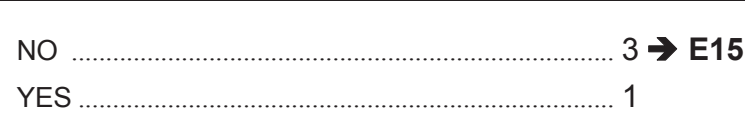 \\
\hline E14. & $\begin{array}{l}\text { HOW CLEAN IS THIS CURTAIN? } \\
\text { (DIRTY=WHEN IT LOOKS UNWASHED, THERE } \\
\text { ARE BLOOD STAINS, OR OTHER DIRT } \\
\text { STICKING TO IT) }\end{array}$ & 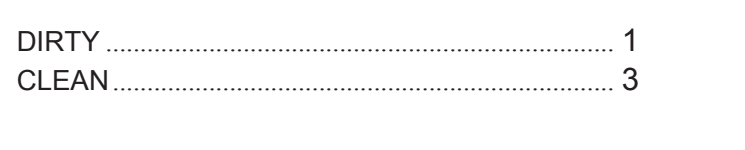 \\
\hline E15. & $\begin{array}{l}\text { WHAT PROVISIONS ARE MADE FOR WASHING } \\
\text { HANDS IN THIS ROOM? }\end{array}$ & $\begin{array}{l}\text { WASHING STAND WITH RUNNING WATER ..... } 1 \\
\text { WASH BASIN WITH CLEAN WATER } \ldots \ldots \ldots \ldots . . . . . \\
\text { NOTHING AVAILABLE }\end{array}$ \\
\hline E16. & IS THERE A WASTEBASKET IN THE ROOM? & $\begin{array}{l}\text { YES } \\
\text { NO }\end{array}$ \\
\hline E17. & $\begin{array}{l}\text { IS THERE A GYNECOLOGICAL EXAMINATION } \\
\text { TABLE IN THIS ROOM? }\end{array}$ & 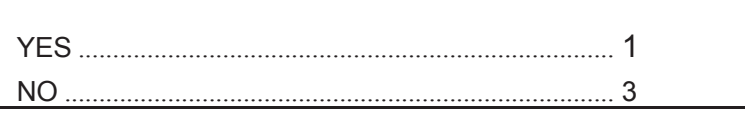 \\
\hline E18. & $\begin{array}{l}\text { Where are the vaccines kept? } \\
\text { (CIRCLE ALL THAT APPLY) }\end{array}$ & 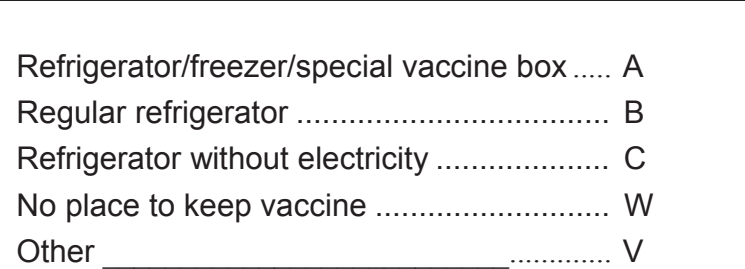 \\
\hline
\end{tabular}


SECTION H : VIGNETTES FACILITY

\section{VIGNETTE FOR PRIVATE PRACTICE}
* Curative Care for Adult
- Curative Care for Adult with Diabetes
* Curative care for children
* Prenatal Care 
Curative Care for Adult

\begin{tabular}{|c|c|c|}
\hline HPR1. & Does this health facility provide curative care for adults? & No \\
\hline HPR2. & Name of Respondent & \\
\hline HPR3. & Can you please tell me your qualifications? & 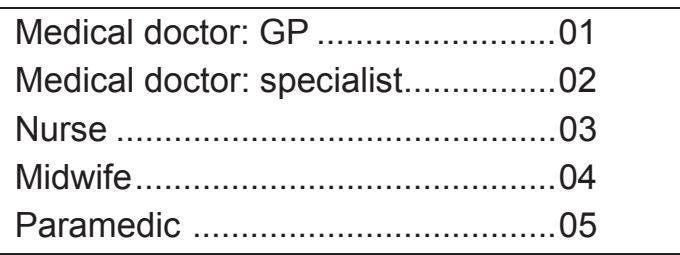 \\
\hline HPR3a & Where did you complete your studies? & 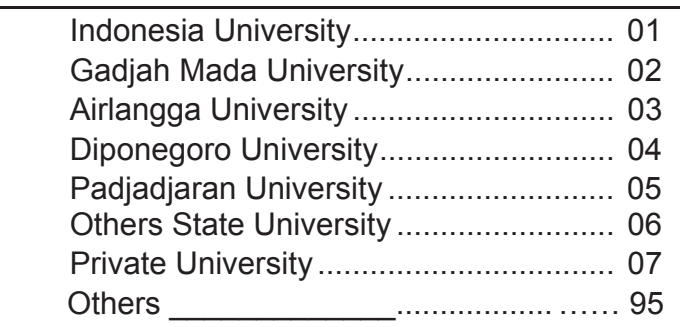 \\
\hline HPR4. & In what year did you complete your studies? & 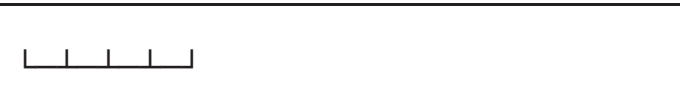 \\
\hline HPR5. & Have you received additional training since you gra & 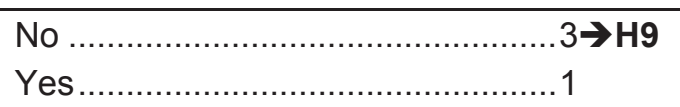 \\
\hline
\end{tabular}

Can you tell me, for each of the following areas, whether you received additional training and, if so, when this training occurred?

\begin{tabular}{|c|c|c|c|c|c|c|c|}
\hline & & \multicolumn{2}{|c|}{ HPR6. } & \multicolumn{2}{|c|}{ HPR7. } & \multicolumn{2}{|c|}{ HPR8. } \\
\hline & & \multicolumn{2}{|c|}{$\begin{array}{l}\text { Have you ever received } \\
\text { training of }[. . .] \text { after you } \\
\text { finished the study? }\end{array}$} & \multicolumn{2}{|c|}{$\begin{array}{l}\text { In the last } 12 \\
\text { months? }\end{array}$} & \multicolumn{2}{|c|}{$\begin{array}{l}\text { In the last } 5 \\
\text { years? }\end{array}$} \\
\hline 1. & $\begin{array}{l}\text { Diagnostic algorithm for adult } \\
\text { diseases }\end{array}$ & 1. Yes & 3. No $\downarrow$ & 1. Yes $\downarrow$ & 3. No & 1. Yes & 3. No \\
\hline 2. & Non-communicable disease & 1. Yes & 3. No $\downarrow$ & 1. Yes $\downarrow$ & 3. No & 1. Yes & 3. No \\
\hline 3. & Respiratory disease & 1. Yes & 3. No $\downarrow$ & 1. Yes $\downarrow$ & 3. No & 1. Yes & 3. No \\
\hline 4. & Antibiotic for respiratory disease & 1. Yes & 3. No $\downarrow$ & 1. Yes $\downarrow$ & 3. No & 1. Yes & 3. No \\
\hline
\end{tabular}

HPR9. For the rest of the interview, we would like to understand the process by which you examine an adult person suffering from cough and fever. We would like to know everything you do, beginning with the arrival of the patient, waiting upon the patient and ending when he/she goes home. I shall describe the patient, and I will ask you a series of questions about activities you perform regularly. Once a section is complete, we are unable to go back and change answers. Now I will read out the case.

\section{INSTRUCTIONS TO INTERVIEWER:}

1. READ OUT CASE 2 TIMES, AND THEN ASK QUESTIONS H11 - H14.

2. FIRST LISTEN TO THE RESPONDENT. ANY RESPONSE THAT HE OR SHE MENTIONS SPONTANEOUSLY SHOULD BE MARKED WITH CODE 1.

3. AFTER THE RESPONDENT IS FINISHED WITH THE WHOLE QUESTIONNAIRE, TELL THE RESPONDENT THAT YOU ARE GOING TO REVIEW THE CASE AGAIN.

4. START FROM THE BEGINNING AND READ THE CASE A SECOND TIME. ASK QUESTIONS H11 - H14.

5. READ OUT ANY RESPONSES THAT HE/SHE DID NOT MENTION SPONTANEOUSLY THE FIRST TIME. CODE THE APPROPRIATE RESPONSE.

HPR10. Pak Widyono came to this facility with a complaint of coughing and a fever. Now I would like to ask you exactly what you would do for this patient..

\begin{tabular}{|c|c|c|c|c|}
\hline \multirow[t]{2}{*}{ HPR11. } & $\begin{array}{l}\text { What questions do you ask the patient about his cough and } \\
\text { fever, and current health? }\end{array}$ & \multirow{2}{*}{$\begin{array}{c}\begin{array}{c}\text { Mentioned } \\
\text { spontaneously }\end{array} \\
1\end{array}$} & \multicolumn{2}{|c|}{ Prompted } \\
\hline & a. How long have you suffered from this condition? & & 2 & 3 \\
\hline & b. Any shortness of breath? & 1 & 2 & 3 \\
\hline & c. Is there any blood when you cough? & 1 & 2 & 3 \\
\hline & d. What was the color of the sputum? & 1 & 2 & 3 \\
\hline & e. Do you have any pain in the chest? & 1 & 2 & 3 \\
\hline & f. Any weight loss? & 1 & 2 & 3 \\
\hline & g. Is cough productive? & 1 & 2 & 3 \\
\hline & h. Any contact with others with respiratory problems/TB? & 1 & 2 & 3 \\
\hline & i. Any night sweats? & 1 & 2 & 3 \\
\hline
\end{tabular}


HPR11. What questions do you ask the patient about his cough and fever, and current health?

spontan

\section{HPR12. What questions do you ask the patient about his medical} history and behavior?
a. Previous TB case or took TB medicine?
b. BCG immunization or ever positive PPD?
${ }^{*}$ Note: $P P D=$ Purified Protein Derivative or Mantoux, examination of TBC
d. History of cardiac problems?
e. History of malignancy or gastric surgery?
f. Medications recently or currently taking?
g. Drug allergies?
h. Smoking history?
i. Number of packages/quantity of smoking?
j. Alcohol use?
k. Live alone or with others?
I. Employment?
m. Family health history?
n. Sanitation, ventilation at home?
c. History of asthma or COPD?
${ }^{*}$ Note: COPD = Chronic Obstructive Pulmonary Disease, chronic lungs disease

HPR13. What do you do when you conduct a physical examination of the patient?
a. Examine general appearance?
b. Take temperature?
c. Listen to respiration?
d. Check for sore throat?
e. Palpitate / feel throat / lymph nodes?
f. Is chest indrawing?
g. Palpate abdomen?
${ }^{*}$ Note: palpation = examination by palpating and pressing
h. Pulse
${ }^{*}$ Note: vital signs = breath, pulse
${ }^{*}$ Note: IPPA = Inspection, Palpation, Percussion, Auscultation
i. Blood Pressure

HPR14. What laboratory examinations would you conduct?

\begin{tabular}{|c|c|}
\hline $\begin{array}{c}\text { Mentioned } \\
\text { spontaneously }\end{array}$ & 2 \\
\hline
\end{tabular}

\begin{tabular}{|c|cc|}
\hline 1 & 2 & 3 \\
\hline 1 & 2 & 3 \\
& & \\
& 2 & 3 \\
1 & & \\
1 & 2 & 3 \\
1 & 2 & 3 \\
1 & 2 & 3 \\
1 & 2 & 3 \\
1 & 2 & 3 \\
1 & 2 & 3 \\
1 & 2 & 3 \\
1 & 2 & 3 \\
1 & 2 & 3 \\
\hline 1 & 2 & 3 \\
\hline 1 & 2 & 3 \\
\hline Mentioned & Prompted \\
\hline spontaneously & & 3 \\
\hline 1 & 2 & \\
\hline
\end{tabular}

\begin{tabular}{|c|cc|}
\hline 1 & 2 & 3 \\
\hline 1 & 2 & 3 \\
1 & 2 & 3 \\
1 & 2 & 3 \\
1 & 2 & 3 \\
1 & 2 & 3 \\
1 & 2 & 3 \\
\hline 1 & 2 & 3 \\
& & \\
\hline 1 & 2 & 3 \\
\hline $\begin{array}{c}\text { Mentioned } \\
\text { spontaneously }\end{array}$ & \multicolumn{2}{|c|}{ Prompted } \\
\hline
\end{tabular}

\begin{tabular}{l|l|ll|}
\hline a. Chest x-ray & 1 & 2 & 3 \\
\hline b. PPD or mantoux test & 1 & 2 & 3 \\
\hline c. Sputum exam for TB & 1 & 2 & 3 \\
\hline d. Routine bloodwork & 1 & 2 & 3 \\
\hline e. Liver function & 1 & 2 & 3 \\
\hline f. CD4/cell count & 1 & 2 & 3 \\
*Note: blood test to see the immune system & 1 & & 3 \\
\hline g. Urinalysis & 1 & 2 & \\
\hline
\end{tabular}


Curative Care for Adult with Diabetes

\begin{tabular}{|c|c|c|}
\hline HPR15. & Does this health facility provide curative care for adults? & 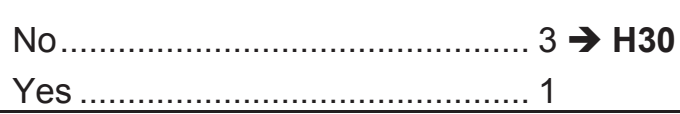 \\
\hline HPR16. & \multicolumn{2}{|l|}{ Name of respondent } \\
\hline HPR17. & Can you please tell me your qualifications? & 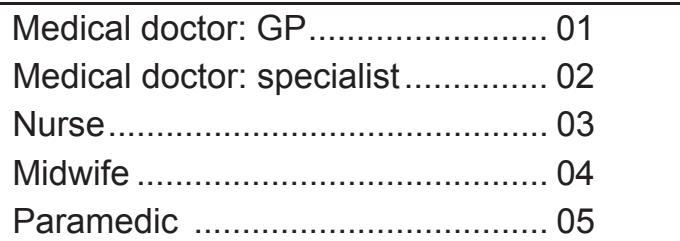 \\
\hline HPR17a & Where did you complete your studies? & 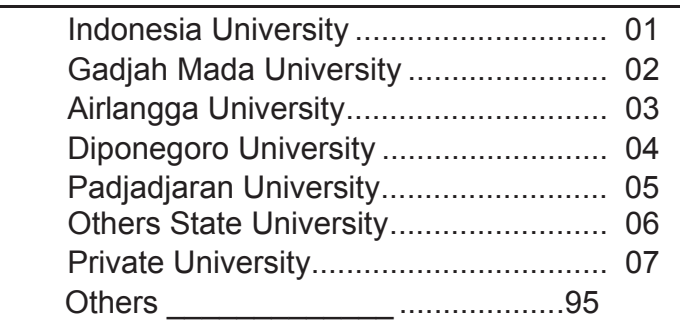 \\
\hline HPR18. & In what year did you complete your studies? & 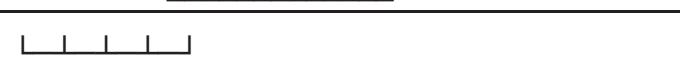 \\
\hline HPR19. & Have you received additional training since you graduated? & 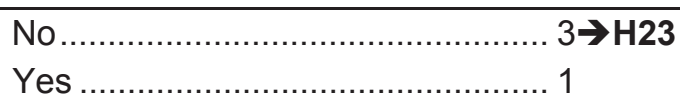 \\
\hline
\end{tabular}

\begin{tabular}{|c|c|c|c|c|c|c|c|}
\hline \multirow[b]{3}{*}{1.} & \multirow[b]{3}{*}{$\begin{array}{l}\text { Diagnostic algorithm for adult } \\
\text { diseases }\end{array}$} & \multirow{2}{*}{\multicolumn{2}{|c|}{$\begin{array}{c}\text { HPR20. } \\
\text { Have you ever } \\
\text { received training of } \\
{[\ldots . .] \text { after you }} \\
\text { finished the study? }\end{array}$}} & \multirow{2}{*}{\multicolumn{2}{|c|}{$\begin{array}{l}\text { HPR21. } \\
\text { In the last } 12 \\
\text { months? }\end{array}$}} & \multirow{2}{*}{\multicolumn{2}{|c|}{$\begin{array}{l}\text { HPR22. } \\
\text { In the last } 5 \text { years? }\end{array}$}} \\
\hline & & & & & & & \\
\hline & & 1. Yes & 3. No $\downarrow$ & 1. Yes $\downarrow$ & 3. No & 1. Yes & 3. No \\
\hline 2. & Non-communicable disease & 1. Yes & 3. No $\downarrow$ & 1. Yes $\downarrow$ & 3. No & 1. Yes & 3. No \\
\hline 3. & Mengenai penyakit diabetes & 1. Yes & 3. No $\downarrow$ & 1. Yes $\downarrow$ & 3. No & 1. Yes & 3. No \\
\hline 4. & $\begin{array}{l}\text { Mengenai obat untuk penyakit } \\
\text { diabetes }\end{array}$ & 1. Yes & 3. No $\downarrow$ & 1. Yes $\downarrow$ & 3. No & 1. Yes & 3. No \\
\hline
\end{tabular}

HPR23. For the rest of the interview, we would like to understand the process by which you examine an adult person suffering from diabetes. We would like to know everything you do, beginning with the arrival of the patient, waiting upon the patient and ending when he/she goes home. I shall describe the patient, and I will ask you a series of questions about activities you perform regularly. Once a section is complete, we are unable to go back and change answers. Now I will read out the case

\section{INSTRUCTIONS TO INTERVIEWER:}

1. READ OUT CASE 2 TIMES, AND THEN ASK QUESTIONS H25 - H29.

2. FIRST LISTEN TO THE RESPONDENT. ANY RESPONSE THAT HE OR SHE MENTIONS SPONTANEOUSLY SHOULD BE MARKED WITH CODE 1.

3. AFTER THE RESPONDENT IS FINISHED WITH THE WHOLE QUESTIONNAIRE, TELL THE RESPONDENT THAT YOU ARE GOING TO REVIEW THE CASE AGAIN.

4. START FROM THE BEGINNING AND READ THE CASE A SECOND TIME. ASK QUESTIONS H25 - H29.

5. READ OUT ANY RESPONSES THAT HE/SHE DID NOT MENTION SPONTANEOUSLY THE FIRST TIME. CODE THE APPROPRIATE RESPONSE.

HPR24. Mr. Widyono came to this facility, and presents to you "to get my sugar checked." He has just moved to the community and has never visited the facility. Now I would like to ask you exactly what you would do for this patient.

\begin{tabular}{|c|c|c|c|c|}
\hline \multirow[t]{2}{*}{ HPR25. } & $\begin{array}{l}\text { What questions do you ask the patient about his present physical } \\
\text { condition, high blood sugar, and medications? }\end{array}$ & \multirow{2}{*}{$\begin{array}{l}\begin{array}{l}\text { Mentioned } \\
\text { spontaneously }\end{array} \\
1\end{array}$} & \multicolumn{2}{|c|}{ Prompted } \\
\hline & a. How long have you suffered from this condition? & & 2 & 3 \\
\hline & b. Medications recently or currently taking? & 1 & 2 & 3 \\
\hline & c. Do you have to urinate frequently? & 1 & 2 & 3 \\
\hline & d. Frequent thirst? & 1 & 2 & 3 \\
\hline & e. Any weight loss? & 1 & 2 & 3 \\
\hline & f. Any sweating? & 1 & 2 & 3 \\
\hline & g. Any anxiety or heart palpitations? & 1 & 2 & 3 \\
\hline & h. Abdominal fullness prematurely after meals? & 1 & 2 & 3 \\
\hline & i. Edema or weight retention? & 1 & 2 & 3 \\
\hline & j. Current treatment for hypertension? & 1 & 2 & 3 \\
\hline
\end{tabular}




\begin{tabular}{|c|c|c|c|c|c|}
\hline \multirow[t]{7}{*}{ HPR25 } & \multicolumn{2}{|r|}{$\begin{array}{l}\text { What questions do you ask the patient about his } \\
\text { present physical condition, high blood sugar, } \\
\text { and medications? }\end{array}$} & \multirow{2}{*}{$\begin{array}{c}\begin{array}{c}\text { Mentioned } \\
\text { spontaneously }\end{array} \\
1\end{array}$} & \multicolumn{2}{|c|}{ Prompted } \\
\hline & & Often feel tingling? & & 2 & 3 \\
\hline & & Wound that stays? & 1 & 2 & 3 \\
\hline & & Often have ulcer? & 1 & 2 & 3 \\
\hline & & Family history & 1 & 2 & 3 \\
\hline & & Feel weary & 1 & 2 & 3 \\
\hline & & Have check blood sugar? & 1 & 2 & 3 \\
\hline \multirow[t]{15}{*}{ HPR26 } & \multicolumn{2}{|c|}{$\begin{array}{l}\text { What questions do you ask Mr. Widyono about his } \\
\text { medical history and behavior? }\end{array}$} & $\begin{array}{c}\text { Mentioned } \\
\text { spontaneously }\end{array}$ & \multicolumn{2}{|c|}{ Prompted } \\
\hline & a. & History of hypertension? & 1 & 2 & 3 \\
\hline & b. & History of high cholesterol? & 1 & 2 & 3 \\
\hline & c. & Co-existing or prior heart condition? & 1 & 2 & 3 \\
\hline & d. & Prior eye examination? & 1 & 2 & 3 \\
\hline & e. & Prior hospitalization? & 1 & 2 & 3 \\
\hline & f. & Prior diabetic coma? & 1 & 2 & 3 \\
\hline & g. & Prior renal failure? & 1 & 2 & 3 \\
\hline & h. & Does he smoke regularly? & 1 & 2 & 3 \\
\hline & i. & Number of packages/quantity of smoking? & 1 & 2 & 3 \\
\hline & j. & Alcohol use? & 1 & 2 & 3 \\
\hline & k. & Immunization history? & 1 & 2 & 3 \\
\hline & I. & Regular exercise? & 1 & 2 & 3 \\
\hline & $\mathrm{m}$. & Questions about nutrion/eating habits? & 1 & 2 & 3 \\
\hline & \multirow{2}{*}{\multicolumn{2}{|c|}{$\begin{array}{l}\text { What do you do when you conduct a physical } \\
\text { examination of the patient? }\end{array}$}} & 1 & 2 & 3 \\
\hline HPR27 & & & $\begin{array}{c}\text { Mentioned } \\
\text { spontaneously }\end{array}$ & \multicolumn{2}{|c|}{ Prompted } \\
\hline & a. & Blood pressure in one arm & 1 & 2 & 3 \\
\hline & b. & Blood pressure in both arms & 1 & 2 & 3 \\
\hline & c. & Listen to chest/heart? & 1 & 2 & 3 \\
\hline & d. & Listen to abdomen? & 1 & 2 & 3 \\
\hline & e. & Examine the feet? & 1 & 2 & 3 \\
\hline & f. & Examine peripheral vascular system? & 1 & 2 & 3 \\
\hline & g. & Check for edema? & 1 & 2 & 3 \\
\hline & h. & Examine prostate? & 1 & 2 & 3 \\
\hline & i. & Pulse & 1 & 2 & 3 \\
\hline & j. & Respiration & 1 & 2 & 3 \\
\hline HPR28 & \multicolumn{2}{|c|}{ What laboratory examinations would you conduct? } & $\begin{array}{c}\text { Mentioned } \\
\text { spontaneously }\end{array}$ & \multicolumn{2}{|c|}{ Prompted } \\
\hline & a. & Chest x-ray? & 1 & 2 & 3 \\
\hline & b. & Blood chemistry: creatinine, glucose? & 1 & 2 & 3 \\
\hline & c. & Sputum exam? & 1 & 2 & 3 \\
\hline & d. & $\begin{array}{l}\text { CBC (Complete Blood Count)? } \\
\text { *Note: blood examination to count the red blood } \\
\text { cells, white blood cells, and blood platelet }\end{array}$ & 1 & 2 & 3 \\
\hline & e. & $\begin{array}{l}\text { Test for triglycerides? } \\
\text { *Note: examination to check the lipid excess in } \\
\text { the blood }\end{array}$ & 1 & 2 & 3 \\
\hline & f. & Ultrasound? & 1 & 2 & 3 \\
\hline & g. & Liver function? & 1 & 2 & 3 \\
\hline & h. & $\begin{array}{l}\text { HgbA1c? } \\
\text { *Note: examination to check the glucose } \\
\text { amount in the haemoglobyn }\end{array}$ & 1 & 2 & 3 \\
\hline & i. & Hepatic enzymes? & 1 & 2 & 3 \\
\hline
\end{tabular}

\begin{tabular}{|c|c|c|c|c|}
\hline \multirow[t]{2}{*}{ HPR29 } & $\begin{array}{l}\text { What advice or future examinatios would you offer for the } \\
\text { patient? }\end{array}$ & \multirow{2}{*}{$\begin{array}{c}\begin{array}{c}\text { Mentioned } \\
\text { spontaneously }\end{array} \\
1\end{array}$} & \multicolumn{2}{|c|}{ Prompted } \\
\hline & a. $\quad$ Recommend stop smoking? & & 2 & 3 \\
\hline & Nutritional advice? & 1 & 2 & 3 \\
\hline & Advice about exercise? & 1 & 2 & 3 \\
\hline & Examine the feet? & 1 & 2 & 3 \\
\hline & Refer to other specialist (eye,foot, or heart)? & 1 & 2 & 3 \\
\hline & $\begin{array}{l}\text { Prescribe anti-hypertensives? } \\
\text { *Note: medicine to control high blood pressure }\end{array}$ & 1 & 2 & 3 \\
\hline & $\begin{array}{l}\text { Prescribe Metformin? } \\
{ }^{*} \text { Note: medicine for diabetes }\end{array}$ & 1 & 2 & 3 \\
\hline & Make an appointment for the next visit? & 1 & 2 & 3 \\
\hline
\end{tabular}


Curative care for children

\begin{tabular}{|c|c|c|}
\hline HPR30 & Does this health facility provide curative care for children? & 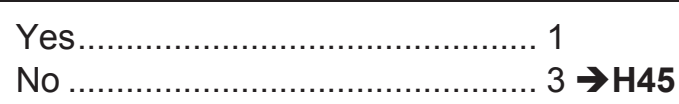 \\
\hline HPR31 & \multicolumn{2}{|l|}{ Name of respondent: } \\
\hline HPR32 & Can you please tell me your qualifications? & 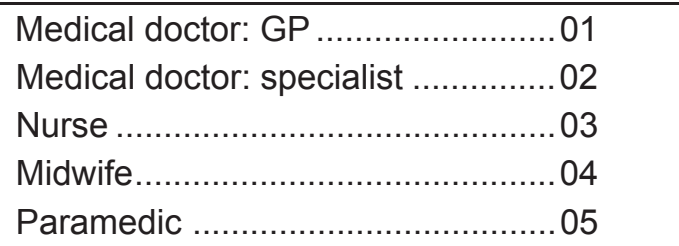 \\
\hline HPR32a & Where did you complete your studies? & 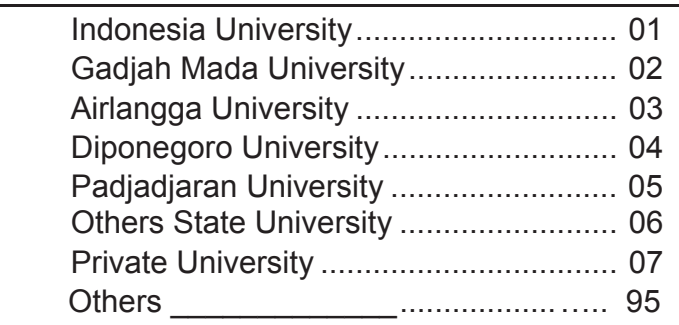 \\
\hline HPR33 & In what year did you complete your studies? & $\begin{array}{llllllllll} & 1 & 1 & 1 & 1\end{array}$ \\
\hline HPR34 & Have you received additional training since you graduated? & 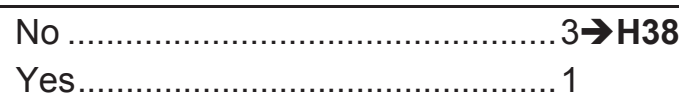 \\
\hline
\end{tabular}

\begin{tabular}{|c|c|c|c|c|c|c|c|}
\hline \multirow[b]{3}{*}{1.} & \multirow[b]{3}{*}{ Child immunization } & \multirow{2}{*}{\multicolumn{2}{|c|}{$\begin{array}{c}\text { HPR35 } \\
\text { Have you ever } \\
\text { received training of } \\
\text { [...] after you } \\
\text { finished the study? }\end{array}$}} & \multirow{2}{*}{\multicolumn{2}{|c|}{$\begin{array}{c}\text { HPR36 } \\
\text { In the last } 12 \\
\text { months? }\end{array}$}} & \multicolumn{2}{|c|}{ HPR37 } \\
\hline & & & & & & In the la & years? \\
\hline & & 1. Yes & 3. No $\downarrow$ & 1. Yes $\downarrow$ & 3. No & 1. Yes & 3. No \\
\hline 2. & $\begin{array}{l}\text { Treatment of Acute Respiratory } \\
\text { Infection }\end{array}$ & 1. Yes & 3. No $\downarrow$ & 1. Yes $\downarrow$ & 3. No & 1. Yes & 3. No \\
\hline 3. & Treatment of diarrhea & 1. Yes & 3. No $\downarrow$ & 1. Yes $\downarrow$ & 3. No & 1. Yes & 3. No \\
\hline 4. & Treatment of malaria & 1. Yes & 3. No $\downarrow$ & 1. Yes $\downarrow$ & 3. No & 1. Yes & 3. No \\
\hline 5. & Nutrition & 1. Yes & 3. No $\downarrow$ & 1. Yes $\downarrow$ & 3. No & 1. Yes & 3. No \\
\hline 6. & HIV transmission in pregnancy & 1. Yes & 3. No $\downarrow$ & 1. Yes $\downarrow$ & 3. No & 1. Yes & 3. No \\
\hline 7. & Prenatal care & 1. Yes & 3. No $\downarrow$ & 1. Yes $\downarrow$ & 3. No & 1. Yes & 3. No \\
\hline
\end{tabular}

HPR38 For the rest of the interview, we would like to understand the process by which you provide curative care for children. We would like to know everything you do, beginning with the arrival of the patient, waiting upon the patient and ending when he/she goes home. I shall describe the patient, and I will ask you a series of questions about activities you perform regularly. Once a section is complete, we are unable to go back and change answers. Now I will read out the case

INSTRUCTIONS TO INTERVIEWER:

1. READ OUT CASE 2 TIMES, AND THEN ASK QUESTIONS H40-H44.

2. FIRST LISTEN TO THE RESPONDENT. ANY RESPONSE THAT HE OR SHE MENTIONS SPONTANEOUSLY SHOULD BE MARKED WITH CODE 1.

3. AFTER THE RESPONDENT IS FINISHED WITH THE WHOLE QUESTIONNAIRE, TELL THE RESPONDENT THAT YOU ARE GOING TO REVIEW THE CASE AGAIN.

4. START FROM THE BEGINNING AND READ THE CASE A SECOND TIME. ASK QUESTIONS H40-H44.

5. READ OUT ANY RESPONSES THAT HE/SHE DID NOT MENTION SPONTANEOUSLY THE FIRST TIME. CODE THE APPROPRIATE RESPONSE:.

HPR39 Mrs. Nani comes to this facility with her daughter, an 8 month old baby. She says that her daughter has had diarrhea for 2 days with vomiting.

\begin{tabular}{|c|c|c|c|c|}
\hline HPR40 & $\begin{array}{l}\text { What are the } 13-14 \text { most important questions you } \\
\text { ask about the diarrhea and vomiting? }\end{array}$ & $\begin{array}{c}\text { Mentioned } \\
\text { spontaneously }\end{array}$ & & \\
\hline & a. When did the diarrhea start? & 1 & 2 & 3 \\
\hline & b. How frequently does diarrhea occur? & 1 & 2 & 3 \\
\hline & What do the feces/vomit look like of smell like & 1 & 2 & 3 \\
\hline & d. Any blood in vomit? & 1 & 2 & 3 \\
\hline & e. Any blood in stools? & 1 & 2 & 3 \\
\hline & f. Any fever? & 1 & 2 & 3 \\
\hline
\end{tabular}




\begin{tabular}{|c|c|c|c|c|}
\hline HPR40. & $\begin{array}{l}\text { What are the } 13-14 \text { most important questions you } \\
\text { ask about the diarrhea and vomiting? }\end{array}$ & $\begin{array}{l}\text { Mentioned } \\
\text { spontaneously }\end{array}$ & \multicolumn{2}{|c|}{ Prompted } \\
\hline & g. Level of activity (active vs listless)? & 1 & 2 & 3 \\
\hline & h. Is the child feeding and drinking? & 1 & 2 & 3 \\
\hline & i. Given any medication already? & 1 & 2 & 3 \\
\hline & j. Any evidence of dehydration? & 1 & 2 & 3 \\
\hline & k. Vomits everything? & 1 & 2 & 3 \\
\hline & I. Has convulsions? & 1 & 2 & 3 \\
\hline & m. Eaten anything unusual? & 1 & 2 & 3 \\
\hline & n. Any ill contacts? & 1 & 2 & 3 \\
\hline & o. Urinating? & 1 & 2 & 3 \\
\hline HPR41 & $\begin{array}{l}\text { What do you ask about the baby's medical history } \\
\text { and environment? }\end{array}$ & $\begin{array}{l}\text { Mentioned } \\
\text { spontaneously }\end{array}$ & \multicolumn{2}{|c|}{ Prompted } \\
\hline & a. History of similar disease? & 1 & 2 & 3 \\
\hline & b. Drug allergies? & 1 & 2 & 3 \\
\hline & c. Any other medical or surgical problems or HIV? & 1 & 2 & 3 \\
\hline & d. Any complications at delivery or prematurity? & 1 & 2 & 3 \\
\hline & e. Access to water or sanitation? & 1 & 2 & 3 \\
\hline & f. Immunization history? & 1 & 2 & 3 \\
\hline & g. Breastfeeding/other fluids? & 1 & 2 & 3 \\
\hline & h. Digestive system normal? & 1 & 2 & 3 \\
\hline & i. Ever had surgery on digestive organs? & 1 & 2 & 3 \\
\hline & k. Eating & 1 & 2 & 3 \\
\hline & I. Baby care & 1 & 2 & 3 \\
\hline HPR42 & $\begin{array}{l}\text { What do you do when you conduct a physical } \\
\text { examination of the child? }\end{array}$ & $\begin{array}{c}\text { Mentioned } \\
\text { spontaneously }\end{array}$ & \multicolumn{2}{|c|}{ Prompted } \\
\hline & a. Check appearance / alertness? & 1 & 2 & 3 \\
\hline & b. Take her temperature? & 1 & 2 & 3 \\
\hline & $\begin{array}{l}\text { c. Examine the crown of the head? } \\
\text { *Note: is it concave? }\end{array}$ & 1 & 2 & 3 \\
\hline & d. Check pulse? & 1 & 2 & 3 \\
\hline & e. Weigh? & 1 & 2 & 3 \\
\hline & f. Check height? & 1 & 2 & 3 \\
\hline & g. Determine capillary refill time/check nailbeds? & 1 & 2 & 3 \\
\hline & h. Examine eyes? & 1 & 2 & 3 \\
\hline & i. $\quad$ Check skin turgor/elasticity? & 1 & 2 & 3 \\
\hline & j. Auscultate abdomen for bowel sounds? & 1 & 2 & 3 \\
\hline & $\begin{array}{l}\text { k. Palpitate abdomen? } \\
{ }^{*} \text { Note: examination of stomach by palpating and } \\
\text { pressing }\end{array}$ & 1 & 2 & 3 \\
\hline & I. $\quad$ Check feces for blood or mucous & 1 & 2 & 3 \\
\hline & m. Check palms of hands? & 1 & 2 & 3 \\
\hline & n. Check for edema in feet? & 1 & 2 & 3 \\
\hline & o. Breathing normally? & 1 & 2 & 3 \\
\hline & p. Blood pressure? & 1 & 2 & 3 \\
\hline HPR43 & What laboratory examinations would you conduct? & $\begin{array}{c}\text { Mentioned } \\
\text { spontaneously }\end{array}$ & \multicolumn{2}{|c|}{ Prompted } \\
\hline & $\begin{array}{l}\text { a. Routine bloodwork/CBC? } \\
{ }^{*} \text { Note: } C B C=\text { Complete Blood Count }\end{array}$ & 1 & 2 & 3 \\
\hline & b. Stool culture? & 1 & 2 & 3 \\
\hline & $\begin{array}{l}\text { c. Blood smear/dipstick for malaria? } \\
\text { *Note: quick test for malaria }\end{array}$ & 1 & 2 & 3 \\
\hline
\end{tabular}




\begin{tabular}{|c|c|c|c|c|}
\hline \multirow[t]{2}{*}{ HPR44 } & $\begin{array}{l}\text { If this child has mild dehydration of viral etiology, } \\
\text { what would you do? }\end{array}$ & \multirow{2}{*}{$\begin{array}{c}\begin{array}{c}\text { Mentioned } \\
\text { spontaneously }\end{array} \\
1\end{array}$} & \multicolumn{2}{|c|}{ Prompted } \\
\hline & a. Recommend to increase fluids? & & 2 & 3 \\
\hline & b. Provide rehydration solution in clinic? & 1 & 2 & 3 \\
\hline & $\begin{array}{l}\text { c. Show how/recommend rehydration solution for } \\
\text { home? }\end{array}$ & 1 & 2 & 3 \\
\hline & d. Recommend vitamin supplements? & 1 & 2 & 3 \\
\hline & e. Recommend medicine for fever? & 1 & 2 & 3 \\
\hline & f. Instruct about returning to clinic if health worsens? & 1 & 2 & 3 \\
\hline & g. Update immunizations? & 1 & 2 & 3 \\
\hline & h. Administrate IV fluids? & 1 & 2 & 3 \\
\hline & Recommend antibiotics? & 1 & 2 & 3 \\
\hline & j. Hospitalize? & 1 & 2 & 3 \\
\hline & k. Continue to breastfeed? & 1 & 2 & 3 \\
\hline
\end{tabular}


Prenatal Care

\begin{tabular}{|c|c|c|}
\hline HPR45 & Does this health facility provide prenatal care? & Yes \\
\hline HPR46 & \multicolumn{2}{|c|}{ Name of respondent $:_{-}$} \\
\hline HPR47 & Can you please tell me your qualifications? & 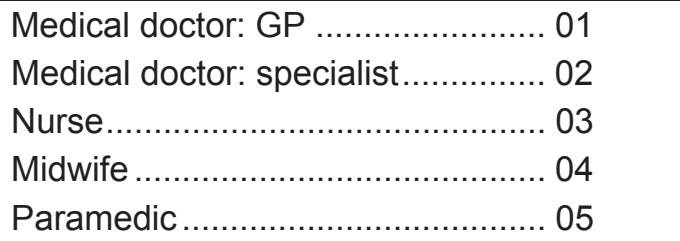 \\
\hline HPR47a & Where did you complete your studies? & 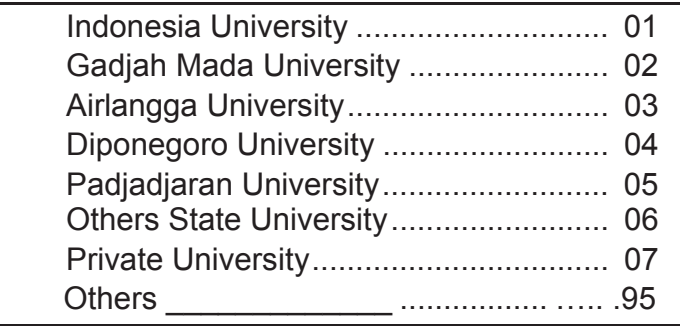 \\
\hline HPR48 & In what year did you complete your studies? & 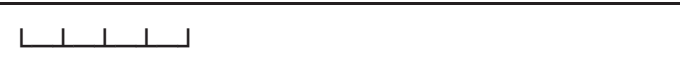 \\
\hline HPR49 & $\begin{array}{l}\text { Have you received additional training since you } \\
\text { graduated? }\end{array}$ & 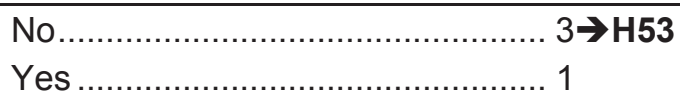 \\
\hline
\end{tabular}

\begin{tabular}{|c|c|c|c|c|c|c|c|}
\hline \multirow[b]{3}{*}{1.} & \multirow[b]{3}{*}{ Safe delivery } & \multirow{2}{*}{\multicolumn{2}{|c|}{$\begin{array}{c}\text { HPR50 } \\
\text { Have you ever } \\
\text { received training of } \\
\text { [...] after you } \\
\text { finished the study? }\end{array}$}} & \multirow{2}{*}{\multicolumn{2}{|c|}{$\begin{array}{c}\text { HPR51 } \\
\text { In the last } 12 \\
\text { months? }\end{array}$}} & \multirow{2}{*}{\multicolumn{2}{|c|}{$\begin{array}{c}\text { HPR52 } \\
\text { In the last } 5 \\
\text { years? }\end{array}$}} \\
\hline & & & & & & & \\
\hline & & 1. Yes & 3. No $\downarrow$ & 1. Yes $\downarrow$ & 3. No & 1. Yes & 3. No \\
\hline 2. & High risk pregnancies & 1. Yes & 3. No $\downarrow$ & 1. Yes $\downarrow$ & 3. No & 1. Yes & 3. No \\
\hline 3. & Assistance during labor & 1. Yes & 3. No $\downarrow$ & 1. Yes $\downarrow$ & 3. No & 1. Yes & 3. No \\
\hline 4. & HIV in pregnancy & 1. Yes & 3. No $\downarrow$ & 1. Yes $\downarrow$ & 3. No & 1. Yes & 3. No \\
\hline 5. & Obstetrical emergencies & 1. Yes & 3. No $\downarrow$ & 1. Yes $\downarrow$ & 3. No & 1. Yes & 3. No \\
\hline 6. & Family planning & 1. Yes & 3. No $\downarrow$ & 1. Yes $\downarrow$ & 3. No & 1. Yes & 3. No \\
\hline 7. & Other & 1. Yes & 3. No $\downarrow$ & 1. Yes $\downarrow$ & 3. No & 1. Yes & 3. No \\
\hline
\end{tabular}

HPR53 For the rest of the interview, we would like to understand the process by which you provide a pregnancy examination. We would like to know everything you do, beginning with the arrival of the patient, waiting upon the patient and ending when she goes home. I shall describe the patient, and I will ask you a series of questions about activities you perform regularly. Once a section is complete, we are unable to go back and change answers. Now I will read out the case

\section{INSTRUCTIONS TO INTERVIEWER:}

1. READ OUT CASE 2 TIMES, AND THEN ASK QUESTIONS H55 - H60.

2. FIRST LISTEN TO THE RESPONDENT. ANY RESPONSE THAT HE OR SHE MENTIONS SPONTANEOUSLY SHOULD BE MARKED WITH CODE 1.

3. AFTER THE RESPONDENT IS FINISHED WITH THE WHOLE QUESTIONNAIRE, TELL THE RESPONDENT THAT YOU ARE GOING TO REVIEW THE CASE AGAIN.

4. START FROM THE BEGINNING AND READ THE CASE A SECOND TIME. ASK QUESTIONS H55 - H60.

5. READ OUT ANY RESPONSES THAT HE/SHE DID NOT MENTION SPONTANEOUSLY THE FIRST TIME. CODE THE APPROPRIATE RESPONSE:

HPR54 Mrs. Ani, a married woman of 26, has not had her period for 3 months. She has come to you for a pregancy examination. This is her first visit. Please recount everything you would do during the pregnancy examination..

\begin{tabular}{|c|c|c|c|c|}
\hline HPR55 & $\begin{array}{l}\text { What are most important questions you would ask Mrs. } \\
\text { Ani about her previous pregnancies and labor? }\end{array}$ & $\begin{array}{l}\text { Mentioned } \\
\text { spontaneously }\end{array}$ & \multicolumn{2}{|c|}{ Prompted } \\
\hline & a. Number of prior pregnancies? & 1 & 2 & 3 \\
\hline & b. Number of living children & 1 & 2 & 3 \\
\hline & c. Number of miscarriages/abortions/stillbirths? & 1 & 2 & 3 \\
\hline & d. Any bleeding during previous labor? & 1 & 2 & 3 \\
\hline
\end{tabular}




\begin{tabular}{|c|c|c|c|c|}
\hline HPR55 & $\begin{array}{l}\text { What are most important questions you would ask } \\
\text { Mrs. Ani about her previous pregnancies and labor? }\end{array}$ & $\begin{array}{l}\text { Mentioned } \\
\text { spontaneously }\end{array}$ & \multicolumn{2}{|c|}{ Prompted } \\
\hline & e. How the last child was delivered? & 1 & 2 & 3 \\
\hline & f. Birth weight of previous child? & 1 & 2 & 3 \\
\hline & g. History of genetic anamolies? & 1 & 2 & 3 \\
\hline & $\begin{array}{l}\text { h. Gynecological history (STIs, pap smear, } \\
\text { contraceptive use, etc.) }\end{array}$ & 1 & 2 & 3 \\
\hline HPR56 & $\begin{array}{l}\text { What are themost important questions you ask Mrs. } \\
\text { Ani about her current pregnancy? }\end{array}$ & $\begin{array}{l}\text { Mentioned } \\
\text { spontaneously }\end{array}$ & \multicolumn{2}{|c|}{ Prompted } \\
\hline & a. Last menstrual date? & 1 & 2 & 3 \\
\hline & b. Any health problems now? & 1 & 2 & 3 \\
\hline & $\begin{array}{l}\text { c. Any obstetric symptoms (contractions, vaginal } \\
\text { bleeding, etc)? }\end{array}$ & 1 & 2 & 3 \\
\hline & d. Any weight loss/gain, nausea, vomiting? & 1 & 2 & 3 \\
\hline & e. Taking any medications now? & 1 & 2 & 3 \\
\hline HPR57 & $\begin{array}{l}\text { What are the most important questions you want to } \\
\text { ask about her medical and social/behavioral history? }\end{array}$ & $\begin{array}{l}\text { Mentioned } \\
\text { spontaneously }\end{array}$ & \multicolumn{2}{|c|}{ Prompted } \\
\hline & a. Any history of high blood pressure? & 1 & 2 & 3 \\
\hline & b. Any history of diabetes? & 1 & 2 & 3 \\
\hline & c. Any previous STI, including HIV+? & 1 & 2 & 3 \\
\hline & d. Any previous IUD or contraceptive use? & 1 & 2 & 3 \\
\hline & e. Tetanus shot in previous pregnancy? & 1 & 2 & 3 \\
\hline & f. Any previous heart disease? & 1 & 2 & 3 \\
\hline & g. Family history of hereditary disease? & 1 & 2 & 3 \\
\hline & h. Ever had malaria? & 1 & 2 & 3 \\
\hline & i. Present or previous smoker? & 1 & 2 & 3 \\
\hline & j. Any history of alcohol use? & 1 & 2 & 3 \\
\hline & k. Assess whether pregnancy is high risk? & 1 & 2 & 3 \\
\hline & I. Ever had surgery? & 1 & 2 & 3 \\
\hline & m. Any history of asthmatism? & 1 & 2 & 3 \\
\hline & n. Any history of kidney disease? & 1 & 2 & 3 \\
\hline HPR58 & $\begin{array}{l}\text { What would you do when you conduct a physical } \\
\text { examination of Mrs. Ani? }\end{array}$ & $\begin{array}{l}\text { Mentioned } \\
\text { spontaneously }\end{array}$ & \multicolumn{2}{|c|}{ Prompted } \\
\hline & a. Body height? & 1 & 2 & 3 \\
\hline & b. Body weight? & 1 & 2 & 3 \\
\hline & c. Take blood pressure? & 1 & 2 & 3 \\
\hline & d. Palpitate abdomen/measure uterine height? & 1 & 2 & 3 \\
\hline & e. Listen to fetal heartbeat? & 1 & 2 & 3 \\
\hline & $\begin{array}{l}\text { f. Pelvic examination? } \\
\text { *Note: internal examination }\end{array}$ & 1 & 2 & 3 \\
\hline & $\begin{array}{l}\text { g. Check for edema? } \\
\text { *Note: swelling or 'odim' }\end{array}$ & 1 & 2 & 3 \\
\hline & h. Upper arm measurement & 1 & 2 & 3 \\
\hline & i. Facial appearance, pale or not & 1 & 2 & 3 \\
\hline
\end{tabular}




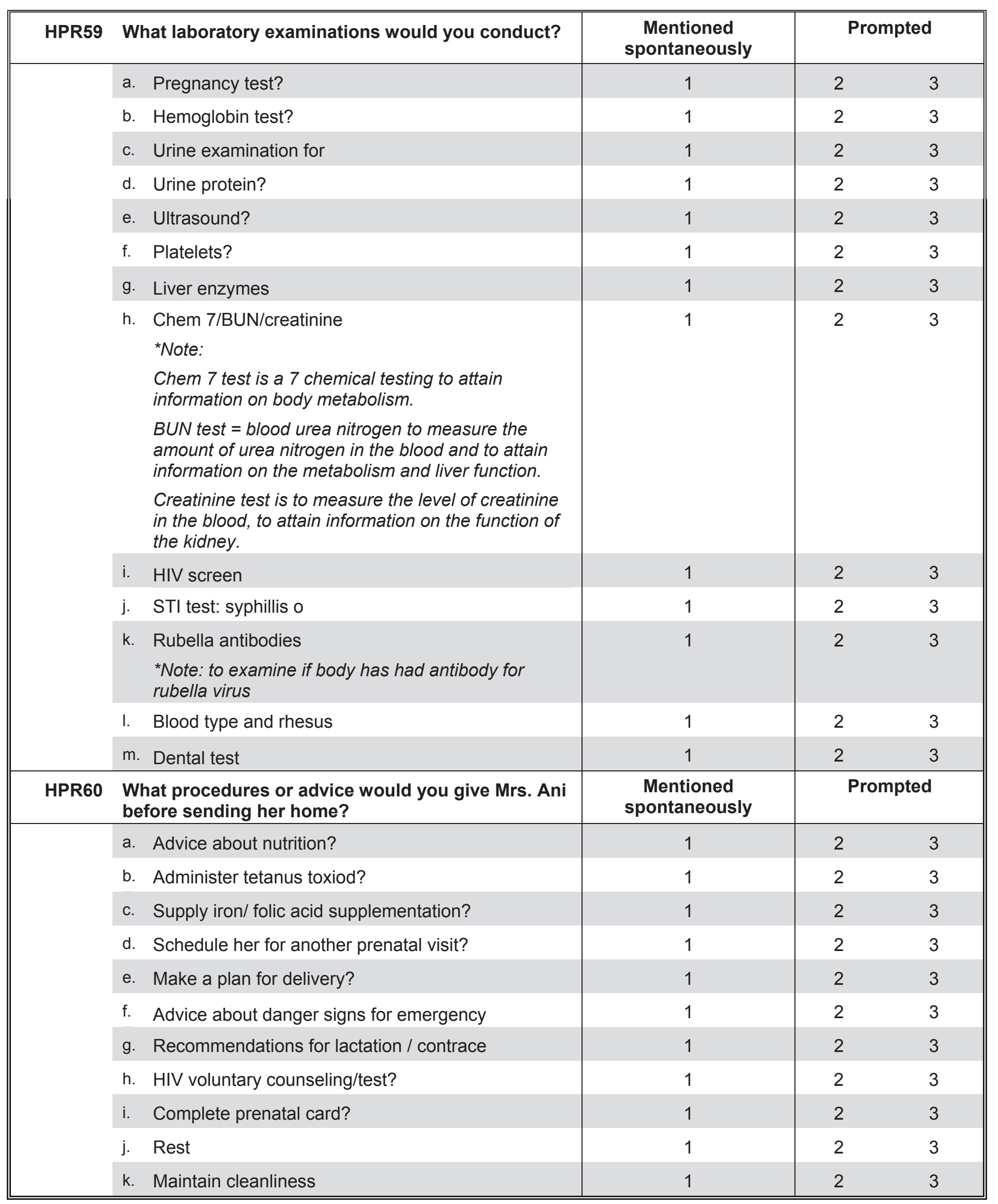




\section{SECTION CP: INTERVIEWER NOTES}

\begin{tabular}{|c|c|c|c|c|c|c|c|}
\hline LANGMAIN (CK1). & \multicolumn{2}{|l|}{ Interview was entirely/mostly conducted in what language? } & \multicolumn{5}{|l|}{$ـ$ other } \\
\hline & Other languaged used (if any): & & \multicolumn{5}{|l|}{$\downarrow \downarrow$ L other } \\
\hline LANGUAGE CODE: & & & & & & & \\
\hline 00. Bahasa Indonesia & 04. Batak & 08. Sasak & 12. Makassar & 16. & Toraja & 20. & Lampung \\
\hline 01. Java & 05. Bugis & 09. Minang & 13. Nias & 17. & Lahat & 95. & Other, \\
\hline 02. Sunda & 06. Cina & 10. Banjar & 14. Palembang & 18. & Other South Sumatera & 96. & NA \\
\hline 03. Bali & 07. Madura & 11. Bima & 15. Sumbawa & 19. & Betawi & & \\
\hline
\end{tabular}

\begin{tabular}{|c|c|c|c|}
\hline RESULT (FP3). RESULTS OF INTERVIEW & REASON (FP4). REASON FOR ANSWERING "2" I "3" IN RESULT. & FP6. MONITORING BY SUPERVISOR & \\
\hline $\begin{array}{l}\text { 1. Completed } \rightarrow \text { FP6 } \\
\text { 2. Partly completed } \\
\text { 3. Not completed } \\
\text { 4. Twin EA with EA } \longleftarrow \perp \rightarrow \text { LP6 }\end{array}$ & $\begin{array}{l}\text { 1. Respondent was travelling/not in location } \\
\text { 2. Respondent was too busy } \\
\text { 3. Respondent refused }\end{array}$ & $\begin{array}{ll}\text { a. } & \text { Observed (sup_obs) .......... } 1 \\
\text { b. } & \text { Checked (sup_edit)............ } 1 \\
\text { c. } & \text { Verified (sup_veri)............... } 1\end{array}$ & $\begin{array}{l}\text { No } \\
3 \\
3 \\
3\end{array}$ \\
\hline
\end{tabular}

INTERVIEWER NOTE: 


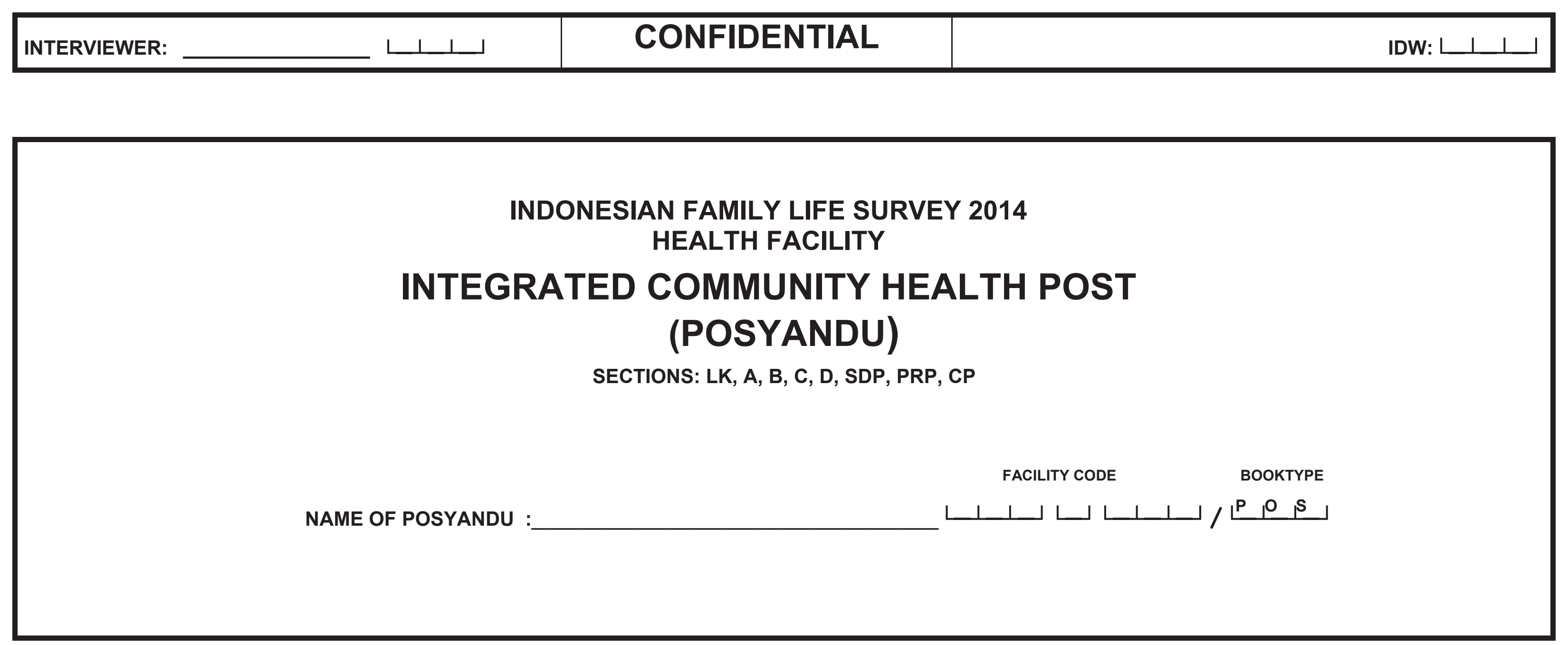




\section{SECTION LK: CONTROL SHEET}

\begin{tabular}{|c|c|c|}
\hline & SAMPLING INFORMATION & CODE \\
\hline LK01. & - & 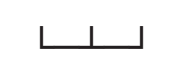 \\
\hline LK02. & Kabupaten/Kota & 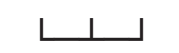 \\
\hline LK03. & Kecamatan & 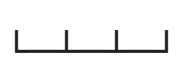 \\
\hline LK04. & Village/Urban Township/Nagari _ & 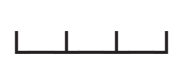 \\
\hline LK05. & Region: 1. Urban 2. Rural & 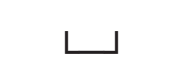 \\
\hline \multirow[t]{3}{*}{ LK08. } & \multicolumn{2}{|l|}{ a. Address: } \\
\hline & \multicolumn{2}{|c|}{ b. Description of location: } \\
\hline & 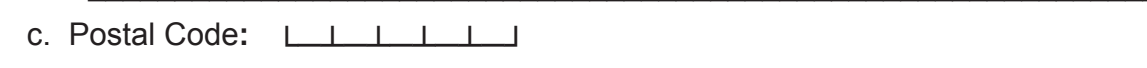 & \\
\hline \multirow[t]{4}{*}{ LK09. } & Phone number: & \\
\hline & 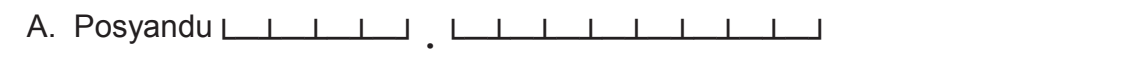 & \\
\hline & 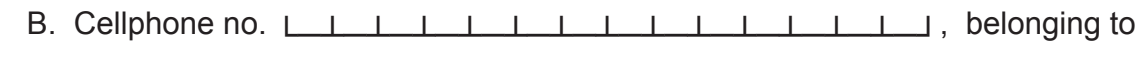 & - \\
\hline & W. NOT APPLICABLE Y. DON'T KNOW & \\
\hline LK09a & E-mail ; & \\
\hline \multirow[t]{2}{*}{ LK14. } & a. Lــ لــ & \\
\hline & b. & \\
\hline
\end{tabular}

\begin{tabular}{||c|c|}
\hline \multicolumn{1}{|c|}{ SUPERVISION } & CODE \\
\hline LK15. Name of Interviewer & \\
\hline LK17. Name of Local Supervisor & LK19. Name of Field Coordinator \\
\hline
\end{tabular}

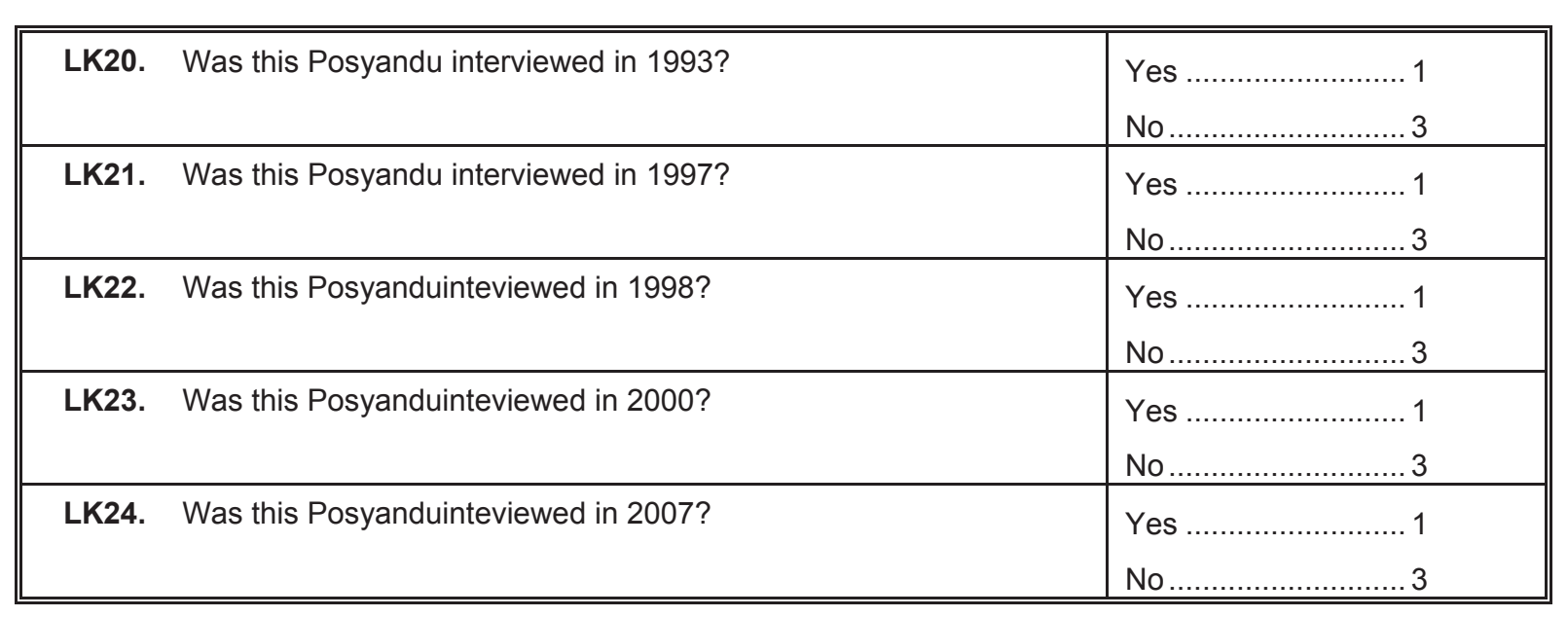




\section{SECTION A: GENERAL}

Section A Respondent

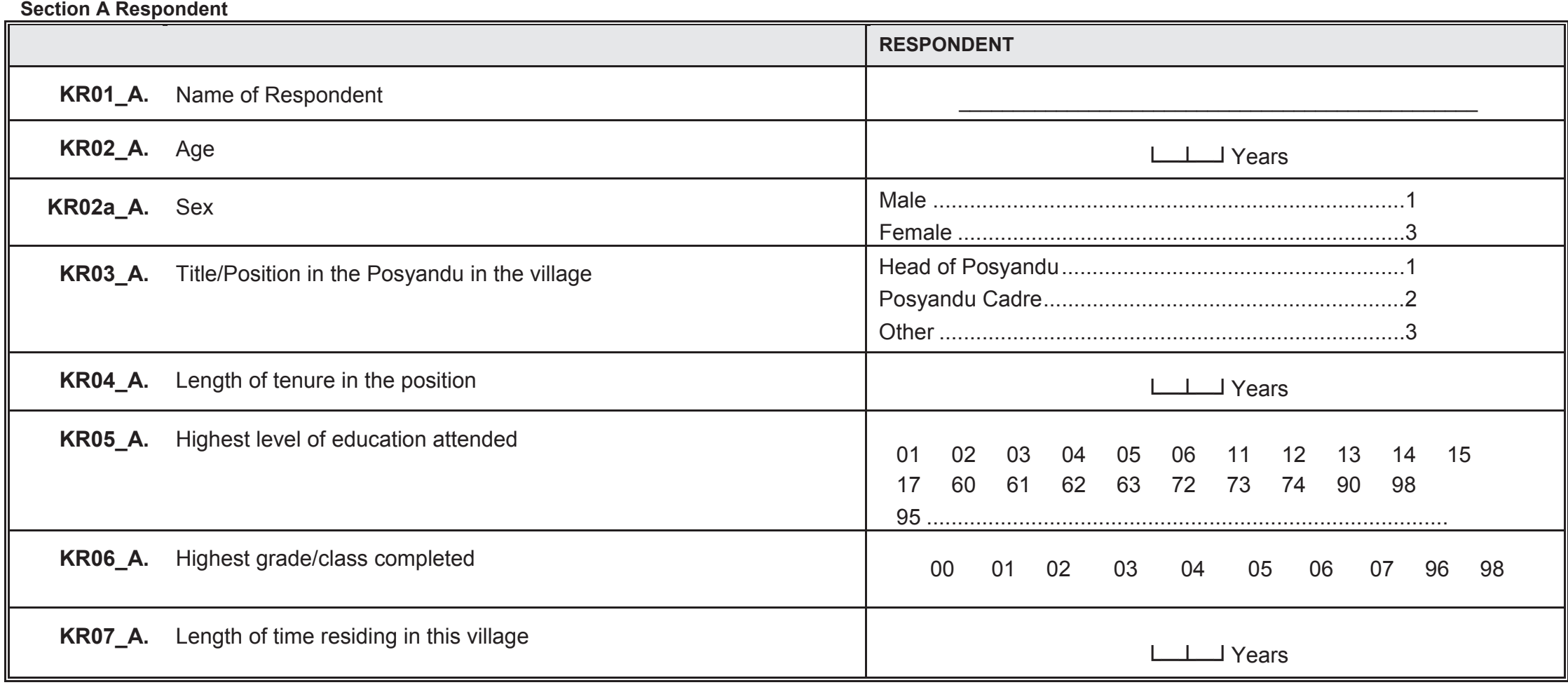

\section{Code KR05_A}

01. No school/Not yet at school

11. Adult Educ. A (KejarPaket A)

Code KR06_A

02. Elementary

00. Never completed class

3. Junior High - General

04. Junior High - Vocational

05. Senior High - General

13. Open University

01.1

14. Islamic School Pesantren

15. Adult Educ. C (KejarPaket C)

6enior High - Vocational

17. School for the disabled

01.1

02. 2

03. 3

D1, D2, D3 (Junior College)

72. Islamic Elementary School(Madrasah Ibtidaiyah)

04. 4

1. University $\mathrm{S} 1$ (Bachelors)

73. Islamic Junior High School (Madrasah Tsanawiya

2. University S2 (Masters)

74. Islamic Senior High School (Madrasah Aliyah)

06. 6

07. Graduated

90. Kindergarten

96. No school

98. DON'T KNOW

98. DON'T KNOW

$$
\text { 95. Other }
$$




\section{SECTION A: GENERAL}

Now we would to ask you about the service and activity of this Posyandu.

\begin{tabular}{|c|c|c|}
\hline A1. & $\begin{array}{l}\text { What year did this Health Post begin } \\
\text { operation? }\end{array}$ & 1. $\begin{array}{llllll} & \perp & \perp & \perp & \text { 8. TIDAK TAHU }\end{array}$ \\
\hline A2. & $\begin{array}{l}\text { Before this Health Post was established, } \\
\text { did this village have a Weighing Post, a } \\
\text { FP Post, or some other community health } \\
\text { post: }\end{array}$ & Yes \\
\hline A2a. & $\begin{array}{l}\text { Is this Posyandufunctioning as a } \\
\text { Posyandu for the Elderly? }\end{array}$ & $\begin{array}{l}\text { Yes } \\
\text { No }\end{array}$ \\
\hline A2b. & $\begin{array}{l}\text { Is there any Posyandufor the Elderly in } \\
\text { this village? }\end{array}$ & Yes \\
\hline A3a. & Is this Health Post open every month? & 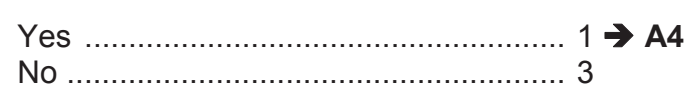 \\
\hline A3b. & $\begin{array}{l}\text { In the last year, how many times was the } \\
\text { Health Post in operation? }\end{array}$ & $\llcorner\perp$ times \\
\hline A4. & $\begin{array}{l}\text { When the Health Post is operating, on } \\
\text { average how many cadres are active? }\end{array}$ & $\llcorner\perp \neg$ cadres \\
\hline A4a. & $\begin{array}{l}\text { Is the PPKBD always present at } \\
\text { Posyandu activities? }\end{array}$ & 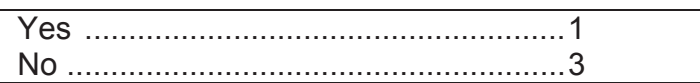 \\
\hline A4b. & $\begin{array}{l}\text { Which classification matches this Health } \\
\text { Post? }\end{array}$ & 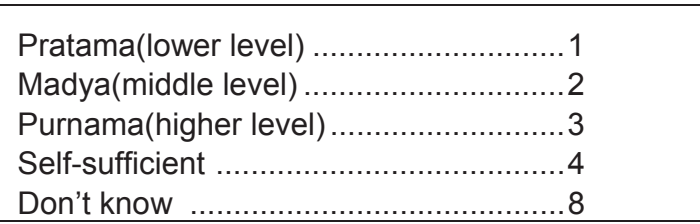 \\
\hline A6. & $\begin{array}{l}\text { What are the opening and closing hours } \\
\text { of this Health Post: }\end{array}$ & 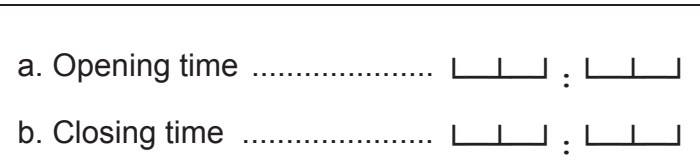 \\
\hline A7. & $\begin{array}{l}\text { When the Health Post is not open, and } \\
\text { someone needs OC pills or oralit, where } \\
\text { can they get them? } \\
\text { (CIRCLE ALL THAT APPLY) }\end{array}$ & $\begin{array}{l}\text { Public Health Center } \\
\text { Public Health Subcenter } \\
\text { Hospital } \\
\text { Clinic } \\
\text { Pharmacy } \\
\text { Private Practice } \\
\text { Cadre's Home } \\
\text { Village Staff } \\
\text { PKK Meeting }\end{array}$ \\
\hline
\end{tabular}

A8. When a mother wants to use the FP method [...], where is she referred to?
Public Health Center
Public Health Subcenter ......... 02
Hospital
Clinic
Private Practice
Not referred............................... 07
Other

A9. In general, where do mothers go for prenatal care in this village (note: past 12 months)?

(CIRCLE ALL THAT APPLY)

A10. In general where do mothers in this village give birth to their babies (note: past 12 months)?

(CIRCLE ALL THAT APPLY)

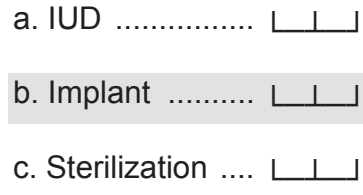

Government $\mathrm{Hosp}$

Private Hospita

Village Maternity Clinic.

Village Maternity Clinic

Clinic/Private Doctor...

Traditional Midwife's House

Health Post.......

Village Midwife . .

Other

Government Hospital.

Private Hospital ..

Maternity Hospital.

Health Center (Puskesmas)

Village Maternity Clinic (Polindes)

Clinic/Private Doctor

Clinic/Private Midwife

Traditional Midwife's House

Own home.......

Family's house.

Other......

\begin{tabular}{l} 
A \\
B \\
C \\
D \\
E \\
F \\
G \\
I \\
J \\
V \\
A \\
B \\
C \\
D \\
E \\
F \\
G \\
H \\
I \\
J \\
V \\
\hline
\end{tabular}




\section{SECTION A: GENERAL}

\begin{tabular}{|c|c|c|}
\hline A11. & $\begin{array}{l}\text { Usually, who assists the mothers in giving } \\
\text { birth to their babies (note: past } 12 \\
\text { months)? } \\
\text { (CIRCLE ALL THAT APPLY) }\end{array}$ & 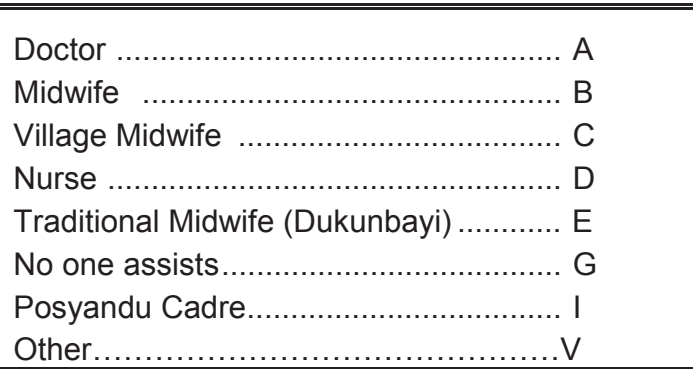 \\
\hline A11a. & $\begin{array}{l}\text { At this time how many traditional } \\
\text { midwives (Dukunbayi) assist birth in this } \\
\text { village? }\end{array}$ & 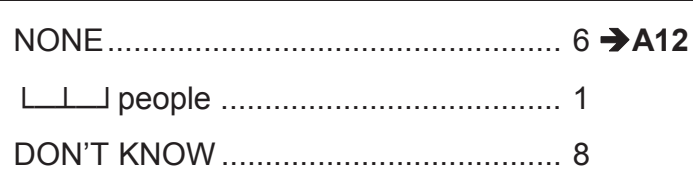 \\
\hline A11b. & $\begin{array}{l}\text { In the last year, what percent of women in } \\
\text { this village are assisted in childbirth by a } \\
\text { traditional midwife? }\end{array}$ & $\begin{array}{r}275 \% \\
50 \%-74 \% \\
25 \%-49 \% \\
25 \% \ldots 1\end{array}$ \\
\hline A12. & $\begin{array}{l}\text { Mention } 3 \text { (three) main problems faced by } \\
\text { this Health Post in the last two years: }\end{array}$ & $\begin{array}{l}\text { A. Lack of fund } \\
\text { B. Lack of medical supply } \\
\text { C. Lack of equipment } \\
\text { D. Lack of active cadres } \\
\text { E. Lack of support from puskesmas } \\
\text { F. Lack of support from village / township } \\
\text { G. No permanent place } \\
\text { H. Lack of interest/ participation } \\
\text { I. Lack of KIA training for cadres } \\
\text { V. Others................................. } \\
\text { W. NO PROBLEM }\end{array}$ \\
\hline A13. & $\begin{array}{l}\text { Mention } 3 \text { (three) main problems that you } \\
\text { faced in mother and child health, in the } \\
\text { last two years: }\end{array}$ & $\begin{array}{l}\text { A. Lack of fund } \\
\text { B. Lack of medical supply } \\
\text { C. Lack of equipment } \\
\text { D. Lack of active cadres } \\
\text { E. Lack of support from puskesmas } \\
\text { F. Lack of support from village / township } \\
\text { G. No permanent place } \\
\text { H. Lack of interest/ participation } \\
\text { I. Lack of KIA training for cadres } \\
\text { V. Others ............................................... } \\
\text { W. NO PROBLEM }\end{array}$ \\
\hline
\end{tabular}




\section{SECTION B : SERVICES AT THE POSYANDU}

Section B Respondent

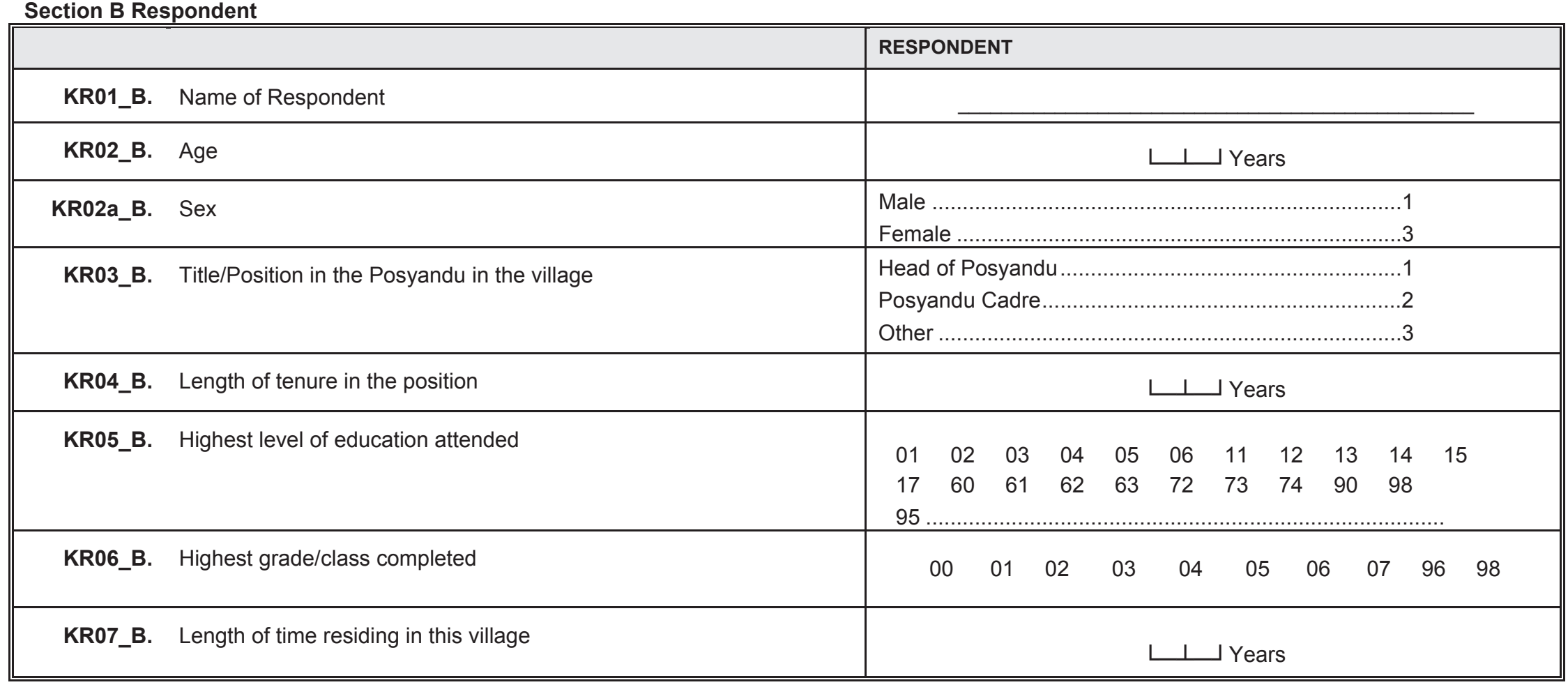

\section{Code KR05_B}

01. No school/Not yet at school

11. Adult Educ. A (KejarPaket A)

Code KR06_B

2. Elementary

Educ. A (KejarPaket A)

00. Never completed class I

03. Junior High - General

04. Junior High - Vocational

55. Senior High - General

12. Adult Educ. B (KejarPaket B)

14. Open University

15. Adult Educ C (Kejantren

17. Adult Educ. C (KejarPaket

. Senior High - Vocational

17. School for the disabled

2. Islamic Elementary School(Madrasah Ibtidaiyah)

73. Islamic Junior High School (Madrasah Tsanawiyah)

61. University S1 (Bachelors)

74. Islamic Senior High School (Madrasah Aliyah)

90. Kindergarten

95. Other

01. 1

02. 2

03. 3

04. 4

05. 5

06. 6

07. Graduated

96. No school

98. DON'T KNOW

63. University S3 (Doctorate)

$$
\text { 5. Other }
$$




\section{SECTION B : SERVICES AT THE POSYANDU}

Now, we would like to ask you about the services available in this Posyandu.

\begin{tabular}{|c|c|c|}
\hline B0a. & How much is the charge for each Posyandu visit? & $\sqcup, \sqcup \sqcup, \quad$ Rp \\
\hline Bob. & In comparison with last year, how much has the service charge changed? & $\begin{array}{l}\text { 1. Increase } \\
\text { 2. No change } \\
\text { 3. Decrease }\end{array}$ \\
\hline
\end{tabular}

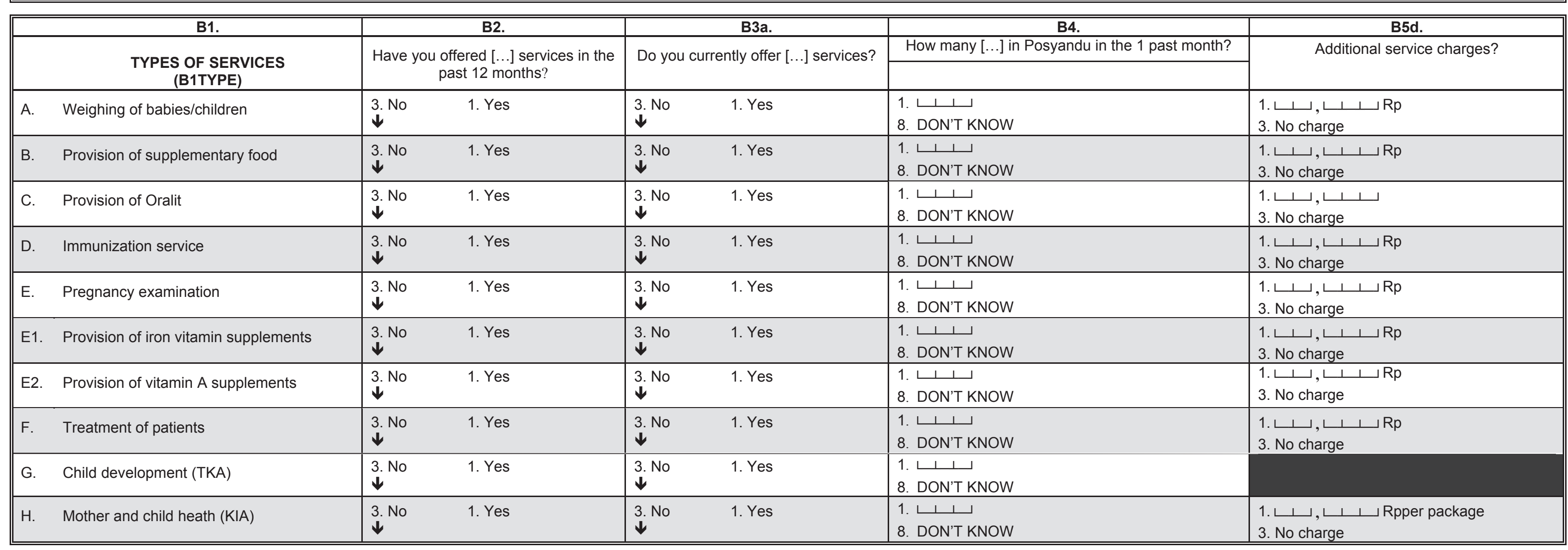




\section{SECTION B : SERVICES AT THE POSYANDU}

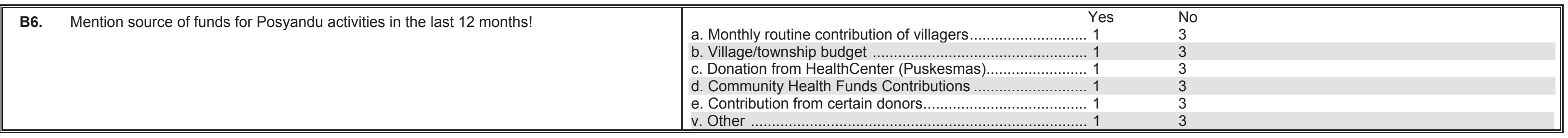

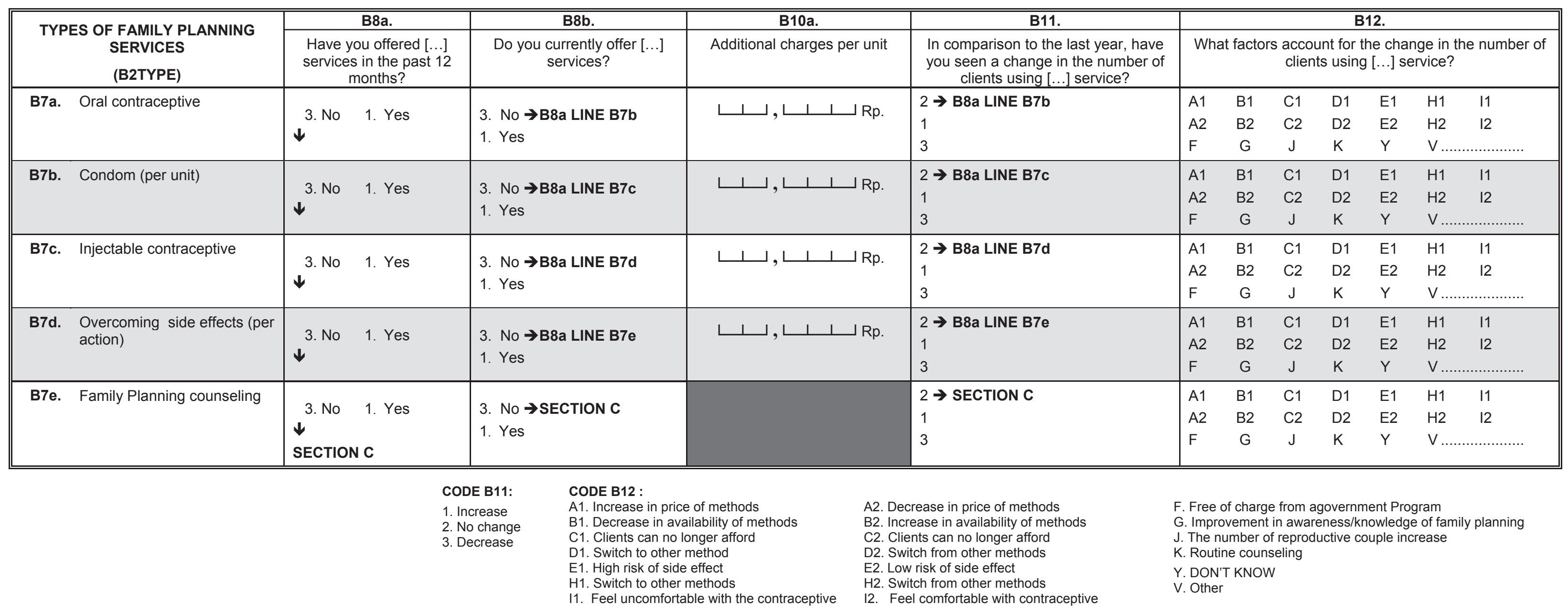




\section{SECTION C: POSYANDU MANPOWER}

Section C Respondent

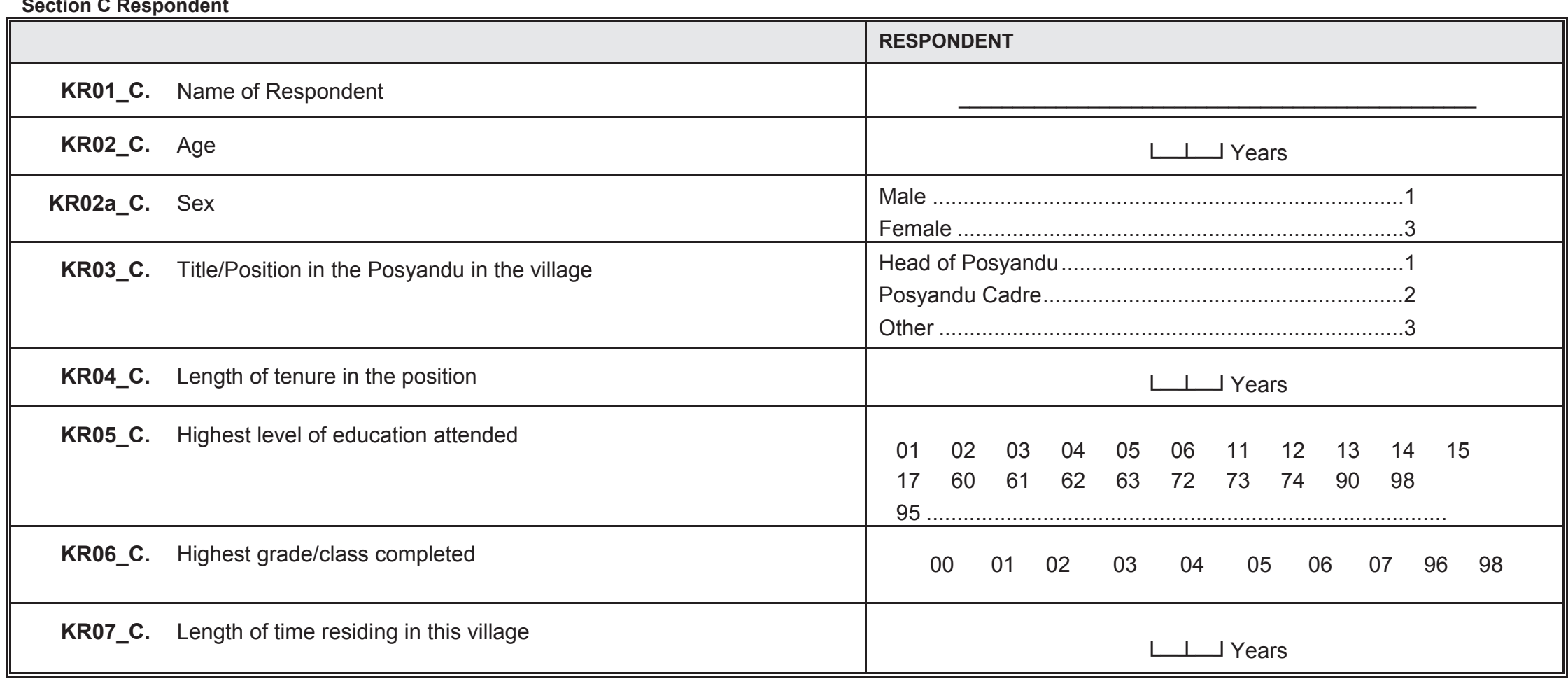

\section{Code KR05_C}

01. No school/Not yet at school

02. Elementary

03. Junior High - General

04. Junior High - Vocational

05. Senior High - General

06. Senior High - Vocational

60. D1, D2, D3 (Junior College)

61. University S1 (Bachelors)

62. University S2 (Masters)

63. University S3 (Doctorate)

11. Adult Educ. A (KejarPaket A)

12. Adult Educ. $B$ (KejarPaket $B$ )

13. Open University

14. Islamic School Pesantren

15. Adult Educ. C (KejarPaket C)

17. School for the disabled

72. Islamic Elementary School(Madrasah Ibtidaiyah)

73. Islamic Junior High School (Madrasah Tsanawiyah)

74. Islamic Senior High School (Madrasah Aliyah)

90. Kindergarten

98. DON'T KNOW

95. Other
Code KR06_C

0. Never completed class

01. 1

02.2

03. 3

04. 4

05. 5

05. 5

07. Graduated

96. No school

98. DON'T KNOW 
SECTION C: POSYANDU MANPOWER

Now, we would like to know about the cadre working for this Posyandu.

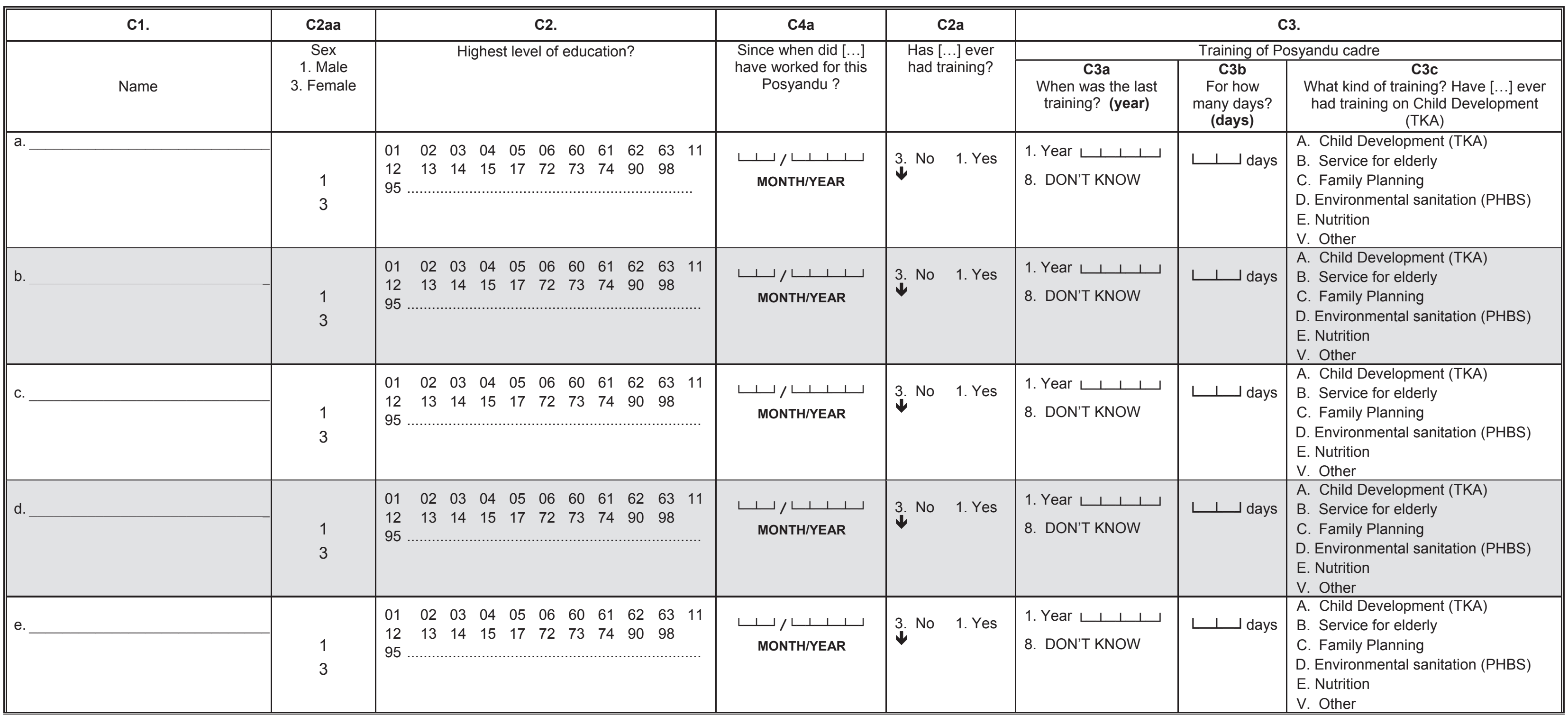




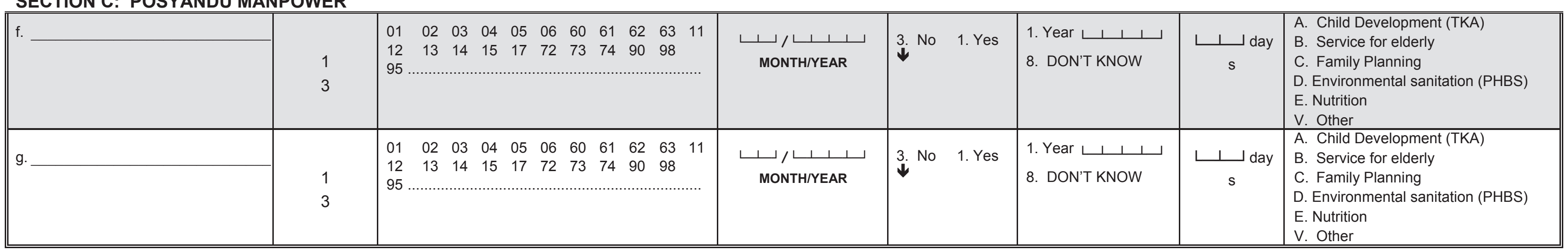

Code C2:

01. No school/Not yet

03. Junior High - Genera

04. Junior High - Vocationa

05. Senior High - General
06. Senior High - Vocational

61. University ( 1 (Bnior College)

62. University S2 (Masters)

63. University S3 (Doctorate)
11. Adult Educ. A (KejarPaket A)

13. Open University

14. Islamic School Pesantren

15. Adult Educ. C (KejarPaket C)
17. School for disable person

72. Islamic Elementary (Madrasah Ibtidaiyah)

7. Islamic Junior High School (Madrasah Tsanawiyah)

90. Kindergarten

95. Other 


\section{SECTION C: POSYANDU MANPOWER}

\begin{tabular}{|c|c|c|}
\hline C4a1. & $\begin{array}{l}\text { In the last } 12 \text { months, did a PLKB staff } \\
\text { visit this Posyandu? }\end{array}$ & 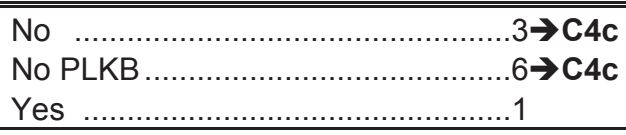 \\
\hline C4b1. & $\begin{array}{l}\text { How many times in the last } 12 \text { months did } \\
\text { a PLKB staff visit this Posyandu? }\end{array}$ & 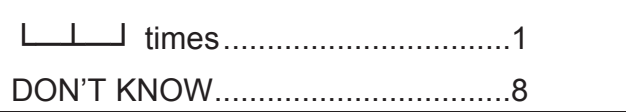 \\
\hline C4b3. & $\begin{array}{l}\text { Relative to previous years since } 2007 \text {, has } \\
\text { there been a change in the visits from } \\
\text { PLKB changed? }\end{array}$ & 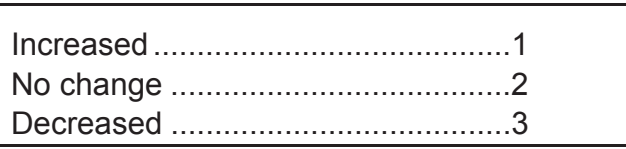 \\
\hline C4c. & $\begin{array}{l}\text { Is there a BinaKeluargaBalita (Child } \\
\text { Development) program at this Posyandu? }\end{array}$ & 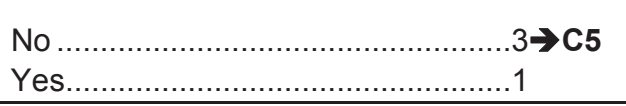 \\
\hline C4d. & $\begin{array}{l}\text { Who implements the BinaKeluargaBalita } \\
\text { program? } \\
\text { (CIRCLE ALL THAT APPLY) }\end{array}$ & 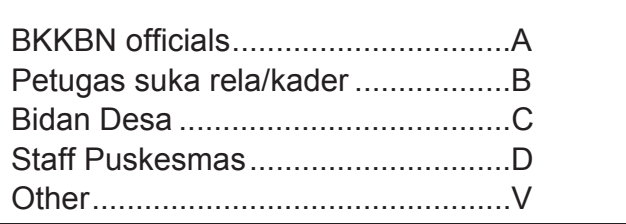 \\
\hline C4e. & $\begin{array}{l}\text { How many times in the last year was the } \\
\text { BinaKeluargaBalita program conducted at } \\
\text { this Posyandu? }\end{array}$ & 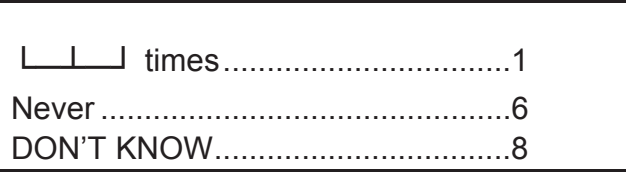 \\
\hline
\end{tabular}

\begin{tabular}{|c|c|c|c|}
\hline \multicolumn{2}{|c|}{$\begin{array}{l}\text { C5. How many times in the last year has } \\
\text { this Posyandu been visited by staff from } \\
\text { the HealthCenter (Puskesmas)? }\end{array}$} & \multicolumn{2}{|c|}{ 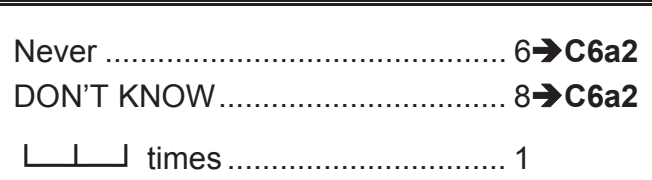 } \\
\hline C6. & Who usually comes? & $\begin{array}{l}\text { Yes } \\
\text { a. Nurse } \ldots \ldots \ldots \ldots \\
\text { b. Midwife }\end{array}$ & $\begin{array}{r}\text { No } \\
3 \\
3 \\
3 \\
3 \\
3\end{array}$ \\
\hline C6a2. & $\begin{array}{l}\text { Relative to previous years since } 2007 \text {, } \\
\text { has there been a change in visits to this } \\
\text { Posyandu from Puskesmas? }\end{array}$ & $\begin{array}{l}\text { Increase } \\
\text { No change }\end{array}$ & \\
\hline
\end{tabular}

\begin{tabular}{|c|c|c|c|c|}
\hline \multirow{3}{*}{$\begin{array}{l}\text { Category of Activities } \\
\text { a. Information on immunization }\end{array}$} & \multirow{2}{*}{\multicolumn{2}{|c|}{$\begin{array}{c}\text { C7. } \\
\text { Are there other visits from HealthCenter personnel outside } \\
\text { Posyandu opening days, for the following activities: }\end{array}$}} & \multirow{2}{*}{\multicolumn{2}{|c|}{$\begin{array}{c}\text { C8. } \\
\text { How many visits in the last year? }\end{array}$}} \\
\hline & & & & \\
\hline & $\begin{array}{l}\text { 3. No } \\
\Downarrow\end{array}$ & 1. Yes & 1. $\downarrow \_\_$t t t $ـ$ times 6 . Never & 8. DON'T KNOW \\
\hline b. Information on KB & 3. No & 1. Yes & 1. $\downarrow \_\_$ل times 6 . Never & 8. DON'T KNOW \\
\hline c. Mobile Medical Team (TMK) & $\begin{array}{l}\text { 3. No } \\
\downarrow\end{array}$ & 1. Yes & 1. $\downarrow \_\_$times 6 . Never & 8. DON'T KNOW \\
\hline d. Sanitation Program & 3. No & 1. Yes & 1. $ـ ل$ لـ times 6. Never & 8. DON'T KNOW \\
\hline e. Information on Mother and Child Health (KIA) & $\begin{array}{l}\text { 3. No } \\
\downarrow\end{array}$ & 1. Yes & 1. $\downarrow \_\_$times 6 . Never & 8. DON'T KNOW \\
\hline f. Services for Posyandu for the elderly & $\begin{array}{l}\text { 3. No } \\
\downarrow \\
\text { SECTI }\end{array}$ & 1. Yes & 1. $\downarrow \_\_$times 6 . Never & 8. DON'T KNOW \\
\hline
\end{tabular}




\section{SECTION D : HEALTH INSTRUMENTS}

Section D Respondent

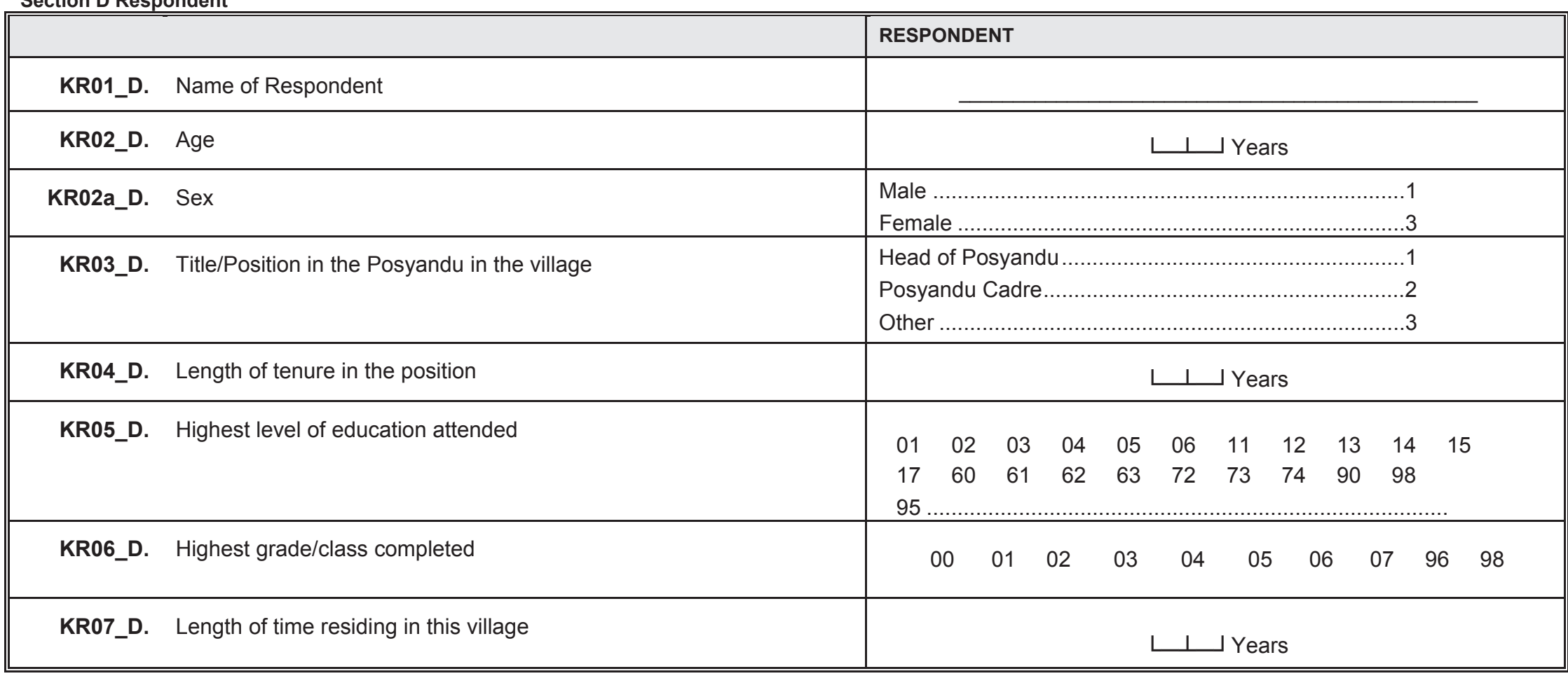

Code KR05_D

01. No school/Not yet at school

02. Elementary

03. Junior High - General

04. Junior High - Vocational

05. Senior High - General

06. Senior High - Vocational

60. D1, D2, D3 (Junior College)

61. University S1 (Bachelors)

62. University S2 (Masters)

63. University S3 (Doctorate)
Code KR06_D

00. Never completed class I

01. 1

02. 2

03. 3

04. 4

05. 5

06. 6

07. Graduated

96. No school

98. DON'T KNOW 


\section{SECTION D: HEALTH INSTRUMENTS}

Kindly give information on health instruments at this Posyandu.

\begin{tabular}{|c|c|c|c|c|c|c|}
\hline $\begin{array}{cc} & \text { D1. } \\
\end{array}$ & \multicolumn{2}{|c|}{ D2. } & D3. & \multicolumn{3}{|c|}{$\begin{array}{lr}\text { D6. } \\
\end{array}$} \\
\hline KINDS OF INSTRUMENTS & Are the $[.$. & uments here? & $\begin{array}{c}\text { How many are there? } \\
\text { (Existing stock at Posyandu) }\end{array}$ & In the last 6 & nths for how many $w$ & has $[\ldots]$ been out of stock? \\
\hline a. Baby scales & 1. Yes & 3. No & & & & \\
\hline b. Height measuring device & 1. Yes & 3. No & & & & \\
\hline c. KMS cards & 3. No $\rightarrow \mathrm{D} 6$ & 1. Yes & 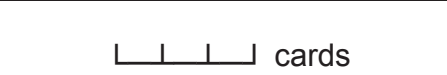 & 1. $\llcorner\perp$ weeks & 6. Never available & 8. DON'T KNOW \\
\hline d. Pregnant Mother cards & 3. No $\rightarrow \mathrm{D} 6$ & 1. Yes & $\llcorner\perp \perp$ cards & 1. $\longleftarrow$ لـ weeks & 6. Never available & 8. DON'T KNOW \\
\hline e. Oral contraceptive pills & 3. No $\rightarrow \mathrm{D} 6$ & 1. Yes & ــــ pill strip & 1. $\llcorner\perp$ weeks & 6. Never available & 8. DON'T KNOW \\
\hline g. Demonstration tools/books & 1. Yes $\rightarrow \mathbf{D} 6$ & 3. No $\downarrow$ & & 1. $\downarrow$ ل weeks & 6. Never available & 8. DON'T KNOW \\
\hline h. Oralit & 3. No $\rightarrow \mathrm{D} 6$ & 1. Yes & ـــــ packages & 1. $\llcorner\perp$ weeks & 6. Never available & 8. DON'T KNOW \\
\hline i. Iron tablets / Sulfas Ferosus & 3. No $\rightarrow \mathrm{D} 6$ & 1. Yes & $\sqcup \perp \perp \_$tablets & 1. $\llcorner\perp$ weeks & 6. Never available & 8. DON'T KNOW \\
\hline j. Vitamin A & 3. No $\rightarrow \mathrm{D} 6$ & 1. Yes & $\perp \perp \_$t tablets & 1. $\llcorner\perp$ weeks & 6. Never available & 8. DON'T KNOW \\
\hline k. Paracetamol/Other lowering fever medicine & 3. No $\rightarrow \mathrm{D} 6$ & 1. Yes & $\downarrow \downarrow \perp \_$tablets & 1. $\longleftarrow$ لـ weeks & 6. Never available & 8. DON'T KNOW \\
\hline I. Children's toys & 1. Yes & 3. No & & & & \\
\hline m. Instruction book for the BinaKeluargaBalita program & 1. Yes & 3. No & & & & \\
\hline n. Adult scale & 1. Yes & 3. No & & & & \\
\hline o. Tensimeter & 1. Yes & 3. No & & & & \\
\hline p. Osteoporosis kit & 3. No $\downarrow$ & 1. Yes & 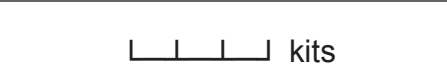 & & & \\
\hline q. Cholesterol kit & 3. No $\downarrow$ & 1. Yes & $\llcorner\perp \perp$ kits & & & \\
\hline r. Stethoscope & 1. Yes & 3. No & & & & \\
\hline s. Thermometer & 1. Yes $\rightarrow \mathrm{D} 6$ & 3. No & $\left\llcorner\perp \perp \_\right.$kits & 1. ــل weeks & 6. Never available & 8. DON'T KNOW \\
\hline
\end{tabular}




\section{SECTION SDP : POSYANDU RESOURCES}

Section SDP Respondent

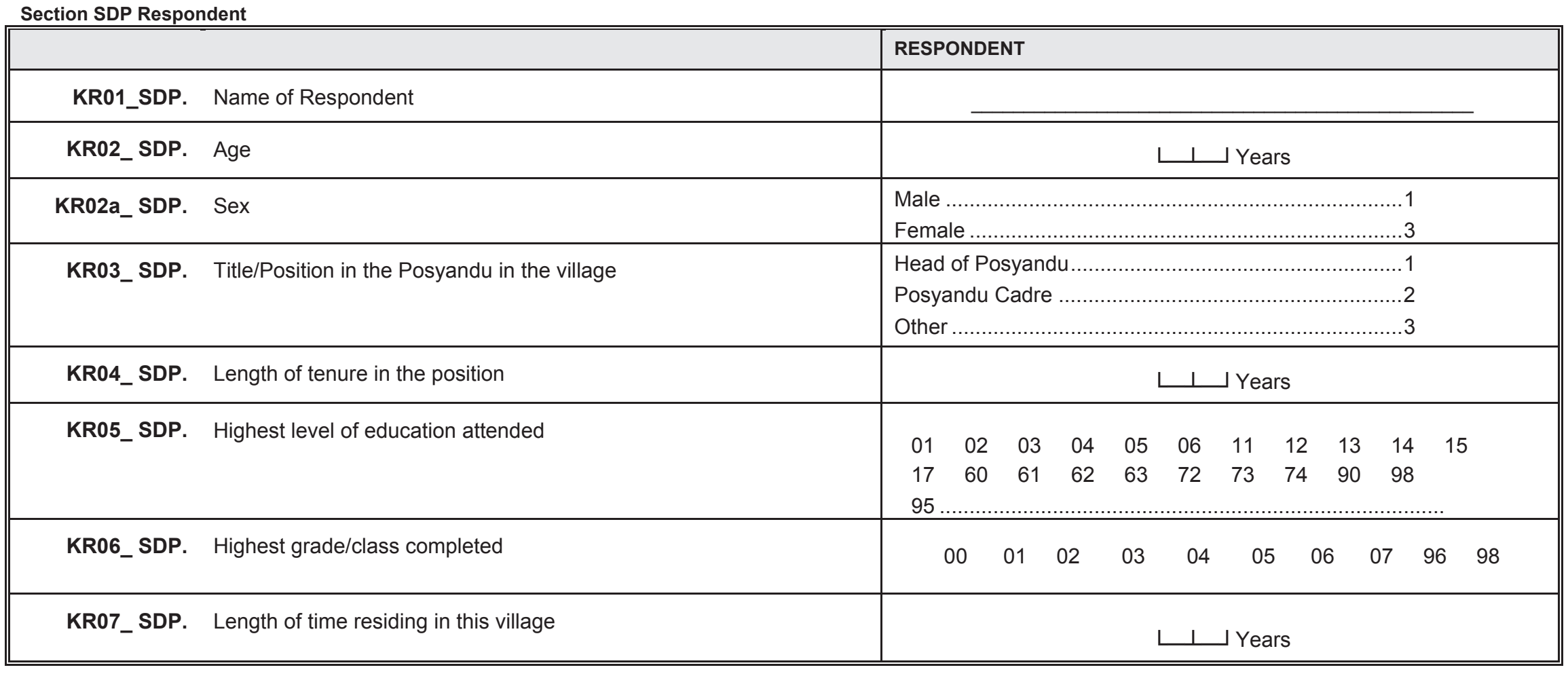

\section{Code KR05_SDP}

01. No school/Not yet at school

02. Elementary

03. Junior High - General

04. Junior High - Vocational

05. Senior High - General

06. Senior High - Vocational

60. D1, D2, D3 (Junior College)

61. University S1 (Bache

62. University S2 (Masters)

63. University S3 (Doctorate)

\section{Code KR06_SDP}

0. Never completed class I

01. 1

02.2

03. 3

04. 4

05. 5

06.6

07. Graduated

96. No school

98. DON'T KNOW 


\section{SECTION SDP : POSYANDU RESOURCES}

Now, we would like to ask resources of this Posyandu.

\begin{tabular}{|c|c|c|}
\hline SDP00. & $\begin{array}{l}\text { INTERVIEWER CHECK A2a=1 (IS THIS POSYANDU IS FUNCTIONING AS ELDERLY } \\
\text { POSYANDU?) }\end{array}$ & 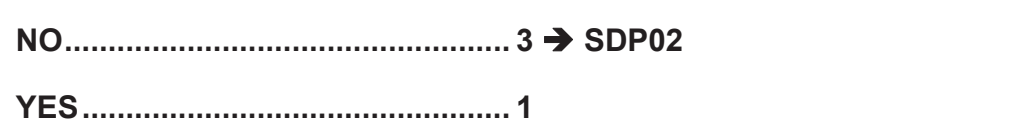 \\
\hline SDP00a. & Can you separate the source of resource for Posyandu and Elderly Posyandu? & $\begin{array}{l}\text { 1. IF YES, SDP is only for POSYANDU } \\
\text { 3. IF NO, SDP is both for POSYANDU AND ELDERLY POSYANDU }\end{array}$ \\
\hline \multirow[t]{4}{*}{ SDP02. } & $\begin{array}{l}\text { What is the value per month of }[\ldots] \text { provided by the community for this Posyandu? } \\
\text { a. Cash }\end{array}$ & 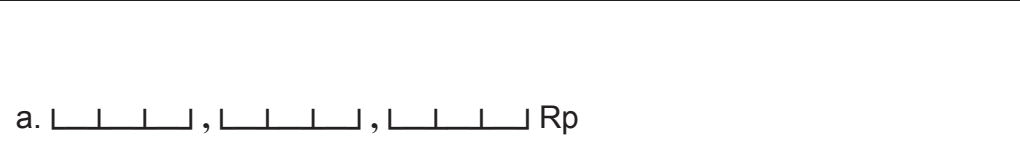 \\
\hline & b. Food & 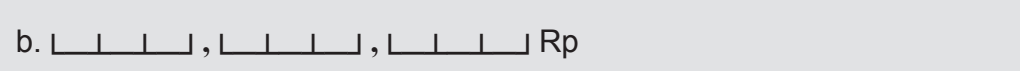 \\
\hline & c. Time spent by volunteers for Posyandu & 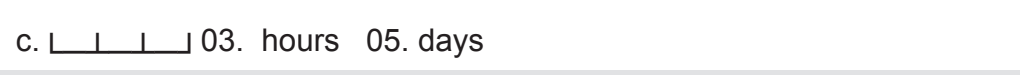 \\
\hline & 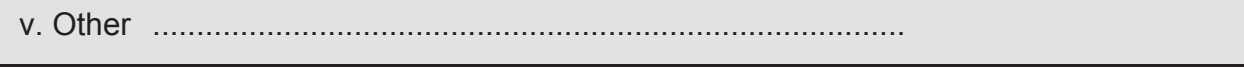 & 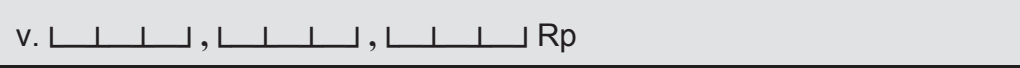 \\
\hline SDP03a. & $\begin{array}{l}\text { In the last } 12 \text { months, has this Posyandu received any resources for preventing } \\
\text { undernourished? }\end{array}$ & 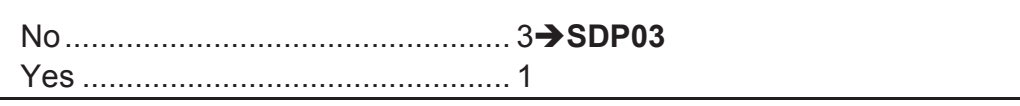 \\
\hline SDP03b. & How many times has this Posyandu receivedresources? & $\sqcup$ times \\
\hline \multirow[t]{4}{*}{ SDP03d. } & $\begin{array}{l}\text { What is the value of }[\ldots] \text { received by Posyandu? } \\
\text { a. Cash }\end{array}$ & 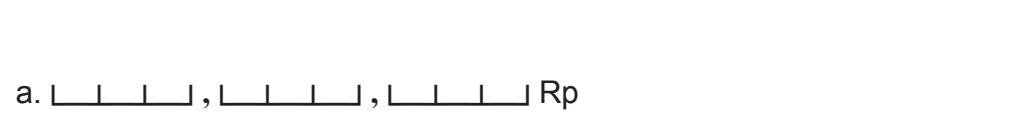 \\
\hline & b. Food & 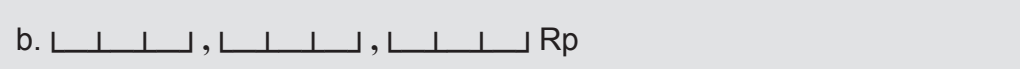 \\
\hline & c. Time spent by volunteers for Posyandu & c. $\longleftarrow \perp \perp \_$ل $1 ـ 3$. hours 05. days \\
\hline & 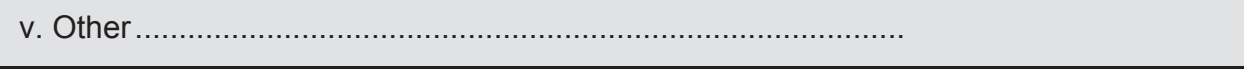 & 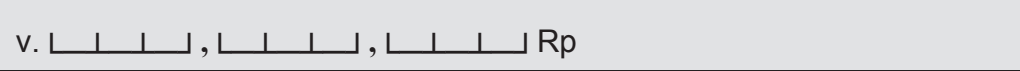 \\
\hline SDP03. & Does this Posyandu receive resources from other parties? & 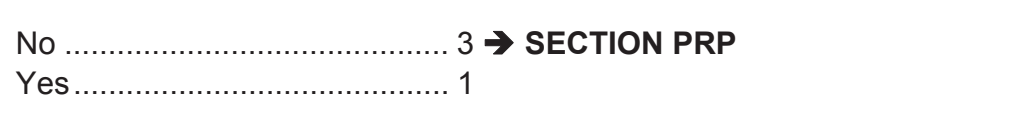 \\
\hline
\end{tabular}




\section{SECTION SDP : POSYANDU RESOURCES}

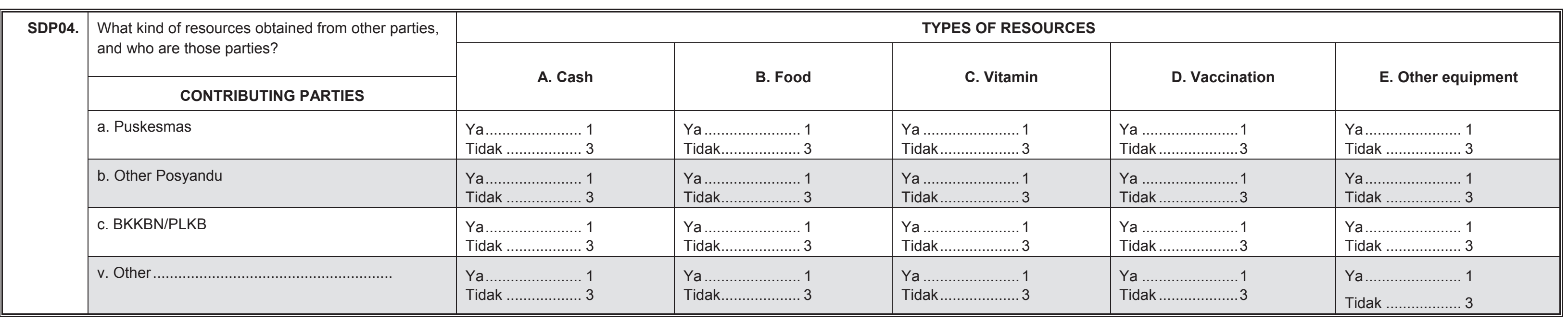




\section{SECTION PRP: POSYANDU REVITALIZATION PROGRAM}

Section PRP Respondent

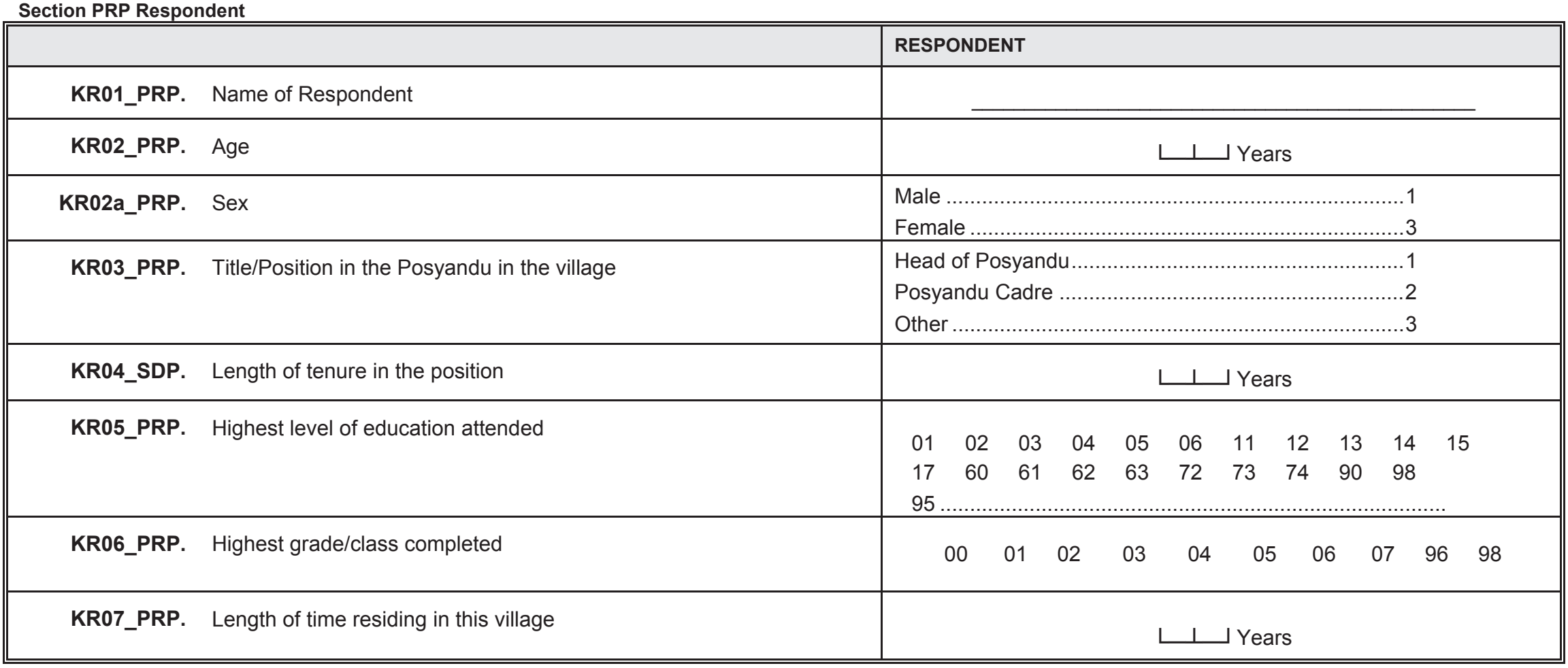

\section{Code KR05 PRP}

01. No

02. Elementary

03. Junior High - General

04. Junior High - Vocational

05. Senior High - General

06. Senior High - Vocational

60. D1, D2, D3 (Junior College)

61. University S1 (Bachelors)

11. Adult Educ. A (KejarPaket A)

Code KR06_PRP

2. University S2 (Masters)

12. Adult Educ. B (KejarPaket $B$ )

13. Open University

. Never completed class

15. Islamic School Pesantren

15. Adult Educ. C (KejarPaket C)

17. School for the disabled

72. Islamic Elementary School(Madrasah Ibtidaiyah)

73. Islamic Junior High School (Madrasah Tsanawiyah)

63. University S3 (Doctorate)

74. Islamic Senior High School (Madrasah Aliyah)

01.1

02. 2

03. 3

04. 4

05. 5

06. 6

07. Graduated

96. No school

98. DON'T KNOW

90. Kindergarten

95. Other 


\section{SECTION PRP: POSYANDU REVITALIZATION PROGRAM}

Now, we would like to ask about the Posyandu Revitalization Program since 2007.

PRP00. Have you ever heard Posyandu revitalization program?

PRP01a. Has there been a Posyandu revitalization program since 2007?

PRP01b. When is the last time this Posyandu received revitalization program?

CAPI CHECK: IF BEFORE 2012 SKIP TO CK1 (LANGMAIN)

\begin{tabular}{|c|}
\hline 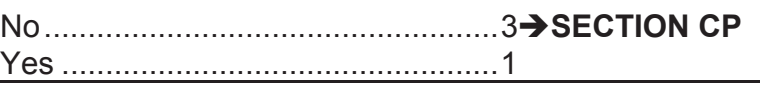 \\
\hline 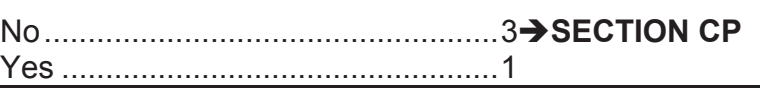 \\
\hline
\end{tabular}

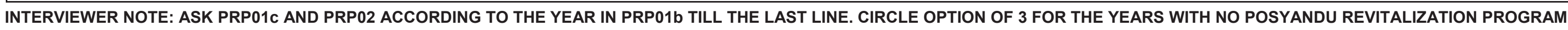

Now we would like to ask about the Posyandu Revitalization Program since 2012.

\begin{tabular}{|c|c|c|c|c|}
\hline \multirow{2}{*}{$\begin{array}{c}\text { YEAR } \\
\text { (PRPTYPE) }\end{array}$} & \multicolumn{2}{|c|}{ PRP01c. } & \multicolumn{2}{|r|}{ PRP02. } \\
\hline & Was there PRP program in $[\ldots]$ ? & & How much money did the Posyandu & revitalization program in $[\ldots]$ ? \\
\hline A. 2014 & 3. No $\downarrow$ & 1.Yes & 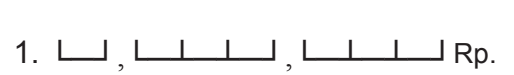 & 8. DON'T KNOW $\downarrow$ \\
\hline B. 2013 & 3. No $\downarrow$ & 1.Yes & 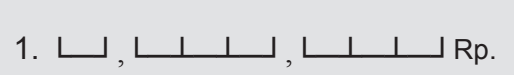 & 8. DON'T KNOW $\downarrow$ \\
\hline C. 2012 & 3. No $\downarrow$ & 1.Yes & 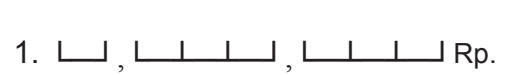 & 8. DON'T KNOW $\downarrow$ \\
\hline
\end{tabular}

PRP03a. Has the money received used for $[\ldots]$ :

. Introduce Education and Information Counseling (KIE)

1. Yes 3.No

to community leader/head of village or township

b. Train cadres

$1+\ldots .1-3$

d. Purchase other supplies for Posyandu

e. Give transport money to cadres

f. Supplementary food distribution program (PMT) 


\section{SECTION CP: INTERVIEWER NOTES}

\begin{tabular}{|c|c|c|c|c|c|c|c|}
\hline LANGMAIN (CK1). & \multicolumn{2}{|l|}{ Interview was entirely/mostly conducted in what language? } & \multicolumn{5}{|l|}{$\downarrow \downarrow$ other } \\
\hline LANGOTHR (CK2). & Other languaged used (if any): & & $\downarrow \downarrow$ ـ other & & & & \\
\hline \multicolumn{8}{|l|}{ LANGUAGE CODE: } \\
\hline 00. Bahasa Indonesia & 04. Batak & Sasak & 12. Makassar & & Toraja & & Lampung \\
\hline 01. Java & 05. Bugis & Minang & 13. Nias & 17. & Lahat & 95. & Other, \\
\hline 02. Sunda & 06. Cina & 10. Banjar & 14. Palembang & 18. & Other South Sumatera & 96. & NA \\
\hline 03. Bali & 07. Madura & 11. Bima & 15. Sumbawa & 19. & Betawi & & \\
\hline
\end{tabular}

\begin{tabular}{|c|c|c|c|}
\hline RESULT (FP3). RESULTS OF INTERVIEW & $\begin{array}{l}\text { REASON (FP4). REASON FOR ANSWERING “2” I } \\
\text { "3" IN RESULT. }\end{array}$ & FP6. MONITORING BY SUPERVISOR & \\
\hline $\begin{array}{l}\text { 1. Completed } \rightarrow \text { FP6 } \\
\text { 2. Partly completed } \\
\text { 3. Not completed } \\
\text { 4. Twin EA with EA } ـ \perp \rightarrow \text { FP6 }\end{array}$ & $\begin{array}{l}\text { 1. Respondent was travelling/not in location } \\
\text { 2. Respondent was too busy } \\
\text { 3. Respondent refused }\end{array}$ & $\begin{array}{ll}\text { a. } & \text { Observed (sup_obs) .......... } 1 \\
\text { b. } & \text { Checked (sup_edit)........... } 1 \\
\text { c. } & \text { Verified (sup_veri).............. } 1\end{array}$ & $\begin{array}{l}\text { No } \\
3 \\
3 \\
3\end{array}$ \\
\hline
\end{tabular}

\section{INTERVIEWER NOTE:}




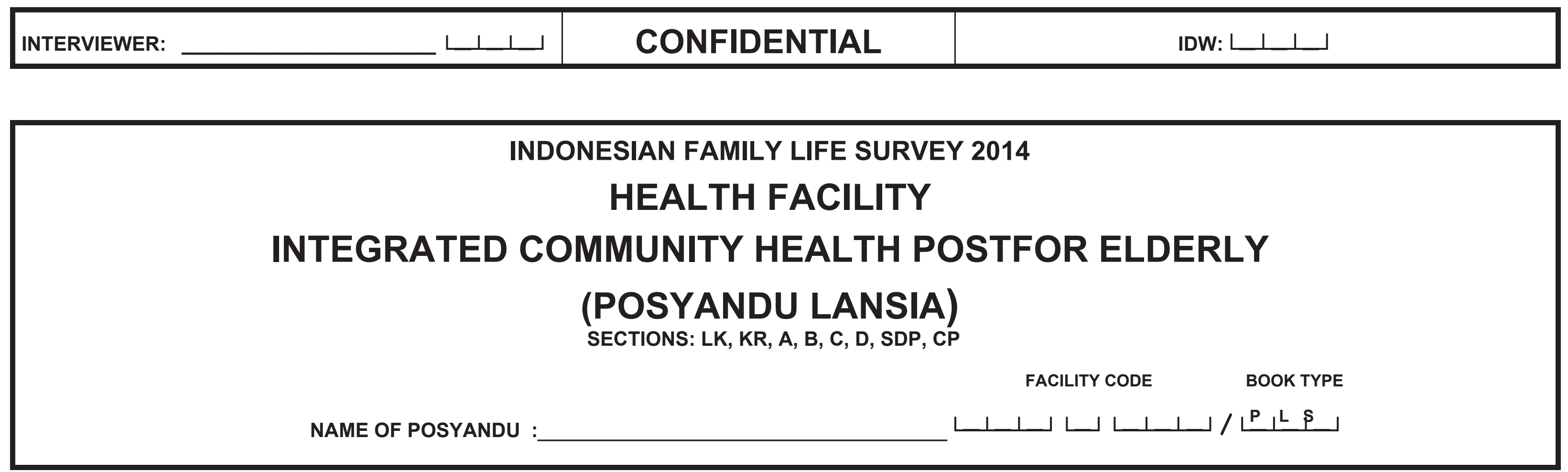




\section{SECTION LK: CONTROL SHEET}

\begin{tabular}{|c|c|c|}
\hline \multicolumn{2}{|r|}{ SAMPLING INFORMATION } & CODE \\
\hline LK01. & Province & $\downarrow$ \\
\hline LK02. & Kabupaten/Kotamadya __ & $\downarrow$ \\
\hline LK03. & Kecamatan & 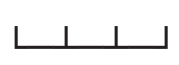 \\
\hline LK04. & Village/Urban Township/Nagari_ & 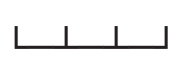 \\
\hline LK05. & Region: 1. Urban 2. Rural & $\sqcup$ \\
\hline \multirow[t]{3}{*}{ LK08. } & \multicolumn{2}{|l|}{ a. Address: } \\
\hline & \multicolumn{2}{|l|}{ b. Description of location: } \\
\hline & 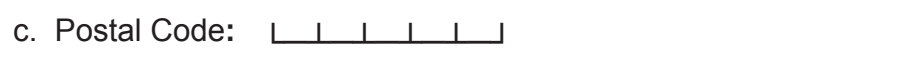 & \\
\hline \multirow[t]{4}{*}{ LK09. } & \multirow{2}{*}{\multicolumn{2}{|c|}{ 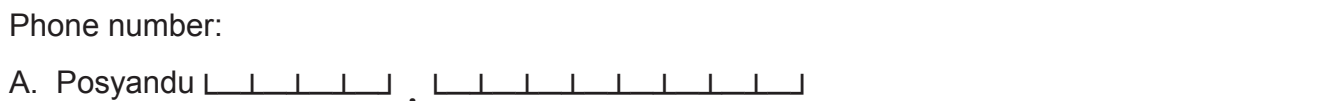 }} \\
\hline & & \\
\hline & \multicolumn{2}{|c|}{ 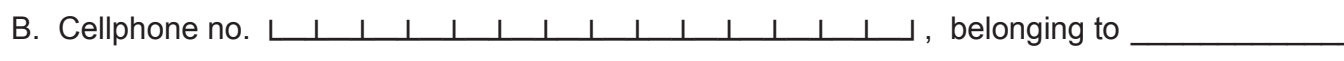 } \\
\hline & \multicolumn{2}{|c|}{ W. NOT APPLICABLE $\quad$ Y. DON'T KNOW } \\
\hline LK09a & \multicolumn{2}{|l|}{ E-mail: } \\
\hline \multirow[t]{2}{*}{ LK14. } & \multicolumn{2}{|l|}{ a. $\llcorner\perp$} \\
\hline & \multicolumn{2}{|l|}{ 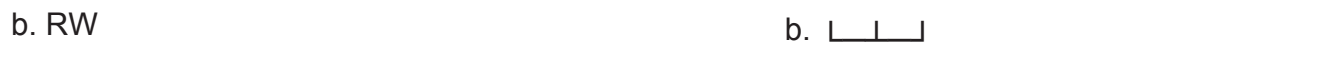 } \\
\hline
\end{tabular}

\begin{tabular}{||c|c||}
\hline \multicolumn{1}{|c|}{ SUPERVISION } & CODE \\
\hline LK15. Name of Interviewer _ & \\
\hline LK17. Name of Local Supervisor & Yes................... \\
\hline LK19. Name of Field Coordinator & No ...................... \\
\hline LK20. Was this Posyandu interviewed in 2007?
\end{tabular}




\section{SECTION A: GENERAL}

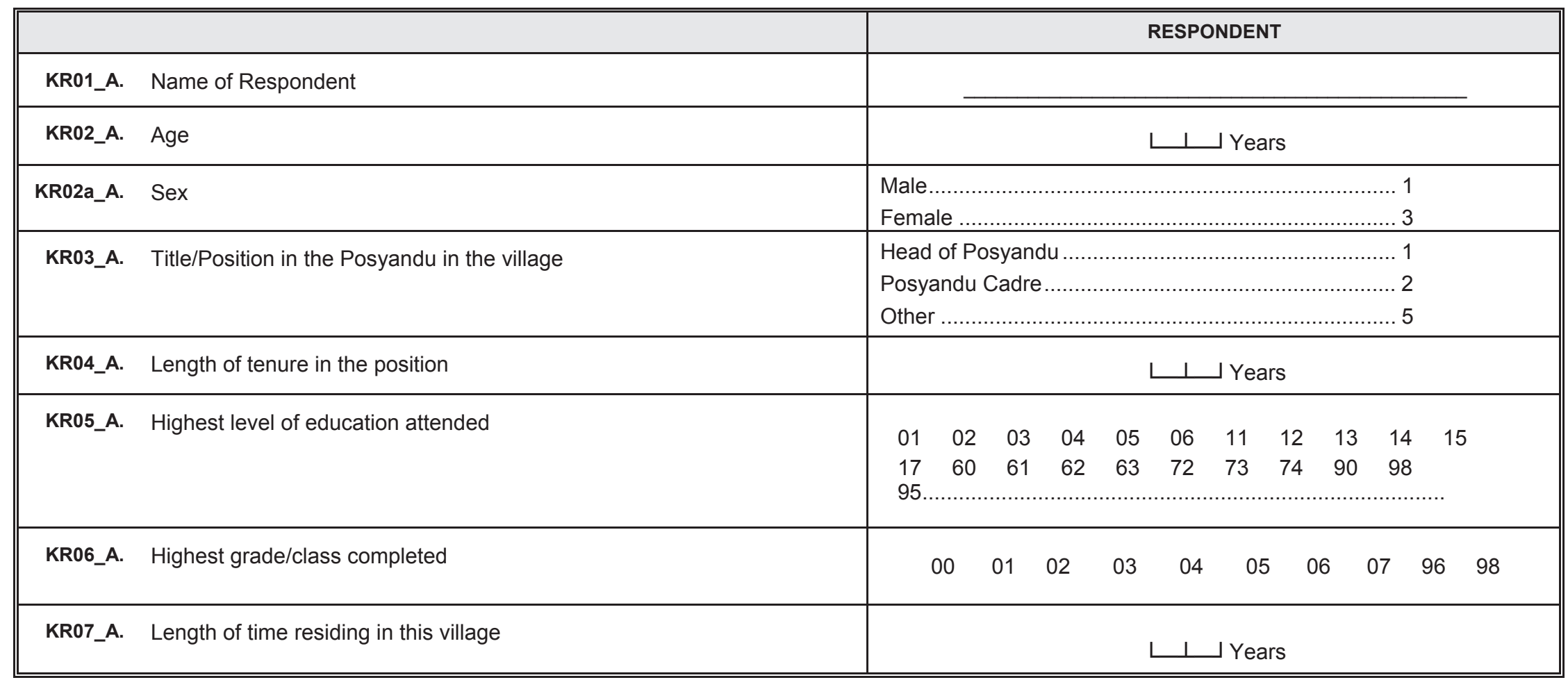

Code KR05_A

01. No school/Not yet at schoo

02. Elementary

03. Junior High - General

04. Junior High - Vocational

05. Senior High - General

06. Senior High - Vocational

60. Senior High - Vocational

61. D1, D2, D3 (Junior College)

62. University S2 (Masters)

62. University S2 (Masters)
63. University S3 (Doctorate)

\section{Code KR06_A}

00. Never completed class I

01.1

02. 2

03. 3

04. 4

06.6

07. Graduated

96 No school

98. DON'T KNOW 


\section{SECTION A: GENERAL}

Now I Would like to ask about services and activities in this Posyandu for the Elderly.

\begin{tabular}{|c|c|c|c|c|}
\hline A1. & $\begin{array}{l}\text { What year did this Posyandu for the Elderly } \\
\text { begin operation? }\end{array}$ & \multicolumn{3}{|l|}{$\begin{array}{l}\text { 1. Year } \underset{1}{\perp} \perp \perp \rightarrow \text { A2a } \\
\text { 8. DON'T KNOW }\end{array}$} \\
\hline A1a. & $\begin{array}{l}\text { How long has this Posyandu for the Elderly } \\
\text { been operating in this village? }\end{array}$ & \multicolumn{3}{|l|}{$\begin{array}{l}\text { 1. } L \perp \text { years } \\
\text { 8. DON'T KNOW }\end{array}$} \\
\hline \multirow[t]{4}{*}{ A1b } & \multirow[t]{4}{*}{ Before becoming a Posyandu for the Elderly? } & & Yes & No \\
\hline & & $\begin{array}{l}\text { Kelompok Bina Keluarga } \\
\text { Lansia }\end{array}$ & 1 & 3 \\
\hline & & Perkumpulan Lansia & 1 & 3 \\
\hline & & $\begin{array}{l}\text { Bentuk lain sebagai wadah } \\
\text { kegiatan lansia }\end{array}$ & 1 & 3 \\
\hline A2a. & $\begin{array}{l}\text { Is this Posyandufor the Elderly is part of } \\
\text { PosyanduBalita (Posyandu for } \leq 5 \text { years } \\
\text { child)? }\end{array}$ & \multicolumn{3}{|l|}{$\begin{array}{l}\text { 1. Yes } \\
\text { 3. No }\end{array}$} \\
\hline A2. & $\begin{array}{l}\text { How many times per month does this health } \\
\text { post meet? }\end{array}$ & \multicolumn{3}{|l|}{$\begin{array}{l}\text { 1. } ـ ل \text { times per month } \\
\text { 2. } ـ \downarrow \text { times per year } \\
\text { 8. DON"T KNOW }\end{array}$} \\
\hline A3. & $\begin{array}{l}\text { In the last year, how many times was the } \\
\text { Posyandu for the Elderly in operation? }\end{array}$ & \multicolumn{3}{|l|}{$\begin{array}{l}\text { 1. } \downarrow \perp \perp 」 \text { times } \\
\text { 8. DON'T KNOW }\end{array}$} \\
\hline A4. & $\begin{array}{l}\text { When the Posyandu for the Elderly is } \\
\text { operating, on average how many cadres are } \\
\text { active? }\end{array}$ & \multicolumn{3}{|l|}{$\downarrow \_\_$cadres } \\
\hline A6. & $\begin{array}{l}\text { What are the opening and closing hours of this } \\
\text { Posyandu for the Elderly: }\end{array}$ & \multicolumn{3}{|c|}{ 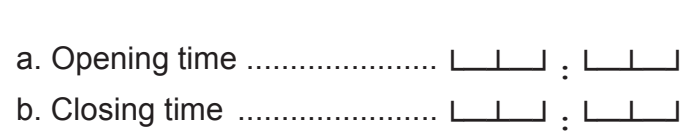 } \\
\hline A7. & $\begin{array}{l}\text { On the day the Posyandu for the Elderly meets } \\
\text { what is the average attendance of the elderlly } \\
\text { at the meeting? }\end{array}$ & \multicolumn{3}{|l|}{$\begin{array}{l}\text { 1. } \perp \perp \_ \text {persons } \\
\text { 8. DON'T KNOW }\end{array}$} \\
\hline A8. & $\begin{array}{l}\text { What is the age of the youngest person who } \\
\text { attends? }\end{array}$ & \multicolumn{3}{|l|}{$\begin{array}{l}\text { 1. } \downarrow \text { ل years } \\
\text { 8. DON'T KNOW }\end{array}$} \\
\hline A9. & $\begin{array}{l}\text { What is the age of the oldest person who } \\
\text { attends? }\end{array}$ & \multicolumn{3}{|l|}{$\begin{array}{l}\text { 1. } L \perp \text { years } \\
\text { 8. DON'T KNOW }\end{array}$} \\
\hline A10. & $\begin{array}{l}\text { What is the charge (or donation) to use the } \\
\text { Posyandu for the Elderly? }\end{array}$ & \multicolumn{3}{|c|}{ 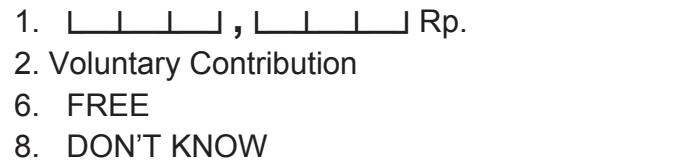 } \\
\hline
\end{tabular}




\section{SECTION B : SERVICES AT THE HEALTH POST FOR THE ELDERLY}

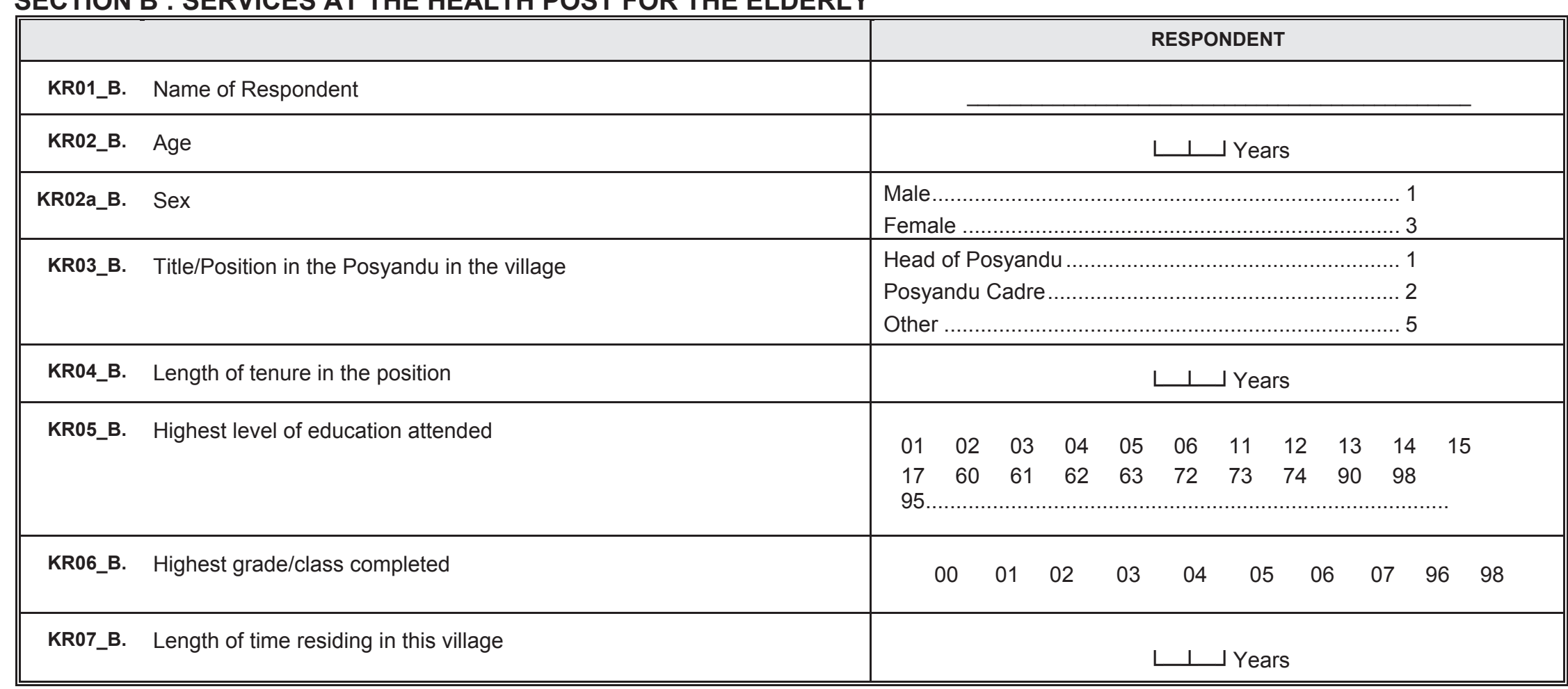

Code KR05_B

01. No school/Not yet at school

02. Elementary

03. Junior High - General

04. Junior High - Vocational

05. Senior High - General

06. Senior High - Vocational

60. D1, D2, D3 (Junior College)

61. University S1 (Bachelors)

62. University S2 (Masters)

63. University S3 (Doctorate)

11. Adult Educ. A (KejarPaket $A)$

2. Adult Educ. B (KejarPaket B)

Open University

15. Adult Educh C (Kesantren

15. Adult Educ. C (KejarPaket $\mathrm{C})$

17. School for the disabled

2. Islamic Elementary School (Madrasah Ibtidaiyah)

73. Islamic Junior High School (Madrasah Tsanawiyah)

74. Islamic Senior High School (Madrasah Aaliyah)

90. Kindergarten

95 Other

\section{Code KR06_B}

00. Never completed class I

$\begin{array}{ll}01 . & 1 \\ 02 . & 2 \\ 03 & 3\end{array}$

02.

04.4

04.4

05. 5

06. 6

07. Graduated

96 No school

98. DON'T KNOW 


\section{SECTION B : SERVICES AT THE HEALTH POST FOR THE ELDERLY}

We would like to ask you about services provided by this Health Post.

\begin{tabular}{|cl|l|}
\hline \multicolumn{1}{|c|}{$\begin{array}{c}\text { BYPE OF SERVICE : } \\
\text { (B1TYPE) }\end{array}$} & Are there [...] services? \\
\hline A. & Weighing & 3. No \\
\hline B. & Measuring Blood Pressure & 3. No \\
\hline C. & Listening to Heartbeat & 1. Yes \\
\hline D. & Checking Eyes & 3. No \\
\hline E. & Checking Ears & 3. No \\
\hline F. & Treatment for minor illnesses & 1. Yes \\
\hline G. & Osteoporosis test & 3. No \\
\hline H. & Supplying Supplementary Food & 3. No \\
\hline I. & Proving iron supplement & 1. Yes \\
\hline J. & Providing vitamin & 3. No \\
\hline K. & Physical Exercise & 3. No \\
\hline L. & Meeting of the elderly (arisan, prayer meeting,etc.) & 1. Yes \\
\hline M & Coordinating activities for the elderly (picnics,etc.) & 3. No \\
\hline N. & $\begin{array}{l}\text { Workshop on Clean and Healthy Living Behavior (diet, } \\
\text { cleanliness, etc.) }\end{array}$ & 3. No \\
\hline O. & Workshop related to ways to earn additional income & 3. No \\
\hline P & Check Hb & 3. No \\
\hline Q & Check cholesterol in the blood & 1. Yes \\
\hline R & Checks blood sugar levels & 1. Yes \\
\hline
\end{tabular}

B3. Who is providing services to the elderly?

B4. Do they receive training for care of the elderly?

B5. What is the average time spent by cadres per month?

36. How many times in the last year has this Posyandu been visited by staff from the HealthCenter(Puskesmas)?

B7. Who usually comes?
A. Doctor
B. Midwife
C. Nurse
Village Midwife
F. Cadre of the Health Post
F. Community members

3. No

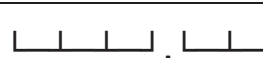

$$
\text { 1. Jam }
$$

Ming

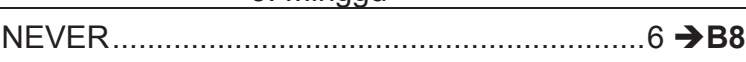

DON'T KNOW ........................................ $8 \rightarrow$ B8

ᄂ $ـ$ times

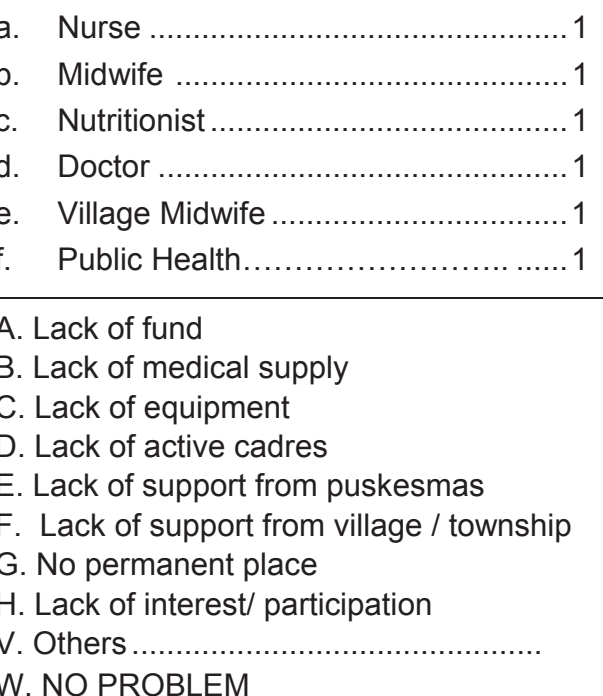

B8. Which of the following problems do
you face at this health post? you face at this health post?

1. Yes 3. No

3

3

3

3

3 


\section{SECTION C : MANPOWERPOST FOR THE ELDERLY}

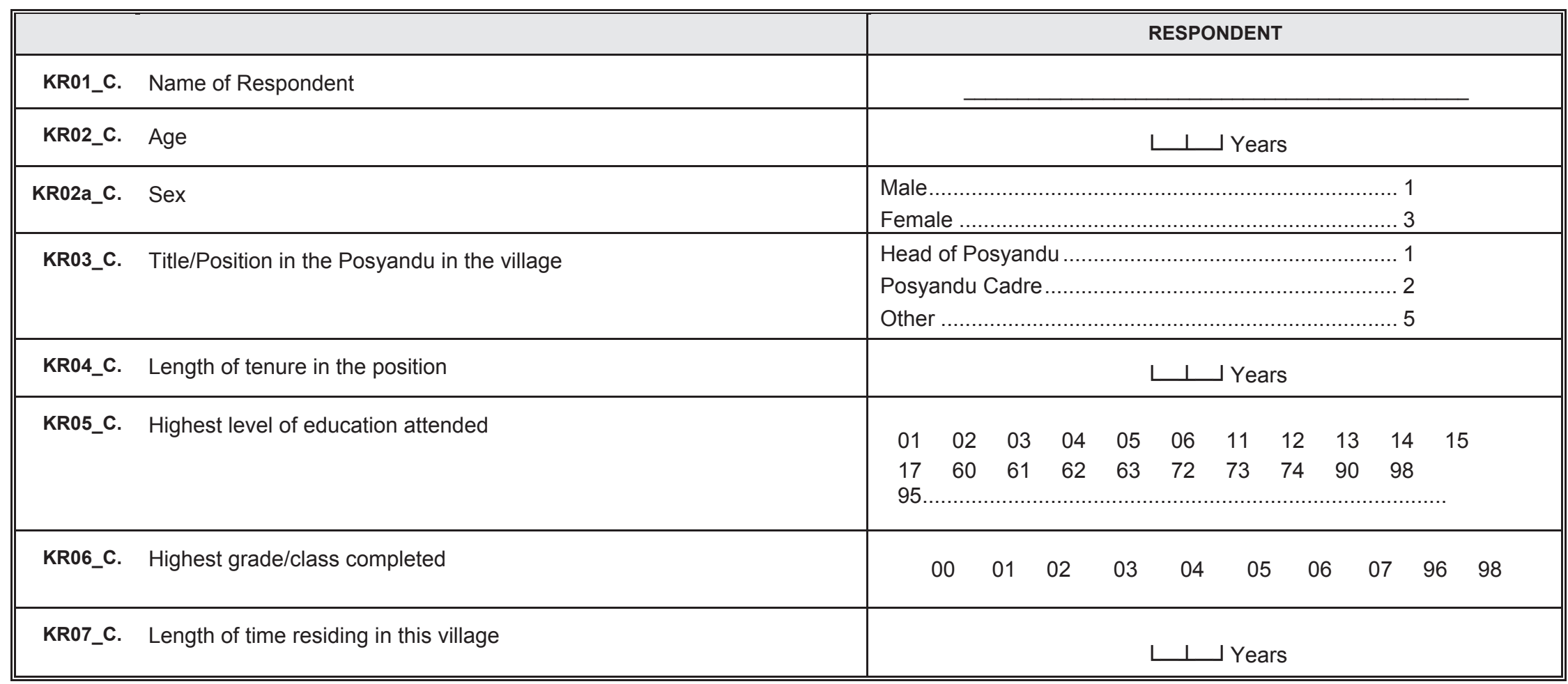

\section{Code KR05_C}

01. No school/Not yet at school 11. Adult Educ. A (KejarPaket A)

02. Elementary

03. Junior High - General

04. Junior High - Vocational

05. Senior High - General

06. Senior High - Vocational

60. D1, D2, D3 (Junior College)

61. University S1 (Bachelors)
62. University S2 (Masters)

63. University S3 (Doctorate)

12. Adult Educ. $B$ (Ke

14. Islamic School Pesantren

15. Adult Educ. C (KejarPaket C)

17. School for the disabled

72. Islamic Elementary School (Madrasah Ibtidaiyah)

73. Islamic Junior High School (Madrasah Tsanawiyah)

74. Islamic Senior High School (Madrasah Aaliyah)

90. Kindergarten

98 DON'T KNOW

95 Other

\section{Code KR06_C}

00. Never completed class

$\begin{array}{ll}01 . & 1 \\ 02 . & 2 \\ 03 . & 3\end{array}$

03. 3

04. 4

05.5

06.

07. Graduated

96 No school

98. DON'T KNOW 
SECTION C : MANPOWERPOST FOR THE ELDERLY

Now, we would like to know about the cadre working for this Posyandu for the Elderly.

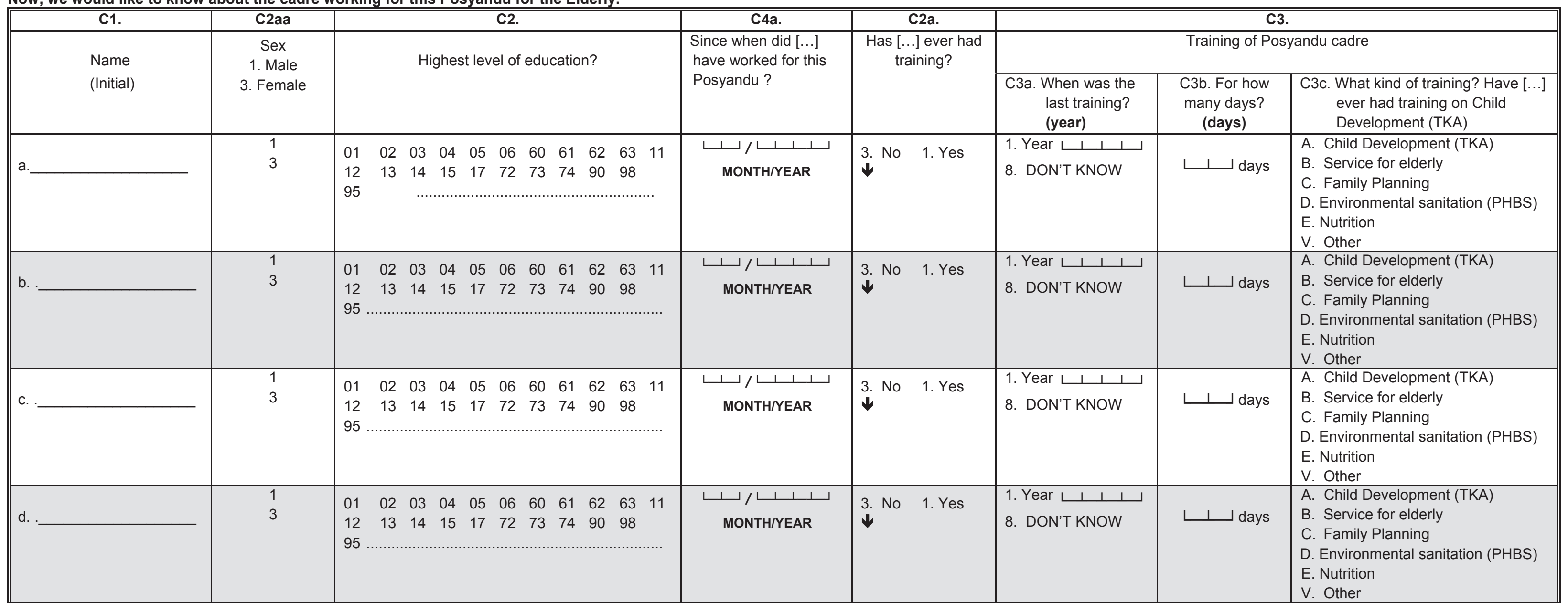

Code C2 :

01. No school/Not yet

02.Elementary School

03. Junior High - General

05. Senior High - General
06. Senior High - Vocational D1, D2, D3 (Junior College)

61. University S1 (Bachelors)

63. University S3 (Doctorate)
11. Adult Educ. A (KejarPaket A) 1. Adult Educ. B (KejarPaket B)

3. Open University

15. Adult Educ. C (KejarPaket C)
17. School for disable person 72. Islamic Elementary (Madrasah Ibtidaiyah)

73. Islamic Junior High School (Madrasah Tsanawiyah)

74. Islamic Senior High Schoo (Madrasah Alliyah)
90. Kindergarten 98. DON'T KNOW 95. Other 
SECTION C : MANPOWERPOST FOR THE ELDERLY

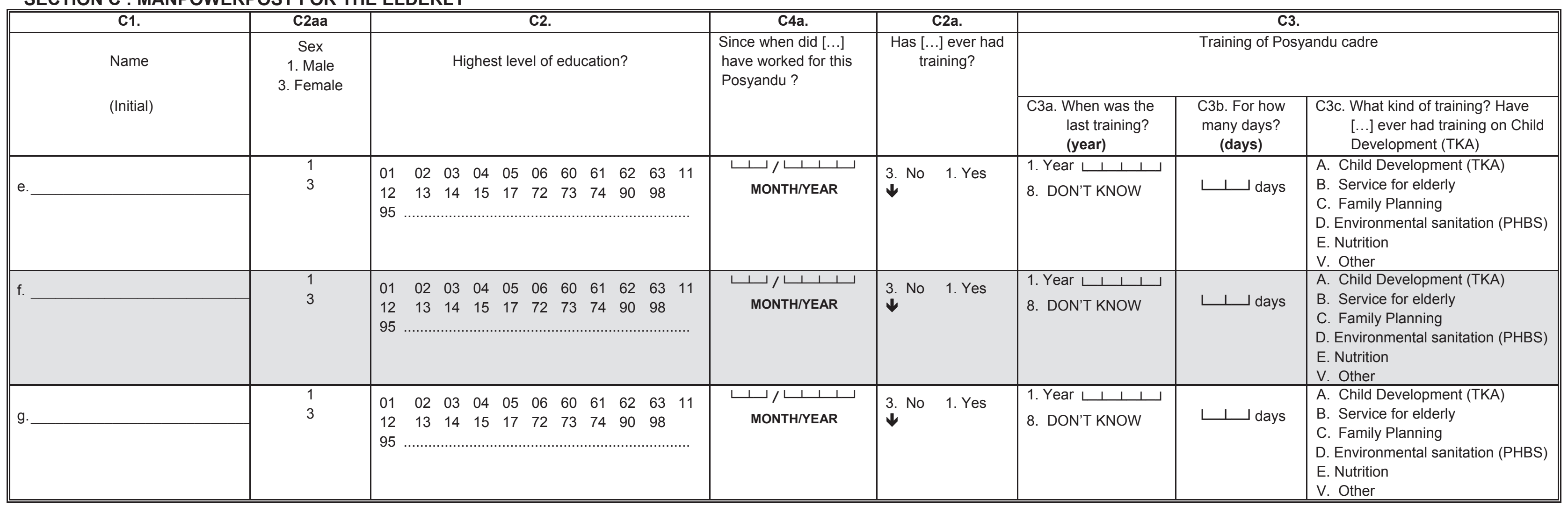

Code $\mathrm{C2}$ :

2. No school/Not yet

03. Junior High - General

04. Junior High - Vocation

05. Senior High - General
06. Senior High - Vocational

61. University $\mathrm{S1}$ (Bachelors)

62. University $S 2$ (Masters)

63. University S3 (Doctorate)
11. Adult Educ. A (KejarPaket A)

2. Adult Educ. B (KejarPaket B)

Open University

15. Adult Educ. C (Kejar
17. School for disable person 2. Islamic Elementary (Madrasah 73. Islamic

Junior High School

(Madrasah Tsanawiyah)

High School (Madrasah Alliyah)
90. Kindergarten 98. DON'T KNOW 95. Other 


\section{SECTION D: HEALTH INSTRUMENTS}

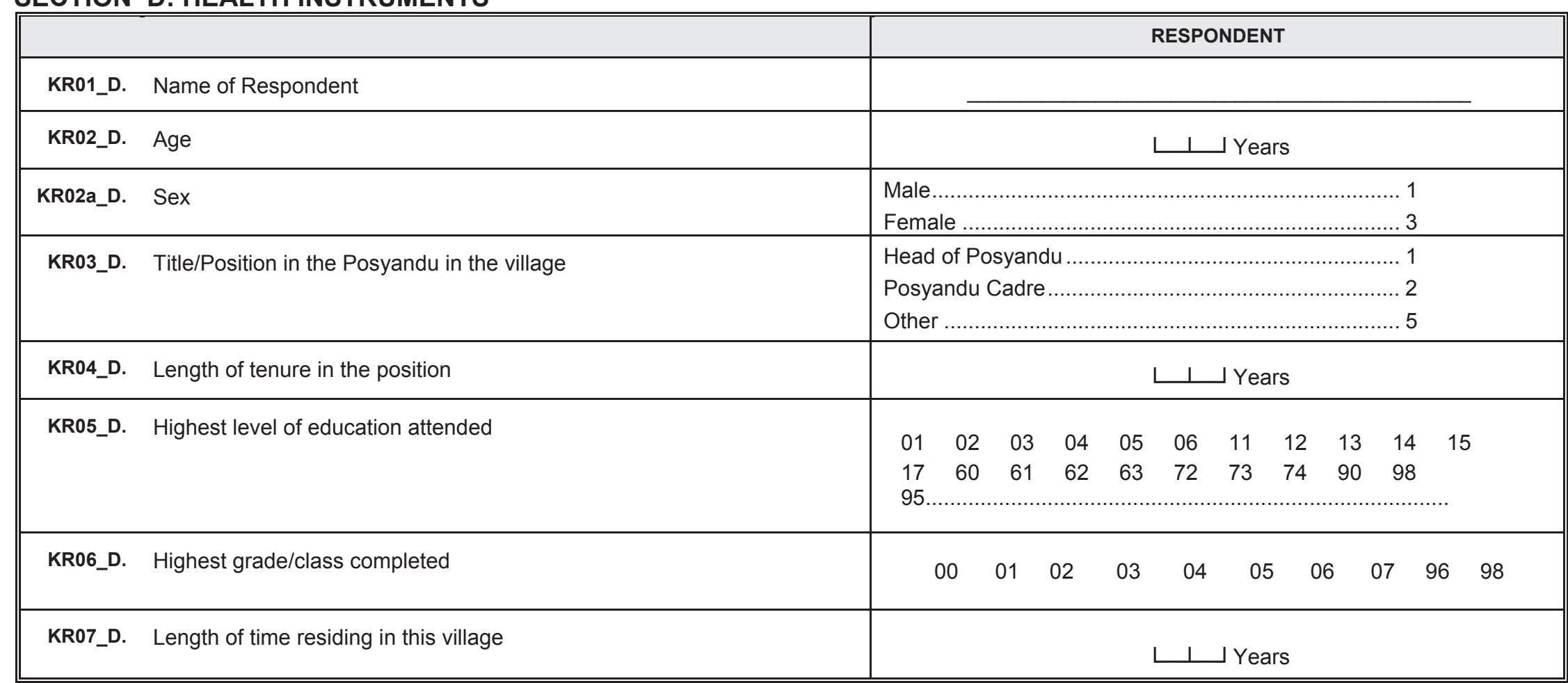

Code KR05_D

01. No school/Not yet at school 11. Adult Educ. A (KejarPaket A)

02. Elementary

03. Junior High - General

04. Junior High - Vocational

05. Senior High - Generat

06. Senior High - Vocational

60. D1, D2, D3 (Junior College)

61. University S1 (Bachelors)

62. University S2 (Masters)

63. University S3 (Doctorate)

Adult Educ. A (KejarPaket $A)$

13. Open University

14. Islamic School Pesantren

15. Adult Educ. C (KejarPaket C)

17. School for the disabled

72. Islamic Elementary School (Madrasah Ibtidaiyah)

73. Islamic Junior High School (Madrasah Tsanawiyah)

74. Islamic Senior High School (Madrasah Aaliyah)

90. Kindergarten

98 DON'T KNOW

95 Other

\section{Code KR06_D}

00. Never completed class

01. 1

02. 2

03. 3

04.4

05.5

07.

07. Graduate

96 No school

98. DON'T KNOW 


\section{SECTION D: HEALTH INSTRUMENTS}

Now we would like to ask about equipment available in this Health Post.

\begin{tabular}{|c|c|c|c|}
\hline & D1. & D2. & D3. \\
\hline & $\begin{array}{c}\text { TYPE OF EQUIPMENT } \\
\text { (DTYPE) }\end{array}$ & $\begin{array}{c}\text { Is [...] available in this } \\
\text { Health Post? }\end{array}$ & Who own[...\} \\
\hline & Weighing scales & 3. No 1. Yes $\rightarrow$ & $\begin{array}{l}\text { 1. Posyandu for the Elderly } \\
\text { 2. Midwife from Puskesmas } \\
\text { 3. RegularPosyandu } \\
\text { 4. Individuals } \\
\text { 5. Others.................................... }\end{array}$ \\
\hline b. & Height measuring device & $\begin{array}{l}\text { 3. No } 1 \text {. Yes } \rightarrow \\
\Downarrow\end{array}$ & $\begin{array}{l}\text { 1. Posyandu for the Elderly } \\
\text { 2. Midwife from Puskesmas } \\
\text { 3. RegularPosyandu } \\
\text { 4. Individuals } \\
\text { 5. Others.................................... }\end{array}$ \\
\hline c. & Blood Pressure Measurement & 3. No 1. Yes $\rightarrow$ & $\begin{array}{l}\text { 1. Posyandu for the Elderly } \\
\text { 2. Midwife from Puskesmas } \\
\text { 3. RegularPosyandu } \\
\text { 4. Individuals } \\
\text { 5. Others................................... }\end{array}$ \\
\hline d. & Eye Checking Device & 3. No 1. Yes $\rightarrow$ & $\begin{array}{l}\text { 1. Posyandu for the Elderly } \\
\text { 2. Midwife from Puskesmas } \\
\text { 3. RegularPosyandu } \\
\text { 4. Individuals } \\
\text { 5. Others.................................... }\end{array}$ \\
\hline e. & Osteoporosis test Kit & 3. No 1. Yes $\rightarrow$ & $\begin{array}{l}\text { 1. Posyandu for the Elderly } \\
\text { 2. Midwife from Puskesmas } \\
\text { 3. RegularPosyandu } \\
\text { 4. Individuals } \\
\text { 5. Others................................... }\end{array}$ \\
\hline f. & Demonstration tools/books & $\begin{array}{l}\text { 3. No } 1 \text {. Yes } \rightarrow \\
\Downarrow\end{array}$ & $\begin{array}{ll}\text { 1. } & \text { Posyandu for the Elderly } \\
\text { 2. } & \text { Midwife from Puskesmas } \\
\text { 3. } & \text { RegularPosyandu } \\
\text { 4. Individuals } \\
\text { 5. Others.................................. }\end{array}$ \\
\hline
\end{tabular}

\begin{tabular}{|c|c|c|c|}
\hline & D1. & D2. & D3. \\
\hline & $\begin{array}{c}\text { TYPE OF EQUIPMENT } \\
\text { (DTYPE) } \\
\end{array}$ & $\begin{array}{c}\text { Is [...] available in this } \\
\text { Health Post? }\end{array}$ & Who own[...] \\
\hline g. & Physical Exercise equipment & 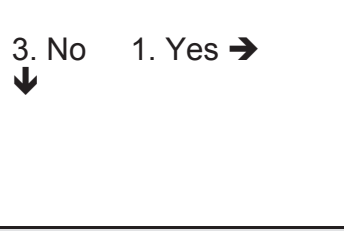 & $\begin{array}{l}\text { 1. Posyandu for the Elderly } \\
\text { 2. Midwife from Puskesmas } \\
\text { 3. RegularPosyandu } \\
\text { 4. Individuals } \\
\text { 5. Others.................................. }\end{array}$ \\
\hline h. & Paracetamol & 3. No 1. Yes $\rightarrow$ & $\begin{array}{l}\text { 1. Posyandu for the Elderly } \\
\text { 2. Midwife from Puskesmas } \\
\text { 3. RegularPosyandu } \\
\text { 4. Individuals } \\
\text { 5. Others.................................... }\end{array}$ \\
\hline i. & Iron Tablets/Sulfas Ferosus & $\begin{array}{l}\text { 3. No } \quad 1 \text {. Yes } \rightarrow \\
\Downarrow\end{array}$ & $\begin{array}{l}\text { 1. Posyandu for the Elderly } \\
\text { 2. Midwife from Puskesmas } \\
\text { 3. RegularPosyandu } \\
\text { 4. Individuals } \\
\text { 5. Others..................................... }\end{array}$ \\
\hline j. & Vitamins & $\begin{array}{l}\text { 3. No } 1 \text {. Yes } \rightarrow \\
\downarrow \\
\text { SDP SECTION }\end{array}$ & $\begin{array}{l}\text { 1. Posyandu for the Elderly } \\
\text { 2. Midwife from Puskesmas } \\
\text { 3. RegularPosyandu } \\
\text { 4. Individuals } \\
\text { 5. Others.................................... }\end{array}$ \\
\hline
\end{tabular}




\section{SECTION SDP : RESOURCES FOR HEALTH POST FOR THE ELDERLY}

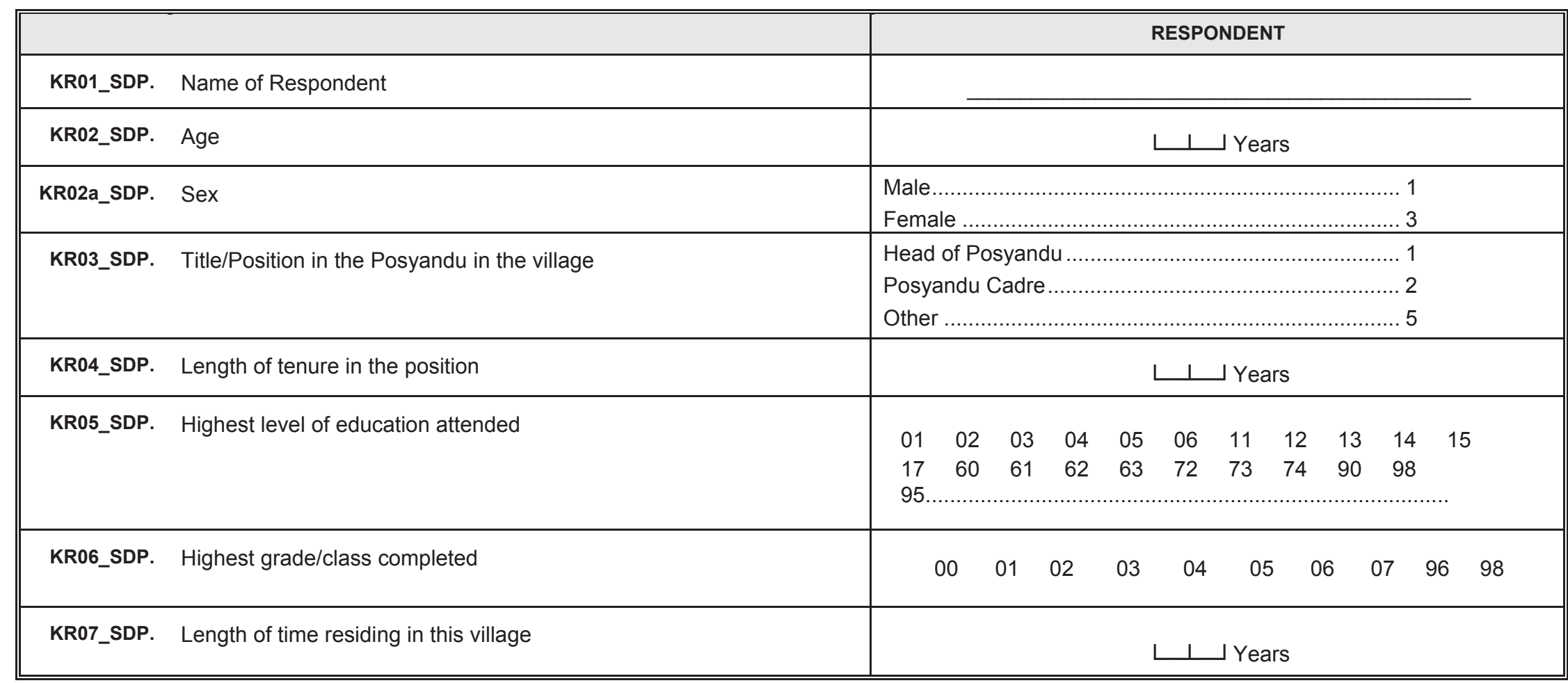

Code KR05_SDP
01. No school/Not yet at school 11. Adult Educ. A (KejarPaket A)
02. Elementary
03. Junior High - General
04. Junior High - Vocational
05. Senior High - General
06. Senior High - Vocational
12. Adult Educ. B (KejarPaket B)
13. Open University
15. Adut Educ C (Kesantren
60. D1, D2, D3 (Juiar College) - 72. Islamic Elementary School (Madasah Ibtidaiyah)
73. Islamic Junior High School (Madrasah Tsanawiyah)
74. Islamic Senior High School (Madrasah Aaliyah)
62. University S2 (Masters)
90. Kindergarten
98 DON'T KNOW

Code KR06_SDP

00. Never completed class I

01.1

01.1

02. 2

03. 3

04.4

05. 5

06. 6

07. Graduated

96 No school

98. DON'T KNOW

95 Other 


\section{SECTION SDP : RESOURCES FOR HEALTH POST FOR THE ELDERLY}

Now we would like to ask you about source of resources for this Health Post

\begin{tabular}{|c|c|c|}
\hline SDP00. & INTERVIEWER CHECK A2a=1 (IS THIS ELDERLY POSYANDU IS PART OF POSYANDU BALITA?) & 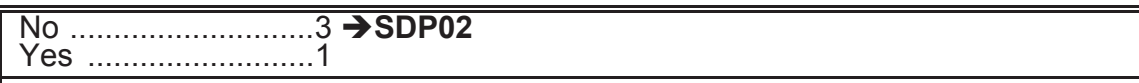 \\
\hline SDP00a. & Can you separate the source of resource for Elderly Posyandu and PosyanduBalita? & $\begin{array}{l}\text { 1. IF YES, SDP IS ONLY FOR POSYANDU } \\
\text { 3. IF NO, SDP IS BOTH FOR POSYANDU AND ELDERLY POSYANDU }\end{array}$ \\
\hline \multirow[t]{3}{*}{ SDP02. } & $\begin{array}{l}\text { What is the value ofi }[\ldots] \text { provided by the community for this Health Post per month? } \\
\text { a. Cash }\end{array}$ & 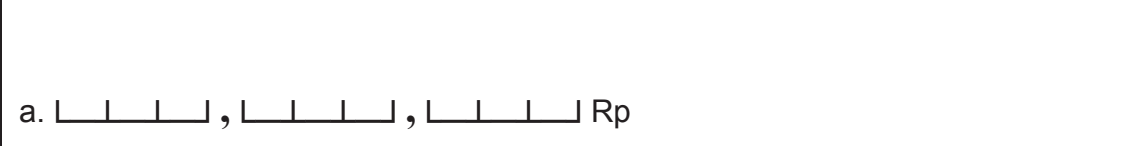 \\
\hline & b. Time & 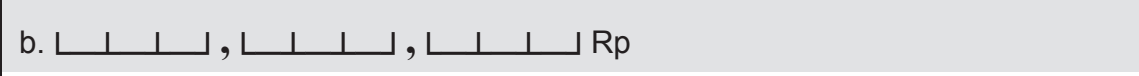 \\
\hline & v. Others & 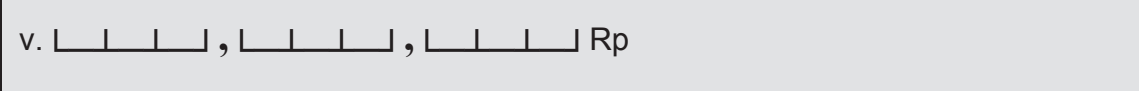 \\
\hline SDP03. & Are there resources received by this Health Post from other source? & $\begin{array}{l}\text { No } \\
\text { Yes } \ldots \ldots \ldots \ldots \ldots \ldots \ldots \ldots \ldots \ldots \ldots \ldots \\
3\end{array}$ \\
\hline
\end{tabular}

\begin{tabular}{|c|c|c|c|c|c|c|}
\hline SDP04. & $\begin{array}{l}\text { What type of resources received from others source and who } \\
\text { provide the resources? }\end{array}$ & \multicolumn{5}{|c|}{ TYPE OF RESOURCES } \\
\hline & SOURCE & A. Cash & B. Food & C. Vitamins & D. Vactination & E. Otherequipment \\
\hline & a. Puskesmas & 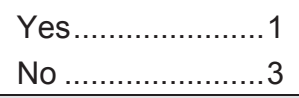 & 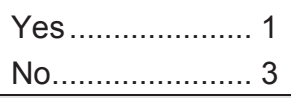 & 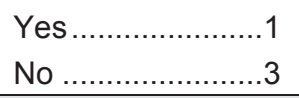 & 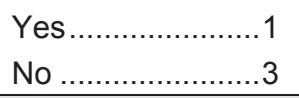 & 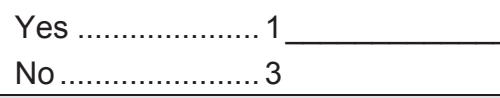 \\
\hline & b. Other Posyandu & 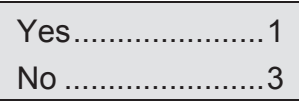 & $\begin{array}{l}\text { Yes } \ldots \ldots \ldots \ldots \ldots \ldots \ldots . . . . .1 \\
\text { No...................... } 3\end{array}$ & 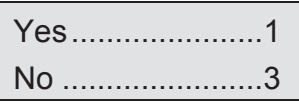 & 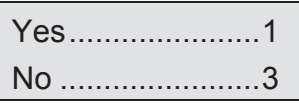 & 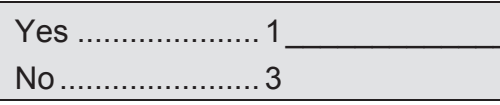 \\
\hline & c. BKKBN/PLKB & 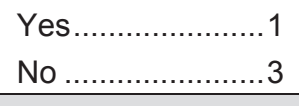 & $\begin{array}{l}\text { Yes } \ldots \ldots \ldots \ldots \ldots \ldots \ldots . . . . . . .1 \\
\text { No.................... } 3\end{array}$ & 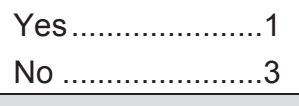 & $\begin{array}{l}\text { Yes } \ldots \ldots \ldots \ldots \ldots \ldots \ldots . . .1 \\
\text { No } \ldots \ldots \ldots \ldots \ldots \ldots \ldots \ldots . . . . .1 \\
\end{array}$ & Yes ............................ \\
\hline & d. Villages/township government & 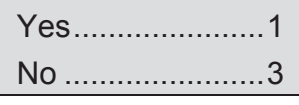 & $\begin{array}{l}\text { Yes } \ldots \ldots \ldots \ldots \ldots \ldots \ldots \ldots . . . .1 \\
\text { No.................... } 3\end{array}$ & 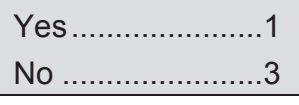 & 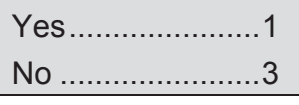 & Yes .......................... \\
\hline & e. Subsidies from government & $\begin{array}{l}\text { Yes } \ldots \ldots \ldots \ldots \ldots \ldots \ldots \ldots . . .1 \\
\text { No } \ldots \ldots \ldots \ldots \ldots \ldots \ldots \ldots \ldots . . .3\end{array}$ & 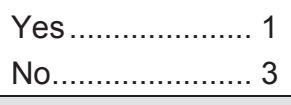 & 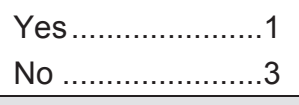 & $\begin{array}{l}\text { Yes ...................... } 1 \\
\text { No ..................... } 3 \\
\end{array}$ & 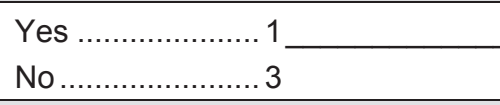 \\
\hline & v. Other & 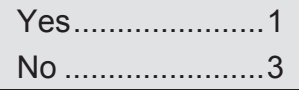 & 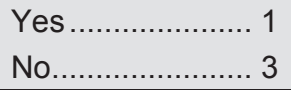 & $\begin{array}{l}\text { Yes ................. } \\
\text { No .................... }\end{array}$ & 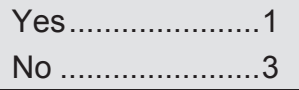 & Yes \\
\hline
\end{tabular}




\section{SECTION CP: INTERVIEWER NOTES}

\begin{tabular}{|c|c|c|c|c|c|c|c|}
\hline LANGMAIN (CK1). & \multicolumn{2}{|l|}{ Interview was entirely/mostly conducted in what language? } & \multicolumn{5}{|l|}{$ـ$ other } \\
\hline & Other languaged used (if any): & & \multicolumn{5}{|l|}{$\downarrow \downarrow$ L other } \\
\hline LANGUAGE CODE: & & & & & & & \\
\hline 00. Bahasa Indonesia & 04. Batak & 08. Sasak & 12. Makassar & 16. & Toraja & 20. & Lampung \\
\hline 01. Java & 05. Bugis & 09. Minang & 13. Nias & 17. & Lahat & 95. & Other, \\
\hline 02. Sunda & 06. Cina & 10. Banjar & 14. Palembang & 18. & Other South Sumatera & 96. & NA \\
\hline 03. Bali & 07. Madura & 11. Bima & 15. Sumbawa & 19. & Betawi & & \\
\hline
\end{tabular}

\begin{tabular}{|c|c|c|c|}
\hline RESULT (FP3). RESULTS OF INTERVIEW & REASON (FP4). REASON FOR ANSWERING “2” I "3" IN RESULT & FP6. MONITORING BY SUPERVISOR & \\
\hline $\begin{array}{l}\text { 1. Completed } \rightarrow \text { FP6 } \\
\text { 2. Partly completed } \\
\text { 3. Not completed } \\
\text { 4. Twin EA with EA } \sqcup, \longrightarrow \text { FP6 }\end{array}$ & $\begin{array}{l}\text { 1. Respondent was travelling/not in location } \\
\text { 2. Respondent was too busy } \\
\text { 3. Respondent refused }\end{array}$ & $\begin{array}{ll} & \\
\text { a. } & \text { Observed (sup_obs) } \ldots . . . . . .1 \\
\text { b. } & \text { Checked (sup_edit)........... } 1 \\
\text { c. } & \text { Verified (sup veri).............. } 1\end{array}$ & $\begin{array}{l}\text { No } \\
3 \\
3 \\
3\end{array}$ \\
\hline
\end{tabular}

INTERVIEWER NOTE: 


\begin{tabular}{|c|c|c|c|}
\hline INTERVIEWER: & 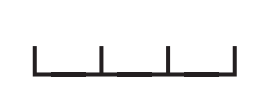 & CONFIDENTIAL & IDW: \\
\hline
\end{tabular}

\section{INDONESIAN FAMILY LIFE SURVEY 2014}

HEALTH FACILITY

\section{TRADITIONAL PRACTICE}

SECTIONS : LK, A, B, C, CP

NAME OF FACILITY:

FACILITY CODE I BOOK TYPE

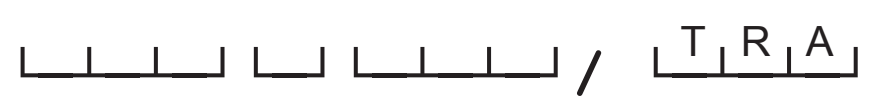




\section{SECTION LK : CONTROL SHEET}

\begin{tabular}{||l|c||}
\hline \multicolumn{1}{|c|}{ SAMPLING INFORMATION } & CODE \\
\hline LK01. Province & \\
\hline LK02. Kabupaten/ Kota & \\
\hline LK03. Kecamatan & 2. Rural \\
\hline LK04. Village/Urban Township & \\
\hline LK05. Region : 1. Urban & \\
\hline
\end{tabular}

\begin{tabular}{|c|c|c|}
\hline & SUPERVISION & CODE \\
\hline LK15. & Name of Interviewer & 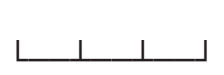 \\
\hline LK17. & Name of Local Supervisor & 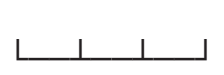 \\
\hline LK19. & Name of Field Coordinator & 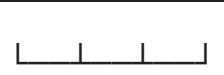 \\
\hline
\end{tabular}

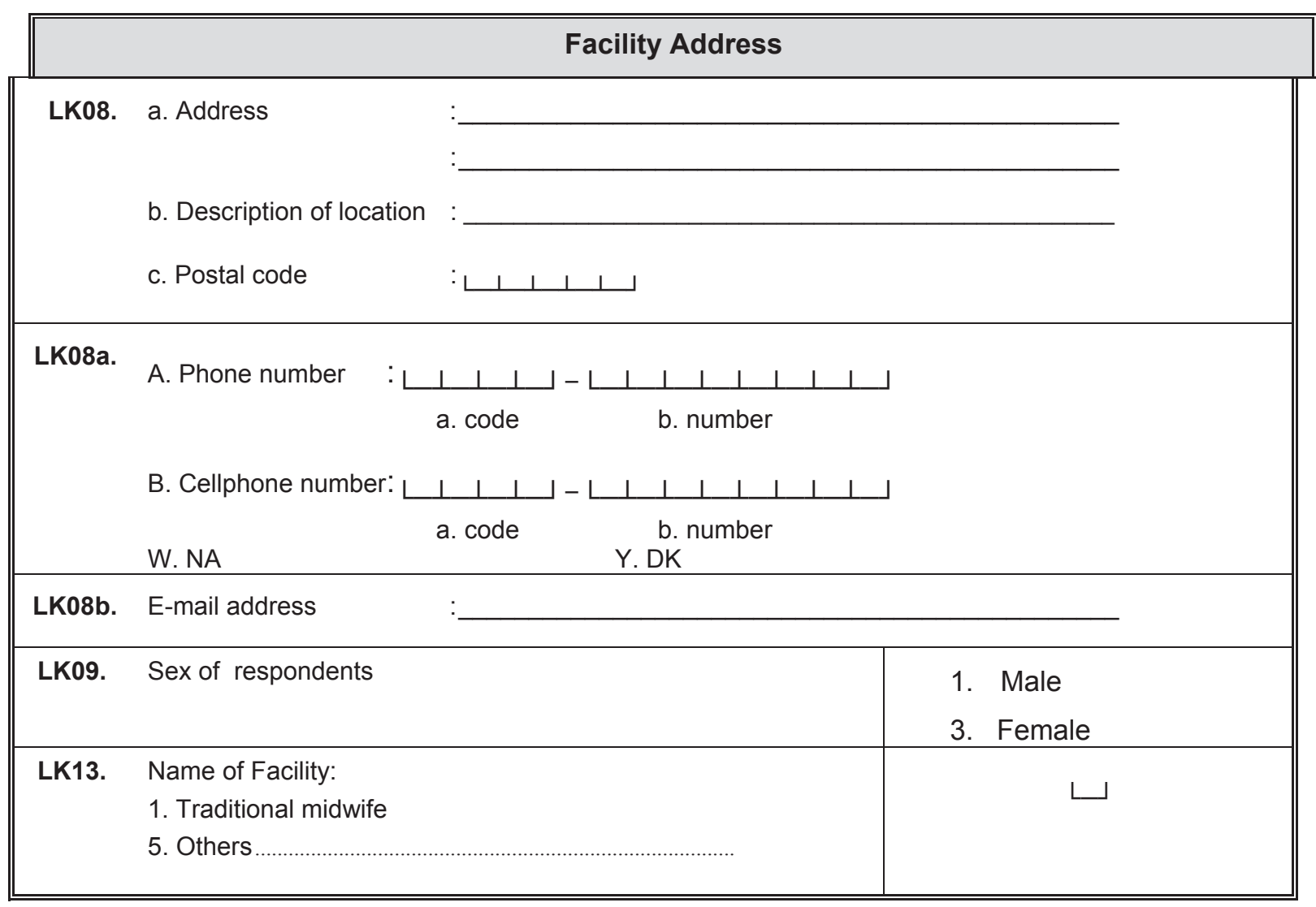




\section{SECTION A : GENERAL}

\begin{tabular}{|c|c|c|}
\hline A00a. & Name & \\
\hline A00. & Sex & $\begin{array}{l}\text { 1. Male } \\
\text { 3. Female }\end{array}$ \\
\hline A00. & $\begin{array}{l}\text { Do you give the following health services to a } \\
\text { patient? }\end{array}$ & 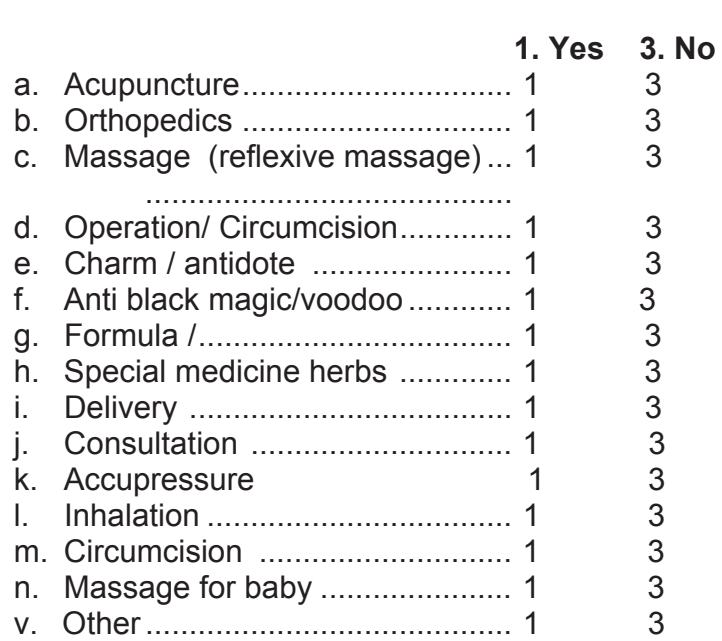 \\
\hline A00x. & $\begin{array}{l}\text { INTERVIEWER CHECK A00: ARE THE } \\
\text { SERVICES PROVIDED ONLY } \\
\text { CHARM/ANTIDOTE (e) OR ANTI BLACK } \\
\text { MAGIC/MAGICAL (f) OR CONSULTATION (j) }\end{array}$ & $\begin{array}{l}\text { ONLY e, f, j } \\
\text { OTHER SERVICES except e, f, j .. } 3\end{array}$ \\
\hline A01. & How old are you? & $\llcorner\perp$ Years \\
\hline A02. & $\begin{array}{l}\text { How long have you been practicing traditional } \\
\text { treatment here? }\end{array}$ & $\downarrow$ Years \\
\hline A03. & $\begin{array}{l}\text { From whom did you learn this traditional } \\
\text { treatment practice? }\end{array}$ & 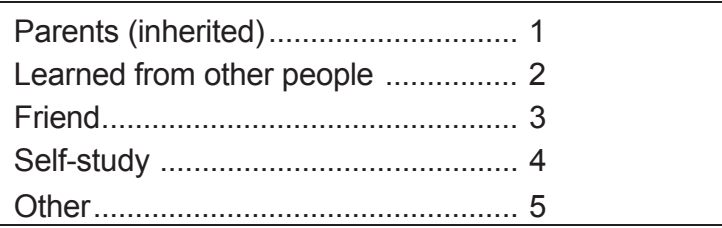 \\
\hline A05a. & $\begin{array}{l}\text { Can you read an Indonesian-language } \\
\text { newspaper? }\end{array}$ & 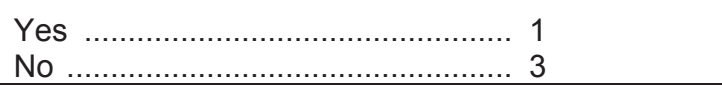 \\
\hline A05b. & $\begin{array}{l}\text { Can you read a newspaper in another } \\
\text { language? }\end{array}$ & $\begin{array}{l}\text { Yes } \\
\text { No } \ldots \ldots \ldots \ldots \ldots \ldots \ldots \ldots \ldots \ldots \\
3\end{array}$ \\
\hline A06a. & Can you write a letter in Indonesian? & Yes \\
\hline A06b. & Can you write a letter in another language? & 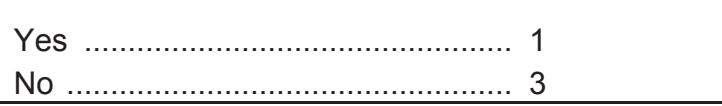 \\
\hline
\end{tabular}

\begin{tabular}{|c|c|c|}
\hline A04a. & $\begin{array}{l}\text { Have you ever attended/are you attending } \\
\text { school? }\end{array}$ & Yes \\
\hline A04. & $\begin{array}{l}\text { What is the highest education level } \\
\text { attended? } \\
\text { [NOTE TO INTERVIEWER: } \\
\text { IF THEY ARE CURRENTLY ATTENDING } \\
\text { SCHOOL, RECORD THE LEVEL THEY } \\
\text { ARE CURRENTLY ATTENDING] }\end{array}$ & 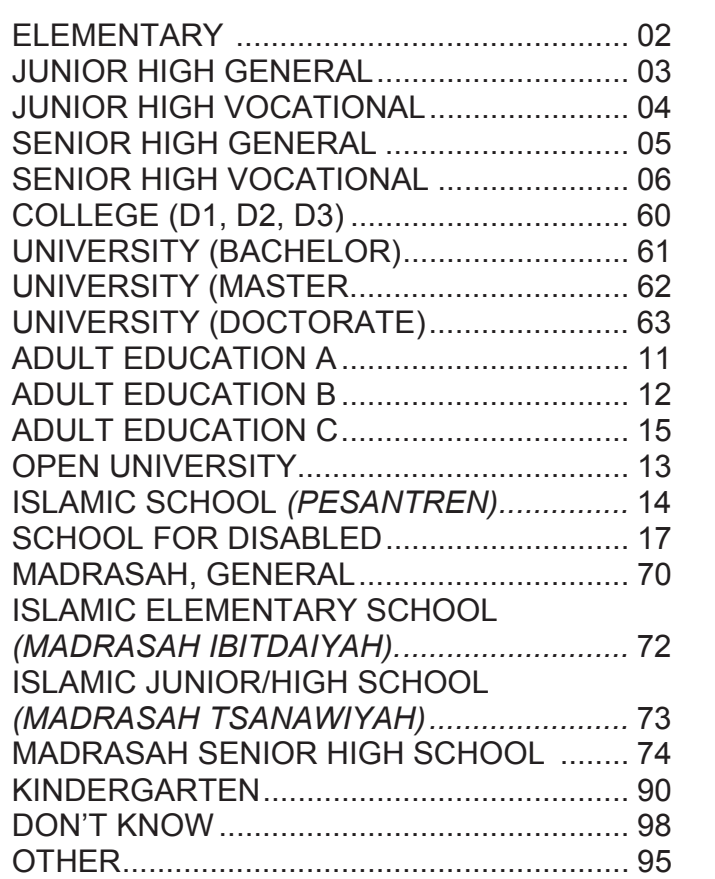 \\
\hline A04b. & $\begin{array}{l}\text { What is the highest grade completed at } \\
\text { that school? }\end{array}$ & 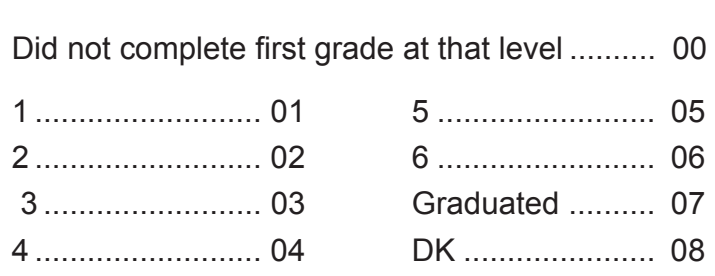 \\
\hline A07. & $\begin{array}{l}\text { In providing service to visitors/patients, are } \\
\text { there specific hours for services? (e.g. } \\
\text { everyday from } 8.00 \text { - } \\
14.00 \text { ) }\end{array}$ & 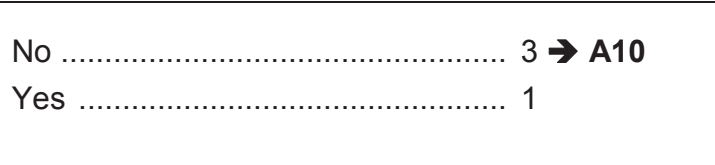 \\
\hline A08. & $\begin{array}{l}\text { If YES, how many days a week do you } \\
\text { provide services to visitors/patients? }\end{array}$ & $\smile$ days a week \\
\hline
\end{tabular}




\section{SECTION A : GENERAL}

A09. How many hours a day do you practice and give services to visitors/patients?

A10. If not, how do you provide services ?

A11. In providing services, what language do you usually use ?

A12. What is your religion?

A13. Besides this practice, do you have other work ?

\begin{tabular}{|c|}
\hline $\begin{array}{c}\longleftarrow \text { hours a day } \\
\rightarrow \text { A11 }\end{array}$ \\
\hline $\begin{array}{l}\text { Open } 24 \text { hours a day } \\
\text { Only by appointment } \\
\text { Other, mention }\end{array}$ \\
\hline Indonesian \\
\hline $\begin{array}{l}\text { Islam } \\
\text { Protestant } \\
\text { Catholic } \\
\text { Hindu } \\
\text { Budha } \\
\text { Kong hu Cu } \\
\text { Other. }\end{array}$ \\
\hline $\begin{array}{l}\text { No } \\
\text { B1TYPE } \\
\text { Yes }\end{array}$ \\
\hline
\end{tabular}

\begin{tabular}{|c|c|c|}
\hline A13a. & What do you produce/do in your work? & \\
\hline A13b. & EDITOR: CODE FOR SECTORS & $\downarrow$ \\
\hline A14. & $\begin{array}{l}\text { Which category best describes the work } \\
\text { you do? }\end{array}$ & 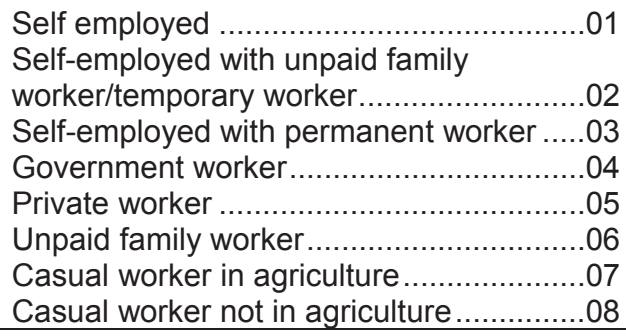 \\
\hline A15. & $\begin{array}{l}\text { How many hours a week do you work } \\
\text { there? }\end{array}$ & 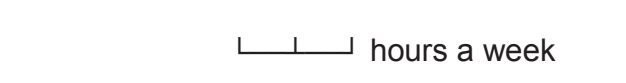 \\
\hline
\end{tabular}

\section{CODE A13b}

01. Farming (including forestry, hunting and fishin

02. Mining (including excavating

03. Manufacturing industry

04. Electricity, gas and water

05. Building construction

06. Large trade, retail trade, restaurants and hotels

07. Transportation, warehousing and communications

08. Finance, insurance, lease of buildings, grounds and business services

09. Social services 


\section{SECTION B : PRACTICE ACTIVITIES}

\begin{tabular}{|c|c|c|c|c|c|c|c|}
\hline \multicolumn{4}{|c|}{ (B1TYPE) } & \multirow{3}{*}{$\begin{array}{c}\text { B2a. } \\
\text { Morning Opening Hour } \\
\_ـ ـ\end{array}$} & \multirow{3}{*}{$\begin{array}{c}\text { B2b. } \\
\text { Morning Closing Hour } \\
\_ـ ـ\end{array}$} & \multirow{3}{*}{ 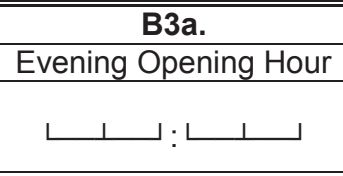 } & \multirow{3}{*}{ 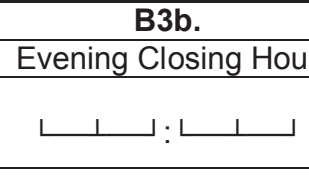 } \\
\hline & Do yo & the las & eek? & & & & \\
\hline & Monday & $\stackrel{\text { 3. No }}{ }^{-10}$ & 1. Yes & & & & \\
\hline & Tuesday & 3. No $^{-12}$ & 1. Yes & لـ & 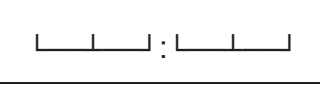 & 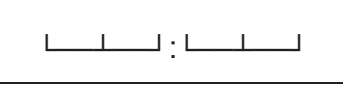 & لـ \\
\hline & Wednesday & 3. No & 1. Yes & 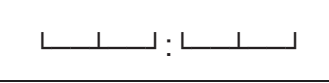 & 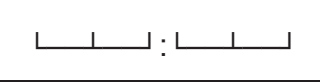 & 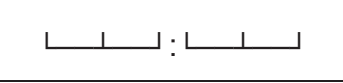 & 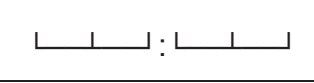 \\
\hline & Thursday & 3. No $^{+}$ & 1. Yes & 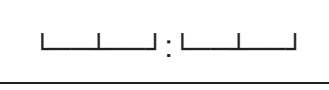 & 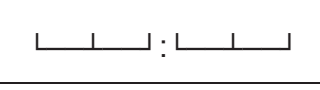 & 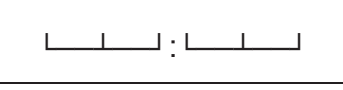 & لـ \\
\hline & Friday & 3. No & 1. Yes & 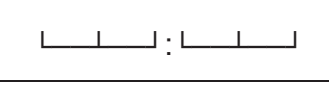 & 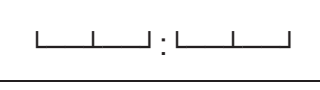 & 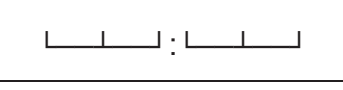 & 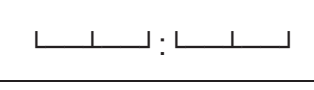 \\
\hline & Saturday & $\stackrel{3}{\Downarrow}^{\mathrm{N} o}$ & 1. Yes & 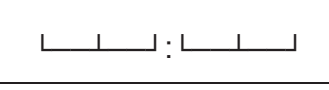 & 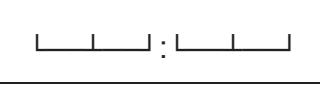 & 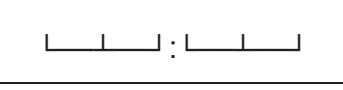 & 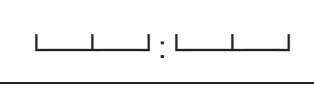 \\
\hline & Sunday & 3. No $^{-12}$ & 1. Yes & لـ & 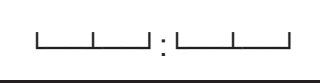 & 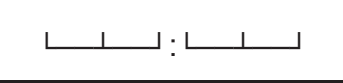 & 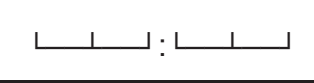 \\
\hline
\end{tabular}




\section{SECTION B : PRACTICE ACTIVITIES}

B05. What disease/problem can you cure/solve ?

\section{[MENTION ALL ITEMS BELOW}

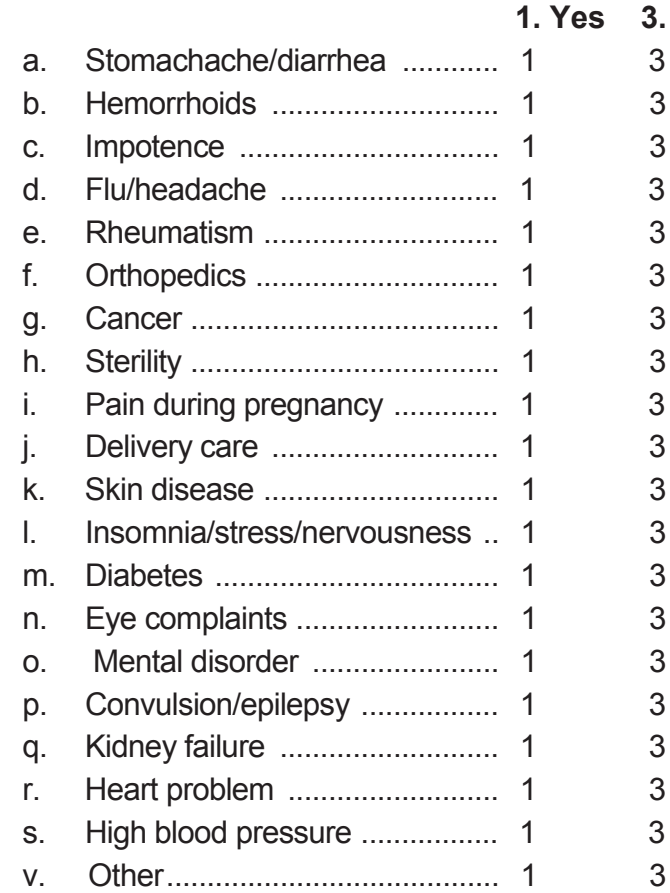

B06. How many patients did you examine/treat in the last one week?

B07. How many patients did you examine/treat in the last one month?

B08. In general, are your patients adults or children? female?

B10. In general, how long does each consultation ast? B15. In general, have your patients ever been you? $\downarrow \perp \perp$ patients

$\longleftarrow, \downarrow \perp \perp \_$patients

Adults ( $\geq 15$ years ) .............................

Children $(<15$ years $)$........................... 3

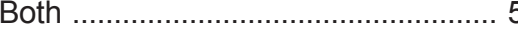

Male …………………………….... 1

Female …................................. 3

$\downarrow$ hours $\longleftarrow \perp$ minutes

Yes ………..................................... 1

No …………………………….... 3
B16. Where is the treatment usually carried out?

B16a.

you use any equipment or tools in

treating your patients?

B16b. What kind of equipment or tools do you normally use?

B16c. What kind of method of treatment do you use ? herbs?
The patient comes to your home

At special place for practice

You visit your patient's home/place .........

At the hospital

No $\quad 3 \rightarrow$ B16c

Yes

Medical ................................................ 1

Using animal as medium ......................... A

Using spells, charm

Using spells, charm …………................. $\mathrm{B}$

Using water as medium .............................. C

Using herb ............................................. D

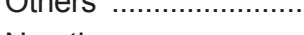

No other

$3 \rightarrow \mathrm{B} 19$

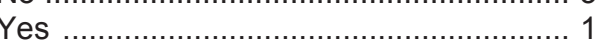




\section{SECTION B : PRACTICE ACTIVITIES}

\begin{tabular}{|c|c|c|c|c|}
\hline B18a. & $\begin{array}{l}\text { Kindly mention the use of the herbs you } \\
\text { use for medicine? }\end{array}$ & 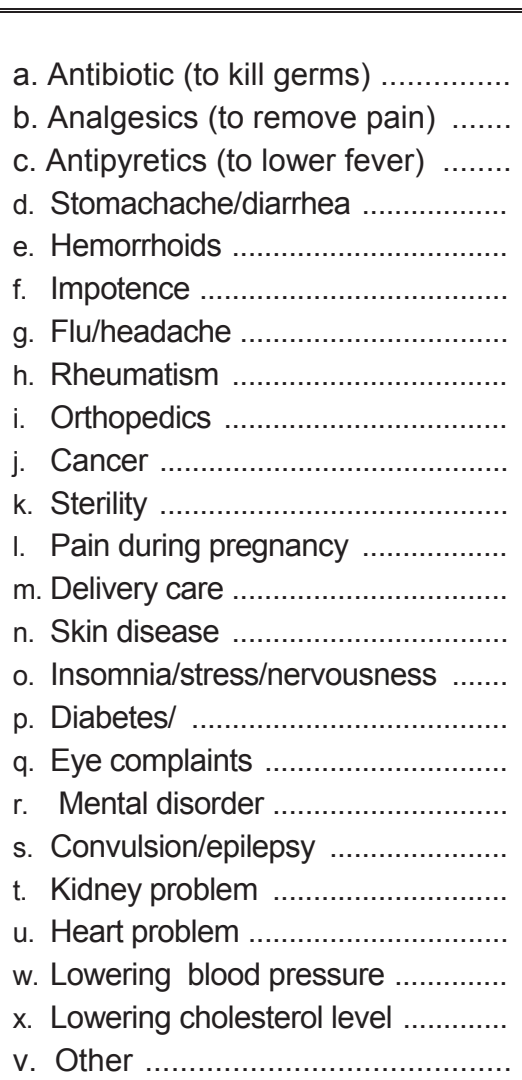 & $\begin{array}{l}1 . \text { Yes } \\
1 \\
1 \\
1 \\
1 \\
1 \\
1 \\
1 \\
1 \\
1 \\
1 \\
1 \\
1 \\
1 \\
1 \\
1 \\
1 \\
1 \\
1 \\
1 \\
1 \\
1 \\
1 \\
1 \\
1\end{array}$ & $\begin{array}{l}\text { 3. No } \\
3 \\
3 \\
3 \\
3 \\
3 \\
3 \\
3 \\
3 \\
3 \\
3 \\
3 \\
3 \\
3 \\
3 \\
3 \\
3 \\
3 \\
3 \\
3 \\
3 \\
3 \\
3 \\
3 \\
3\end{array}$ \\
\hline B19. & Do you also give modern medicine? & \multicolumn{3}{|c|}{ 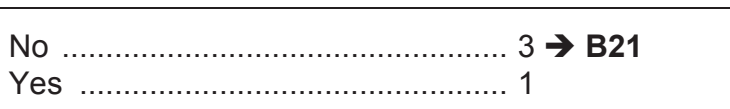 } \\
\hline B20. & $\begin{array}{l}\text { If "Yes", did you ever provide the } \\
\text { following medicine : } \\
\text { MENTION ALL ITEM BELOW }\end{array}$ & $\begin{array}{l}\text { a. Antibiotic (to kill germs) } \\
\text { b. Analgesics (to remove pain) ......... } \\
\text { c. Antipyretics (to lower fever) } \\
\text { d. Anti -TBC } \\
\text { e. Oralite } \\
\text { v. Other }\end{array}$ & $\begin{array}{l}1 . \text { Yes } \\
1 \\
1 \\
1 \\
1 \\
1 \\
1\end{array}$ & $\begin{array}{l}\text { 3. No } \\
3 \\
3 \\
3 \\
3 \\
3 \\
3\end{array}$ \\
\hline B21. & Do you provide FP services? & \multicolumn{3}{|c|}{$\begin{array}{l}\text { No } \\
\text { Yes }\end{array}$} \\
\hline
\end{tabular}

B22. If "Yes", what kind of FP services do you give?

a. Medicinal herbs

b. Other traditional

. Modern (pill, injection, condom) ...

Now we want to ask about fees that you charge or that you received from your patients.

\begin{tabular}{|c|c|c|}
\hline B11. & $\begin{array}{l}\text { Do you usually charge a fee for your } \\
\text { services? }\end{array}$ & 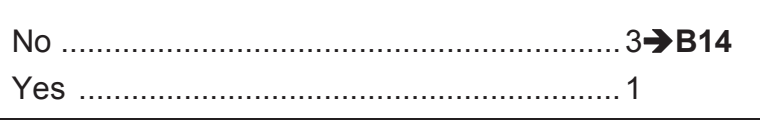 \\
\hline B12. & If you do, how much is the usual charge? & 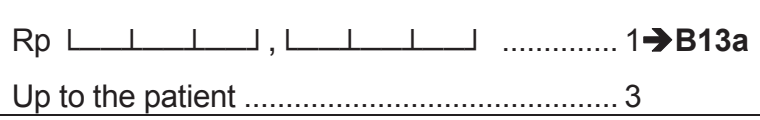 \\
\hline B13. & $\begin{array}{l}\text { Kindly mention the lowest and highest } \\
\text { amount your patients have given you? }\end{array}$ & 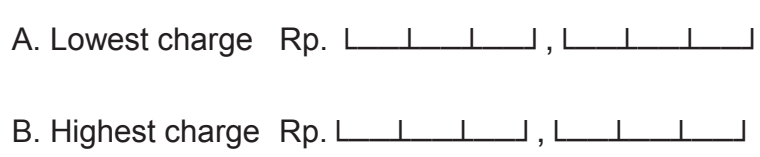 \\
\hline B13a & Does the charge include medicine? & Yes No \\
\hline B13b. & $\begin{array}{l}\text { How much do you usually charge } \\
\text { visitors/patients for medicinal herbs / } \\
\text { medicine or other prescriptions? }\end{array}$ & 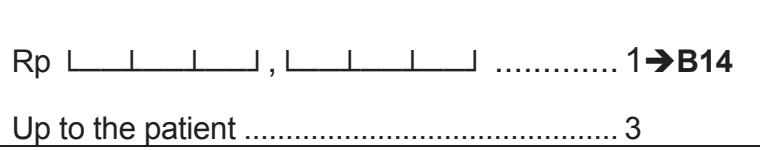 \\
\hline B13c. & $\begin{array}{l}\text { Kindly mention the lowest and highest } \\
\text { amount your patients have given you for } \\
\text { medicinal herbs/medicine or other } \\
\text { prescriptions? }\end{array}$ & 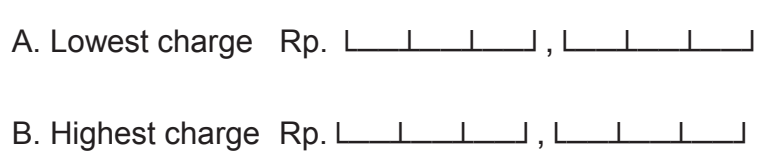 \\
\hline B14. & $\begin{array}{l}\text { Do the patients usually give }[\ldots] \text { as a } \\
\text { token of gratefulness? }\end{array}$ & 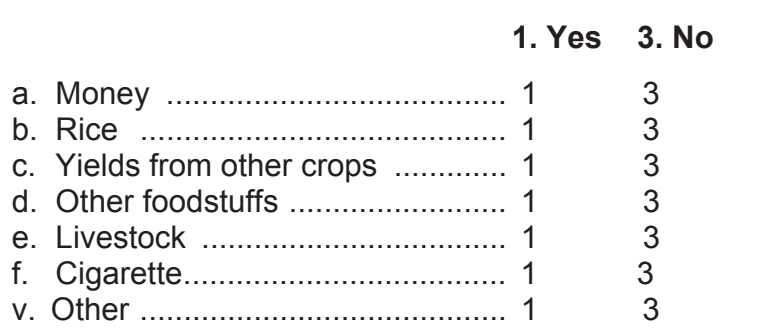 \\
\hline B14a. & How much is the value of those gifts? & 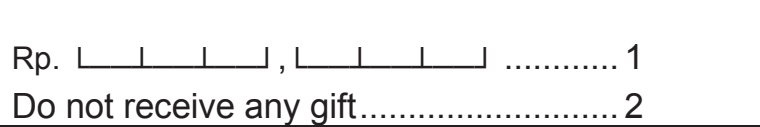 \\
\hline
\end{tabular}




\section{SECTION C (TRADITIONAL MIDWIFE)}

COO. INTERVIEWER CHECK POINT :

ARE YOU A TRADITIONAL MIDWIFE ?

Now we want to ask about services you provide as a traditional midwife.

\begin{tabular}{|c|c|c|}
\hline C01. & $\begin{array}{l}\text { Have you ever received training as a } \\
\text { traditional midwife? }\end{array}$ & No \\
\hline $\mathrm{Co2}$. & $\begin{array}{l}\text { In what year was your most recent } \\
\text { training? } \\
\text { IF FORGOTTEN, ASK HOW MANY } \\
\text { YEARS AGO IT WAS }\end{array}$ & 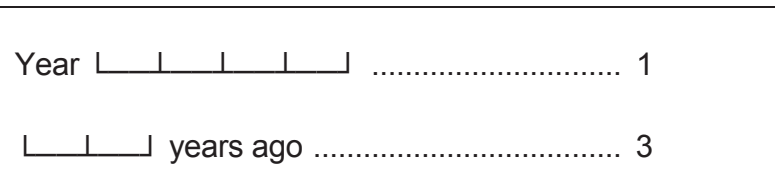 \\
\hline C03. & Who organized the most recent training ? & 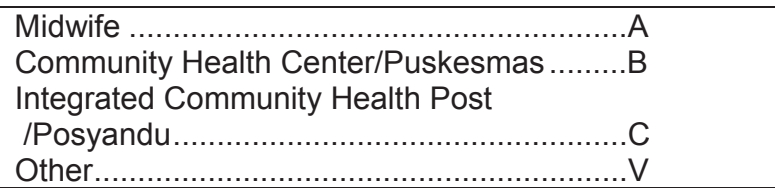 \\
\hline Co4. & $\begin{array}{l}\text { For how many days did you participate in } \\
\text { this training? }\end{array}$ & $\left\llcorner \_\_\right.$days \\
\hline C05. & $\begin{array}{l}\text { Where do you usually provide delivery } \\
\text { services? }\end{array}$ & 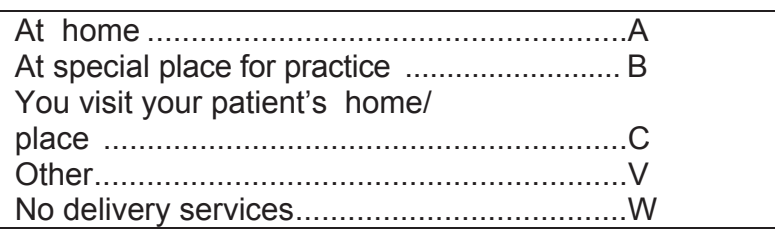 \\
\hline C06. & Do you also give TT immunization? & $\begin{array}{l}\text { Yes } \\
\text { No } \ldots \ldots \ldots \ldots \ldots \ldots \ldots \ldots \ldots \ldots \ldots \ldots \ldots \ldots \ldots \ldots \ldots \ldots \ldots \ldots \ldots \ldots .1 \\
\end{array}$ \\
\hline C07. & What is the usual fee for delivery charge? & 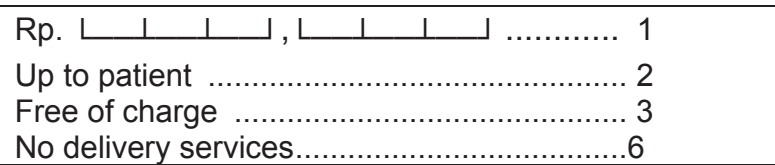 \\
\hline C07aa & $\begin{array}{l}\text { How much was the lowest and the highest } \\
\text { payment your patient ever paid you? }\end{array}$ & 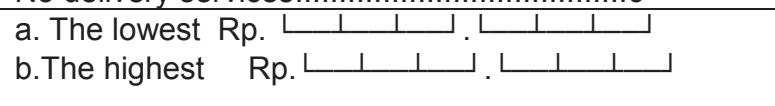 \\
\hline C07a. & $\begin{array}{l}\text { Do you provide traditional post natal care } \\
\text { for mother after delivery }\end{array}$ & 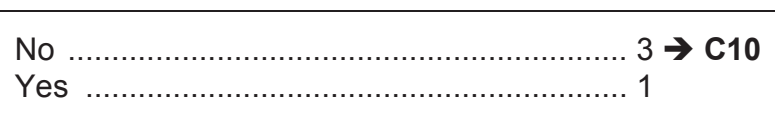 \\
\hline
\end{tabular}

\begin{tabular}{|c|c|c|}
\hline C08. & $\begin{array}{l}\text { In general, what is the fee for mother care } \\
\text { after delivery? }\end{array}$ & 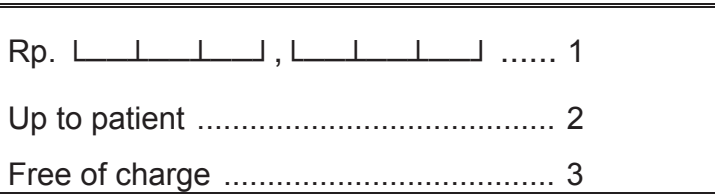 \\
\hline C08a & $\begin{array}{l}\text { How much was the lowest and the highest } \\
\text { payment your patient ever paid you? }\end{array}$ & 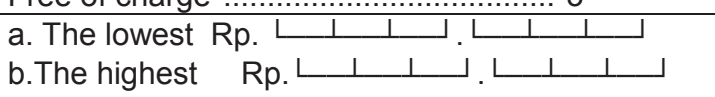 \\
\hline C09. & $\begin{array}{l}\text { For how long do you provide mother care } \\
\text { services after delivery? }\end{array}$ & $\left\llcorner \_\_\right.$days \\
\hline C10. & $\begin{array}{l}\text { Do you also provide care for new born } \\
\text { baby? }\end{array}$ & $\begin{array}{l}\text { No } \\
\text { Yes } \ldots \ldots . .1\end{array}$ \\
\hline C10a. & $\begin{array}{l}\text { For how long do you provide services for } \\
\text { new born baby? }\end{array}$ & $\downarrow \_$ل days \\
\hline C11. & $\begin{array}{l}\text { How much do you charge per visit for the } \\
\text { baby's care? }\end{array}$ & 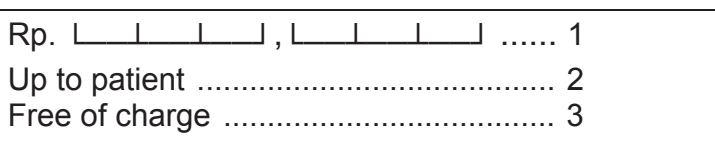 \\
\hline C11a & $\begin{array}{l}\text { How much was the lowest and the highest } \\
\text { payment your patient ever paid you? }\end{array}$ & 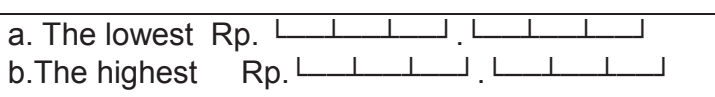 \\
\hline C12. & $\begin{array}{l}\text { Is immunization included in the baby's } \\
\text { care? }\end{array}$ & No \\
\hline C13. & What are the charges of immunization? & 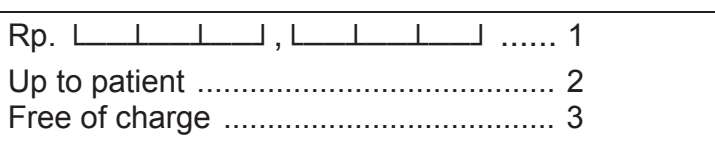 \\
\hline C13a & $\begin{array}{l}\text { How much was the lowest and the highest } \\
\text { payment your patient ever paid you? }\end{array}$ & 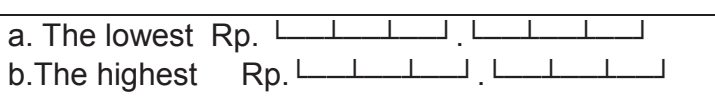 \\
\hline C14. & $\begin{array}{l}\text { Do you have the following instruments ? } \\
\text { MENTION ALL ITEM BELOW }\end{array}$ & 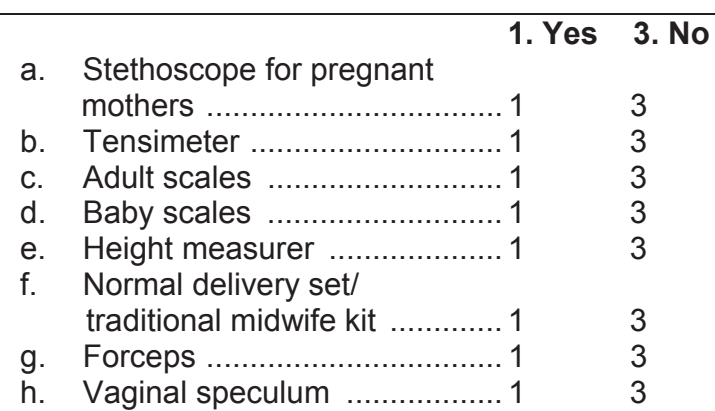 \\
\hline
\end{tabular}




\section{SECTION CP: INTERVIEWER NOTES}

\begin{tabular}{|c|c|c|c|c|c|c|c|}
\hline \multirow{2}{*}{$\begin{array}{l}\text { LANGMAIN (CK1). } \\
\text { LANGOTHR (CK2). }\end{array}$} & \multicolumn{2}{|l|}{ Interview was entirely/mostly conducted in what language? } & \multicolumn{5}{|l|}{$\llcorner$ ـ other } \\
\hline & Other languaged used (if any): & & \multicolumn{5}{|l|}{$\downarrow \downarrow \downarrow \downarrow$ other } \\
\hline LANGUAGE CODE: & & & & & & & \\
\hline 00. Bahasa Indonesia & 04. Batak & 08. Sasak & 12. Makassar & 16. & Toraja & & Lampung \\
\hline 01. Java & 05. Bugis & 09. Minang & 13. Nias & 17. & Lahat & 95. & Other, \\
\hline 02. Sunda & 06. Cina & 10. Banjar & 14. Palembang & 18. & Other South Sumatera & 96. & NA \\
\hline 03. Bali & 07. Madura & 11. Bima & 15. Sumbawa & 19. & Betawi & & \\
\hline
\end{tabular}

\begin{tabular}{|c|c|c|c|}
\hline RESULT (FP3). RESULTS OF INTERVIEW & REASON (FP4). REASON FOR ANSWERING “2” I "3" IN RESULT. & FP6. MONITORING BY SUPERVISOR & \\
\hline $\begin{array}{l}\text { 1. Completed } \rightarrow \text { FP6 } \\
\text { 2. Partly completed } \\
\text { 3. Not completed } \\
\text { 4. Twin EA with EA } \downarrow \perp \rightarrow F P 6\end{array}$ & $\begin{array}{l}\text { 1. Respondent was travelling/not in location } \\
\text { 2. Respondent was too busy } \\
\text { 3. Respondent refused }\end{array}$ & $\begin{array}{ll}\text { a. } & \text { Observed (sup_obs) .......... } 1 \\
\text { b. } & \text { Checked (sup_edit)........... } 1 \\
\text { c. } & \text { Verified (sup_veri)............. } 1\end{array}$ & $\begin{array}{l}\text { No } \\
3 \\
3 \\
3\end{array}$ \\
\hline
\end{tabular}

\section{INTERVIEWER NOTE:}




\begin{tabular}{|l|l|l|}
\hline INTERVIEWER: & CONFIDENTIAL & IDW: $\llcorner\perp\llcorner$ \\
\hline
\end{tabular}

\section{INDONESIAN FAMILY LIFE SURVEY 2014 \\ COMMUNITY AND FACILITY SURVEY SCHOOL QUESTIONNAIRE A}

SCHOOL PRINCIPAL BOOK

SECTION: LK, A, B, SC, E, H, CP

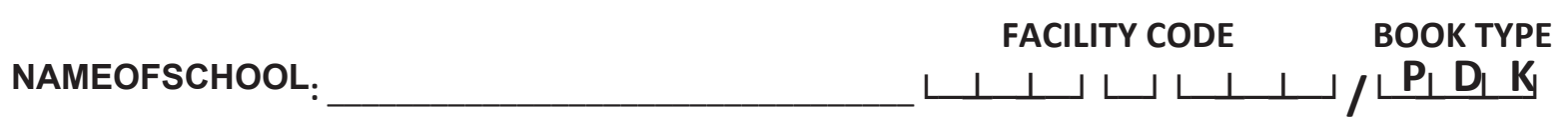




\section{SECTION LK: CONTROL SHEET}

\begin{tabular}{|c|c|c|c|}
\hline & \multicolumn{2}{|r|}{ Sampling Information } & CODE \\
\hline LK01. & \multicolumn{2}{|l|}{ Province } & 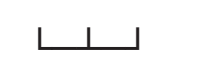 \\
\hline LK02. & \multicolumn{2}{|l|}{ Kabupaten/ Kota } & $\llcorner\perp$ \\
\hline LK03. & \multicolumn{2}{|l|}{ Kecamatan } & ᄂ \\
\hline LK04. & \multicolumn{2}{|c|}{ Desa/Kelurahan/Nagari } & $\downarrow \downarrow$ \\
\hline LK05. & \multicolumn{2}{|c|}{ Region: 1. Urban $\quad$ 2. Rural } & $\sqcup$ \\
\hline LK07a. & \multicolumn{3}{|c|}{$\begin{array}{l}\text { Location of facility: a. Latitude } \\
\qquad \begin{array}{l}\text { b. Longitude } \\
\text { c. Accuracy } \\
\text { d. Elevation }\end{array}\end{array}$} \\
\hline LK08. & \multicolumn{3}{|c|}{$\begin{array}{ll}\text { a. Address } & : \\
\text { b. Description of location: } & : 1 \\
\text { c.Postal code } & : L 1 / L\end{array}$} \\
\hline LK09. & \multicolumn{3}{|c|}{ 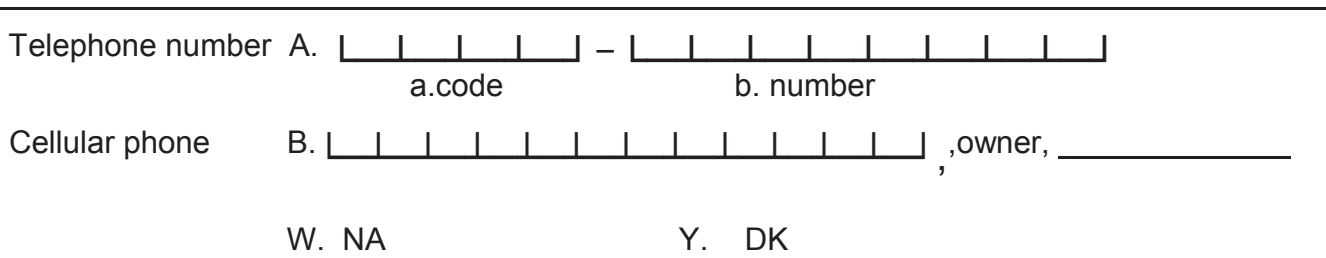 } \\
\hline LK09a & \multirow{2}{*}{\multicolumn{3}{|c|}{ 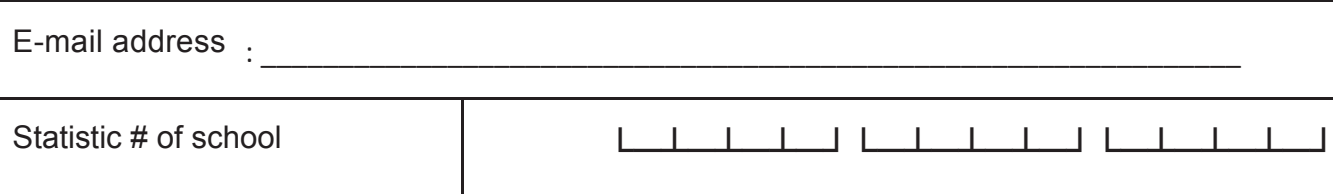 }} \\
\hline LK10. & & & \\
\hline
\end{tabular}

\begin{tabular}{|c|c|c|}
\hline & Supervision & CODE \\
\hline LK15. & Name of Interviewer & ل \\
\hline LK17. & Name of Local Supervisor & لـ \\
\hline LK19. & Name of Field Coordinator & L 11 \\
\hline
\end{tabular}

\begin{tabular}{|c|c|c|}
\hline LK20 & Was the school ever interviewed in $1993 ?$ & 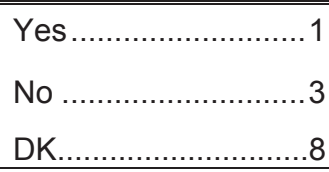 \\
\hline LK21 & Was the school ever interviewed in $1997 ?$ & 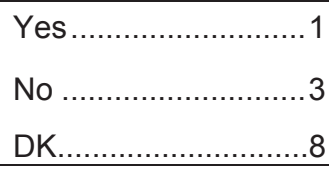 \\
\hline LK22 & Was the school ever interviewed in $1998 ?$ & 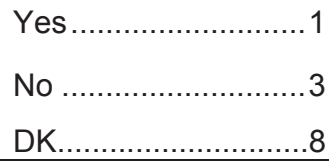 \\
\hline LK23 & Was the school ever interviewed in $2000 ?$ & 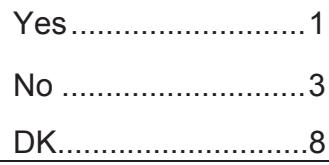 \\
\hline LK24 & Was the school ever interviewed in $2007 ?$ & 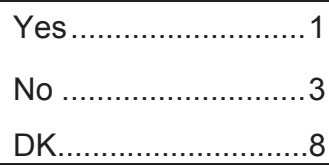 \\
\hline
\end{tabular}


SECTION A: SCHOOL PRINCIPAL

\begin{tabular}{|c|c|}
\hline KRTYPE & $\begin{array}{l}\text { RESPONDENT A } \\
\text { A. PRINCIPAL }\end{array}$ \\
\hline KR1_A Respondent & \\
\hline $\begin{array}{ll}\text { KR1a_A } & \begin{array}{l}\text { Posttion/occupation in } \\
\text { this School: }\end{array}\end{array}$ & $\begin{array}{lllll}01 & 02 & 03 & 04 & 05 \\
06 & 07 & 08 & 95 & \end{array}$ \\
\hline KR2_A Age : & $\llcorner\perp$ Years \\
\hline $\begin{array}{ll}\text { KR2a_A } & \begin{array}{l}\text { Length of tenure in } \\
\text { Position s: }\end{array}\end{array}$ & 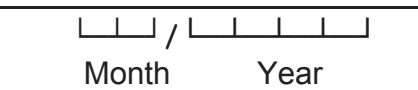 \\
\hline $\begin{array}{ll}\text { KR3_A } & \text { INTERVIEWER CHECK : } \\
& \text { SEX OF RESPONDENT }\end{array}$ & 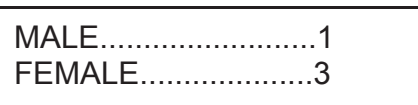 \\
\hline $\begin{array}{l}\text { KR3a_A Highest level of education } \\
\text { attended: }\end{array}$ & 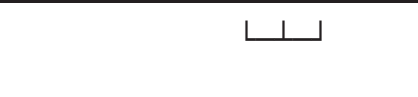 \\
\hline KR4_A Highest class completed & $\begin{array}{ccccc}00 & 01 & 02 & 03 & 04 \\
05 & 06 & 07 & 98 & 95\end{array}$ \\
\hline $\begin{array}{l}\text { KR5_A Length of time lived in the } \\
\text { village: }\end{array}$ & 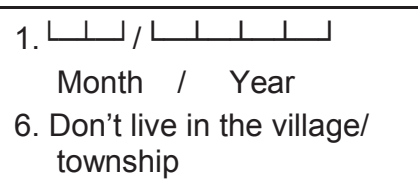 \\
\hline $\begin{array}{l}\text { KR6_AHow did }[\ldots] \text { come to assume } \\
\text { to the position? }\end{array}$ & $\begin{array}{l}\text { 1. Elected } \\
\text { 2. Appointed by Goverment } \\
\quad \text { / Education District Office } \\
\text { 3. Appointed byFoundation } \\
\text { 5.Other }\end{array}$ \\
\hline
\end{tabular}

Code for position in village (KR1a)

01. Village/township heads

03. Head of Government Administration

04. Head of Village Development

05. Head of Welfare

06. Head of Financial Matters

07. Head of General Affairs
Codes for highest level of education

$$
\text { (KR3a): }
$$

01. No school/not yet in school

02. Elementary school

03. Junior High - General

5. High School - General

06. High School - Vocational

60. D1, D2, D3 (Junior College)

62. University $\mathrm{S1}$ (Bachelor)

63. University S3 (Ph D.)
11. Kejar Paket $A$ (Adult Education $A$ )

12. Kejar Paket $B$ (Adult Education $B$ )

13. Open Univeristy

4. Islamic School (Pondok Pensantren)

15. Kejar Paket C (Adult Education C)

2. Islamic Elementary School (Madrasah Ibtidaiyah)

74. Islamic Senior High School (Madrasah Allyah)

9. DON'T KNOW

95. Other
Codes for highest grade (KR4):

00. Never completed class

01.1

03.

04.4

06. 6

07. Graduated

07. Graduate

96. No school 


\section{SECTION A: SCHOOL PRINCIPAL}

If the respondent is not School Principal, the questions should be asked with respect to the School Principal

\begin{tabular}{|c|c|c|}
\hline A1. & $\begin{array}{l}\text { a. Name of School Principal: } \\
\text { b. If Respondent is the School Principal } \\
\text { 1.Yes } \rightarrow \text { A } 3 \\
\text { 3.No, he is not the School Principal }\end{array}$ & 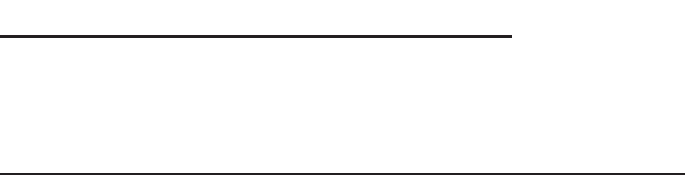 \\
\hline A2. & $\begin{array}{l}\text { a. Name of Respondent : } \\
\text { b. Position of Respondent : }\end{array}$ & \\
\hline A3. & $\begin{array}{l}\text { When were you (the School Principal) born? } \\
\text { (IF HE/SHE HAS FORGOTTEN THE BIRTH } \\
\text { DATE, PLEASE ASK THE PRESENT AGE) }\end{array}$ & 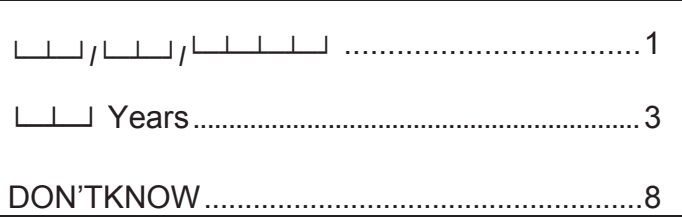 \\
\hline A4. & Sex of the School Principal & 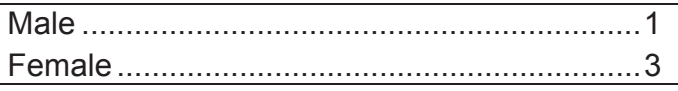 \\
\hline A5. & $\begin{array}{l}\text { When was the School Principal appointed } \\
\text { Principal of this school? }\end{array}$ & Month \\
\hline A6. & $\begin{array}{l}\text { What is the highest level of education from } \\
\text { which the School Principal graduated? }\end{array}$ & 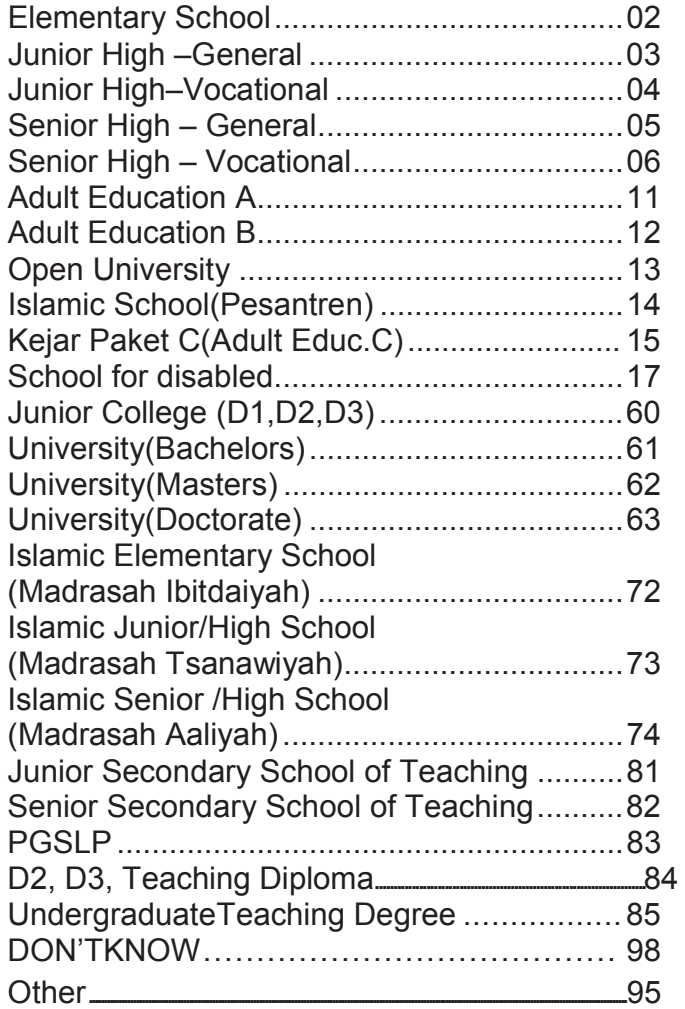 \\
\hline
\end{tabular}

\begin{tabular}{|c|c|c|}
\hline A6a & $\begin{array}{l}\text { What is the highest grade/class completed by } \\
\text { the School Principal? }\end{array}$ & 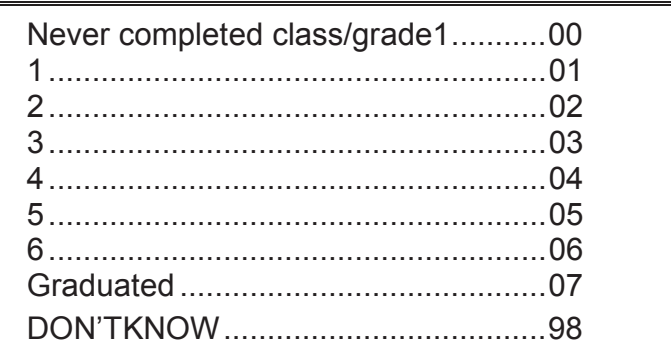 \\
\hline A7 & $\begin{array}{l}\text { Please mention the year you/School Principal } \\
\text { graduated from highest level of education? }\end{array}$ & 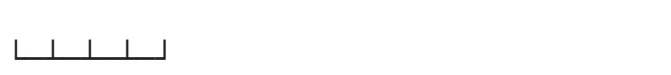 \\
\hline A8. & $\begin{array}{l}\text { Who appointed you /School Principal as a } \\
\text { School Principal at this school? }\end{array}$ & 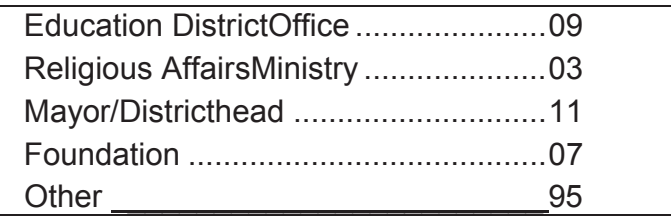 \\
\hline A8a & $\begin{array}{l}\text { Approximately how much is the net salary from } \\
\text { the job as School Principal? }\end{array}$ & 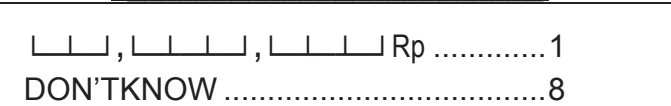 \\
\hline A8aa & $\begin{array}{l}\text { How much do you earn per month as school } \\
\text { principal of this school? }\end{array}$ & 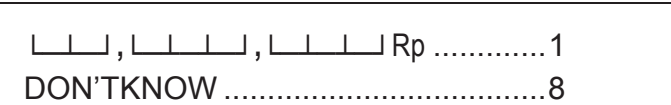 \\
\hline A8b & $\begin{array}{l}\text { A side the main salary, did you/he/she } \\
\text { also received the allowance / } \\
\text { subsidy,i. e.: }\end{array}$ & 1. Yes \\
\hline & 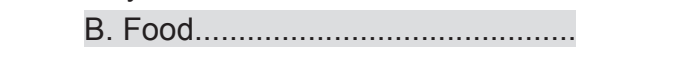 & 1.Yes \\
\hline & C.Housing & 1. Yes \\
\hline & D. Transportation ................................. & 1.Yes \\
\hline & $\begin{array}{l}\text { E .Health Insurance................................ } \\
\text { G. Structural Benefitt. }\end{array}$ & 1. Yes \\
\hline & H. Professional Benefit....................... & 1.Yes \\
\hline & Familv Benefit & 1.Yes \\
\hline & J. Incentive........... & 1.Yes \\
\hline & K. Certification Allowance & 3. No \\
\hline & V. Other & 1. Yes \\
\hline A9. & $\begin{array}{l}\text { Aside from being a School Principal here, } \\
\text { were you/he/she ever a School Principal } \\
\text { else where? }\end{array}$ & 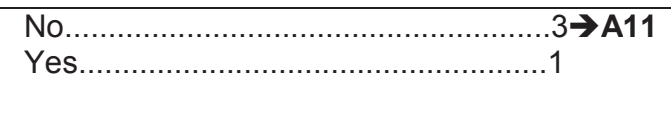 \\
\hline A10. & $\begin{array}{l}\text { How long did you serve as a School } \\
\text { Principal at those other schools? }\end{array}$ & 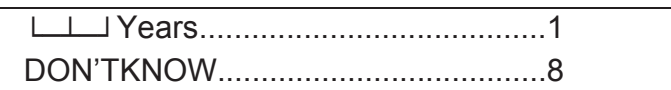 \\
\hline
\end{tabular}




\begin{tabular}{|c|c|c|}
\hline A11. & $\begin{array}{l}\text { Aside from being a School } \\
\text { Principal in this school, do you } \\
\text { hold any other job? }\end{array}$ & $\begin{array}{l}\text { No } \\
\text { Yes }\end{array}$ \\
\hline A12. & $\begin{array}{l}\text { If yes, howmany hours } \\
\text { perweek do you spend in that } \\
\text { job? }\end{array}$ & $\begin{array}{l}\text { 1. } L \perp ل \text { hours per week } \\
\text { 8. DON'TKNOW }\end{array}$ \\
\hline A12d. & $\begin{array}{l}\text { What is the status of your other } \\
\text { job? }\end{array}$ & 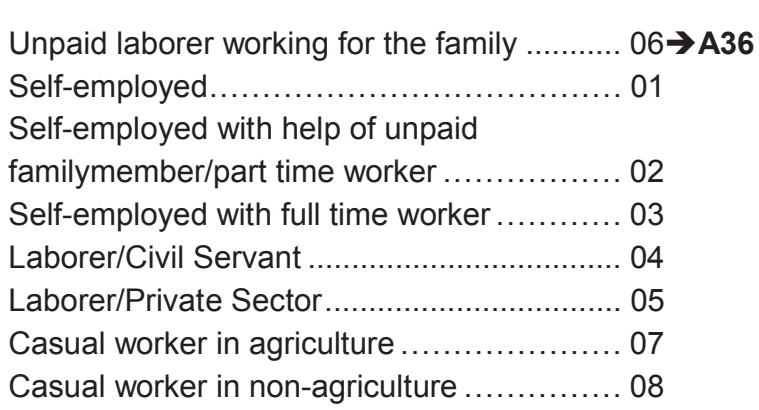 \\
\hline A12e. & $\begin{array}{l}\text { Approximately, how much } \\
\text { is your net salary from } \\
\text { your other job? }\end{array}$ & 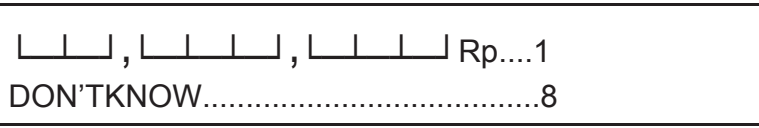 \\
\hline A36. & $\begin{array}{l}\text { Is Mr/ Mrs knows about the } \\
\text { minimum standart of grade } \\
\text { school service? }\end{array}$ & 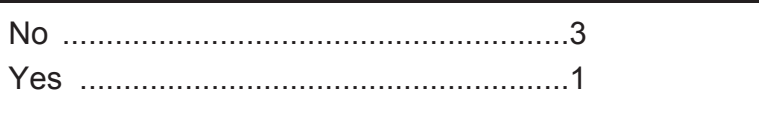 \\
\hline
\end{tabular}

\begin{tabular}{|c|c|c|}
\hline \multirow{2}{*}{\multicolumn{2}{|c|}{ ATYPE }} & A38 \\
\hline & & $\begin{array}{l}\text { The previous school year }(2013 \text { / 2014) did this } \\
\text { school have }[\ldots] \text { ? }\end{array}$ \\
\hline A & $\begin{array}{l}\text { Mid-term working plan/ Rencana Kerja } \\
\text { Jarak Menengah ( RKJM ) }\end{array}$ & Yes \\
\hline$B$ & $\begin{array}{l}\text { Annual working plan/ Rencana Kerja } \\
\text { Tahunan (RKT) }\end{array}$ & Yes \\
\hline C & $\begin{array}{l}\text { School Budget / Rencana anggaran } \\
\text { sekolah (RKAS)/Document of School } \\
\text { Budget /Dokumen Perencanaan } \\
\text { Anggaran ( DPA) }\end{array}$ & 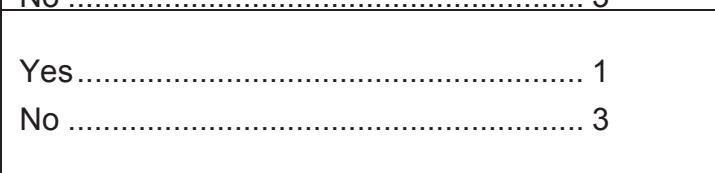 \\
\hline
\end{tabular}


SECTION A: SCHOOL PRINCIPAL

\begin{tabular}{|c|c|c|c|c|}
\hline & A13c. & A13d. & $\begin{array}{ll}\text { A13e. } \\
\end{array}$ & A13f. \\
\hline ATYPE & $\begin{array}{l}\text { In the last } 5 \text { years, did you } \\
\text { ever attend any [...] } \\
\text { training in keeping with the } \\
\text { duties of School Principal? }\end{array}$ & $\begin{array}{l}\text { When did you participate in the } \\
\text { last training[...]? }\end{array}$ & $\begin{array}{c}\text { How long did you participate in this } \\
\text { training? } \\
\text { (INTERVIEWER NOTE: IF THE RESPONDENT } \\
\text { HAS ATTENDED MORE THAN ONE TRAINING IN THE LAST } 5 \\
\text { YEARS, RECORD THE INFORMATION REGARDING THE MOST } \\
\text { RECENT TRAINING.) }\end{array}$ & $\begin{array}{l}\text { How many hours per week did you attend the } \\
\text { training? }\end{array}$ \\
\hline a. Management & $\begin{array}{l}\text { 1.Yes } \rightarrow \\
\text { 3.No } \downarrow\end{array}$ & 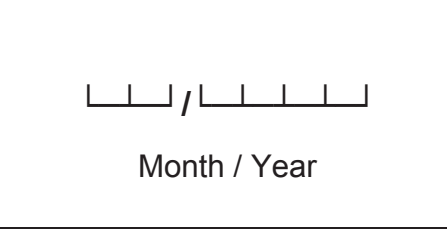 & $\begin{array}{ll}\text { 1. } & \perp-1 \text {. days } \\
\text { 4. weeks } \\
\text { 5. months } \\
\text { 8. DON'TKNOW }\end{array}$ & $\begin{array}{l}\text { 1. } \downarrow \downarrow \downarrow \text { hours per week } \\
\text { 8. DON'TKNOW }\end{array}$ \\
\hline b. Teaching skills & $\begin{array}{l}\text { 1.Yes } \rightarrow \\
\text { 3.No } \downarrow\end{array}$ & 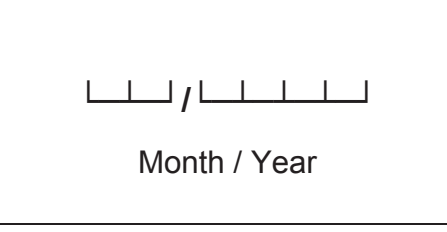 & 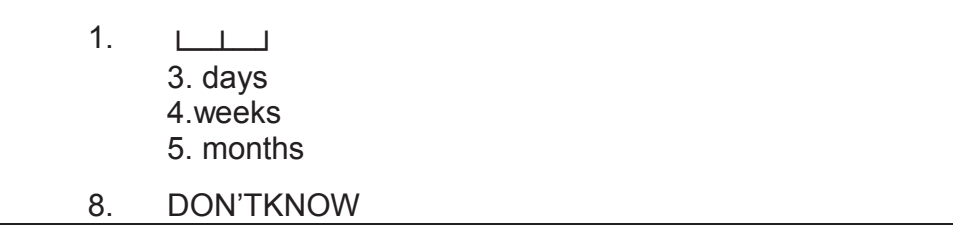 & $\begin{array}{l}\text { 1. } \sqcup \downarrow \text { hours per week } \\
\text { 8. DON'TKNOW }\end{array}$ \\
\hline c. Subject-related & $\begin{array}{l}\text { 1.Yes } \rightarrow \\
\text { 3.No } \downarrow\end{array}$ & 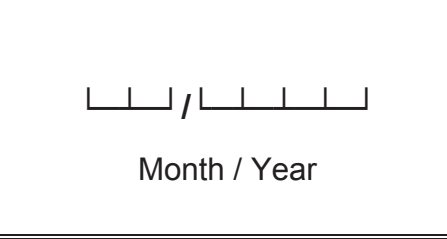 & \begin{tabular}{ll} 
1. & \multicolumn{1}{|c}{ 3. days } \\
& 4.weeks \\
& 5. months \\
8. & DON'TKNOW \\
\end{tabular} & $\begin{array}{l}\text { 1. } \sqcup \sqcup \text { hours per week } \\
\text { 8. DON'TKNOW }\end{array}$ \\
\hline
\end{tabular}




\begin{tabular}{|c|c|c|c|c|c|}
\hline \multirow[t]{14}{*}{ A15. } & $\begin{array}{l}\text { During the } 2013 / 2014 \text { School Year, did } \\
\text { you engage in the following activities: }\end{array}$ & 1.Yes & 3.No & 6.NA & 8.DON'TKNOW \\
\hline & a. Act as a substitute teacher ....................... & a. 1 & 3 & 6 & 8 \\
\hline & $\begin{array}{l}\text { b. Supervise teachers in class } \\
\text { preparation }\end{array}$ & b. 1 & 3 & 6 & 8 \\
\hline & c. Supervise the teaching process in & & & & \\
\hline & 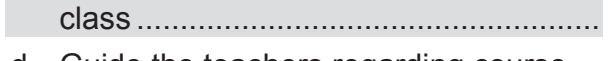 & c. 1 & 3 & 6 & 8 \\
\hline & $\begin{array}{l}\text { d. Guide the teachers regarding course } \\
\text { material }\end{array}$ & d. 1 & 3 & 6 & 8 \\
\hline & e. Discuss the curriculum ............................ & e. 1 & 3 & 6 & 8 \\
\hline & f. Organize meetings with the parents ...... & f. 1 & 3 & 6 & 8 \\
\hline & g. Make comparative studies .......................... & g. 1 & 3 & 6 & 8 \\
\hline & $\begin{array}{l}\text { h. Attend seminars and meetings .......... } \\
\text { i. Discuss school planning with the }\end{array}$ & h. 1 & 3 & 6 & 8 \\
\hline & Regional Office or Foundation .......... & i. 1 & 3 & 6 & 8 \\
\hline & 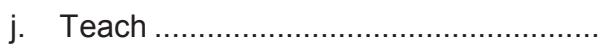 & j. 1 & 3 & 6 & 8 \\
\hline & $\begin{array}{l}\text { k. Conduct the meeting with the school } \\
\text { committee }\end{array}$ & k. 1 & 3 & 6 & 8 \\
\hline & $\begin{array}{l}\text { I. Follow the meeting with teacher and } \\
\text { principal from the other shool.............. }\end{array}$ & I. 1 & 3 & 6 & 8 \\
\hline A22. & $\begin{array}{l}\text { Are you aware of theTeacher Certification } \\
\text { program? }\end{array}$ & $\begin{array}{l}\text { No........ } \\
\text { Yes...... }\end{array}$ & …....... & $\ldots \ldots \ldots$ & $\ldots \ldots \ldots \ldots . .1$ \\
\hline A23. & $\begin{array}{l}\text { Has there been as socialization about the } \\
\text { Teacher Certification program? }\end{array}$ & $\begin{array}{l}\text { No........ } \\
\text { Yes....... }\end{array}$ & …......... & (n................... & $\begin{array}{ll}\ldots \ldots \ldots .3 \\
\ldots \ldots \ldots \ldots .1\end{array}$ \\
\hline A24. & $\begin{array}{l}\text { Doyou know about the procedure } \\
\text { (including the criteria) to obtain Teacher } \\
\text { Certification? }\end{array}$ & $\begin{array}{l}\text { No........ } \\
\text { Yes....... }\end{array}$ & …...... & ............. & $\ldots \ldots \ldots . .3$ \\
\hline A25. & $\begin{array}{l}\text { How many teachers have applied for the } \\
\text { Certification or have submitted the } \\
\text { required documents for Certification? }\end{array}$ & $\begin{array}{l}\text { 1. } L \perp \\
\text { 8.DK }\end{array}$ & \lrcorner tea & & \\
\hline A26. & $\begin{array}{l}\text { How many teachers have received } \\
\text { Certification? }\end{array}$ & $\begin{array}{l}\text { 1. L } \\
\text { 6.NA } \rightarrow \\
\text { 8.DK } \rightarrow\end{array}$ & $\begin{array}{l}\sqcup \text { tec } \\
27 \\
27\end{array}$ & & \\
\hline A26a. & What are the lesson teacher? & $\begin{array}{ll}\text { A. } & \mathrm{S} \\
\text { B. } & \mathrm{E} \\
\text { C. } & \mathrm{M} \\
\text { D. } & \text { In } \\
\text { E. } & \mathrm{C} \\
\text { V. } & \mathrm{O} \\
\text { W. } & \mathrm{N}\end{array}$ & $\begin{array}{l}\text { ence } \\
\text { glish } \\
\text { them } \\
\text { onesi } \\
\text { iss tea } \\
\text { eers } \\
\text { NE }\end{array}$ & & \\
\hline
\end{tabular}

\begin{tabular}{|c|c|c|}
\hline A27. & $\begin{array}{l}\text { What curriculum do you use } \\
\text { in this school for the current } \\
\text { academic year? }\end{array}$ & 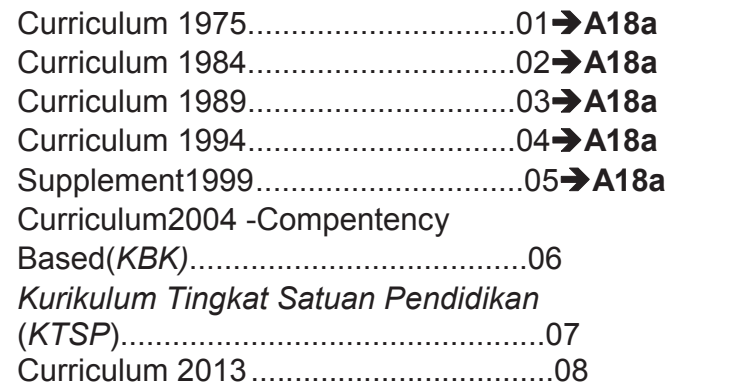 \\
\hline A28. & $\begin{array}{l}\text { What is/are the local } \\
\text { contents of the } \\
\text { KTSP/KBK in this school? }\end{array}$ & $\begin{array}{l}\text { A. Language (local/foreign language) } \\
\text { B. Science (prepare to UAN/UN, etc.) } \\
\text { C. Vocational skills } \\
\text { D.Tourism } \\
\text { V.Other }\end{array}$ \\
\hline A34. & $\begin{array}{l}\text { Does teacher gives Mr/Mrs } \\
\text { the copy of student test } \\
\text { result every semester than } \\
\text { Mr/Mrs report that result to } \\
\text { the official education at } \\
\text { kabupaten/city? }\end{array}$ & $\begin{array}{l}\text { Yes, for the headmaster or the head of official......1 } \\
\text { Yes, for headmaster only }\end{array}$ \\
\hline A18a. & $\begin{array}{l}\text { INTERVIEWER NOTE: } \\
\text { IS THIS SCHOOL A } \\
\text { PRIMARY SCHOOL? }\end{array}$ & 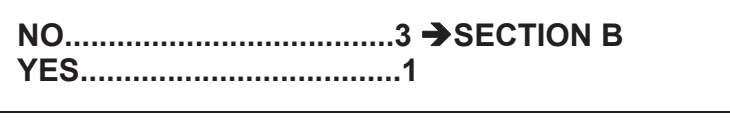 \\
\hline A19. & $\begin{array}{l}\text { Is there a school lunch } \\
\text { program } \\
\text { (PMTAS) in this school? }\end{array}$ & 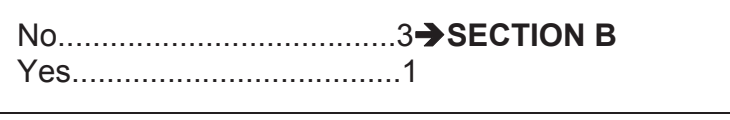 \\
\hline A19a. & $\begin{array}{l}\text { How many days aweek } \\
\text { does this school offer a } \\
\text { PMTAS snack? }\end{array}$ & $\sqcup$ days \\
\hline
\end{tabular}




\section{SECTION A: SCHOOL PRINCIPAL}

\begin{tabular}{|c|c|c|c|c|c|c|c|}
\hline A2TYPE & Never & $\begin{array}{c}\text { More than } 1 \text { times per } \\
\text { mounth }\end{array}$ & $\begin{array}{c}\text { More than } 1 \text { times per } \\
\text { week }\end{array}$ & 1 times per week & 2 times per week & 3 times per week & 4 times per week \\
\hline a. Milk & 1 & 2 & 3 & 4 & 5 & 6 & 7 \\
\hline c. Cake/bread & 1 & 2 & 3 & 4 & 5 & 6 & 7 \\
\hline d. Cassava/rice & 1 & 2 & 3 & 4 & 5 & 6 & 7 \\
\hline e. Noodles & 1 & 2 & 3 & 4 & 5 & 6 & 7 \\
\hline g. Eggs & 1 & 2 & 3 & 4 & 5 & 6 & 7 \\
\hline h. Tempe/tofu. & 1 & 2 & 3 & 4 & 5 & 6 & 7 \\
\hline i. Fish/meat & 1 & 2 & 3 & 4 & 5 & 6 & 7 \\
\hline
\end{tabular}

A21. Where does the funding come from for the school lunch program? (CIRCLE ALL THAT APPLY)

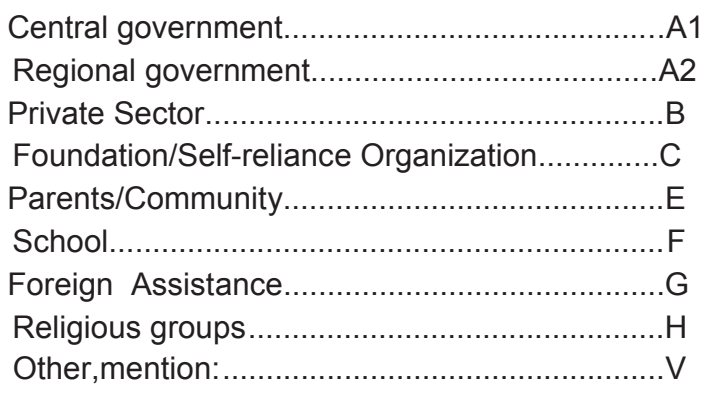




\section{SECTION B: SCHOOL}

\begin{tabular}{|c|c|}
\hline $\begin{array}{l}\text { KR1_B } \\
\text { Respondent (CAPI WILL SKIP DIRECTLY } \\
\text { TO B1 IF THE NAME OF RESPONDENT } \\
\text { HAVE EVER ANSWERED IN THE PREVIOUS } \\
\text { SECTION) }\end{array}$ & \\
\hline $\begin{array}{l}\text { KR1a_B Posttion/occupation in } \\
\text { this School: }\end{array}$ & $\begin{array}{lllll}01 & 02 & 03 & 04 & 05 \\
06 & 07 & 08 & 95 & \end{array}$ \\
\hline KR2_B & $\llcorner\sqcup$ Years \\
\hline $\begin{array}{ll}\text { KR2a_B } & \text { Length of tenure in } \\
& \text { Position s: }\end{array}$ & 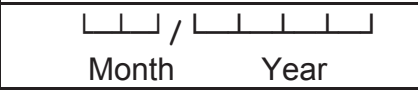 \\
\hline $\begin{array}{ll}\text { KR3_B } & \text { INTERVIEWER CHECK : } \\
& \text { SEX OF RESPONDENT }\end{array}$ & 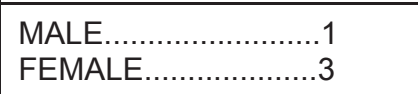 \\
\hline KR3a_B Highest level of education attended: & 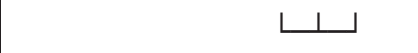 \\
\hline KR4_B Highest class completed & $\begin{array}{ccccc}00 & 01 & 02 & 03 & 04 \\
05 & 06 & 07 & 98 & 95 \\
\end{array}$ \\
\hline KR5_B Length of time lived in the village : & 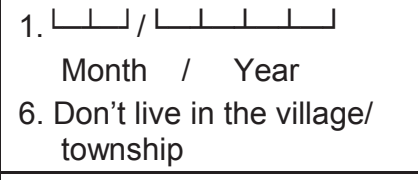 \\
\hline $\begin{array}{l}\text { KR6_B How did [...] come to assume to the } \\
\text { position? }\end{array}$ & $\begin{array}{l}\text { 1. Elected } \\
\text { 2. Appointed by Goverment } \\
\text { / Education District Office } \\
\text { 3. Appointed byFoundation } \\
\text { 5.Other }\end{array}$ \\
\hline
\end{tabular}

Code for position in village (KR1a)

08. Village/township heads

010. Head of Government Administration

010. Head of Government Administ
011 . Head of Village Development

012. Head of Welfare

013. Head of Financial Matters

014. Head of General Affairs

95. Other
Codes for highest level of education

KR3a):

01. No school/not yet in school

03. Elementary school

04. Junior High - Goneral

05. High School - General

06. High School - Vocational

60. D1, D2, D3 (Junior College)

61. University S1 (Bachelor)

2. University S2 (Masters)

63. University S3 (Ph.D.)
11. Kejar Paket $\mathrm{A}$ (Adult Education $\mathrm{A}$ )

Kejar Paket B (Adult Education B)

3. Open Univeristy
4. Islamic School (Pondok Pensantren)

5. Kejar Paket C (Adult Education C)

17. School for the disabled

72. Islamic Elementary School (Madrasah Ibtidaiyah)

74. Islamic Senior High School (Madrasah Alliyah)

90. Kindergarten

95. Other
Codes for highest grade (KR4):

00. Never completed class 1

01.

02.

03. 3

04. 4

05. 5

06. 6

07. Graduated

96. No school

98. DON'T KNOW 


\begin{tabular}{|c|c|c|}
\hline B1. & What year was this school founded? & 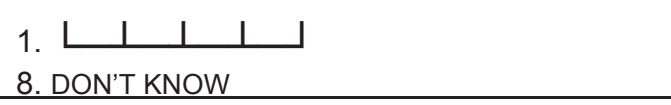 \\
\hline B1e. & What is the classification of the school? & 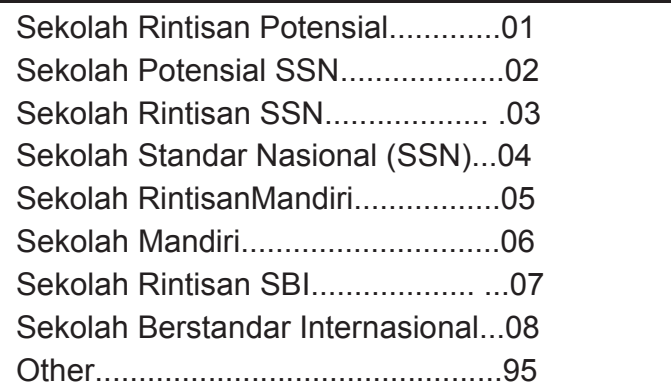 \\
\hline B1f. & $\begin{array}{l}\text { Since when was the school classified } \\
\text { as the above? }\end{array}$ & 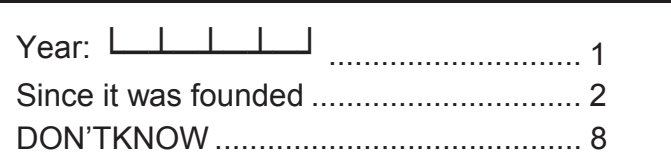 \\
\hline B1g. & $\begin{array}{l}\text { Does this school have an } \\
\text { international class? }\end{array}$ & 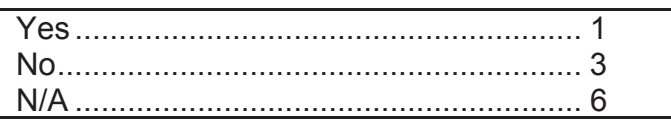 \\
\hline B1h. & $\begin{array}{l}\text { Does this school have an Accelaration } \\
\text { Class? }\end{array}$ & 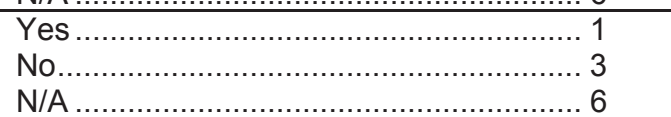 \\
\hline B1i. & What is the accreditation of this school? & 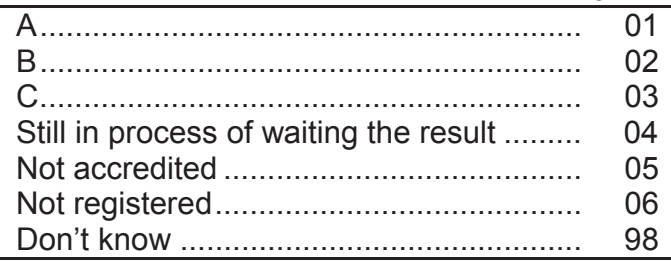 \\
\hline B1j & Teaching language used in this school? & $\begin{array}{ll}\text { A. } & \text { Indonesian } \\
\text { B. English } \\
\text { C. Local Language / Vernacular } \\
\text { D. Mandarin } \\
\end{array}$ \\
\hline B1c. & $\begin{array}{l}\text { Has this school ever been closed? } \\
\text { If yes, howmany year was this } \\
\text { school closed? }\end{array}$ & $\begin{array}{l}\text { 1. } L \perp ل \text { Years } \\
\text { 3. Never been closed for an academic year }\end{array}$ \\
\hline B2a. & $\begin{array}{l}\text { Did this school ever close down for at } \\
\text { least } 1 \text { week in the } 2013 / 2014 \text { school } \\
\text { year, not including regularly } \\
\text { scheduled holidays/vacations? } \\
\text { (EXCLUDINGSCHEDULEDSCHOO } \\
\text { L HOLIDAYS) }\end{array}$ & $\begin{array}{l}\text { No } \\
\text { DON'TKNOW } \\
\text { Yes }\end{array}$ \\
\hline
\end{tabular}

\begin{tabular}{|c|c|c|}
\hline \multirow[t]{2}{*}{ B3. } & \multirow[t]{2}{*}{$\begin{array}{l}\text { If yes, mention the period when } \\
\text { the school activities stopped: }\end{array}$} & 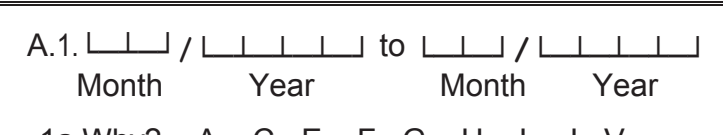 \\
\hline & & \\
\hline & \multirow{2}{*}{$\begin{array}{l}\text { CODE: } \\
\text { A. Smoke } \\
\text { C. Budgetary short fall } \\
\text { E. Earthquake } \\
\text { F. Landslide } \\
\text { G. Flood } \\
\text { H. Tsunami } \\
\text { I. Fire } \\
\text { J. Strong winds } \\
\text { V. Other }\end{array}$} & 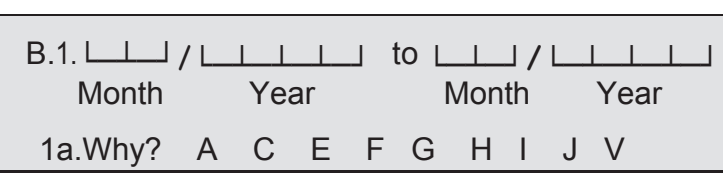 \\
\hline & & 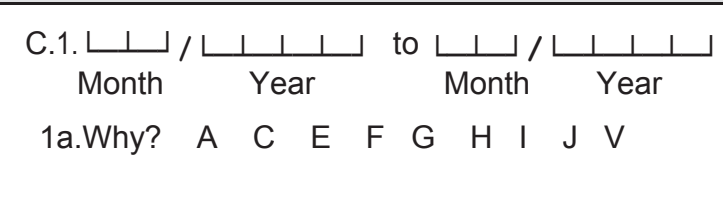 \\
\hline B3a & $\begin{array}{l}\text { During school year } 2013 / 2014 \text { were } \\
\text { any of the school session sheld } \\
\text { outside this school building? }\end{array}$ & 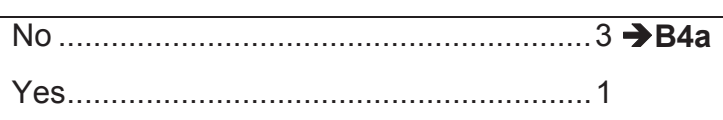 \\
\hline \multirow[t]{4}{*}{ B3b. } & \multirow{4}{*}{$\begin{array}{l}\text { If yes, mention how long the } \\
\text { school held sessions outside the } \\
\text { school building? } \\
\text { CODE: } \\
\text { A. Smoke } \\
\text { C. Budgetaryshortfall } \\
\text { E. Earthquake } \\
\text { F. Landslide } \\
\text { G. Flood } \\
\text { H. Tsunami } \\
\text { I. Fire } \\
\text { J. Strong winds } \\
\text { V. Others }\end{array}$} & $\begin{array}{l}\text { A } 1 . \sqcup \perp \text { 1. days } \\
\text { 2.weeks }\end{array}$ \\
\hline & & $\begin{array}{l}\text { 1a.Why? A C E F G H I J V } \\
\text { B 1. } \downarrow \text { 1. days } \\
\text { 2.weeks }\end{array}$ \\
\hline & & $\begin{array}{l}\text { 1a.Why? A C E F G H I J V } \\
\text { C. 1. } \downarrow \text { 1. days } \\
\text { 2.weeks }\end{array}$ \\
\hline & & 1a.Why? A C E F G H I J V \\
\hline B4a. & Who administers this school? & 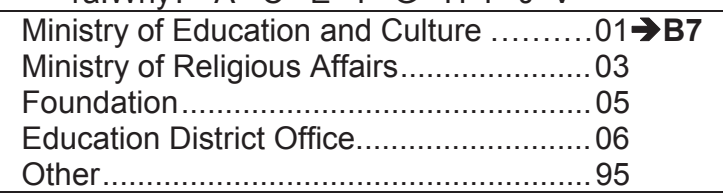 \\
\hline B5. & $\begin{array}{l}\text { Does this school have a religious } \\
\text { orientation? }\end{array}$ & 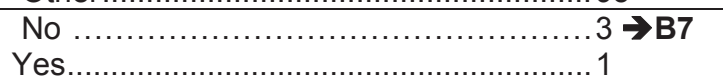 \\
\hline & If yes, state the religious orientation: & 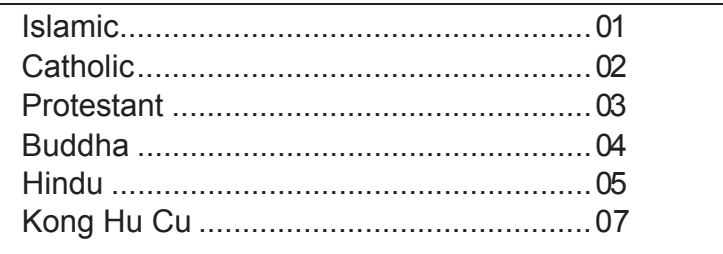 \\
\hline
\end{tabular}




\section{SECTION B: SCHOOL}

\begin{tabular}{|c|c|c|c|}
\hline B7. & $\begin{array}{l}\text { What is the language predominantly } \\
\text { used in teaching at this school? }\end{array}$ & $\begin{array}{l}\text { 00. Indonesian } \\
\text { 01. Javanese } \\
\text { 02. Sundanese } \\
\text { 03. Balinese } \\
\text { 04. Batak } \\
\text { 05. Bugis } \\
\text { 06. Chinese } \\
\text { 07. Maduranese } \\
\text { 08. Sasak } \\
\text { 09. Minang } \\
\text { 10. Banjar } \\
\text { 11. Bima }\end{array}$ & $\begin{array}{l}\text { 12. Makassar } \\
\text { 13. Nias } \\
\text { 14. Palembang } \\
\text { 15. Sumbawa } \\
\text { 16. Toraja } \\
\text { 17. Lahat } \\
\text { 18. Other South Sumatra } \\
\text { 19. Betawi } \\
\text { 20. Lampung } \\
\text { 96. No other } \\
\text { 95. Other }\end{array}$ \\
\hline B8. & $\begin{array}{l}\text { During School Year 2013/2014, how } \\
\text { many weeks was this school in } \\
\text { session? }\end{array}$ & \multicolumn{2}{|l|}{$\downarrow \downarrow$ weeks } \\
\hline B9. & $\begin{array}{l}\text { During School Year } 2013 / 2014 \text {, how } \\
\text { many days perweek was this school in } \\
\text { session? }\end{array}$ & \multicolumn{2}{|l|}{$\left\llcorner \_\right.$days } \\
\hline B10. & $\begin{array}{l}\text { What time is this schoolheld? } \\
\text { A. In the morning } \\
\text { B. In the afternoon }\end{array}$ & \multicolumn{2}{|c|}{ 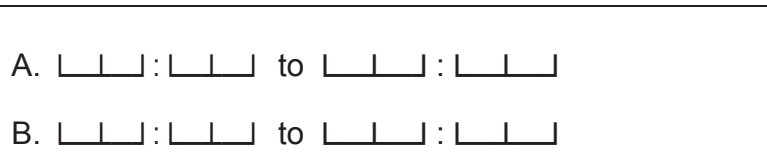 } \\
\hline B81 & 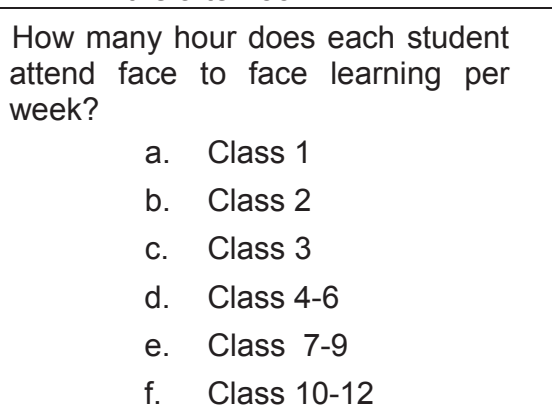 & \multicolumn{2}{|c|}{$\begin{array}{l}\text { a. } 1 . \sqcup \sqcup \text { 6. No class } \\
\text { b. } 1 . \sqcup \text { 6. No class } \\
\text { c. } 1 . \sqcup \text { 6. No class } \\
\text { d. } 1 . \sqcup \text { 6. No class } \\
\text { e.1. } \sqcup \text { 6. No class } \\
\text { f. } 1 . \sqcup \text { 6. No class }\end{array}$} \\
\hline B11. & $\begin{array}{l}\text { Is this school building shared with } \\
\text { other schools? }\end{array}$ & \multicolumn{2}{|c|}{ 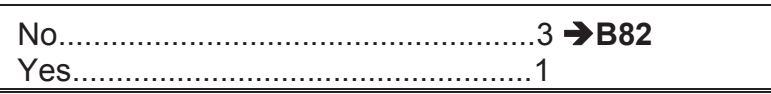 } \\
\hline
\end{tabular}

Please list the other schools that use this building?

\begin{tabular}{|c|c|c|c|c|}
\hline & \multicolumn{2}{|l|}{ B11b. } & \multicolumn{2}{|l|}{ B11e. } \\
\hline $\begin{array}{c}\text { TYPE OFSCHOOL } \\
\text { (B2TYPE) }\end{array}$ & Is it used by a [ & & Status of Schc & \\
\hline a.Elementary School & 3.No $\Downarrow$ & 1.Yes & 1. Public & 3. Private \\
\hline b.Junior High School & 3.No $\downarrow$ & 1.Yes & 1. Public & 3. Private \\
\hline c.Senior High School & 3.No $\downarrow$ & 1.Yes & 1. Public & 3. Private \\
\hline v.Other & 3.No $\downarrow$ & 1.Yes & 1. Public & 3. Private \\
\hline
\end{tabular}

\begin{tabular}{|c|c|c|}
\hline B82 & $\begin{array}{l}\text { What is the average distant of the } \\
\text { house of the pupils to the school? }\end{array}$ & 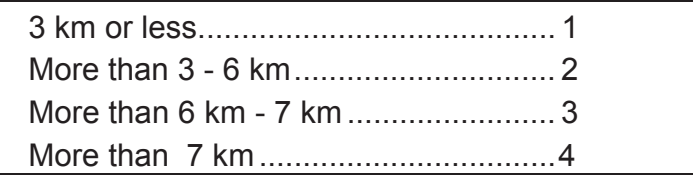 \\
\hline B15a & $\begin{array}{l}\text { Does this school use a certain textbook } \\
\text { package? }\end{array}$ & 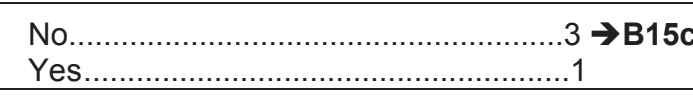 \\
\hline B15f & $\begin{array}{l}\text { What are the textbooks provided by } \\
\text { theschool for the students? }\end{array}$ & $\begin{array}{l}\text { A. BAHASA INDONESIA } \\
\text { B. ENGLISH } \\
\text { C. MATH } \\
\text { D. SCIENCE } \\
\text { E. SOCIAL SCIENCE } \\
\text { V. Other }\end{array}$ \\
\hline B15g & $\begin{array}{l}\text { How many books rovided for each } \\
\text { student? } \\
\text { INTERVIEWER CHECKS: } \\
\text { 1. one set for elementary school : } \\
\text { Indonesian, Mathematic, science, dan } \\
\text { social science. } \\
\text { 2. one set for junior high school: } \\
\text { Indonesian, English, Mathematic, Science, } \\
\text { Social Science. }\end{array}$ & $\begin{array}{l}\text { 1. One set for one student } \\
\text { 2. One set for two students } \\
\text { 3. One set for more than two students } \\
\text { 4. Less than one set for one student. } \\
\text { 5. Less than one set for more than one student. }\end{array}$ \\
\hline B15b. & $\begin{array}{l}\text { How big is the problem in making these } \\
\text { book packages available? }\end{array}$ & 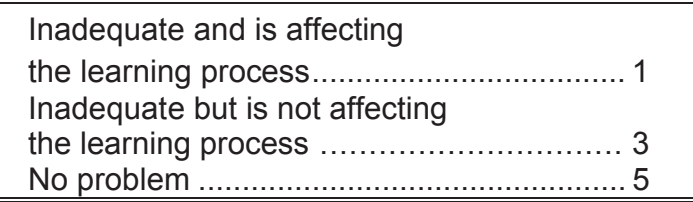 \\
\hline
\end{tabular}




\section{SECTION B: SCHOOL}

\begin{tabular}{|c|c|c|}
\hline B15ba. & $\begin{array}{l}\text { What is the percentage of students who } \\
\text { bought the textbook packages? }\end{array}$ & $\left\llcorner\perp \_\right.$percent \\
\hline B15c & $\begin{array}{l}\text { Does this school use other } \\
\text { supplementarybooks? }\end{array}$ & 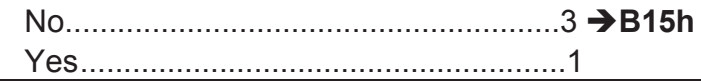 \\
\hline B15d. & $\begin{array}{l}\text { How big is the problem in making these } \\
\text { supplementary books available? }\end{array}$ & 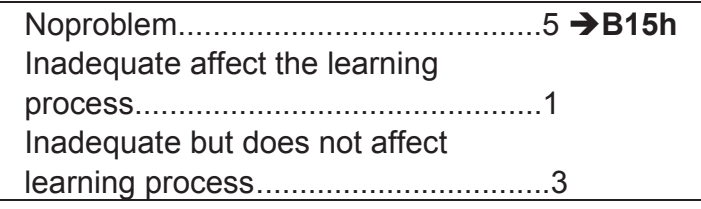 \\
\hline B15e. & $\begin{array}{l}\text { What is the percentage of students } \\
\text { who bought the supplementary books? }\end{array}$ & $\llcorner\perp \perp$ percent \\
\hline B15h. & $\begin{array}{l}\text { Does the school have enrichment } \\
\text { books } \\
\text { (ENRICHMENT BOOK IS A BOOK TO } \\
\text { ENRICH KNOWLEDGE OF THE } \\
\text { STUDENTS AND TEACHERS) }\end{array}$ & No \\
\hline B15i & $\begin{array}{l}\text { How many enrichment books provided } \\
\text { by this school? }\end{array}$ & $\begin{array}{l}\text { 1. Less than } 100 \text { books } \\
\text { 2. More than } 100 \text { books } \\
\text { 3. More than } 200 \text { books }\end{array}$ \\
\hline B15j. & $\begin{array}{l}\text { Does this school have refference } \\
\text { books such as atlas, dictionary, etc? }\end{array}$ & 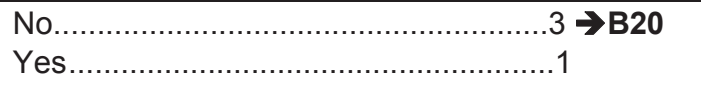 \\
\hline B15k. & $\begin{array}{l}\text { How many refference books provided } \\
\text { by this school? }\end{array}$ & $\begin{array}{l}\text { 1. Less than } 10 \text { books } \\
\text { 2. } 11-20 \text { books } \\
\text { 3. } 3 \text {. More than } 20 \text { books }\end{array}$ \\
\hline
\end{tabular}

Does this school have the following facilities

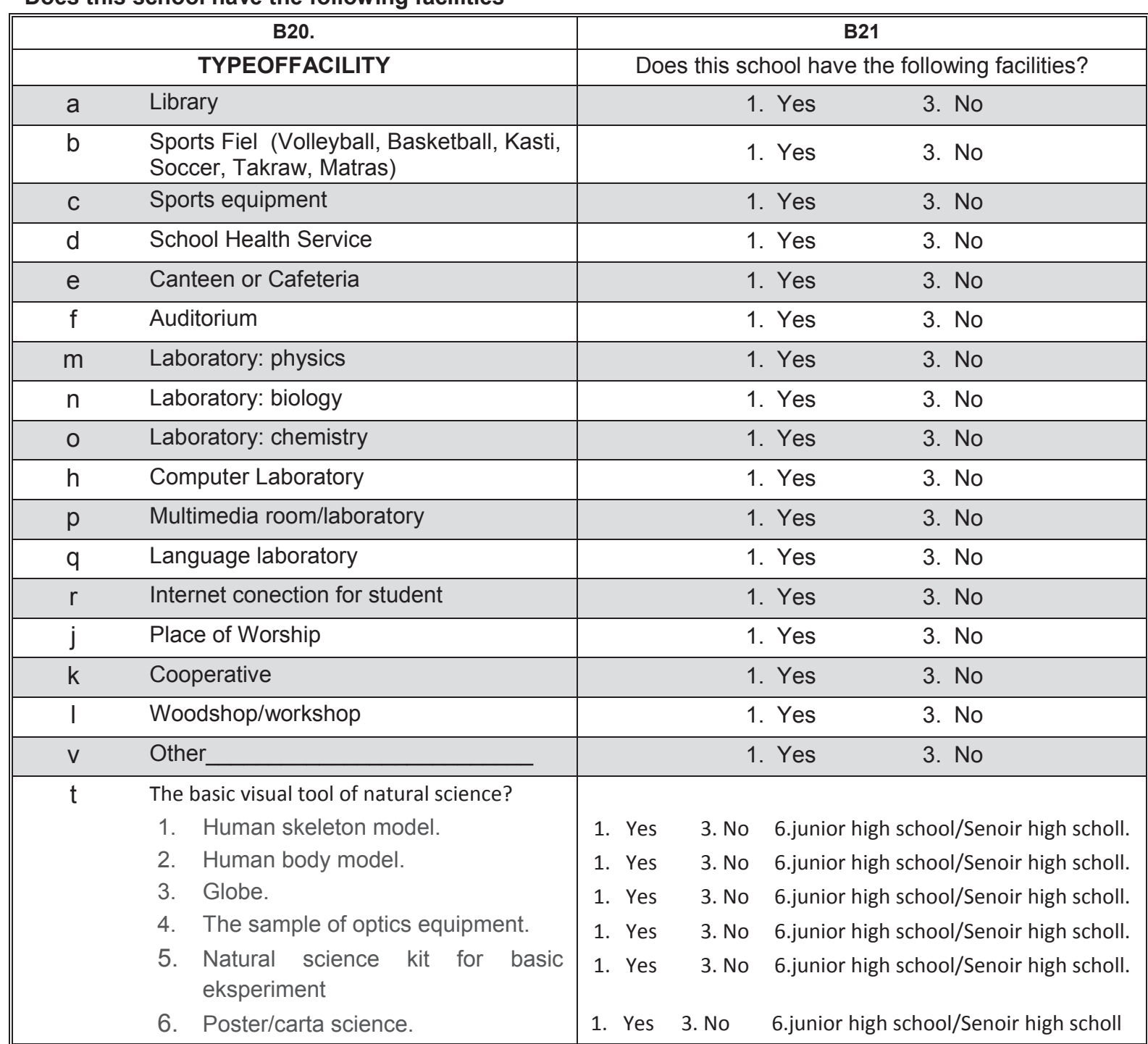




\section{SECTION B: SCHOOL}

\begin{tabular}{|c|c|c|}
\hline B30a. & $\begin{array}{l}\text { What is the minimum National } \\
\text { Examination (UAN) score required for } \\
\text { new entrants to this school for the school } \\
\text { year 2013/2014? }\end{array}$ & 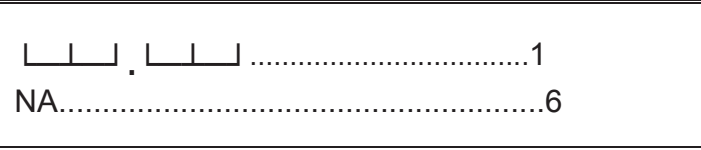 \\
\hline B30b & $\begin{array}{l}\text { What is the average National } \\
\text { Examination (UAN) score among the } \\
\text { incoming student for the school year } \\
\text { 2013/2014? }\end{array}$ & 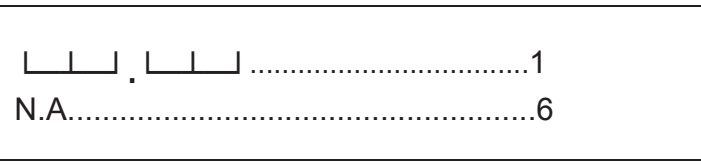 \\
\hline B30c & $\begin{array}{l}\text { What is the total number of the student } \\
\text { that registered in this school for the } \\
\text { school year } 2013 / 2014 \text { ? }\end{array}$ & 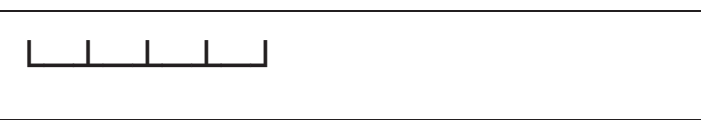 \\
\hline B31 & $\begin{array}{l}\text { Does this school have teachers/staff } \\
\text { for guidance and counseling? }\end{array}$ & 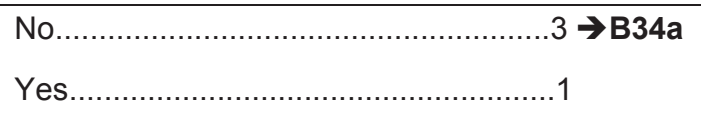 \\
\hline B32 & $\begin{array}{l}\text { Is guidance and counseling activity } \\
\text { routinely conducted? }\end{array}$ & 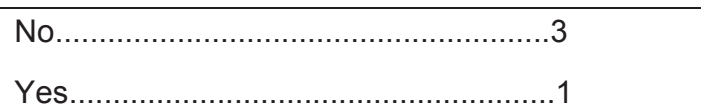 \\
\hline B33 & $\begin{array}{l}\text { What forms does the counseling take at } \\
\text { this school? }\end{array}$ & 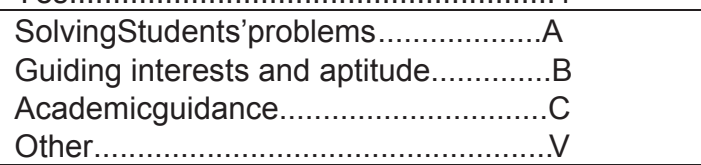 \\
\hline B34a. & $\begin{array}{l}\text { What is the total number of classrooms } \\
\text { in this scholl? }\end{array}$ & $\llcorner\perp$ Classrooms \\
\hline B35 & $\begin{array}{l}\text { In the last } 5 \text { years were additional } \\
\text { classrooms built(RKB)? }\end{array}$ & 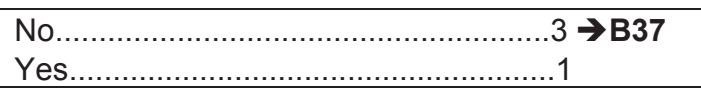 \\
\hline B36. & $\begin{array}{l}\text { Howmany additional classroomswere } \\
\text { built? }\end{array}$ & $\llcorner\perp$ Classrooms \\
\hline B37. & $\begin{array}{l}\text { Does this school still need additional } \\
\text { classrooms? }\end{array}$ & 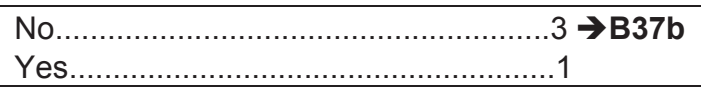 \\
\hline B37a. & $\begin{array}{l}\text { Howmany additional classrooms are } \\
\text { needed? }\end{array}$ & $\llcorner\perp$ Classrooms \\
\hline B37b. & $\begin{array}{l}\text { What is the total number of the } \\
\text { laboratories in this school? }\end{array}$ & $\begin{array}{l}\square \\
\text { NA }\end{array}$ \\
\hline B37e & $\begin{array}{l}\text { Does this school have science lab and at } \\
\text { least one set of science practice } \\
\text { instrument? }\end{array}$ & 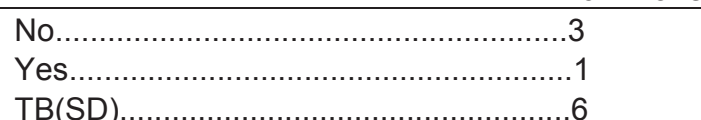 \\
\hline B37c. & $\begin{array}{l}\text { Does this school still need additional } \\
\text { laboratory? }\end{array}$ & 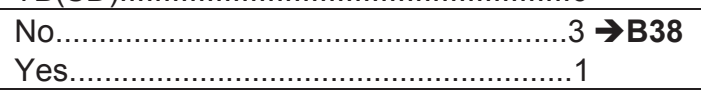 \\
\hline B37d & $\begin{array}{l}\text { Howmany additional laboratories are } \\
\text { needed? }\end{array}$ & $\llcorner\perp$ Laboratories \\
\hline B38 & $\begin{array}{l}\text { Does this school have any } \\
\text { extracurricular activities? }\end{array}$ & No \\
\hline
\end{tabular}

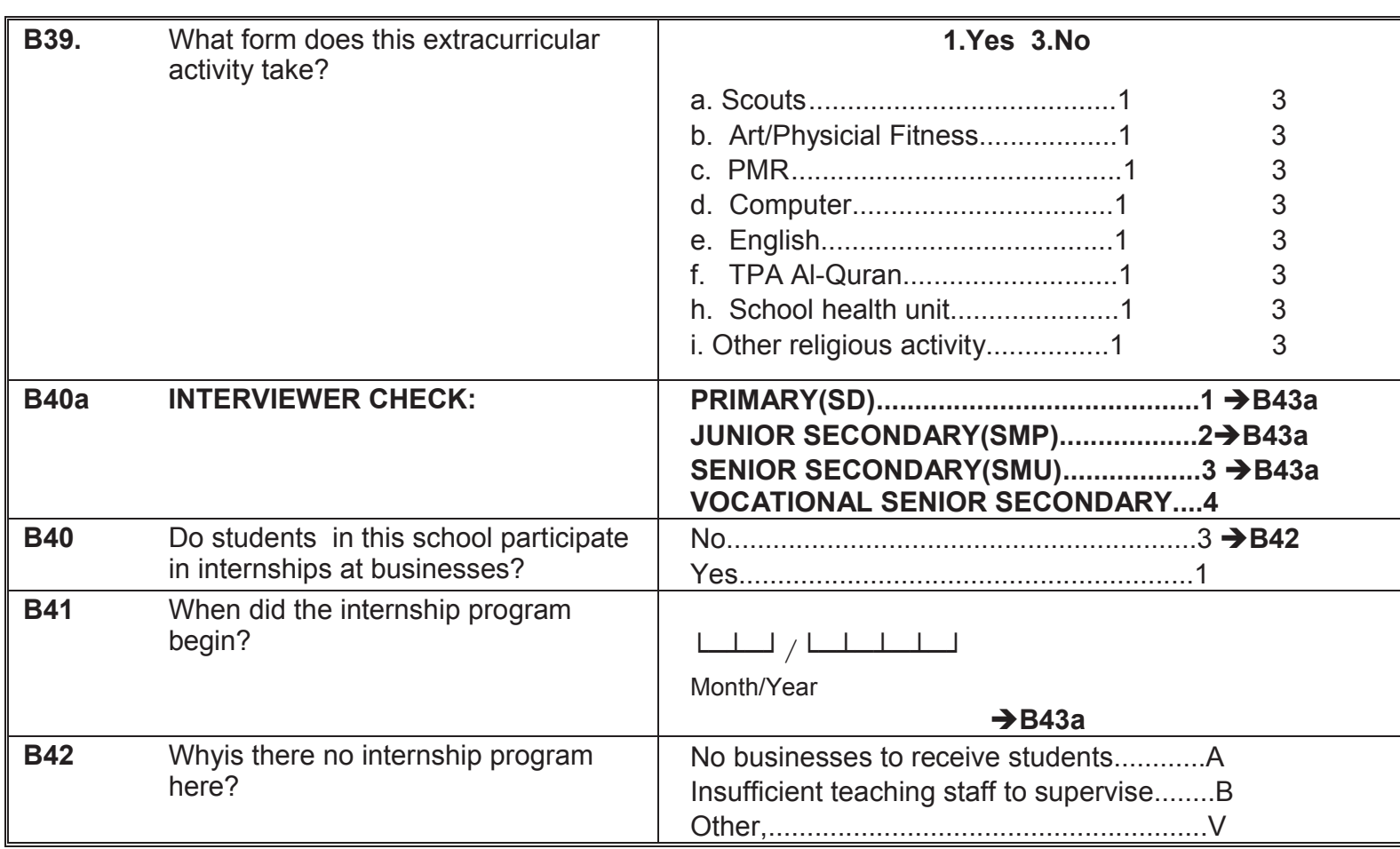


SECTION B: SCHOOL

Now we would like to ask about scholarship /assistance programs in this school

\begin{tabular}{|c|c|c|c|c|c|c|c|c|c|c|c|c|c|c|}
\hline \multirow{3}{*}{$\begin{array}{l}\text { SCHOLARSHIPPROGRAM } \\
\text { (B5TYPE) }\end{array}$} & \multirow[b]{2}{*}{\begin{tabular}{l}
\multicolumn{1}{c}{ B43a. } \\
Are scholarship \\
lassistance \\
programs of this \\
type [...]available to \\
children in this \\
community?
\end{tabular}} & \multicolumn{4}{|c|}{\begin{tabular}{|c|} 
B43b. \\
What are the sources of
\end{tabular}} & \multirow{2}{*}{$\begin{array}{l}\text { B44. } \\
\text { Did any students in your school } \\
\text { receive scholarships from [...] } \\
\text { inthe } 2013 / 2014 \text { school year? }\end{array}$} & \multirow[b]{2}{*}{\begin{tabular}{l}
\multicolumn{1}{c}{ B45. } \\
Howmanyreceived \\
scholarships \\
from[...]in the \\
$2013 / 2014$ school \\
year?
\end{tabular}} & \multicolumn{6}{|c|}{ B46. } & B47. \\
\hline & & & $\begin{array}{l}\text { ire } \\
\text { nola }\end{array}$ & $\begin{array}{l}\text { ourc } \\
\text { p? }\end{array}$ & $\mathrm{s}$ of & & & $\begin{array}{l}\text { What } \\
\text { afore } \\
\text { schol } \\
\text { take? } \\
\text { CIRCL }\end{array}$ & $\begin{array}{l}\text { forr } \\
\text { ner } \\
\text { arst } \\
\text { E A }\end{array}$ & $\begin{array}{l}\text { did } \\
\text { ione } \\
\text { p } \\
\text { L T }\end{array}$ & & AP & & $\begin{array}{l}\text { For the average student in your } \\
\text { school what was the } \\
\text { value of this scholarship for the } \\
\text { school year 2013/2014? }\end{array}$ \\
\hline & $\begin{array}{l}\text { 1.Yes } \\
\text { 3. No } \downarrow \\
\text { 5. No heard of } \downarrow \\
\text { 8.DON'T KNOW } \downarrow \\
\end{array}$ & $\begin{array}{l}A \\
E\end{array}$ & $\begin{array}{l}B \\
F\end{array}$ & $\begin{array}{l}\mathrm{C} \\
\mathrm{V}\end{array}$ & D & $\begin{array}{l}\text { 8. DON'T KNOW } \rightarrow \text { B43a ROWb } \\
\text { 3. No } \rightarrow \text { B43aROWb } \\
\text { 1.Yes }\end{array}$ & 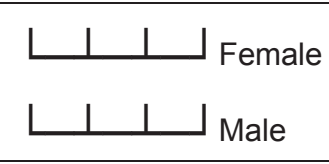 & A & B & C & D & $\mathrm{E}$ & $\mathrm{v}$ & 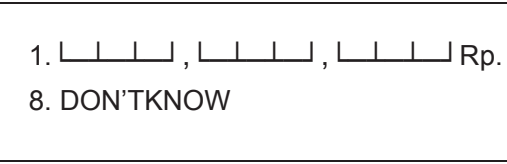 \\
\hline b.Talent and merit scholarship & $\begin{array}{l}\text { 1.Yes } \\
\text { 3. No } \downarrow \\
\text { 5. No heard of } \downarrow \\
\text { 8.DON'T KNOW } \downarrow\end{array}$ & $\begin{array}{l}\text { A } \\
E\end{array}$ & $\begin{array}{l}B \\
F\end{array}$ & $\begin{array}{l}\mathrm{C} \\
\mathrm{v}\end{array}$ & D & $\begin{array}{l}\text { 8. DON'T KNOW } \rightarrow \text { B43a ROWc } \\
\text { 3. No } \rightarrow \text { B43aROWc } \\
\text { 1. Yes }\end{array}$ & 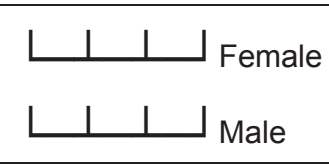 & A & B & C & D & $\mathrm{E}$ & $\mathrm{v}$ & 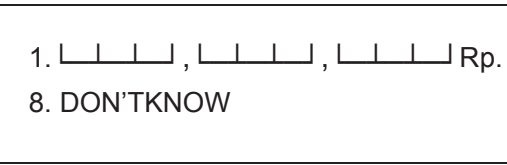 \\
\hline c. Retrieval scholarship & $\begin{array}{l}\text { 1.Yes } \\
\text { 3. No } \downarrow \\
\text { 5. No heard of } \downarrow \\
\text { 8.DON'T KNOW } \downarrow\end{array}$ & $\begin{array}{l}\text { A } \\
E\end{array}$ & $\begin{array}{l}B \\
F\end{array}$ & $\begin{array}{l}\mathrm{C} \\
\mathrm{V}\end{array}$ & D & $\begin{array}{l}\text { 8. DON'T KNOW } \rightarrow \text { B43a ROWd } \\
\text { 3. No } \rightarrow \text { B43aROWd } \\
\text { 1.Yes }\end{array}$ & 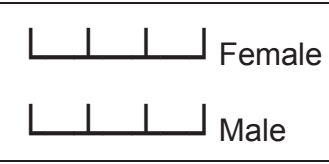 & A & B & c & $D$ & $\mathrm{E}$ & $\mathrm{v}$ & 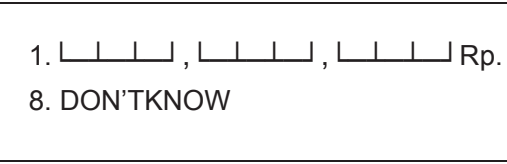 \\
\hline d. Transition scholarship & $\begin{array}{l}\text { 1.Yes } \\
\text { 3. No } \downarrow \\
\text { 5. No heard of } \downarrow \\
\text { 8.DON'T KNOW } \downarrow\end{array}$ & $\begin{array}{l}\text { A } \\
E\end{array}$ & $\begin{array}{l}B \\
F\end{array}$ & $\begin{array}{l}\mathrm{C} \\
\mathrm{V}\end{array}$ & D & $\begin{array}{l}\text { 8. DON'T KNOW } \rightarrow \text { B43a ROWe } \\
\text { 3. No } \rightarrow \text { B43a ROWe } \\
\text { 1.Yes }\end{array}$ & 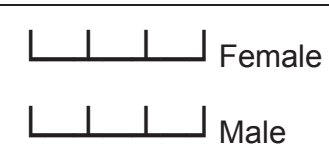 & A & B & c & D & $\mathrm{E}$ & $\mathrm{V}$ & $\begin{array}{l}\text { 1. } \sqcup \perp \perp \_, \sqcup \perp \perp, \downarrow \perp \perp \_ \text {Rp. } \\
\text { 8. DON'TKNOW }\end{array}$ \\
\hline
\end{tabular}

\section{CodesforB43b}

A. Central government

A. Rebupaton/Kota government

D. Privatenon-religious

E. GNOTA

F. School Committee

V. Other

\section{CodesforB46:}

A. Registration fee waiver

Conther school feewaiver

C. Money

D. Schoolbooks
En Uniform

V. Other 


\section{SECTION B: SCHOOL}

Now,we would like to ask about the budget of and source of revenue for the School.

\begin{tabular}{|c|c|c|}
\hline & B6TYPE & Academic Year 2013/2014 \\
\hline B75. & What is the budget of the school? & $\begin{array}{l}\text { 1. Document } \\
\text { 6. Do not have budget } \rightarrow \text { B52aa }\end{array}$ \\
\hline \multirow[t]{15}{*}{ B76. } & Revenue & \\
\hline & T.Total & 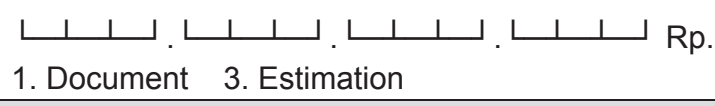 \\
\hline & APBN & \\
\hline & A. BOS & 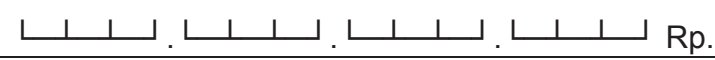 \\
\hline & 1. BOS Central & 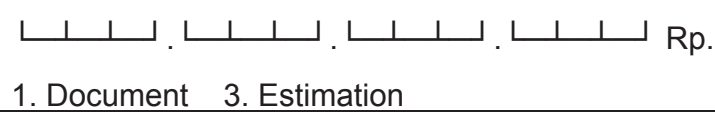 \\
\hline & 2. BOS Province & 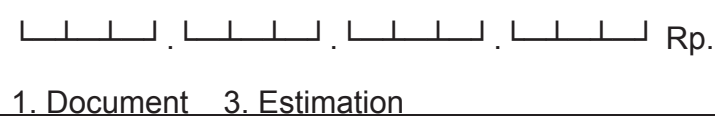 \\
\hline & B. School Grant & 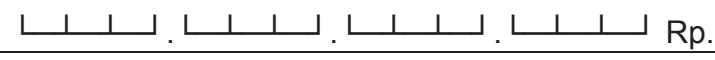 \\
\hline & 1. Deconcentration Funds & 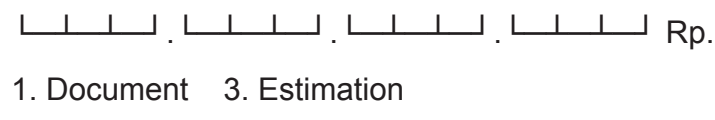 \\
\hline & 2. Dana Alokasi Khusus & 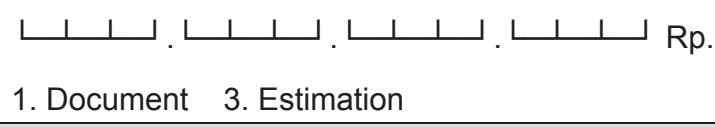 \\
\hline & APBD & \\
\hline & C. Routine & 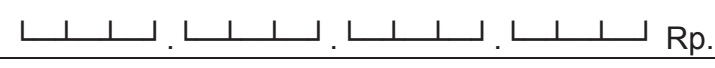 \\
\hline & D. BOP & 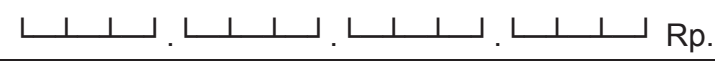 \\
\hline & 1. BOS Kabupaten/Kota & 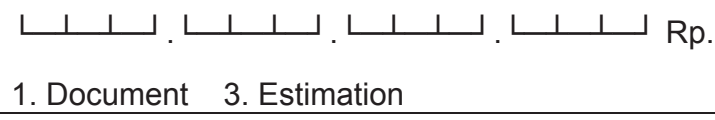 \\
\hline & $\begin{array}{l}\text { 2. Funding assistance tasks } \\
\text { (Dana Tugas Pembantuan) }\end{array}$ & 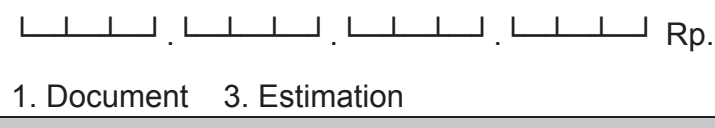 \\
\hline & E. School comittee & 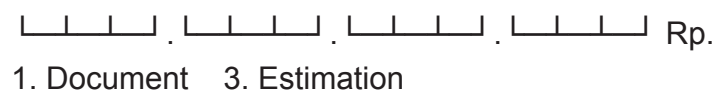 \\
\hline
\end{tabular}

\begin{tabular}{||l|l|l||}
\hline \hline B78 & $\begin{array}{l}\text { Do you have the authorityto real } \\
\text { locate between posts of expenditure } \\
\text { without approval from the Dinas or } \\
\text { any other parties? }\end{array}$ & $\begin{array}{l}\text { Yes................................................ } \\
\text { No......................................... }\end{array}$ \\
\hline
\end{tabular}




\section{SECTION B: SCHOOL}

\begin{tabular}{|c|c|c|}
\hline B52aa. & $\begin{array}{l}\text { Did this school receive School } \\
\text { Operational Fund during } \\
\text { 2013/2014? }\end{array}$ & 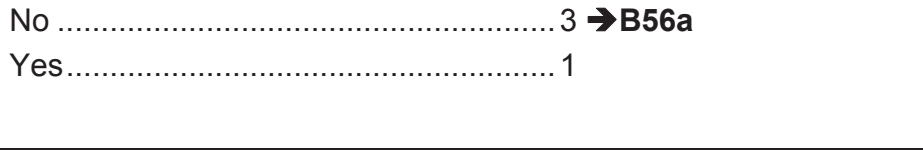 \\
\hline \multirow[t]{4}{*}{ B54aa. } & \multirow[t]{4}{*}{$\begin{array}{l}\text { What did you do with the fund and } \\
\text { how much did you spend? }\end{array}$} & 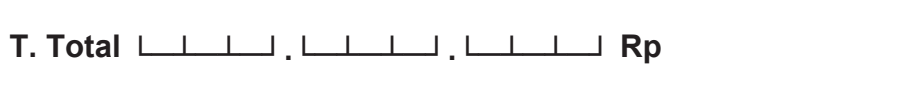 \\
\hline & & 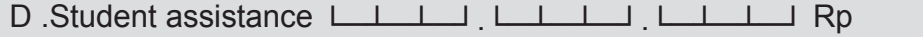 \\
\hline & & 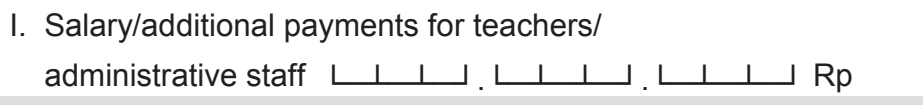 \\
\hline & & V.Other,__ ᄂ \\
\hline
\end{tabular}

\begin{tabular}{|c|c|c|}
\hline B79. & $\begin{array}{l}\text { How about the utilization of BOS fund } \\
2013 \text { by this scholl? } \\
\text { INTERVIEWER'S NOTE: DO NOT } \\
\text { READ OUT THE OPTIONS }\end{array}$ & $\begin{array}{l}\text { A. } \text { BUYING/STOCK OF TEXT BOOK. } \\
\text { B. ACCEPTANCE OF NEW STUDENT AT } \\
\text { EDUCATION YEAR 2013/2014. } \\
\text { C. COSTING REMEDIAL LESSON, ENRICHMENT, } \\
\text { STABILIZATION, DAN EXTRA CURRICULAR. } \\
\text { D. COSTING FOR TEST, THE REPORT OF THE } \\
\text { TEST. } \\
\text { E. BUYING SCHOOL TOOL THAT HAS RUN OUT. } \\
\text { F. BUYING ENERGY AND SERVICE INCLUDE NEW } \\
\text { PAIRS. } \\
\text { G. FOR THE REPARATION COSTS AND TREATMENT } \\
\text { OF SCHOOL.UNTUK BIAYA PERBAIKAN DAN } \\
\text { PERAWATAN SEKOLAH } \\
\text { H. COSTING HONORARIUM HONORER TEACHER } \\
\text { DAN HONORER EDUCATION ENERGY. } \\
\text { I. DEVELOPMENT PROFESSION OF THE TEACHER } \\
\text { SUCH AS TRAINING, KKG/MGMP AND } \\
\text { KKS/MKKS. } \\
\text { J. INFORMATION ABOUT TRANSFORMATION } \\
\text { EFFORT TO POOR STUDENT. } \\
\text { K. FOR FUND BOS MANAGEMENT. } \\
\text { L. BUYING COMPUTER } \\
\text { M. BUYING ALAT PERAGA, MEDIA PEMBELAJARAN } \\
\text { V. OTHER }\end{array}$ \\
\hline
\end{tabular}




\begin{tabular}{|c|c|c|c|c|c|c|c|}
\hline \multirow{3}{*}{ B10TYPE } & \multicolumn{7}{|c|}{ B56a. } \\
\hline & \multicolumn{7}{|c|}{$\begin{array}{l}\text { Who makes decisions about: } \\
\text { (CIRCLE ALL THAT APPLY ON EACH LINE) }\end{array}$} \\
\hline & $\begin{array}{l}\text { EDUCATION DISTRICT } \\
\text { OFFICE (DINAS) }\end{array}$ & $\begin{array}{l}\text { RELIGIOUS } \\
\text { AFFAIRS }\end{array}$ & $\begin{array}{l}\text { PRIVATE } \\
\text { FOUNDATION }\end{array}$ & $\begin{array}{l}\text { SCHOOL } \\
\text { COMMITTEE }\end{array}$ & SCHOOL & $\begin{array}{c}\text { REGIONAL } \\
\text { DEVELOPMENT } \\
\text { BOARD (BAPPEDA) }\end{array}$ & NA \\
\hline A. Books to use in this school & A & B & C & $\mathrm{D}$ & E & $\mathrm{F}$ & W \\
\hline B. Curriculum to use in this school & A & B & C & $\mathrm{D}$ & $E$ & $\mathrm{~F}$ & W \\
\hline C. Local content in the curriculum to use in thisschool & A & B & C & $\mathrm{D}$ & $\mathrm{E}$ & $\mathrm{F}$ & W \\
\hline D. Hiring of the permanent teachers & A & B & C & $\mathrm{D}$ & $\mathrm{E}$ & $\mathrm{F}$ & W \\
\hline E. Hiring of the assistant teachers (guru bantu) & A & B & C & $\mathrm{D}$ & $\mathrm{E}$ & $\mathrm{F}$ & W \\
\hline F. Hiring of the honoraria teachers(guru honorer) & A & B & C & $\mathrm{D}$ & $\mathrm{E}$ & $\mathrm{F}$ & W \\
\hline $\begin{array}{l}\text { G. How the salaryof permanent teachers should be based on (e.g., based on } \\
\text { experience, quality,etc.)? }\end{array}$ & A & $\mathrm{B}$ & $\mathrm{C}$ & D & $\mathrm{E}$ & $\mathrm{F}$ & W \\
\hline $\begin{array}{l}\text { I. How the salary of honoraria teachers should be based on (e.g., based on } \\
\text { experience, quality,etc.)? }\end{array}$ & A & $\mathrm{B}$ & $\mathrm{C}$ & $\mathrm{D}$ & $\mathrm{E}$ & $\mathrm{F}$ & W \\
\hline J. Incentive of the permanent teachers & A & $\mathrm{B}$ & $\mathrm{C}$ & $\mathrm{D}$ & $\mathrm{E}$ & $\mathrm{F}$ & W \\
\hline K. Incentive of the assistant teachers & A & $\mathrm{B}$ & $\mathrm{C}$ & $\mathrm{D}$ & $E$ & $\mathrm{~F}$ & W \\
\hline L. Incentive of the honoria teachers & A & $\mathrm{B}$ & C & $\mathrm{D}$ & $E$ & $\mathrm{~F}$ & W \\
\hline M. Student admission fees & A & B & C & D & $\mathrm{E}$ & $\mathrm{F}$ & W \\
\hline $\mathbf{N}$. When building maintanance isneeded & A & B & C & $\mathrm{D}$ & $\mathrm{E}$ & $\mathrm{F}$ & W \\
\hline o. Teachers who should obtain Teacher Certification & A & $\mathrm{B}$ & $\mathrm{C}$ & $\mathrm{D}$ & $E$ & $\mathrm{~F}$ & W \\
\hline
\end{tabular}




\section{SECTION B: SCHOOL}

\begin{tabular}{|c|c|c|}
\hline B68 & $\begin{array}{l}\text { In comparis on to the } 2012 / 2013 \\
\text { academic year,howwell did the students } \\
\text { in this school do in the UAN 2013/2014? }\end{array}$ & 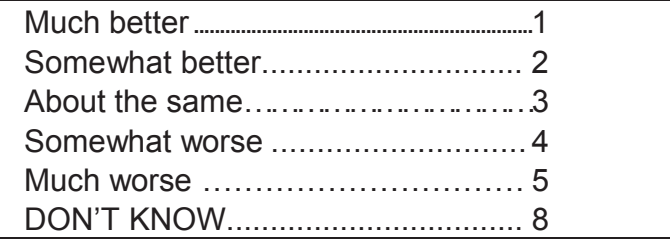 \\
\hline B68a. & $\begin{array}{l}\text { What percentage of students passed in } \\
\text { UAN in the last twoyears? }\end{array}$ & 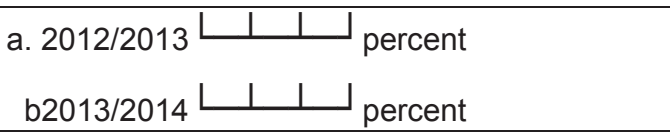 \\
\hline B68b. & $\begin{array}{l}\text { What is the Grade Point Average (GPA) } \\
\text { of the graduating class for thisschool? }\end{array}$ & 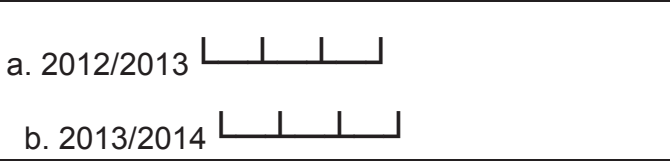 \\
\hline B69. & $\begin{array}{l}\text { In comparis onto the } 2013 / 2014 \\
\text { academic year,are the percentage of } \\
\text { students who dropped out of school } \\
\text { higher, lower or about the same? }\end{array}$ & 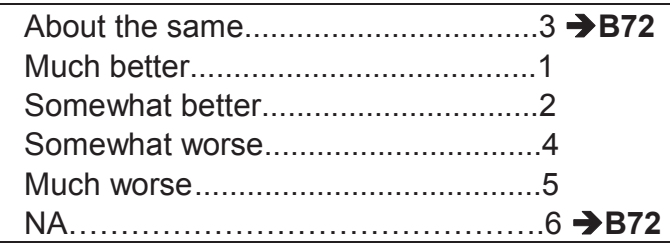 \\
\hline \multirow[t]{2}{*}{ B70. } & \multirow[t]{2}{*}{$\begin{array}{l}\text { In your opinion, what factors account for } \\
\text { the change in the percentage of } \\
\text { students who dropped out of school? }\end{array}$} & $\begin{array}{l}\text { Fewerwere ill................................ } 1 \\
\text { Parents/studentscan afford topay......B1 } \\
\text { More parents want their children } \\
\text { to work and earnwages...................... } 1 \\
\text { Improvement in education facilities....D1 } \\
\text { Improvement in teaching qualities......E1 } \\
\text { DON'TKNOW................................ } 1 \\
\text { Other }\end{array}$ \\
\hline & & $\begin{array}{l}\text { Morewere ill.................................. } 22 \\
\text { Parents/studentscan't afford topay.... B2 } \\
\text { Less parents want their children } \\
\text { to work and earn wages...................... } 2 \\
\text { Deterioration in education facilities...... } 2 \\
\text { Deterioration in teaching qualities.......E2 } \\
\text { DON'TKNOW........................... } \\
\text { Other }\end{array}$ \\
\hline
\end{tabular}

\begin{tabular}{|c|c|c|}
\hline B72. & $\begin{array}{l}\text { Is there a change in the percentage of } \\
\text { students who register at the beginning } \\
\text { of this academic year compared to the } \\
2013 / 2014 \text { academic year? }\end{array}$ & 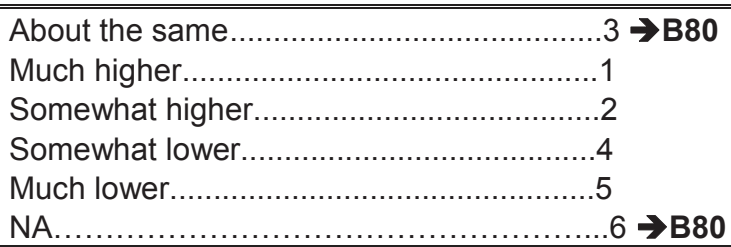 \\
\hline \multirow[t]{2}{*}{ B73. } & \multirow[t]{2}{*}{$\begin{array}{l}\text { Inyour opinion, what factors account for } \\
\text { the change? } \\
\text { CIRCLE ALL THAT APPLY }\end{array}$} & $\begin{array}{l}\text { Fewerwere ill........................................... } 1 \\
\text { Parents/students can afford to pay............ } \\
\text { More parents want their children to } \\
\text { work and earnwages............................. } 1 \\
\text { Improvement in education facilities............ } 1 \\
\text { Improvement in teaching qualities.............E1 } \\
\text { DON'TKNOW..................................... } 1 \\
\text { Other,mention }\end{array}$ \\
\hline & & $\begin{array}{l}\text { Morewere ill....................................... } \\
\text { Parents/studentscannot afford topay........B2 } \\
\text { Less parents want their children to } \\
\text { work and earnwages............................ } 2 \\
\text { Deterioration in education facilities........... } 2 \\
\text { Deterioration in teaching qualities..............E2 } \\
\text { DON'TKNOW.................................... } \\
\text { Other }\end{array}$ \\
\hline B80 & $\begin{array}{l}\text { Averagely, how many time } \\
\text { doessupervisor visit the school each } \\
\text { month to carry out supervision and } \\
\text { givecounseling in } 2013 / 2014 \text { ? }\end{array}$ & $\begin{array}{l}\text { More than once in a month } \\
\text { About once in a month } \\
\text { About once in } 2-3 \text { months } \\
\text { About twice in a year }\end{array}$ \\
\hline
\end{tabular}




\section{SECTION SC:SCHOOL COMMITTE}

\begin{tabular}{|c|c|}
\hline $\begin{array}{l}\text { KR1_SC } \text { Respondent (CAPI WILL SKIP DIRECTLY } \\
\text { TO B1 IF THE NAME OF RESPONDENT } \\
\text { HAVE EVER ANSWERED IN THE PREVIOUS } \\
\text { SECTION) }\end{array}$ & \\
\hline $\begin{array}{l}\text { KR1a_SC Posttion/occupation in } \\
\text { this School: }\end{array}$ & $\begin{array}{lllll}01 & 02 & 03 & 04 & 05 \\
06 & 07 & 08 & 95 & \end{array}$ \\
\hline KR2_SC Age : & $\sqcup$ \ـ Years \\
\hline $\begin{array}{l}\text { KR2a_SC Length of tenure in } \\
\text { Position s: }\end{array}$ & \begin{tabular}{llll|}
$\perp \perp / L$ & $\perp$ & $\perp$ & $\perp$ \\
Month & & Year
\end{tabular} \\
\hline $\begin{array}{r}\text { KR3_SC INTERVIEWER CHECK : } \\
\text { SEX OF RESPONDENT }\end{array}$ & 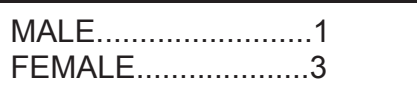 \\
\hline KR3a_SC Highest level of education attended: & \llcorner \\
\hline KR4_SC Highest class completed: & $\begin{array}{ccccc}00 & 01 & 02 & 03 & 04 \\
05 & 06 & 07 & 98 & 95 \\
\end{array}$ \\
\hline KR5_SC Length of time lived in the village : & 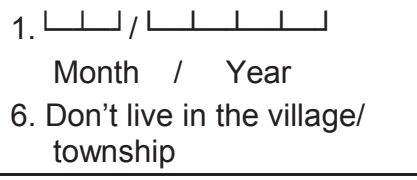 \\
\hline $\begin{array}{l}\text { KR6_SC How did [...] come to assume to the } \\
\text { position? }\end{array}$ & $\begin{array}{l}\text { 1. Elected } \\
\text { 2. Appointed by Goverment } \\
\text { / Education District Office } \\
\text { 3. Appointed byFoundation } \\
\text { 5.Other }\end{array}$ \\
\hline
\end{tabular}

Code for position in village (KR1a)

015. Village/township heads

016. Village secretary

17. Head of Government Administration

018. Head of Village Development

020. Head of Financial Matters

021. Head of General Affairs

\section{Codes for highest level of education}

(KR3a):

01. No school/not yet in school

02. Elementary school

03. Junior High - General

05. High School - Geational

06. High School - Vocational

60. D1, D2, D3 (Junior College)

61. University S1 (Bachelor)

62. University S2 (Masters)

63. University S3 (Ph.D.)
11. Kejar Paket A (Adult Education A)

2. Kejar Paket B (Adult Education B)

13. Open Univeristy
14. Islamic School (Pondok Pensantren)

15. Kejar Paket C (Adult Education C)

17. School for the disabled

72. Islamic Elementary School (Madrasah Ibtidaiyah)

73. Islamic Junior High School (Madrasah Tsanawiyah)

74. Islamic Senior High School (Madrasah Alliyah)

90. Kindergarten

95. Other
Codes for highest grade (KR4):

00. Never completed class

01. 1

02. 2

03. 3

04. 4

05. 5

06. 6

07. Graduated

96. No schoo

98. DON'T KNOW 


\section{SECTION SC:SCHOOL COMMITTEE}

Nowwewould like to askabout the School Committee in this school.

Sc01. Is there a School Committee for this school?

SC02. When was the School Committee for this school first established?

$\llcorner\perp /\llcorner\perp \perp \perp$

Month/ Year

SC03. Who are the members of the School Committee?

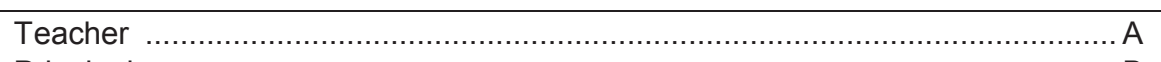

Principal ...........

Student's parent ...Tumon

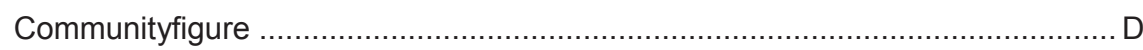

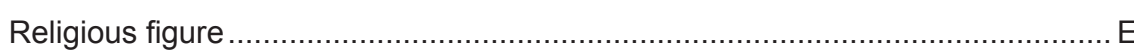

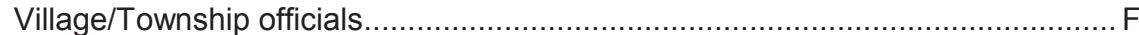

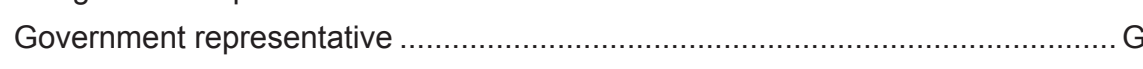

Other

$\rightarrow$ NEXTSECTION

SC04. Howmany people are in the School Committee including the head?

SC05. What is the source of funding for the School Committee's activities?

$\llcorner\sqcup$ people

Student'sparent

School budget ..........A

SC06. Howmany times did the School Committee hold meetings with the school in the last 12 months?

SC07. Howmany times did the School Committee hold meetings with the students' parents in the last 12 months?

$\left\llcorner\downarrow_{\text {times }}\right.$

\begin{tabular}{|ll}
\hline SC08. Has the School Committee been involved indetermining the school program? \\
\hline
\end{tabular}

SC09. Has the School Committee been involved in increasing the welfare of the teachers?

SC10. Has the School Committee been involved in the procurement of books and teaching equipment?

SC11. Has the School Committee tried to raise fund from the school from outside sources?

SC12. Has the School Committee been involved in physical rehabilitation/construction of the school?

SC13. Has the School Committee been giving inputs about the teaching process at the school?

$\llcorner\sim$ times

No

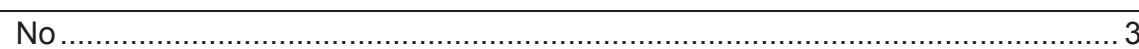

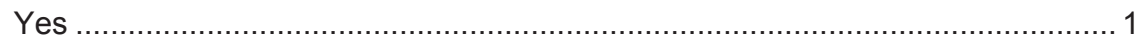

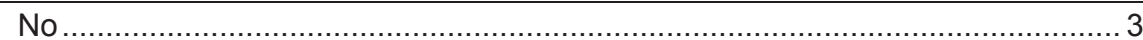

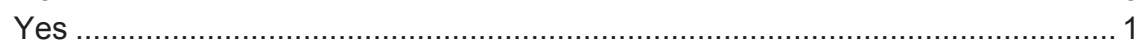

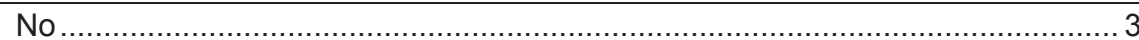

Yes

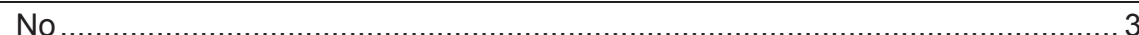

Yes

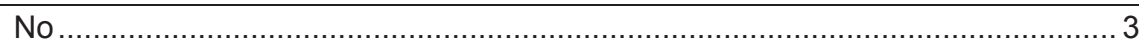

Yes 
SECTION E:AVERAGE EXPENDITURE OF EACH STUDENT IN SCHOOL YEAR 2013/2014

KR1_E
Respondent (CAPI WILL SKIP DIRECTL
TO B1 IF THE NAME OF RESPONDENT

HAVE EVER ANSWERED IN THE PREVIOUS

SECTION)

\begin{tabular}{|c|c|c|}
\hline KR1a_E & $\begin{array}{l}\text { Posttion/occupation in } \\
\text { this School: }\end{array}$ & $01 \quad 02 \quad 03 \quad 04 \quad 05$ \\
$06 \quad 07 \quad 0895$
\end{tabular}

Code for position in village (KR1a):

022. Village/township heads

023. Village secretary

024. Head of Government Administration

025. Head of Village Developmen

026. Head of Welfare

028. Head of General Affairs

95. Other
Codes for highest level of education (KR3a):

01. No school/not yet in school

02. Elementary school

03. Junior High - General

04. Junior High - Vocational

05. High School - General

66. High School - Vocational

61. University S1 (Bachelor)

62. University S2 (Masters)

63. University S3 (Ph.D.)
11. Kejar Paket $\mathrm{A}$ (Adult Education $\mathrm{A}$ )

12. Kejar Paket B (Adult Education B)

13. Open Univeristy

14. Islamic School (Pondok Pensantren)

15. Kejar Paket C (Adult Education C)

72. Islamic Elementary School (Madrasah Ibtidaiyah)

73. Islamic Junior High School (Madrasah Tsanawiyah)

74. Islamic Senior High School (Madrasah Alliyah)

90. Kindergarten

95. Other
Codes for highest grade (KR4):

00. Never completed class

01. 1

03.3

04.4

05. 5

06. 6

07. Graduated

96. No school

98. DON'T KNOW 
EDUCATION EXPENSE INCURRED BY STUDENTS.

\begin{tabular}{|c|c|c|}
\hline & FORM OFSTUDENT EXPENDITURES (ETYPE) & TOTAL EXPENDITURES (IN RUPIAH) 2013/2014] \\
\hline \multirow[t]{7}{*}{ E1A. } & New student school fees in school year 2013/2014 & \\
\hline & t. TOTAL & 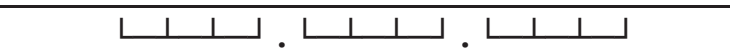 \\
\hline & a. Registration Fees (base/facilityfees) for school year 2013/2014: & 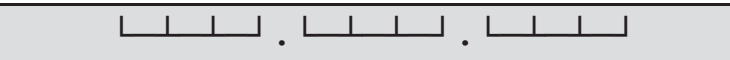 \\
\hline & b4. School committee fee per month per student & 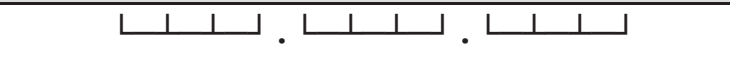 \\
\hline & b2. SPP per month per student & 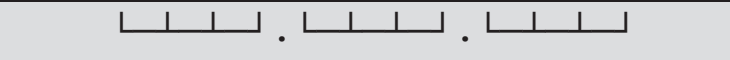 \\
\hline & b3. Other fees (fieldwork/skills, OSIS) entire year per student & 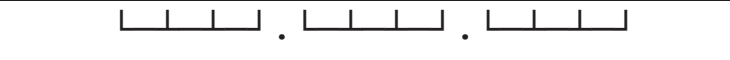 \\
\hline & c3. UAS per student per year & 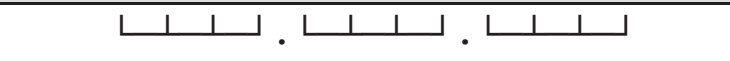 \\
\hline \multirow[t]{7}{*}{ E1B. } & Fees for continuing students in school year 2013/2014 & \\
\hline & t. TOTAL & 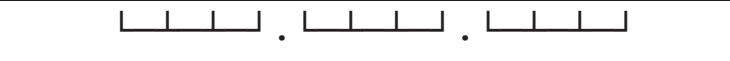 \\
\hline & a. Registration Fees (base/facility fees) for school year 2013/2014: & 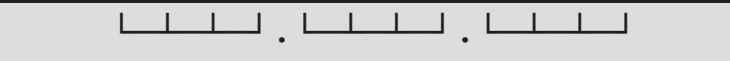 \\
\hline & b4. School committee fee per month per student & 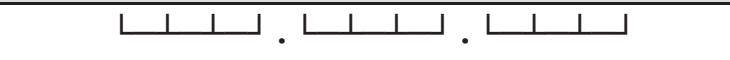 \\
\hline & b2. SPP per month per student & 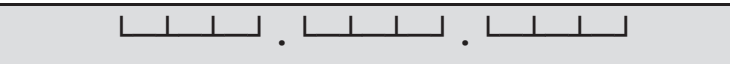 \\
\hline & c1. EHB per student per year & 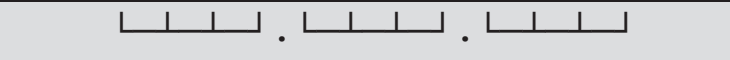 \\
\hline & c3. UAS per student per year & 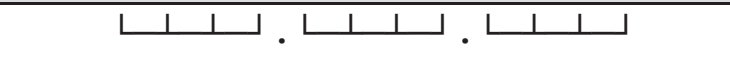 \\
\hline \multirow[t]{3}{*}{ E2. } & School supplies and materialexpenditures per year 2013/2014: & \\
\hline & a. Books, writing materials and school supplies(work/book/guide/lecture notes) & 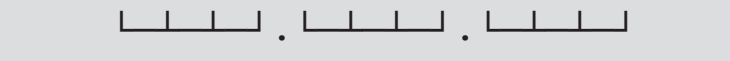 \\
\hline & b. School and physicial education uniform & 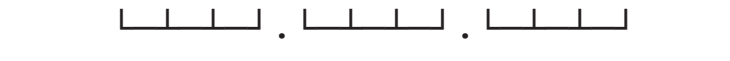 \\
\hline E3. & UAN/UN per student per year & $\begin{array}{llllllllll}1 & 1 & 1 & 1\end{array}$ \\
\hline E4. & Extracurricular activities feesper student per year & 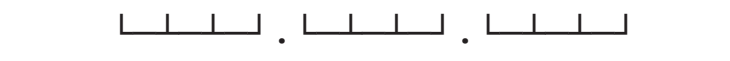 \\
\hline E5. & Magazine & $\begin{array}{lllllllll}1 & 1 & \perp\end{array}$ \\
\hline E7. & Irrregular contribution & $\begin{array}{lllllll}\perp & 1\end{array}$ \\
\hline E8. & Field trips & 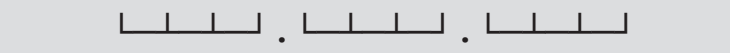 \\
\hline
\end{tabular}




\section{SECTION H: DIRECT OBSERVATION}

\begin{tabular}{|c|c|c|}
\hline & \multicolumn{2}{|l|}{ HOMEROOM TEACHER } \\
\hline H1. WERE THERE ANY OTHER PEOPLE PRESENT IN THE ROOM DURING INTERVIEW? & $\begin{array}{l}\text { NO } \\
\text { YES }\end{array}$ & \\
\hline \multirow[t]{6}{*}{ H2. WHO EXACTLY WERE PRESENT DURING INTERVIEW? } & 1.YES & 3.NO \\
\hline & a. SCHOOL PRINCIPAL & 3 \\
\hline & b. DEPUTY SCHOOL PRINCIPAL & 3 \\
\hline & c. TEACHERS & 3 \\
\hline & d. FOUNDATION OFFICER & 3 \\
\hline & 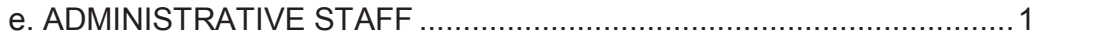 & 3 \\
\hline \multirow[t]{5}{*}{ H3. DID THOSE PRESENT ALSO ANSWER THE QUESTION IN THIS INTERVIEW? } & 1.YES & 3.NO \\
\hline & a. SCHOOL PRINCIPAL & 3 \\
\hline & b. DEPUTY SCHOOL PRINCIPAL & 3 \\
\hline & c. TEACHERS …… & 3 \\
\hline & 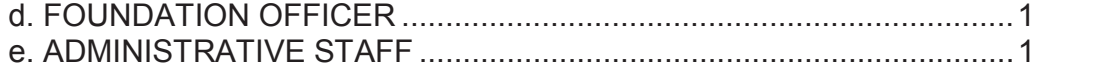 & $\begin{array}{l}3 \\
3\end{array}$ \\
\hline \multirow[t]{2}{*}{ H4. WERE THE RESPONDENTS ABLE TO ANSWER THE QUESTIONS FLUENTLY? } & NO & \\
\hline & YES & \\
\hline
\end{tabular}




\section{SECTION CP:INTERVIEW SESSION NOTES}

\begin{tabular}{|c|c|c|c|c|c|c|}
\hline LANGMAIN (CK1). & \multicolumn{2}{|l|}{ Interview was entirely/mostly conducted in what language? } & \multicolumn{4}{|l|}{$\downarrow \downarrow \downarrow \downarrow$ other_ } \\
\hline LANGOTHR (CK2). & Other languaged used (if any): & & $\perp$ ـ other_ & & & \\
\hline \multicolumn{7}{|l|}{ LANGUAGE CODE: } \\
\hline 00. Bahasa Indonesia & 04. Batak & Sasak & 12. Makassar & 16. Toraja & 20. & Lampung \\
\hline 01. Java & 05. Bugis & Minang & 13. Nias & 17. Lahat & 95. & Other, \\
\hline 02. Sunda & 06. Cina & Banjar & 14. Palembang & 18. Other South Sumatera & 96. & NA \\
\hline 03. Bali & 07. Madura & 11. Bima & 15. Sumbawa & 19. Betawi & & \\
\hline
\end{tabular}

\section{RESULT (FP3). RESULTS OF INTERVIEW}

REASON (FP4). REASON FOR ANSWERING "2" / "3" IN RESULT.

FP6. MONITORING BY SUPERVISOR

\section{Completed $\rightarrow$ FP6}

2. Partly completed

3. Not completed

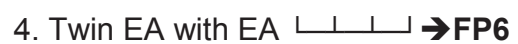

3. Respondent refused

a. Observed (sup_obs)......... 1

b. Checked (sup_edit) .............. 1

c. Verified (sup_veri) ................ 1

rant

INTERVIEWER NOTE: 


\section{INDONESIAN FAMILY LIFE SURVEY 2014 COMMUNITY AND FACILITY SURVEY SCHOOL QUESTIONNAIRE B}

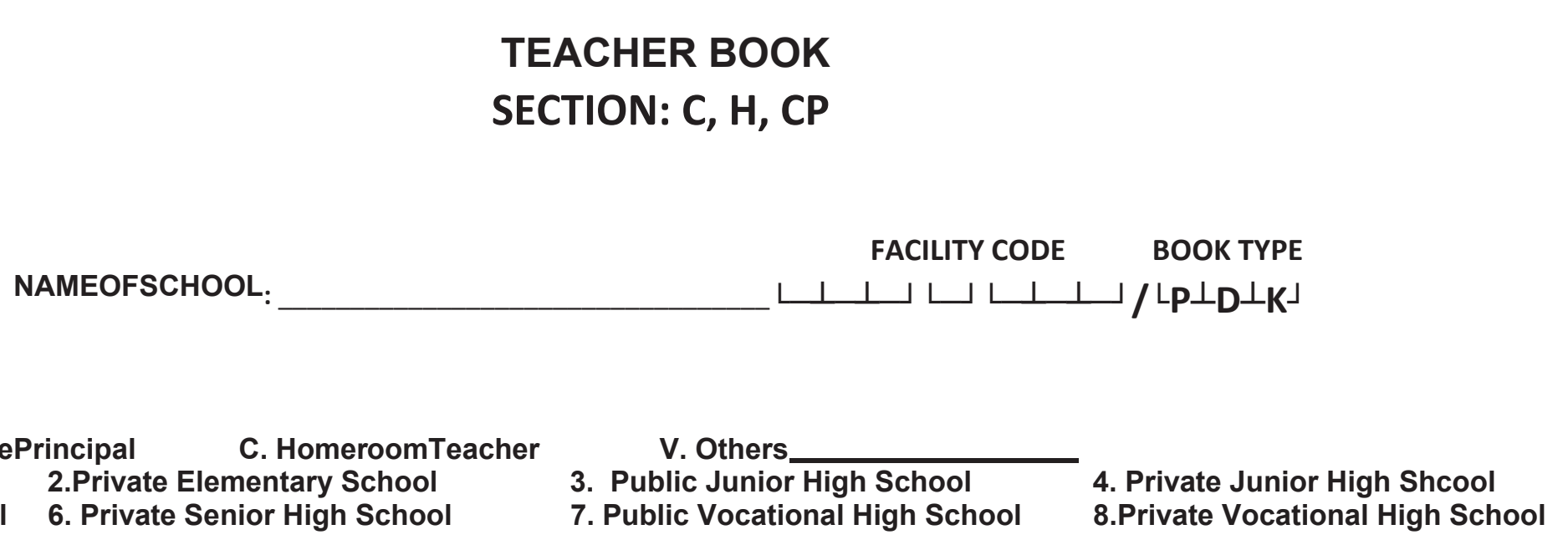




\section{SECTION C: TEACHER}

Section C Respondent

\begin{tabular}{|c|c|c|}
\hline & KRTYPE & $\begin{array}{c}\text { RESPONDENT C } \\
\text { C.TEACHER/HOMEROOM TEACHER }\end{array}$ \\
\hline KR1_C. & Respondent & \\
\hline KR1a_C. & Position/occupation in this School: & $\begin{array}{lllll}01 & 02 & 03 & 04 & 05 \\
06 & 07 & 08 & 95 & \end{array}$ \\
\hline KR2_C. & Age : & $\llcorner\sqcup$ Years \\
\hline KR2a_C. & Length of tenure in position: & $\begin{array}{llll}\sqcup \downarrow / & \perp & \perp & \perp \\
\text { Month } & \text { Year }\end{array}$ \\
\hline KR3_C. & INTERVIEWER CHECK: SEX OF RESPONDENT & 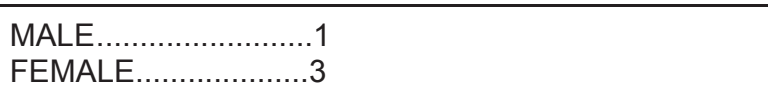 \\
\hline KR3a_C. & Highest level of education attended & \llcorner \\
\hline KR4_C. & Highest class completed: & $\begin{array}{ccccc}00 & 01 & 02 & 03 & 04 \\
05 & 06 & 07 & 98 & 95 \\
\end{array}$ \\
\hline KR5_C. & Length of time lived in the village : & 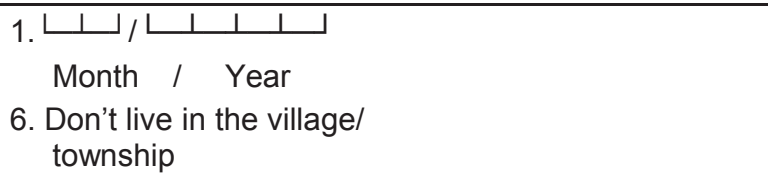 \\
\hline KR6_C. & How did $[\ldots]$ come to assume to the position? & $\begin{array}{l}\text { 1. Elected } \\
\text { 2. Appointed by Government / Education District Office } \\
\text { 3. Appointed byFoundation } \\
\text { 5. Other }\end{array}$ \\
\hline
\end{tabular}

\section{Code for position in village (KR1a_C):}

01. The Principal

21. The Vice Principal of the Curricullum

24. The Vice Principl of the Sublic Relations

05. The Vice Principal of the basic facilities

(Sarana dan prasarana)

The Principal of Administration and

Finance/Tata Usaha

07. Other Vice Principal
08. Teacher/Homeroom Teach

98. Teach
Codes for highest level of education

(KR3a):

01. No school/not yet in schoo

03 . Junior High school

04. Junior High - Vocationa

05. High School - General

06. High School - Vocational

60. D1, D2, D3 (Junior College)

61. University S1 (Bachelor)

62. University S2 (Masters)

63. University S3 (Ph.D.)
11. Kejar Paket $A$ (Adult Education A)

2. Kejar Paket B (Adult Education B)

14. Islamic School (Pondok Pensantren)

15. Kejar Paket C (Adult Education C)

17. School for the disabled

72. Islamic Elementary School (Madrasah Ibtidaiyah)

73. Islamic Junior High School (Madrasah Tsanawiyah)

74. Islamic Senior High School (Madrasah Alliyah)

90. Kindergarten

95. Other
Codes for highest grade (KR4):

00 . Never completed class 1

01.

02. 2

03. 3

05. 5

06. 6

07. Graduated

96. No school

98. DON'T KNOW 


\section{SECTION C: TEACHER}

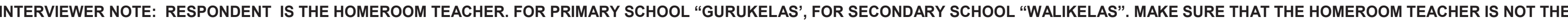
SCHOOL COUNSELLOR, NOT GRADE 6 " GURU KELAS” FOR ELEMENTARY SCHOOL, AND NOT GRADE 3 "WALI" FOR SECONDARY SCHOOL.

\begin{tabular}{|c|c|c|}
\hline C1. & Name of Teacher? & \\
\hline C2. & $\begin{array}{l}\text { When were you born? } \\
\text { (IF HE/SHE HAS FORGOTTEN THE } \\
\text { DATE, PLEASE ASK THE PRESENT } \\
\text { AGE) }\end{array}$ & 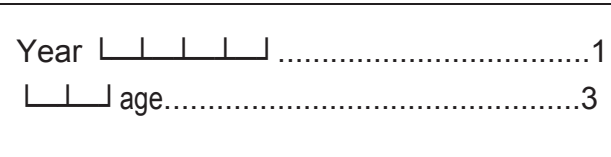 \\
\hline C3. & Sex of Respondent? & $\begin{array}{l}\text { Male } \\
\text { Female }\end{array}$ \\
\hline C4a & $\begin{array}{l}\text { What courses do you teach at this } \\
\text { school? }\end{array}$ & 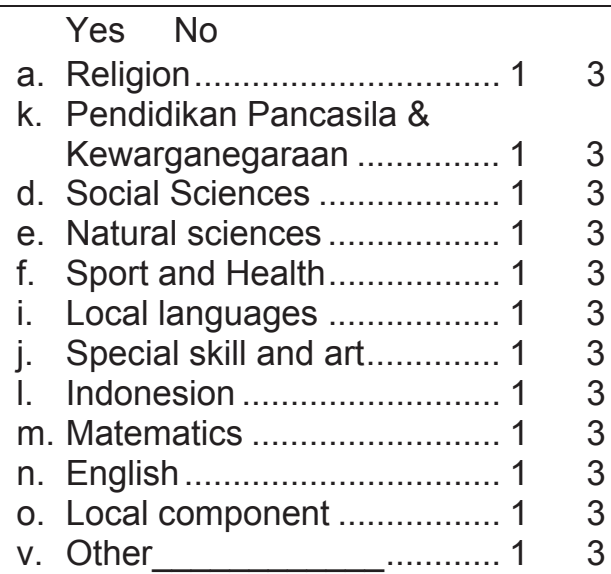 \\
\hline
\end{tabular}

\begin{tabular}{|c|c|c|}
\hline C5. & $\begin{array}{l}\text { What is the highest level of education you } \\
\text { graduated from? }\end{array}$ & 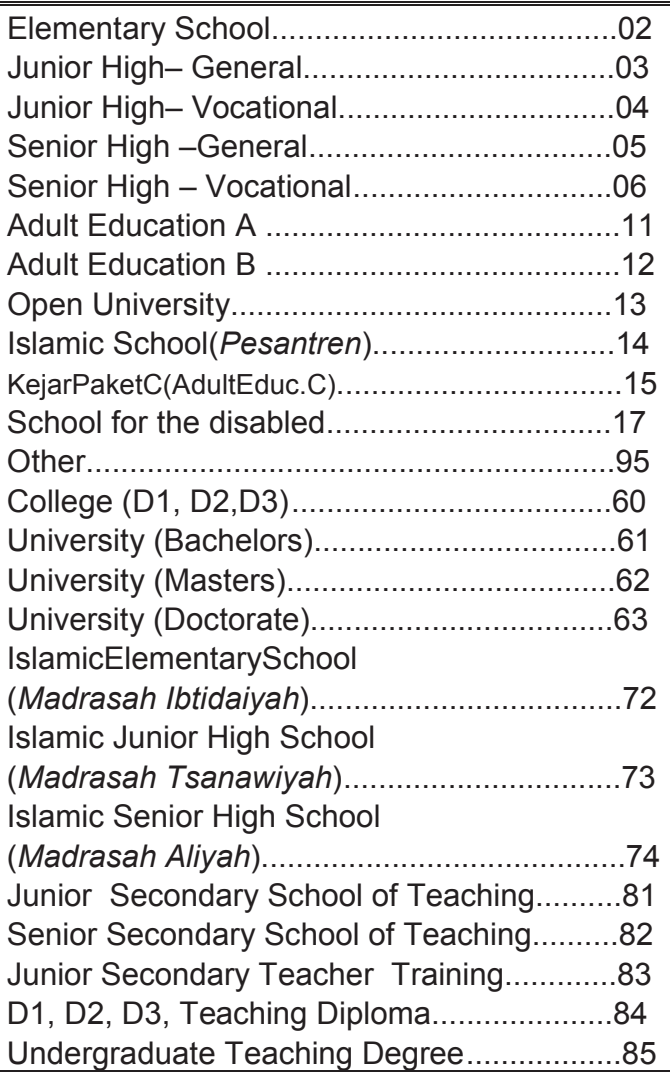 \\
\hline C5a & $\begin{array}{l}\text { State the highest level/class you have } \\
\text { completed? }\end{array}$ & 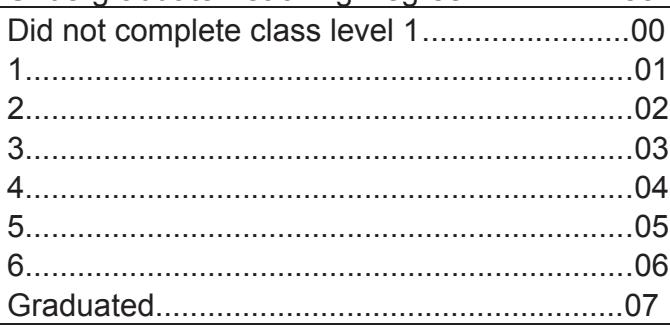 \\
\hline C6 & $\begin{array}{l}\text { Please state the year of your graduation } \\
\text { from the highest level of education you } \\
\text { completed: }\end{array}$ & Year \\
\hline
\end{tabular}




\section{SECTION C: TEACHER}

\begin{tabular}{|c|c|c|}
\hline$\overline{C 7}$ & $\begin{array}{l}\text { Please state the number of years you } \\
\text { have been teaching: }\end{array}$ & $\llcorner\sqcup$ Years \\
\hline C8 & $\begin{array}{l}\text { Howmany hours perweek do you work at } \\
\text { this school? }\end{array}$ & $\begin{array}{l}\sqcup \perp \text { hours perweek } \\
\text { houl }\end{array}$ \\
\hline $\mathrm{C8a}$ & $\begin{array}{l}\text { Howmany hours perweek doyou spent } \\
\text { teaching in class? }\end{array}$ & $\begin{array}{l}\sqcup \sqcup \sqcup \\
\text { hours perweek }\end{array}$ \\
\hline C9 & $\begin{array}{l}\text { Please state your monthly net salary from } \\
\text { teaching at this school: }\end{array}$ & 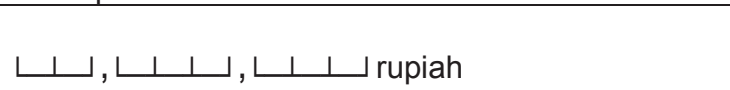 \\
\hline C8aa & $\begin{array}{l}\text { Please state your monthly income from } \\
\text { teaching at this school: }\end{array}$ & $\left\llcorner\perp,\left\llcorner\perp \perp,\left\llcorner\perp \perp \_\right.\right.\right.$rupiah \\
\hline C10 & $\begin{array}{l}\text { Do you receive any teacher's allowance } \\
\text { from this school? }\end{array}$ & 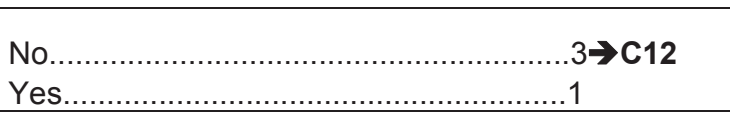 \\
\hline C11. & $\begin{array}{l}\text { What type allowance do you receive from } \\
\text { this school? }\end{array}$ & 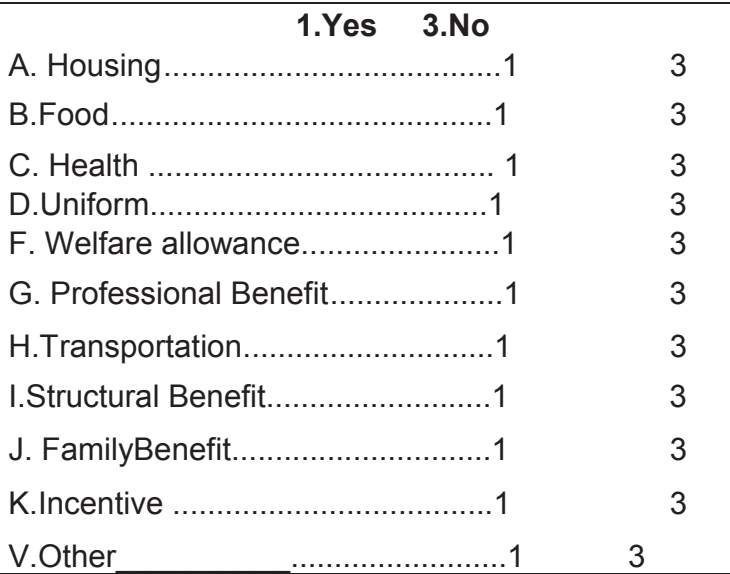 \\
\hline C12 & $\begin{array}{l}\text { Apart from teaching at this school, do you } \\
\text { have any other jobs? }\end{array}$ & 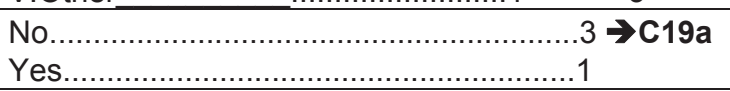 \\
\hline C13. & $\begin{array}{l}\text { How many hours perweek do you spend } \\
\text { on that job? }\end{array}$ & $\sqcup$ hours perweek \\
\hline C17a & $\begin{array}{l}\text { What is your employment status at your } \\
\text { other job? }\end{array}$ & 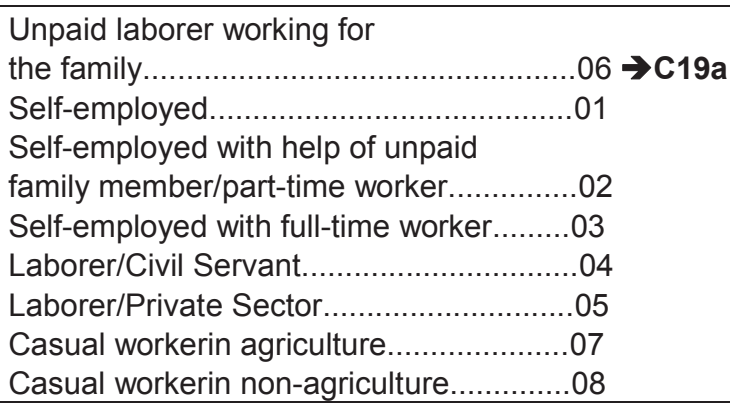 \\
\hline C18 & $\begin{array}{l}\text { What is your monthly gross income from } \\
\text { your other job? }\end{array}$ & $\begin{array}{l}\text { 1. Total } \sqcup \perp,\left\llcorner\perp \perp, \sqcup \perp \perp \_ \text {Rupiah }\right. \\
\text { 8. DON'TKNOW }\end{array}$ \\
\hline
\end{tabular}

\begin{tabular}{|c|c|c|c|c|}
\hline & C19a. & A19b. & C20a. & C20c. \\
\hline C1TYPE & $\begin{array}{c}\text { In the last } 5 \\
\text { years, did you } \\
\text { ever attend any } \\
{[\ldots . .] \text { training in }} \\
\text { keeping with the } \\
\text { duties of School } \\
\text { Principal? }\end{array}$ & $\begin{array}{c}\text { When didyou } \\
\text { participate in the last } \\
\text { training[...]? }\end{array}$ & $\begin{array}{c}\text { Howlong didyou } \\
\text { participate in this } \\
\text { training? } \\
\text { (INTERVIEWER } \\
\text { NOTE:IF THE } \\
\text { RESPONDENT HAS } \\
\text { ATTENDED MORE } \\
\text { THAN ONE } \\
\text { TRAINING IN THE } \\
\text { LAST 5 YEARS, } \\
\text { RECORD THE } \\
\text { INFORMATION } \\
\text { REGARDING THE } \\
\text { MOST RECENT } \\
\text { TRAINING.) }\end{array}$ & $\begin{array}{l}\text { Howmany hours } \\
\text { perweek did you } \\
\text { attend the } \\
\text { training? }\end{array}$ \\
\hline a. Management & $\underset{\Downarrow}{3 . \text { No } 1 . Y e s \rightarrow}$ & 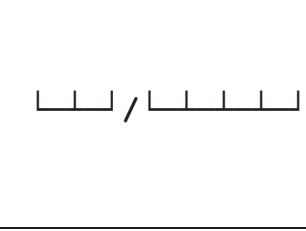 & $\begin{array}{l}\text { 1. } \frac{1}{\text { 3. days }} \\
\text { 4.weeks } \\
\text { 5. months } \\
\text { 8. DON'TKNOW }\end{array}$ & $\begin{array}{l}\text { 1. } \downarrow \perp \text { hours } \\
\text { perweek } \\
\text { 8. DON'TKNOW }\end{array}$ \\
\hline b. Teaching skills & $\underset{\downarrow}{\text { 3.No } 1 . Y e s \rightarrow}$ & 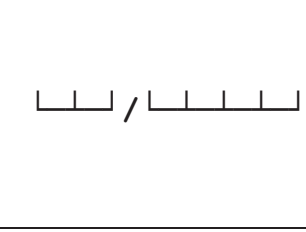 & 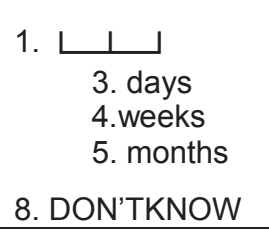 & $\begin{array}{l}\text { 1. } \downarrow \text { hours } \\
\text { perweek } \\
\text { 8. DON'TKNOW }\end{array}$ \\
\hline c. Subject-related & 3.No 1.Yes $\rightarrow$ & 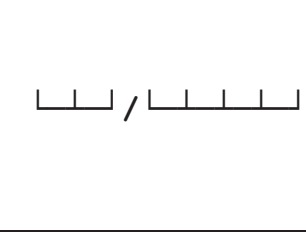 & $\begin{array}{l}\text { 1. L } 1 \text {. } \\
\text { 3. days } \\
\text { 4.weeks } \\
\text { 5. months } \\
\text { 8. DON'TKNOW }\end{array}$ & $\begin{array}{l}\text { 1. } \downarrow \text { hours } \\
\text { perweek } \\
\text { 8. DON'TKNOW }\end{array}$ \\
\hline
\end{tabular}

\begin{tabular}{|c|c|c|}
\hline C21a. & $\begin{array}{l}\text { What curriculum do you use } \\
\text { inteaching your courses? }\end{array}$ & 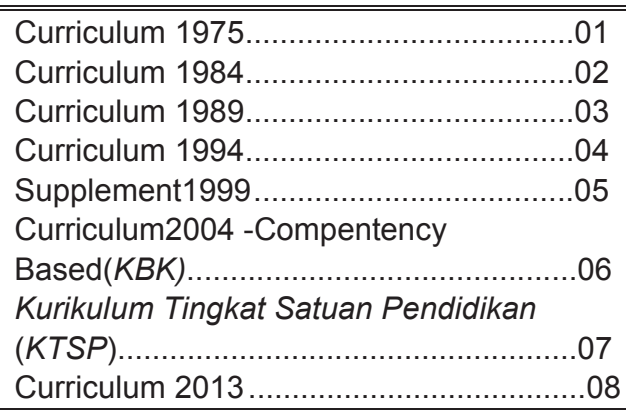 \\
\hline C22a. & $\begin{array}{l}\text { What year were the handbooks or } \\
\text { compulsory reading books for the } \\
\text { subjects you are using published? }\end{array}$ & 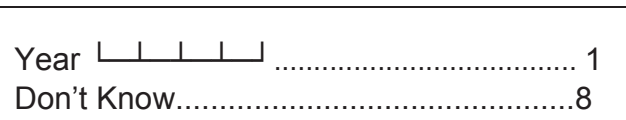 \\
\hline
\end{tabular}




\section{SECTION C: TEACHER}

C23a. When you areteaching your

courses in the class, beside

using the handbooks or the

compulsory reading books, do

books or materials?

C24a. Please state the supporting

materials that you use in teaching your courses.

C37. Do you always prepare before teaching?

C25. Howmany students are in this class?

No................................................... C37

Yes....................................................

a. School Work Sheet.................... 1. Yes 1 3. No

i. Magazine/newspapers/articles.

C26. On average, howmany students attend this class eachday?

C27a. What is the total number of students in this class that receive books from related to your courses from the following sources: b. Textbooks............................... 1

c. Calculator.................................. 1

d. Computer ................................... 1

e. Dictionary.

f. Writing tools $\mathrm{MN}$

g. Demonstration Tools.................... 1

v. Others.....................................1

Yes, made RPP ..................................... 1

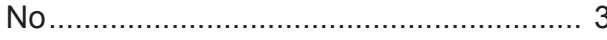

\section{$\sqcup \perp \downarrow$ students}

\section{$\sqcup \perp$ students}

3

3 3

(n)

s

a. Free of charge from the school......... $\downarrow \perp \perp$

Persons

b. Borrow from the school......................

c. Purchase fromschool....................... $\llcorner\perp \perp$

d. Make photocopies........................... ᄂ

e. Purchase outside of theschool........ $\downarrow \perp \perp$

g. Obtain from sibling, friend...................

f. Obtain outside the school................. $\downarrow \perp \perp$

\begin{tabular}{|c|c|c|}
\hline C28a. & $\begin{array}{l}\text { CAPI CHECK: C27a, C27a LINE } \\
\text { B >0? }\end{array}$ & 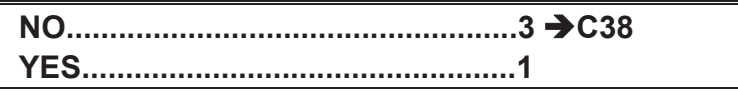 \\
\hline C28. & $\begin{array}{l}\text { Can the afore mentioned books } \\
\text { be taken home at night? }\end{array}$ & 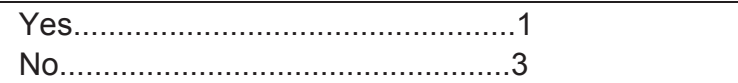 \\
\hline C38 & $\begin{array}{l}\text { Do you give a test to the student } \\
\text { weekly and have notes about the } \\
\text { resutls? }\end{array}$ & 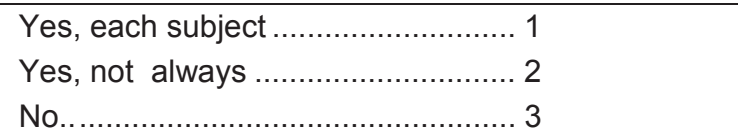 \\
\hline C29. & $\begin{array}{l}\text { Have you everheard about the } \\
\text { Teacher Certification program? }\end{array}$ & 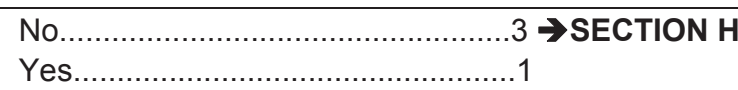 \\
\hline
\end{tabular}

\begin{tabular}{|c|c|c|}
\hline C29a. & $\begin{array}{l}\text { Have you everheard about the } \\
\text { Teacher Certification program? }\end{array}$ & $\begin{array}{l}\text { Yes. } \\
\text { No }\end{array}$ \\
\hline C30. & Have you beencertified? & 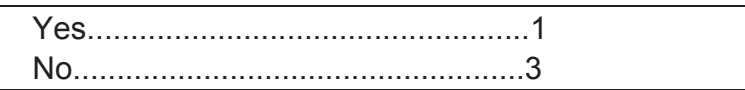 \\
\hline c31. & $\begin{array}{l}\text { Do you know about the procedure } \\
\text { to obtain Teacher Certification? }\end{array}$ & 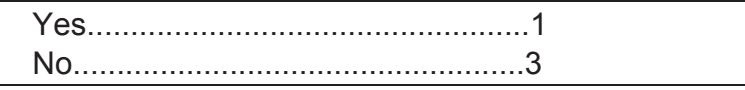 \\
\hline C27b & $\begin{array}{l}\text { Do you allocate additional } \\
\text { teaching hours to fulfill } \\
\text { certification requirement? How } \\
\text { many hours? }\end{array}$ & 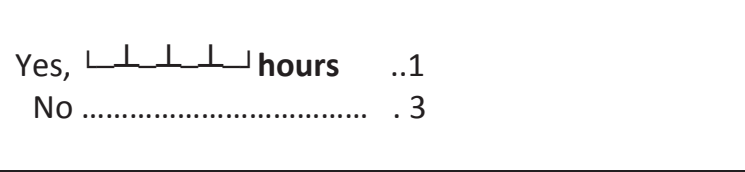 \\
\hline C27c & $\begin{array}{l}\text { How do you fulfill teaching hour } \\
\text { requirement? }\end{array}$ & $\begin{array}{l}\text { 1. Teaching in other school } \\
\text { 2. Joint teaching with other teacher } \\
\text { 3. Teaching Adult Education ABC/PKBM } \\
\text { 5. Other }\end{array}$ \\
\hline C32. & $\begin{array}{l}\text { Have you applied for the } \\
\text { Certificate? }\end{array}$ & 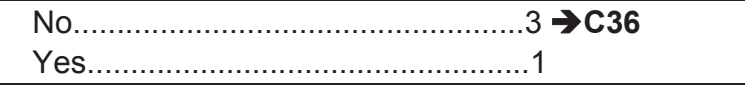 \\
\hline C33. & $\begin{array}{l}\text { Howmuch did/will it cost to obtain } \\
\text { the certificate? }\end{array}$ & 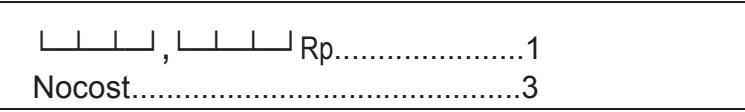 \\
\hline C34. & $\begin{array}{l}\text { Has there been an increase in } \\
\text { your income or allowance from } \\
\text { teaching after you have been } \\
\text { certified? }\end{array}$ & 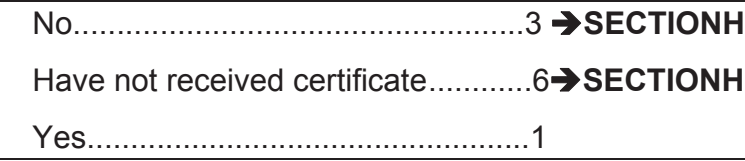 \\
\hline C35. & By howmuch was the increase? & 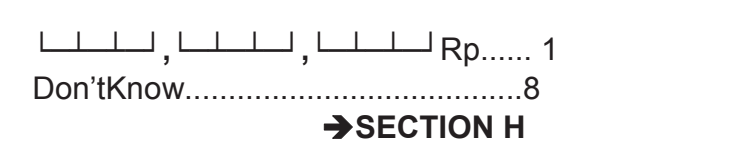 \\
\hline C36. & $\begin{array}{l}\text { Do you have any plan to register } \\
\text { the teacher certification in the } \\
\text { future? }\end{array}$ & Yes \\
\hline
\end{tabular}




\section{SECTION H: OBSERVATION SHEET}

\begin{tabular}{|c|c|c|}
\hline & HOMEROOM TEACHER & \\
\hline H1. WERE THERE ANY OTHER PEOPLE PRESENT IN THE ROOM DURING INTERVIEW? & NO & \\
\hline H2. WHO EXACTLY WERE PRESENT DURING INTERVIEW? & 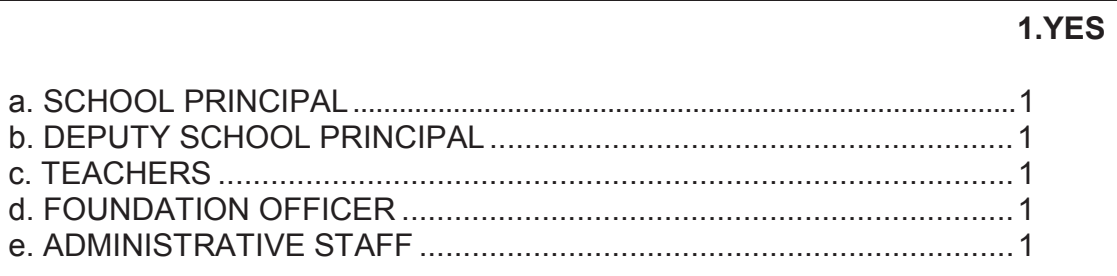 & $\begin{array}{l}3 . N O \\
3 \\
3 \\
3 \\
3 \\
3\end{array}$ \\
\hline H3. DID THOSE PRESENTALSO ANSWER THE QUESTION INTHIS INTERVIEW? & 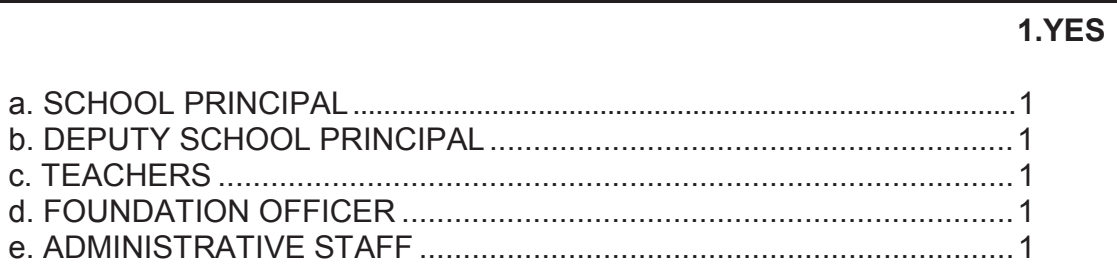 & $\begin{array}{l}3 . N O \\
3 \\
3 \\
3 \\
3 \\
3\end{array}$ \\
\hline H4. WERE THE RESPONDENTS ABLE TO ANSWER THE QUESTIONS FLUENTLY? & $\begin{array}{l}\text { NO } \\
\text { YES }\end{array}$ & \\
\hline
\end{tabular}




\section{SECTION CP: INTERVIEWER NOTES}

\begin{tabular}{|c|c|c|c|c|c|c|c|}
\hline LANGMAIN (CK1). & Interview was entirely/mostly conducted in what language? & & L 1 other & & & & \\
\hline LANGOTHR (CK2). & Other languaged used (if any): & & $\perp$ ـ other & & & & \\
\hline LANGUAGE CODE: & & & & & & & \\
\hline 00. Bahasa Indonesia & 04. Batak & 08. Sasak & 12. Makassar & 16. & Toraja & 20. & Lampung \\
\hline 01. Java & 05. Bugis & 09. Minang & 13. Nias & 17. & Lahat & 95. & Other, \\
\hline 02. Sunda & 06. Cina & 10. Banjar & 14. Palembang & 18. & Other South Sumatera & 96. & NA \\
\hline 03. Bali & 07. Madura & 11. Bima & 15. Sumbawa & 19. & Betawi & & \\
\hline
\end{tabular}

\begin{tabular}{|c|c|c|c|}
\hline RESULT (FP3). RESULTS OF INTERVIEW & REASON (FP4). REASON FOR ANSWERING “2” / "3" IN RESULT. & FP6. MONITORING BY SUPERVISOR & \\
\hline $\begin{array}{l}\text { 1. Completed } \rightarrow \text { FP6 } \\
\text { 2. Partly completed } \\
\text { 3. Not completed } \\
\text { 4. Twin EA with EA } \downarrow \neg \rightarrow \text { FP6 }\end{array}$ & $\begin{array}{l}\text { 1. Respondent was travelling/not in location } \\
\text { 2. Respondent was too busy } \\
\text { 3. Respondent refused }\end{array}$ & $\begin{array}{ll} & \text { Yes } \\
\text { a. } & \text { Observed (sup_obs) .......... } 1 \\
\text { b. } & \text { Checked (sup_edit)............ } 1 \\
\text { c. } & \text { Verified (sup_veri)............. } 1\end{array}$ & $\begin{array}{l}\text { No } \\
3 \\
3 \\
3\end{array}$ \\
\hline
\end{tabular}

\section{INTERVIEWER NOTE:}




\title{
INDONESIAN FAMILY LIFE SURVEY 2014 \\ COMMUNITY AND FACILITY SURVEY SCHOOL QUESTIONNAIRE C
}

\author{
SCHOOL TEST BOOK \\ SECTION: F, CP \\ NAMEOFSCHOOL: \\ FACILITY CODE BOOK TYPE

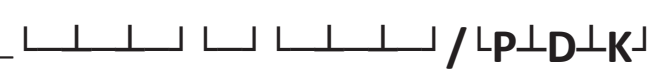


SECTION F: STATISTICS AND FINAL EXAMINATION GRADES

\begin{tabular}{|c|c|c|}
\hline & KRTYPE & RESPONDENT \\
\hline KR1_F. & Respondent & \\
\hline KR1a_F. & Posttion/occupation in this School: & $\begin{array}{lllll}01 & 02 & 03 & 04 & 05 \\
06 & 07 & 08 & 95 & \end{array}$ \\
\hline KR2_F. & Age : & $\sqcup \downarrow$ Years \\
\hline KR2a_F. & Length of tenure inpositions: & 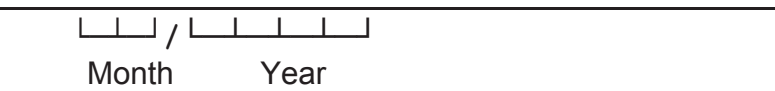 \\
\hline KR3_F. & INTERVIEWER CHECK: SEX OF RESPONDENT & 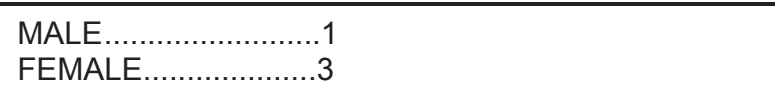 \\
\hline KR3a_F. & Highest level of education attended: & $\sqcup$ \\
\hline KR4_F. & Highest class completed & $\begin{array}{ccccc}00 & 01 & 02 & 03 & 04 \\
05 & 06 & 07 & 98 & 95\end{array}$ \\
\hline KR5_F. & Length of time lived in the village : & 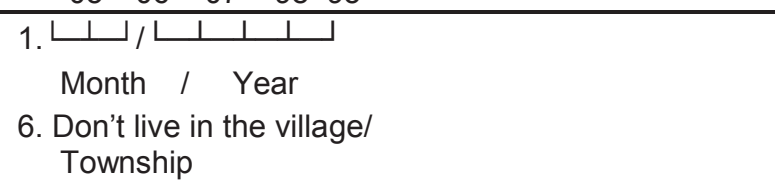 \\
\hline KR6_F. & How did $[\ldots]$ come to assume to the position? & $\begin{array}{l}\text { 1. Elected } \\
\text { 2. Appointed by Government / Education District Office } \\
\text { 3. Appointed byFoundation } \\
\text { 5.Other }\end{array}$ \\
\hline
\end{tabular}

\section{Code for position in village (KR1a_F):}

01. The Principal

03. The Vice Principal of the Curricullum

4. The Vice Principal of the Sublic Relations

5. The Vice Principal of the basic facilities

(Sarana dan prasarana)

6. The Principal of Administration and

Finance/Tata Usaha

07. Other Vice Principal

08. Teacher/Homeroom Teacher
Codes for highest level of education

01. No school/not yet in school

2. Elementary school

03. Junior High - General

05. High School - General

06. High School - Vocational

60. D1, D2, D3 (Junior College)

62. University $S 2$ (Machelor)

63. University S3 (Ph D.)
11. Kejar Paket A (Adult Education $A$ )

13. Open Univeristy

14. Islamic School (Pondok Pensantren)

5. Kejar Paket C (Adult Education C)

17. School for the disabled

72. Islamic Elementary School (Madrasah Ibtidaiyah)

73. Islamic Junior High School (Madrasah Tsanawiya

. Kindergarten

98. DON'T KNOW

95. Other
Codes for highest grade (KR4 F)

00. Never completed class 1

02.

03.3

04. 4

05. 5

7. Graduated

98. DON'T KNOW 
We would like to have information regarding the repor t of the UAN Score for the school year 2013/2014. In this study, we need the tests results of 25 students who will be selected at random. The sample selection stages areas follows:

How to collect the national final examination mark for the school year 2013/2014

1. Collect the data from school for students who followed final examination test for mathematic and Indonesian subjects.

2. Grouped each mark based on the subject and arranged based on examination number, for senior high school if each major has each series number then it is compounded from science major added with social mayor or language major.

3. It is needed to get all final examination marks for all students who followed national examination.

4. If it's possible the marks of final examination can be coppied to be borrowed to the base camp. Therefore if it is possible it can be entered in base camp to short the interview time in facilities so that move to other facilities.

5. The data entry for final examination marks should be suitable with the selected number based on random rules that is done automatically by CAPI

6. For entry the final examination marks, if there is selected number but has no final examination marks then it will be replaced with the previous number (it is the rules applied previously)

This proses is done automatically by CAP

\section{FIRST ROUND}

1. Count the number of students who have taken the Ebtanas test for Indonesian Language and Mathematics. For example, participants for Indonesian Language= $\mathbf{5 3}$ students, therefore $(\mathbf{N})=\mathbf{5 3}$ students. Write the figure 53 in column $1 \mathrm{a}$.

2. The interval of the selection[ I ] is a rounding up of $(N / 25)$. Example $N=53$, round up from $(53 / 25)=3$. Write the figure 3 in column $2 a$.

3. The Consecutive Numbers of the First Selected Participants are:I,2l, kl.(with $\mathrm{kl} \leq \mathrm{N})$

In case $N=53$, the First Round of Selected Participants have the Numbers: 3, 6, 9, 48, and 51.So the number of Selected Participants of the First round is: $\mathbf{k}=(53 / 3)=17$. Write the figure 17 in column $3 a$.

\section{EXAMINE:}

Is the number of Selected Participants $(\mathbf{K})=25 ?$

If Yes ----------------------> STOP

No -------------------------->continue to stage 5

5. Thus, the number of participants still needed $=X=(25-k)$

In this case it has been calculated (seestage 3$)$, that $k=17$ So, $X=(25-17)=8$.

6. EXAMINE: if, $X=1$---------> To stage 7 if $X>1$ to stage 8

7. Because $X \neq 1$, for the purpose of determining the Number of the Final Selected Participants, another student is needed. Thus the Final Selected Participant is the Participant with the Number ( I - ( $\mathbf{N}-\mathbf{k I})$ ).

8. SECOND ROUND:

If at the sixth stage, $X>1---------->$, then

Determine a New Interval [ IB ] which constitutes a rounding down from $\{\mathbf{N} /(\mathbf{2 5 - k})\}$

In case $\mathbf{N}=53$, IB $=$ rounding down $\{53 /(25-17)\}=7$. Write number 6 in column $4 a$

9. The number of the First Selected Participant [NPP]= IB - ( $\mathbf{N}-\mathbf{k l})$

In example $\mathbf{N}=53, \mathbf{N P P}=6-(53-51)=4$. Write number 4 in column 5 a.

10. The Consecutive Order of the Selected Participants in the Second Round is: NPP,NPP+IB,NPP + 2IB,ect. In example $\mathrm{N}=53$,the Consecutive Order of Selected Participants in the Second Round is:4, 10, 16, ..

11. BEFORE WRITING DOWN THE EBTANAS SCORES, EXAMINE:IF THERE ARE ANY SELECTED PARTICIPANTS IN THE FIRST ROUND. IF YES, REPLACE THE NUMBER BY THE NUMBER OF A PREVIOUS PARTICIPANT.

12. If the selected number has no data, replaced with the next number. For population $\leq 25(\mathrm{~N} \leq 25)$ with selected number without data, enter "996" (because previous number has been selected)

13. Subsequently, write the UAN SCORE for eachselected participantin the second round 


\begin{tabular}{|c|c|c|c|c|}
\hline & \multicolumn{2}{|c|}{ A. INDONESIAN LANGUAGE } & \multicolumn{2}{|c|}{ B. MATHEMATICS } \\
\hline F1. Number of UAN participants =[N] & \multicolumn{2}{|c|}{ 1a. $\llcorner\perp \perp$} & \multicolumn{2}{|c|}{ 1b. $L \perp$} \\
\hline $\begin{array}{l}\text { F2. Number of UAN participants divided } \\
25=[\mathrm{I}] \text {. Please round up. }\end{array}$ & \multicolumn{2}{|c|}{ 2a. $\llcorner\perp \perp$} & \multicolumn{2}{|c|}{ 2b. $\downarrow$} \\
\hline $\begin{array}{l}\text { F3. Number of selected participants= } \\
\text { rounding downward of }(\mathbf{N} / \mathrm{I})=\mathbf{k}\end{array}$ & \multicolumn{2}{|c|}{ 3a. $\left\llcorner\perp \perp \_ـ\right.$} & \multicolumn{2}{|c|}{ 3b. $\downarrow$} \\
\hline $\begin{array}{l}\text { F4.SECOND: } \\
\quad \text { IB = rounding down of }\{\mathrm{N} /(25-\mathbf{k})\}\end{array}$ & \multicolumn{2}{|c|}{ 4a. $\llcorner\perp \perp$} & \multicolumn{2}{|c|}{ 4b. $\downarrow$} \\
\hline \multirow{2}{*}{$\begin{array}{l}\text { F5. Number of First Selected Participant }= \\
{[\text { [NPP] }=\{\text { IB }-(\mathbf{N}-\mathbf{k I})\}}\end{array}$} & \multicolumn{2}{|c|}{ 5a. $\llcorner\perp \perp$} & \multicolumn{2}{|c|}{ 5b. } \\
\hline & SelectedStudents & Student Marks & SelectedStudents & Student Marks \\
\hline & 1. L L & 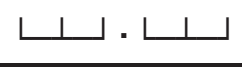 & 1. L $\downarrow \perp$ & 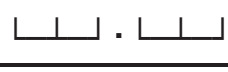 \\
\hline & 2. $\downarrow$ & 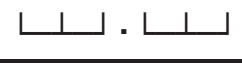 & 2. $\downarrow$ & 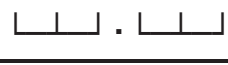 \\
\hline & 3. $\downarrow \perp$ & $\llcorner\llcorner. \sqcup \sqcup$ & 3. $\downarrow \perp \perp$ & $\llcorner\llcorner.\llcorner\perp$ \\
\hline & 4. $L \perp \perp \perp$ & 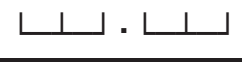 & 4. $\downarrow$ & 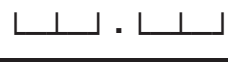 \\
\hline & 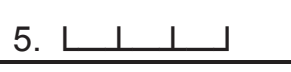 & 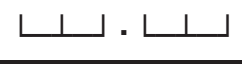 & 5. $\downarrow$ & $\llcorner\perp$. لــ \\
\hline & 6. Lلـ & 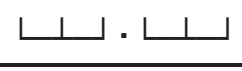 & 6. $\downarrow \perp \perp$ & 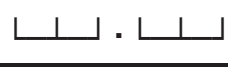 \\
\hline & 7. $\downarrow$ & 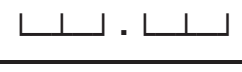 & 7. $\mid \begin{array}{llll} & \perp\end{array}$ & 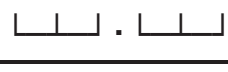 \\
\hline & 8. ᄂ $\downarrow \perp$ & 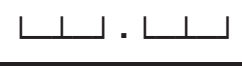 & 8. $\llcorner\perp \perp$ & 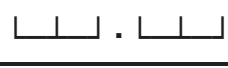 \\
\hline & 9. $L \perp \perp \perp$ & 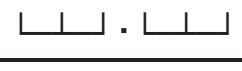 & 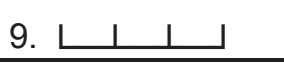 & $\llcorner\perp$. $\cdot ـ$ \\
\hline & 10. 1 & $\sqcup \sqcup$ & 10. $ا ل \perp$ & لــ \\
\hline & 11. $ـ ل ـ$ & 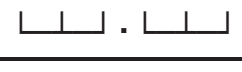 & 11. $ـ \perp$ & 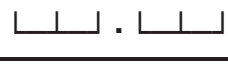 \\
\hline & 12. $\downarrow \perp$ & 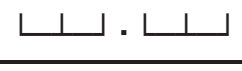 & 12. ᄂ & 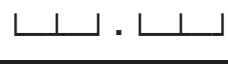 \\
\hline & 13. L $\downarrow \perp$ & 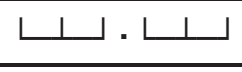 & 13. ᄂ $\downarrow \perp$ & لــ \\
\hline & 14. L لــلـ & $ـ \downarrow$ & 14. $ا ل \perp$ & 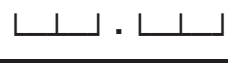 \\
\hline & 15. لــلـ لــالـا & $ـ \downarrow$ & 15. & 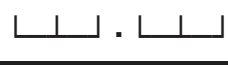 \\
\hline & 16. $ا \perp \perp$ & $\llcorner\perp$. & 16. ᄂ لـ & ــ . لــ \\
\hline & 17. ᄂ لــلـ & لـ & 17. ᄂ لــ & $\sqcup \sqcup$ \\
\hline & 18. $ا$ & 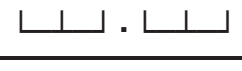 & 18. $\downarrow \perp \perp$ & $\llcorner\sqcup .\llcorner\perp$ \\
\hline & 19. لــلـ لــالـا & 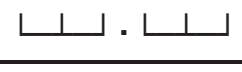 & 19. $ا$ & $\llcorner\perp .\llcorner\perp$ \\
\hline & 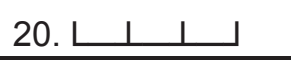 & 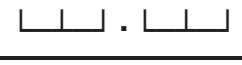 & 20. L لـ & $\left\llcorner \_\right.$. $\longleftarrow$ \\
\hline & 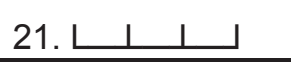 & لــ & 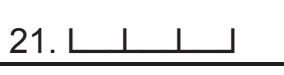 & 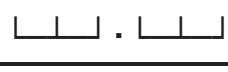 \\
\hline & 22. ᄂ $\downarrow$ & 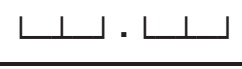 & 22. ᄂ $\downarrow \perp$ & 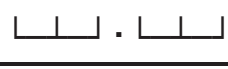 \\
\hline & 23. & $\llcorner\perp . \sqcup \sqcup$ & 23. $ا \quad \perp$ & 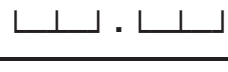 \\
\hline & 24. $ا$ & $\sqcup \sqcup$ & 24. $ا \perp \perp$ & $ـ \downarrow$ \\
\hline & 25. لــــــــا لــا & 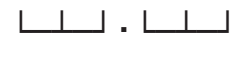 & 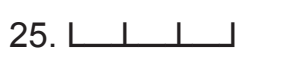 & لـ \\
\hline
\end{tabular}




\section{SECTION CP: INTERVIEW NOTES}

\begin{tabular}{|c|c|c|c|c|c|c|c|}
\hline LANGMAIN (CK1). & Interview was entirely/mostly conducted in what language? & & $\perp$ other_ & & & & \\
\hline LANGOTHR (CK2). & Other languaged used (if any): & & 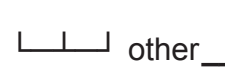 & & & & \\
\hline LANGUAGE CODE: & & & & & & & \\
\hline 00. Bahasa Indonesia & 04. Batak & Sasak & 12. Makassar & 16. & Toraja & 20. & Lampung \\
\hline 01. Java & 05. Bugis & 09. Minang & 13. Nias & 17. & Lahat & 95. & Other, \\
\hline 02. Sunda & 06. Cina & 10. Banjar & 14. Palembang & 18. & Other South Sumatera & 96. & NA \\
\hline 03. Bali & 07. Madura & 11. Bima & 15. Sumbawa & 19. & Betawi & & \\
\hline
\end{tabular}

\begin{tabular}{|c|c|c|c|}
\hline RESULT (FP3). RESULTS OF INTERVIEW & REASON (FP4). REASON FOR ANSWERING “2” / “3” IN RESULT. & FP6. MONITORING BY SUPERVISOR & \\
\hline $\begin{array}{l}\text { 1. Completed } \rightarrow \text { FP6 } \\
\text { 2. Partly completed } \\
\text { 3. Not completed } \\
\text { 4. Twin EA with EA } \downarrow \perp \_\rightarrow \text { FP6 }\end{array}$ & $\begin{array}{l}\text { 1. Respondent was travelling/not in location } \\
\text { 2. Respondent was too busy } \\
\text { 3. Respondent refused }\end{array}$ & $\begin{array}{ll} & \text { Yes } \\
\text { a. } & \text { Observed (sup_obs)......... } 1 \\
\text { b. } & \text { Checked (sup_edit) ........... } 1 \\
\text { c. } & \text { Verified (sup_veri) ............. } 1\end{array}$ & $\begin{array}{l}\text { No } \\
3 \\
3 \\
3\end{array}$ \\
\hline
\end{tabular}

\section{INTERVIEWER NOTE:}




\section{INDONESIAN FAMILY LIFE SURVEY 2014 \\ COMMUNITY AND FACILITY SURVEY \\ SCHOOL QUESTIONNAIRE D}

OBSERVATIONS BOOK

SECTION: D, G, H, CP

NAME OF SCHOOL:

FACILITY CODE BOOK TYPE $\llcorner\perp \perp\llcorner\llcorner\perp \perp / L \mathbf{L} \perp \mathbf{D} \perp \mathbf{K}\lrcorner$ 


\section{SECTION D: OBSERVATION}

DIRECT OBSERVATION IN CLASS, TEACHER ROOM, AND SCHOOL AREA.

ELEMENTARYIJUNIOR HIGH SCHOOL: Choose grade having the biggest number of classes, then observe the first Class found in the grade.

(VOCATIONAL) SENIOR HIGH SCHOOL: Choose field/department having the biggest number of classes, then observe THE FIRSTCLASS FOUND IN THE FIELD/DEPARTMENT.

\section{QUESTION NO.D7,D8,D9, D13 AND D19a MUST BE ASKED}

\begin{tabular}{|c|c|c|}
\hline \multicolumn{2}{|r|}{ KRTYPE } & \multirow[t]{2}{*}{ RESPONDENT } \\
\hline KRP1_D. & Respondent & \\
\hline KRP1a_D. & Posttion/occupation in this School: & $\begin{array}{lllll}01 & 02 & 03 & 04 & 05 \\
06 & 07 & 08 & 95 & \end{array}$ \\
\hline KRP2_D. & Age : & $\llcorner\perp$ Years \\
\hline KRP2a_D. & Length of tenure inpositions: & $\begin{array}{llll}\perp / L & \perp & \perp \\
\text { Month } & \text { Year }\end{array}$ \\
\hline KRP3_D. & INTERVIEWER CHECK: SEX OF RESPONDENT & $\begin{array}{l}\text { MALE } \ldots \ldots \ldots \ldots \ldots \ldots \ldots \ldots \ldots . .1 \\
\text { FEMALE } \ldots \ldots \ldots \ldots \ldots \ldots \ldots \ldots \ldots . . .3\end{array}$ \\
\hline KRP3a_D. & Highest level of education attended & $\perp$ \\
\hline KRP4_D. & Highest class completed: & $\begin{array}{ccccc}00 & 01 & 02 & 03 & 04 \\
05 & 06 & 07 & 98 & 95 \\
\end{array}$ \\
\hline KRP5_D. & Length of time lived in the village : & 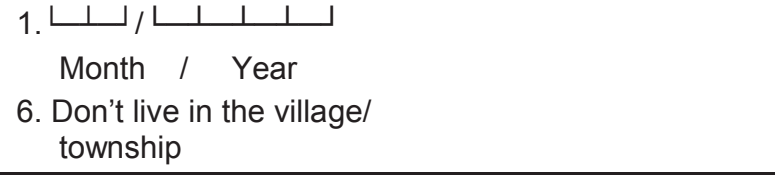 \\
\hline KRP6_D. & How did $[\ldots]$ come to assume to the position? & $\begin{array}{l}\text { 1. Elected } \\
\text { 2. Appointed by Government / Education District Office } \\
\text { 3. Appointed byFoundation } \\
\text { 5. Other }\end{array}$ \\
\hline
\end{tabular}

Code for position in village (KR1a_D): $\quad$ Codes for highest level of education

01. Village/township heads
02. Village secretary

03. Head of Government Administration

04. Head of Village Development

05. Head of Welfare

06. Head of Financial Matters

07. Head of General Affairs

95. Other

01. No school/not yet in school

04. Junior High - General

\section{(KR3a_D):}

02. Elementary schoo

05. High School - General

06. High School - Vocational

60. D1, D2, D3 (Junior College)

61. University S1 (Bachelor)

62. University S2 (Masters)

63. University S3 (Ph.D.)
11. Kejar Paket A (Adult Education A)

13. Open Univeristy

14. Islamic School (Pondok Pensantren)

15. Kejar Paket C (Adult Education $C$ )

17. School for the disabled

72. Islamic Elementary School (Madrasah Ibtidaiyah)

73. Islamic Junior High School (Madrasah Tsangal

74. Islamic Senior High School (Madrasah Alliyah)

90. Kindergarten

95. Other
Codes for highest grade (KR4 D):

00. Never completed class 1

01.

02. 2

03. 3

05. 5

06.

07. Graduated

96. No school

98. DON'T KNOW 


\section{SECTION D: OBSERVATION}

\begin{tabular}{|c|c|c|}
\hline D1. & $\begin{array}{l}\text { IN THE CLASSROOM, IS THERE A } \\
\text { DESK FOR THE TEACHER? }\end{array}$ & YES \\
\hline D2. & $\begin{array}{l}\text { PLEASE COUNT THE NUMBER OF } \\
\text { SEATS IN THIS CLASSROOM! } \\
\text { (IF ONE BENCH CAN BE USED FOR } \\
\text { SIX STUDENTS,THEN THE COUNT IS } \\
6 \text { SEATS) }\end{array}$ & Lلــل SEATS \\
\hline D3. & $\begin{array}{l}\text { THE NUMBER OF BENCHES } \\
\text { OCCUPIED BY STUDENTS IN THIS } \\
\text { CLASS. }\end{array}$ & Lلــل SEATS \\
\hline D4. & $\begin{array}{l}\text { ARE THE BLACKBOARDS, CHALK, } \\
\text { AND ERASERS IN THE CLASSROOM } \\
\text { USABLE? }\end{array}$ & YES \\
\hline D5. & $\begin{array}{l}\text { IS TEACHING IN THIS CLASSROOM } \\
\text { EVER DISRUPTED BY INADEQUATE } \\
\text { LIGHTING FROM THE MAIN SOURCE } \\
\text { OF LIGHT,LIKE WINDOW, DOOR,AND } \\
\text { OPENING? }\end{array}$ & YES \\
\hline D6. & $\begin{array}{l}\text { DOES THIS CLASSROOM USE ANY } \\
\text { ELECTRIC LIGHTING? }\end{array}$ & $\begin{array}{l}\text { NO } \\
\text { YES }\end{array}$ \\
\hline D7. & $\begin{array}{l}\text { If yes, what is the main source of } \\
\text { electricity? }\end{array}$ & 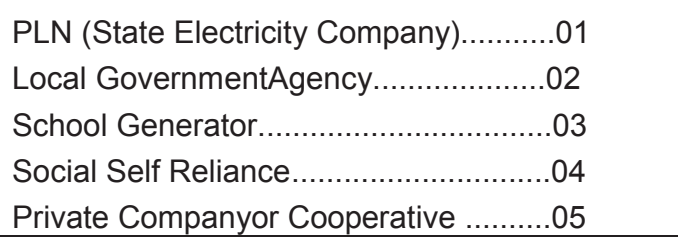 \\
\hline D8. & $\begin{array}{l}\text { Did this classroom ever lose electrical } \\
\text { power, and did this disrupt the study } \\
\text { activities? }\end{array}$ & $\begin{array}{l}\text { Yes. } \\
\text { No }\end{array}$ \\
\hline D9. & $\begin{array}{l}\text { When dsruptions occur, is a substitute } \\
\text { electricity source available? }\end{array}$ & Yes \\
\hline
\end{tabular}

\begin{tabular}{|c|c|c|}
\hline D10. & $\begin{array}{l}\text { PLEASE DESCRIBE THE FLOOR IN THIS } \\
\text { CLASSROOM. }\end{array}$ & 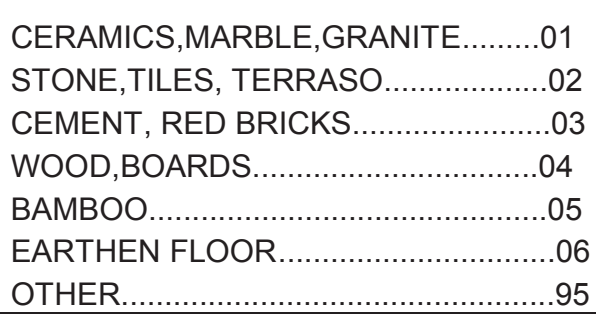 \\
\hline D11 & $\begin{array}{l}\text { PLEASE DESCRIBE THE WALLS IN THIS } \\
\text { CLASSROOM. }\end{array}$ & 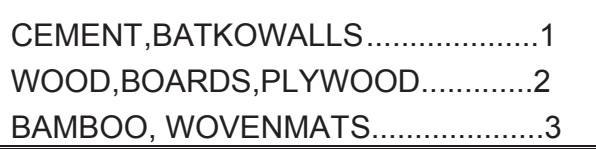 \\
\hline
\end{tabular}

CONCRETE ..................................... 0 WOOD,SHINGLES............................... 02 CORRUGATED IRON...........................03

ROOFTILES

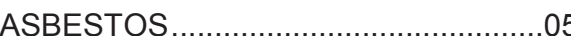

FOLIAGE,PALMFIBER ........................... .06

D13. During the rainy season, did this classroom experience problems with

D27. DO YOU HAVE DESIGNATED ROOM FOR THE TEACHERS?

D27a. DOES IT PROVIDE ENOUGH TABLES AND CHAIRS FOR ALL TEACHERS?

D28. DOES PRINCIPAL HAVE HIS OWN ROOM SEPARATED FROM TEACHERS ROOM?

D14. IS THERE A TOILET FOR THE TEACHERS?

WHAT IS THE CONDITION OF THE TOILET?

1.Yes 3.No

NO . Flains.............................. 3

YES

NO ..................................................

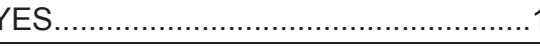

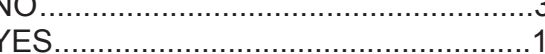

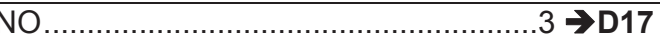

YES ..............................................

DK (LOCKED, ETC.) …...................... $8 \rightarrow$ D17

CLEAN AND FUNCTIONING................1

UNCLEAN BUT FUNCTIONING...........2

NOT FUNCTIONING 


\section{SECTION D: OBSERVATION}

\begin{tabular}{|c|c|c|}
\hline D16. & $\begin{array}{l}\text { IS THERE RUNNING WATER IN THE } \\
\text { TOILET }\end{array}$ & YES $\mathrm{Y}$ \\
\hline D17. & $\begin{array}{l}\text { IS THERE A TOILET FOR THE } \\
\text { STUDENTS? }\end{array}$ & 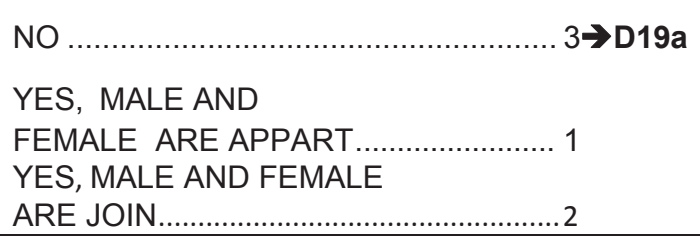 \\
\hline D18. & $\begin{array}{l}\text { WHAT IS THE CONDITION OF THE } \\
\text { TOILET? }\end{array}$ & 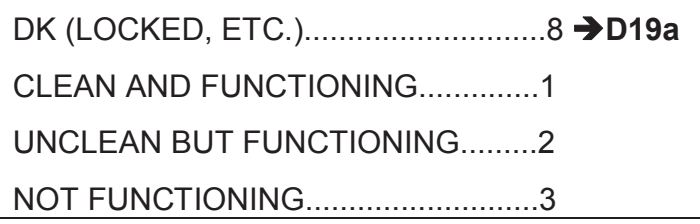 \\
\hline D19. & $\begin{array}{l}\text { IS THERE RUNNING WATER IN THE } \\
\text { TOILET }\end{array}$ & 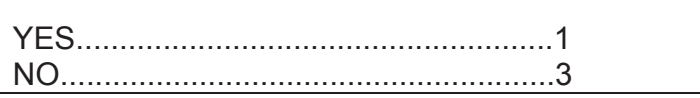 \\
\hline D19a. & $\begin{array}{l}\text { State the mainwater source used by the } \\
\text { school? }\end{array}$ & 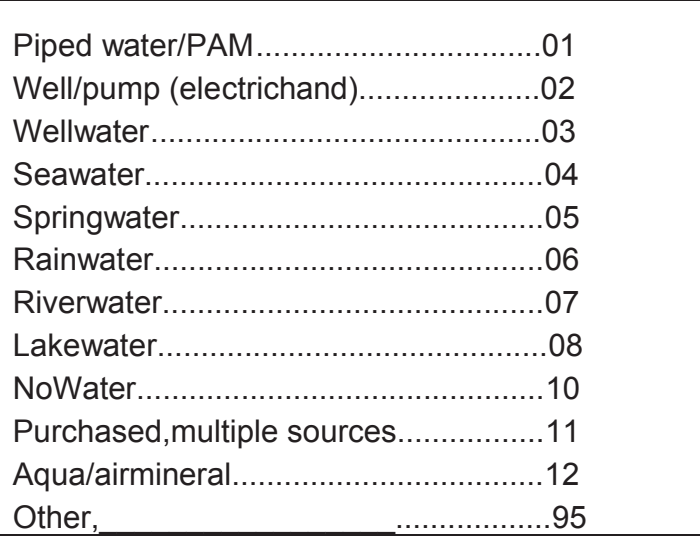 \\
\hline D20. & $\begin{array}{l}\text { ARE STUDENTS WORK DISPLAYED } \\
\text { IN THE SCHOOL/ CLASSROOMS? }\end{array}$ & 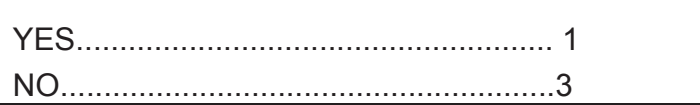 \\
\hline D27. & $\begin{array}{l}\text { IS THERE ANY NOTICE BOARD THAT } \\
\text { DISPLAYED THE INFORMATION } \\
\text { ABOUT BOS INCLUDING THE } \\
\text { REALIZATION REPORTOF BOS } \\
\text { FUND? }\end{array}$ & 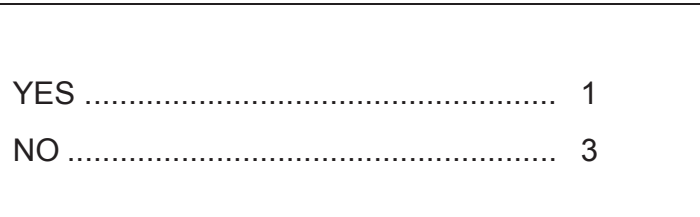 \\
\hline
\end{tabular}




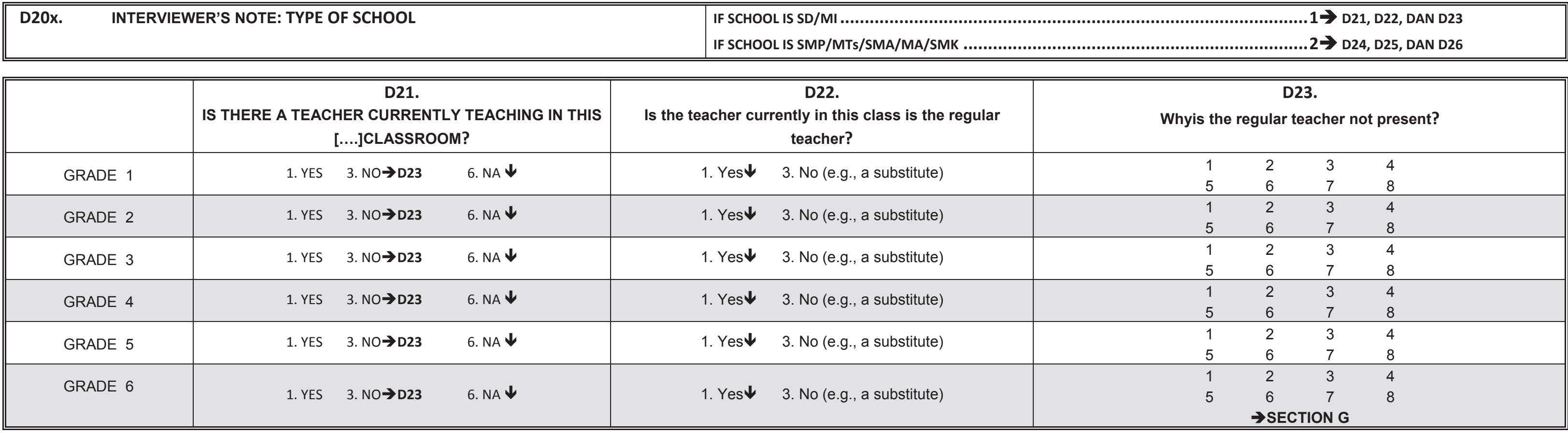

\begin{tabular}{|c|c|c|c|c|}
\hline \multirow[b]{2}{*}{ GRADE 1/7/10 } & \multicolumn{3}{|c|}{$\begin{array}{c}\text { D24. } \\
\text { IS THERE A TEACHER CURRENTLY TEACHING IN THIS } \\
{[\ldots . .] \text { CLASSROOM? }}\end{array}$} & \multirow{2}{*}{$\begin{array}{r}\text { Is the teacher cu } \\
\text { 1. Yes } \downarrow\end{array}$} \\
\hline & 1. YES & 3. $\mathrm{NO} \rightarrow \mathrm{D} 26$ & 6. NA $\downarrow$ & \\
\hline GRADE 2/8/11 & 1. YES & 3. $\mathrm{NO} \rightarrow \mathrm{D} 26$ & 6. NA $\downarrow$ & 1. Yes $\downarrow$ \\
\hline GRADE 3/9/12 & 1. YES & 3. $\mathrm{NO} \rightarrow \mathrm{D} 26$ & 6. NA $\downarrow$ & 1. Yes $\downarrow$ \\
\hline \multicolumn{2}{|c|}{$\begin{array}{l}\text { Codefor D23 and D26 } \\
\text { 1. Assigned by the school to do other task } \\
\text { 2. Assigned by the Dinas todo other task } \\
\text { 3. On leave/vacation } \\
\text { 4.Sick }\end{array}$} & \multicolumn{2}{|c|}{$\begin{array}{l}\text { 5. Family is sick } \\
\text { 6. Other authorized absence } \\
\text { 7. Late } \\
\text { 8. Absent without authorization }\end{array}$} & \\
\hline
\end{tabular}

D25.

tlyin this class is the regula teacher?
D26.

Whyis the regular teacher not present? 


\section{SECTIONG: NUMBER OF TEACHERS AND STUDENTS BY GRADE}

\begin{tabular}{|c|c|c|}
\hline \multicolumn{2}{|r|}{ KRTYPE } & \multirow[t]{2}{*}{ RESPONDENT } \\
\hline KRP1_G. & Respondent & \\
\hline KRP1a_G. & Posttion/occupation in this School: & $\begin{array}{lllll}01 & 02 & 03 & 04 & 05 \\
06 & 07 & 08 & 95 & \end{array}$ \\
\hline KRP2_G. & Age : & $\llcorner$ \ـears \\
\hline KRP2a_G. & Length of tenure inpositions: & 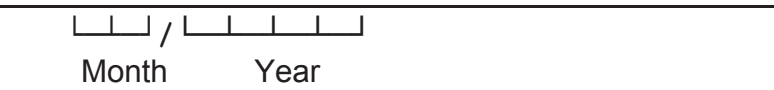 \\
\hline KRP3_G. & INTERVIEWER CHECK: SEX OF RESPONDENT & $\begin{array}{l}\text { MALE } \ldots \ldots \ldots \ldots \ldots \ldots \ldots \ldots . .1 \\
\text { FEMALE } \ldots \ldots \ldots \ldots \ldots \ldots \ldots \ldots . . .3\end{array}$ \\
\hline KRP3a_G. & Highest level of education attended: & 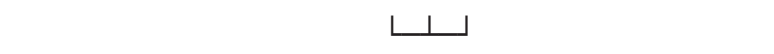 \\
\hline KRP4_G. & Highest class completed: & $\begin{array}{ccccc}00 & 01 & 02 & 03 & 04 \\
05 & 06 & 07 & 98 & 95 \\
\end{array}$ \\
\hline KRP5_G. & Length of time lived in the village : & 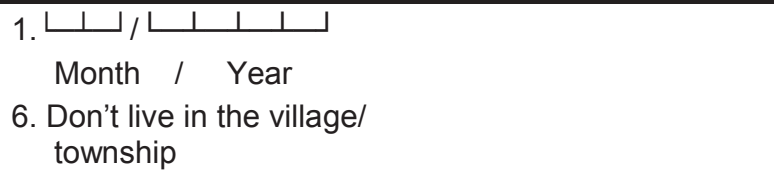 \\
\hline KRP6_G. & How did $[\ldots]$ come to assume to the position? & $\begin{array}{l}\text { 1. Elected } \\
\text { 2. Appointed by Government / Education District Office } \\
\text { 3. Appointed byFoundation } \\
\text { 5. Other }\end{array}$ \\
\hline
\end{tabular}

Code for position in village (KR1a D):

08. Village/township heads

010. Head of Government Administration

011. Head of Village Development

012. Head of Welfare

013. Head of Financial Matters

014. Head of General Affairs

\section{Codes for highest level of education}

01 . No school/not yet in school
02 . Elementary school

11. Kejar Paket A (Adult Education A)

03. Junior High - General

05. High School - General

06. High School - Vocational

60. D1, D2, D3 (Junior College)

61. University S1 (Bachelor)

62. University S2 (Masters)

98. DON'T KNOW
13. Open Univeristy

14. Islamic School (Pondok Pensantren)

15. Kejar Paket C (Adult Education C)

7. School for the disabled

2. Islamic Elementary School (Madrasah Ibtidaiyah)

73. Islamic Junior High School (Madrasah Tsanawiyah)

Kindergarten

95. Other
Codes for highest grade (KR4 D):

00. Never completed class 1

01.

02. 2

03. 3

05.5

06.6

07. Graduated

96. No school

98. DON'T KNOW 


\section{YEAR 2013/2014}

\begin{tabular}{|c|c|c|c|c|c|c|}
\hline \multirow{2}{*}{$\begin{array}{c}\text { SCHOOL PRINCIPAL } \\
\text { GRADE }\end{array}$} & \multicolumn{3}{|c|}{ 1. PRIMARY } & \multicolumn{3}{|c|}{ 2. JUNIOR AND SENIOR HIGH } \\
\hline & $\begin{array}{l}\text { G1a. TOTAL } \\
\text { TEACHERS }\end{array}$ & $\begin{array}{l}\text { G2a. TOTAL } \\
\text { STUDENTS }\end{array}$ & $\begin{array}{c}\text { G5a. TOTAL } \\
\text { PARALEL CLASS }\end{array}$ & $\begin{array}{l}\text { G1b. TOTAL } \\
\text { TEACHERS }\end{array}$ & G2b. TOTAL STUDENTS & $\begin{array}{c}\text { G5b. TOTAL PARALEL } \\
\text { CLASS }\end{array}$ \\
\hline 1 & & & & & & \\
\hline 2 & & & & & & \\
\hline 3 & & & & & & \\
\hline 4 & & & & & & \\
\hline 5 & & & & & & \\
\hline 6 & & & & & & \\
\hline TOTAL & & & & & & \\
\hline
\end{tabular}

\begin{tabular}{|c|c|c|c|c|}
\hline YEAR 2013/2014 & G6a. & G6b. & G6c. & G6d. \\
\hline $\begin{array}{c}\text { NUMBER OF TEACHERSWITH THE } \\
\text { HIGHEST DEGREE }\end{array}$ & PERMANENT TEACHERS & $\begin{array}{l}\text { ASSISTANT TEACHERS (GURU } \\
\text { BANTU)- CENTRAL }\end{array}$ & $\begin{array}{l}\text { ASSISTANT TEACHERS (GURU } \\
\text { BANTU)- REGIONAL }\end{array}$ & HONORARY TEACHERS \\
\hline \multicolumn{5}{|l|}{ BELOW DIPLOMA 1} \\
\hline \multicolumn{5}{|l|}{ DIPLOMA 1} \\
\hline \multicolumn{5}{|l|}{ DIPLOMA 2} \\
\hline \multicolumn{5}{|l|}{ DIPLOMA 3} \\
\hline \multicolumn{5}{|l|}{ BACHELOR DEGREE } \\
\hline \multicolumn{5}{|l|}{ HIGHER THAN BACHELOR DEGREE } \\
\hline TOTAL & & & & \\
\hline
\end{tabular}




\section{SECTIONH:OBSERVATIONSHEET}

\begin{tabular}{|c|c|c|}
\hline & HOMEROOM TEACHER & \\
\hline H1. WERE THERE ANY OTHER PEOPLE PRESENT IN THE ROOM DURING INTERVIEW? & $\begin{array}{l}\text { NO } \\
\text { YES }\end{array}$ & \\
\hline H2. WHO EXACTLY WERE PRESENT DURING INTERVIEW? & 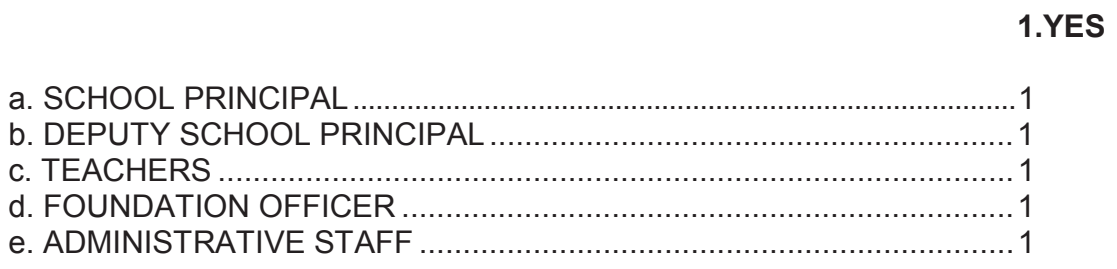 & $\begin{array}{l}3 . N O \\
3 \\
3 \\
3 \\
3 \\
3\end{array}$ \\
\hline H3. DID THOSE PRESENT ALSO ANSWER THE QUESTION IN THIS INTERVIEW? & 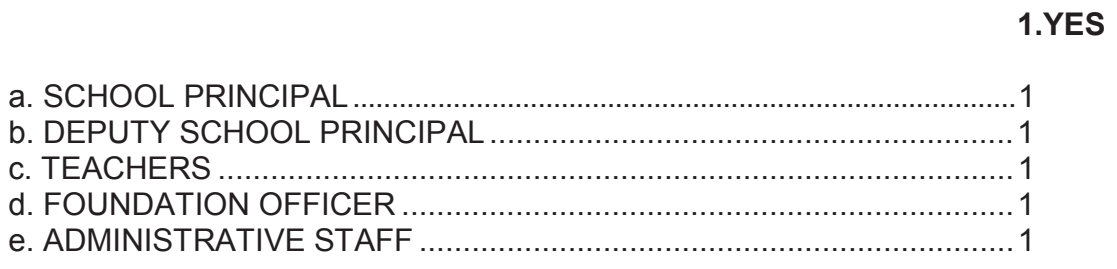 & $\begin{array}{l}3 . N O \\
3 \\
3 \\
3 \\
3 \\
3\end{array}$ \\
\hline H4. WERE THE RESPONDENTS ABLE TO ANSWER THE QUESTIONS FLUENTLY? & $\begin{array}{l}\text { NO } \\
\text { YES }\end{array}$ & \\
\hline
\end{tabular}




\section{SECTION CP: INTERVIEW NOTES}

\begin{tabular}{|c|c|c|c|c|c|c|}
\hline LANGMAIN (CK1). & \multicolumn{2}{|l|}{ Interview was entirely/mostly conducted in what language? } & \multicolumn{4}{|l|}{$\llcorner\perp$ other } \\
\hline LANGOTHR (CK2). & Other languaged used (if any): & & $\left\llcorner \_\right.$L other_ & & & \\
\hline \multicolumn{7}{|l|}{ LANGUAGE CODE: } \\
\hline 00. Bahasa Indonesia & 04. Batak & Sasak & 12. Makassar & 16. Toraja & 20. & Lampung \\
\hline 01. Java & 05. Bugis & 09. Minang & 13. Nias & 17. Lahat & 95. & Other, \\
\hline 02. Sunda & 06. Cina & 10. Banjar & 14. Palembang & 18. Other South Sumatera & 96. & NA \\
\hline 03. Bali & 07. Madura & 11. Bima & 15. Sumbawa & 19. Betawi & & \\
\hline
\end{tabular}

\begin{tabular}{|c|c|c|c|}
\hline RESULT (FP3). RESULTS OF INTERVIEW & REASON (FP4). REASON FOR ANSWERING “2” I “3” IN RESULT. & FP6. MONITORING BY SUPERVISOR & \\
\hline $\begin{array}{l}\text { 1. Completed } \rightarrow \text { FP6 } \\
\text { 2. Partly completed } \\
\text { 3. Not completed } \\
\text { 4. Twin EA with EA ـــ ـ ـ } \longrightarrow \text { FP6 }\end{array}$ & $\begin{array}{l}\text { 1. Respondent was travelling/not in location } \\
\text { 2. Respondent was too busy } \\
\text { 3. Respondent refused }\end{array}$ & $\begin{array}{lll} & & \text { Yes } \\
\text { a. } & \text { Observed (sup_obs)......... } 1 \\
\text { b. } & \text { Checked (sup_edit) ........... } 1 \\
\text { c. } & \text { Verified (sup_veri) .............. } 1\end{array}$ & $\begin{array}{l}\text { No } \\
3 \\
3 \\
3\end{array}$ \\
\hline
\end{tabular}

\section{INTERVIEWER NOTE:}




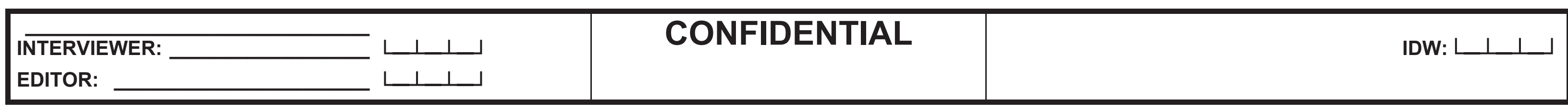

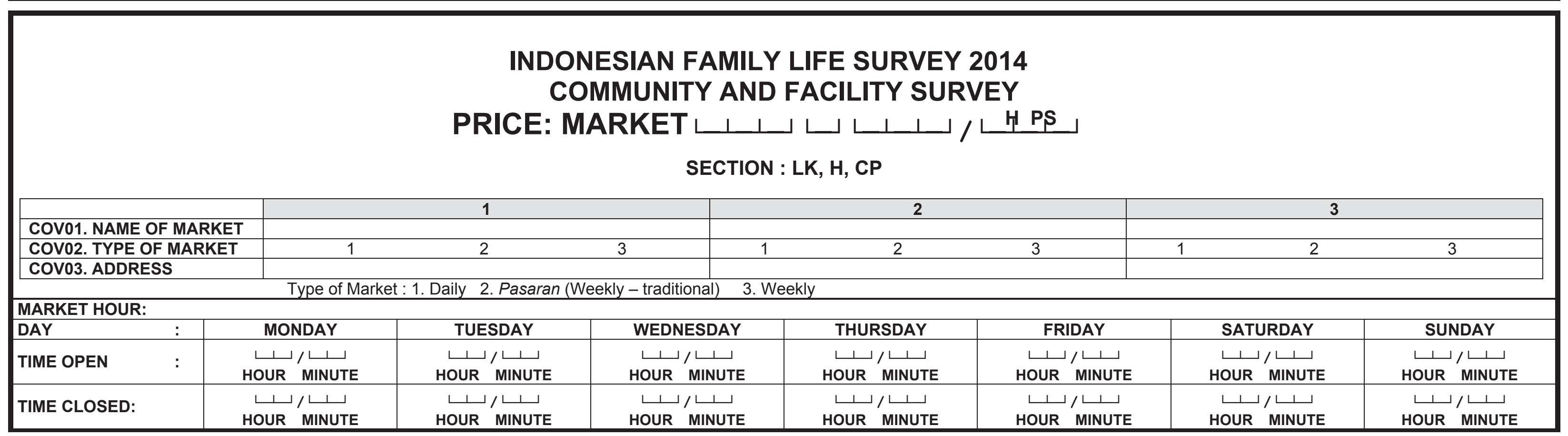




\begin{tabular}{|c|c|}
\hline SAMPLING INFORMATION & CODE \\
\hline LK01. Province_ & 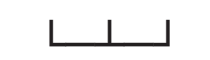 \\
\hline LK02. Kabupaten/Kotamadya & 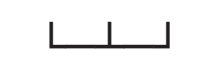 \\
\hline LK03. Kecamatan & 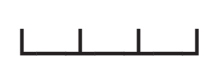 \\
\hline LK04. Village/Urban Township & 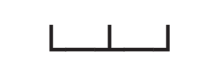 \\
\hline LK05. Region: 1. Urban 2. Rural & L \\
\hline
\end{tabular}

\begin{tabular}{|c|c|c|}
\hline & SUPERVISION: & CODE \\
\hline LK15. & Name of Interviewer : & 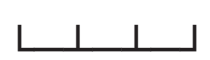 \\
\hline LK16. & Name of Editor : & 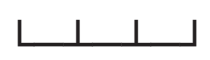 \\
\hline LK17. & Name of Local Supervisor: & 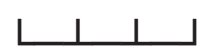 \\
\hline LK19. & Name of Field Coordinator: & $1 \quad 1 \quad 1$ \\
\hline
\end{tabular}

\begin{tabular}{|c|c|}
\hline & MARKETADDRESS \\
\hline LK07c. & 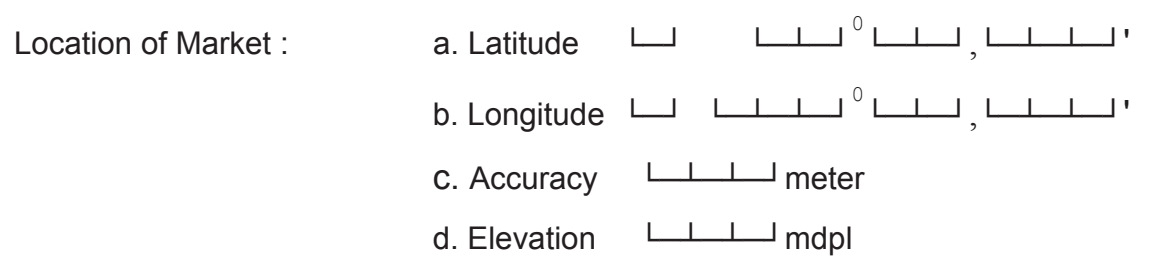 \\
\hline LK08. & $\begin{array}{ll}\text { a. Address } & : \\
\text { b. Description of location } & : \\
\text { d. Postal code } & : L 1 / L 1 / L\end{array}$ \\
\hline LK09. & 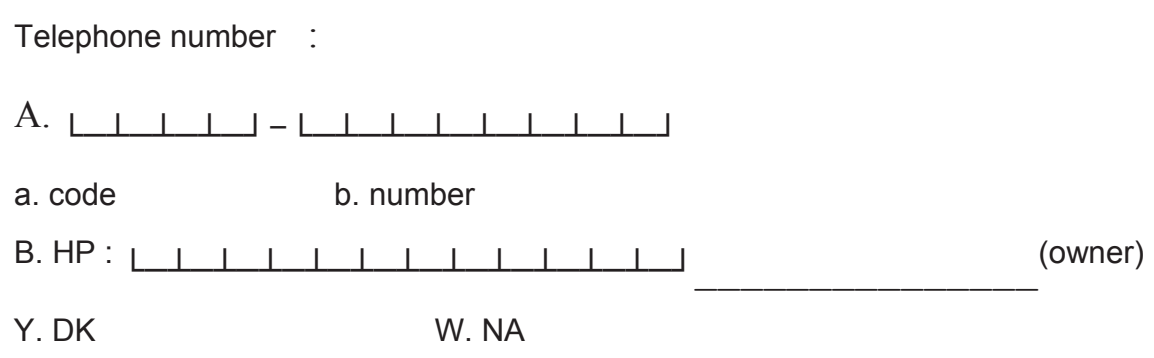 \\
\hline
\end{tabular}

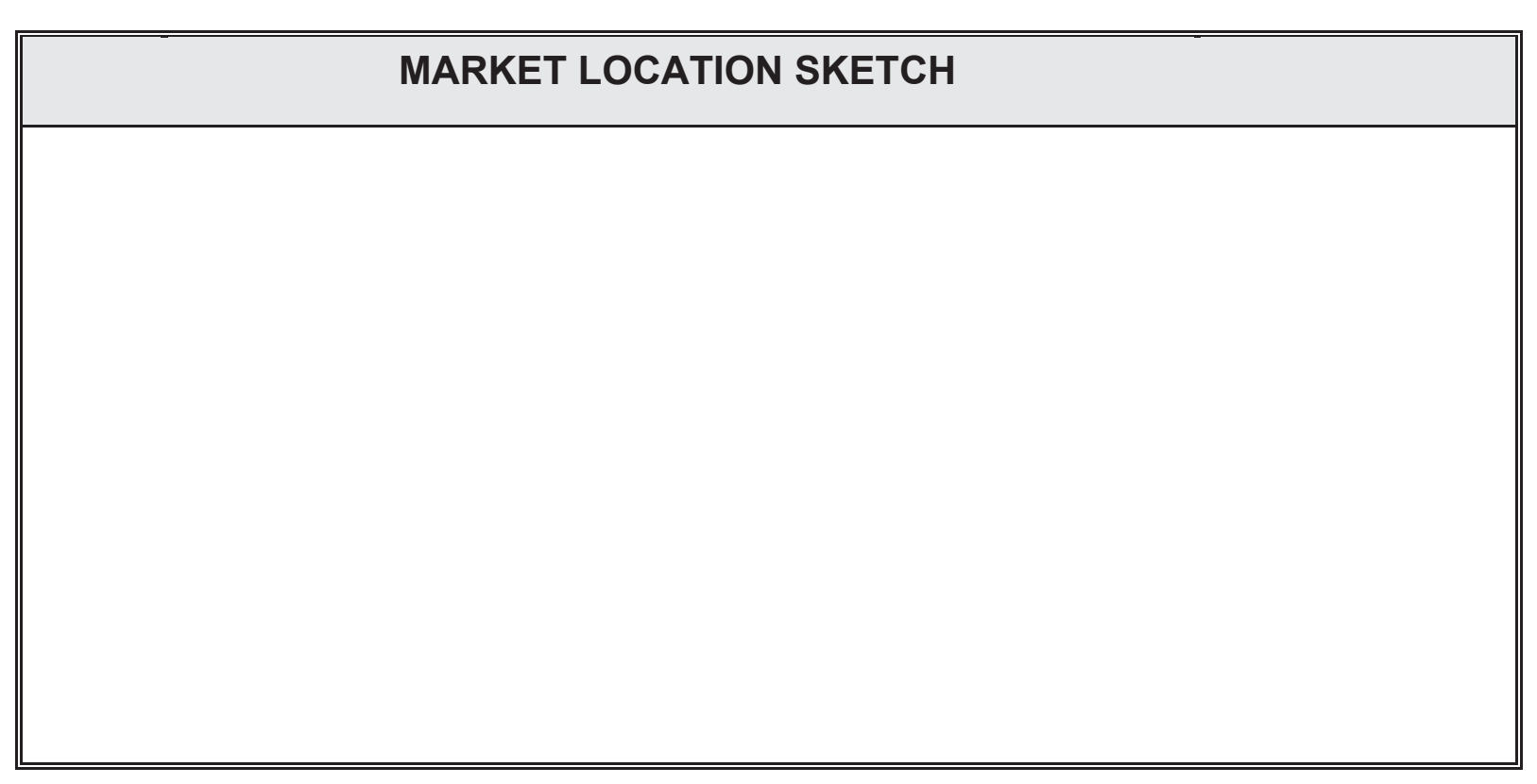




\section{SECTION H : PRICE}

\begin{tabular}{|c|c|c|c|c|c|}
\hline \multirow[b]{2}{*}{$\begin{array}{l}\text { FOOD TYPE } \\
\text { (HTYPE) }\end{array}$} & $\mathrm{H03a}$. & H01. & $\begin{array}{ll}\mathrm{H} 02 . \\
\end{array}$ & H03. & H04. \\
\hline & Is [...] available for sale? & $\begin{array}{l}\text { What is the price per } \\
\text { unit (see H02) now? }\end{array}$ & $\begin{array}{l}\text { UNIT FOR H01 } \\
\text { INTERVIEWER NOTE: } \\
\text { IF THE RESPONDENT ISN'T ABLE TO PROVIDE } \\
\text { INFORMATION ABOUT THE PRICE PER KG OR PER } \\
\text { LITER, YOU MUST PROBE AND RECORD } \\
\text { INFORMATION ON THE QUANTITY AND UNITS OF } \\
\text { MEASUREMENT IN H03 AND H04 }\end{array}$ & $\begin{array}{l}\text { How much is contained in } \\
\text { one unit? }\end{array}$ & $\begin{array}{l}\text { In what units is the quantity } \\
\text { measured? }\end{array}$ \\
\hline 1. Rice: high quality & $\begin{array}{l}\text { 1. Rojolele } \\
\text { 2. Mentik } \\
\text { 5. Other ....................... } \\
\text { 6. No stock } \downarrow \\
\text { 8. Not sold } \downarrow\end{array}$ & 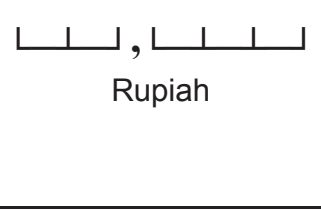 & 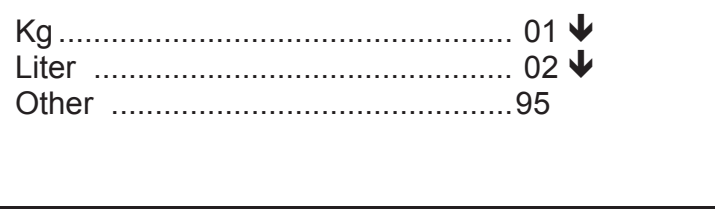 & 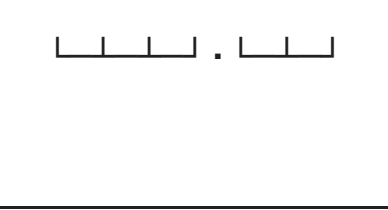 & 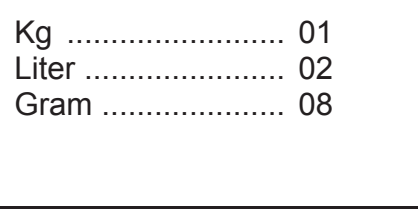 \\
\hline 2. Rice: average quality & $\begin{array}{l}\text { 1. Cisadane } \\
\text { 5..Other ........................ } \\
\text { 7. No stock } \downarrow \\
\text { 8. Not sold } \downarrow\end{array}$ & 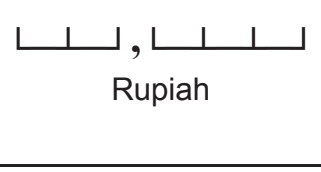 & 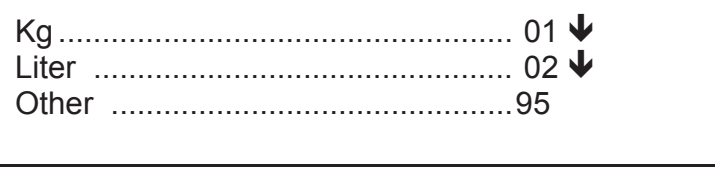 & 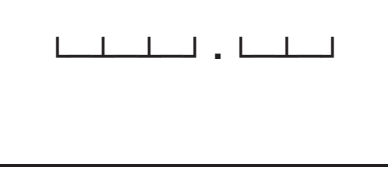 & 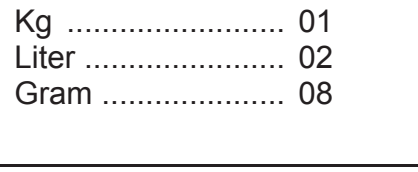 \\
\hline 3. Rice : low quality & $\begin{array}{l}\text { 1. IR64 } \\
\text { 5. Other ........... } \\
\text { 8. No stock } \downarrow \\
\text { 8. Not sold } \downarrow\end{array}$ & 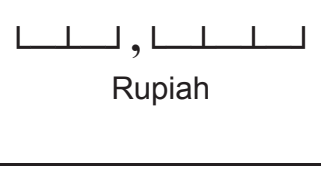 & 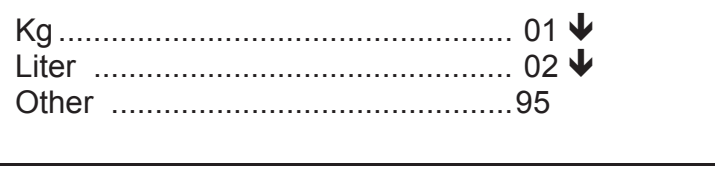 & 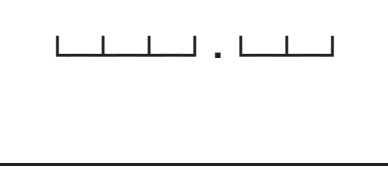 & 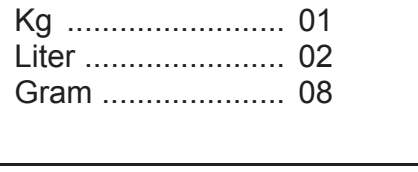 \\
\hline 4. Corn & $\begin{array}{l}\text { 1. Yes } \\
\text { 6. No stock } \downarrow \\
\text { 8. Not sold } \downarrow \\
\end{array}$ & 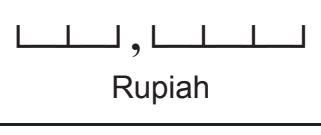 & Kg ............................................. $01 \downarrow$ & 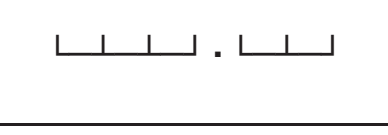 & 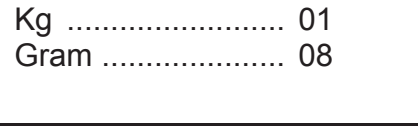 \\
\hline 5. Cassava & $\begin{array}{l}\text { 1. Yes } \\
\text { 6. Not stock } \downarrow \\
\text { 8. Not sold } \downarrow\end{array}$ & 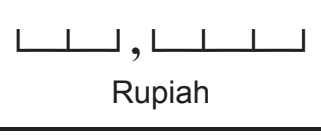 & Kg & & $\begin{array}{l}\mathrm{Kg} \ldots \ldots \ldots \ldots \ldots \ldots \ldots \ldots \ldots . . . . \\
\mathrm{Gram}\end{array}$ \\
\hline 6. Tapioca & $\begin{array}{l}\text { 1. Yes } \\
\text { 6. Not stock } \downarrow \\
\text { 8. Not sold } \downarrow\end{array}$ & 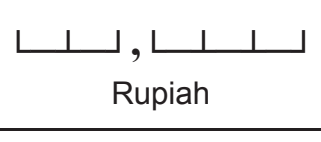 & Kg & 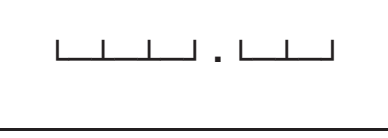 & 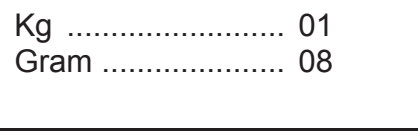 \\
\hline 7. Beef (good quality, boneless) & $\begin{array}{l}\text { 1. Yes } \\
\text { 6. Not stock } \downarrow \\
\text { 8. Notsold } \downarrow\end{array}$ & 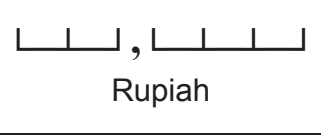 & Kg & 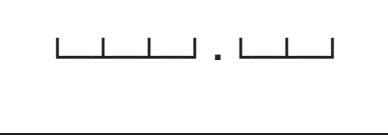 & 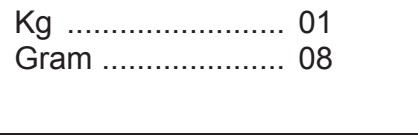 \\
\hline 8. Chicken (one chicken already cleaned) & $\begin{array}{l}\text { 1. AyamRas } \\
\text { 2. AyamKampung } \\
\text { 5. Others..................... } \\
\text { 6. No stock } \downarrow \\
\text { 8. Not sold } \downarrow\end{array}$ & 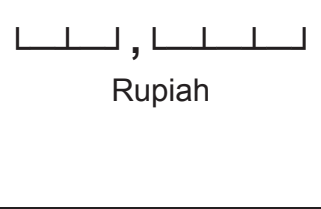 & Kg & $\downarrow \downarrow$ & 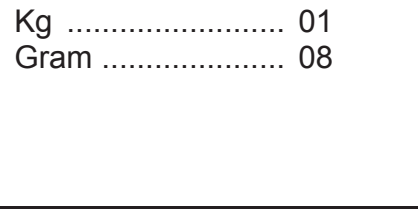 \\
\hline
\end{tabular}




\section{SECTION H : PRICE}

\begin{tabular}{|c|c|c|c|c|c|}
\hline & H03a.. & H01. & H02. & H03. & H04. \\
\hline $\begin{array}{l}\text { FOOD TYPE } \\
\text { (HTYPE) }\end{array}$ & Is [...] available for sale? & $\begin{array}{l}\text { What is the price per } \\
\text { unit (see } \mathrm{H} 02 \text { ) now? }\end{array}$ & $\begin{array}{l}\text { UNIT FOR H01 } \\
\text { INTERVIEWER NOTE: } \\
\text { IF THE RESPONDENT ISN'T ABLE TO PROVIDE } \\
\text { INFORMATION ABOUT THE PRICE PER KG OR PER } \\
\text { LITER, YOU MUST PROBE AND RECORD } \\
\text { INFORMATION ON THE QUANTITY AND UNITS OF } \\
\text { MEASUREMENT IN HO3 AND H04 }\end{array}$ & $\begin{array}{l}\text { How much is contained in } \\
\text { one unit? }\end{array}$ & $\begin{array}{l}\text { In what units is the quantity } \\
\text { measured? }\end{array}$ \\
\hline 9. Goat (good quality, boneless) & $\begin{array}{l}\text { 1. Yes } \\
\text { 6. No stock } \downarrow \\
\text { 8. Not sold } \downarrow\end{array}$ & 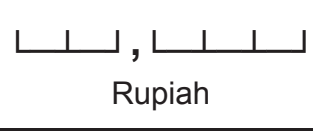 & $\begin{array}{l}\mathrm{Kg} \\
\mathrm{Other}\end{array}$ & 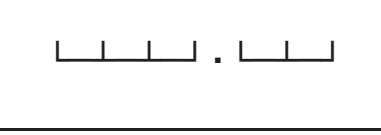 & 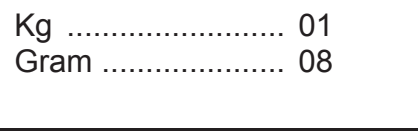 \\
\hline 10. Fresh fish (whole) & $\begin{array}{l}\text { 1. Kembung } \\
\text { 2. Tongkol } \\
\text { 3. Mujair } \\
\text { 4. Mas } \\
\text { 5. Others .......... } \\
\text { 6. No stock } \downarrow \\
\text { 8. Not sold } \downarrow\end{array}$ & 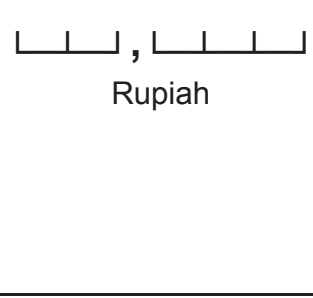 & Kg & 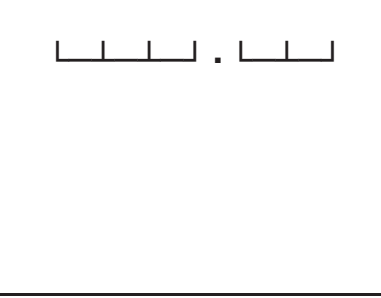 & 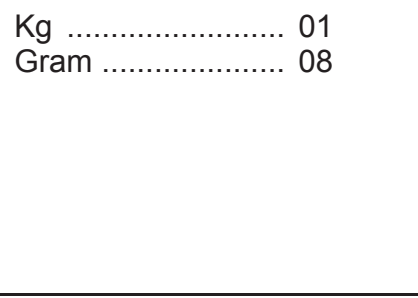 \\
\hline 11. Dried or salted fish & $\begin{array}{l}\text { 1. Layur } \\
\text { 2. Teri } \\
\text { 3. Pindang } \\
\text { 5. Others } \\
\text { 6. No stock } \downarrow \\
\text { 8. Not sold } \downarrow \\
\end{array}$ & 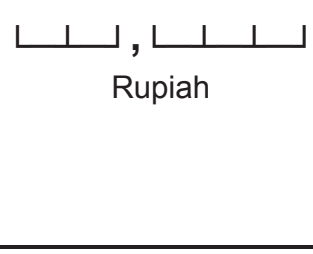 & Kg & 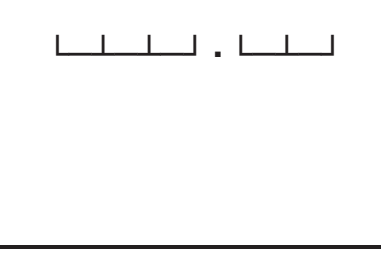 & 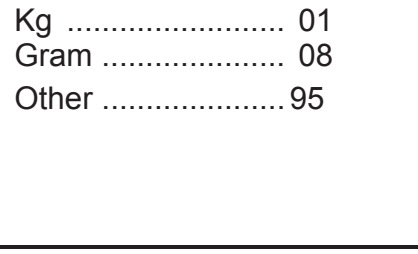 \\
\hline 12. Wheat flour & $\begin{array}{l}\text { 1. Yes } \\
\text { 6. No stock } \downarrow \\
\text { 8. Not sold } \downarrow\end{array}$ & 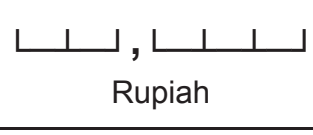 & Kg & 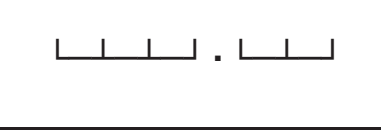 & 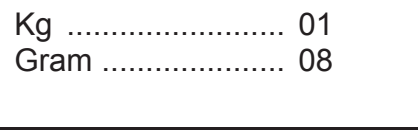 \\
\hline 13. Cooking oil & $\begin{array}{l}\text { 1. Palm oil } \\
\text { 2. Coconut oil } \\
\text { 5. Others ..................... } \\
\text { 6. No stock } \downarrow \\
\text { 8. Not sold } \downarrow \\
\end{array}$ & 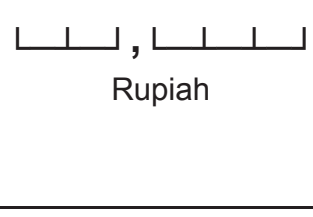 & 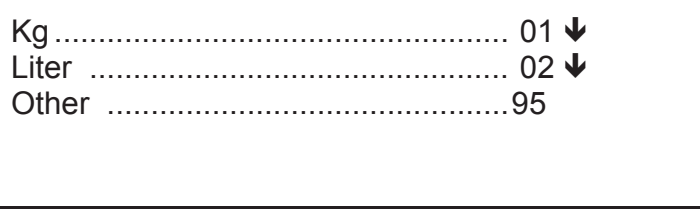 & 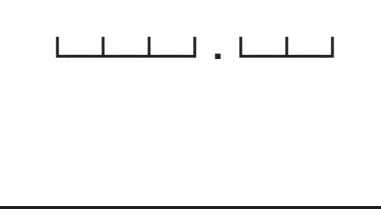 & 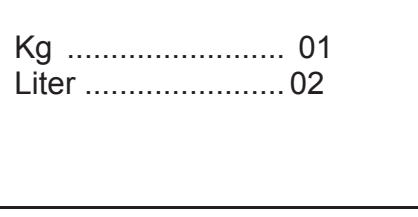 \\
\hline 14. White sugar & $\begin{array}{l}\text { 1. Local } \\
\text { 2. Import } \\
\text { 5. Other } \\
\text { 6. No stock } \downarrow \\
\text { 8. Not sold } \downarrow \\
\end{array}$ & 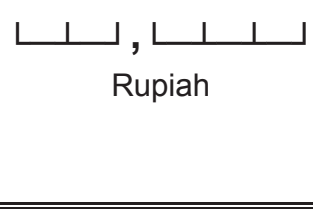 & Kg & ᄂ & 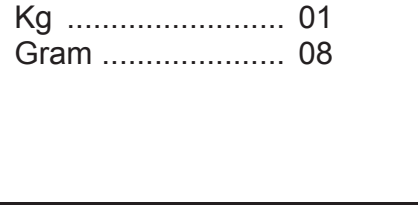 \\
\hline
\end{tabular}




\section{SECTION H : PRICE}

\begin{tabular}{|c|c|c|c|c|c|}
\hline \multirow[b]{2}{*}{$\begin{array}{l}\text { FOOD TYPE } \\
\text { (HTYPE) }\end{array}$} & H03a. & H01. & $\begin{array}{l}\mathrm{H} 02 . \\
\end{array}$ & H03. & ( H04. \\
\hline & Is [...] available for sale? & $\begin{array}{l}\text { What is the price per } \\
\text { unit (see } \mathrm{H} 02 \text { ) now? }\end{array}$ & $\begin{array}{l}\text { UNIT FOR H01 } \\
\text { INTERVIEWER NOTE: } \\
\text { IF THE RESPONDENT ISN'T ABLE TO PROVIDE } \\
\text { INFORMATION ABOUT THE PRICE PER KG OR PER } \\
\text { LITER, YOU MUST PROBE AND RECORD } \\
\text { INFORMATION ON THE QUANTITY AND UNITS OF } \\
\text { MEASUREMENT IN HO3 AND H04 }\end{array}$ & $\begin{array}{l}\text { How much is contained in } \\
\text { one unit? }\end{array}$ & $\begin{array}{l}\text { In what units is the quantity } \\
\text { measured? }\end{array}$ \\
\hline 15. Salt & $\begin{array}{l}\text { 1. Garam (fine) } \\
\text { 2. Garam (coarse) } \\
\text { 5. Others...................... } \\
\text { 6. No stock } \downarrow \\
\text { 8. Not sold } \downarrow\end{array}$ & 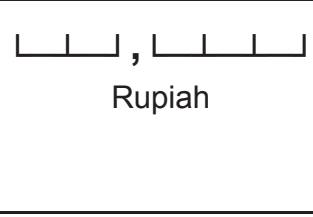 & 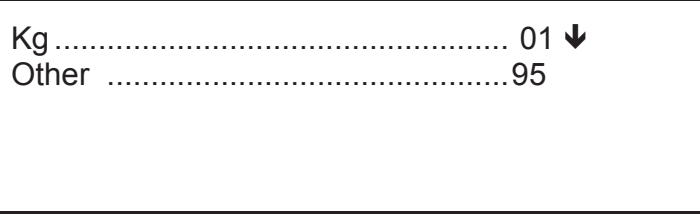 & 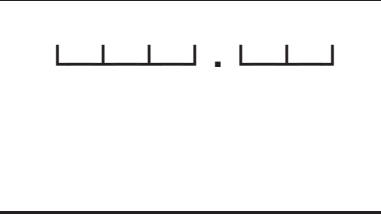 & 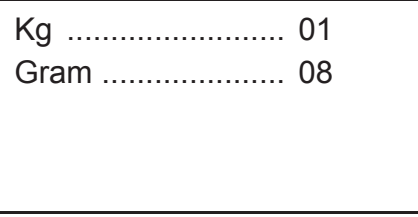 \\
\hline 16. Infant porridge & $\begin{array}{l}\text { 1. SUN } \\
\text { 2. PROMINA } \\
\text { 5. Others.................... } \\
\text { 6. No stock } \downarrow \\
\text { 8. Not sold } \downarrow \\
\end{array}$ & 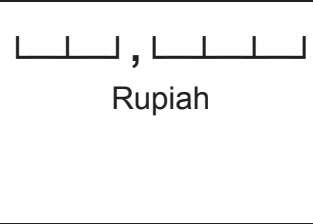 & 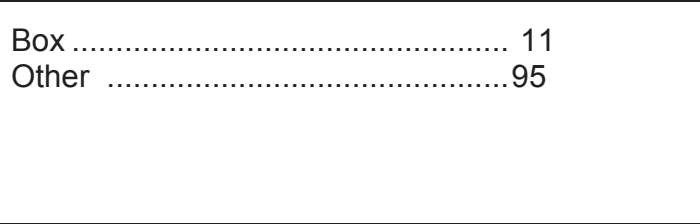 & 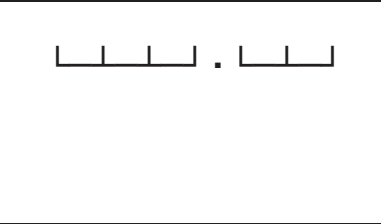 & $\begin{array}{ll}\mathrm{Kg} \\
\mathrm{Gram}\end{array}$ \\
\hline 17. Infant formula & $\begin{array}{l}\text { 1. SGM } \\
\text { 2. Frisian Flag - Children } \\
\text { 3. Dancow - Children } \\
\text { 5. Others.................... } \\
\text { 6. No stock } \downarrow \\
\text { 8. Not sold } \downarrow \\
\end{array}$ & 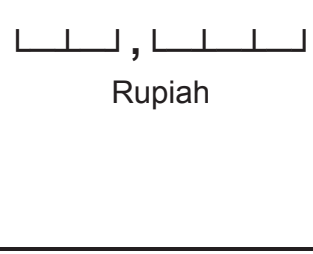 & 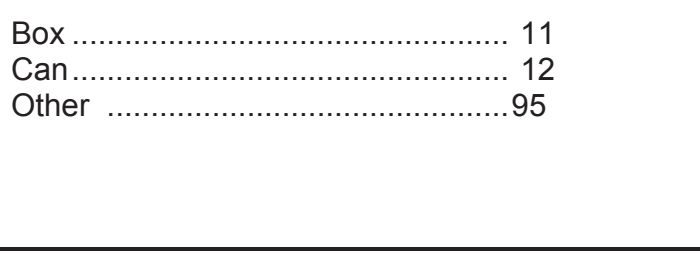 & 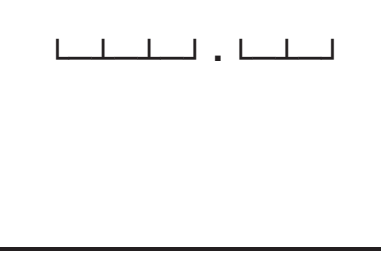 & $\mathrm{Kg}$ \\
\hline 18. Sweetened condensed milk & $\begin{array}{l}\text { 1. Frisian Flag } \\
\text { 2. Indomilk } \\
\text { 3. Nona } \\
\text { 5. Others.................... } \\
\text { 6. No stock } \downarrow \\
\text { 8. Not sold } \downarrow \\
\end{array}$ & 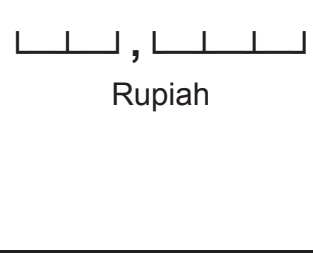 & 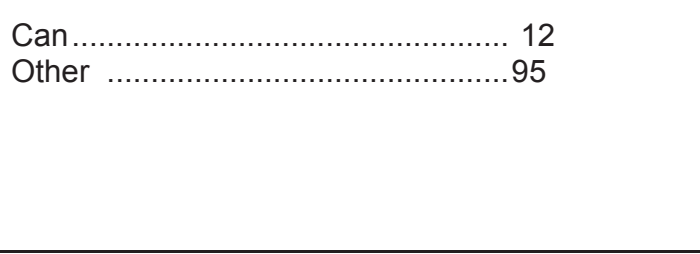 & 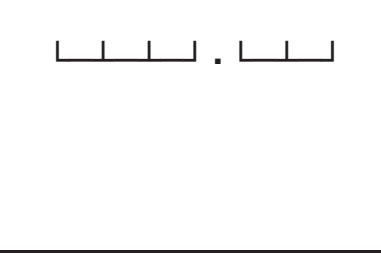 & 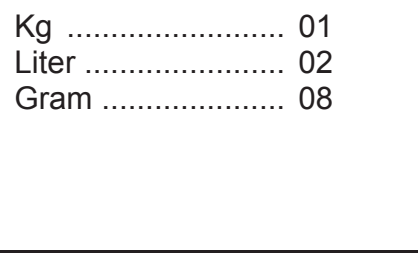 \\
\hline 19. Powdered Milk & $\begin{array}{l}\text { 1. Dancow } \\
\text { 2. Indomilk } \\
\text { 5. Others............................. } \\
\text { 6. No stock } \downarrow \\
\text { 8. Not sold } \downarrow\end{array}$ & 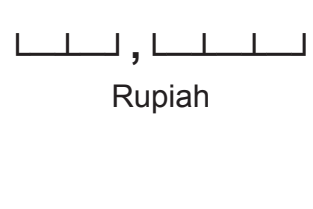 & $\begin{array}{l}\text { Box } \\
\text { Can } \\
\text { Other }\end{array}$ & 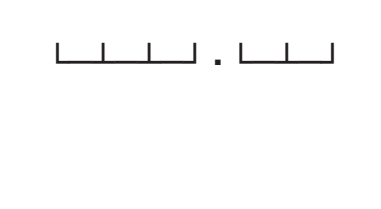 & 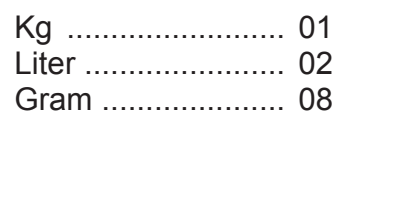 \\
\hline 20. Noodles & $\begin{array}{l}\text { 1. Supermie } \\
\text { 2. Sarimie } \\
\text { 3. Indomie } \\
\text { 5. Others.................... } \\
\text { 6. No stock } \downarrow \\
\text { 8. Not sold } \downarrow\end{array}$ & 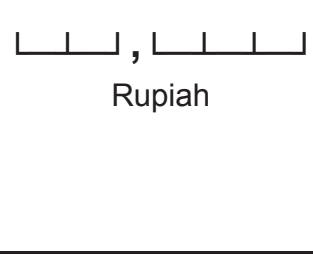 & $\begin{array}{l}\text { Pack } \\
\text { Other }\end{array}$ & 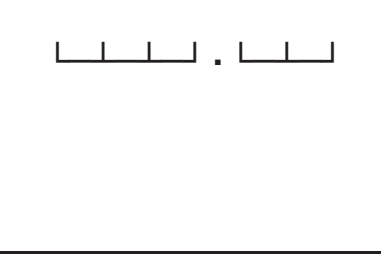 & $\begin{array}{ll}\mathrm{Kg} \\
\mathrm{Gram}\end{array}$ \\
\hline
\end{tabular}




\section{SECTION H : PRICE}

\begin{tabular}{|c|c|c|c|c|c|c|}
\hline \multirow{2}{*}{\multicolumn{2}{|c|}{$\begin{array}{c}\text { FOOD TYPE } \\
\text { (HTYPE) }\end{array}$}} & H03a. & H01. & $\mathrm{H02}$. & H03. & H04. \\
\hline & & Is [...] available for sale? & $\begin{array}{l}\text { What is the price per } \\
\text { unit (see H02) now? }\end{array}$ & $\begin{array}{l}\text { UNIT FOR H01 } \\
\text { INTERVIEWER NOTE: } \\
\text { IF THE RESPONDENT ISN'T ABLE TO PROVIDE } \\
\text { INFORMATION ABOUT THE PRICE PER KG OR PER } \\
\text { LITER, YOU MUST PROBE AND RECORD } \\
\text { INFORMATION ON THE QUANTITY AND UNITS OF } \\
\text { MEASUREMENT IN H03 AND H04 }\end{array}$ & $\begin{array}{l}\text { How much is contained in } \\
\text { one unit? }\end{array}$ & $\begin{array}{l}\text { In what units is the quantity } \\
\text { measured? }\end{array}$ \\
\hline & Tofu & $\begin{array}{l}\text { 1. White tofu } \\
\text { 2. Yellow tofu } \\
\text { 5. Others ....................... } \\
\text { 6. No stock } \downarrow \\
\text { 8. Not sold } \downarrow \\
\end{array}$ & 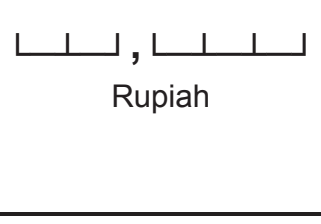 & 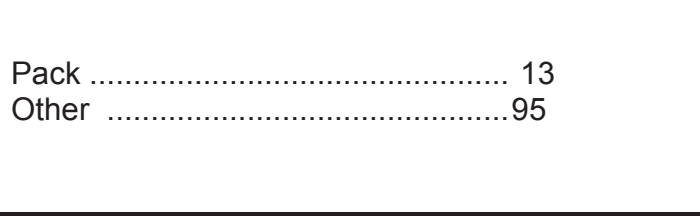 & 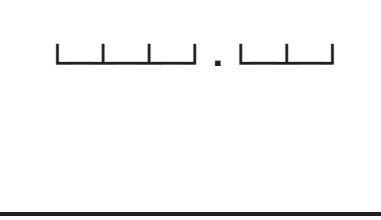 & 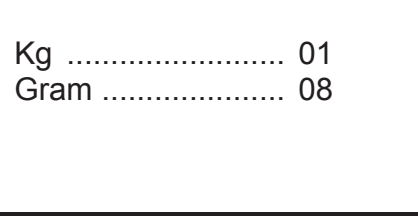 \\
\hline & Bean Curd & $\begin{array}{l}\text { 1. Yes } \\
\text { 5. Others } \\
\text { 6. No stock } \downarrow \\
\text { 8. Not sold } \downarrow\end{array}$ & 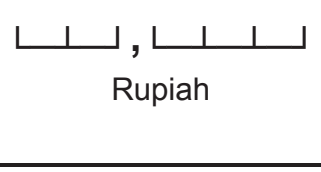 & 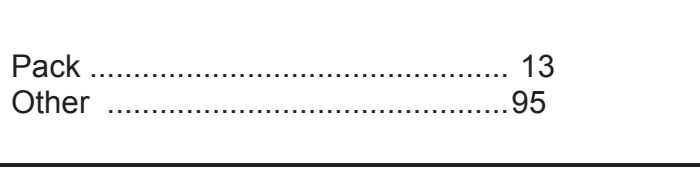 & 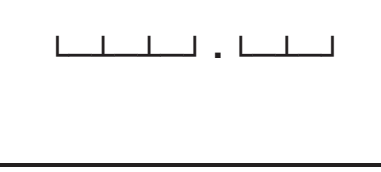 & 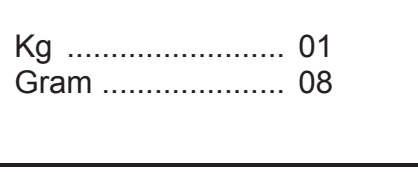 \\
\hline & Filtered clove cigarettes & $\begin{array}{l}\text { 1. GudangGaram } \\
\text { 2. Djarum Super } \\
\text { 3. Sampurna } \\
\text { 4. Marlboro clove } \\
\text { 5. Others ............. } \\
\text { 6. Not Sold } \downarrow \\
\text { 8. Out of stock } \downarrow \\
\end{array}$ & 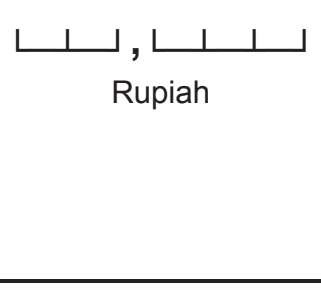 & 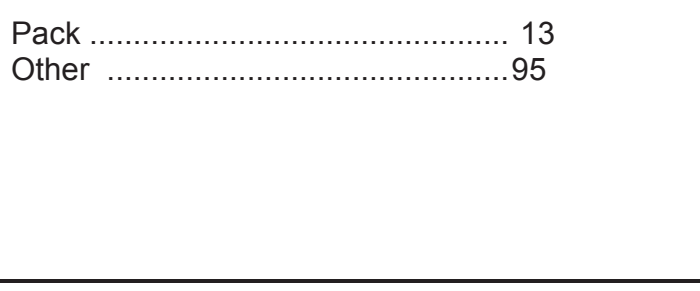 & 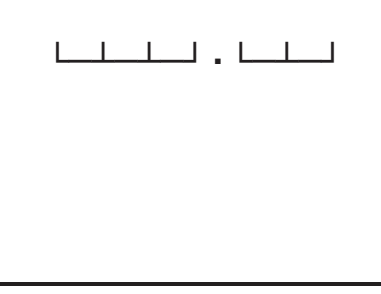 & Sticks ....................... 01 \\
\hline & Filtered cigarettes & $\begin{array}{l}\text { 1. Kansas } \\
\text { 2. Marlboro } \\
\text { 3. Lucky Strike } \\
\text { 6. Not Sold } \downarrow \\
\text { 8. Out of stock } \downarrow\end{array}$ & 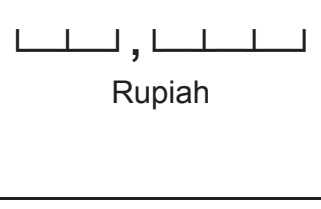 & 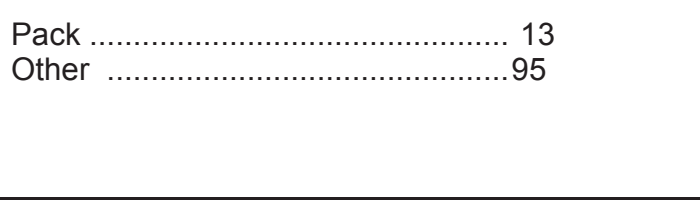 & 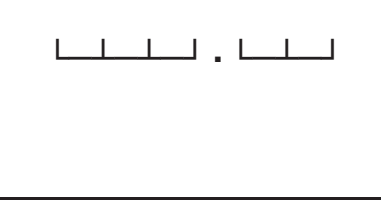 & Sticks ........................ 01 \\
\hline & Potato & $\begin{array}{l}\text { 1. Yes } \\
\text { 6. No stock } \downarrow \\
\text { 8. Not sold } \downarrow \\
\end{array}$ & 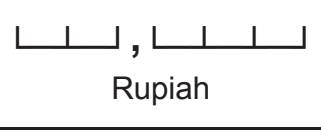 & 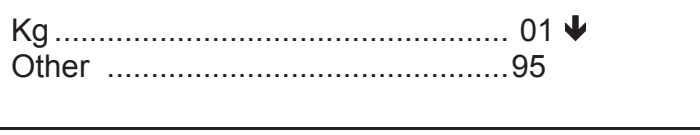 & 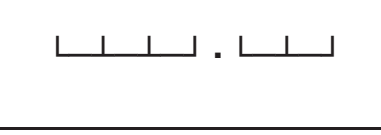 & $\begin{array}{l}\mathrm{Kg} \ldots \ldots \ldots \ldots \ldots \ldots \ldots \ldots \ldots \ldots \ldots \ldots \\
\mathrm{Gram}\end{array}$ \\
\hline & Kangkung (Water Spinach) & $\begin{array}{l}\text { 1. Yes } \\
\text { 6. No stock } \downarrow \\
\text { 8. Not sold } \downarrow \\
\end{array}$ & 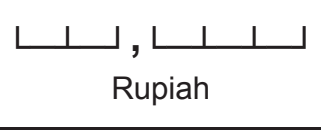 & 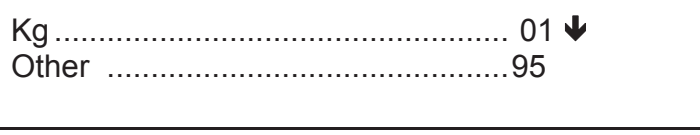 & 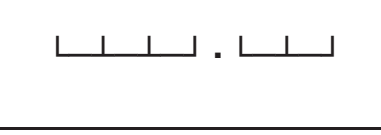 & $\begin{array}{l}\mathrm{Kg} \ldots \ldots \ldots \ldots \ldots \ldots \ldots \ldots \ldots \ldots \ldots \ldots \\
\mathrm{Gram}\end{array}$ \\
\hline & Spinach & $\begin{array}{l}\text { 1. Without } \mathrm{root} / \mathrm{piece} \\
\text { 2. With } \mathrm{root} / \mathrm{bunch} \\
\text { 6. No stock } \downarrow \\
\text { 8. Not sold } \downarrow\end{array}$ & 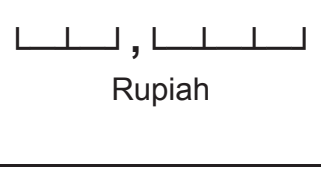 & Kg & 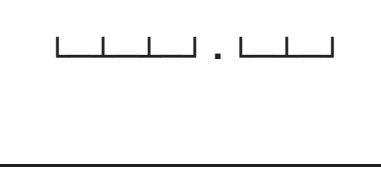 & $\begin{array}{l}\mathrm{Kg} \ldots \ldots \ldots \ldots \ldots \ldots \ldots \ldots \ldots \ldots \\
\mathrm{Gram}\end{array}$ \\
\hline
\end{tabular}




\section{SECTION H : PRICE}

\begin{tabular}{|c|c|c|c|c|c|c|}
\hline \multirow{2}{*}{\multicolumn{2}{|c|}{$\begin{array}{l}\text { FOOD TYPE } \\
\text { (HTYPE) }\end{array}$}} & \multirow{2}{*}{$\begin{array}{c}\text { H03a. } \\
\text { Is }[\ldots] \text { available for sale? }\end{array}$} & \multirow{2}{*}{$\begin{array}{l}\text { H01. } \\
\text { What is the price per } \\
\text { unit (see H02) now? }\end{array}$} & \multirow[b]{2}{*}{$\begin{array}{l}\text { HO2. } \\
\text { UNIT FOR H01 } \\
\text { INTERVIEWER NOTE: } \\
\text { IF THE RESPONDENT ISN'T ABLE TO PROVIDE } \\
\text { INFORMATION ABOUT THE PRICE PER KG OR PER } \\
\text { LITER, YOU MUST PROBE AND RECORD } \\
\text { INFORMATION ON THE QUANTITY AND UNITS OF } \\
\text { MEASUREMENT IN H03 AND H04 } \\
\end{array}$} & \multirow{2}{*}{$\begin{array}{c}\text { H03. } \\
\text { How much is contained in } \\
\text { one unit? }\end{array}$} & \multirow{2}{*}{$\begin{array}{l}\text { H04. } \\
\text { In what units is the quantity } \\
\text { measured? }\end{array}$} \\
\hline & & & & & & \\
\hline 28. & Cassava leaves & $\begin{array}{l}\text { 1. Yes } \\
\text { 6. No stock } \downarrow \\
\text { 8. Not sold } \downarrow\end{array}$ & 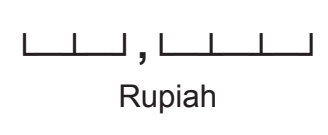 & Kg & 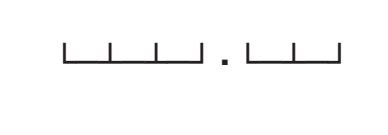 & $\begin{array}{l}\mathrm{Kg} \ldots \ldots \ldots \ldots \ldots \ldots \ldots \ldots \ldots \ldots . . .0 \\
\text { Gram } 01\end{array}$ \\
\hline & Bananas & $\begin{array}{l}\text { 1. Ambon } \\
\text { 2. Susu } \\
\text { 5. Others ........ } \\
\text { 6. No stock } \downarrow \\
\text { 8. Not sold } \downarrow\end{array}$ & 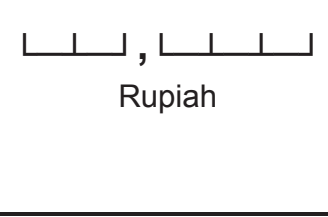 & Kg & 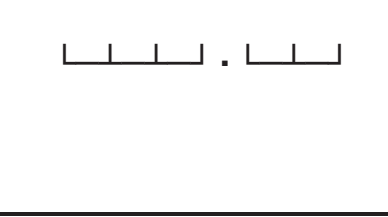 & $\mathrm{Kg}$ \\
\hline & Papaya & $\begin{array}{l}\text { 1. Yes } \\
\text { 6. No stock } \downarrow \\
\text { 8. Not sold } \downarrow\end{array}$ & 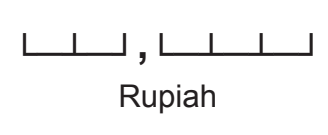 & $\begin{array}{l}\text { Kg } \\
\text { Other }\end{array}$ & 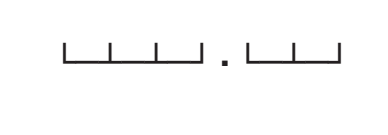 & 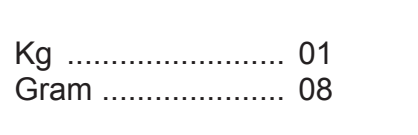 \\
\hline & Bottled Drinking water & $\begin{array}{l}\text { 1. Aqua } \\
\text { 2. Aquaria } \\
\text { 3. } 2 \text { Tang } \\
\text { 5. Others ........ } \\
\text { 6. No stock } \downarrow \\
\text { 8. Not sold } \downarrow\end{array}$ & 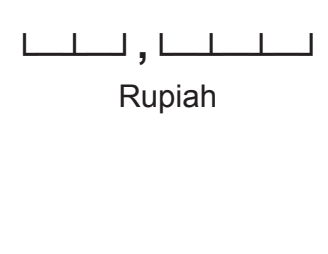 & $\begin{array}{l}\text { Liter } \\
\text { Bottle } \\
\text { Other }\end{array}$ & 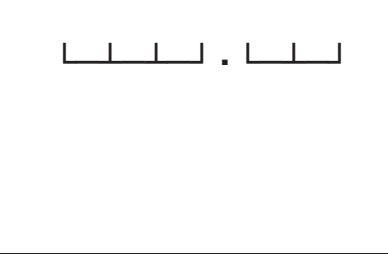 & Liter …………....... 02 \\
\hline & Kerosene & $\begin{array}{l}\text { 1. Yes } \\
\text { 6. No stock } \downarrow \\
\text { 8. Not sold } \downarrow\end{array}$ & 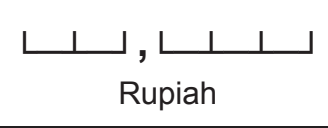 & Liter & 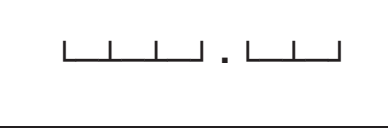 & Liter ……............... 02 \\
\hline & Gasoline by retail & $\begin{array}{l}\text { 1. Yes } \\
\text { 6. No stock } \downarrow \\
\text { 8. Not sold } \downarrow\end{array}$ & 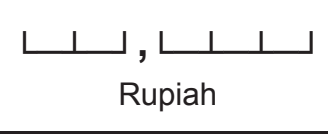 & Liter & 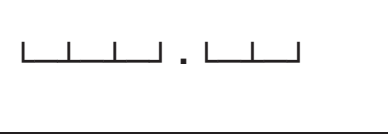 & Liter ………............ 02 \\
\hline 35. & Bath soap & $\begin{array}{l}\text { 1. Lux } \\
\text { 2. Lifeboy } \\
\text { 3. Giv } \\
\text { 5. Other } \\
\text { 6. No......................... stock } \downarrow \\
\text { 8. Notsold } \downarrow\end{array}$ & 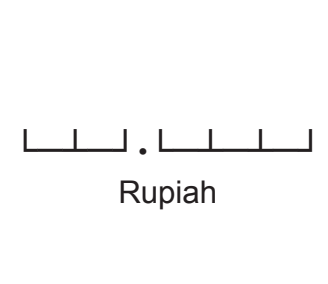 & Bar & 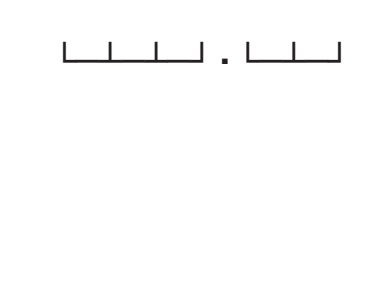 & 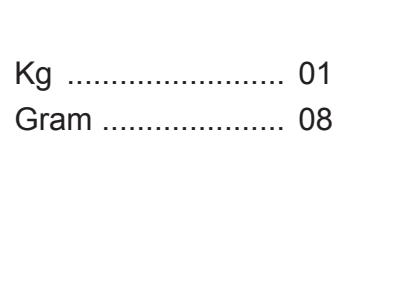 \\
\hline
\end{tabular}




\section{SECTION H : PRICE}

\begin{tabular}{|c|c|c|c|}
\hline \multirow[b]{2}{*}{$\begin{array}{c}\text { ITEM } \\
\text { (HTYPE) }\end{array}$} & H03a. & $\begin{array}{l}\text { H01. } \\
\end{array}$ & H02. \\
\hline & Is $[\ldots]$ available for sale? & What is the price per unit (see $\mathrm{H} 02$ ) now? & Unit \\
\hline 36. Cotton cloth & $\begin{array}{l}\text { 1. Yes } \\
\text { 6. No stock } \downarrow \\
\text { 8. Notsold } \downarrow\end{array}$ & 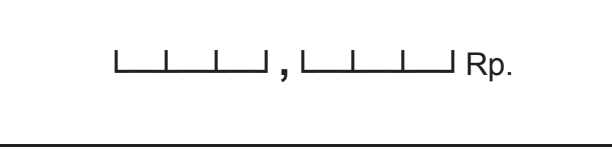 & per meter \\
\hline 37. Women's blouse & $\begin{array}{l}\text { 1. Yes } \\
\text { 6. No stock } \downarrow \\
\text { 8. Notsold } \downarrow\end{array}$ & 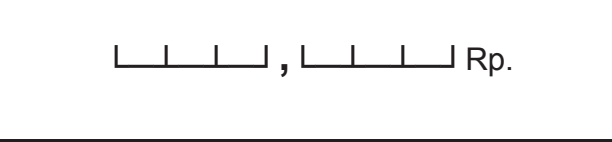 & per piece \\
\hline 38. Men's trousers & $\begin{array}{l}\text { 1. Yes } \\
\text { 6. No stock } \downarrow \\
\text { 8. Notsold } \downarrow\end{array}$ & 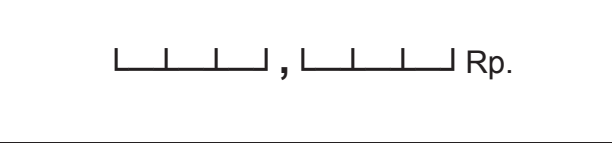 & per piece \\
\hline 39. Women's skirt & $\begin{array}{l}\text { 1. Yes } \\
\text { 6. No stock } \\
\text { 8. Notsold } \downarrow\end{array}$ & 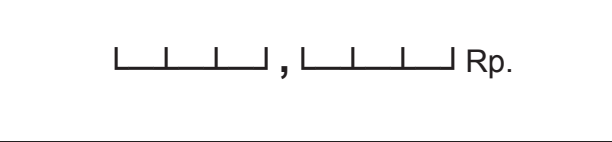 & per piece \\
\hline 40. Plastic sandals (for adult) & $\begin{array}{l}\text { 1. Yes } \\
\text { 6. No stock } \downarrow \\
\text { 8. Notsold } \downarrow\end{array}$ & 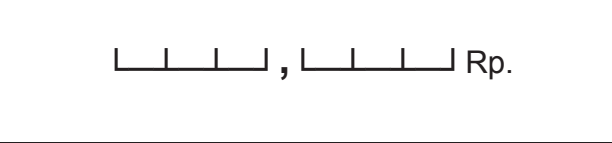 & per pair \\
\hline 41. Writing book & $\begin{array}{l}\text { 1. Yes } \\
\text { 6. No stock } \\
\text { 8. Notsold } \downarrow\end{array}$ & $\left\llcorner\perp \_\right.$Rp. & per piece \\
\hline
\end{tabular}




\section{SECTION CP: INTERVIEWER NOTES}

RESULT (FP3). INTERVIEW RESULTS CODE

1. Completed $\rightarrow$ FPG

2. Partially completed

3. Not completed
REASON (FP4). REASON FOR “2"|"3" IN RESULT

\section{Respondent is traveling}

2. Respondent is too busy

Refused
FP6. MONITORING BY LOCAL SUPERVISOR

a. Observed (sup obs)

Checked (sup_obs)

c. Verified (sup_verif)

No

FILL IN THIS SECTION AS SOON AS INTERVIEW HAS BEEN COMPLETED

\begin{tabular}{|c|c|c|c|c|c|}
\hline CP10. & $\begin{array}{l}\text { WAS INTERVIEW INTERUPTED BY: } \\
\text { COULD BE MORE THAN ONE: }\end{array}$ & CP11. & $\begin{array}{l}\text { WHAT IS YOUR EVALUATION ON THE ANSWER OF } \\
\text { RESPONDENT? }\end{array}$ & CP12. & $\begin{array}{l}\text { WHAT IS YOUR EVALUATION ON THE SERIOUSNESS AND } \\
\text { ATTENTIVENESS OF RESPONDENT? }\end{array}$ \\
\hline & $\begin{array}{l}\text { A. NONE } \\
\text { B. CUSTOMERS } \\
\text { C. FAMILY MEMBERS } \\
\text { D. OTHERS }\end{array}$ & & $\begin{array}{l}\text { 1. VERY GOOD } \\
\text { 2. GOOD } \\
\text { 3. FAIR } \\
\text { 4. BAD } \\
\text { 5. VERY BAD }\end{array}$ & & $\begin{array}{l}\text { 1. VERY GOOD } \\
\text { 2. GOOD } \\
\text { 3. FAIR } \\
\text { 4. BAD } \\
\text { 5. VERY BAD }\end{array}$ \\
\hline
\end{tabular}

NOTE : 


\begin{tabular}{|c|c|c|}
\hline INTERVIEWER: _ L & CONFIDENTIAL & 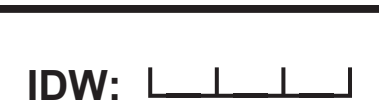 \\
\hline
\end{tabular}

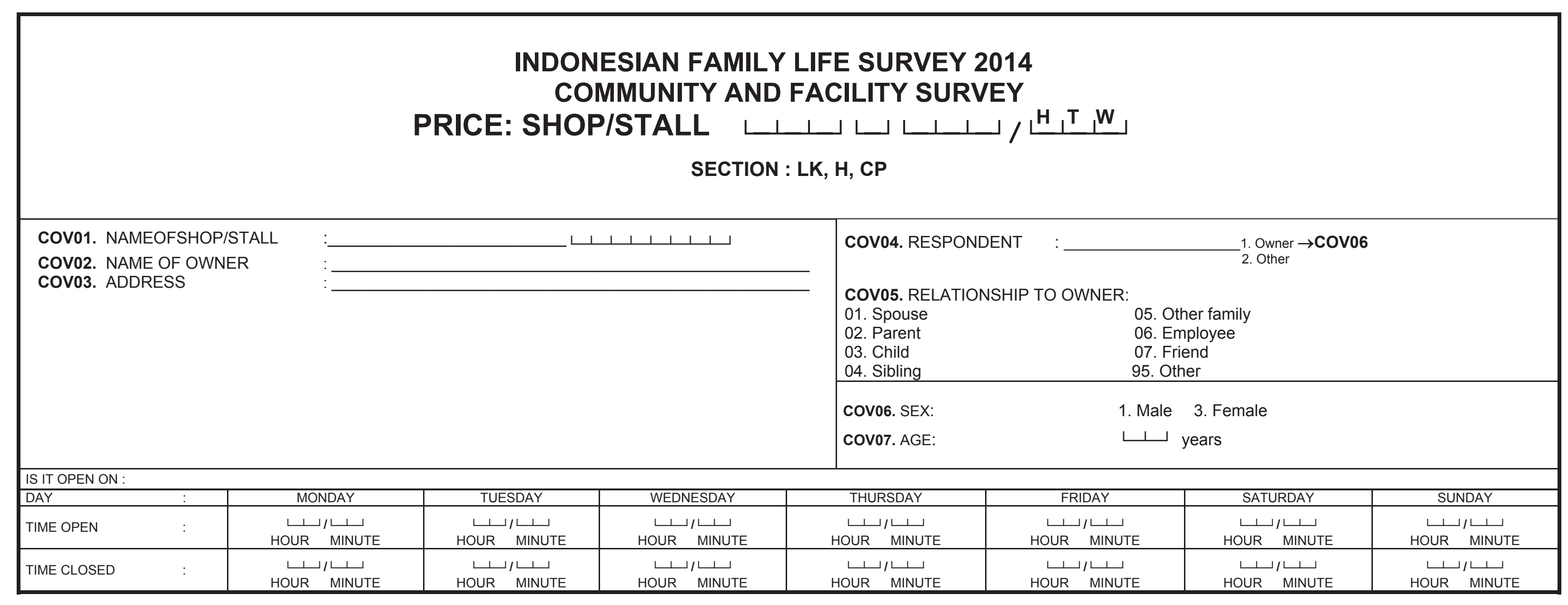




\begin{tabular}{|c|c|}
\hline SAMPLING INFORMATION & CODE \\
\hline LK01. Province & 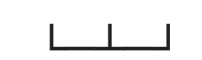 \\
\hline LK02. Kabupaten/Kotamadya & لــــــــــــ \\
\hline LK03. Kecamatan & 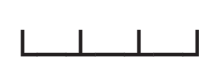 \\
\hline Village/Urban Township & 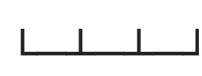 \\
\hline LK05. Region: 1. Urban 2. Rural & 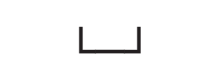 \\
\hline
\end{tabular}

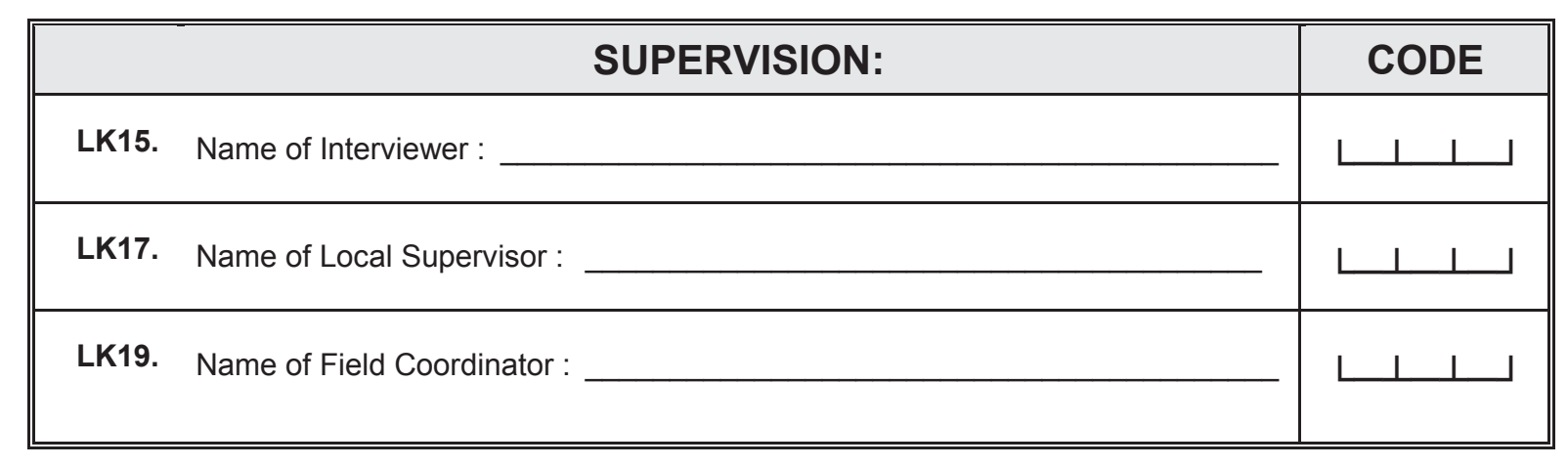

\section{ADDRESS OF MAIN INSTRUMENT}

LK08. a. Address

b. Description of location :

d. Postal code : : :

LK09. Telephone number :

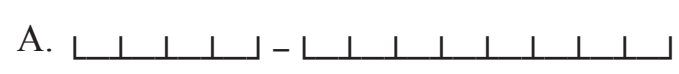

$\begin{array}{ll}\text { a. code } & \text { b. number }\end{array}$

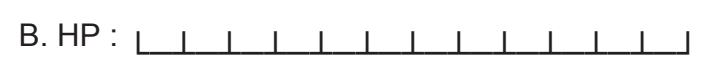

(owner)

$\begin{array}{ll}\text { Y. DK W. NA } & \text { WK }\end{array}$

LK09a E-mail Address 


\section{SECTION H: PRICES}

\begin{tabular}{|c|c|c|c|c|c|}
\hline & H03a. & H01. & $\begin{array}{l}\mathrm{H} 02 . \\
\end{array}$ & H03. & H04. \\
\hline $\begin{array}{l}\text { TYPE OF FOOD } \\
\text { (HPJ3TYPE) }\end{array}$ & Is $[\ldots]$ available for sale? & $\begin{array}{l}\text { What is the price per unit } \\
\text { (see H02) now? }\end{array}$ & $\begin{array}{l}\text { UNIT FOR H01 } \\
\text { INTERVIEWER NOTE: } \\
\text { IF THE RESPONDENT ISN'T ABLE TO PROVIDE } \\
\text { INFORMATION ABOUT THE PRICE PER KG OR } \\
\text { PER LITER, YOU MUST PROBE AND RECORD } \\
\text { INFORMATION ON THE QUANTITY AND UNITS OF } \\
\text { MEASUREMENT IN H03 AND H04 }\end{array}$ & $\begin{array}{l}\text { How much is contained in } \\
\text { one unit? }\end{array}$ & $\begin{array}{l}\text { In what units is the quantity } \\
\text { measured? }\end{array}$ \\
\hline 2. Rice: average quality & $\begin{array}{l}\text { 1. Cisadane } \rightarrow \\
\text { 5. Others............................ } \\
\text { 5. No stock } \downarrow \\
\text { 8. Not sold } \downarrow \\
\end{array}$ & 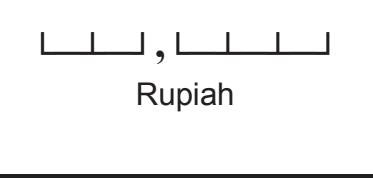 & 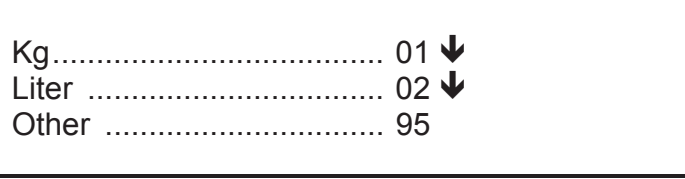 & $\downarrow \downarrow$ & $\begin{array}{l}\text { Kg } \\
\text { Liter }\end{array}$ \\
\hline 7. Beef (good quality, boneless) & $\begin{array}{l}\text { 1. Yes } \\
\text { 6. No stock } \downarrow \\
\text { 8. Not sold } \downarrow \\
\end{array}$ & 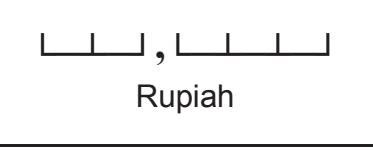 & 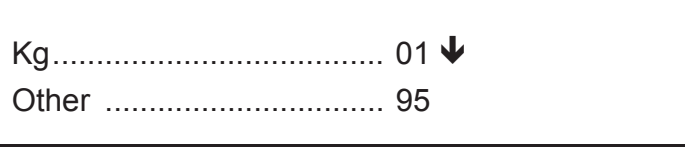 & $\downarrow \downarrow$ & 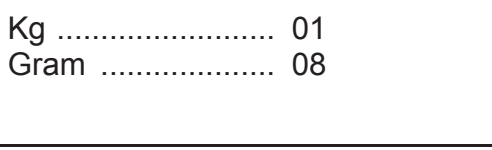 \\
\hline 11. Dried or salted fish & $\begin{array}{l}\text { 1. Layur } \\
\text { 2. Teri } \\
\text { 3. Pindang } \\
\text { 5. Others.................. } \\
\text { 6. No stock } \downarrow \\
\text { 8. Not sold } \downarrow\end{array}$ & 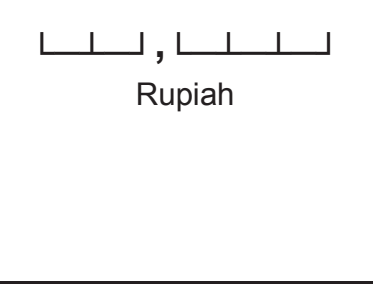 & 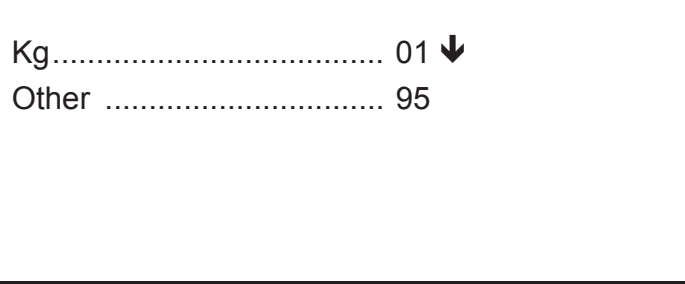 & 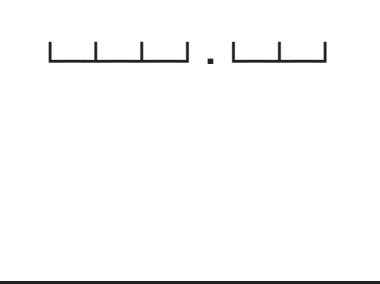 & 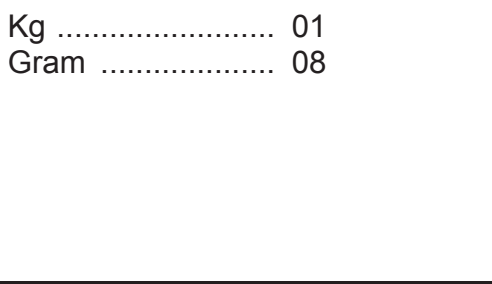 \\
\hline 13. Cooking oil & $\begin{array}{l}\text { 1. Palm oil } \\
\text { 2. Cocounut oil } \\
\text { 5. Others................... } \\
\text { 6. No stock } \downarrow \\
\text { 8. Not sold } \downarrow \\
\end{array}$ & 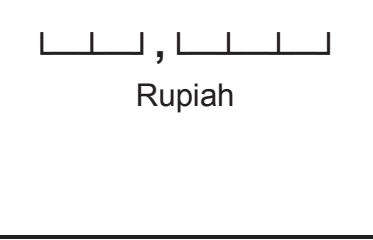 & 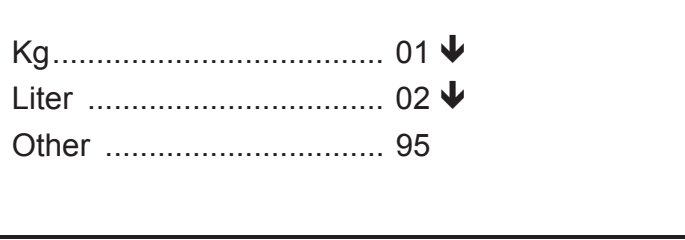 & 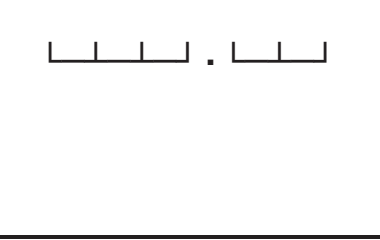 & $\begin{array}{lll}\mathrm{Kg} & \ldots & \ldots \\
\mathrm{C}\end{array}$ \\
\hline 14. White sugar & $\begin{array}{l}\text { 1. Local } \\
\text { 2. Imported } \\
\text { 5. Others } \\
\text { 6. No stock.......... } \\
\text { 8. Not sold } \downarrow \\
\end{array}$ & 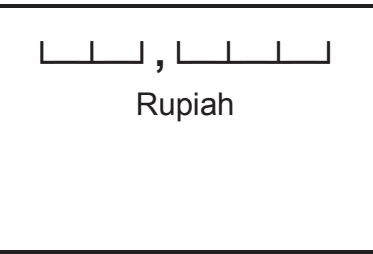 & 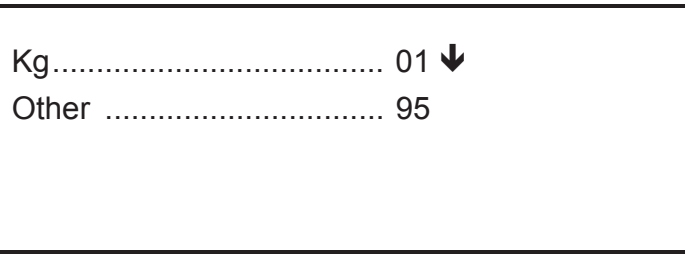 & 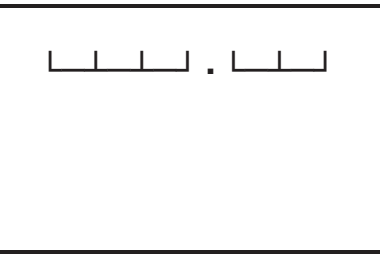 & $\begin{array}{l}\mathrm{Y} \text { Kg } \\
\mathrm{Gg}\end{array}$ \\
\hline 15. Salt & $\begin{array}{l}\text { 1. Table salt } \\
\text { 2. Block Salt } \\
\text { 5. Others................ } \\
\text { 6. No stock } \downarrow \\
\text { 8. Not sold } \downarrow \\
\end{array}$ & 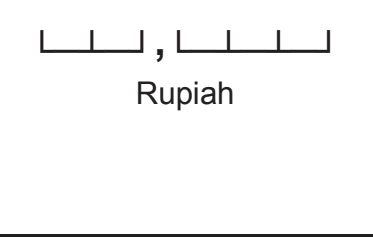 & 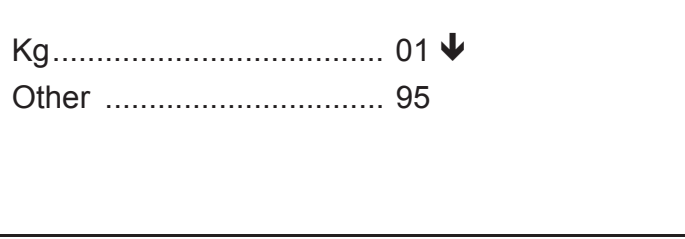 & 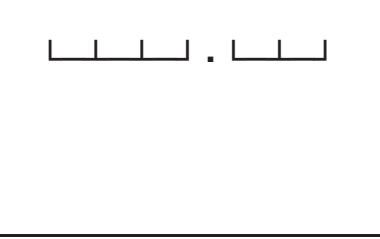 & 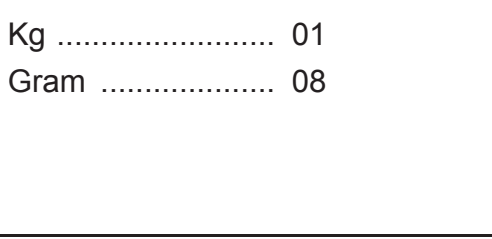 \\
\hline
\end{tabular}




\section{SECTION H: PRICES}

\begin{tabular}{|c|c|c|c|c|c|}
\hline & H03a. & H01. & $\begin{array}{l}\mathrm{H} 02 . \\
\end{array}$ & H03. & H04. \\
\hline $\begin{array}{l}\text { TYPE OF FOOD } \\
\text { (HPJ3TYPE) }\end{array}$ & Is [...] available for sale? & $\begin{array}{l}\text { What is the price per unit } \\
\text { (see H02) now? }\end{array}$ & $\begin{array}{l}\text { UNIT FOR H01 } \\
\text { INTERVIEWER NOTE: } \\
\text { IF THE RESPONDENT ISN'T ABLE TO PROVIDE } \\
\text { INFORMATION ABOUT THE PRICE PER KG OR } \\
\text { PER LITER, YOU MUST PROBE AND RECORD } \\
\text { INFORMATION ON THE QUANTITY AND UNITS OF } \\
\text { MEASUREMENT IN H03 AND H04 }\end{array}$ & $\begin{array}{l}\text { How much is contained in } \\
\text { one unit? }\end{array}$ & $\begin{array}{l}\text { In what units is the quantity } \\
\text { measured? }\end{array}$ \\
\hline 18. Sweetened condensed milk & $\begin{array}{l}\text { 1. Susu Bendera Putih } \\
\text { 2. Indomilk } \\
\text { 3. Nona } \\
\text { 5. Others................. } \\
\text { 6. No stock } \downarrow \\
\text { 8. Not sold } \downarrow\end{array}$ & 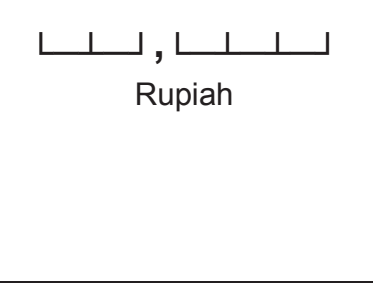 & 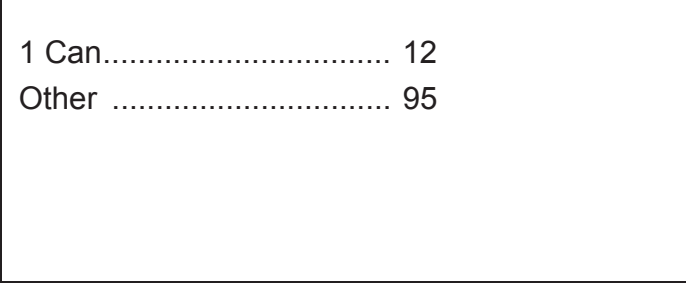 & 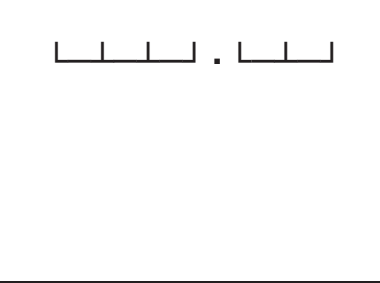 & 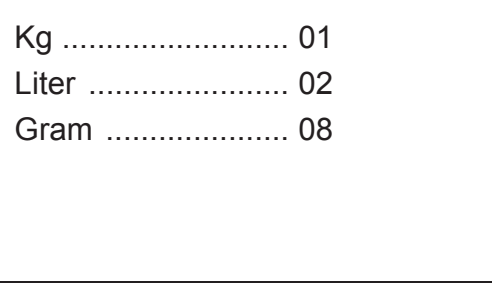 \\
\hline 19. Powdered Milk & $\begin{array}{l}\text { 1. Dancow } \\
\text { 2. Indomilk } \\
\text { 5. Others................ } \\
\text { 6. No stock } \downarrow \\
\text { 8. Not sold } \downarrow \\
\end{array}$ & 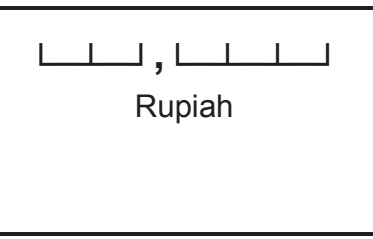 & 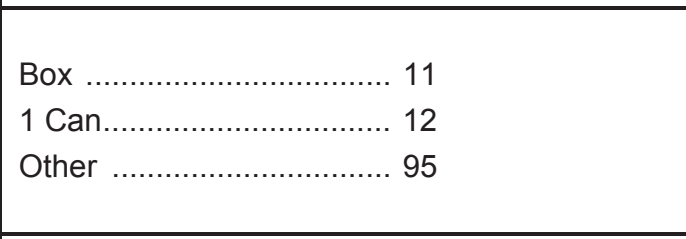 & 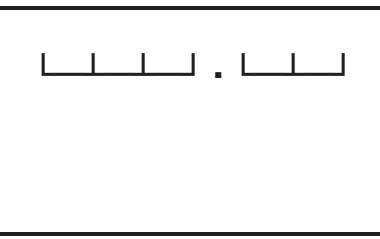 & 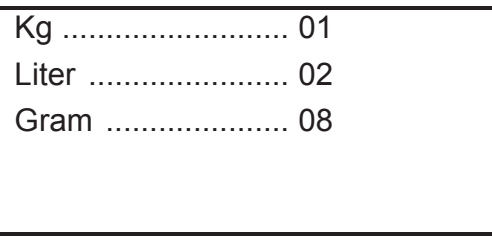 \\
\hline 20. Noodles & $\begin{array}{l}\text { 1. Supermie } \\
\text { 2. Sarimie } \\
\text { 3. Indomi } \\
\text { 5. Others } \\
\text { 6. No stock......... } \\
\text { 8. Not sold } \downarrow\end{array}$ & 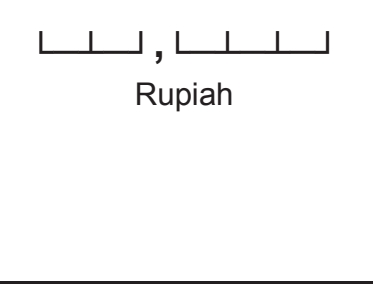 & 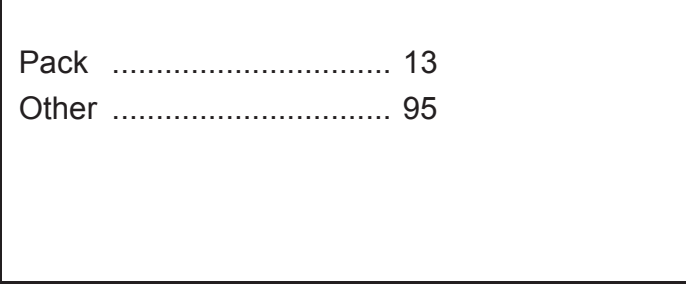 & 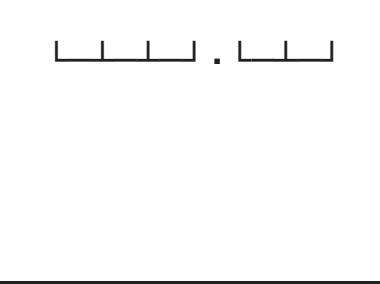 & 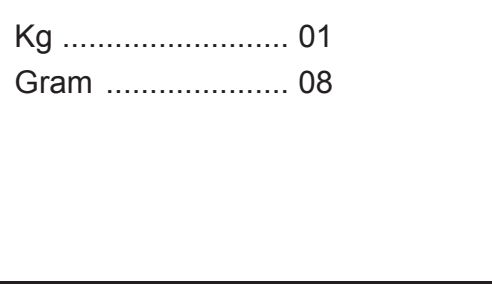 \\
\hline 21. Tofu & $\begin{array}{l}\text { 1. White tofu } \\
\text { 2. Yellow tofu } \\
\text { 5. Others................ } \\
\text { 6. No stock } \downarrow \\
\text { 8. Not sold } \downarrow\end{array}$ & 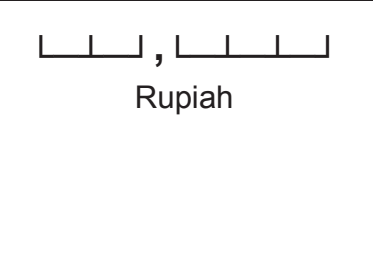 & $\begin{array}{l}\text { Pack } \\
\text { Other } \ldots \ldots \ldots . .1\end{array}$ & 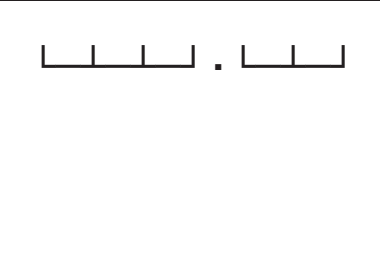 & 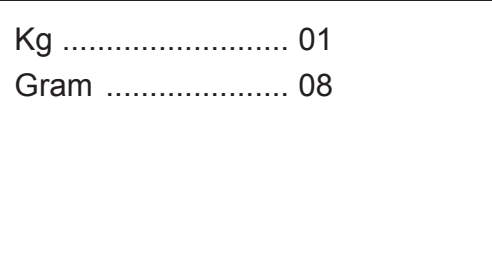 \\
\hline 22. Bean Curd & $\begin{array}{l}\text { 1. SUN } \\
\text { 5. Others ................. } \\
\text { 6. No stock } \downarrow \\
\text { 8. Not sold } \downarrow\end{array}$ & 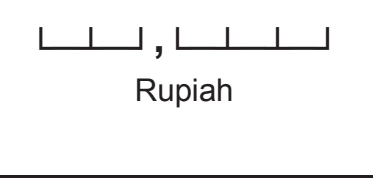 & $\begin{array}{l}\text { Pack } \\
\text { Other } \ldots \ldots \ldots \ldots . . .1\end{array}$ & 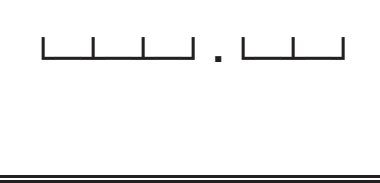 & 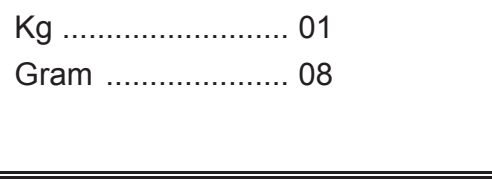 \\
\hline
\end{tabular}




\section{SECTION H: PRICES}

\begin{tabular}{|c|c|c|c|c|c|}
\hline & H03a. & H01. & $\mathrm{H} 02$. & $\mathrm{H03.}$ & H04. \\
\hline $\begin{array}{l}\text { TYPE OF FOOD } \\
\text { (HPJ3TYPE) }\end{array}$ & Is $[\ldots]$ available for sale? & $\begin{array}{l}\text { What is the price per unit } \\
\text { (see H02) now? }\end{array}$ & $\begin{array}{l}\text { UNIT FOR H01 } \\
\text { INTERVIEWER NOTE: } \\
\text { IF THE RESPONDENT ISN'T ABLE TO PROVIDE } \\
\text { INFORMATION ABOUT THE PRICE PER KG OR } \\
\text { PER LITER, YOU MUST PROBE AND RECORD } \\
\text { INFORMATION ON THE QUANTITY AND UNITS OF } \\
\text { MEASUREMENT IN H03 AND H04 }\end{array}$ & $\begin{array}{l}\text { How much is contained in } \\
\text { one unit? }\end{array}$ & $\begin{array}{l}\text { In what units is the quantity } \\
\text { measured? }\end{array}$ \\
\hline 23. Filtered clove cigarettes & $\begin{array}{l}\text { 1. GUDANG GARAM } \\
\text { 2. JARUM SUPER } \\
\text { 3. DJISAMSOE } \\
\text { 4. MARLBORO KRETEK } \\
\text { 5. Others................ } \\
\text { 6. No stock } \downarrow \\
\text { 8. Not sold } \downarrow \\
\end{array}$ & 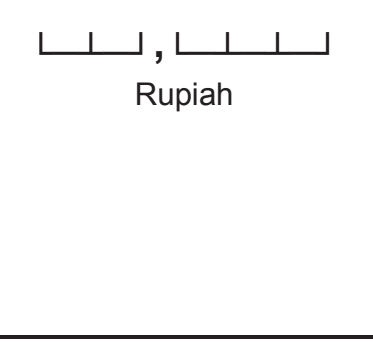 & 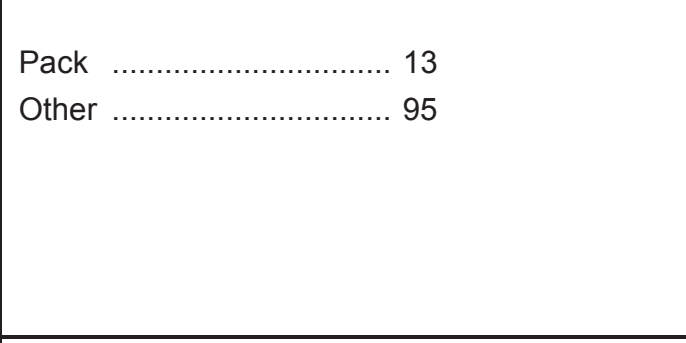 & 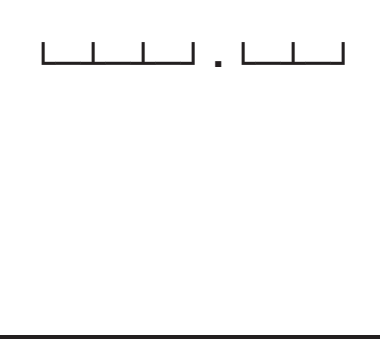 & Stick .......................... 01 \\
\hline 24. Filtered cigarettes & $\begin{array}{l}\text { 1. KANSAS } \\
\text { 2. MARLBORO } \\
\text { 3. LUCKY STRIKE } \\
\text { 5. Others................ } \\
\text { 6. No stock } \downarrow \\
\text { 8. Not sold } \downarrow \\
\end{array}$ & 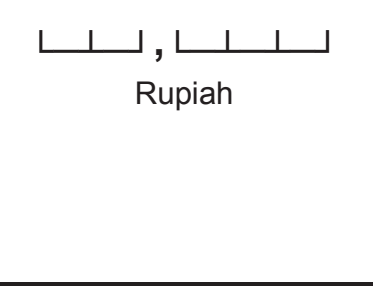 & 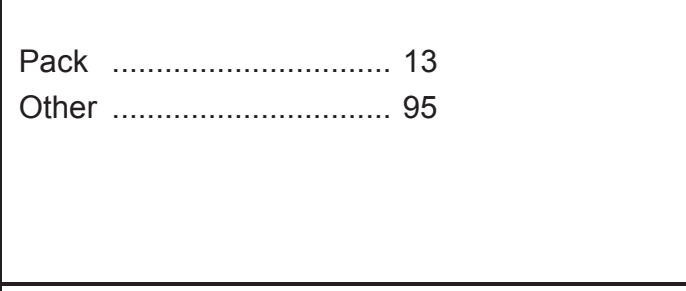 & 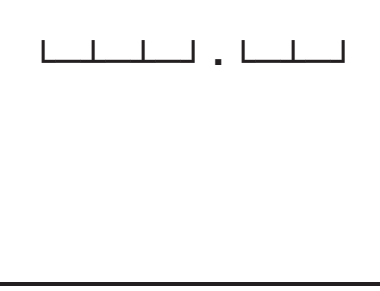 & Stick ……............... 01 \\
\hline 26. Kangkong (Water Spinach) & $\begin{array}{l}\text { 1. Yes } \\
\text { 6. No stock } \downarrow \\
\text { 8. Not sold } \downarrow\end{array}$ & 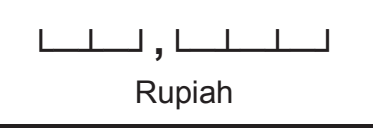 & 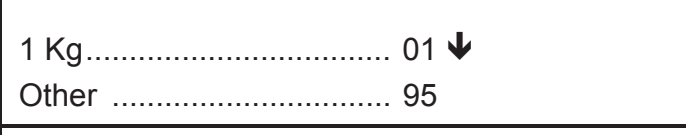 & 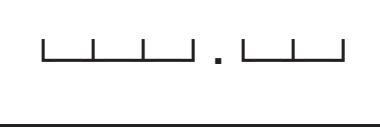 & $\begin{array}{l}\mathrm{Kg} \ldots \ldots \ldots \ldots \ldots \ldots \\
\mathrm{Gram}\end{array}$ \\
\hline 29. Bananas & $\begin{array}{l}\text { 1. Ambon } \\
\text { 2. Susu } \\
\text { 5. Others................ } \\
\text { 6. No stock } \downarrow \\
\text { 8. Not sold } \downarrow \\
\end{array}$ & 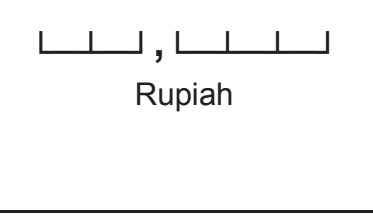 & $1 \mathrm{Kg}$ & 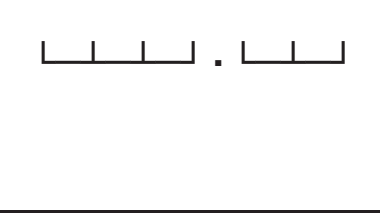 & 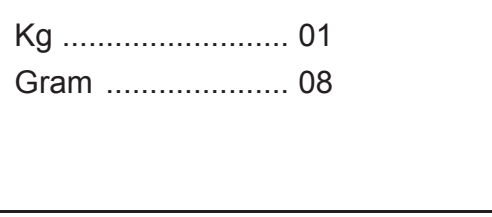 \\
\hline 30. Papaya & $\begin{array}{l}\text { 1. Yes } \\
\text { 6. No stock } \downarrow \\
\text { 8. Not sold } \downarrow\end{array}$ & 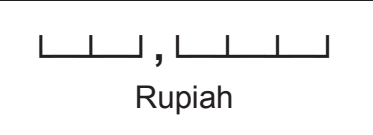 & 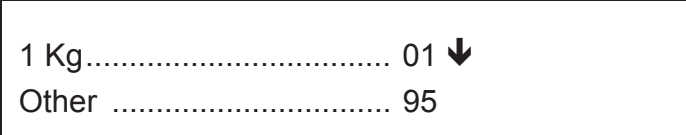 & 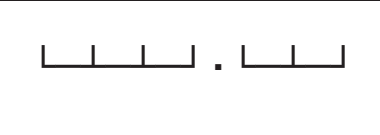 & 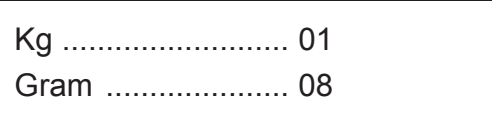 \\
\hline
\end{tabular}




\section{SECTION H: PRICES}

\begin{tabular}{|c|c|c|c|c|c|}
\hline & H03a. & H01. & $\mathrm{H} 02$. & $\mathrm{H03.}$ & H04. \\
\hline $\begin{array}{l}\text { TYPE OF FOOD } \\
\text { (HPJ3TYPE) }\end{array}$ & Is $[\ldots]$ available for sale? & $\begin{array}{l}\text { What is the price per unit } \\
\text { (see H02) now? }\end{array}$ & $\begin{array}{l}\text { UNIT FOR H01 } \\
\text { INTERVIEWER NOTE: } \\
\text { IF THE RESPONDENT ISN'T ABLE TO PROVIDE } \\
\text { INFORMATION ABOUT THE PRICE PER KG OR } \\
\text { PER LITER, YOU MUST PROBE AND RECORD } \\
\text { INFORMATION ON THE QUANTITY AND UNITS OF } \\
\text { MEASUREMENT IN H03 AND H04 }\end{array}$ & $\begin{array}{l}\text { How much is contained in } \\
\text { one unit? }\end{array}$ & $\begin{array}{l}\text { In what units is the quantity } \\
\text { measured? }\end{array}$ \\
\hline 31. Bottled Drinking water & $\begin{array}{l}\text { 1. Aqua } \\
\text { 2. Aquaria } \\
\text { 3. } 2 \text { Tang } \\
\text { 5. Others............... } \\
\text { 6. No stock } \downarrow \\
\text { 8. Not sold } \downarrow \\
\end{array}$ & 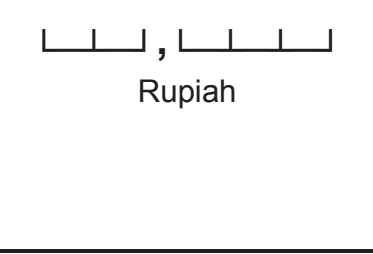 & 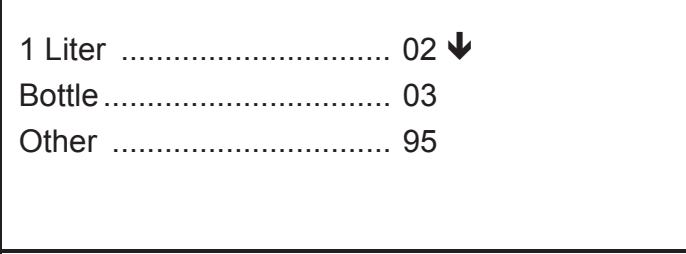 & 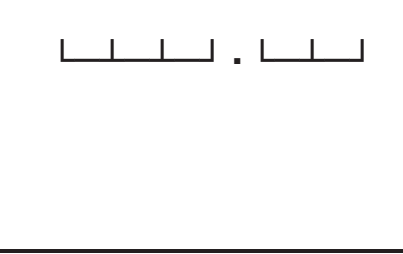 & Liter ........................ 02 \\
\hline 32. Kerosene & $\begin{array}{l}\text { 1. Yes } \\
\text { 6. No stock } \downarrow \\
\text { 8. Not sold } \downarrow\end{array}$ & 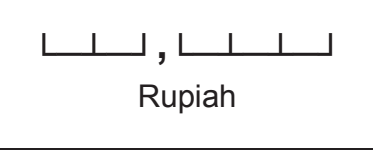 & 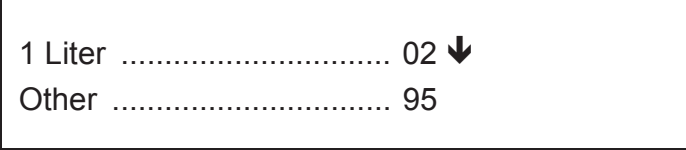 & 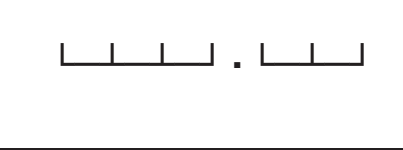 & Liter .......................... 02 \\
\hline 32a Gasoline by retail & $\begin{array}{l}\text { 1. Yes } \\
\text { 6. No stock } \downarrow \\
\text { 8. Not sold } \downarrow\end{array}$ & 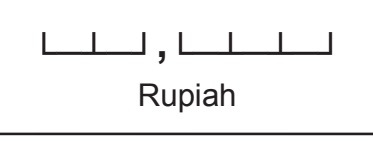 & Liter & 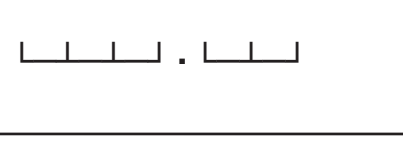 & Liter ......................... 02 \\
\hline 35. Bath soap & $\begin{array}{l}\text { 1. Lux } \\
\text { 2. Lifebuoy } \\
\text { 3. Giv } \\
\text { 5. Other } \\
\text { 6. No stock } \downarrow \\
\text { 8. Not sold } \downarrow\end{array}$ & 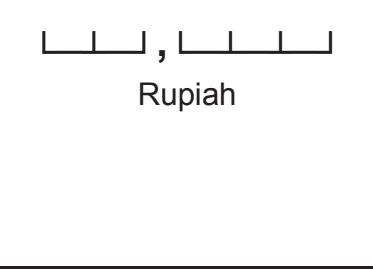 & $\begin{array}{l}1 \text { Stick } \ldots \ldots \ldots \ldots \ldots . . .04 \\
\text { Other }\end{array}$ & 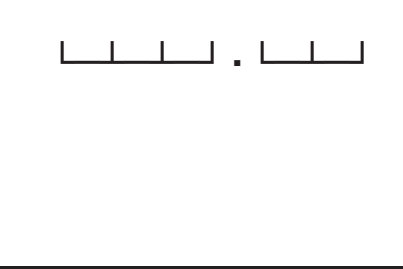 & 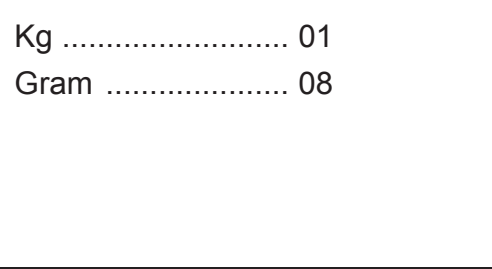 \\
\hline
\end{tabular}




\section{SECTION H: PRICES}

PRICE OF NON FOOD

Now, we would like to ask about the price of non foods as follows.

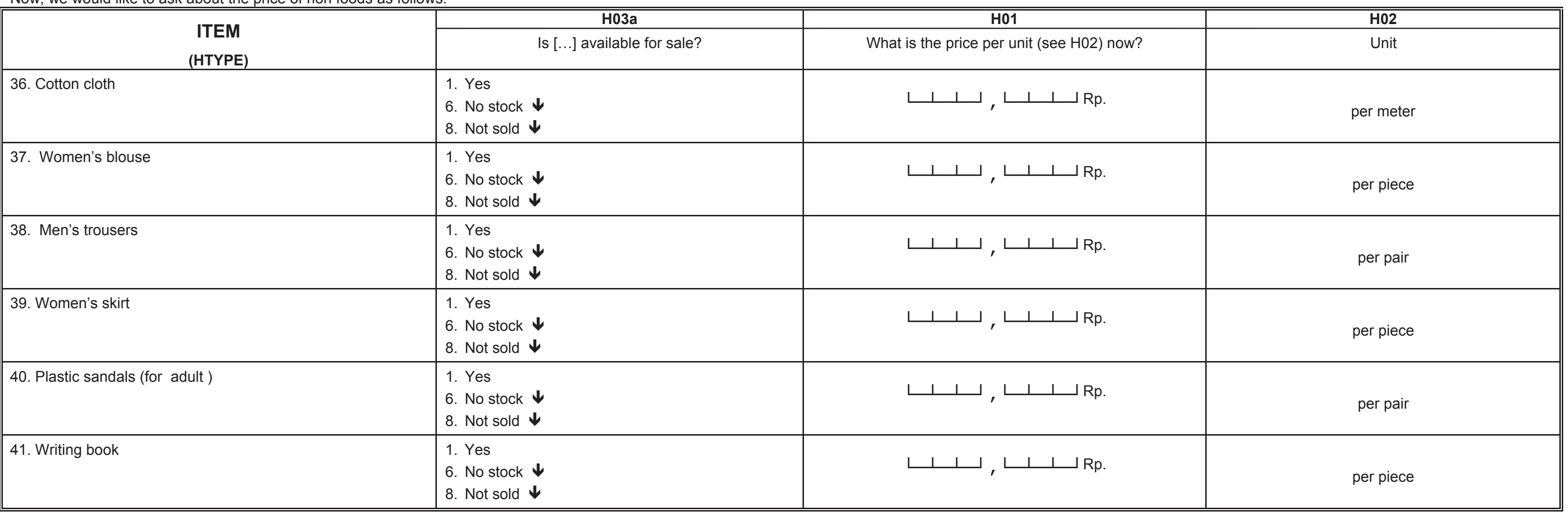


RESULT (FP3). INTERVIEW RESULTS CODE

\section{Completed $\rightarrow$ FP6}

2. Partially completed

3. Not completed
REASON (FP4). REASON FOR "2"|"3" IN RESULT 1. Respondent is traveling

2. Respondent is too busy

Refused
FP6. MONITORING BY LOCAL SUPERVISOR

a. Observed (sup obs)

Checked (sup_obs)

c. Verified (sup_verif)

\begin{tabular}{||l|l|}
\hline CP10. & WAS INTERVIEW INTERUPTED BY: \\
COULD BE MORE THAN ONE: & C \\
\hline $\begin{array}{l}\text { A. NONE } \\
\text { B. CUSTOMERS } \\
\text { C. FAMILY MEMBERS } \\
\text { D. OTHERS_ }\end{array}$ & \\
\hline \hline
\end{tabular}

\begin{tabular}{|c|c|}
\hline CP11. & $\begin{array}{l}\text { WHAT IS YOUR EVALUATION ON THE ANSWER OF } \\
\text { RESPONDENT? }\end{array}$ \\
\hline & $\begin{array}{l}\text { 1. VERY GOOD } \\
\text { 2. GOOD } \\
\text { 3. FAIR } \\
\text { 4. BAD } \\
\text { 5. VERY BAD }\end{array}$ \\
\hline
\end{tabular}
CP12. WHAT IS YOUR EVALUATION ON THE SERIOUSNESS AND
ATTENTIVENESS OF RESPONDENT?

NOTE :
1. VERY GOOD
2. GOOD
4. BAD
5. VERY BAD 
INDONESIAN FAMILY LIFE SURVEY 2014

\section{COMMUNITY AND FACILITY PEOPLE CHARACTERISTICS}

\section{SERVICE AVAILIBILITY ROSTER (SAR)}

NAME OF VILLAGE/KELURAHAN : —


EA $ᄂ \perp \_$STRATA $\longleftarrow$ PUSKESMAS/PRIVATE PRACTICE/POSYANDU/POSYANDU LANSIA/PRAKTEK TRADISIONAL

\begin{tabular}{|c|c|c|c|c|c|c|c|c|c|c|c|c|c|c|c|}
\hline & J01 \& J02 & J04a & J15 & J05 \& J06 & J07 & J08 & J09 & J10 & J11 & J11a & J12 & $\mathrm{J} 13$ & J14 & J16 & J17 \\
\hline $\begin{array}{l}N \\
\text { U } \\
M \\
\text { B } \\
\text { B } \\
\text { E } \\
\text { R }\end{array}$ & $\begin{array}{l}\text { Name and Address } \\
\text { and Specific } \\
\text { Information on } \\
\text { Location }\end{array}$ & $\begin{array}{l}\text { CODE } \\
\text { OF } \\
\text { FACILI } \\
\text { TY }\end{array}$ & $\begin{array}{l}\text { INTERVIEWER } \\
\text { NOTE ; } \\
\text { WHAT IS THIS } \\
\text { FACILITY } \\
\text { DUPLICATED ? } \\
\text { FILL THE CODE } \\
\text { OF FACILITY } \\
\text { DUPLICATED }\end{array}$ & $\begin{array}{l}\text { Desa and } \\
\text { kecamatan }\end{array}$ & $\begin{array}{l}\text { Still } \\
\text { operating } \\
\text { services? } \\
\text { 0. DK } \\
\text { Health Fac. } \\
\text { 1. Yes } \\
\text { 3. No } \\
\text { 8. DK Open }\end{array}$ & $\begin{array}{l}\text { What year did } \\
\text { close? }\end{array}$ & $\begin{array}{l}\text { Distance to } \\
\text { facility from } \\
\text { kepala Desa / } \\
\text { Kelurahan } \\
\text { office? }\end{array}$ & $\begin{array}{l}\text { Cost of transport } \\
\text { From Kepala Desa / } \\
\text { Kelurahan office }\end{array}$ & $\begin{array}{l}\text { On way } \\
\text { time from } \\
\text { Desa / } \\
\text { Kelurahan } \\
\text { office to this } \\
\text { facility }\end{array}$ & $\begin{array}{l}\text { On way time } \\
\text { from Desa / } \\
\text { Kelurahan to } \\
\text { this facility, } \\
\text { using } \\
\text { motorized } \\
\text { transport - } \\
\text { motorcycle or } \\
\text { motorboat? }\end{array}$ & $\begin{array}{l}\text { When did this } \\
\text { facility open? } \\
\text { If TT, Please } \\
\text { ask what year } \\
\text { this facility } \\
\text { operated? }\end{array}$ & $\begin{array}{l}\text { LOCATION } \\
\text { a. LATITUDE } \\
\text { b. LONGITUDE }\end{array}$ & $\begin{array}{l}\text { INTERVIEW } \\
\text { ER NOTE : } \\
\text { a. } \\
\text { INFORMATI } \\
\text { ON OF THE } \\
\text { LOCATION } \\
\text { b. TYPE OF } \\
\text { FACILITY }\end{array}$ & $\begin{array}{l}\text { INTER } \\
\text { VIEW } \\
\text { ED } \\
\text { STAT } \\
\text { US }\end{array}$ & $\begin{array}{l}\text { Did this } \\
\text { facility } \\
\text { have an } \\
\text { interview } \\
\text { in 1993, } \\
1997, \\
1998, \\
2000 ? \\
\text { reformat }\end{array}$ \\
\hline & $\begin{array}{l}\text { Nama } \\
\text { Almat } \\
\text { Desa : } \\
\text { 1. } \\
\text { 3. Sama dengan Wilcah } \\
\text { Kec: } \\
\text { 1. } \\
\text { 3. Sama dengan Wilcah } \\
\text { Kab: } \\
\text { 1. } \\
\text { 3. Sama dengan Wilcah } \\
\text { Prop: } \\
\text { 1. } \\
\text { 3. Sama dengan Wilcah } \\
\text { Pat }\end{array}$ & & $\begin{array}{l}\text { 1. Yes } \\
\text { Code of facility } \\
\rightarrow \text { NEXT ROW } \\
\text { 3. No }\end{array}$ & & $\begin{array}{l}\text { 0. } \rightarrow \mathbf{J 1 3} \\
\text { 1. Yes } \rightarrow \mathrm{J09} \\
\text { 8. DK } \rightarrow \mathrm{J} 13 \\
\text { 3. No } \\
\text { 2. Moved } \\
\rightarrow \text { J09 }\end{array}$ & $\begin{array}{l}\text { 1. Year } \\
\qquad \sqcup \sqcup \sqcup, ~ \sqcup \sqcup \text { years } \\
\text { 3. DK } \\
\downarrow \\
\text { NEXT ROW }\end{array}$ & 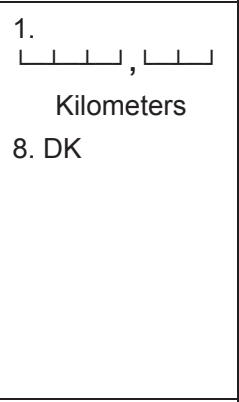 & 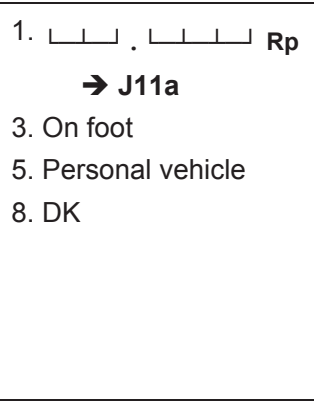 & 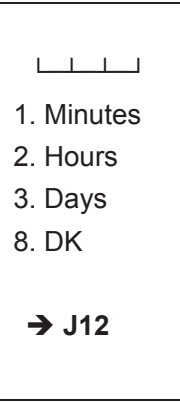 & $\begin{array}{l}111 \text {. Minutes } \\
\text { 2. Hours } \\
\text { 3. Days } \\
\text { 8. DK }\end{array}$ & 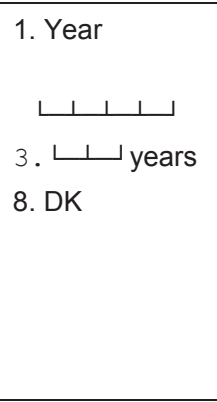 & 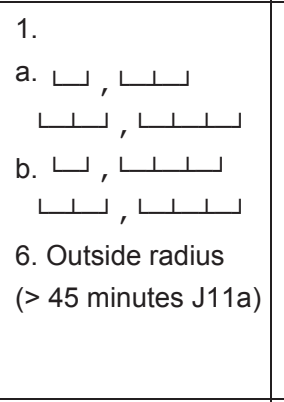 & $\begin{array}{l}\text { a. } \\
\\
\end{array}$ & $\begin{array}{l}\text { 1. Yes } \\
\text { 3. No }\end{array}$ & $\begin{array}{l}\text { 1. Yes } \\
\text { 3. No }\end{array}$ \\
\hline & $\begin{array}{l}\text { Nama } \\
\text { Almat } \\
\text { Desa } \\
\text { 1. } \\
\text { 3. Sama dengan Wilcah } \\
\text { Kec: } \\
\text { 1. } \\
\text { 3. Sama dengan Wilcah } \\
\text { Kab: } \\
\text { 1 } \\
\text { 3. Sama dengan Wilcah } \\
\text { Prop: } \\
\text { 1. } \\
\text { 3. Sama dengan Wilcah } \\
\end{array}$ & & $\begin{array}{l}\text { 1. Yes } \\
\text { Code of facility } \\
\rightarrow \text { NEXT ROW } \\
\text { 3. No }\end{array}$ & & $\begin{array}{l}\text { 0. } \rightarrow \mathbf{J} \mathbf{J 1 3} \\
\text { 1. Yes } \rightarrow \mathrm{J} 09 \\
\text { 8. DK } \rightarrow \mathrm{J} 13 \\
\text { 3. No } \\
\text { 2. Moved } \\
\rightarrow \text { J09 }\end{array}$ & 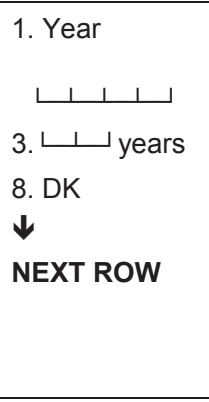 & $\begin{array}{l}\text { Kilometers } \\
\text { 8. DK ـــــــــــــــ }\end{array}$ & $\begin{array}{l}\text { 1. } \quad \rightarrow \text { J11a } \\
\text { 3. On foot } \\
\text { 5. Personal vehicle } \\
\text { 8. DK }\end{array}$ & 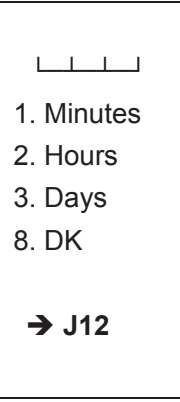 & 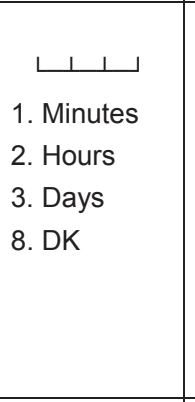 & 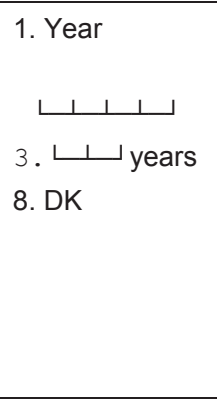 & 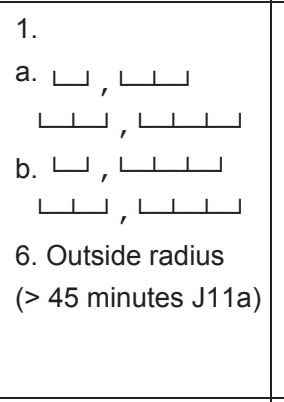 & a. & $\begin{array}{l}\text { 1. Yes } \\
\text { 3. No }\end{array}$ & $\begin{array}{l}\text { 1. Yes } \\
\text { 3. No }\end{array}$ \\
\hline & $\begin{array}{l}\text { Nama } \\
\text { Alamat } \\
\text { Desa : } \\
\text { 1. } \\
\text { 3. Sama dengan Wilcah } \\
\text { Kec: } \\
\text { 1. } \\
\text { 3. Sama dengan Wilcah } \\
\text { Kab: } \\
\text { 1. Sama dengan Wilcah } \\
\text { 3rop: } \\
\text { 1. } \\
\text { 3. Sama dengan Wilcah }\end{array}$ & & $\begin{array}{l}\text { 1. Yes } \\
\text { Code of facility } \\
\rightarrow \text { NEXT ROW } \\
\text { 3. No }\end{array}$ & & $\begin{array}{l}\text { 0. } \rightarrow \mathbf{J} \mathbf{1 3} \\
\text { 1. Yes } \rightarrow \mathrm{J} 09 \\
\text { 8. DK } \rightarrow \mathrm{J} 13 \\
\text { 3. No } \\
\text { 2. Moved } \\
\rightarrow \text { J09 }\end{array}$ & $\begin{array}{l}\text { 1. Year } \\
\text { 3. } \longleftarrow \perp \_ \text {years } \\
\text { 8. DK } \\
\downarrow \\
\text { NEXT ROW }\end{array}$ & 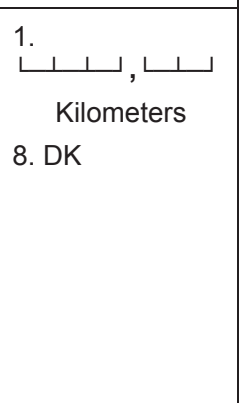 & 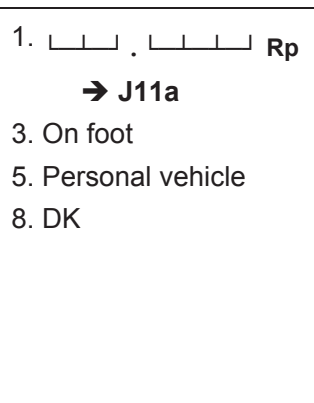 & 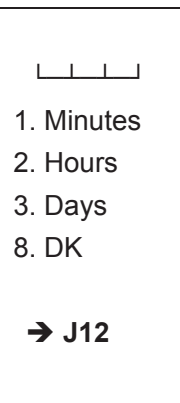 & 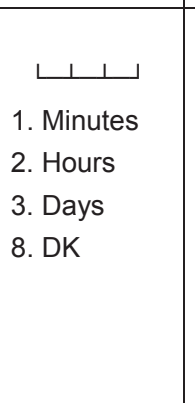 & 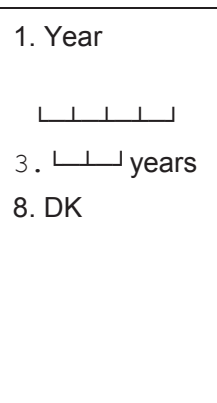 & 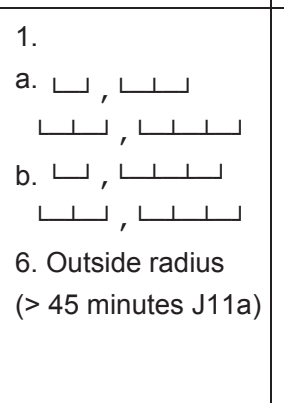 & a. & $\begin{array}{l}\text { 1. Yes } \\
\text { 3. No }\end{array}$ & $\begin{array}{l}\text { 1. Yes } \\
\text { 3. No }\end{array}$ \\
\hline
\end{tabular}

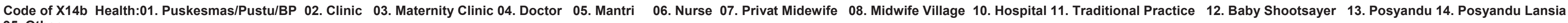
95. Other

IFLS CODE 


\begin{tabular}{|c|c|c|c|c|c|c|c|c|c|c|c|c|c|}
\hline \multirow{2}{*}{\multicolumn{2}{|c|}{ X01 \& X02 }} & \multirow[b]{2}{*}{$\mathrm{X03}$} & \multirow[b]{2}{*}{ X04a } & \multirow[b]{2}{*}{ X15 } & \multirow[b]{2}{*}{ X05 \& X06 } & \multirow[b]{2}{*}{ X09 } & \multicolumn{3}{|l|}{ TYPE OF FACILITY } & \multicolumn{4}{|c|}{$\begin{array}{l}\text { 1. Puskesmas/Puskesmas Pembantu } \\
\text { 2. Doctor/Clinic/Maternity Clinic/Midwife/Midwife Village/Nurse/Mantri } \\
\text { 3. Tradicional Practise } \\
\text { 4. Integrated Health Post (Posyandu) } \\
\text { 5. Integrated Health Post for Elderly (Posyandu Lansia) } \\
\text { 6. Hospital }\end{array}$} \\
\hline & & & & & & & X10 & $\mathrm{X} 11$ & $\mathrm{X} 11 \mathrm{a}$ & $\mathrm{X} 12$ & $\mathrm{X} 13$ & X14 & $\mathrm{X} 16$ \\
\hline $\begin{array}{l}N \\
U \\
M \\
B \\
B \\
E \\
R\end{array}$ & $\begin{array}{l}\text { Name and Address of } \\
\text { Health Faclity }\end{array}$ & $\begin{array}{l}\text { SOURCE OF } \\
\text { INFORMATIO } \\
\mathrm{N} \\
\text { 4. NCR } \\
\text { SECCION } \\
\text { PP } \\
\text { 3. PKK } \\
\text { 2. HEAD OF } \\
\text { VILLAGE/ } \\
\text { KELURAH- } \\
\text { AN }\end{array}$ & $\begin{array}{l}\text { CODE OF } \\
\text { FACILITY }\end{array}$ & $\begin{array}{l}\text { INTERVIEWER } \\
\text { NOTE: } \\
\text { WHAT IS THIS } \\
\text { FACILITY } \\
\text { DUPLICATED? } \\
\text { FILL THE CODE } \\
\text { OF FACILITY } \\
\text { DUPLICATED }\end{array}$ & $\begin{array}{l}\text { Desa dan } \\
\text { Kecamatan }\end{array}$ & $\begin{array}{l}\text { Distance to } \\
\text { facility from } \\
\text { kepala Desa / } \\
\text { Kelurahan } \\
\text { office? }\end{array}$ & $\begin{array}{l}\text { Cost of transport } \\
\text { From Kepala Desa } \\
\text { / Kelurahan office }\end{array}$ & $\begin{array}{l}\text { On way } \\
\text { time from } \\
\text { Desa / } \\
\text { Kelurahan } \\
\text { office to } \\
\text { this facility }\end{array}$ & $\begin{array}{l}\text { On way } \\
\text { time from } \\
\text { Desa / } \\
\text { Kelurahan } \\
\text { to this } \\
\text { facility, } \\
\text { using } \\
\text { motorized } \\
\text { transport - } \\
\text { motorcycle } \\
\text { or } \\
\text { motorboat? }\end{array}$ & $\begin{array}{l}\text { When did this facility } \\
\text { open? } \\
\text { If TT, Please ask } \\
\text { what year this } \\
\text { facility operated? }\end{array}$ & $\begin{array}{l}\text { LOCATION } \\
\text { c. LATITUDE } \\
\text { d. LONGITUDE }\end{array}$ & $\begin{array}{l}\text { INTERVIEWER } \\
\text { NOTE : } \\
\text { a. INFORMATION } \\
\text { OF THE } \\
\text { LOCATION } \\
\text { b. TYPE OF } \\
\text { FACILITY }\end{array}$ & $\begin{array}{l}\text { INTERVI } \\
\text { EWED } \\
\text { STATUS }\end{array}$ \\
\hline & $\begin{array}{l}\text { Nama } \\
\text { Alamat } \\
\text { Desa : } \\
\text { 1. } \\
\text { 3. Sama dengan Wilcah } \\
\text { Kec: } \\
\text { 1. } \\
\text { 3. Sama dengan Wilcah } \\
\text { Kab: } \\
\text { 1 } \\
\text { 3. Sama dengan Wilcah } \\
\text { Prop: } \\
\text { 1. Sama dengan Wilcah } \\
\text { 3. Sama }\end{array}$ & $\begin{array}{l}\text { 4. PP } \\
\text { 3. PKK } \\
\text { 2. KD }\end{array}$ & 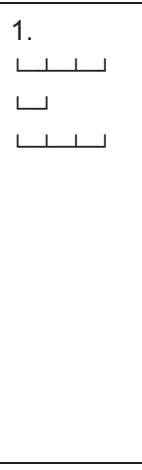 & 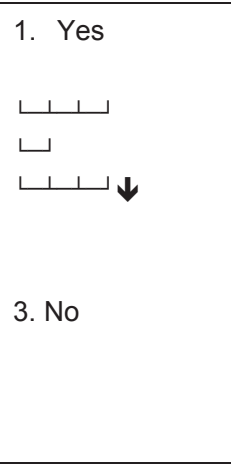 & $\begin{array}{l}\text { a. Village } \\
\frac{1 .}{\text { 3. Same }} \\
\text { 8. DK } \\
\text { b. Kecamatan } \\
11 . \\
\overline{13 . \text { Same }} \\
\text { 98. DK }\end{array}$ & 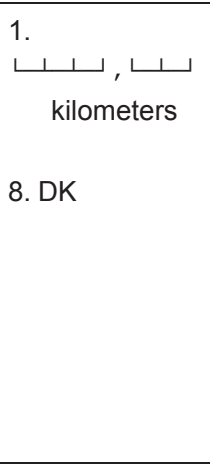 & 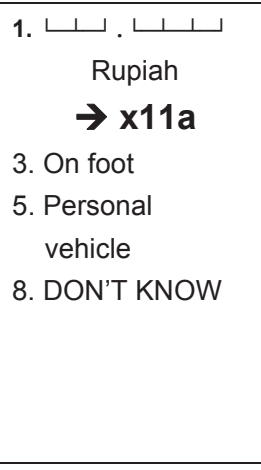 & $\begin{array}{l}\qquad \perp 1 \_ \\
\text {1. Minutes } \\
\text { 2. Hours } \\
\text { 3. Days } \\
\text { 8. TT } \\
\rightarrow \times 12\end{array}$ & $\begin{array}{l}\qquad \perp 1 \_ \\
\text {1. Minutes } \\
\text { 2. Hours } \\
\text { 3. Days } \\
\text { 8. TT }\end{array}$ & 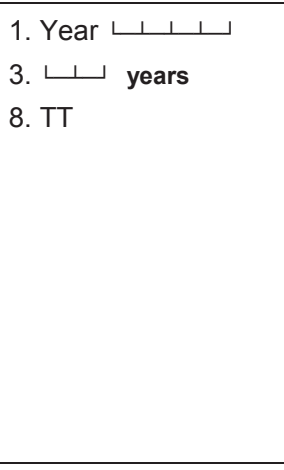 & 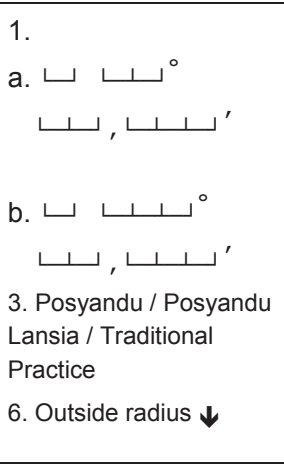 & $\begin{array}{l}\text { a. } \\
\\
\\
\text { b. } \longleftarrow \text {. } \\
\end{array}$ & $\begin{array}{l}\text { 1. Yes } \\
\text { 3. No }\end{array}$ \\
\hline & $\begin{array}{l}\text { Nama } \\
\text { Alamat } \\
\text { Desa : } \\
\text { 1. } \\
\text { 3. Sama dengan Wilcah } \\
\text { Kec: } \\
\text { 1. } \\
\text { 3. Sama dengan Wilcah } \\
\text { Kab: } \\
\text { 1 } \\
\text { 3. Sama dengan Wilcah } \\
\text { Prop: } \\
\text { 1. Sama dengan Wilcah } \\
\text { 3. Sal }\end{array}$ & $\begin{array}{l}\text { 4. PP } \\
\text { 3. PKK } \\
\text { 2. } \mathrm{KD}\end{array}$ & 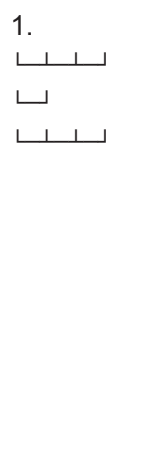 & $\begin{array}{l}\text { 2. Yes } \\
\sqcup \sqcup \\
\sqcup \\
\sqcup \sqcup \sqcup \downarrow\end{array}$ & $\begin{array}{l}\text { a. Village } \\
\frac{1 .}{\text { 3. Same }} \\
\text { 8. DK } \\
\text { b. Kecamatan } \\
11 . \\
\overline{13 . \text { Same }} \\
\text { 98. DK }\end{array}$ & 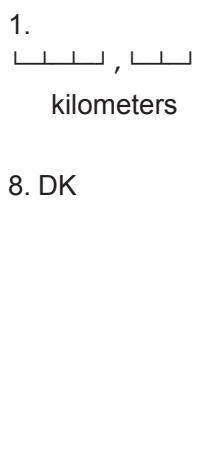 & $\begin{array}{l}\text { 1. } \quad \text { Rupiah } \\
\quad \rightarrow \text { x11a } \\
\text { 3. On foot } \\
\text { 5. Personal } \\
\text { vehicle } \\
\text { 8. DON'T KNOW }\end{array}$ & $\begin{array}{l}\qquad \_1 \text {. } \\
\text { 1. Minutes } \\
\text { 2. Hours } \\
\text { 3. Days } \\
\text { 8. TT } \\
\rightarrow \times 12\end{array}$ & $\begin{array}{l}\qquad \perp\lrcorner 1 \\
\text { 1. Minutes } \\
\text { 2. Hours } \\
\text { 3. Days } \\
\text { 8. TT }\end{array}$ & $\begin{array}{l}\text { 1. Year } \sqcup \sqcup \sqcup \text {. } \sqcup \text { years } \\
\text { 3. } \sqcup \sqcup \text { yeas } \\
\text { 8. TT }\end{array}$ & 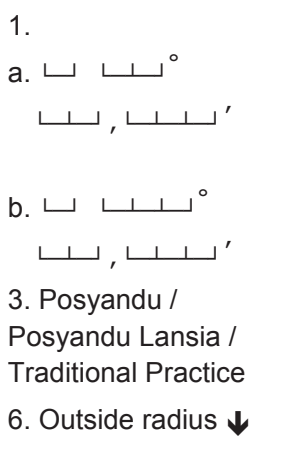 & $\begin{array}{l}\text { a. } \\
\\
\\
\text { b. } \longleftarrow \\
\end{array}$ & $\begin{array}{l}\text { 1. Yes } \\
\text { 3. No }\end{array}$ \\
\hline
\end{tabular}

Code of X14b Health:

01. Puskesmas/Pustu/BP

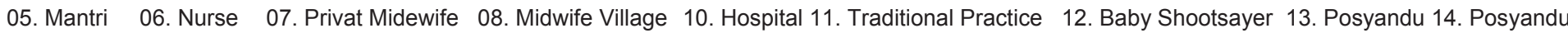

\section{PREPRINTED}

SERVICE AVAILABILITY ROSTER - PREPRINTED 


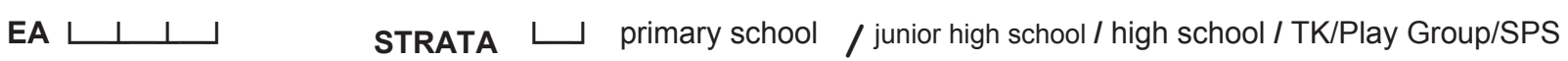

\begin{tabular}{|c|c|c|c|c|c|c|c|c|c|c|c|c|c|c|c|}
\hline & J01 \& J02 & J04a & J15 & J05 \& J06 & J07 & J08 & J09 & J10 & J11 & J11a & J12 & J13 & J14 & J16 & J17 \\
\hline $\begin{array}{l}N \\
\text { U } \\
M \\
\text { M } \\
\text { E } \\
\text { R }\end{array}$ & $\begin{array}{l}\text { Name and Address } \\
\text { and Specific } \\
\text { Information on } \\
\text { Location }\end{array}$ & $\begin{array}{l}\text { CODE } \\
\text { OF } \\
\text { FACILI } \\
\text { TY }\end{array}$ & $\begin{array}{l}\text { INTERVIEWER } \\
\text { NOTE ; } \\
\text { WHAT IS THIS } \\
\text { FACILITY } \\
\text { DUPLICATED? } \\
\text { FILL THE CODE } \\
\text { OF FACILITY } \\
\text { DUPLICATED }\end{array}$ & $\begin{array}{l}\text { Desa and } \\
\text { kecamatan }\end{array}$ & $\begin{array}{l}\text { Still } \\
\text { operating } \\
\text { services? } \\
\text { 0. DK } \\
\text { Health Fac. } \\
\text { 1. Yes } \\
\text { 3. No } \\
\text { 8. DK Open }\end{array}$ & $\begin{array}{l}\text { What year did } \\
\text { close? }\end{array}$ & $\begin{array}{l}\text { Distance to } \\
\text { facility from } \\
\text { kepala Desa / } \\
\text { Kelurahan } \\
\text { office? }\end{array}$ & $\begin{array}{l}\text { Cost of transport } \\
\text { From Kepala Desa / } \\
\text { Kelurahan office }\end{array}$ & $\begin{array}{l}\text { On way } \\
\text { time from } \\
\text { Desa / } \\
\text { Kelurahan } \\
\text { office to this } \\
\text { facility }\end{array}$ & $\begin{array}{l}\text { On way time } \\
\text { from Desa / } \\
\text { Kelurahan to } \\
\text { this facility, } \\
\text { using } \\
\text { motorized } \\
\text { transport - } \\
\text { motorcycle or } \\
\text { motorboat? }\end{array}$ & $\begin{array}{l}\text { When did this } \\
\text { facility open? } \\
\text { If TT, Please } \\
\text { ask what year } \\
\text { this facility } \\
\text { operated? }\end{array}$ & $\begin{array}{l}\text { LOCATION } \\
\text { a. LATITUDE } \\
\text { b. LONGITUD } \\
\text { E }\end{array}$ & $\begin{array}{l}\text { INTERVIEW } \\
\text { ER NOTE : } \\
\text { a. } \\
\text { INFORMATI } \\
\text { ON OF THE } \\
\text { LOCATION } \\
\text { b. TYPE OF } \\
\text { FACILITY }\end{array}$ & $\begin{array}{l}\text { INTER } \\
\text { VIEW } \\
\text { ED } \\
\text { STAT } \\
\text { US }\end{array}$ & $\begin{array}{l}\text { Did this } \\
\text { facility } \\
\text { have an } \\
\text { interview } \\
\text { in 1993, } \\
1997, \\
1998, \\
2000 ? \\
\text { reformat }\end{array}$ \\
\hline & $\begin{array}{l}\text { Name: } \\
\text { Address: } \\
\text { Village : } \\
\text { 1. } \\
\text { 3. Same as EA } \\
\text { Kec: } \\
\text { 1. } \\
\text { Kab: } \\
1 . \\
\text { 3. Same as EA } \\
\text { Prov: } \\
\text { 1. } \\
\text { 3. Same as EA }\end{array}$ & & $\begin{array}{l}\text { 1. Yes } \\
\text { Code of facility } \\
\rightarrow \text { NEXT ROW } \\
\text { 3. No }\end{array}$ & & $\begin{array}{l}\text { 0. } \rightarrow \mathbf{J 1 3} \\
\text { 1. Yes } \rightarrow \mathrm{J} 09 \\
\text { 8. DK } \rightarrow \mathrm{J13} \\
\text { 3. No } \\
\text { 2. Moved } \\
\rightarrow \mathbf{J} \mathbf{0 9}\end{array}$ & 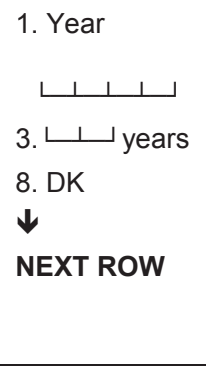 & $\begin{array}{l}\text { Kilometers } \\
\text { 8. DK ـــــــــــــ }\end{array}$ & 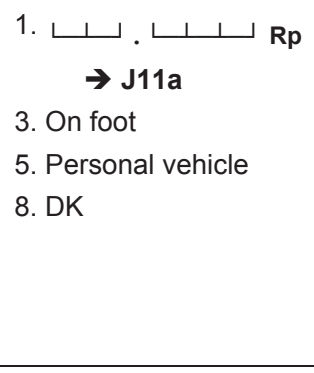 & 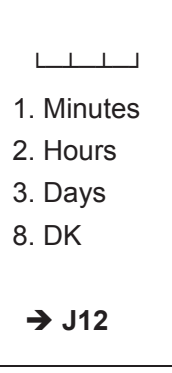 & $\begin{array}{l}1 ـ 1 ـ 1 ـ 1 \text { 1. Minutes } \\
\text { 2. Hours } \\
\text { 3. Days } \\
\text { 8. DK }\end{array}$ & 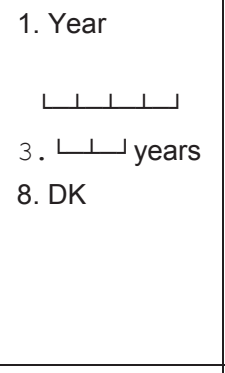 & 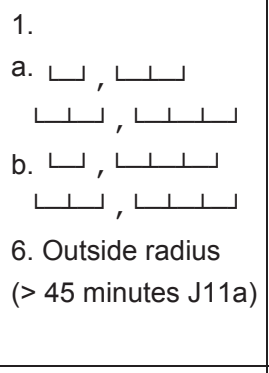 & $\begin{array}{l}\text { a. } \\
\\
\text { b. } \sqcup \text { L }\end{array}$ & $\begin{array}{l}\text { 1. Yes } \\
\text { 3. No }\end{array}$ & $\begin{array}{l}\text { 1. Yes } \\
\text { 3. No }\end{array}$ \\
\hline & $\begin{array}{l}\text { Name: } \\
\text { Address: } \\
\text { Village : } \\
\text { 1. } \\
\text { 3. Same as EA } \\
\text { Kec: } \\
\text { 1. } \\
\text { Kab: } \\
1 \text { 3. Same as EA } \\
\text { Prov: } \\
\text { 1. } \\
\text { 3. Same as EA }\end{array}$ & & $\begin{array}{l}\text { 1. Yes } \\
\text { Code of facility } \\
\rightarrow \text { NEXT ROW } \\
\text { 3. No }\end{array}$ & & $\begin{array}{l}\text { 0. } \rightarrow \mathbf{J} \mathbf{J 1 3} \\
\text { 1. Yes } \rightarrow \mathrm{J} 09 \\
\text { 8. DK } \rightarrow \mathrm{J} 13 \\
\text { 3. No } \\
\text { 2. Moved } \\
\rightarrow \mathrm{J} \mathbf{0 9}\end{array}$ & 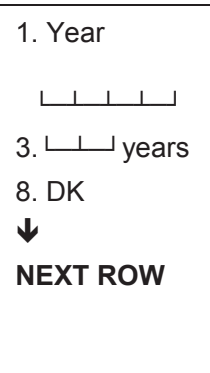 & 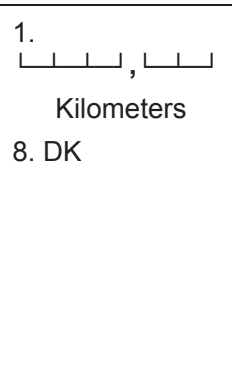 & $\begin{array}{l}\text { 1. } \quad \rightarrow \text { J11a } \\
\text { 3. On foot } \\
\text { 5. Personal vehicle } \\
\text { 8. DK }\end{array}$ & \begin{tabular}{l}
\multicolumn{1}{c}{$\perp \perp \perp$ 1. Minutes } \\
2. Hours \\
3. Days \\
8. DK \\
$\rightarrow \mathrm{J12}$
\end{tabular} & $\begin{array}{l}1 \perp 1 ـ 1 \text { 1. Minutes } \\
\text { 2. Hours } \\
\text { 3. Days } \\
\text { 8. DK }\end{array}$ & 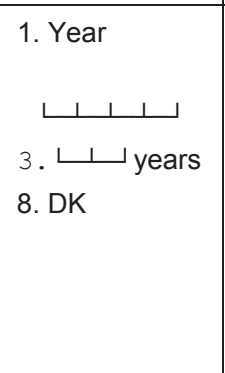 & 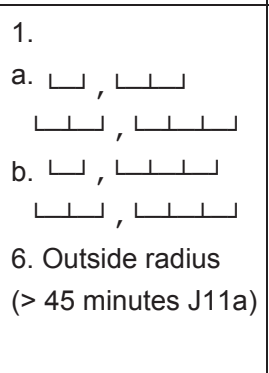 & a. & $\begin{array}{l}\text { 1. Yes } \\
\text { 3. No }\end{array}$ & $\begin{array}{l}\text { 1. Yes } \\
\text { 3. No }\end{array}$ \\
\hline & $\begin{array}{l}\text { Name: } \\
\text { Address: } \\
\text { Village : } \\
\text { 1. } \\
\text { 3. Same as EA } \\
\text { Kec: } \\
\text { 1. } \\
\text { Kab: } \\
1 \text { 3. } \\
\text { 3ame as EA } \\
\text { Prov: } \\
\text { 1. } \\
\text { 3. Same as EA } \\
\end{array}$ & & $\begin{array}{l}\text { 1. Yes } \\
\text { Code of facility } \\
\rightarrow \text { NEXT ROW } \\
\text { 3. No }\end{array}$ & & $\begin{array}{l}\text { 0. } \rightarrow \mathbf{J} \mathbf{J 1 3} \\
\text { 1. Yes } \rightarrow \mathrm{J} 09 \\
\text { 8. DK } \rightarrow \mathrm{J13} \\
\text { 3. No } \\
\text { 2. Moved } \\
\rightarrow \mathrm{J} 09\end{array}$ & 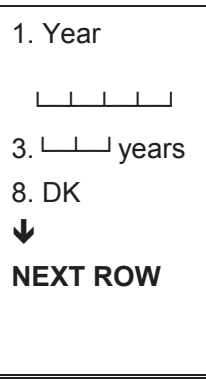 & 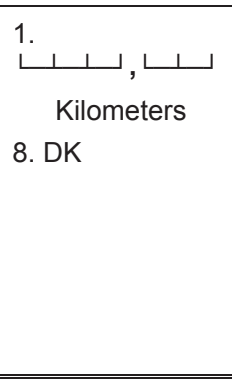 & $\begin{array}{l}\text { 1. } \rightarrow \text { J11a } \\
\quad \rightarrow \perp \perp \text { Rp } \\
\text { 3. On foot } \\
\text { 5. Personal vehicle } \\
\text { 8. DK }\end{array}$ & 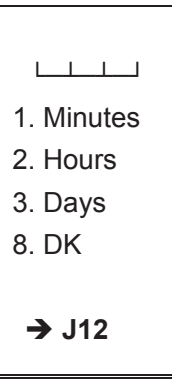 & 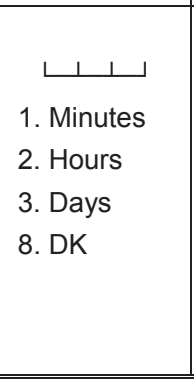 & 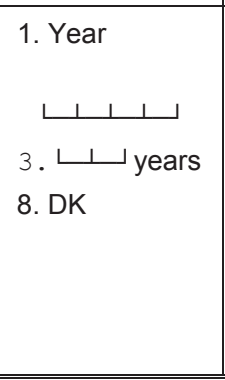 & 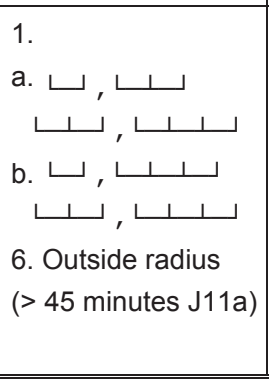 & a. & $\begin{array}{l}\text { 1. Yes } \\
\text { 3. No }\end{array}$ & $\begin{array}{l}\text { 1. Yes } \\
\text { 3. No }\end{array}$ \\
\hline
\end{tabular}




\begin{tabular}{|c|c|c|c|c|c|c|c|c|c|c|c|c|c|}
\hline & & & & & & & \multicolumn{3}{|l|}{ TYPE OF FACILITY } & \multicolumn{4}{|c|}{$\begin{array}{l}\text { 1. primary school (SD/MI) } \\
\text { 2. junior high school (SMP/MTs) } \\
\text { 3. high school (SMA/MA/SMK) } \\
\text { 4. TK/Play Group/SPS } \\
\end{array}$} \\
\hline & X01 \& X02 & $\mathrm{X} 03$ & X04a & X15 & X05 \& X06 & X09 & $\mathrm{X} 10$ & X11 & $\mathrm{X} 11 \mathrm{a}$ & $\mathrm{X} 12$ & $\mathrm{X} 13$ & X14 & $\mathrm{X} 16$ \\
\hline $\begin{array}{l}N \\
U \\
M \\
M \\
E \\
\text { R }\end{array}$ & $\begin{array}{l}\text { Name and Address of } \\
\text { Health Faclity }\end{array}$ & $\begin{array}{l}\text { SOURCE OF } \\
\text { INFORMATIO } \\
\mathrm{N} \\
\text { 4. NCR } \\
\text { SECCION } \\
\text { PP } \\
\text { 3. PKK } \\
\text { 2. HEAD OF } \\
\text { VILLAGE/ } \\
\text { KELURAH- } \\
\text { AN }\end{array}$ & $\begin{array}{l}\text { CODE OF } \\
\text { FACILITY }\end{array}$ & $\begin{array}{l}\text { INTERVIEWER } \\
\text { NOTE: } \\
\text { WHAT IS THIS } \\
\text { FACILITY } \\
\text { DUPLICATED? } \\
\text { FILL THE CODE } \\
\text { OF FACILITY } \\
\text { DUPLICATED }\end{array}$ & $\begin{array}{l}\text { Desa dan } \\
\text { Kecamatan }\end{array}$ & $\begin{array}{l}\text { Distance to } \\
\text { facility from } \\
\text { kepala Desa / } \\
\text { Kelurahan } \\
\text { office? }\end{array}$ & $\begin{array}{l}\text { Cost of transport } \\
\text { From Kepala Desa } \\
\text { / Kelurahan office }\end{array}$ & $\begin{array}{l}\text { On way } \\
\text { time from } \\
\text { Desa / } \\
\text { Kelurahan } \\
\text { office to } \\
\text { this facility }\end{array}$ & $\begin{array}{l}\text { On way } \\
\text { time from } \\
\text { Desa / } \\
\text { Kelurahan } \\
\text { to this } \\
\text { facility, } \\
\text { using } \\
\text { motorized } \\
\text { transport - } \\
\text { motorcycle } \\
\text { or } \\
\text { motorboat? }\end{array}$ & $\begin{array}{l}\text { When did this facility } \\
\text { open? } \\
\text { If TT, Please ask } \\
\text { what year this } \\
\text { facility operated? }\end{array}$ & $\begin{array}{ll}\text { LOCATION } & \\
\text { c. } & \text { LATIT } \\
\text { d. } & \text { LONE } \\
& \text { ITUD } \\
& \text { E }\end{array}$ & $\begin{array}{l}\text { INTERVIEWER } \\
\text { NOTE : } \\
\text { a. INFORMATION } \\
\text { OF THE } \\
\text { LOCATION } \\
\text { b. TYPE OF } \\
\text { FACILITY }\end{array}$ & $\begin{array}{l}\text { INTERVI } \\
\text { EWED } \\
\text { STATUS }\end{array}$ \\
\hline & $\begin{array}{l}\text { Name: } \\
\text { Address: } \\
\text { Village : } \\
\text { 1. } \\
\text { 3. Same as EA } \\
\text { Kec: } \\
\text { Ka } \\
\text { Kab: } \\
1 . \\
\text { 3. Same as EA } \\
\text { Prov: } \\
\text { 1. } \\
\text { 3. Same as EA }\end{array}$ & $\begin{array}{l}\text { 4. PP } \\
\text { 3. PKK } \\
\text { 2. KD }\end{array}$ & 速 & $\begin{array}{l}\text { 3. Yes } \\
\sqcup \sqcup \\
\sqcup \\
\sqcup \sqcup \downarrow \\
\text { 3. No }\end{array}$ & $\begin{array}{l}\text { a. Village } \\
\frac{1 .}{\text { 3. Same }} \\
\text { 8. DK } \\
\text { b. Kecamatan } \\
11 . \\
\overline{\text { 13. Same }} \\
\text { 98. DK }\end{array}$ & 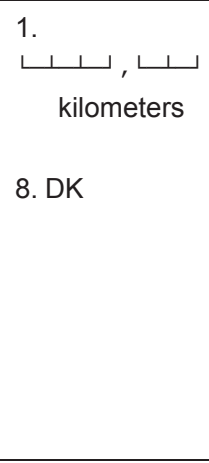 & 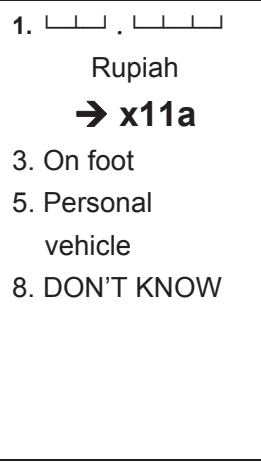 & $\begin{array}{l}\qquad \perp 1 \_ \\
\text {1. Minutes } \\
\text { 2. Hours } \\
\text { 3. Days } \\
\text { 8. TT } \\
\rightarrow \mathbf{x 1 2}\end{array}$ & $\begin{array}{l}\qquad \perp \perp \text { \ـ } \\
\text { 1. Minutes } \\
\text { 2. Hours } \\
\text { 3. Days } \\
\text { 8. TT }\end{array}$ & 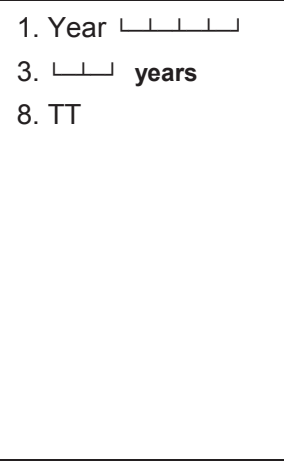 & 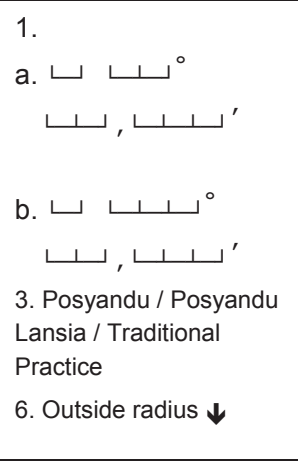 & a. & $\begin{array}{l}\text { 1. Yes } \\
\text { 3. No }\end{array}$ \\
\hline & $\begin{array}{l}\text { Name: } \\
\text { Address: } \\
\text { Village : } \\
\text { 1. } \\
\text { 3. Same as EA } \\
\text { Kec: } \\
\text { 1. } \\
\text { Kab: } \\
1 . \\
\text { 3. Same as EA } \\
\text { Prov: } \\
\text { 1. } \\
\text { 3. Same as EA }\end{array}$ & $\begin{array}{l}\text { 4. PP } \\
\text { 3. PKK } \\
\text { 2. KD }\end{array}$ & 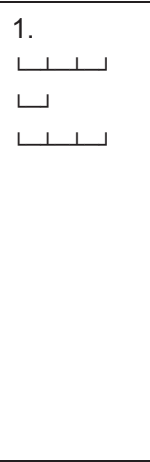 & $\begin{array}{l}\text { 4. Yes } \\
\sqcup \sqcup \\
\sqcup \\
\sqcup \sqcup \sqcup \downarrow\end{array}$ & $\begin{array}{l}\text { a. Village } \\
\frac{1 .}{\text { 3. Same }} \\
\text { 8. DK } \\
\text { b. Kecamatan } \\
11 . \\
\text { 13. Same } \\
\text { 98. DK }\end{array}$ & 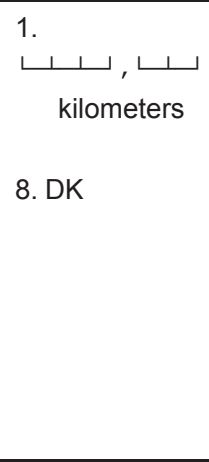 & 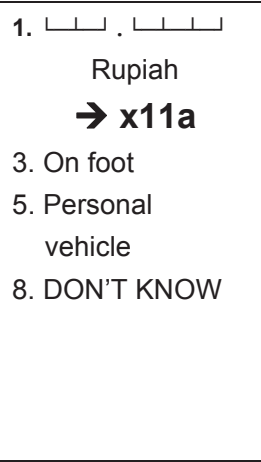 & $\begin{array}{l}\qquad \perp 1 \_ \\
\text {1. Minutes } \\
\text { 2. Hours } \\
\text { 3. Days } \\
\text { 8. TT } \\
\rightarrow \times 12\end{array}$ & $\begin{array}{l}\qquad \perp 1 \sqsubset \\
\text { 1. Minutes } \\
\text { 2. Hours } \\
\text { 3. Days } \\
\text { 8. TT }\end{array}$ & 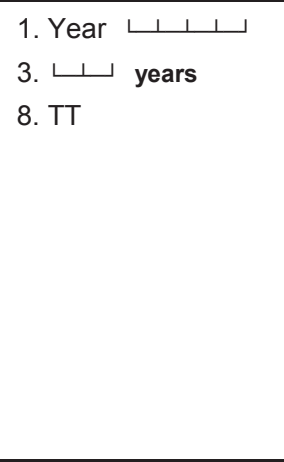 & $\begin{array}{l}\text { 1. } \\
\text { a. } \sqcup \sqcup^{\circ} \\
\text { b. } \sqcup, \sqcup \sim^{\prime} \\
\sqcup \sqcup, \sqcup \sim^{\prime} \\
\text { 3. Posyandu / } \\
\text { Posyandu Lansia / } \\
\text { Traditional Practice } \\
\text { 6. Outside radius } \downarrow\end{array}$ & a. & $\begin{array}{l}\text { 1. Yes } \\
\text { 3. No }\end{array}$ \\
\hline
\end{tabular}


INTERVIEWER NOTE: 
INDONESIA FAMILY LIFE SURVEY 2014

\section{DESA/KELURAHAN CHARACTERISTICS} FOR NON-IFLS VILLAGE

SECTION : LK, IR, S, A, B, C, D, I, J, F, E, SW, PAP, TR, W

VILLAGE CODE

NAME OF DESA/KELURAHAN :

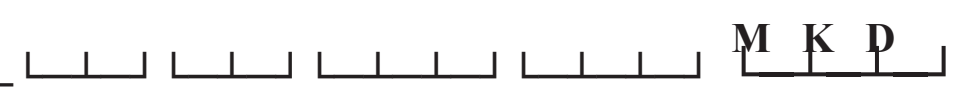


SECTION LK (CONTROL SHEET)

\begin{tabular}{|ll|c||}
\hline \multicolumn{1}{|c|}{ SAMPLING INFORMATION } & CODE \\
\hline LK01. $\quad$ Province & \\
\hline LK02. $\quad$ Kabupaten/Kota & \\
\hline LK03. Kecamatan & \\
\hline LK04. Village/Urban Township /Nagari & \\
\hline LK05. $\quad$ Region: 1. Urban 2. Rural & \\
\hline \hline
\end{tabular}

\begin{tabular}{|l|c|c|}
\hline \multicolumn{1}{|c|}{ SUPERVISION: } & CODE \\
\hline LK15. & Name of Interviewer : & \\
\hline LK16. $\quad$ Name of Local Supervisor : & \\
\hline LK17. $\quad$ Name of Field Coordinator : & \\
\hline
\end{tabular}

\section{VILLAGE/TOWNSHIP ADDRESS}

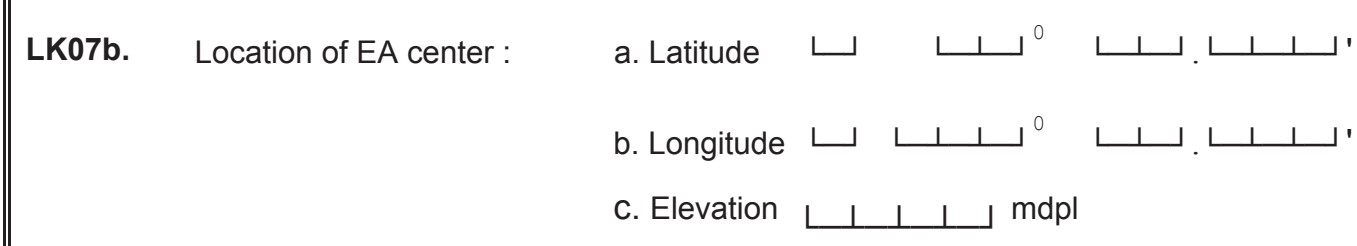

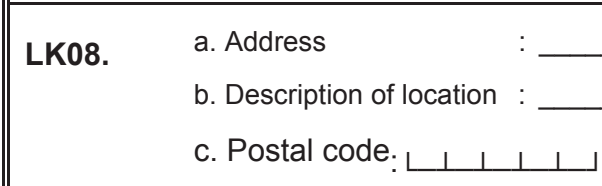

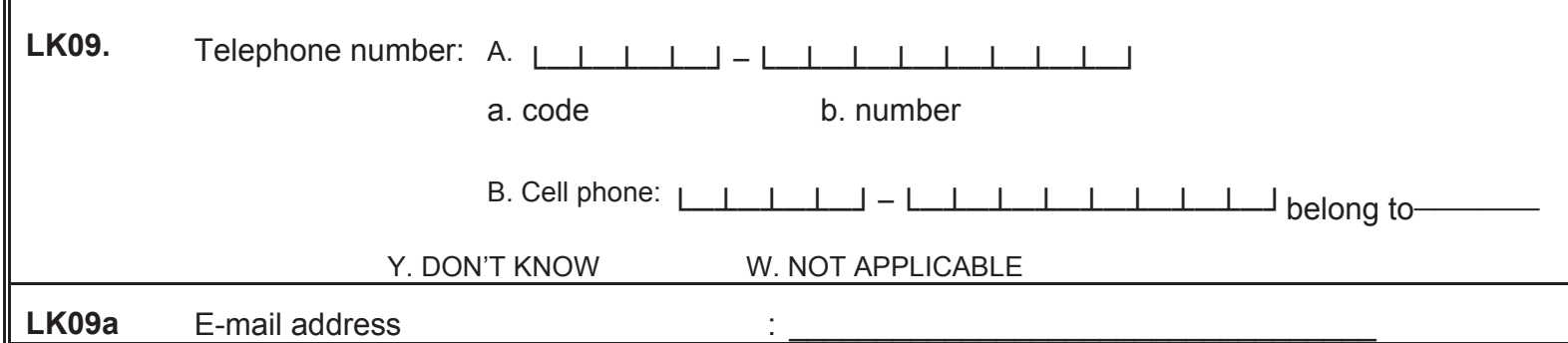


We would like to ask about the structure of the village office

\begin{tabular}{|c|c|c|c|}
\hline & & VILLAGE HEAD & VILLAGE SECRETARY \\
\hline & IR1. & IR2. & IR3. \\
\hline 1. & Name of Respondent : & & \\
\hline 1a. & Position in the village & $\begin{array}{lllll}01 & 02 & 03 & 04 & 05\end{array}$ & $\begin{array}{lllll}01 & 02 & 03 & 04 & 05\end{array}$ \\
\hline & & $07 \quad 95 \ldots \ldots$ & $07 \quad 95 \ldots \ldots$. \\
\hline 2. & Age & $\perp$ Years & $\perp$ Years \\
\hline 2a. & Length of tenure in positions: & 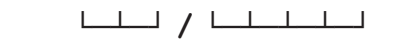 & 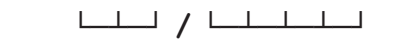 \\
\hline & & Month $\quad$ Year & Month Year \\
\hline 3. & $\begin{array}{l}\text { INTERVIEWER CHECK : } \\
\text { SEX OF RESPONDENT }\end{array}$ & 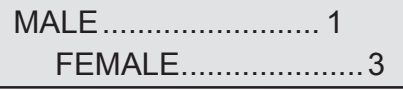 & 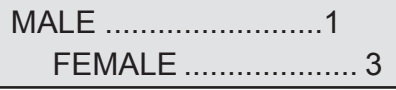 \\
\hline $3 a$. & $\begin{array}{l}\text { Highest level of education } \\
\text { attended: }\end{array}$ & لــ لــا & 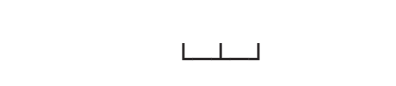 \\
\hline 4. & Highest class completed: & $\begin{array}{ccccc}00 & 01 & 02 & 03 & 04 \\
05 & & & 06 & 07\end{array}$ & $\begin{array}{ccccc}00 & 01 & 02 & 03 & 04 \\
05 & & & 06 & 07\end{array}$ \\
\hline 5. & $\begin{array}{l}\text { Length of time lived in the } \\
\text { village : }\end{array}$ & 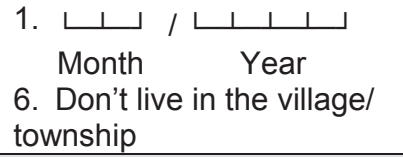 & 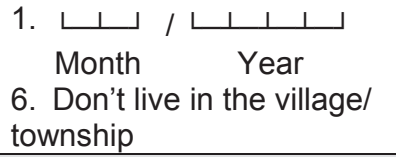 \\
\hline 6. & $\begin{array}{l}\text { How did }[\ldots] \text { come to } \\
\text { assume to the position }\end{array}$ & $\begin{array}{l}\text { 1. Elected } \\
\text { 2. Appointed by residence } \\
\text { 3. Appointed by camat } \\
\text { 4. Appointed by } \\
\text { Bupati/Walikota } \\
\text { 5. Other }\end{array}$ & $\begin{array}{l}\text { 1. Elected } \\
\text { 2. Appointed by residence } \\
\text { 3. Appointed by camat } \\
\text { 4. Appointed by } \\
\text { Bupati/Walikota } \\
\text { 5. Other }\end{array}$ \\
\hline
\end{tabular}

Code for position in village (1a):

01. Village/township heads

02. Village secretary

03. Head of Government Administration

05. Head of Welfare

06. Head of Financial Matters

07. Head of General Affairs

95. Other
11. Kejar Paket A (Adult Education A)

12. Kejar Paket $B$ (Adult Education $B$ )

01. No school/not yet in school

02. Elementary school

04. Junior High - Vocational

05. High School - General

06. High School - Vocational

60. D1, D2, D3 (Junior College)

61. University S1 (Bachelor)

62. University $\mathrm{S2}$ (Masters)

63. University S3 (Ph.D.)
13. Open Univeristy

14. Islamic School (Pondok Pensantren)
15. Kejar Paket C (Adult Education C)

17. School for the disabled

72. Islamic Elementary School (Madrasah Ibtidaiyah)

73. Islamic Junior High School (Madrasah Tsanawiyah)

74. Islamic Senior High School (Madrasah Alliyah)

98. DON'T KNOW

95. Other
Codes for highest grade (4):

00. Never completed class 1

01.

$\begin{array}{ll}02 . & 2 \\ 03 . & 3\end{array}$

04. 4

06. 6

07. Graduated

96. No school 


\section{SECTION S (POPULATION CHARACTERISTICS)}

We would like to ask about the population of this village.

\begin{tabular}{|c|c|c|}
\hline s31. & $\begin{array}{l}\text { What is the total population in this } \\
\text { village/township? }\end{array}$ & 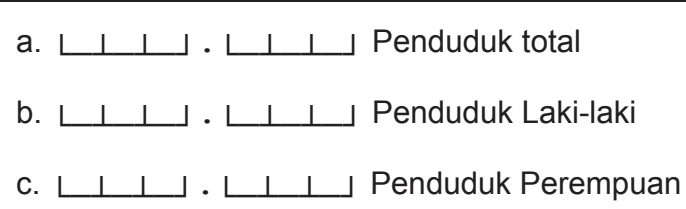 \\
\hline s1 & Size of village/township & $\sqcup \sqcup . \sqcup \perp \perp, \sqcup \perp$ hektar \\
\hline
\end{tabular}

\begin{tabular}{|c|c|c|c|c|c|c|c|c|c|}
\hline & \multicolumn{8}{|c|}{ S1A. What is ethnic composition in this Village/Township? } & $\%$ \\
\hline 1. & $\begin{array}{l}\text { Largest ethnic } \\
\text { group : }\end{array}$ & $\begin{array}{c}A \text { B } \\
\text { I } \\
\text { Q } \\
\text { D1 }\end{array}$ & $\begin{array}{l}\mathrm{C} \\
\mathrm{J} \\
\mathrm{R} \\
\mathrm{E} 1\end{array}$ & $\begin{array}{l}D \\
K \\
S \\
\quad F 1\end{array}$ & $\begin{array}{l}\text { E } \\
L \\
T\end{array}$ & $\begin{array}{r}F \\
M \\
U \\
G 1\end{array}$ & $\begin{array}{l}\mathrm{G} \\
\mathrm{N} \\
\mathrm{A} 1 \\
\mathrm{~V}\end{array}$ & $\begin{array}{l}\mathrm{H} \\
\mathrm{O} \\
\mathrm{B} 1\end{array}$ & 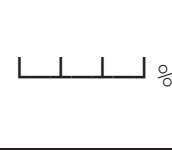 \\
\hline 2. & $\begin{array}{l}\text { Second largest } \\
\text { ethnic group: }\end{array}$ & $\begin{array}{c}\mathrm{A} \text { B } \\
\mathrm{I} \\
\mathrm{Q} \\
\mathrm{D} 1 \\
\end{array}$ & $\begin{array}{l}\mathrm{C} \\
\mathrm{J} \\
\mathrm{R} \\
\mathrm{E} 1\end{array}$ & $\begin{array}{l}\mathrm{D} \\
\mathrm{K} \\
\mathrm{S} \\
\mathrm{F} 1 \\
\end{array}$ & $\begin{array}{l}\text { E } \\
L \\
T\end{array}$ & $\begin{array}{r}F \\
M \\
U \\
G 1 \\
\end{array}$ & $\begin{array}{l}\mathrm{G} \\
\mathrm{N} \\
\mathrm{A} 1 \\
\mathrm{~V}\end{array}$ & $\begin{array}{l}\mathrm{H} \\
\mathrm{O} \\
\mathrm{B} 1\end{array}$ & 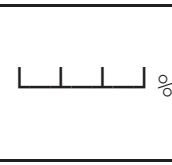 \\
\hline 3 & $\begin{array}{l}\text { Third largest } \\
\text { ethnic group: }\end{array}$ & $\begin{array}{c}\text { A } B \\
\text { I } \\
\text { Q } \\
\text { D1 }\end{array}$ & $\begin{array}{l}\mathrm{C} \\
\mathrm{J} \\
\mathrm{R} \\
\mathrm{E} 1\end{array}$ & $\begin{array}{l}\mathrm{D} \\
\mathrm{K} \\
\mathrm{S} \\
\mathrm{F} 1 \\
\end{array}$ & $\begin{array}{l}\text { E } \\
\text { L } \\
T\end{array}$ & $\begin{array}{r}F \\
M \\
U \\
G 1\end{array}$ & $\begin{array}{l}\mathrm{G} \\
\mathrm{N} \\
\mathrm{A} 1 \\
\mathrm{~V}\end{array}$ & $\begin{array}{l}\mathrm{H} \\
\mathrm{O} \\
\mathrm{B} 1\end{array}$ & 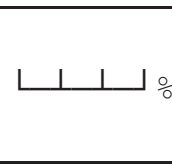 \\
\hline
\end{tabular}

\begin{tabular}{|c|c|c|}
\hline S2a & $\begin{array}{l}\text { What is the religious composition of the } \\
\text { villagers in this village/kelurahan? What are } \\
\text { the percentages? }\end{array}$ & 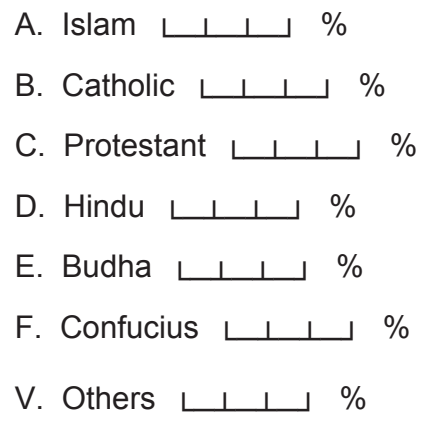 \\
\hline
\end{tabular}

\begin{tabular}{|c|c|c|}
\hline \multicolumn{3}{|l|}{ KODE A12 } \\
\hline & Bima-Dompu . & Ambon.... \\
\hline Sunda & Makassar....... & Manado.. \\
\hline 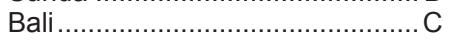 & Nias ................... & Aceh ............................ \\
\hline & 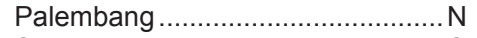 & Sumatera Sel. lainnya .....C1 \\
\hline Bugis ........ & 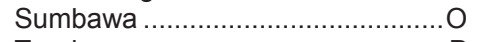 & Banten ..........................D1 \\
\hline Tionghoa. & Toraja ............. & Cirebon .................... \\
\hline Madura......... & Betawi & Gorontalo.........................F1 \\
\hline Sasak......... & Dayak & Kutai .............................. G1 \\
\hline Minang ...................... & Melayu................. & Lainnya................................ V \\
\hline Baniar & Komering ............ & \\
\hline
\end{tabular}


SECTION S (POPULATION CHARACTERISTICS)

\begin{tabular}{|c|c|c|c|c|}
\hline & \multirow[b]{2}{*}{ SECTORS : } & \multicolumn{2}{|c|}{ S39. } & S40. \\
\hline & & \multicolumn{2}{|c|}{$\begin{array}{l}\text { What are the enterprises of } \\
\text { the village/township } \\
\text { population? }\end{array}$} & $\begin{array}{l}\text { Which are the three most } \\
\text { predominant sources of } \\
\text { income for the } \\
\text { village/township population? } \\
\text { (ONLY THREE ANSWER) }\end{array}$ \\
\hline 01. & $\begin{array}{l}\text { Farming (including forestry, } \\
\text { hunting and fishing) }\end{array}$ & 1. Yes & 3. No & \multirow{10}{*}{ a. First $\llcorner\perp$} \\
\hline 02. & Mining (including excavating) & 1. Yes & 3. No & \\
\hline 03. & Manufacturing industry & 1. Yes & 3. No & \\
\hline 04. & Electricity, gas and water & 1. Yes & 3. No & \\
\hline 05. & Building construction & 1. Yes & 3. No & \\
\hline 06. & $\begin{array}{l}\text { Large trade, retail trade, } \\
\text { restaurants and hotels }\end{array}$ & 1. Yes & 3. No & \\
\hline 07. & $\begin{array}{l}\text { Transportation, warehousing } \\
\text { and communications }\end{array}$ & 1. Yes & 3. No & \\
\hline 08. & $\begin{array}{l}\text { Finance, insurance, lease of } \\
\text { buildings, grounds and } \\
\text { business services }\end{array}$ & 1. Yes & 3. No & \\
\hline 09. & Social services & 1. Yes & 3. No & \\
\hline & Other & 1. Yes & 3. No & \\
\hline
\end{tabular}




\section{SECTION A (TRANSPORTATION ANDINFRASTRUCTURE)}

A8. What is the predominant type of road in this village?

A8a. Can the main road in the village be traveled by a motorized vehicle?

A.9 In this last year, how many months was the main road/water way able to be traveled by motor vehicle?

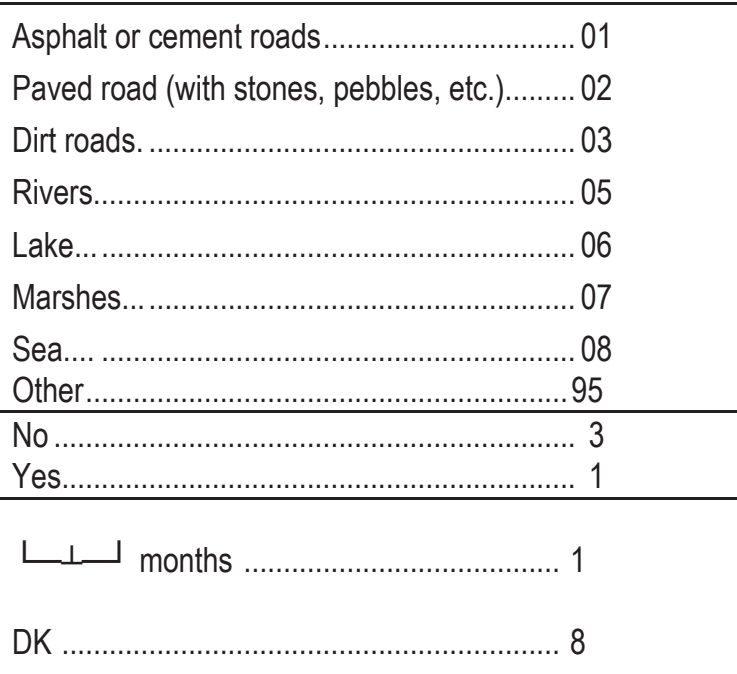

\section{SEKSI B (ELECTRICITY AND INTERNET)}

\begin{tabular}{|c|c|c|}
\hline B1. & $\begin{array}{l}\text { Is there any electricity available in this } \\
\text { village? }\end{array}$ & $\begin{array}{l}\text { No } \\
\text { Yes }\end{array}$ \\
\hline B1a. & $\begin{array}{l}\text { What percent of the households in this } \\
\text { village use electricity? } \\
\text { । }\end{array}$ & 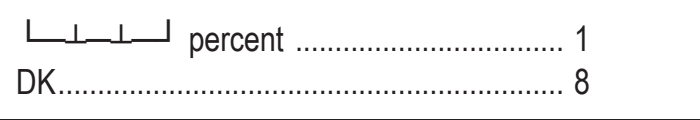 \\
\hline B2a & $\begin{array}{l}\text { Is internet access available in this village } \\
\text { office? }\end{array}$ & 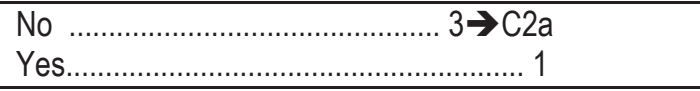 \\
\hline B2b & $\begin{array}{l}\text { In what year did this village office first } \\
\text { have internet access? }\end{array}$ & 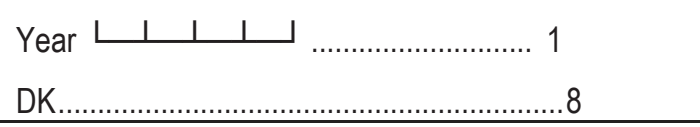 \\
\hline B2C & $\begin{array}{l}\text { How is this village office connected to } \\
\text { the internet? }\end{array}$ & 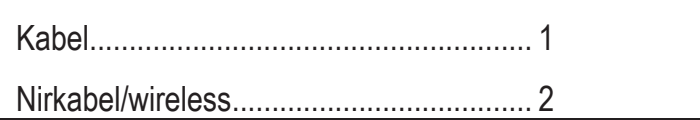 \\
\hline B2d & $\begin{array}{l}\text { In this village office, what do you usually use } \\
\text { the internet access for? }\end{array}$ & $\begin{array}{l}\text { A. Sending and receiving emails } \\
\text { B. Sending and receiving documents/attachments } \\
\text { C. Browsing/searching (e.g. news portal, Google) } \\
\text { D. E-government } \\
\text { E. Video/streaming (e.g. Youtube) } \\
\text { V. Other }\end{array}$ \\
\hline
\end{tabular}

\section{SECTION C (WATER AND SANITATION)}

C2a. Where does the village population get water for drinking and cooking? a. PAM piped water directly to the house D. PAM piped water from a public hydrant/stand pipe c. PAM piped water purchased from a vendor Pump water (electrical/manual)

Spring water

Rain water

River wate

(purchased)

(non-PAM)

8a. Other, drinking and cooking water

\section{SECTION D (MAIN CROPS, FARM AND NON-FARM WAGES)}

\begin{tabular}{|c|c|c|}
\hline D2. & $\begin{array}{l}\text { Mention three main crops cultivated in } \\
\text { this village in the last } 12 \text { months? }\end{array}$ & 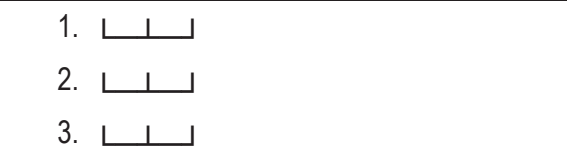 \\
\hline D4. & $\begin{array}{l}\text { How many times was paddy harvested in } \\
\text { this village in the last } 12 \text { months? }\end{array}$ & $\sqcup$ ـ times \\
\hline D19A & $\begin{array}{l}\text { What are the average daily wages for adult } \\
\text { male farm laborers for harvesting? }\end{array}$ & $\begin{array}{l}\text { 1. } \longleftarrow \perp \perp \_,\left\llcorner\perp \perp \_ \text {Rp. }\right. \\
\text { 6. NA } \\
\text { 8. DK }\end{array}$ \\
\hline D19B & $\begin{array}{l}\text { What are the average daily wages for adult } \\
\text { female farm laborers for harvesting? }\end{array}$ & 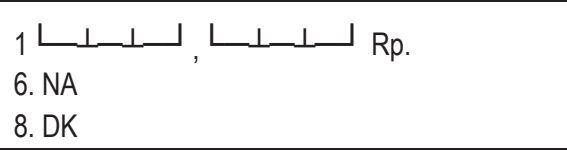 \\
\hline D20a & $\begin{array}{l}\text { What was the average yield per hectare of } \\
\text { rice in irrigated farm in this village in the } \\
\text { last harvest? }\end{array}$ & 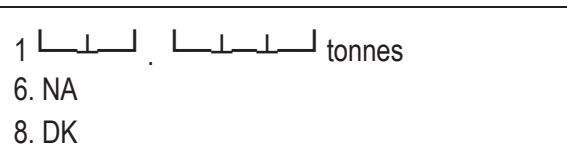 \\
\hline D28. & $\begin{array}{l}\text { Is there a factory (plant) in this village } \\
\text { including cottage/home industry? }\end{array}$ & 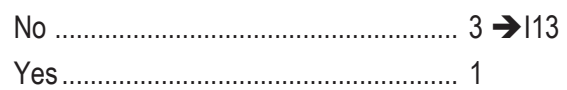 \\
\hline
\end{tabular}

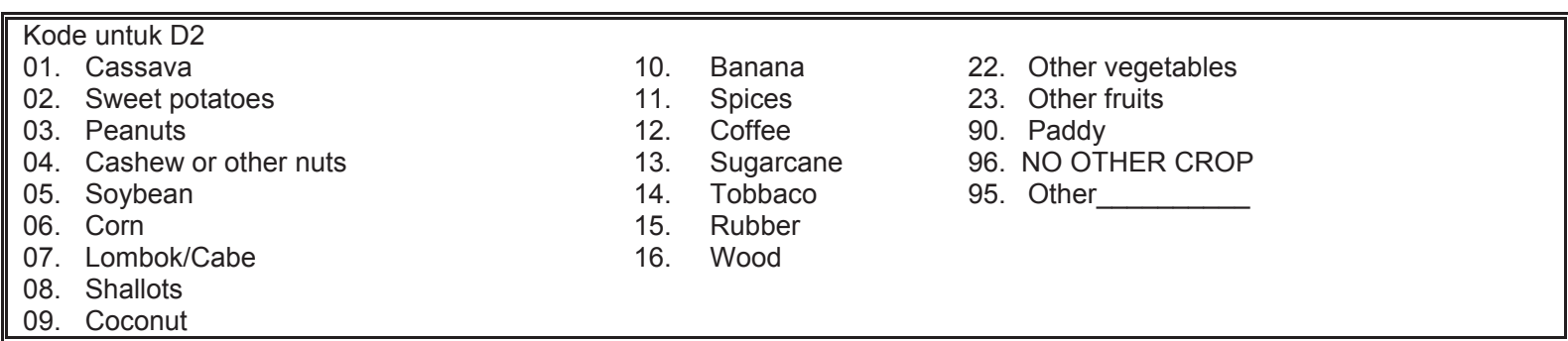




\section{D31a.}

D31b.

\begin{tabular}{|l|l} 
What are the daily wages for adult male non-farm laborers? & What are the daily wages for adult female non-farm
\end{tabular}

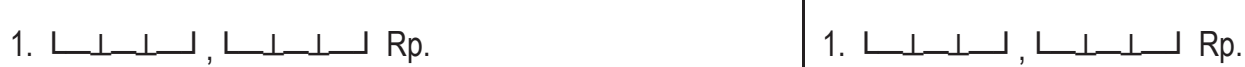

\begin{tabular}{l|l|} 
6. NA & 6. NA \\
\hline
\end{tabular}

\begin{tabular}{l|l|l} 
8. DK & 6. DK \\
\hline
\end{tabular} 


\begin{tabular}{|c|c|c|c|c|c|}
\hline & & Pre-elementary schools & SD & SMP & SMA \\
\hline I13. & $\begin{array}{l}\text { With regards to the }[\ldots] \text { available to the } \\
\text { community in this village/township, how } \\
\text { many [...] can be used by the people from } \\
\text { this village/township? } \\
\text { INCLUDING THOSE LOCATED OUTSIDE } \\
\text { THE VILLAGE }\end{array}$ & $\begin{array}{l}\text { 1. } ـ \perp \text { schools } \\
\text { 3. NONE } \\
\text { 8. DK }\end{array}$ & $\begin{array}{l}\text { 1. ـــ schools } \\
\text { 3. NONE } \\
\text { 8. DK }\end{array}$ & $\begin{array}{l}\text { 1. ـــ schools } \\
\text { 3. NONE } \\
\text { 8. DK }\end{array}$ & $\begin{array}{l}\text { 1. } \longleftarrow \perp \_ \text {schools } \\
\text { 3. NONE } \\
\text { 8. DK }\end{array}$ \\
\hline
\end{tabular}

\section{SECTION J (HEALTH FACILITY)}

\begin{tabular}{|c|c|c|}
\hline $\mathrm{J} 10 \mathrm{a}$ & $\begin{array}{l}\text { Is there a Village Midwife (Bidan Desa) located in this } \\
\text { village? }\end{array}$ & 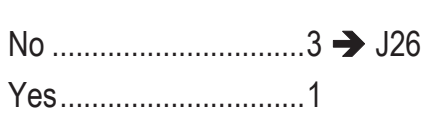 \\
\hline J10b. & How many Village Midwives are located in this village? & $\begin{array}{l}\text { 1. Lــ modwives } \\
\text { 8. DK }\end{array}$ \\
\hline
\end{tabular}

\begin{tabular}{|c|c|c|c|c|c|c|c|}
\hline & (JTYPE) & $\begin{array}{l}\text { Aa.Integrated Community } \\
\text { Health Post } \\
\text { (Posyandu) }\end{array}$ & $\begin{array}{l}\text { Ab.Integrated Community } \\
\text { Health Post for the } \\
\text { elderly (Posyandu } \\
\text { Lansia) }\end{array}$ & $\begin{array}{l}\text { A. Health Centers } \\
\text { (Puskesmas) and } \\
\text { Health Sub-centers } \\
\text { (Puskesmas } \\
\text { Pembantu/Pustu) }\end{array}$ & $\begin{array}{l}\text { B. Private health } \\
\text { practitioner such as } \\
\text { doctors, clinics, } \\
\text { medical posts, } \\
\text { maternity clinics, } \\
\text { nurses, midwives, } \\
\text { medical assistants, } \\
\text { and other paramedical } \\
\text { workers } \\
\end{array}$ & $\begin{array}{l}\text { C. Traditional health } \\
\text { workers, such as: } \\
\text { midwives, Chinese } \\
\text { healer (sinshe), } \\
\text { shaman, etc. }\end{array}$ & $\begin{array}{l}\text { D. Public or private } \\
\text { hospital }\end{array}$ \\
\hline J26. & $\begin{array}{l}\text { With regards to the }[\ldots . .] \text { available to the } \\
\text { community in this village/township, how } \\
\text { many [...] can be used by the people } \\
\text { from this village/township? } \\
\text { INCLUDING THOSE LOCATED OUTSIDE } \\
\text { THE VILLAGE }\end{array}$ & $\begin{array}{l}\text { 1. } \longleftarrow \perp \_ \text {facilities } \\
\text { 3. NONE } \\
\text { 8. DK }\end{array}$ & 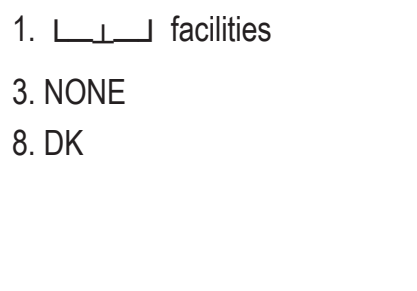 & 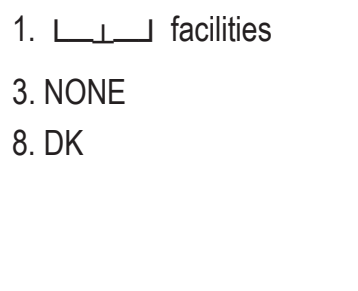 & $\begin{array}{l}\text { 1. } \longleftarrow \perp \_ \text {facilities } \\
\text { 3. NONE } \\
\text { 8. DK }\end{array}$ & $\begin{array}{l}\text { 1. } \longleftarrow \perp \_ \text {facilities } \\
\text { 3. NONE } \\
\text { 8. DK }\end{array}$ & $\begin{array}{l}\text { 1. } \longleftarrow \perp \_ \text {facilities } \\
\text { 3. NONE } \\
\text { 8. DK }\end{array}$ \\
\hline
\end{tabular}




\section{SECTION F (NATURAL DISASTERS)}

\begin{tabular}{|c|c|c|c|c|c|c|}
\hline \multirow{2}{*}{\multicolumn{2}{|c|}{ (F TYPE) }} & \multicolumn{2}{|c|}{ F01 } & F02 & F03 & F03a \\
\hline & & \multicolumn{2}{|c|}{$\begin{array}{l}\text { Were there any } \\
\text { natural } \\
\text { disasters[...] in } \\
\text { this village in the } \\
\text { last } 5 \text { years? }\end{array}$} & $\begin{array}{l}\text { How many } \\
\text { times has [...] } \\
\text { disaster } \\
\text { occurred in } \\
\text { this village? }\end{array}$ & $\begin{array}{l}\text { When was the most } \\
\text { severe }[\ldots] \text { disaster } \\
\text { occurred? }\end{array}$ & $\begin{array}{l}\text { How many casualties } \\
\text { were resulted from the } \\
\text { most severe disaster? }\end{array}$ \\
\hline A & Flood & 3. No & 1. Yes & ــ times & $\underset{\text { Month / }}{\left\llcorner \_ \text {Year }\right.}$ & 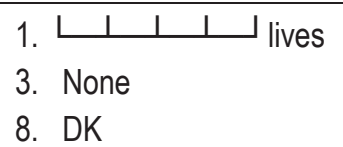 \\
\hline B & Earthquake & $\begin{array}{l}\text { 3. No } \\
\downarrow\end{array}$ & 1. Yes & $\begin{array}{l}\llcorner\perp \dashv \\
\text { times }\end{array}$ & 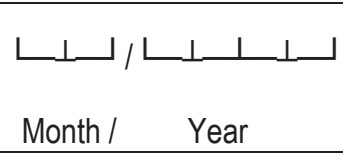 & $\begin{array}{l}\text { 1. } \downarrow \perp \perp \perp \vdash \text { lives } \\
\text { 3. None } \\
\text { 8. DK }\end{array}$ \\
\hline C & Landslide & $\begin{array}{l}\text { 3. No } \\
\downarrow\end{array}$ & 1. Yes & $\begin{array}{l}\llcorner\perp \dashv \\
\text { times }\end{array}$ & 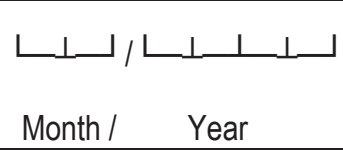 & $\begin{array}{l}\text { 1. } \downarrow \perp|\perp| \downarrow|l| l|l| l|l| l \\
\text { 3. None } \\
\text { 8. DK }\end{array}$ \\
\hline & $\begin{array}{l}\text { Volcano } \\
\text { Eruption }\end{array}$ & $\begin{array}{l}\text { 3. No } \\
\downarrow\end{array}$ & 1. Yes & $\begin{array}{l}\llcorner\perp \dashv \\
\text { times }\end{array}$ & 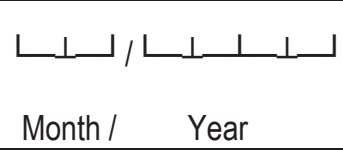 & $\begin{array}{l}\text { 1. } \downarrow \perp|\perp| \downarrow|l| l|l| l|l| l \\
\text { 3. None } \\
\text { 8. DK }\end{array}$ \\
\hline $\mathrm{E}$ & Tsunami & $\begin{array}{l}\text { 3. No } \\
\downarrow\end{array}$ & 1. Yes & $\begin{array}{l}\llcorner\perp \dashv \\
\text { times }\end{array}$ & 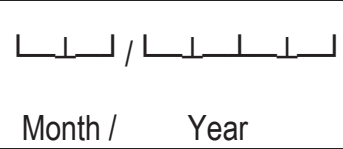 & $\begin{array}{l}\text { 1. } \downarrow \perp|\perp| \downarrow|l| l|l| l|l| l \\
\text { 3. None } \\
\text { 8. DK }\end{array}$ \\
\hline $\mathrm{F}$ & Drought & $\begin{array}{l}\text { 3. No } \\
\downarrow\end{array}$ & 1. Yes & $\begin{array}{l}\llcorner\perp \dashv \\
\text { times }\end{array}$ & 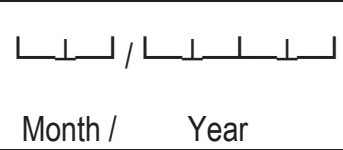 & $\begin{array}{l}\text { 1. } \downarrow \perp|\perp| \downarrow|l| l \text { lives } \\
\text { 3. None } \\
\text { 8. DK }\end{array}$ \\
\hline G & Forest fire & $\begin{array}{l}\text { 3. No } \\
\downarrow\end{array}$ & 1. Yes & $\begin{array}{l}\llcorner\perp \dashv \\
\text { times }\end{array}$ & 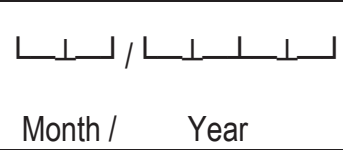 & $\begin{array}{l}\text { 1. } \downarrow \perp|\perp| \downarrow|l| l \text { lives } \\
\text { 3. None } \\
\text { 8. DK }\end{array}$ \\
\hline $\mathrm{H}$ & Fire & $\begin{array}{l}\text { 3. No } \\
\downarrow\end{array}$ & 1. Yes & $\begin{array}{l}\llcorner\perp \dashv \\
\text { times }\end{array}$ & 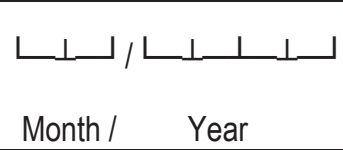 & $\begin{array}{l}\text { 1. } \downarrow|\perp| \perp \mid l \text { lives } \\
\text { 3. None } \\
\text { 8. DK }\end{array}$ \\
\hline
\end{tabular}

\begin{tabular}{|c|c|c|}
\hline E15a. & $\begin{array}{l}\text { In the past } 5 \text { years, did this village ever } \\
\text { experienced any smoke clouds (from } \\
\text { forest fire)? }\end{array}$ & 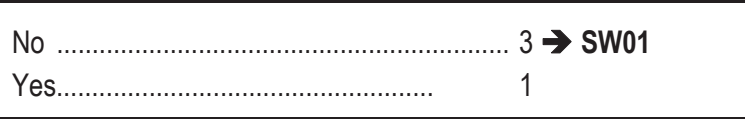 \\
\hline E16a. & $\begin{array}{l}\text { When did this village experienced } \\
\text { smoke clouds for the first time? }\end{array}$ & 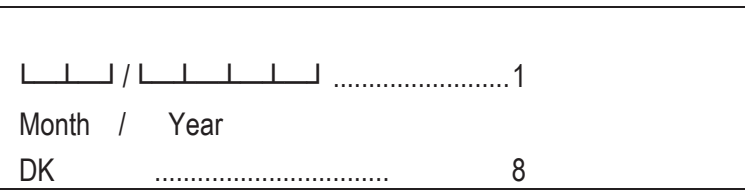 \\
\hline E17a. & $\begin{array}{l}\text { When did this village experienced } \\
\text { smoke clouds for the last time? }\end{array}$ & 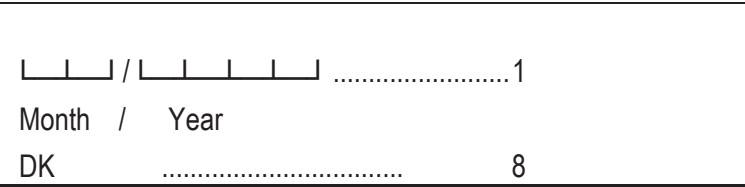 \\
\hline E26. & $\begin{array}{l}\text { Approximately how far in front of you } \\
\text { could you see clearly at that time (In the } \\
\text { past } 5 \text { years)? }\end{array}$ & 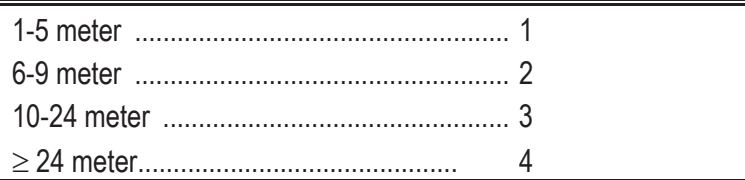 \\
\hline E26a. & $\begin{array}{l}\text { Approximately how long does the } \\
\text { smoke last before it stopped } \\
\text { completely? }\end{array}$ & $\perp$ 1. days 2. weeks 3. months \\
\hline E27. & $\begin{array}{l}\text { At the time when the smoke clouds } \\
\text { were at their worst in the last } 6 \text { months, } \\
\text { how were the normal daily activities of } \\
\text { residents of your community affected? } \\
\text { (CIRCLE ALL THAT APPLY) }\end{array}$ & 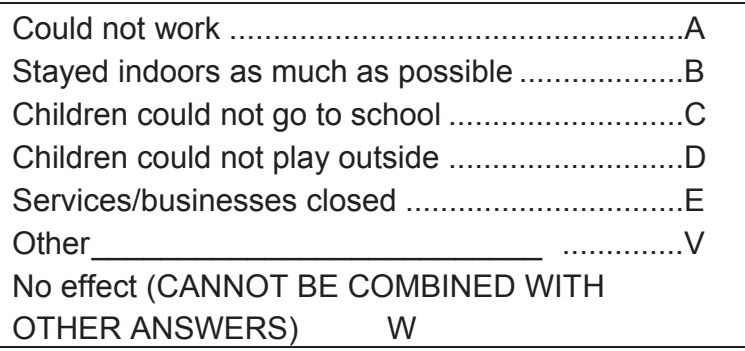 \\
\hline E28. & $\begin{array}{l}\text { At the time when the smoke clouds } \\
\text { were at their worst (in the last } 6 \\
\text { months), did residents in your } \\
\text { community have to wear masks? }\end{array}$ & 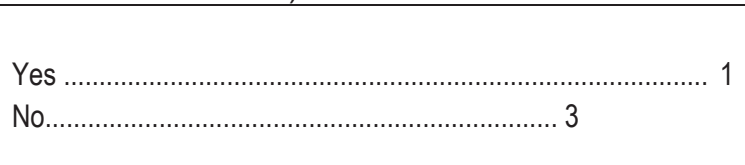 \\
\hline
\end{tabular}

\section{SEKSI SW (KESEJAHTERAAN)}

SW01. If you consider a scale of 1 to 6 , where 1 represents the village where the population is poorest, and 6 represents the the village where the population is richest, what is the number that best represents your village, considering the current conditions of people in your village?

\begin{tabular}{lrrrrrr}
\multicolumn{2}{l}{ Poorest } & & & & Richest \\
1 & 2 & 3 & 4 & 5 & 6 \\
8. & DK & & & &
\end{tabular}


SECTION PAP (POVERTY ALLEVIATION PROGRAM)

\begin{tabular}{|c|c|c|c|c|c|c|c|c|}
\hline \multirow{2}{*}{\multicolumn{2}{|c|}{$\begin{array}{r}\text { PROGRAM } \\
\text { (PAP1TYPE) }\end{array}$}} & PAP7. & PAP7a & PAP7c. & PAP7d. & PAP8 & PAP14 & PAP17a \\
\hline & & $\begin{array}{l}\text { Was there } \\
\text { [...] program } \\
\text { in this village } \\
\text { since 2007? }\end{array}$ & $\begin{array}{l}\text { When was the first time the } \\
\text { program begin? }\end{array}$ & $\begin{array}{l}\text { Is the program still } \\
\text { running? }\end{array}$ & When did the program stop? & $\begin{array}{l}\text { What percent of } \\
\text { households were } \\
\text { recipients/participants of } \\
\text { the program? }\end{array}$ & $\begin{array}{l}\text { Before the [...] program was } \\
\text { implemented, has there been } \\
\text { information campaign of the } \\
\text { program to the community in this } \\
\text { village? }\end{array}$ & $\begin{array}{l}\text { Apakah ada keluhan / protes dari masyarakat } \\
\text { sehubungan dengan pemilihan mereka yang } \\
\text { memenuhi syarat untuk menerima }[\ldots . .] \text { ? }\end{array}$ \\
\hline 1 & Jamkesda & $\begin{array}{l}\text { 1. Yes } \\
\text { 3. No } \downarrow\end{array}$ & 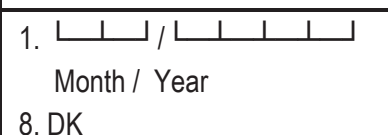 & $\begin{array}{l}\text { 1. Yes } \rightarrow \text { PAP8 } \\
\text { 3. No } \\
\text { 8. DK }\end{array}$ & 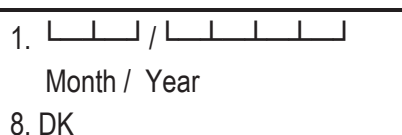 & 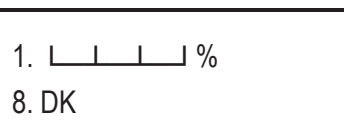 & $\begin{array}{ll}\text { 1. } & \text { Yes } \\
\text { 3. } & \text { No }\end{array}$ & $\begin{array}{ll}\text { 1. } & \text { Yes } \\
\text { 3. } & \text { No }\end{array}$ \\
\hline 2. & Jamkesmas & $\begin{array}{l}\text { 1. Yes } \\
\text { 3. No } \downarrow\end{array}$ & 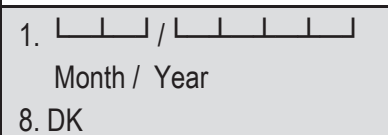 & $\begin{array}{l}\text { 1. Yes } \rightarrow \text { PAP8 } \\
\text { 3. No } \\
\text { 8. DK }\end{array}$ & 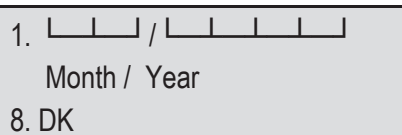 & 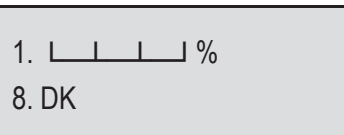 & $\begin{array}{ll}\text { 1. } & \text { Yes } \\
\text { 3. } & \text { No }\end{array}$ & $\begin{array}{ll}\text { 1. } & \text { Yes } \\
\text { 3. } & \text { No }\end{array}$ \\
\hline $2 a$ & Jampersal & $\begin{array}{l}\text { 1. Yes } \\
\text { 3. No } \downarrow\end{array}$ & 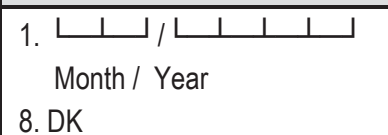 & $\begin{array}{l}\text { 1. Yes } \rightarrow \text { PAP8 } \\
\text { 3. No } \\
\text { 8. DK }\end{array}$ & 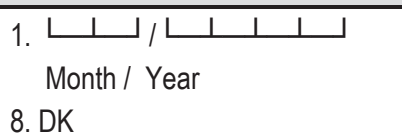 & 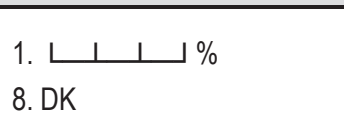 & $\begin{array}{ll}\text { 1. } & \text { Ya } \\
\text { 3. } & \text { Tidak }\end{array}$ & $\begin{array}{ll}\text { 1. } & \text { Ya } \\
\text { 3. } & \text { Tidak }\end{array}$ \\
\hline $2 b$ & $\begin{array}{l}\text { JKN (Jaminan Kesehatan } \\
\text { Nasional) }\end{array}$ & $\begin{array}{l}\text { 1. Yes } \\
\text { 3. No } \downarrow\end{array}$ & 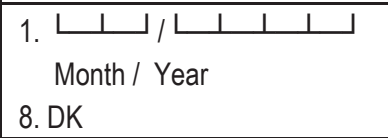 & $\begin{array}{l}\text { 1. Yes } \rightarrow \text { PAP8 } \\
\text { 3. No } \\
\text { 8. DK }\end{array}$ & $\begin{array}{l}\text { 1. } \begin{array}{l}\text { Month / Year } \\
\text { 8. DK }\end{array} \\
\text { DK }\end{array}$ & 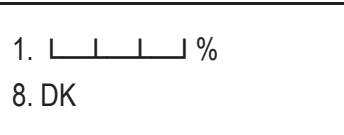 & $\begin{array}{ll}\text { 1. } & \text { Ya } \\
\text { 3. } & \text { Tidak }\end{array}$ & $\begin{array}{ll}\text { 1. } & \text { Ya } \\
\text { 3. } & \text { Tidak }\end{array}$ \\
\hline 3. & Raskin & $\begin{array}{l}\text { 1. Yes } \\
\text { 3. No } \downarrow\end{array}$ & 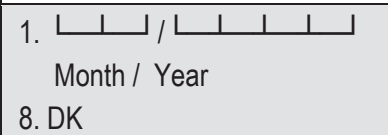 & $\begin{array}{l}\text { 1. Yes } \rightarrow \text { PAP8 } \\
\text { 3. No } \\
\text { 8. DK }\end{array}$ & 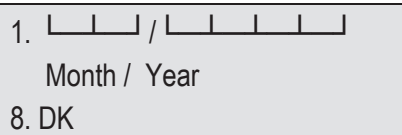 & 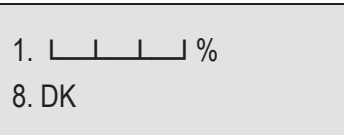 & $\begin{array}{ll}\text { 1. } & \text { Ya } \\
\text { 3. } & \text { Tidak }\end{array}$ & $\begin{array}{ll}\text { 1. } & \text { Ya } \\
\text { 3. } & \text { Tidak }\end{array}$ \\
\hline 4. & Operasi Pasar Beras & $\begin{array}{l}\text { 1. Yes } \\
\text { 3. No } \downarrow\end{array}$ & $\begin{array}{l}\text { 1. } \begin{array}{l}\text { Month / Year } \\
\text { 8. DK }\end{array} \\
\text { 8. }\end{array}$ & $\begin{array}{l}\text { 1. Yes } \rightarrow \text { PAP8 } \\
\text { 3. No } \\
\text { 8. DK }\end{array}$ & 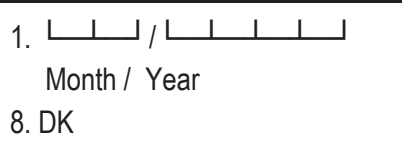 & & & \\
\hline 5. & $\begin{array}{l}\text { Operasi Pasar untuk } \\
\text { Minyak Tanah, Gula, atau } \\
\text { Minyak Goreng }\end{array}$ & $\begin{array}{l}\text { 1. Yes } \\
\text { 3. No } \downarrow\end{array}$ & 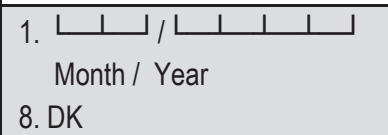 & $\begin{array}{l}\text { 1. Yes } \rightarrow \text { PAP8 } \\
\text { 3. No } \\
\text { 8. DK }\end{array}$ & 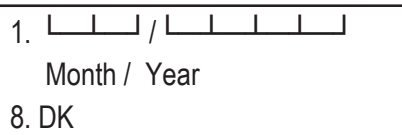 & & & \\
\hline 6. & $\begin{array}{l}\text { PKPS BBM-BLT (Bantuan } \\
\text { Langsung Tunai) }\end{array}$ & $\begin{array}{l}\text { 1. Yes } \\
\text { 3. No } \downarrow\end{array}$ & 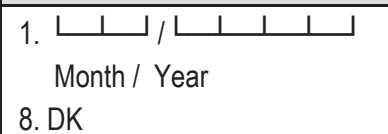 & $\begin{array}{l}\text { 1. Yes } \rightarrow \text { PAP8 } \\
\text { 3. No } \\
\text { 8. DK }\end{array}$ & 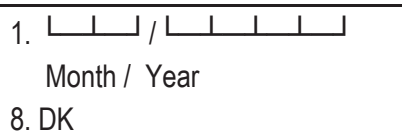 & 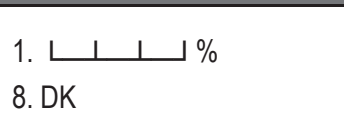 & $\begin{array}{ll}\text { 1. } & \text { Yes } \\
\text { 3. No } & \text { No }\end{array}$ & $\begin{array}{ll}\text { 1. } & \text { Yes } \\
\text { 3. } & \text { No }\end{array}$ \\
\hline 7. & $\begin{array}{l}\text { Program Keluarga } \\
\text { Harapan }\end{array}$ & $\begin{array}{l}\text { 1. Yes } \\
\text { 3. No } \downarrow\end{array}$ & 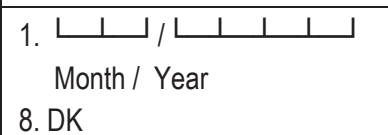 & $\begin{array}{l}\text { 1. Yes } \rightarrow \text { PAP8 } \\
\text { 3. No } \\
\text { 8. DK }\end{array}$ & 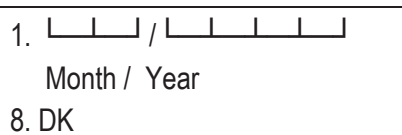 & 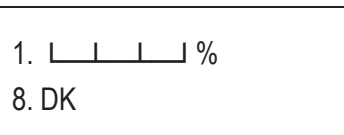 & $\begin{array}{ll}\text { 1. } & \text { Yes } \\
\text { 3. } & \text { No }\end{array}$ & $\begin{array}{ll}\text { 1. } & \text { Yes } \\
\text { 3. } & \text { No }\end{array}$ \\
\hline 8 & PNPM MANDIRI & $\begin{array}{l}\text { 1. Yes } \\
\text { 3. No } \downarrow\end{array}$ & 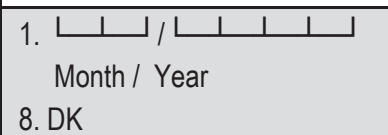 & $\begin{array}{l}\text { 1. Yes } \rightarrow \text { PAP8 } \\
\text { 3. No } \\
\text { 8. DK }\end{array}$ & 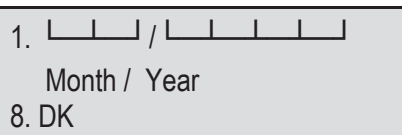 & 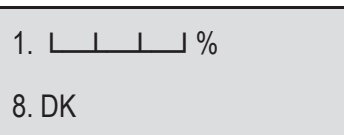 & $\begin{array}{ll}\text { 1. } & \text { Yes } \\
\text { 3. } & \text { No }\end{array}$ & $\begin{array}{ll}\text { 1. } & \text { Yes } \\
\text { 3. } & \text { No }\end{array}$ \\
\hline 9 & $\begin{array}{l}\text { Bantuan Langsung } \\
\text { Sementara Masyarakat ( } \\
\text { BLSM ) }\end{array}$ & $\begin{array}{l}\text { 1. Yes } \\
\text { 3. No } \downarrow\end{array}$ & 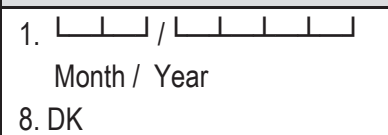 & $\begin{array}{l}\text { 1. Yes } \rightarrow \text { PAP8 } \\
\text { 3. No } \\
\text { 8. DK }\end{array}$ & 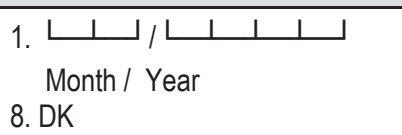 & 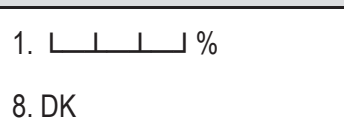 & $\begin{array}{ll}\text { 1. } & \text { Yes } \\
\text { 3. } & \text { No }\end{array}$ & $\begin{array}{ll}\text { 1. } & \text { Yes } \\
\text { 3. No }\end{array}$ \\
\hline
\end{tabular}




\begin{tabular}{|c|c|c|c|c|c|c|c|c|c|}
\hline & PROGRAM & PAP7. & & $\begin{array}{l}\text { PAP7a } \\
\end{array}$ & PAP7C. & PAP7d. & PAP8 & PAP14 & PAP17a \\
\hline 10 & $\begin{array}{l}\text { Bantuan Siswa Miskin ( } \\
\text { BSM ) }\end{array}$ & $\begin{array}{l}\text { 1. Yes } \\
\text { 3. No }\end{array}$ & $\downarrow$ & $\begin{array}{l}\text { 1. } \amalg \text { Month / Year } \\
\text { 8. DK }\end{array}$ & $\begin{array}{l}\text { 1. Yes } \rightarrow \text { PAP8 } \\
\text { 3. No } \\
\text { 8. DK }\end{array}$ & 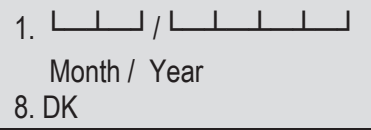 & $\begin{array}{l}\text { 1. } \sqcup \perp \text { \% } \\
\text { 8. DK }\end{array}$ & $\begin{array}{ll}\text { 1. } & \text { Yes } \\
\text { 3. } & \text { No }\end{array}$ & $\begin{array}{ll}\text { 1. } & \text { Yes } \\
\text { 3. } & \text { No }\end{array}$ \\
\hline 11 & $\begin{array}{l}\text { Pengurangan Pekerja } \\
\text { Anak - Program Keluarga } \\
\text { Harapan (PPA-PKH) }\end{array}$ & $\begin{array}{l}\text { 1. Yes } \\
\text { 3. No }\end{array}$ & $\downarrow$ & 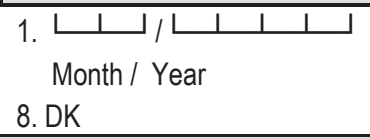 & $\begin{array}{l}\text { 1. Yes } \rightarrow \text { PAP8 } \\
\text { 3. No } \\
\text { 8. DK }\end{array}$ & 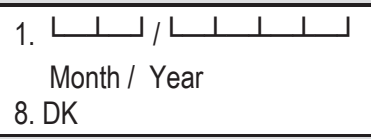 & & & \\
\hline 12 & $\begin{array}{l}\text { Jaminan Sosial } \\
\text { Penyandang cacat / } \\
\text { Jaminan Sosial Bagi } \\
\text { Penyandang Cacat Berat } \\
\text { (JSPACAJJSODK) }\end{array}$ & $\begin{array}{l}\text { 1. Yes } \\
\text { 3. No }\end{array}$ & $\downarrow$ & 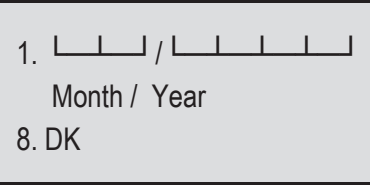 & $\begin{array}{l}\text { 1. Yes } \rightarrow \text { PAP8 } \\
\text { 3. No } \\
\text { 8. DK }\end{array}$ & 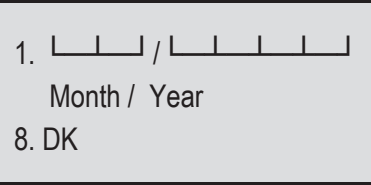 & & & \\
\hline 13 & $\begin{array}{l}\text { Jaminan Sosial Lanjut } \\
\text { Usia ( JSLU/ASLUT ) }\end{array}$ & $\begin{array}{l}\text { 1. Yes } \\
\text { 3. No }\end{array}$ & $\downarrow$ & $\begin{array}{l}\text { 1. } \amalg \text { Month / Year } \\
\text { 8. DK }\end{array}$ & $\begin{array}{l}\text { 1. Yes } \rightarrow \text { PAP8 } \\
\text { 3. No } \\
\text { 8. DK }\end{array}$ & 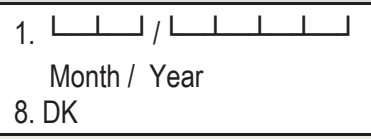 & & & \\
\hline 14 & $\begin{array}{l}\text { Kelompok Usaha } \\
\text { Bersamal/Usaha Ekonomi } \\
\text { Produktif ( KUBE/UEP ) }\end{array}$ & $\begin{array}{l}\text { 1. Yes } \\
\text { 3. No }\end{array}$ & $\downarrow$ & 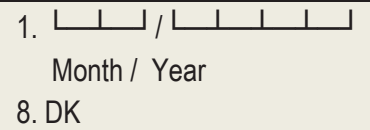 & $\begin{array}{l}\text { 1. Yes } \rightarrow \text { PAP8 } \\
\text { 3. No } \\
\text { 8. DK }\end{array}$ & 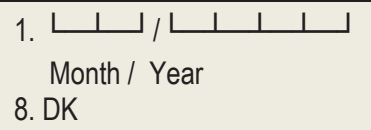 & & & \\
\hline 15 & $\begin{array}{l}\text { Program Perbaikan } \\
\text { Rumah (RTLH (Rumah } \\
\text { Tidak Layak Huni)/ Bedah } \\
\text { Rumah ) }\end{array}$ & $\begin{array}{l}\text { 1. Yes } \\
\text { 3. No }\end{array}$ & $\downarrow$ & $\begin{array}{l}\text { 1. } \sqcup \sqcup / \text { Month / Year } \\
\text { 8. DK }\end{array}$ & $\begin{array}{l}\text { 1. Yes } \rightarrow \text { PAP8 } \\
\text { 3. No } \\
\text { 8. DK }\end{array}$ & 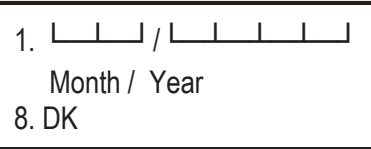 & & & \\
\hline 16 & $\begin{array}{l}\text { Program Kesejahteraan } \\
\text { Sosial Anak ( PKSA ) }\end{array}$ & $\begin{array}{l}\text { 1. Yes } \\
\text { 3. No }\end{array}$ & $\downarrow$ & 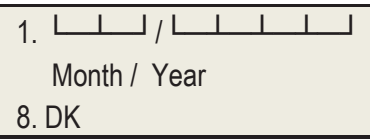 & $\begin{array}{l}\text { 1. Yes } \rightarrow \text { PAP8 } \\
\text { 3. No } \\
\text { 8. DK }\end{array}$ & $\begin{array}{l}\text { 1. } \begin{array}{l}\perp \\
\text { Month / Year } \\
\text { 8. DK }\end{array} \\
\end{array}$ & & & \\
\hline & $\begin{array}{l}\text { Kartu Perlindungan Sosial } \\
\text { (KPS ) }\end{array}$ & $\begin{array}{l}\text { 1. Yes } \\
\text { 3. No }\end{array}$ & $\downarrow$ & $\begin{array}{l}\text { 1. } \sqcup \sqcup / \text { Month / Year } \\
\text { 8. DK }\end{array}$ & $\begin{array}{l}\text { 1. Yes } \rightarrow \text { PAP8 } \\
\text { 3. No } \\
\text { 8. DK } \\
\end{array}$ & 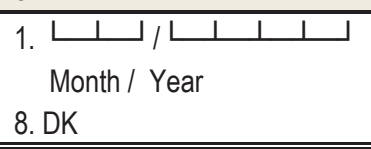 & & & \\
\hline
\end{tabular}




\begin{tabular}{|c|c|c|c|}
\hline PAP20a & $\begin{array}{l}\text { Was there Regional health insurance } \\
\text { program(jamkesda) in your kabupaten ? }\end{array}$ & \multicolumn{2}{|c|}{$\begin{array}{l}\text { No } \\
\text { Yes }\end{array}$} \\
\hline PAP20b & $\begin{array}{l}\text { Was there Regional health insurance } \\
\text { program ( jamkesda )in the last } 12 \text { months? }\end{array}$ & \multicolumn{2}{|c|}{$\begin{array}{ll}\text { No } & 3 \text { PPAP24a } \\
\text { Yes } & 1\end{array}$} \\
\hline PAP21 & $\begin{array}{l}\text { From which facilities is someone who has a } \\
\text { Regional health insurance program ( } \\
\text { jamkesda ) able to obtain services? } \\
\text { CIRCLE ALL THAT APPLY }\end{array}$ & \multicolumn{2}{|c|}{$\begin{array}{l}\text { A. Puskesmas } \\
\text { B. Puskesmas Pembantu } \\
\text { C. Village Midwife } \\
\text { D. Private Practice } \\
\text { E. Public Hospital } \\
\text { F. Private Hospital }\end{array}$} \\
\hline PAP24a & $\begin{array}{l}\text { Was there care and childbirth insurance } \\
\text { program ( Jampersal) at yours kabupaten ? }\end{array}$ & \multicolumn{2}{|c|}{ 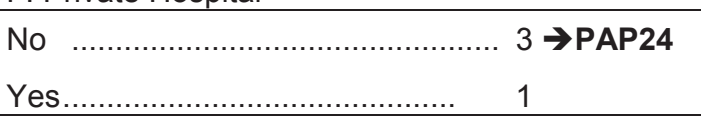 } \\
\hline PAP25a & $\begin{array}{l}\text { Was there care and childbirth insurance } \\
\text { program ( Jampersal) in the last } 12 \\
\text { months? }\end{array}$ & \multicolumn{2}{|c|}{$\begin{array}{ll}\text { No } & \text {. } \\
\text { Yes } & \end{array}$} \\
\hline PAP27a & $\begin{array}{l}\text { From which facilities is someone who has a } \\
\text { jampersal able to obtain services? } \\
\text { CIRCLE ALL THAT APPLY }\end{array}$ & \multicolumn{2}{|c|}{$\begin{array}{l}\text { A. Puskesmas } \\
\text { B. Puskesmas Pembantu } \\
\text { C. Village Midwife } \\
\text { D. Private Practice } \\
\text { E. Public Hospital } \\
\text { F. Private Hospital }\end{array}$} \\
\hline PAP24. & $\begin{array}{l}\text { Was there Jamkesmas program in the last } \\
12 \text { months? }\end{array}$ & \multicolumn{2}{|c|}{ No } \\
\hline PAP25 & $\begin{array}{l}\text { From which facilities is someone who has a } \\
\text { Jamkesmas able to obtain services? } \\
\text { CIRCLE ALL THAT APPLY }\end{array}$ & \multicolumn{2}{|c|}{$\begin{array}{l}\text { A. Puskesmas } \\
\text { B. Puskesmas Pembantu } \\
\text { C. Village Midwife } \\
\text { D. Private Practice } \\
\text { E. Public Hospital } \\
\text { F. Private Hospital } \\
\end{array}$} \\
\hline PAP28. & $\begin{array}{l}\text { Was there Raskin program in the last } 12 \\
\text { months? }\end{array}$ & \multicolumn{2}{|c|}{$\begin{array}{l}\text { No } \ldots \ldots \ldots \ldots \ldots \ldots \ldots \ldots \ldots \ldots \ldots . . .1 \\
\text { Yes }\end{array}$} \\
\hline PAP31. & $\begin{array}{l}\text { On average, in the last } 12 \text { months was the } \\
\text { percentage of households that received } \\
\text { RASKIN rice? }\end{array}$ & 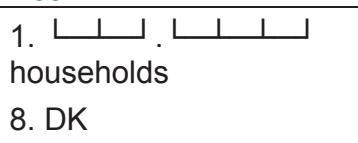 & 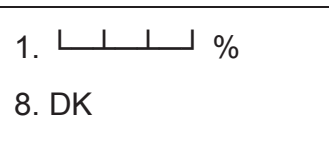 \\
\hline PAP32. & $\begin{array}{l}\text { When was the last time RASKIN rice was } \\
\text { distributed in the last } 12 \text { months? }\end{array}$ & \multicolumn{2}{|c|}{ 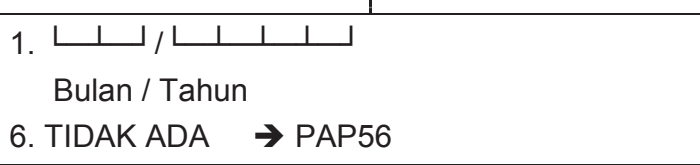 } \\
\hline PAP36. & $\begin{array}{l}\text { What was the average amount of rice } \\
\text { received by all RASKIN household } \\
\text { recipients? }\end{array}$ & \multicolumn{2}{|c|}{ 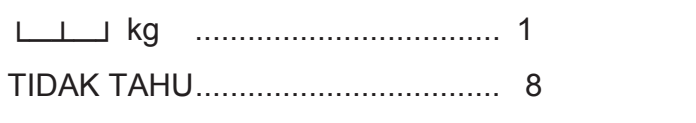 } \\
\hline
\end{tabular}

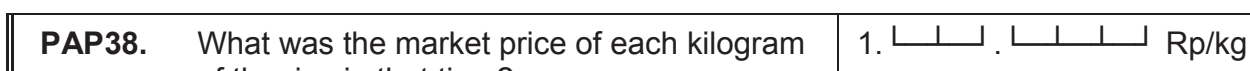
of the rice in that time?

8. DK

\begin{tabular}{|c|c|c|c|c|}
\hline \multirow[t]{3}{*}{ PAP56. } & \multirow[t]{3}{*}{$\begin{array}{l}\text { Was there ever BLSM program in this } \\
\text { village since } 2012 \text { ? }\end{array}$} & \multicolumn{3}{|c|}{$\begin{array}{l}\text { 3. No } \rightarrow \text { PAP66a } \\
\text { 1. Yes }\end{array}$} \\
\hline & & 2013 & 1. Ya, $\sqcup$ times & 3. No \\
\hline & & 2012 & 1. $\mathrm{Ya}, \sqcup$ times & 3. No \\
\hline PAP58. & $\begin{array}{l}\text { When was the last time household in this } \\
\text { village received the SLT funds / BLSM } \\
\text { funds? }\end{array}$ & \multicolumn{3}{|c|}{ 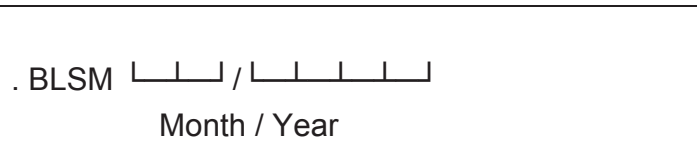 } \\
\hline PAP59. & $\begin{array}{l}\text { How many percent household in this village } \\
\text { received the SLT funds / BLSM funds? }\end{array}$ & \multicolumn{3}{|c|}{ BLSM $\left\llcorner\perp \_\_\right.$percent } \\
\hline PAP60. & $\begin{array}{l}\text { How many rupiah did each household at the } \\
\text { last time receive the SLT funds / BLSM } \\
\text { funds? }\end{array}$ & BLSM Rp & $\perp .\llcorner\perp \perp$ & \\
\hline
\end{tabular}

\begin{tabular}{|c|c|c|}
\hline PAP66a & $\begin{array}{l}\text { Was there KPS ( Social Security Card ) } \\
\text { program in this village? }\end{array}$ & $\begin{array}{l}\text { Tidak } \\
\text { Ya }\end{array}$ \\
\hline PAP67a & When was the first time the program begin? & 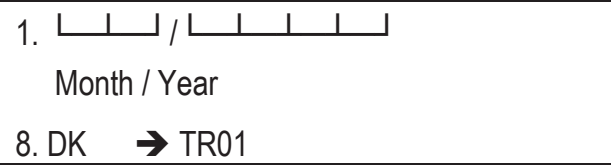 \\
\hline PAP68a & $\begin{array}{l}\text { What assistance programs are the social } \\
\text { security card used? }\end{array}$ & $\begin{array}{l}\text { A. RASKIN } \\
\text { B. BLSM } \\
\text { C. BSM ( Bantuan Siswa Miskin ) } \\
\text { D. PKH ( Program Keluarga Harapan ) } \\
\text { V. Others } \\
\end{array}$ \\
\hline PAP69a & $\begin{array}{l}\text { How many percent household in this village } \\
\text { received the KPS program? }\end{array}$ & $\begin{array}{l}\text { 1. } \downarrow \perp \perp \_ \text {percent } \\
\text { 8. DK }\end{array}$ \\
\hline
\end{tabular}


SECTION TR ( CONFLICT AND TRUST)

\begin{tabular}{|c|c|c|c|c|c|c|c|c|c|c|c|c|c|c|c|c|c|c|c|}
\hline & \multicolumn{3}{|c|}{ TR01. } & TR02. & TR02a & \multicolumn{11}{|c|}{ TR03. } & \multicolumn{3}{|c|}{ TR04. } \\
\hline Type of conflicts & $\begin{array}{l}\text { Has }[\ldots] \\
\text { village/to }\end{array}$ & $\begin{array}{l}\text { occured in } t \\
\text { wnshop sin }\end{array}$ & $\begin{array}{l}\text { this } \\
\text { ice } 2007 ?\end{array}$ & $\begin{array}{l}\text { Was there violence in } \\
\text { the }[\ldots . .] \text { ? }\end{array}$ & $\begin{array}{l}\text { Was there anyone dead } \\
\text { in (...) }\end{array}$ & Who us & we & nvo & $d$ in & e effort & to reso & Ive t & he con & ict? & & & $\begin{array}{l}\text { Since } 2 \\
\text { of this } \\
\text { increas } \\
\text { decrea }\end{array}$ & $\begin{array}{l}\text { has } \\
\text { of ce } \\
\text { he }\end{array}$ & $\begin{array}{l}\text { umber } \\
\text { been } \\
\text { or }\end{array}$ \\
\hline $\begin{array}{l}\text { a. Conflicts on land/building } \\
\text { between citizen and } \\
\text { government }\end{array}$ & 3. No & 8. DK $_{\downarrow}$ & 1. Yes $\rightarrow$ & $\begin{array}{l}\text { 1. Yes } \\
\text { 3. No }\end{array}$ & $\begin{array}{l}\text { 1. Yes } \\
\text { 3. No }\end{array}$ & Y & A & B & C & $\begin{array}{c}\mathrm{D} \\
\mathrm{K}\end{array}$ & $\begin{array}{l}\mathrm{E} \\
\mathrm{V}\end{array}$ & $\mathrm{F}$ & G & $\mathrm{H}$ & I & J & 1 & 2 & 3 \\
\hline $\begin{array}{l}\text { b. Conflicts on land building } \\
\text { between citizens }\end{array}$ & 3. No & ${ }^{8 .} \mathrm{DK} \downarrow$ & 1. Yes $\rightarrow$ & $\begin{array}{l}\text { 1. Yes } \\
\text { 3. No }\end{array}$ & $\begin{array}{l}\text { 1. Yes } \\
\text { 3. No }\end{array}$ & $Y$ & A & B & $\mathrm{C}$ & $\mathrm{D}$ & $\mathrm{E}$ & $\mathrm{F}$ & G & $\mathrm{H}$ & I & J & 1 & 2 & 3 \\
\hline $\begin{array}{l}\text { c. Conflicts arising from } \\
\text { abuses of power/authority }\end{array}$ & 3. No & 8. DK $\downarrow$ & 1. Yes $\rightarrow$ & $\begin{array}{l}\text { 1. Yes } \\
\text { 3. No }\end{array}$ & $\begin{array}{l}\text { 1. Yes } \\
\text { 3. No }\end{array}$ & $Y$ & A & B & C & $\mathrm{D}$ & $\mathrm{E}$ & $\mathrm{F}$ & G & $\mathrm{H}$ & I & $\mathrm{J}$ & 1 & 2 & 3 \\
\hline $\begin{array}{l}\text { d. Conflicts related to } \\
\text { election of public officials } \\
\text { or general election }\end{array}$ & 3. No & 8. DK $\downarrow$ & 1. Yes $\rightarrow$ & $\begin{array}{l}\text { 1. Yes } \\
\text { 3. No }\end{array}$ & $\begin{array}{l}\text { 1. Yes } \\
\text { 3. No }\end{array}$ & $Y$ & A & B & C & ${ }^{\mathrm{D}} \mathrm{K}$ & $\mathrm{E}_{\mathrm{V}}$ & $\mathrm{F}$ & G & $\mathrm{H}$ & I & J & 1 & 2 & 3 \\
\hline $\begin{array}{l}\text { e. Conflicts between } \\
\text { members of different } \\
\text { ethnic groups }\end{array}$ & 3. No & 8. DK $\downarrow$ & 1. Yes $\rightarrow$ & $\begin{array}{l}\text { 1. Yes } \\
\text { 3. No }\end{array}$ & $\begin{array}{l}\text { 1. Yes } \\
\text { 3. No }\end{array}$ & Y & A & B & C & $\mathrm{D}$ & $\mathrm{E}$ & $\mathrm{F}$ & G & $\mathrm{H}$ & I & $\mathrm{J}$ & 1 & 2 & 3 \\
\hline $\begin{array}{l}\text { f. Conflicts between } \\
\text { members of different } \\
\text { religions/faiths }\end{array}$ & $\stackrel{3 .}{\downarrow}^{\text {No }}$ & $\downarrow^{8 . \mathrm{DK}}$ & 1. Yes $\rightarrow$ & $\begin{array}{l}\text { 1. Yes } \\
\text { 3. No }\end{array}$ & $\begin{array}{l}\text { 1. Yes } \\
\text { 3. No }\end{array}$ & Y & $A$ & B & C & ${ }^{\mathrm{D}}$ & $\mathrm{E}$ & $\mathrm{F}$ & G & $\mathrm{H}$ & I & $\mathrm{J}$ & 1 & 2 & 3 \\
\hline $\begin{array}{l}\text { g. Conflict between community } \\
\text { and private } \\
\text { business/corporation }\end{array}$ & $\begin{array}{l}\text { 3. No } \\
\downarrow \\
\text { TR05 }\end{array}$ & $\begin{array}{c}\text { 8. DK } \\
\text { TR05 } \\
\end{array}$ & 1. Yes $\rightarrow$ & $\begin{array}{l}\text { 1. Yes } \\
\text { 3. No }\end{array}$ & $\begin{array}{l}\text { 1. Yes } \\
\text { 3. No }\end{array}$ & Y & $A$ & B & C & ${ }^{D}$ & $\mathrm{E}_{\mathrm{V}}$ & $\mathrm{F}$ & G & $\mathrm{H}$ & I & $\mathrm{J}$ & 1 & 2 & 3 \\
\hline
\end{tabular}

Codes for TR03

A. Law expert

B. Village /Adat/Religious leaders

C. Village officials

G. Lawyers/paralegal

H. Subdistrict officials

j. District officials

Codes for TR04:

D. NGO

K. Judicial Court

1. Increasing

F. Family member or Friend

K. Judicial Court
Y. DON'T KNOW

Other

2. Same

3. Decreasing

V. Other


TRUST

\begin{tabular}{|c|c|c|}
\hline TR05. & How safe do you consider this village? & 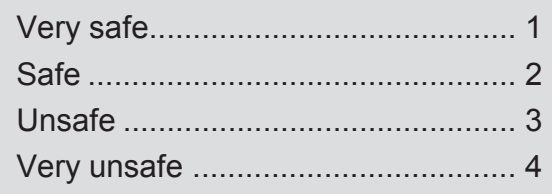 \\
\hline TR07. & $\begin{array}{l}\text { In most part of the village, is it safe to } \\
\text { walk alone at night? }\end{array}$ & $\begin{array}{l}\text { Very safe } \\
\text { Safe } \\
\text { Unsafe } \\
\text { Very unsafe }\end{array}$ \\
\hline TR07a & $\begin{array}{l}\text { In most part of the village, is it safe for a } \\
\text { woman to walk alone at night? }\end{array}$ & $\begin{array}{l}\text { Very safe } \\
\text { Safe } \\
\text { Unsafe } \\
\text { Very unsafe }\end{array}$ \\
\hline
\end{tabular}




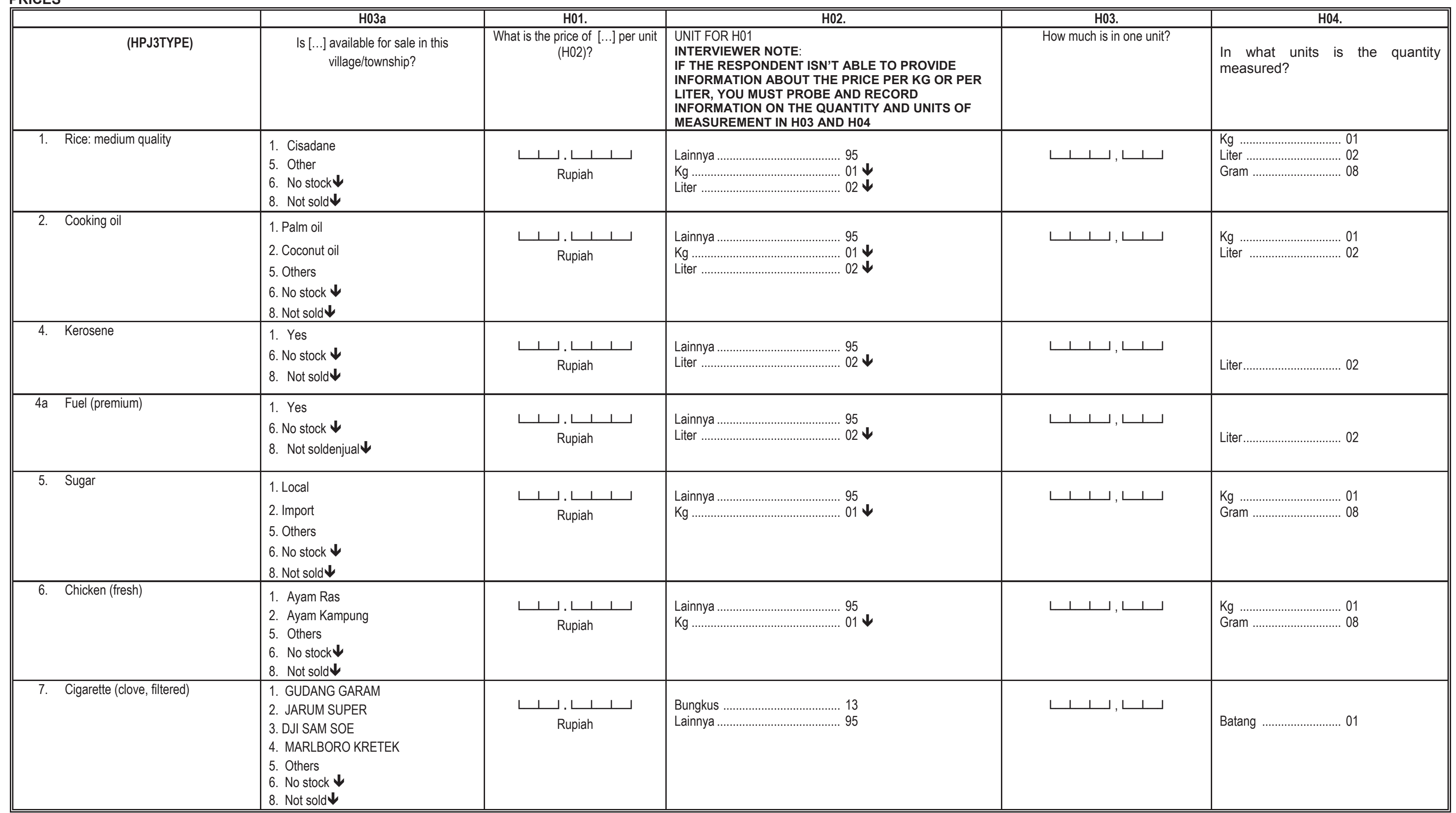




\begin{tabular}{|c|c|c|}
\hline & QUESTION NUMBERS & NOTES \\
\hline CP1. Questions wit & & \\
\hline CP2. Questions the & & \\
\hline Questions an & & \\
\hline CP4. Other notes & & \\
\hline
\end{tabular}

\begin{tabular}{|c|c|c|c|c|c|c|c|}
\hline \multirow{2}{*}{$\begin{array}{l}\text { LANGMAIN (CK1). } \\
\text { LANGOTHR (CK2). }\end{array}$} & \multicolumn{2}{|l|}{ Interview was entirely/mostly conducted in what language? } & \multicolumn{5}{|l|}{$\downarrow \sqcup$ - } \\
\hline & Other languaged used (if any): & & \multicolumn{5}{|l|}{ 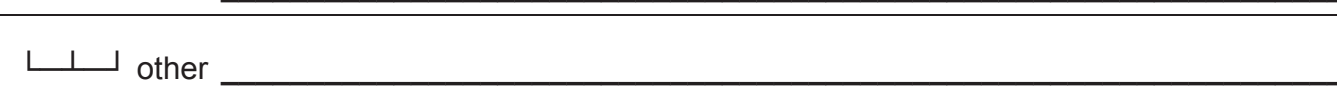 } \\
\hline \multicolumn{8}{|l|}{ LANGUAGE CODE: } \\
\hline 00. Bahasa Indonesia & 04. Batak & Sasak & 12. Makassar & 16. & Toraja & & Lampung \\
\hline 01. Java & 05. Bugis & 09. Minang & 13. Nias & 17. & Lahat & 95. & Other, \\
\hline 02. Sunda & 06. Cina & 10. Banjar & 14. Palembang & 18. & Other South Sumatera & 96. & NA \\
\hline Bali & 07. Madura & 11. Bima & 15. Sumbawa & 19. & Betawi & & \\
\hline
\end{tabular}

\begin{tabular}{|c|c|c|c|c|}
\hline \multirow{2}{*}{\begin{tabular}{l}
\multicolumn{1}{c}{$\begin{array}{c}\text { C1. RESULT OF } \\
\text { INTERVIES }\end{array}$} \\
1. Completed $\rightarrow$ C4 \\
2.Partially completed \\
3. Not completed
\end{tabular}} & \multirow{2}{*}{$\begin{array}{l}\text { C2. REASON FOR ANSWER “3"/"“2” IN C1 } \\
\text { 1. Respondent was travelling } \\
\text { 2. Respondent was too busy } \\
\text { 3. Responden refused } \\
\text { 5. Other: }\end{array}$} & \multicolumn{2}{|c|}{ C4. MONITORING BY SUPERVISOR } & C5. CLOSEST EA \\
\hline & & $\begin{array}{l}\text { a. Observed (sup_obs } \\
\text { b. Checked (sup_edit) } \\
\text { c. Verified (sup_verif) }\end{array}$ & $\begin{array}{c}\text { Yes } \\
1 \\
1 \\
1\end{array}$ & 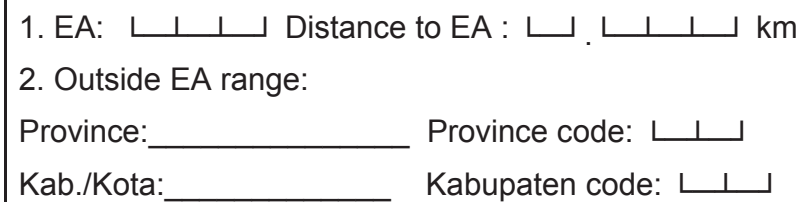 \\
\hline
\end{tabular}


COMFAS 2014 